\title{
Akteursorientierte multimodale Straßenverkehrssimulation
}

\author{
Dissertation \\ zur Erlangung des Doktorgrades \\ der Naturwissenschaften
}

vorgelegt beim Fachbereich Informatik und Mathematik

der Johann Wolfgang Goethe - Universität

in Frankfurt am Main

\author{
von \\ Jörg Dallmeyer \\ aus Hanau
}

Frankfurt (2013)

(D 30) 
vom Fachbereich Informatik und Mathematik der Johann Wolfgang Goethe-Universität als Dissertation angenommen

Dekan: $\quad$ Prof. Dr. Thorsten Theobald

Gutachter: $\quad$ Prof. Dr. Ingo J. Timm

Prof. Dr. Lars Hedrich

Datum der Disputation: 09.07.2013 


\section{Danksagung}

Während meiner Zeit als Doktorand an der Professur für Wirtschaftsinformatik und Simulation der Goethe-Universität Frankfurt am Main wurde ich von verschiedenen Personen und Institutionen unterstützt.

An erster Stelle möchte ich mich bei meinem Doktorvater Prof. Dr. Ingo Timm für seine fortwährende Unterstützung auch über räumliche Distanzen hinweg bedanken. Seine Ratschläge haben diese Arbeit häufig gelenkt und abgerundet.

Mein Dank geht ebenso an Herrn Prof. Dr. Lars Hedrich, der seit meiner Zeit als studentische Hilfskraft in seiner Professur für Entwurfsmethodik ein Ansprechpartner für mich geblieben ist.

Mein Dank geht an die Stiftung Polytechnische Gesellschaft Frankfurt am Main (SPTG), die mir ein MainCampus doctus Stipendium gewährte und mir somit eine finanzielle und ideelle Förderung zukommen ließ. Besonders hervorheben möchte ich den persönlichen Einsatz von Herrn Dr. Wolfgang Eimer und Herrn Tobias Ullrich.

Ebenso möchte ich mich bei der Goethe Graduate Academy GRADE (vormals Otto Stern School for Integrated Doctoral Education (OSS)) bedanken, die mir ein Stipendium zur Anschubfinanzierung als Promotionsstudent gewährte und mir die Teilnahme an verschiedenen Seminaren ermöglichte.

Herzlichen Dank an meine aktuellen und ehemaligen Kollegen Dr. habil. Andreas Lattner, Prof. Dr. René Schumann, Dimitrios Paraskevopoulos, Tjorben Bogon, Yann Lorion, Pascal Katzenbach und Marion Terrell für zahlreiche Diskussionen und Unterstützungen. Besonders hervorheben möchte ich hierbei Dr. habil. Andreas Lattner, der stets ein offenes Ohr hatte und sich Zeit für Beratungen und Kooperationen nahm.

Durch eine Kooperation mit Prof. Dr. Guido Cervone (George Mason University) 
entstanden die Ergebnisse der letzten Fallstudie dieser Arbeit. Diese Kooperation wurde durch fruchtbare Diskussionen begleitet. Vielen Dank hierfür.

Ich möchte Herrn Carsten Taubert danken, dessen Masterarbeit ich betreuen konnte. Seine Arbeit hat zur Verbesserung des entwickelten Systems beigetragen.

Im Rahmen dieser Arbeit konnte ich Daten verwenden, die mir vom Hessischen Landesamt für Bodenmanagement und Geoinformation und von Hessen Mobil Straßen- und Verkehrsmanagement (vormals Hessische Straßen- und Verkehrsverwaltung HSVV) zur Verfügung gestellt wurden. Vielen Dank für diese freundliche Unterstützung. Gesondert hervorheben möchte ich den regen Austausch mit Frau Dagmar Holst von Hessen Mobil.

Die Vereinigung von Freunden und Förderern der Goethe-Universität bezuschusste mehrfach Reisen zu Tagungen und ermöglichte somit den wissenschaftlichen Austausch über die Grenzen der Bundesrepublik hinweg. Vielen Dank dafür. Aus gleichem Grunde geht mein Dank an die Hermann-Willkomm-Stiftung.

Ein herzliches Dankeschön möchte ich Herrn Prof. Dr. Jürgen Bereiter-Hahn aussprechen, der während meiner Zeit als Stipendiat der SPTG eine Mentorentätigkeit für mich übernahm.

Seit Beginn des Studiums an der Goethe-Universität bin ich mit Frau Claudia Stockhausen und Herrn Julius von Rosen befreundet. Ich denke, wir haben uns gegenseitig motiviert und unterstützt. Danke.

Meine Eltern - Heike und Uwe Dallmeyer - haben mir diesen Weg der Ausbildung ermöglicht. Vielen Dank für die fortwährende Geduld und Unterstützung jedweder Art.

Besonders bedanken möchte ich mich bei meiner Frau - Ina Dallmeyer - die mich in den vergangenen Jahren stets unterstützte und mir die nötige Motivation gab. Ohne sie wäre diese Arbeit mit Sicherheit nicht entstanden. Vielen Dank. 


\section{Kurzzusammenfassung}

Die vorliegende Dissertation behandelt die Entwicklung eines Verkehrssimulationssystems, welches vollautomatisch aus Landkarten Simulationsgraphen erstellen kann. Der Fokus liegt bei urbanen Simulationsstudien in beliebigen Gemeinden und Städten. Das zweite fundamentale Standbein dieser Arbeit ist daher die Konstruktion von Verkehrsmodellen, die die wichtigsten Verkehrsteilnehmertypen im urbanen Bereich abbilden. Es wurden Modelle für Autos, Fahrräder und Fußgänger entwickelt.

Die Betrachtung des Stands der Forschung in diesem Bereich hat ergeben, dass die Verknüpfung von automatischer Grapherstellung und Modellen, die die Wechselwirkungen der verschiedenen Verkehrsteilnehmertypen abbilden, von keinem vorhandenen System geleistet wird. Es gibt grundlegend zwei Gruppen von Verkehrssimulationssystemen. Zum Einen existieren Systeme, die hohe Genauigkeiten an Simulationsergebnissen erzielen und dafür exakte (teil-)manuelle Modellierung der Gegebenheiten im zu simulierenden Bereich benötigen. Es werden in diesem Bereich meist Verkehrsmodelle simuliert, die die Verhaltensweisen der Verkehrsteilnehmer sehr gut abbilden und hierfür einen hohen Berechnungsaufwand benötigen. Auf der anderen Seiten existieren Simulationssysteme, die Straßengraphen automatisch erstellen können, darauf jedoch sehr vereinfachte Verkehrsmodelle simulieren. Es werden meist nur Autobewegungen simuliert. Der Nutzen dieser Herangehensweise ist die Möglichkeit, sehr große Szenarien simulieren zu können.

Im Rahmen dieser Arbeit wird ein System mit Eigenschaften beider grundlegenden Ansätze entwickelt, um multimodalen innerstädtischen Verkehr auf Basis automatisch erstellter Straßengraphen simulieren zu können. Die Entwicklung eines neuen Verkehrssimulationssystems erschien notwendig, da sich zum Zeitpunkt der Literaturbetrachtung kein anderes vorhandenes System für die Nutzung zur Erfüllung der genannten Zielstellung eignete. Das im Rahmen dieser Arbeit entwickelte System heißt MAINSIM! (MultimodAle INnerstädtische VerkehrsSIMulation).

${ }^{1}$ http://www.mainsim.de 
Die Simulationsgraphen werden aus Kartenmaterial von OpenStreetMap extrahiert. Kartenmaterial wird zuerst in verschiedene logische Layer separiert und anschließend zur Bestimmung eines Graphen des Straßennetzes genutzt. Eine Gruppe von Analyseschritten behebt Ungenauigkeiten im Kartenmaterial und ergänzt Informationen, die während der Simulation benötigt werden (z.B. die Verbindungsrichtung zwischen zwei Straßen). Das System verwendet Geoinformationssystemkomponenten zur Verarbeitung der Geodaten. Dies birgt den Vorteil der einfachen Erweiterbarkeit um zusätzliche Datenquellen.

Die Verkehrssimulation verwendet mikroskopische Verhaltensmodelle. Jeder einzelne Verkehrsteilnehmer wird somit simuliert. Das Modell für Autos basiert auf dem in der Verkehrsforschung weit genutzten Nagel-Schreckenberg-Modell. Es verfügt jedoch über zahlreiche Modifikationen und Erweiterungen, um das Modell auch abseits von Autobahnen nutzen zu können und weitere Verhaltensweisen zu modellieren. Das Fahrradmodell entsteht durch geeignete Parametrisierung aus dem Automodell. Zur Entwicklung des Fußgängermodells wurde Literatur über das Verhalten von Fußgängern diskutiert, um daraus geeignete Eigenschaften (z.B. Geschwindigkeiten und Straßenüberquerungsverhaltensmuster) abzuleiten. MAINSIM ermöglicht folglich die Betrachtung des Verkehrsgeschehens auch aus der Sicht der Gruppe der Fußgänger oder Fahrradfahrer und kann deren Auswirkungen auf den Straßenverkehr einer ganzen Stadt bestimmen.

Das Automodell wurde auf Autobahnszenarien und innerstädtischen Straßengraphen evaluiert. Es konnte die gut verstandenen Zusammenhänge zwischen Verkehrsdichte, -fluss und -geschwindigkeit reproduzieren. Zur Evaluierung von Fahrradmodellen liegen nach dem besten Wissen des Autors keine Studien vor. Daher wurden an dieser Stelle der Einfluss der Fahrradfahrer auf den Straßenverkehr und die von Fahrrädern gefahrenen Geschwindigkeiten untersucht. Das Fußgängermodell konnte die aus der Literaturbetrachtung ermittelten Verhaltensweisen abbilden.

Nachdem die wichtigsten Komponenten von MAINSIM untersucht wurden, begannen Fallstudien, die verschiedene Gebiete abdecken. Die wichtigsten Ergebnisse aus diesem Teil der Arbeit sind:

- Es ist möglich, mit Hilfe maschineller Lernverfahren Staus innerhalb Frankfurts vorherzusagen.

- Nonkonformismus bezüglich der Verkehrsregeln kann je nach Verhalten den Verkehrsfluss empfindlich beeinflussen, kann aber auch ohne Effekt bleiben. 
- Mit Hilfe von Kommunikationstechniken könnte in der Zukunft die Routenplanung von Autos verbessert werden. Ein Verfahren auf Basis von Pheromonspuren wurde im Rahmen dieser Arbeit untersucht.

- MAINSIM eignet sich zur Simulation großer Szenarien. In der letzten Fallstudie dieser Arbeit wurde der Autoverkehr eines Simulationsgebietes um Frankfurt am Main herum mit ca. 1,6 Mio. Trips pro Tag simuliert. Da MAINSIM über ein Kraftstoffverbrauchs- und $\mathrm{CO}_{2}$-Emissionsmodell verfügt, konnten die $\mathrm{CO}_{2}$-Emissionen innerhalb von Frankfurt ermittelt werden. Eine angekoppelte Simulation des Wetters mit Hilfe einer atmosphärischen Simulation zeigte, wie sich die Gase innerhalb Frankfurts verteilen.

Für den professionellen Einsatz in der Verkehrsforschung muss das entwickelte Simulationssystem um eine Methode zur Kalibrierung auf Sensordaten im Simulationsgebiet erweitert werden. Die vorhandenen Ampelschaltungen bilden nicht reale Ampeln ab. Eine Erweiterung des Systems um die automatische Integrierung maschinell lesbarer Schaltpläne von Ampeln im Bereich des Simulationsgebietes würde die Ergebnisgüte weiter erhöhen.

MAINSIM hat mehrere Anwendungsgebiete. Es können sehr schnell Simulationsgebiete modelliert werden. Daher bietet sich die Nutzung für Vorabstudien an. Wenn große Szenarien simuliert werden müssen, um z.B. die Verteilung der $\mathrm{CO}_{2}{ }^{-}$ Emissionen innerhalb einer Stadt zu ermitteln, kann MAINSIM genutzt werden. Es hat sich im Rahmen dieser Arbeit gezeigt, dass Fahrräder und Fußgänger einen Effekt auf die Mengen des Kraftstoffverbrauchs von Autos haben können. Es sollte bei derartigen Szenarien folglich ein Simulationssysytem genutzt werden, welches die relevanten Verkehrsteilnehmertypen abbilden kann. Zur Untersuchung weiterer wissenschaftlicher Fragestellungen kann MAINSIM beliebig erweitert werden. 



\section{Inhaltsverzeichnis}

Danksagung iii

Kurzzusammenfassung v v v v v

Inhaltsverzeichnis xii

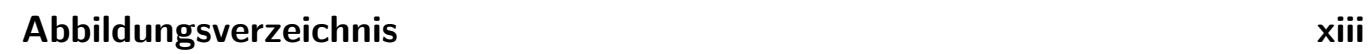

Tabellenverzeichnis xvii

Algorithmenverzeichnis $\quad$ xix

\begin{tabular}{|ll}
\hline Einleitung und Grundlagen & 1
\end{tabular}

1 Einleitung 3

1.1 Motivierendes Beispiel . . . . . . . . . . . . . . . . . . . . 4

1.2 Problemfelder . . . . . . . . . . . . . . . . . . . . . 5

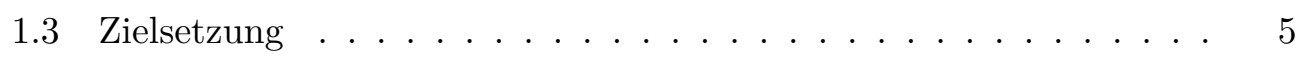

1.4 Anforderungsanalyse $\ldots \ldots \ldots \ldots \ldots$

1.5 Beitrag zum Stand der Forschung . . . . . . . . . . . . . . 8

$1.6 \quad$ Gliederung der Arbeit $\ldots \ldots \ldots \ldots$

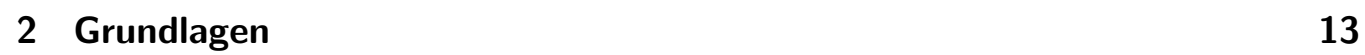

2.1 Straßenkarten und Geodaten . . . . . . . . . . . . . . . . . . . . . . . . . 13

$2.2 \quad$ Verkehrsforschungsgrundlagen . . . . . . . . . . . . . . . . . . . . . . . . . . . . . .

2.3 Simulation . . . . . . . . . . . . . . . . . . . . . . 16

2.4 Verkehrssimulation . . . . . . . . . . . . . . . . . . . . . . . . . . . . . . 19 


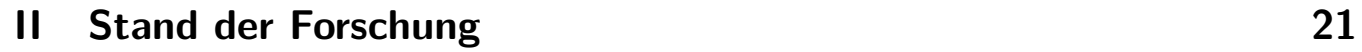

$\begin{array}{lll}3 & \text { Verkehrssimulationsmodelle } & 23\end{array}$

3.1 Modelle zur Fahrzeugmodellierung . . . . . . . . . . . . . . . . 23

3.2 Fahrradmodelle . . . . . . . . . . . . . . . . . . . . . . . 40

3.3 Fußgängermodelle $\ldots \ldots \ldots \ldots$. . . . . . . . . . . . . . . 42

3.4 Detailmodellierung . . . . . . . . . . . . . . . . . . . . . . . . . . . . 49

$3.5 \quad$ Diskussion . . . . . . . . . . . . . . . . . . . . . . . . 54

4 Verkehrssimulationssysteme $\quad \mathbf{5 5}$

4.1 Einordnung in den wissenschaftlichen Kontext. . . . . . . . . . . . 55

4.2 Corsim . . . . . . . . . . . . . . . . . . . . . . . 57

4.3 Transims $\ldots \ldots \ldots \ldots \ldots \ldots$

4.4 Matsim . . . . . . . . . . . . . . . . . . . . 62

$4.5 \quad \operatorname{vissim} \ldots \ldots \ldots \ldots$

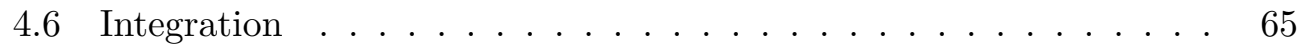

4.7 Hutsim $\ldots \ldots \ldots \ldots \ldots \ldots$

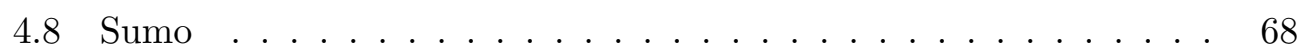

4.9 Weitere Simulationssysteme $\ldots \ldots \ldots$

4.10 Bewertung . . . . . . . . . . . . . . . . . . 71

\begin{tabular}{lll}
\hline III Konzept & 75
\end{tabular}

5 Akteursorientierte multimodale Straßenverkehrssimulation 77

5.1 Architektur . . . . . . . . . . . . . . . . . . 78

5.2 Router . . . . . . . . . . . . . . . . . . . 80

5.3 verkehrsmodell $\ldots \ldots \ldots \ldots \ldots$. . . . . . . . . . . . . 81

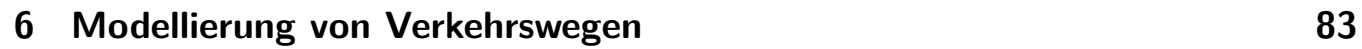

6.1 OpenStreetMap als Datenquelle . . . . . . . . . . . . . . . . . . 84

6.2 Ausschnittbestimmung aus OpenStreetMap . . . . . . . . . . . . 88

$6.3 \quad$ Filterung relevanter Informationen und Aufteilung in Layer . . . . 90

6.4 Generierung eines ExtendedGraph . . . . . . . . . . . . . . . . . 91

6.5 Graphanpassungen für urbane Szenarien . . . . . . . . . . . . . . . 94

\begin{tabular}{lll}
\hline 7 & Modellierung multimodalen Verkehrs & 97
\end{tabular}

7.1 Raumkontinuierliche Modellierung des Automobilverkehrs . . . . . 97

7.2 Modellierung des Fahrradverkehrs . . . . . . . . . . . . . . . 110

7.3 Bimodulares Fußgängermodell . . . . . . . . . . . . . . . . . . . . . 111

7.4 Vorfahrtsregeln . . . . . . . . . . . . . . . . . . . . . . . . . 119

7.5 Ampelschaltungen $\ldots \ldots \ldots \ldots$. . . . . . . . . . . 120 
7.6 Routing . . . . . . . . . . . . . . . . . . . . . . 121

IV Prototypische Implementierung und Modellevaluierung 127

\begin{tabular}{llr}
\hline & Prototypische Implementierung & 129
\end{tabular}

8.1 Designentscheidungen und Architektur . . . . . . . . . . . . . . . . 129

8.2 Ablauf einer Simulationsstudie . . . . . . . . . . . . . . . . . 131

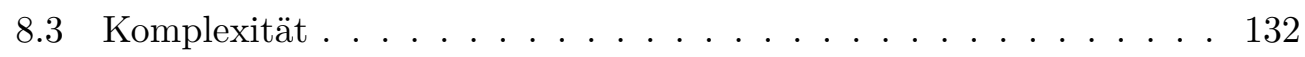

$8.4 \quad$ Skalierbarkeit . . . . . . . . . . . . . . . . . . . . . . . . . . 134

$\begin{array}{llr}9 & \text { Evaluierung der Verkehrsmodelle } & 139\end{array}$

9.1 Modellkalibrierung . . . . . . . . . . . . . . . . . . . . . . . . 139

9.2 Automodell . . . . . . . . . . . . . . . . . . . . . . . . . 141

9.3 Fahrrad . . . . . . . . . . . . . . . . . . . . . . . . . . 152

9.4 Fußgänger . . . . . . . . . . . . . . . . . . . . . . . 155

9.5 Zusammenfassung . . . . . . . . . . . . . . . 163

\begin{tabular}{lll}
\hline V Fallstudien & 165
\end{tabular}

10 Lernen in Verkehrsszenarien 167

10.1 Verwandte Arbeiten . . . . . . . . . . . . . . . . . . 167

10.2 Verkehrsbeeinflussung in Autobahnszenarien . . . . . . . . . . 168

10.3 Stauprognose in urbanen Szenarien . . . . . . . . . . . . . . . 176

10.4 Zusammenfassung und Ausblick . . . . . . . . . . . . . . . . 182

11 Approximation von Fußgängereffekten 183

11.1 Protokollierung von Fußgängereinflüssen . . . . . . . . . . . . . 183

11.2 Lernen von Wahrscheinlichkeitsverteilungen . . . . . . . . . . . . . 184

11.3 Anwendung . . . . . . . . . . . . . . . . . . . . . 186

11.4 Diskussion und Ausblick . . . . . . . . . . . . . . . . . . . 188

$\begin{array}{lr}12 \text { Nonkonformismus } & 189\end{array}$

12.1 Überholverbot für $\mathrm{Lkw}$. . . . . . . . . . . . . . . . . . . . . . . . 190

12.2 Aufbau der urbanen Studien . . . . . . . . . . . . . . . . . . . 192

12.3 Drängelnde Fahrradfahrer . . . . . . . . . . . . . . . . . . . 193

12.4 Aggressive Fußgänger $\ldots \ldots \ldots$

12.5 Zusammenfassung und Ausblick . . . . . . . . . . . . . . . . . 195

13 Ameisen-inspirierte Routingmethode $\quad 199$

13.1 Kontextuelle Einordnung. . . . . . . . . . . . . . . . . . . 200

13.2 Erweiterte Routingmethode . . . . . . . . . . . . . . . . . 201 
13.3 Parameterbestimmung . . . . . . . . . . . . . . . . . . . . . . 201

13.4 Vergleich mit anderen Verfahren . . . . . . . . . . . . . . . . 203

13.5 Diskussion und Ausblick . . . . . . . . . . . . . . . . . . . 207

14 Benzinverbrauch und Emissionen 209

14.1 Einleitung . . . . . . . . . . . . . . . . . . . . . 210

14.2 Verkehrssimulation und Benzinverbrauch . . . . . . . . . . . . . 210

14.3 Verbauchsmodell . . . . . . . . . . . . . . . . . . . . . . . 211

14.4 Vergleich zu NEFZ . . . . . . . . . . . . . . . . . . . . . . . . . . . 213

14.5 Autobahn-Plausibilitätsstudie . . . . . . . . . . . . . . . 215

14.6 Fallstudie im urbanen Verkehr. . . . . . . . . . . . . . . . . . 217

14.7 Zusammenfassung und Ausblick . . . . . . . . . . . . . . 220

15 Wind und Wetter 223

15.1 Relevante Literatur. . . . . . . . . . . . . . . . . . . . . . . 224

15.2 Nutzung von Quelle-Ziel-Informationen. . . . . . . . . . . . . 226

15.3 Herausforderungen des Szenarios . . . . . . . . . . . . . . . . 228

15.4 Anbindung an atmosphärische Simulation . . . . . . . . . . . . 229

15.5 Windverteilung im Messbereich . . . . . . . . . . . . . . 230

15.6 Ergebnisse . . . . . . . . . . . . . . . . . . . . . . . 232

15.7 Zusammenfassung und Ausblick . . . . . . . . . . . . . . 235

\begin{tabular}{|l|l|}
\hline VI Zusammenfassung und Ausblick & 239
\end{tabular}

16 Zusammenfassung und Ausblick 241

\begin{tabular}{|lr}
\hline VII Anhänge & 247
\end{tabular}

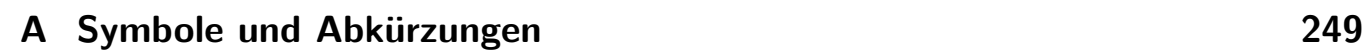

\begin{tabular}{ll}
\hline B Sicherheitsabstand & 251
\end{tabular}

C Fußgängerbewegung: Algorithmus auf ZA-Basis 253

\begin{tabular}{ll|}
\hline D KFZ-NEFZ-Daten & 257
\end{tabular}

\begin{tabular}{lr}
\hline Literaturverzeichnis & 259
\end{tabular}

\begin{tabular}{ll}
\hline Lebenslauf & 301
\end{tabular}

xii 


\section{Abbildungsverzeichnis}

$2.1 \quad$ Fundamentaldiagramm $\ldots \ldots \ldots \ldots \ldots \ldots$

3.1 Autonummerierung in Fahrzeugfolgemodellen . . . . . . . . . . 26

3.2 Beispielsequenz: Zellularautomat Rule 184 . . . . . . . . . . . . 32

3.3 Nagel-Schreckenberg-Modell (NSM) $\ldots \ldots \ldots$. . . . . . . . . 34

3.4 Beispielsequenz: NSM $\ldots \ldots \ldots \ldots$. . . . . . . . . . . . . . . . . . 35

3.5 NSM: Unfälle nach Boccara et al. . . . . . . . . . . . . . . . . 37

3.6 NSM: Fehlklassifikation nach Boccara et al. . . . . . . . . . . . . 37

3.7 Überholmanöver Auto/Fahrrad . . . . . . . . . . . . . . . . . . . . 41

3.8 Verhaltensmodellierungsebenen für Fußgänger . . . . . . . . . . . . 43

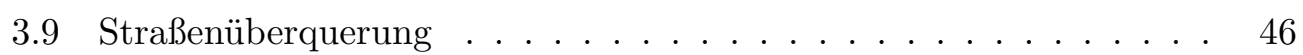

3.10 Abbiegevorgang . . . . . . . . . . . . . . . . . . . 50

5.1 Architektur . . . . . . . . . . . . . . . . . . . . . . 79

5.2 Architektur: Router . . . . . . . . . . . . . . . . 81

5.3 Architektur: Verkehrsmodell . . . . . . . . . . . . . . . . . 82

6.1 Vorgehen bei der Generierung eines Graphen aus OSM . . . . . . . 83

6.2 OSM-Entitäten . . . . . . . . . . . . . . . . . . . . . 85

6.3 OSM-Ausschnitt . . . . . . . . . . . . . . . . . . . . . . . 86

6.4 Attribute von EdgeInformation und NodeInformation . . . . . . . 92

6.5 Modellierung von Straßen mittels Kanten . . . . . . . . . . . . . . 93

$7.1 \mathrm{Zu}$ starkes Bremsen durch zu dichtes Auffahren . . . . . . . . . . . 98

7.2 Simulationssteuerung: Updatezyklus . . . . . . . . . . . . . . . . 100

7.3 Verteilung von AMax . . . . . . . . . . . . . . . . . . . . . . 102

7.4 Sicherheitsabstand . . . . . . . . . . . . . . . . . . . 103

7.5 Spurwechsel . . . . . . . . . . . . . . . . . . . . . . . . . . 106

7.6 Überholvorgang . . . . . . . . . . . . . . . . . . . . . . . . . . . 107

7.7 Positionierung von Fußgängerüberwegen . . . . . . . . . . . . . . 113

7.8 Spurbildung für Fußgängerüberwege $\ldots \ldots \ldots$. . . . . . . . . 114 
7.9 Nachbarschaft und Lücken auf Fußgängerüberwegen . . . . . . . . 116

7.10 Straßenüberquerung $\ldots \ldots \ldots \ldots \ldots$

7.11 Lookup table für NI-IDs $\ldots \ldots \ldots$. . . . . . . . . . . . 122

8.1 Architektur von MAINSIM . . . . . . . . . . . . . . . . . 130

8.2 Vollständiger Updatezyklus der Simulationssteuerung. . . . . . . . 133

8.3 Simulationsgeschwindigkeitsskalierung nach Verkehrsmengen. . . . 135

8.4 Menge an Interaktionen von Autos mit anderen Verkehrsteilnehmern. 136

8.5 Simulationsgeschwindigkeitsskalierung bei variierenden Graphgrößen. 138

$9.1 \quad$ Fundamentaldiagramm einspurig . . . . . . . . . . . . . . . . . 143

9.2 Dichte-Geschwindigkeit einspurig . . . . . . . . . . . . . . . . . 144

9.3 Fluss-Geschwindigkeit einspurig . . . . . . . . . . . . . . . . . . . . 145

9.4 Raum-Zeit-Diagramm einspurig . . . . . . . . . . . . . . . . . . 146

9.5 Fundamentaldiagramm zweispurig $\ldots \ldots \ldots \ldots \ldots$

$9.6 \quad$ Zusammenhang Geschwindigkeit, Dichte und Fluss zweispurig . . . 147

9.7 Raum-Zeit-Diagramm zweispurig . . . . . . . . . . . . . . . . . 147

9.8 Dichte-Geschwindigkeitsdiagramme im urbanen Bereich . . . . . . 148

9.9 Visualisierung der Stadt Hanau . . . . . . . . . . . . . . . . . . . . 149

9.10 Beschleunigungsverhalten von $\mathrm{Pkw}$. . . . . . . . . . . . . . 150

9.11 Vergleich der Straßennutzungshäufigkeiten . . . . . . . . . . . . . 151

9.12 Fahrradgeschwindigkeiten . . . . . . . . . . . . . . 153

9.13 Fahrradeinfluss auf den Straßenverkehr. . . . . . . . . . . . . 155

9.14 Verteilung der Fußgängergeschwindigkeiten in der Simulation . . . 156

9.15 Verteilung der Aggressivität der Fußgänger . . . . . . . . . . . . . . 157

9.16 Exemplarischer Verlauf des $A F$. . . . . . . . . . . . . . 158

9.17 Geschwindigkeit-Dichte-Diagramm für Fußgängerüberwege. . . . . . 159

9.18 Geschwindigkeit-Dichte-Diagramm für Fußgängerüberwege urban . 161

9.19 Fußgängereinfluss auf Straßenverkehr . . . . . . . . . . . . . . . 162

10.1 Ablauf des Lernvorgangs nach [Lattner et al., 2011] . . . . . . . . . 170

10.2 Generierung von Trainingsdaten nach [Lattner et al., 2011] . . . . 171

10.3 Ergebnisse: Statisches Szenario . . . . . . . . . . . . . . . 173

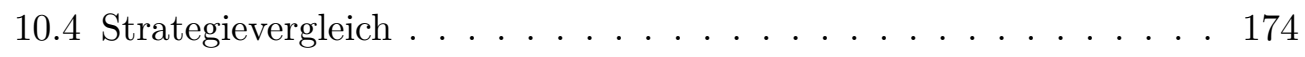

10.5 Kartenausschnitt . . . . . . . . . . . . . . . . . . . . . 174

10.6 Ergebnisse: Dynamisches Szenario … . . . . . . . . . 176

10.7 Kartenausschnitt im Osten Frankfurts . . . . . . . . . . . . . 177

10.8 Lernprozess . . . . . . . . . . . . . . . . . . . . . . . . . . . 179

10.9 Verteilung der Trainingsdaten $\ldots \ldots \ldots \ldots$. . . . . . . . 180

11.1 Wahrscheinlichkeitsverteilungsapproximation . . . . . . . . 185 
11.2 Histogrammbasierte Wahrscheinlichkeitsverteilungsapproximation. 186

11.3 Vergleich der Reisedauern von Autos . . . . . . . . . . . . . . . 187

12.1 Simulationsergebnisse: Autobahn . . . . . . . . . . . . . . . . . 191

12.2 Kartenausschnitt Trier . . . . . . . . . . . . . . . . . . . . . . . 192

12.3 Simulationsergebnisse: Trier: Fahrrad . . . . . . . . . . . . . . . . 193

12.4 Simulationsergebnisse: Trier: Fußgänger $\ldots$. . . . . . . . . . . 195

13.1 Stadt Erlensee: Gesamtstraßenlänge $142 \mathrm{~km}, 937 \mathrm{EIs}$ und $714 \mathrm{NIs}$. 202

13.2 Vergleich der Routingmethoden: Erlensee . . . . . . . . . . . . . 204

13.3 Vergleich der Straßennutzungshäufigkeiten . . . . . . . . . . . . . 205

13.4 Synthetischer Straßengraph . . . . . . . . . . . . . . . . 207

14.1 Kraftstoffverbrauch auf Autobahn bei steigender Verkehrsdichte. . 215

14.2 Kraftstoffverbrauch auf Autobahn: Einfluss von Lkw. . . . . . . . . 217

14.3 Kartenausschnitt der Stadt Hanau: Digitales Geländemodell . . . . 218

14.4 Vergleich: Experimenteller urbaner Kraftstoffverbrauch. . . . . . . 218

14.5 Kartenausschnitt der Stadt Hanau: Emissionskarte. . . . . . . . . . 220

15.1 Simulationsbereich Frankfurt und Umgebung . . . . . . . . . . . . 224

15.2 Skalierungsfaktoren von QZM . . . . . . . . . . . . . . . . . 228

15.3 Windsensordaten [Cervone et al., 2013]. . . . . . . . . . . . . . . 231

15.4 Emissionen durch Straßenverkehr . . . . . . . . . . . . . . . . . 233

$15.5 \mathrm{CO}_{2}$-Konturen: 1. März $2011(\log (\mathrm{kg}))$ [Cervone et al., 2013]. . . . 234

$15.6 \mathrm{CO}_{2}$-Konturen: 17. Oktober $2011(\log (\mathrm{kg}))$ Cervone et al., 2013]. . 235

15.7 Verteilung der $\mathrm{CO}_{2}$-Emissionen für verschiedene Tage. . . . . . . . 236

15.8 Verteilung der $\mathrm{CO}_{2}$-Emissionen für das Jahr 2011 . . . . . . . . . 237

B.1 Auswirkung des Sicherheitsabstands . . . . . . . . . . . . . 252

C.1 ZA-Fußgängermodell: Spurwechselkonflikte . . . . . . . . . . . . 255 



\section{Tabellenverzeichnis}

$3.1 \quad$ Modal Split nach Verkehrsaufkommen . . . . . . . . . . . . . . 24

3.2 Typische Modellparameter für IDM $\ldots \ldots \ldots \ldots$

3.3 Zustandsübergangsfunktion: Zellularautomat Rule 184 . . . . . . . 31

3.4 Mögliche Geschwindigkeiten im NSM . . . . . . . . . . . . . . . 33

3.5 Fußgängergeschwindigkeiten: Kreuzungen . . . . . . . . . . . . . . 44

3.6 Fußgängergeschwindigkeiten: Gehwege . . . . . . . . . . . . . . . 45

3.7 Abbiegevorgang: Lückengrößen . . . . . . . . . . . . . . . . . 51

$4.1 \quad$ Kontextuelle Einordnung betrachteter Verkehrssimulationssysteme 56

4.2 Vergleich der besprochenen Verkehrssimulationssysteme . . . . . . 72

6.1 Notation: Laufzeitberechnung für OSM-Ausschnittsbestimmung . . 88

$6.2 \quad$ OSM: Anzahl Elemente nach Typen $\ldots \ldots \ldots \ldots$

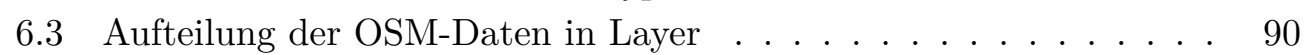

8.1 Kenngrößen der Simulationsgraphen für Skalierungstests..$\ldots 137$

$9.1 \quad$ Modellkalibrierungsebenen . . . . . . . . . . . . . . . . . . . . . 140

9.2 Fahrradeinfluss auf Straßenverkehr . . . . . . . . . . . . . . . 154

9.3 Fußgängereinfluss auf Straßenverkehr . . . . . . . . . . . . . . . . 161

9.4 Fußgängereinfluss auf Straßenverkehr: t-Tests . . . . . . . . . . . . 163

10.1 Wahrheitsmatrix in statischem Lernszenario . . . . . . . . . . . . 173

10.2 Wahrheitsmatrix in dynamischem Lernszenario . . . . . . . . . . 176

10.3 Urbanes Lernszenario: Einstellungen . . . . . . . . . . . . . . . . . 178

10.4 Wahrheitsmatrizen berechneter Klassifikatoren auf einem Testset. 181

11.1 Mittlere Reisedauern für Autos und Fahrräder. . . . . . . . . . . . 187

13.1 Vergleich mittlerer Reisedauern: Erlensee . . . . . . . . . . . . . 204

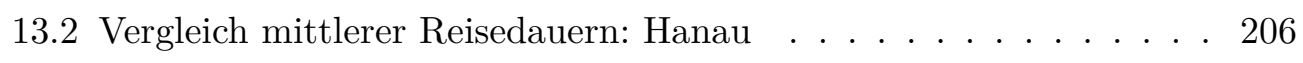

13.3 Vergleich mittlerer Reisedauern: Synthetischer Graph. . . . . . . . 207 
14.1 Ermittelte Parameter für das Treibstoffverbrauchsmodell. . . . . . 212

14.2 Wirkungsgrad und Emissionen nach Kraftstoffart. . . . . . . . . . 213

14.3 Vergleich mit NEFZ: Verwendete Fahrzeuge. . . . . . . . . . . . . . 214

14.4 Vergleich mit NEFZ: Simulationsergebnisse . . . . . . . . . . . . 215

D.1 Länge, Masse und NEFZ-Verbrauchsdaten typischer Fahrzeuge . . 258 


\section{Algorithmenverzeichnis}

3.1 Nagel-Schreckenberg-Modell . . . . . . . . . . . . . . . . . . . 33

$6.1 \quad$ Bestimmung eines Kartenausschnitts aus einer OSM-Datei . . . . . 88

7.1 Automodell: Raumkontinuierlicher Ansatz . . . . . . . . . . . . . . 100

7.2 Automodell: Berechnung des Sicherheitsabstands . . . . . . . . . . 103

7.3 Automodell: Trödelwahrscheinlichkeit . . . . . . . . . . . . . . . 104

$7.4 \quad$ Fußgängermodell: Bewegung auf EI . . . . . . . . . . . . . . . . . . 113

$7.5 \quad$ Fußgängermodell: update()-Methode der way-Objekte . . . . . . 115

C.1 ZA-Fußgängermodell: update() . . . . . . . . . . . . . . . 254 



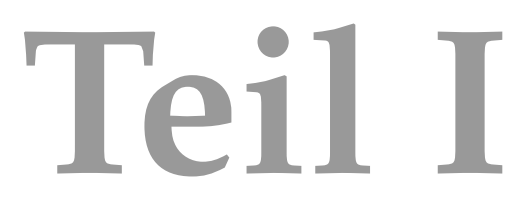

Einleitung und Grundlagen 



\section{Einleitung}

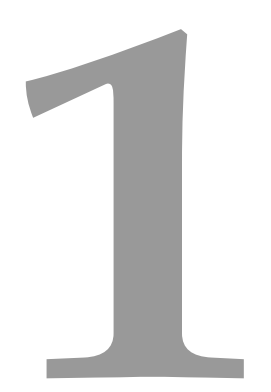

Mobilität ist für eine moderne Gesellschaft ein sehr wichtiges Thema. In Deutschland hat zwischen 1995 und 2007 der motorisierte Individualverkehr um 54,9 Mrd. Pkm (Personenkilometer) auf 885,4 Mrd. Pkm zugenommen. Die Zunahme zwischen 2005 und 2007 lag bei 9,7 Mrd. Pkm. Die transportierten Gütermengen auf deutschen Straßen stiegen im selben Zeitraum um 46,9 Mio. t auf 3.393,9 Mio. $t$ an Kolodziej, 2009. Diese Zahlen machen deutlich, dass eine effiziente Nutzung der Infrastruktur notwendig ist, da eine Erweiterung des Straßennetzes nicht in dieser Geschwindigkeit standhalten kann.

Die Modellbildung und Simulation ist im wissenschaftlichen Bereich ein bedeutender Zweig, da nicht jeder Sachverhalt analytisch zu erfassen ist. Dynamische Systeme können oftmals in Simulationsmodellen abgebildet und anschließend wissenschaftlich untersucht werden. Mit Hilfe eines realistischen Simulationsmodells kann Systemverhalten vorhergesagt werden und Änderungen am Modell können kostengünstig und ohne schwerwiegende Folgen untersucht werden.

Die Leistungsfähigkeit moderner Computer ermöglicht die Entwicklung von Simulationssystemen zur Analyse des Straßenverkehrs. In der Verkehrsplanung werden Simulationsmodelle als Entscheidungshilfe eingesetzt. Ein Beispiel ist das Verständnis über die Stauentstehung auf Autobahnen, welches einen gezielten Eingriff in den Straßenverkehr zur Stauabmilderung ermöglicht.

Eine Optimierung des Straßenverkehrs zur Minimierung von Staus bedeutet für 
den Einzelnen einen geringeren Zeitaufwand und verminderte Kosten für den Kraftstoffverbrauch. Umweltbelastungen können analog reduziert werden.

Für die Verkehrsflussmodellierung weiterer Verkehrsarten wie Radfahrer, Läufer oder Inline-Skater existieren im Gegensatz zur Verkehrsplanung nahezu keine Verkehrsflussmodelle, obwohl dafür durchaus Bedarf besteht. [...] Treiber und Kesting, 2010, S. 59]

In der Verkehrsforschung wird der Fokus meist auf motorisierten Verkehr gelegt, da der Einfluss von Fahrrädern und Fußgängern auf den Straßenverkehr geringer ist und zusätzlichen Modellierungs- und Berechungsaufwand hervorrufen würde. Es wäre interessant, den Einfluss von nicht-motorisiertem Verkehr auf den Automobilverkehr zu untersuchen und zu prüfen, ob dieser tatsächlich vernachlässigbar ist. Fußgänger betätigen Ampeln oder nutzen Zebrastreifen; Fahrräder müssen häufig überholt werden, doch dies ist nicht immer möglich.

\subsection{Motivierendes Beispiel}

Zur Bestimmung der Luftqualität soll eine Schadstoffuntersuchung über der gesamten Fläche einer Stadt durchgeführt werden. Das Ziel der Untersuchung ist die Bestimmung von besonders belasteten Gebieten und die Prüfung, ob in diesen Gebieten Kindergärten, Spielplätze oder Grundschulen vorhanden sind. Anschließend kann untersucht werden, wie ausgewählte Regionen entlastet werden könnten. Die Messung von Schadstoffwerten muss über einen längeren Zeitraum geschehen, um Durchschnittswerte bilden zu können und nicht auf Grund eines außergewöhnlichen Messtages falsche Schlüsse zu folgern. Eine flächenmäßige Untersuchung lässt sich aus Kostengründen nicht durchführen. Abhilfe kann in diesem Beispiel eine Simulationsstudie schaffen, die den Verkehr der Stadt simuliert.

Wenn Informationen über die Bewegungen der Bürger einer Stadt gegeben sind, können diese simuliert werden. Es sollen die grundlegenden Individualverkehrsarten Auto, Fahrrad und Fußgänger berücksichtigt werden. Dies ermöglicht zusätzlich die Bestimmung des Einflusses von Fahrrädern und Fußgängern auf die Emissionsverteilung. 


\subsection{Problemfelder}

Aus dem im vorherigen Abschnitt genannten motivierendem Beispiel ergeben sich verschiedene Problemfelder. Der Modellierungsaufwand zur Durchführung von Verkehrssimulationen kann sehr groß sein, wenn detaillierte Modelle des Straßennetzes erstellt werden müssen. Für einen Simulationsbereich der Größenordung der Stadt Frankfurt am Main ist dies nicht mehr kosteneffizient möglich.

Es müssen Informationen über die Bewegungen der Verkehrsteilnehmer verarbeitet werden können, um während einer Simulation realistische Verkehrsaufkommen zu erhalten. Höheninformationen werden benötigt, um Steigungen im Straßennetz bestimmen zu können. Diese sind zur Berechnung des Kraftstoffverbrauchs und der damit verbundenen Emissionen notwendig.

Zur Untersuchung der Wechselwirkungen zwischen den verschiedenen Verkehrsteilnehmertypen werden Modelle benötigt, die deren Verhalten abbilden. Das Konzept muss flexibel gefasst sein, sodass weitere Modelle integriert werden können beispielsweise Lkw oder Busse.

\subsection{Zielsetzung}

Die Zielsetzung der vorliegenden Arbeit ist die Konzeptualisierung eines Simulationssystems, welches die Untersuchung des Einflusses nicht-motorisiertem Verkehrs auf den Automobilverkehr in urbanen Szenarien unter Berücksichtung des Kraftstoffverbrauchs und der $\mathrm{CO}_{2}$-Emissionen ermöglicht. Hierfür müssen die im Abschnitt 1.2 ermittelten Problemfelder untersucht werden.

Es soll ein Konzept entwickelt werden, welches aus Geodaten einen Simulationsgraphen extrahieren kann. Auf diese Weise kann der Modellierungsaufwand für das Straßennetz auf ein Minimum reduziert werden und die Untersuchung großer Szenarien wird ermöglicht. Hierbei muss auf etwaige Unzulänglichkeiten des Kartenmaterials Rücksicht genommen werden. Unvollständige Daten (z.B. fehlende Geschwindigkeitsbegrenzungen) müssen ergänzt, benötigte Zusatzinformationen (z.B. Abbiegerichtungen) extrahiert werden.

Das Konzept muss entstehende Anforderungen bezüglich multimodalen Verkehrs berücksichtigen. Diese entstehen bereits bei der Extraktion einer Graphdatenstruktur. Beispielsweise müssen Straßentypen unterschieden werden können, damit 
Fahrräder nicht auf Autobahnen fahren. Es werden Modelle benötigt, die die genannten Verkehrsteilnehmertypen abbilden können und deren Interaktionen miteinander berücksichtigen. Die Modelle und die dazugehörige Simulationssteuerung müssen eine möglichst geringe Berechnungskomplexität aufweisen, um Szenarien der Größenordnung ganzer Städte simulieren zu können.

Mit dem im Rahmen dieser Arbeit entwickelten Konzept sollen ferner die folgenden Fragestellungen untersucht werden können:

- Wie können maschinelle Lernverfahren im Rahmen von Verkehrssimulationsstudien genutzt werden, um Wissen aus dem Verhalten der Verkehrsteilnehmer zu extrahieren?

- Welchen Effekt haben die einzelnen Verkehrsteilnehmergruppen auf den Straßenverkehr und wie wirkt sich Nonkonformismus bezüglich der Verkehrsregeln aus? Welche Vorteile haben Regelbrecher und welche Nachteile entstehen für die restlichen Verkehrsteilnehmer?

- Wie kann der Verkehr möglichst effizient geroutet werden, um Staus und damit erhöhte Reisedauern und verstärkten Kraftstoffverbrauch zu vermindern?

- Welchen Einfluss hat nicht-motorisierter Verkehr auf den Kraftstoffverbrauch der Autos und wie verteilen sich die $\mathrm{CO}_{2}$-Emissionen in einer Stadt?

- Genügt es, nur den Verkehr eines bestimmten Kartenausschnitts zu simulieren, um eine Emissionslandkarte des Simulationsbereiches zu erstellen oder muss zusätzlich auch der Einfluss des Wetters im Simulationsbereich berücksichtigt werden, um aussagekräftige Resultate zu erhalten?

\subsection{Anforderungsanalyse}

Aus der Zielsetzung ergeben sich Anforderungen, die ein Konzept erfüllen muss. Die grundlegensten Anforderungen werden im Folgenden aufgelistet:

AutoGraph Das zur Simulation verwendete Straßennetz muss automatisch generiert werden können. Dies ist notwendig, da Straßenkarten von Städten den manuell leistbaren Modellierungsumfang sprengen. Es entstehen Graphen mit mehreren tausend Knoten und Kanten. Nach einer automatischen Grapherstellung können mit Hilfe von Zusatzmodellen weitere Informationen in 
den Graph integriert werden. Als Datenquelle können digitale Straßenkarten genutzt werden. Die Nutzung von Zufallsgraphen ist nicht ausreichend, da realistischer Straßenverkehr auf diese Weise nicht betrachtet werden kann.

Skalierbarkeit Das Simulationssystem soll genutzt werden können, um ganze Städte bzw. Gruppen von Ortschaften zu simulieren. Es muss folglich gut skalierbar und effizient bezüglich der Berechungskomplexität im Hinblick auf Zeit- und Speicherbedarf sein.

Verkehrsmodelle In urbanen Szenarien sollen die Wechselwirkungen der verschiedenen Verkehrsteilnehmer untersucht werden. Hierfür müssen die wichtigsten Arten von Verkehrsteilnehmern (Pkw, Lkw, Fahrrad, Fußgänger) modelliert werden. Die Wechselwirkungen zwischen den verschiedenen Verkehrsteilnehmertypen müssen so modelliert werden, dass realistische Kenngrößen aus Simulationsläufen extrahiert werden können. Als Beispiel kann die Straßenüberquerung von Fußgängern genannt werden. Ein Fußgänger muss dabei auf den Verkehr achten und kann gegebenenfalls Autos bei der Straßenüberquerung behindern. Es genügt somit nicht, die Straße zu überqueren, indem er zum Zeitpunkt $t$ von der einen Seite der Straße verschwindet und zu $t+\Delta$ auf der anderen Seite wieder erscheint, ohne "gelaufen“ zu sein.

Zusatzmodule Das System muss erweiterbar sein, um konkrete Szenarien untersuchen zu können. Es könnten Modelle über die Verteilung der Dichte an Straftaten für verschiedene Stadtteile geladen werden oder die Benzinverbrauchsberechnung mit Hilfe eines Digitalen Geländemodells erweitert werden.

Die Anforderung Verkehrsmodelle wird präzisiert zu Pkw, Lkw, Fahrräder und Fußgänger, sowie deren Wechselwirkungen. Pkw und Lkw sind unterschiedliche Auto-Arten, Fahrräder und Fußgänger nutzen teilweise andere Wege und bewegen sich mit anderen Geschwindigkeiten als Autos. Fußgänger überqueren zusätzlich Straßen. Die im Verlauf entstehenden Wechselwirkungen ergeben grundlegend das städtische Verkehrsbild und müssen daher Berücksichtigung finden. Es entsteht die Anforderung einer durchgehenden Modellierung der verschiedenen Verkehrsteilnehmergruppen auf einem gemeinsamen modellierten Straßennetz. Dies vereinfacht die Modellierung der Wechselwirkungen während der Simulation der Akteursbewegungen. 


\subsection{Beitrag zum Stand der Forschung}

Im Rahmen dieser Arbeit wird ein Konzept entwickelt, welches die genannten Anforderungen erfüllt. Das Konzept wird durch eine prototypische Implementierung untersucht. Dabei entsteht das Verkehrssimulationssystem MAINSIM (MultimodAle INnerstädtische StraßenverkehrsSIMulation), welches durch Verarbeitungskomponenten und Analysemodule aus einer Landkarte von OpenStreetMap automatisch einen Straßengraphen berechnet, der zur Verkehrssimulation im innerstädtischen Bereich und außerorts verwendet werden kann. Es werden mikroskopische Verkehrsmodelle vorgeschlagen, die die Klassen Auto, Fahrrad und Fußgänger mit Abstufungen modellieren können. Ein besonderer Fokus wird hierbei auf die Modellierung der Wechselwirkungen zwischen den drei Verkehrsteilnehmertypen gelegt.

Das entwickelte System zeichnet sich infolge der Nutzung von Geoinformationssystemkomponenten durch eine einfache Erweiterbarkeit um zusätzliche Datenquellen aus.

In einer Auswahl an Fallstudien wird ein Überblick über die Möglichkeiten von MAINSIM gegeben. Das umfangreichste untersuchte Szenario ist die Simulation des Straßenverkehrs Frankfurt am Mains und seiner umliegenden Städte zur Ermittelung der $\mathrm{CO}_{2}$-Emissionen innerhalb Frankfurts. Die Ankopplung an ein atmosphärisches Simulationssystem ermöglicht die Berücksichtigung von Wettereinflüssen auf die Gasausbreitung.

Im Folgenden werden die im Rahmen dieser Arbeit entstandenen Veröffentlichungen aufgelistet. Die Auflistung ist chronologisch nach den Entstehungs- bzw. Einreichungsdaten geordnet und spiegelt nicht die Erscheinungsreihenfolge wider. Teile dieser Dissertation basieren auf den folgenden Arbeiten.

[Dallmeyer et al., 2011] In dieser Arbeit wird die Vorgehensweise beschrieben, um aus Kartenmaterial eine Graphdatenstruktur zu bestimmen, die zur Verkehrssimulation unter besonderer Berücksichtigung multimodalen Verkehrs zur Simulation von urbanen Szenarien geeignet ist. Eine raumkontinuierliche Verallgemeinerung des $N S M$ wird diskutiert und es wird aufgezeigt, wie das Modell genutzt werden kann, um neben Autos auch Fahrräder zu modellieren.

[Lattner et al., 2011] Anwendung von Maschinellen Lernverfahren auf Simulationsergebnisse zur Generierung von Klassifikatoren, die in Abhängigkeit zur 
Verkehrssituation Aktionen vorschlagen, um den Verkehrsfluss zu maximieren. Zwei Fallstudien untersuchen regulative Maßnahmen für Autobahnszenarien.

[Dallmeyer et al., 2012a] Vorhersage von Staus in der Straße „Am Erlenbruch“ im Osten Frankfurts anhand der Verkehrsmengen in umliegenden Gebieten mittels maschineller Lernverfahren.

[Dallmeyer et al., 2012b] Vorstellung eines mikroskopischen Simulationsmodells für Fußgängerbewegungen in urbanen Szenarien. Die Plausibilität des Modells sowie der Einfluss der simulierten Fußgänger auf die Reisedauern von simulierten Autos konnten gezeigt werden. Diese Arbeit wurde mit einem Best Paper Award auf der SummerSim'12 ausgezeichnet.

[Lattner et al., 2012] Abschätzung der Einflüsse von Fußgängern auf den StraBenverkehr über Bestimmung von Wahrscheinlichkeitsverteilungen, wann Fußgänger Straßen überqueren und Ampeln drücken. Evaluierung in der mittelgroßen Stadt Hanau.

[Dallmeyer et al., 2012e] Erweiterung des Automodells um ein Modell für den Kraftstoffverbrauch und $\mathrm{CO}_{2}$-Ausstoß. Visualisierung der $\mathrm{CO}_{2}$-Verteilung in der Stadt Hanau.

[Dallmeyer et al., 2012d] Vorstellung eines durch Ameisen inspirierten Routingverfahrens und Vergleich mit bestehenden Verfahren in einem synthetischen Szenario und auf Straßenkarten in einer Kleinstadt und einer mittelgroßen Stadt. Eine erweiterte Fassung dieser Arbeit befindet sich derzeit in Begutachtung für das Journal JITS Special Issue on Agents in Traffic and Transportation (selected papers from the 7th Workshop on Agents in Traffic and Transportation - ATT 2012).

[Dallmeyer et al., 2012c] Fallstudien, die den Einfluss von Regelbruch im Straßenverkehr untersuchen: Welche Vorteile haben nonkonforme Verkehrsteilnehmer und welchen Einfluss hat dieses Verhalten auf die restlichen Verkehrsteilnehmer?

[Dallmeyer und Timm, 2012] Vorstellung des Simulationssystems und seiner grundlegenden Funktionalitäten im Rahmen einer Demonstration.

[Cervone et al., 2013 Simulation des Straßenverkehrs in Frankfurt am Main und umliegenden Städten und Gemeinden. Nutzung von Quelle-Ziel-Relationen zur Tripgenerierung. In dieser Arbeit werden die $\mathrm{CO}_{2}$-Emissionen des Stra- 
ßenverkehrs innerhalb Frankfurt am Mains gemessen. Anschließend wird der Einfluss des Wetters auf die Ausbreitung der Gase anhand realer Wetterdaten simuliert. Es handelt sich um einen eingeladenen Buchbeitrag, der 2013 erscheinen wird.

\subsection{Gliederung der Arbeit}

Diese Arbeit ist wie folgt gegliedert:

Teil I schließt in Kapitel 2 mit einem Überblick über Grundlagen, die zum Verständnis dieser Arbeit benötigt werden.

Teil [I] betrachtet den Stand der Forschung im Bereich der Verkehrssimulation. Es wird zwischen Verkehrssimulationsmodellen in Kapitel 3 und Verkehrssimulationssystemen in Kapitel 4 unterschieden.

In Teil III wird das grundlegende Konzept, welches im Rahmen dieser Arbeit entwickelt wurde, diskutiert. Die gewählte Architektur wird in Kapitel 5 gezeigt. Kapitel 6 beschreibt, wie Verkehrswege aus Kartenmaterialien extrahiert werden können. In Kapitel 7 wird die Modellierung multimodalen Verkehrs dargelegt.

Die Umsetzung des Konzepts wird in Teil[IV]besprochen. Kapitel 8 beschreibt die prototypische Implementierung. In Kapitel 9 wird diskutiert, wie die entwickelten Modelle kalibriert und grundlegend evaluiert werden.

Teil V dieser Arbeit behandelt durchgeführte Fallstudien. In Kapitel 10 wird eine Anwendung für maschinelle Lernverfahren in verschiedenen Experimenten im urbanen Bereich und auf Autobahnen aufgezeigt. Die Effekte, die Fußgänger auf den Straßenverkehr haben, werden in Kapitel 11 mittels Wahrscheinlichkeitsverteilungen und Dummy-Fußgängern nachgebildet. Kapitel 12 untersucht anhand dreier Beispiele, welchen Effekt Regelbruch im Straßenverkehr für den Regelbrecher und die restlichen Verkehrsteilnehmer hat. Eine Routingmethode, die sich am Verhalten von Ameisen orientiert, um die Verkehrsinfrastruktur optimal zu nutzen, wird in Kapitel 13 vorgestellt. Kapitel 14 beschreibt die Bestimmung eines Kraftstoffverbrauchs- und $\mathrm{CO}_{2}$-Emissionsmodells, sowie dessen Kalibrierung und Nutzung. In Kapitel 15 wird schließlich MAINSIM mittels Quelle-Ziel-Relationen kalibriert und zur Simulation eines großen Szenarios, welches die Stadt Frankfurt am Main und umliegende Städte umfasst, genutzt. Die $\mathrm{CO}_{2}$-Emissionen von Fahrzeugen innerhalb eines Kerngebiets in Frankfurt werden protokolliert. Anschlie- 
ßend wird die Verflüchtigung der Emissionen durch Wettereinflüsse berechnet.

In verschiedenen Fallstudien wird teils der Verkehr in identischen Straßennetzen simuliert. Dennoch weichen die ermittelten Geschwindigkeiten bzw. Reisedauern teils voneinander ab. Dies liegt einerseits an unterschiedlichen Verkehrsmengen und -zusammensetzungen. Andererseits wurde MAINSIM während der Durchführung der Studien weiterentwickelt. Einen großen Einfluss auf die fahrbaren Geschwindigkeiten hat beispielsweise die Analyse der Straßenkrümmungen, die erst für die Fallstudien der Kapitel 13 und 15 genutzt wurde.

Teil[VI gibt in Kapitel 16 eine Zusammenfassung der vorliegenden Arbeit und eine Diskussion über zukünftige verwandte wissenschaftliche Fragestellungen. 



\section{Grundlagen}

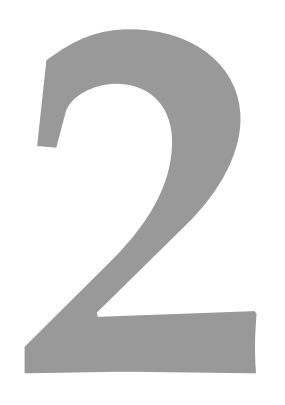

Um Simulationsmodelle entwickeln zu können, werden Datenquellen benötigt, die reale Straßenverläufe abbilden. Wichtige Grundlagen aus dem Bereich der StraBenkarten und Geoinformatik werden daher in Abschnitt 2.1 beschrieben. Darauf aufbauend können Grundlagen der Verkehrsforschung (Abschnitt 2.2), der Computersimulation (Abschnitt 2.3), sowie der Verkehrssimulation im Speziellen (Abschnitt 2.4 genutzt werden, um Verkehrssimulationssysteme zu entwickeln.

\subsection{Straßenkarten und Geodaten}

Straßenkarten gehören zur Gruppe der Geodaten. Geodaten werden grundlegend zwischen Raster- und Vektordaten unterschieden.

Rasterdaten zerlegen einen Kartenausschnitt mit Hilfe eines Rasters in Zellen. Jede Zelle kann verschiedene Eigenschaften annehmen, z.B. eine Höhe über Normalnull oder einen mittleren Niederschlag. Rasterdaten können in gängigen Rasterbildformaten gespeichert werden.

Vektordaten setzen geometrische Objekte aus Punktkoordinaten zusammen. Eine Folge von Punkten kann einen Straßenzug oder einen Fluss darstellen - ein geschlossener Linienzug ein Polygon. Es existieren verschiedene Formate für Geo- 
vektorinformationen. Im Rahmen dieser Arbeit wird der Quasi-Standard Shapefile verwendet.

Geodaten werden in verschiedene Layer aufgeteilt, sodass sie logisch gruppiert sind. Eine mögliche Aufteilung wäre nach Polygonen, Linienzügen und Punkten. Die Aufteilung in Layer bietet zusätzlich die Möglichkeit, bei der Datenvisualisierung die Layer übereinander zu rendern.

Für die Entwicklung eines Verkehrssimulationssystems werden vorrangig Straßenkarten benötigt. Es gibt unterschiedliche Gruppen von Straßenkarten. Kommerzielle Karten - z.B. von Navteq - werden für Navigationssysteme - z.B. von Garmin und BMW - verwendet. Kostenlos nutzbar, jedoch nicht frei herunterladbar, sind die Straßenkarten von Google Maps. In der Geoinformationswirtschaft haben offene Systeme in der Vergangenheit an Bedeutung gewonnen Neis und Zipf, 2008. Mit OSM (OpenStreetMap) ist ein Sytem entstanden, das ähnlich zu Wikipedia jedermann an der Bearbeitung und Gewinnung von Daten teilnehmen lässt. Es handelt sich um den größten freien Geodatenbestand.

Zur Angabe von Koordinaten auf der Erdoberfläche werden meist sphärische Koordinaten genutzt. Eine Einführung in diese Systematik gibt Hennermann, 2006, S.86ff]. Im Rahmen dieser Arbeit werden die Grenzen von Kartenausschnitten im Format WGS84 (World Geodetic System 1984) angegeben. Die Koordinaten [länge, breite $]=[8,0046850,47,8739607]$ bezeichnen beispielsweise die Position des Gipfels des Feldbergs im Taunus. Koordinaten in WGS84 können rund um den Globus angegeben werden. Das GPS arbeitet ebenfalls mit WGS84-Koordinaten. Eine Transformation in andere Koordinatensysteme ist möglich.

Intern wird im Rahmen dieser Arbeit mit kartesischen Koordinaten gearbeitet. Dies ist möglich, indem der Erdball in Abschnitte unterteilt wird, für die jeweils eine eigene Projektion berechnet wird. Abweichungen müssen dennoch in Kauf genommen werden. Der Vorteil dieser Koordinaten ist jedoch, dass Positionen und Distanzen ohne weitere Umrechnungen metrisch angegeben werden können. Für das Bundesland Hessen ist die korrekte Projektion EPSG:31463 (European Petroleum Survey Group Geodesy). Weitere Informationen können Bartelme, 2005, S. $217 \mathrm{ff}]$ entnommen werden.

Zur Nutzung von Höheninformationen werden Digitale Geländemodelle (DGM) genutzt. Ein DGM gibt für Koordinatenpaare die Höhe über Normalnull an. Die Höhe für nicht gegebene Koordinaten muss interpoliert werden. 


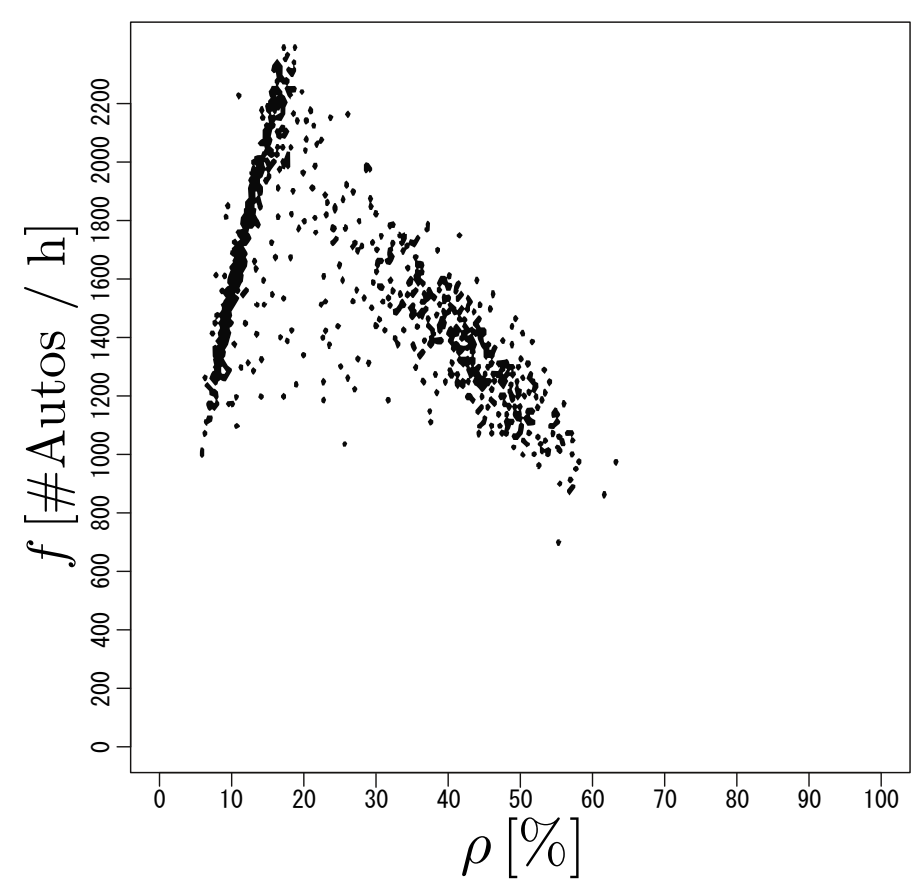

Abbildung 2.1: Fundamentaldiagramm des Verkehrsflusses nach Hall et al., 1986.

\subsection{Verkehrsforschungsgrundlagen}

Mit der Ausbreitung des Automobils in der ersten Hälfte des 20. Jahrhunderts begann der Bereich der Verkehrsforschung zu entstehen. Schon sehr früh wurden Studien durchgeführt, die durch Verkehrsbeobachtung grundlegende Zusammenhänge z.B. zwischen Verkehrsdichte $\rho(x, t)$, Verkehrsfluss $f(x, t)$ und mittlerer Geschwindigkeit $V(x, t)$ am Ort $x$ zum Zeitpunkt $t$ untersuchten (vgl. Greenshield, 1935 und Wardrop, 1952).

Die Verkehrsdichte wird in verschiedenen Arbeiten entweder in prozentualer Auslastung der Straße oder in einer Anzahl an Fahrzeugen pro Streckenabschnittslänge angegeben. Der Verkehrsfluss $f(x, t)$ [\#Autos $\cdot h^{-1}$ ] definiert die Anzahl an Autos, die pro Zeiteinheit (z.B. Stunde oder Sekunde) eine Position passiert haben. Der Verkehrsfluss lässt sich mittels einer Kontaktschwelle auf einer Straße bestimmen. Die Verkehrsdichte kann über zwei Kontaktschwellen bestimmt werden, mit deren Hilfe die Anzahl an Fahrzeugen, die in das Messgebiet eintreten bzw. es wieder verlassen, gemessen wird.

Abbildung 2.1 zeigt den Zusammenhang zwischen Verkehrsdichte und Verkehrsfluss. Die Daten wurden anhand von Messungen im Straßenverkehr ermittelt. Der 
Verlauf des Diagramms ist in der Verkehrsforschung grundlegend - es wird daher als Fundamentaldiagramm bezeichnet. Bei sehr geringen Verkehrsdichten passieren wenige Autos die Flussmessschwelle. Bei steigender Verkehrsdichte steigt auch der Verkehrsfluss, bis eine kritische Verkehrsdichte mit maximalem $f$ erreicht ist.

An dieser Stelle ist die mittlere Geschwindigkeit $V(x, t)$ der Autos auf Grund von Wechselwirkungen mit anderen Autos und zu haltenden Sicherheitsabständen bereits stark verringert. Dieser Effekt führt bei weiterem Anstieg von $\rho$ zu einer Verringerung von $f$. Bei $\rho=100 \%$ wird $f=0$, da sich kein Auto mehr bewegen kann.

Der Zusammenhang zwischen Verkehrsdichte, Verkehrsfluss und mittlerer Geschwindigkeit kann mit der hydrodynamischen Formel abgeschätzt werden:

$$
\rho(x, t)=\frac{f(x, t)}{V(x, t)}
$$

Hierbei muss $V(x, t)$ das räumliche Mittel der Geschwindigkeit zum Zeitpunkt $t$ bestimmen und nicht das zeitliche Mittel der Geschwindigkeit am Ort $x$ Treiber und Kesting, 2010.

Bei Untersuchungen über Verkehrsmengen wird häufig die Fahrzeugfolgedauer angegeben. Sie bezeichnet den zeitlichen Abstand zwischen zwei aufeinander folgenden Fahrzeugen an einer Messstelle. Sie kann bei der Bestimmung des Verkehrsflusses zusätzlich angegeben werden.

\subsection{Simulation}

$\mathrm{Zu}$ analysierende Sachverhalte sind nicht immer mit vertretbarem Aufwand analytisch zu untersuchen. Dies tritt häufig auf, wenn nichtlineare Verknüpfungen der Systemelemente zu komplexen Wirkungsstrukturen führen Bossel, 2004. In diesem Fall kann eine Simulationsstudie Abhilfe schaffen.

\section{Definition 2.3.1 (Simulation)}

Simulation ist die Nachbildung eines dynamischen Prozesses in einem Modell, um zu Erkenntnissen zu gelangen, die auf die Wirklichkeit übertragbar sind (VDI-Richtline). Suhl und Mellouli, 2007 
Simulationen werden verwendet, wenn die reale Durchführung eines Experiments zu kostspielig oder moralisch nicht vertretbar wäre. Heutige Verfahren ermöglichen realistische Crash-Tests mit Hilfe von Computermodellen bereits vor Produktion eines Prototypen. Somit können Fehler im Entwicklungsprozess frühzeitig erkannt und behoben werden (siehe Frisch, 2004). In vielen Bereichen werden Simulationssysteme zur Ausbildung von Fachpersonal verwendet. Angehende Chirurgen können beispielsweise Operationen mit Hilfe von VR-Systemen (Virtuelle Realität) trainieren Satava, 2001.

Nach Law, 2007 muss grundlegend zwischen den folgenden Simulationsarten unterschieden werden:

Statisch / Dynamisch Ein statisches Simulationsmodell berechnet den Zustand des zu simulierenden Systems zu einem Zeitpunkt. Dynamische Simulationsmodelle berücksichtigen Veränderungen über die Zeit und können somit größere Zeitspannen modellieren.

Deterministisch / Stochastisch In deterministischen Modellen werden nicht vorhersagbare Schwankungen nicht abgebildet. Stochastische Modelle verwenden hierfür Wahrscheinlichkeitsverteilungen.

Kontinuierlich / Diskret Physikalische Kenngrößen - z.B. Temperatur, Gewicht oder Zeit - haben kontinuierliche Werte. In Simulationsmodellen werden diese häufig diskretisiert. Ein Simulationsmodell kann den Folgezustand eines Systems (z.B. die Position eines Balles) in unterschiedlichen Größenordnungen bestimmen. Wenn die Position mit $1 \mathrm{~Hz}$ aktualisiert wird, würde die Simulation als zeitdiskret eingeordnet werden. Je kleiner die Zeitscheiben werden, desto zeitkontinuierlicher wird die Simulation. Die Position des Balles (seiner Geschwindigkeit, Beschleunigung, Verformung, ...) kann analog verortet werden.

Auch Mischungen von kontinuierlichen und diskreten Simulationsteilen werden eingesetzt (hybride Simulation). Ein Spezialfall der Diskreten Simulation ist die Ereignisorientierte Simulation. Die Simulationszeit wird verwendet, um Ereignisse hervorzurufen, die ihrerseits den Simulationszustand verändern Ross, 2006. Ein Beispiel ist die Simulation einer Warteschlange. Ereignis 1 könnte das Eintreten in die Schlange sein, ein weiteres Ereignis 2 das Verlassen der Schlange. Wenn Ereignis 1 auftritt, folgt nach einer bestimmten Zeit Ereignis 2.

Wenn ein System aus einer Vielzahl sich gegenseitig beeinflussender Subsysteme besteht - z.B. die Simulation eines Aktienmarktes - so entsteht das Gesamtverhal- 
ten - z.B. der DAX - aus dem Zusammenspiel der Verhaltensweisen der Subsysteme - z.B. der Anleger. Das Gesamtverhalten lässt sich oftmals nicht vorhersagen, ohne die Verhaltensweisen der enthaltenen Subsysteme zu simulieren. Einzelne Simulationsentitäten können als Agenten modelliert werden. Einen Überblick liefert Yilmaz und Ören, 2009. Die Agententechnologie eignet sich beispielsweise für sozialwissenschaftliche Untersuchungen Gilbert, 2007.

\section{Definition 2.3.2 (Agent)}

Ein Agent ist ein Computersystem, das sich in einer Umgebung befindet und die Fähigkeit hat, autonome Aktionen in dieser Umgebung durchzuführen, um seine gesetzten Ziele zu erreichen. (nach Wooldridge, 2009]).

Nach Definition 2.3.2 lassen sich beispielsweise eigenständige Staubsauger als Agenten auffassen. Im Rahmen dieser Arbeit werden Verkehrsteilnehmer als Akteure oder Agenten aufgefasst. Verkehrsteilnehmer müssen weitere Eigenschaften besitzen, um komplexe urbane Verkehrsszenarien modellieren zu können. Es wird zwischen Agenten und intelligenten Agenten unterschieden. Nach Timm, 2004 wird die folgende Definition 2.3 .3 von weiten Teilen der Agentenforschung akzeptiert.

Definition 2.3.3 (Intelligenter Agent)

Ein intelligenter Agent ist eine Software, die

- Autonom (agiert selbständig)

- Sozial (Interaktionen mit anderen Agenten werden genutzt, um Ziele zu erreichen)

- Reaktiv (ist in der Lage, die Umgebung wahrzunehmen und auf Änderungen zu reagieren, um die definierten Ziele zu erreichen)

- Proaktiv (führt Aktionen aus, um Ziele zu erreichen)

ist. (nach Wooldridge und Jennings, 1995)

Das Zusammenspiel einer Menge von Agenten kann zu emergentem Verhalten führen. Es entstehen Situationen, die nicht durch das Verhalten einzelner Agenten entstehen, sondern durch deren Wechselwirkungen. Ein Beispiel negativer Emergenz ist ein Stau. Das Zusammenspiel staatenbildender Insekten kann zu positiver Emergenz führen - beispielsweise die Ermittelung kürzester Wege durch Ameisen (vgl. Kennedy et al., 2001). 
Multi-Agenten-Simulationen (MAS) werden in verschiedenen Bereichen eingesetzt. Die Granularität kann hierbei zwischen makroskopischen (z.B. Simulation der Wechselwirkungen zwischen Städten [Sanders, 2007]) und mikroskopischen Systemen (z.B. Simulation eines Spielplatzes Koizumi et al., 2010) unterschieden werden. Wenn sowohl makroskopische als auch mikroskopische Modelle verwendet werden, kann ein Übergang zwischen beiden mittels mesoskopischer Modellierung geschehen (z.B. makroskopische Modellierung des Autoverkehrs einer Autobahn und mikroskopische Modellierung einer konkreten Auf- oder Abfahrt Rose, 2003).

\subsection{Verkehrssimulation}

Im Rahmen dieser Arbeit liegt der Fokus auf Systemen, die zur Simulation von Verkehr im Allgemeinen und urbanen Verkehr im Speziellen genutzt werden können. Hierbei liegt ein Augenmerk auf Simulatiossystemen, die mit einem GIS (Geoinformationssysteme) gekoppelt sind. Dies ermöglicht die Nutzung von Straßenkarten zur Erstellung eines Simulationsgraphen zur agentenbasierten Verkehrssimulation Schüle et al., 2004.

Im Bereich der Verkehrssimulation wurden erste Untersuchungen bereits Anfang der 1930er Jahre durchgeführt (vgl. Greenshield, 1935), da eine steigende Verkehrsdichte nach Prognosemethoden für Verkehrsaufkommen und Modellen zur Optimierung der Verkehrsleittechnik verlangte. Damalige Verkehrsmodelle waren makroskopisch.

Makroskopische Kenngrößen (Verkehrsfluss, Verkehrsdichte usw.) werden hierbei mittels physikalischer Flussmodelle ermittelt. Lighthill und Whitham haben die Kontinuitätsgleichung eingeführt, nach der der Verkehrsfluss und die durchschnittliche Geschwindigkeit proportional zur Verkehrsdichte sind. Unabhängig von dieser Arbeit hat Richards identische Überlegungen angestellt, sodass das Modell Lighthill-Whitham-Richards-Modell (LWR) genannt wird Lighthill und Whitham, 1955, Richards, 1956. Erweiterte Modelle nehmen die durchschnittliche Geschwindigkeit als dynamische Variable in das Simulationsmodell auf und berücksichtigen die beschränkte Beschleunigungsfähigkeit von Automobilen Kerner und Konhäuser, 1994, Treiber et al., 1999. Makroskopische Verkehrsmodelle lassen sich zur Vorhersage makroskopischer Kenngrößen kalibrieren, sofern ausreichend große Zeiträume und Streckenabschnitte betrachtet werden.

Eine feinere Granularität ermöglichen mikroskopische Simulationsmodelle, die die 
Bewegung einzelner Verkehrsteilnehmer modellieren. Heterogene Fahrverhaltensweisen lassen sich simulieren und makroskopische Kenngrößen können extrahiert werden. Der Zusammenschluss aus Fahrzeug und Fahrer wird meistens als eine Entität betrachtet und kann durch Agenten modelliert werden Kesting et al., 2009. Ein Überblick wird in Kesting et al., 2008 gegeben.

Bei der Betrachtung von Verkehrsmodellen werden häufig Zeitpunkte $t$ verwendet. Es sei beispielsweise $x_{n}(t)$ die Position des Autos $n$ zum Zeitpunkt $t$ und $\dot{x}_{n}(t)$ dessen Geschwindigkeit. Der Zeitpunkt $t$ bezieht sich auf die Simulationszeit. Diese korrelliert nicht mit der Uhrzeit. Ein Simulationslauf wird für gewöhnlich zum Zeitpunkt $t=0$ gestartet und die Simulationszeit erhöht sich während der Simulation. Je nach Simulationsart können hierbei äquidistante Zeitschritte genutzt werden. Eine gängige Praxis ist, pro simulierter Sekunde einmal alle Zustände der Verkehrsteilnehmer zu aktualisieren, um der typischen menschlichen Reaktionszeit Rechnung zu tragen. Es werden jedoch auch andere - meist geringere Zeitscheiben verwendet, um feingranularer modellieren zu können.

Eine besondere Gruppe der Verkehrssimulationssysteme sind multimodale Systeme. Sie berücksichtigen multiple Arten von Simulationsentitäten, z.B. Autos, Fahrräder und Fußgänger. Die genutzten Modelle sind in der Regel mikroskopisch, da makroskopische Modelle die benötigten punktuellen Wechselwirkungen zwischen den Verkehrsteilnehmertypen nicht modellieren können.

Innerhalb mikroskopischer Modelle können unterschiedliche Detaillierungsgrade erzielt werden. High-Fidelity-Modelle können teilweise sehr komplexe Wirkungszusammenhänge abbilden. Je nach Anwendungsgebiet können Parameter wie z.B. der Winkel des Gaspedals simuliert werden. 


\section{Teil II}

Stand der Forschung 



\section{Verkehrssimulationsmodelle}

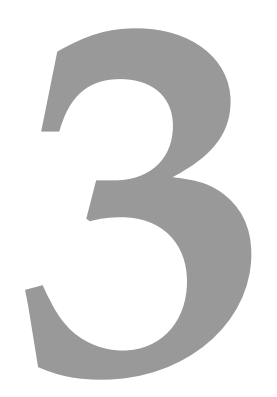

Dieses Kapitel diskutiert den Stand der Forschung zu Simulationsmodellen für unterschiedliche Verkehrsteilnehmertypen. In der realen Welt werden in urbanen Szenarien nicht nur Autos genutzt. Tabelle 3.1 zeigt beispielhaft anhand einer Auswahl deutscher Städte, welche Art der Fortbewegung wie häufig ist. Es werden die Fortbewegungsmethoden „Auto“, „Öffentliche Verkehrsmittel“, „Fahrrad" und „zu Fuß“ unterschieden.

Zur Berücksichtigung von öffentlichen Verkehrsmitteln müssen deren Fahrrouten definiert sein. Berücksichtigt werden ferner Autos (Abschnitt 3.1), Fahrräder (Abschnitt 3.2) und Fußgänger (Abschnitt 3.3. Jede der drei Gruppen kann in Untergruppen präzisiert werden und heterogene Verkehrsteilnehmer modellieren. Busse können als Spezialfall eines Autos definiert werden. Dieses Kapitel schließt in Unterabschnitt 3.5 mit einer Diskussion der vorgestellten Modelle.

\subsection{Modelle zur Fahrzeugmodellierung}

Zur Simulation des Automobilverkehrs können grundlegend drei verschiedene Betrachtungsweisen genutzt werden: Makroskopische, mesoskopische und mikroskopische Verkehrsmodelle.

In makroskopischen Modellen wird der Verkehr mit Hilfe von Flüssigkeitsmodellen 


\begin{tabular}{lcccc} 
& 43 & 12 & 22 & 23 \\
\hline Bamberg & 34 & 28 & 12 & 26 \\
\hline Berlin & 46 & 14 & 17 & 23 \\
\hline Bremen & 46 & 3 & 29 & 9 \\
\hline Coesfeld & 49 & 19 & 10 & 20 \\
\hline Dortmund & 40 & 5 & 24 & 31 \\
\hline Greifswald & 42 & 18 & 13 & 27 \\
\hline Halle & 44 & 18 & 16 & 22 \\
\hline Karlsruhe & 47 & 12 & 17 & 24 \\
\hline Kiel & 58 & 10 & 8 & 24 \\
\hline Koblenz & 59 & 11 & 10 & 20 \\
\hline Ludwigsburg & 43 & 28 & 14 & 15 \\
\hline Nürnberg & & &
\end{tabular}

Tabelle 3.1: Modal Split nach Verkehrsaufkommen: Prozentualer Anteil der gezeigten Verkehrsteilnehmertypen. Beispieldaten für ausgewählte deutsche Städte. Adaptiert nach Kolodziej et al., 2009.

oder gaskinetischen Gleichungen beschrieben. Anhand wichtiger Parameter - z.B. der Anzahl der Spuren oder der Verkehrsdichte - lassen sich über Zeit und Raum gemittelte Kenngrößen vorhersagen - z.B. die mittlere Geschwindigkeit des Verkehrs. Makroskopische Modelle eignen sich zur Simulation großer Verkehrsmengen, da Verkehr anhand weniger Kenngrößen beschrieben werden kann. Makroskopische Modelle benötigen jedoch größere Zeiträume und Strecken, um gemittelte Ergebnisse berechnen zu können.

Auf der gegenüberliegenden Seite beschreiben mikroskopische Verkehrsmodelle das Verhalten jedes einzelnen Verkehrsteilnehmers. Aus dem Zusammenspiel einer Gruppe von Verkehrsteilnehmern entsteht das makroskopische Verhalten auf den simulierten Straßen. Makroskopische Kenngrößen können durch Mittelung (z.B. mittlere Fließgeschwindigkeit) oder Zählungen (z.B. Verkehrsfluss) aggregiert werden. Mikroskopische Verkehrsmodelle können verschiedene Verkehrsteilnehmertypen berücksichtigen. Makroskopische Modelle beschränken sich üblicherweise auf den Automobilverkehr.

Mesoskopische Verkehrsmodelle verbinden makroskopische und mikroskopische Elemente in einem Simulationssystem. Es könnte beispielsweise der Verkehr in einem Stadtgebiet mikroskopisch simuliert werden und der umliegende Verkehr (Zu- und Abflüsse aus dem betrachteten Gebiet) makroskopisch. 
Die verschiedenen Betrachtungsweisen eignen sich für unterschiedliche Aufgabenstellungen. Ein makroskopisches Modell eignet sich beispielsweise gut zur Modellierung von Autobahnverkehr über große Strecken mit geringem Berechnungsaufwand. Es lässt sich jedoch nicht zur Simulation von innerstädtischem Verkehr mit seinen komplexen Wechselwirkungen zwischen verschiedenen Verkehrsteilnehmern und häufigen Störungen durch Ampelschaltungen verwenden. An dieser Stelle müssen mikroskopische Modelle genutzt werden.

Wenn nur Teilaspekte des Verkehrs betrachtet werden sollen, eignen sich mesoskopische Ansätze. Für eine Straße kann die mittlere Durchfahrtsdauer makroskopisch ermittelt werden. Ein mikroskopisch simulierter Bus kann an diesen Stellen mit der makroskopisch ermittelten Geschwindigkeit fahren und dennoch an Bushaltestellen anhalten. Auf diese Weise können Busfahrpläne bis zu einem gewissen Grade auf Plausibilität geprüft werden.

Zur Simulation des Verkehrs in urbanen Szenarien werden mikroskopische Modelle genutzt, da sie verschiedene Aspekte des Verkehrs, z.B. Vorfahrtsregeln oder die Routenwahl von simulierten Autofahrern, modellieren können.

Mikroskopische Modelle können in zwei grundlgegende Gruppen unterteilt werden. In Unterabschnitt 3.1.1 werden Fahrzeugfolgemodelle untersucht. Modelle auf Basis Zellulärer Automaten werden in Abschnitt 3.1.2 beschrieben. Stellvertretend für Modelle dieser Gruppe wird hierbei der Fokus auf das Nagel-Schreckenberg-Modell gelegt, welches ein gut verstandenes und viel genutztes Modell ist. Im Unterabschnitt 3.1 .3 wird kurz auf weitere Modellgruppen eingegangen und diskutiert, welche Aspekte der vorgestellten Modelle im Rahmen dieser Arbeit übernommen werden können bzw. abgewandelt werden müssen.

\subsubsection{Fahrzeugfolgemodelle}

Fahrzeugfolgemodelle sind mikroskopische Verkehrsmodelle, die die Verhaltensweisen der simulierten Autos in Relation zu den vorausfahrenden Fahrzeugen auf ihrer Spur modellieren. Diese Modellklasse basiert auf Differntialgleichungen und hat das Ziel, sprunghafte Geschwindigkeitsänderungen zu vermeiden, um Fahrverhaltensweisen realistisch abbilden zu können. Ein besonders einfaches Modell wird in McCartney und Carey, 1999 vorgeschlagen, um Studenten für Verkehrsmodelle und Differentialgleichungen zu begeistern. Nach Helbing, 2001 wurden die ersten Fahrzeugfolgemodelle von Reuschel Reuschel, 1950a, Reuschel, 1950b und Pipes Pipes, 1953 publiziert. Die Autos sind im Modell wie in Abbildung 3.1 


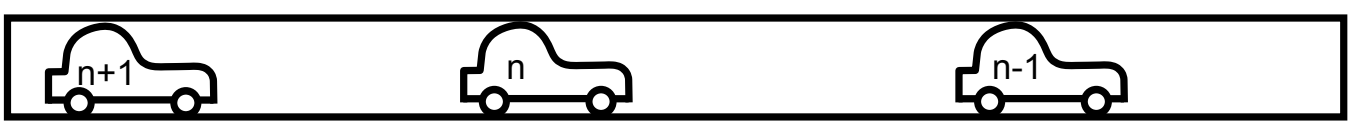

Abbildung 3.1: Die Autos werden in der Reihenfolge, in der sie sich auf der Straße befinden, sortiert. Auto $n$ muss auf das Fahrverhalten von Auto $n-1$ reagieren.

dargestellt, nummeriert.

Ein Auto $n$ bestimmt seine Geschwindigkeit hierbei in Abhängigkeit zu seinem Vordermann $n-1$ nach Gleichung 3.1 .

$$
\begin{aligned}
\ddot{x}_{n}(t+T) & =\frac{v_{n}^{0}+\xi_{n}(t)-\dot{x}_{n}(t)}{\tau_{n}}+f_{n,(n-1)}(t) \\
\text { mit } x_{n}(t)= & \text { Position von Auto } n \text { zum Zeitpunkt } t \\
\dot{x}_{n}(t)= & \text { Geschwindigkeit von Auto } n \text { zum Zeitpunkt } t \\
\ddot{x}_{n}(t)= & \text { Beschleunigung von Auto } n \text { zum Zeitpunkt } t \\
T= & \text { Reaktionszeit } \\
v_{n}^{0}= & \text { Wunschgeschwindigkeit von Auto } n \\
\xi_{n}(t)= & \text { Verhaltensschwankung von Auto } n \text { zum Zeitpunkt } t \\
\tau_{n}= & \text { Relaxationszeit von Auto } n \\
f_{n,(n-1)}(t)= & \text { Abstoßung zwischen Auto } n \text { und } n-1 \\
& \text { zum Zeitpunkt } t
\end{aligned}
$$

Mit Hilfe dieses ersten Fahrzeugfolgemodells können divergierende Verhaltensweisen modelliert werden. Jedes Auto folgt dabei seinem Vordermann. Ein weiteres Modell dieser Klasse ist das Gazis-Herman-Rothery-Modell (GHR) Chen et al., 2005. Es ist ein Modell, das in den vergangenen Jahrzehnten von verschiedenen Forschergruppen erweitert wurde Al-Jameel, 2009. In Gazis et al., 1959 wird ein erster Ansatz vorgestellt, der darauf basiert, dass jedes simulierte Auto seine Geschwindigkeit in Relation zur Geschwindigkeitsdifferenz zum Vordermann anpasst. Heutige Modelle auf der Grundlage von GHR basieren auf der in Gleichung 3.2 dargestellten Differentialgleichung nach Gazis et al., 1961. 


$$
\begin{aligned}
\ddot{x}_{n}(t+T) & =\frac{a \dot{x}_{n}^{m}(t+T) \Delta \dot{x}(t)}{(\Delta x(t))^{l}} \\
\text { mit } a & =\text { Proportionalitätskonstante } \\
m, l & =\text { Verhaltensparameter } \\
\Delta x(t) & =x_{n-1}(t)-x_{n}(t) \\
\Delta \dot{x}(t) & =\dot{x}_{n-1}(t)-\dot{x}_{n}(t)
\end{aligned}
$$

Die Proportionalitätskonstante $a$ bestimmt die Reaktionsstärke des simulierten Autos $n$. Im GHR-Modell reagiert ein simuliertes Auto $n$ auf jede Geschwindigkeitsänderung des Autos $n-1$.

Es handelt sich um ein rein physikalisches Modell, welches in verschiedenen Situationen unrealistische Verhaltensweisen modelliert und zu Auffahrunfällen führen kann. Es können Beschleunigungs- und Bremswerte entstehen, die reale Fahrzeugfähigkeiten übersteigen. Das Auto $n$ wirkt auf $n+1$ außerdem anziehend Helbing, 1997b. Gleichung 3.2 ist nicht plausibel, wenn kein Auto $n$ existiert. In diesem Fall beschleunigt Auto $n+1$ nie.

Eine Erweiterung des GHR-Modells ist das Optimal-Velocity Modell (OVM), welches in Gleichung 3.3 gezeigt wird Bando et al., 1994. Jedes Auto bestimmt seine optimale Geschwindigkeit in Relation zu dem vorausfahrenden Auto.

$$
\begin{aligned}
\ddot{x}_{n}(t) & =\frac{V(\Delta x(t))-\dot{x}_{n-1}(t)}{\tau} \\
\text { mit } V(\Delta x(t)) & =\text { Optimal Velocity Funktion } \\
\tau & =\text { Sensitivitätskonstante }
\end{aligned}
$$

Das Modell konnte mit den in Gleichung 3.4 gezeigten Parametern auf japanischen Autobahnverkehr kalibriert werden Bando et al., 1998.

$$
V(\Delta x(t))=16,8 \cdot[\tanh (0,0860(\Delta x(t)-25))+0,913]
$$


Das OVM ist empfindlich bezüglich seiner Optimal Velocity Funktion Bando et al., 1995. Es produziert teils unrealistisch starke Bremsungen und führt dennoch zu Unfällen, wenn ein Auto auf einen stehenden Vordermann auffährt Helbing und Tilch, 1998. Um dies zu vermeiden, muss $\tau$ sehr gering gewählt werden. Dies geschieht jedoch auf Kosten unrealistisch starker Beschleunigungswerte Helbing, 2001. Zhao und Gao erweitern das Modell um den Geschwindigkeitsunterschied $\Delta \dot{x}(t)=\dot{x}_{n-1}(t)-\dot{x}_{n}(t)$ zum Vordermann und vermeiden dadurch unrealistisch starke Bremsungen und Auffahrunfälle [Zhao und Gao, 2005.

Treiber und Helbing schlagen das Intelligent Driver Modell (IDM) vor, welches durch Gleichung 3.5 definiert wird Treiber und Helbing, 1999, Treiber et al., 2000.

$$
\begin{aligned}
\ddot{x}_{n}(t) & =a_{n} \cdot[\underbrace{1-\left(\frac{\dot{x}_{n}(t)}{v_{n}^{0}}\right)^{\delta}}_{v^{+}}-\underbrace{\left(\frac{s_{n}^{*}}{s}\right)^{2}}_{v^{-}}] \\
s_{n}^{*} & =s_{n}^{0}+\max \left(0, \dot{x}_{n}(t) T_{n}^{0}-\frac{\dot{x}_{n}(t) \Delta \dot{x}(t)}{2 \sqrt{a_{n} b}}\right) \\
\text { mit } v^{+} & =\text {Beschleunigungsverhalten } \\
v^{-} & =\text {Bremsverhalten } \\
a_{n} & =\text { Maximale Beschleunigung von Auto } n \\
\delta & =\text { Beschleunigungsexponent } \\
s_{n}^{*} & =\text { Wunschabstand zu Vordermann von Auto } n \\
s & =x_{n-1}(t)-x_{n}(t) \\
s_{n}^{0} & =\text { Minimaler Sicherheitsabstand von Auto } n \\
T_{n}^{0} & =\text { Zeitl. Wunschabstand zu Vordermann von Auto } n \\
b & =\text { Komfortable Verzögerung }
\end{aligned}
$$

Die Wahl von $s_{n}^{*}$ führt dazu, dass ein langsamer Vordermann einen erhöhten Wunschabstand und ein schneller Vordermann einen verringerten Wunschabstand bewirkt. Schnelle Vordermänner verringern den Einfluss von $s_{n}^{*}$ auf die Geschwindigkeitswahl.

Typische Modellparameter werden in Tabelle 3.2 gezeigt. Wenn Auto $n$ keinen Vordermann hat, wird $s=\infty$ und somit $v^{-}=0$. Das Auto verringert durch den 


\begin{tabular}{lll}
\hline Parameter & Autobahn & Stadtverkehr \\
\hline Wunschgeschwindigkeit $v^{0}[\mathrm{~km} / \mathrm{h}]$ & 130 & 50 \\
Folgezeit $T^{0}[\mathrm{~s}]$ & 1,5 & 1,5 \\
Minimalabstand $s^{0}[\mathrm{~m}]$ & 3 & 2 \\
Beschleunigungsexponent $\delta$ & 4 & 4 \\
Beschleunigung $a_{n}\left[\mathrm{~m} / \mathrm{s}^{2}\right]$ & 1,0 & 1,5 \\
Verzögerung $b\left[\mathrm{~m} / \mathrm{s}^{2}\right]$ & 2 & 2 \\
\hline
\end{tabular}

Tabelle 3.2: Typische Modellparameter für IDM |Kesting et al., 2010, Treiber, 2011

Term $v^{+}$die Differenz seiner Geschwindigkeit $\dot{x}_{n}(t)$ zu seiner Wunschgeschwindigkeit $v_{n}^{0}$. Der Verlauf der Beschleunigung wird durch den Exponenten $\delta$ bestimmt. Wenn ein Vordermann vorhanden ist, wird die Beschleunigung zusätzlich von dessen Position und Geschwindigkeit durch $v^{-}$beeinflusst. Jedes Auto $n$ versucht, seinen Wunschabstand $s_{n}^{*}$ zum Vordermann zu halten.

Das IDM führt auf einspurigen Straßen zu realistischem Fahrverhalten. Bei mehrspurigem Verkehr können Spurwechsel abrupte Verzögerungen hervorrufen, die einerseits ungewollt und andererseits unrealistisch stark ausfallen. In Kesting et al., 2010 wird die Beschleunigungsfunktion des IDM daher erweitert. Das IDM ist deterministisch und eignet sich mit Erweiterungen zur adaptiven Temporegelung [Treiber und Helbing, 2002]. Zufällige Einflüsse werden nicht modelliert.

In Treiber et al., 2006 wird eine Erweiterung des IDM vorgeschlagen, die Reaktionszeiten, Schätzfehler, zeitliches Vorausschauen und die Berücksichtigung mehrerer vorherfahrender Fahrzeuge modelliert. Es entsteht ein gleichmäßigeres Fahrverhalten. Dies ist vor allem relevant, da das IDM auch im Hinblick auf die Nutzung als Fahrassistenzsystem entwickelt wird.

In Wiedemann, 1974 wurde ausgehend vom GHR-Modell ein psychischphysikalisches Modell entwickelt, indem eine Wahrnehmungsschwelle hinzugefügt wurde. Das Modell wird Wiedemann-Modell genannt. Ein Auto $n+1$ reagiert demnach nur noch auf, mit Hilfe eines Schwellwertes angegebene, „spürbare“ Änderungen der Geschwindigkeit des Vordermanns $n$. Der Schwellwert ist vom Abstand $x_{n}(t)-x_{n+1}(t)$ abhängig.

Das Wiedemann-Modell ist ein High-Fidelity-Modell und kann zur Berücksichtigung verschiedenster Fahrdetails erweitert werden [Schnieder und Becker, 2007. Der Berechnungsaufwand ist verglichen mit anderen Modellen jedoch sehr hoch. Das Modell wird daher vorwiegend verwendet, wenn kleinere Streckenabschnit- 
te, z.B. ein detailliert modellierter Ausschnitt eines Stadtstraßennetzes, analysiert werden sollen.

Ein Fahrzeugfolgemodell, das nicht menschliches Verhalten detailliert modelliert, sondern nur generelle Annahmen über den Verkehrsfluss verarbeitet, ist das KraußModell Krauß, 1997]. Es wird über Gleichung 3.7 definiert.

$$
\begin{aligned}
\dot{x}_{n}(t+\Delta t) & =\max \left[0, \dot{x}_{\mathrm{des}}(t)-\eta\right] \\
\dot{x}_{\mathrm{des}}(t) & =\min \left[\dot{x}_{\max }, \dot{x}(t)+a(\dot{x}(t)) \Delta t, \dot{x}_{\mathrm{safe}}(t)\right] \\
\dot{x}_{\mathrm{safe}}(t) & =\dot{x}_{n-1}(t)+\frac{\left[x_{n-1}(t)-x_{n}(t)\right]-g_{\mathrm{des}}(t)}{\tau_{b}+\tau} \\
x(t+\Delta t) & =x(t)+v \Delta t \\
g_{\mathrm{des}}(t) & =\tau \dot{x}_{n-1}(t) \\
\text { mit } \eta>0 & =\text { Zufällige Abweichung } \\
a(\dot{x}) & =\text { Beschleunigungsvermögen } \\
\tau & =\text { Reaktionszeit } \\
\tau_{b} & =\frac{0,5 \cdot\left(\dot{x}_{n}(t)+\dot{x}_{n-1}(t)\right)}{b}
\end{aligned}
$$

Die grundlegende Idee des Modells ist trivial: Fahre so schnell wie möglich, in Abhängigkeit zur Wunschgeschwindigkeit $\dot{x}_{\text {des }}(t)$ und zur Geschwindigkeit, die ein sicheres Fahren ermöglicht $\dot{x}_{\text {safe }}(t)$. Die Definition von $\dot{x}_{\text {safe }}(t)$ modelliert Wechselwirkungen zwischen Auto $n$ und seinem Vordermann $n-1$ in Relation zur Geschwindigkeit des Vordermanns und der Abweichung der Distanz zum Vordermann $x_{n-1}(t)-x_{n}(t)$ zur Wunschdistanz $g_{\text {des }}(t)$. Die Reaktionsstärke hängt von der Reaktionszeit, der mittleren Geschwindigkeit von Auto $n$ und $n-1$, sowie der komfortablen Verzögerung $b$ ab. Die Wunschdistanz $g_{\text {des }}(t)$ hängt nicht von der Geschwindigkeit von Auto $n$, sondern der seines Vordermanns $n-1$ ab.

In Krauß et al., 1997, Krauß, 1998 wurde gezeigt, dass das Krauß-Modell grundlegende Zusammenhänge des Verkehrsflusses reproduzieren kann und auf mehrspurigen Verkehr erweitert werden kann. Die Simulationsgeschwindigkeit ist jedoch um einen Faktor $\approx 2$ langsamer, als unter Verwendung des im nächsten Abschnitt beschriebenen Nagel-Schreckenberg-Modells.

Der Berechnungsaufwand von Fahrzeugfolgemodellen ist im Vergleich zu anderen Ansätzen hoch. Simulationsszenarien mit großen Mengen an Autos sind daher 


\begin{tabular}{|l|c|c|c|c|c|c|c|c|}
\hline aktueller Zustand & $1 \mathbf{1 1}$ & $1 \mathbf{1 0}$ & $1 \mathbf{0 1}$ & $1 \mathbf{0 0}$ & $0 \mathbf{1 1}$ & $0 \mathbf{1 0}$ & $0 \mathbf{0 1}$ & $0 \mathbf{0 0}$ \\
\hline Folgezustand & 1 & 0 & 1 & 1 & 1 & 0 & 0 & 0 \\
\hline
\end{tabular}

Tabelle 3.3: Zustandsübergangsfunktion: Zellularautomat Rule 184

schwierig durchführbar.

\subsubsection{Nagel-Schreckenberg-Modell}

Im Kontrast zu Fahrzeugfolgemodellen ist das Nagel-Schreckenberg-Modell darauf ausgelegt, mit einfachen Verhaltensregeln große Mengen von Fahrzeugen simulieren zu können. Das Modell bedient sich der Technologie der zellulären Automaten. Zelluläre Automaten werden zur Modellierung räumlich und zeitlich diskreter Systeme verwendet.

\section{Definition 3.1.1 (Zellulärer Automat)}

Ein Zellular- oder zellulärer Automat (ZA) ist ein gedachter Parallelrechner, dessen Einzelrechner, die (Automaten-)Zellen, in einem regulären Gitter angeordnet sind. Alle Zellen führen das gleiche Programm aus. Dieses beschreibt einen Automat mit endlich vielen Zuständen, der mit Nachbarzellen kommuniziert. Goos, 1998, S. 75]

Das zu modellierende System wird in Zellen $z$ zerlegt, die einen Zustand aus einer Zustandsmenge annehmen können. Eine Zustandsübergangsfunktion bestimmt in Abhängigkeit des Zustands von $z$ und den Zuständen der Zellen in einer endlichen Nachbarschaft von $z$ zum Zeitpunkt $t$ den Folgezustand für den Zeitpunkt $t+1$ Batty, 2007.

Steve Wolfram beschrieb einen zellulären Automaten, der Rule 184 genannt wird, da $184_{(10)}=10111000_{(2)}$ und dies dem Muster der Zustandsübergangsfunktion entspricht, die in Tabelle 3.3 dargestellt wird. Der Automat beschreibt ein eindimensionales Verkehrsflussproblem Wang et al., 1998. Viele weitere und komplexere Verkehrsmodelle bauen auf Rule 184 auf Nagel, 1996.

Jede Zelle kann einen der Zustände 0 (leer) oder 1 (belegt) annehmen. Der Folgezustand für eine Zelle wird durch ihren eigenen Zustand (in Tabelle 3.3 fett markiert) und die Zustände der direkt benachbarten Zellen links und rechts beeinflusst. Für jede Zelle des Systems wird parallel der Zustandsübergang berechnet. In Abbildung 3.2 wird eine Simulation von 10 Durchläufen auf einem Feld mit einer Länge 


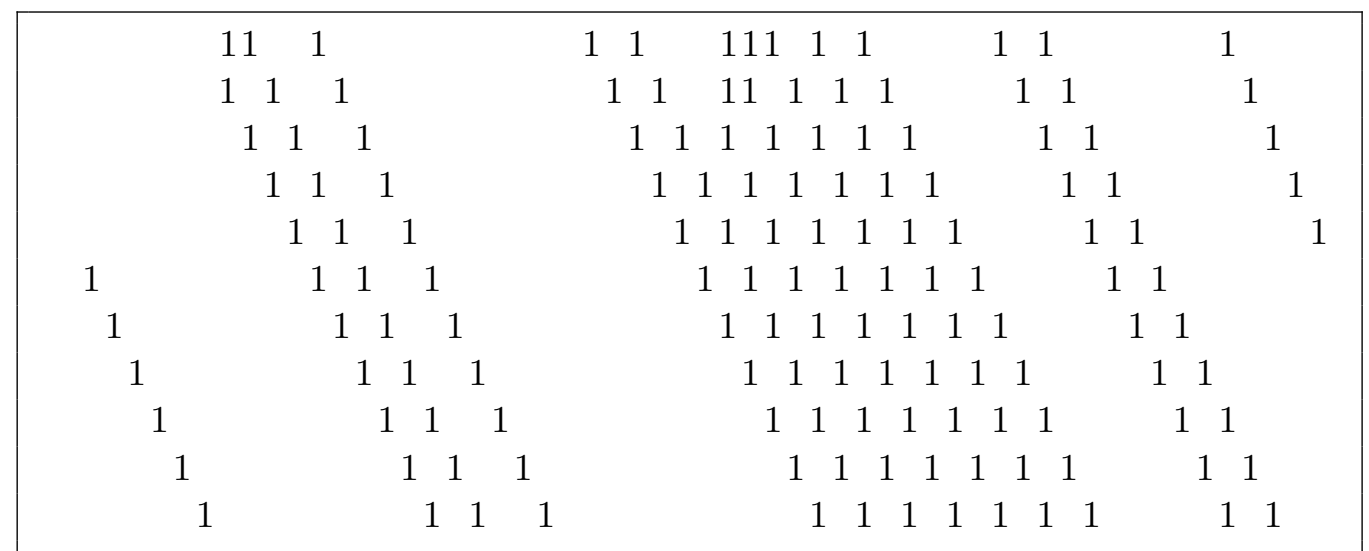

Abbildung 3.2: Beispielsequenz: Zellularautomat Rule 184. Da das Verhalten der Zellen rein deterministisch ist, wird ein anfangs vorhandener Stau schnell aufgelöst und die Zellen "fahren“ in einer Kolonne mit konstanten Abständen hintereinander her.

von 55 Zellen gezeigt. Die erste Zeile stellt den Startzustand dar, wobei jede mit einer 1 markierte Zelle belegt ist. Jede weitere Zeile $n$ zeigt den Folgezustand, der aus dem Zustand zum Zeitpunkt $n-1$ hervorgeht. Belegte Zellen wandern immer weiter nach rechts, bis sie auf eine weitere belegte Zelle „auflaufen“, sodass ein Stau sich rückwärts ausbreitet.

Wenn man annimmt, dass jede belegte Zelle mit einem Auto belegt ist, so stellt Rule 184 ein sehr einfaches Modell zur Verkehrssimulation auf einer einspurigen Straße dar, bei der jedes Auto eine Maximalgeschwindigkeit von $1\left[\right.$ Zelle $\left.^{\text {Zeiteinheit }}{ }^{-1}\right]$ hat. Der Zellularautomat Rule 184 wird daher auch Traffic Rule genannt.

Fukui und Ishibashi erweiterten Rule 184 um Geschwindigkeiten von bis $\mathrm{zu} \quad v_{\max }=5\left[\right.$ Zelle $\cdot$ Zeiteinheit $\left.^{-1}\right]$. Autos fahren stets mit $v=$ $\min \left(v_{\max }, d i s t\right)$, wobei dist die Anzahl der freien Zellen bis zum Vordermann ist Fukui und Ishibashi, 1996]. In [Nagatani, 1996 wird der Ansatz beschrieben, dass ein Auto seine Geschwindigkeit um $a$ erhöht, wenn dist größer als der Sicherheitsabstand ist. Im gegenteiligen Fall wird die Geschwindigkeit um $a$ verringert. Nagel und Schreckenberg erweiterten Rule 184 zu einem mikroskopischen Verkehrssimulationsmodell Fuks und Boccara, 1998. Das Modell konnte sich als Quasi-Standard durchsetzen und wurde vielfach erweitert und für verschiedene Situationen angepasst. Dieser Abschnitt diskutiert das NSM daher detailliert.

Das von Nagel und Schreckenberg entwickelte Nagel-Schreckenberg-Modell (NSM) zerteilt Straßen in Zellen mit einer Länge von je 7,5 m. Diese Zellenlänge repräsentiert die Länge eines Autos (ca. 4,5 m) und je etwas Raum vor und hinter dem 


\begin{tabular}{|l|c|c|c|c|c|c|}
\hline$v[$ Zellen $/ \mathrm{s}]$ & 0 & 1 & 2 & 3 & 4 & 5 \\
\hline$v[\mathrm{~km} / \mathrm{h}]$ & 0 & 27 & 54 & 81 & 108 & 135 \\
\hline
\end{tabular}

Tabelle 3.4: Mögliche Geschwindigkeiten im NSM

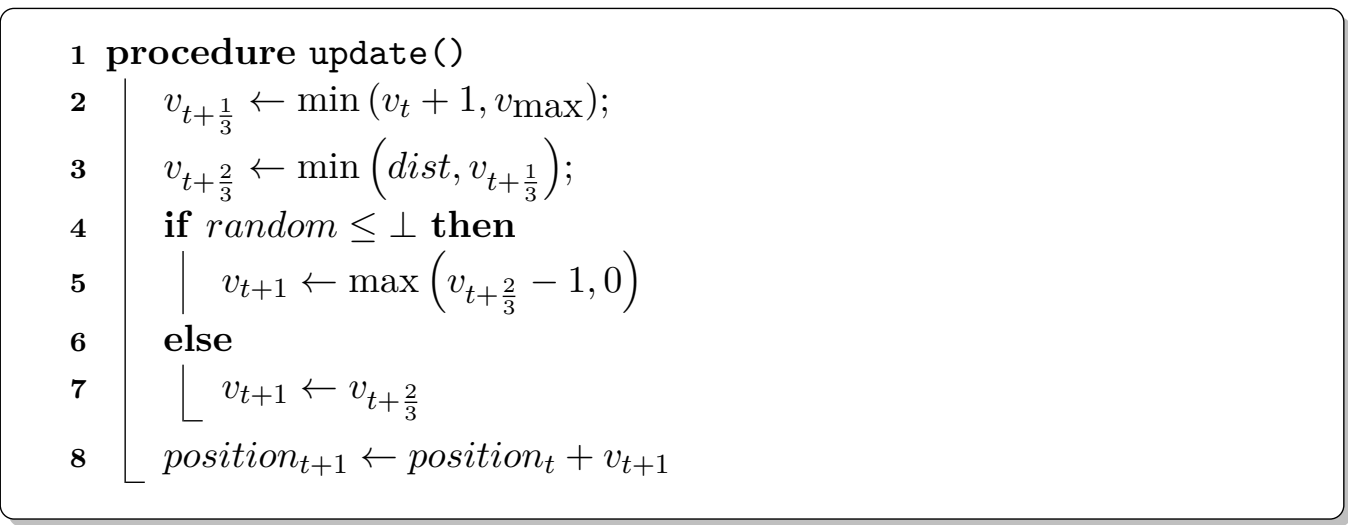

Algorithmus 3.1: Nagel-Schreckenberg-Modell Nagel und Schreckenberg, 1992

Auto. Autos können sich mit Geschwindigkeiten $v\left[\right.$ Zelle $\cdot$ Zeiteinheit $\left.^{-1}\right]$ fortbewegen. Die Maximalgeschwindigkeit der Autos wird in der Regel auf 5 festgelegt.

Das NSM ist zeitdiskret und jeder simulierte Zeitschritt beträgt $1 \mathrm{~s}$ Realzeit, sodass die in Tabelle 3.4 dargestellten Geschwindigkeiten erreicht werden können. In jedem Zeitschritt $t+1$ aktualisieren alle simulierten Autos ihre Zustände, bestehend aus der aktuellen Geschwindigkeit $v_{t}$ und der Position position $t$ parallel nach Algorithmus 3.1 .

In den Zeilen 2 bis 7 wird die Geschwindigkeit des Autos aktualisiert. Zuerst wird in Zeile 2 beschleunigt, sofern nicht bereits die maximale Geschwindigkeit $v_{\max }$ erreicht wurde. Anschließend wird in Zeile 3 die Geschwindigkeit gegebenenfalls auf den Abstand zum Vordermann dist verringert. Dieser Schritt garantiert die Kollisionsfreiheit - es finden keine Unfälle statt. Mit einer bestimmten Wahrscheinlichkeit $\perp$ wird in den Zeilen 4 und 5 gebremst (getrödelt). Der Parameter $\perp$ liegt in den meisten Arbeiten im Bereich von 0, 3. Abschließend wird das Auto um seine neue Geschwindigkeit verschoben. Das grundlegende Verfahren wird in Abbildung 3.3 verdeutlicht.

Abbildung 3.4 ermöglicht einen einfachen Vergleich zum zellulären Automaten Rule 184. Wenn $\perp=0$, sowie $v_{\max }=1$ gesetzt wird, so ergibt sich Rule 184 als Spezialfall von NSM.

Das beschriebene Modell wurde breit untersucht und seine Eignung zur realis- 


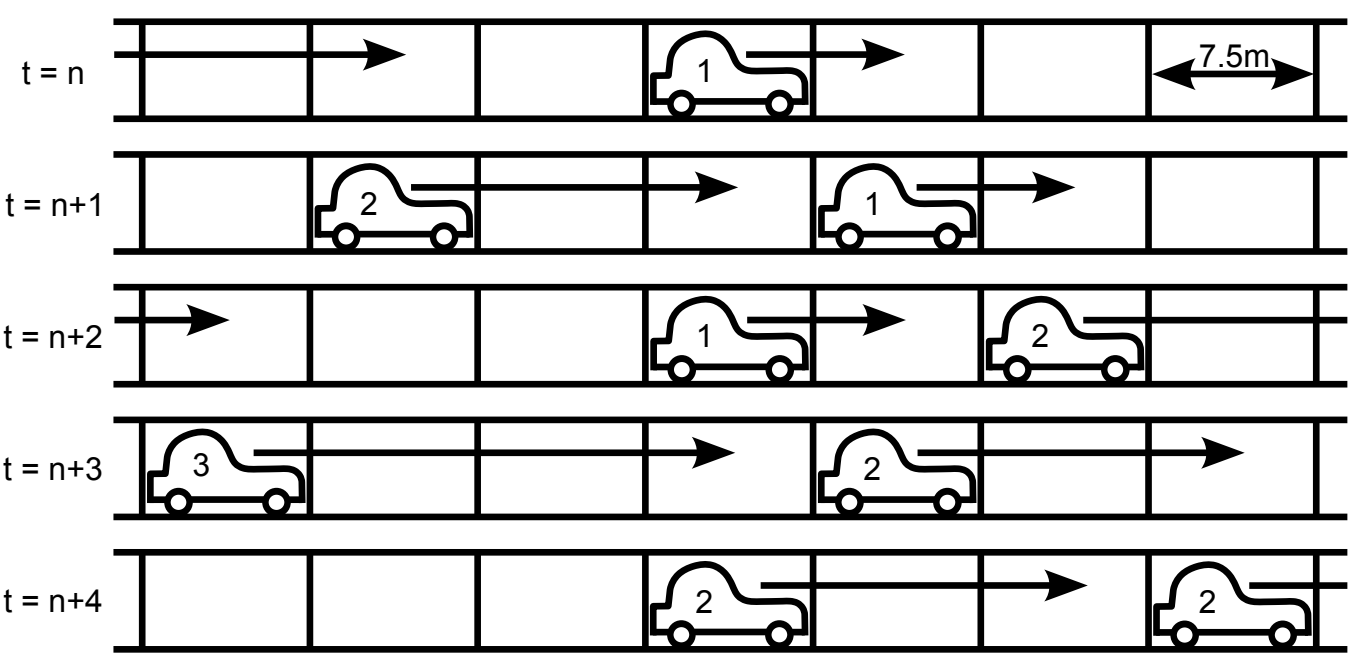

Abbildung 3.3: Nagel-Schreckenberg-Modell (NSM). Die Autos sind jeweils mit ihrer neuen Geschwindigkeit beschriftet. Das einzige zum Zeitpunkt $n$ sichtbare Auto trödelt in Zeitpunkt $n+1$ - es hätte beschleunigen können, da der Sicherheitsabstand zu einem vorausfahrenden Fahrzeug eine Beschleunigung nicht behindert hätte.

tischen Abbildung des Autobahnverkehrs konnte gezeigt werden Wagner, 1995. Mit Hilfe des NSM konnte erstmals eine Simulation des gesamten Autobahnverkehrs für Deutschland in Echtzeit unter Verwendung einer Mikrosimulation realisiert werden Rickert und Wagner, 1996, Rickert et al., 1996. Das NSM war das erste Verkehrsmodell, welches einen Stau ohne äußere Einflüsse entstehen lassen konnte („Stau aus dem Nichts“). Im Folgenden werden Erweiterungen und Modifikationen besprochen, die das Modell realistischer machen und die Verwendung für innerstädtische Szenarien ermöglichen.

Die in Tabelle 3.4 aufgezählten erreichbaren Geschwindigkeiten innerhalb des NSM stellen eine Einschränkung des Ansatzes dar. Für innerstädtische Szenarios wird in Esser und Schreckenberg, 1997 eine Maximalgeschwindigkeit von $v_{\max }=2$ vorgeschlagen ${ }^{1}$ In Fouladvand et al., 2005 wird die Zellengröße auf 5,6 m verringert, um eine feinere Granularität der Geschwindigkeiten zu erreichen. Zusätzlich wird die Simulationsgeschwindigkeit halbiert, indem zwei Simulationsiterationen eine Sekunde Realzeit simulieren. Es entstehen Geschwindigkeitsschritte von 10, 08 km/h. Die Zellengröße kann bei geeigneter Kalibrierung des Systems beliebig klein werden Krauss et al., 1996. Die Zellengröße von $0,5 \mathrm{~m}$ hat sich als weiterer Standard etabliert und wird als Kerner-Klenov-Wolf-Modell (KKW-Modell) bezeichnet Kerner et al., 2002.

\footnotetext{
${ }^{1} v_{\max }=2$ Zellen $\cdot \mathrm{s}^{-1} \stackrel{\wedge}{=} 54 \mathrm{~km} / \mathrm{h}$
} 


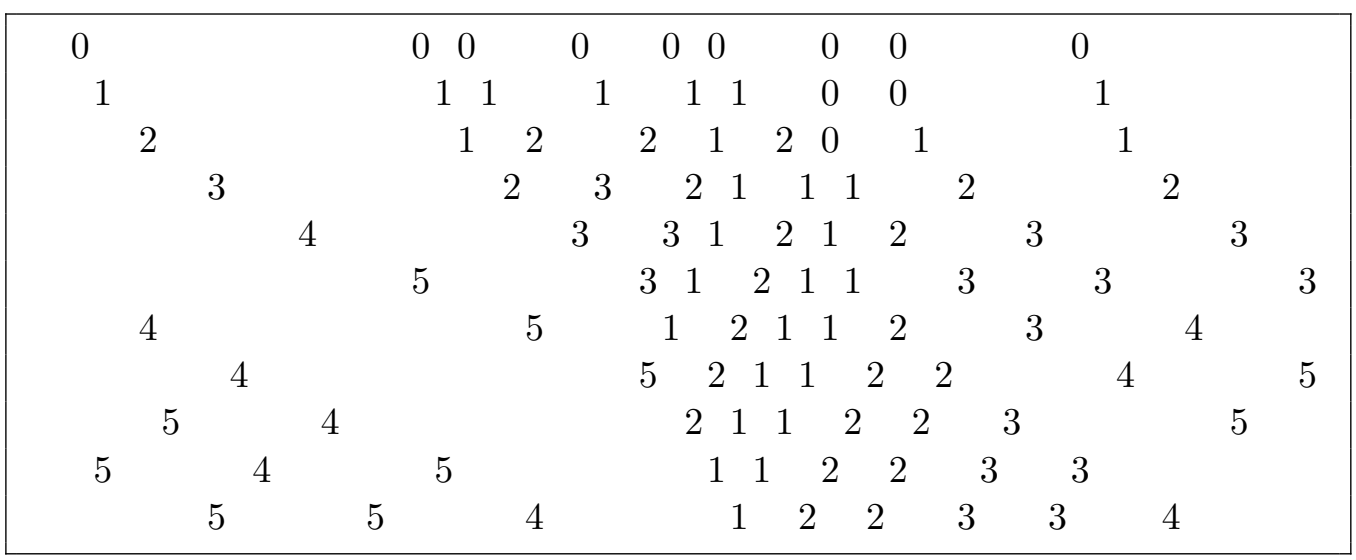

Abbildung 3.4: Beispielsequenz: NSM. Gestartet wurde der Durchlauf mit stehenden Autos. Die Ziffern geben die jeweiligen Geschwindigkeiten an. Die Wahrscheinlichkeit zu trödeln wurde auf 0,3 gesetzt. Es ist zu sehen, dass das Auto, das links gestartet ist, bei freier Fahrt bis zur Maximalgeschwindigkeit 5 beschleunigt hat, anschließend auf eine Gruppe anderer Autos auffuhr und deshalb die Geschwindigkeit senken musste. Das Auto, das ganz rechts gestartet ist, trödelte zweimal während des Durchlaufs.

Durch Verkehrsbeobachtungen konnte ermittelt werden, dass Autofahrer dazu tendieren, mit einer höheren Wahrscheinlichkeit zu trödeln, wenn sie $v=0$ haben, als wenn sie fahren. Es wurde in [Takayasu und Takayasu, 1993] eine Startverzögerung eingeführt, deren Einfluss in Helbing, 1997a bestätigt wurde. In das NSM kann diese Erweiterung trivial integriert werden, indem der konstante Faktor $\perp$ durch eine Funktion $\perp(v)$ ersetzt wird Knospe et al., 2000. Bisher antizipieren simulierte Autofahrer nicht, wie sich der Verkehr entwickeln wird. Eine einfache Erweiterung des Modells um das Bremslicht des Vordermanns bewirkt eine bessere Anpassung an Messdaten Hafstein et al., 2003. Die Erweiterung kann in das Modell durch eine weitere Anpassung der $\perp(v)$-Funktion integriert werden.

Das Modell muss auf mehrere Spuren erweitert werden. Hierfür werden Spurwechselregeln benötigt. In Nagel et al., 1998, Knospe et al., 2002 wird der Spurwechsel in zwei Phasen getrennt. Zuerst muss geprüft werden, ob ein Spurwechsel erwünscht ist. Ein Spurwechsel nach links ist erwünscht, wenn ein Vordermann auf der aktuellen oder linken Spur langsamer als die eigene Wunschgeschwindigkeit fährt 2. Nun muss geprüft werden, ob ein Spurwechsel nach links möglich ist. Hierfür muss eine ausreichend große Lücke vorhanden sein. Nach vorne muss der Sicherheitsabstand eingehalten werden. Ein von hinten herannahendes Fahrzeug muss seine Bewegung durchführen können, ohne seinerseits durch den eigenen Spurwechsel einen zu geringen Sicherheitsabstand zu erhalten. Der Wunsch nach

\footnotetext{
${ }^{2}$ Es ist auf deutschen Straßen in der Regel nicht erlaubt, rechts zu überholen.
} 
rechts zu wechseln entsteht, wenn es keinen Grund mehr gibt, links zu fahren (Rechtsfahrgebot). Es darf also weder auf der linken, noch auf der rechten Spur ein Fahrzeug geben, dass derzeit überholt werden soll. Das Sicherheitskriterium wird abermals durch das Vorhandensein einer ausreichenden Lücke definiert. Das Vorbeifahren auf der rechten Spur wird ab einer fixen Geschwindigkeit und darunter ermöglicht, um die volle Nutzung aller Spuren zu ermöglichen, wenn der Verkehr stockt Wagner et al., 1996.

Das NSM ist kollisionsfrei. Um Unfälle zu modellieren, wurde ein Unaufmerksamkeits- bzw. Leichtsinnigkeitsfaktor $\perp^{\prime}$ eingeführt, der äquivalent zur Trödelwahrscheinlichkeit $\perp$ angewendet wird Boccara et al., 1997. In jeder Iteration ist jedes simulierte Auto $k$ mit der Wahrscheinlichkeit $\perp^{\prime}$ unaufmerksam und nimmt an, dass der Vordermann $k+1$ seine Geschwindigkeit zum Zeitpunkt $t$ beibehalten hat (siehe Gleichung 3.8),

$$
v(k+1, t)=v(k+1, t-1)
$$

ohne dies zu überprüfen. Falls $v(k, t)<v_{\max }$, beschleunigt Auto $k$ in diesem Fall leichtsinnig und kann somit in Iteration $t$ seine Geschwindigkeit um maximal zwei Geschwindigkeitsschritte erhöhen, da diese Beschleunigung entkoppelt vom Beschleunigungsschritt im NSM geschieht. Es sei $d(k, t)$ die Distanz zwischen den Autos $k$ und $k+1$. Falls die Bedingungen der Gleichung 3.9 erfüllt sind, findet mit Wahrscheinlichkeit $\perp^{\prime}$ zum Zeitpunkt $t$ ein Unfall statt.

$$
d(k, t-1) \leq v_{\max } \quad v(k+1, t-1)>0 \quad v(k+1, t)=0
$$

Abbildung 3.5 verdeutlicht das Auftreten von Unfällen anhand der von Boccara et al. festgesetzten Maximalgeschwindigkeit $v_{\max }=3$.

Die in Gleichung 3.9 definierten Regeln sind ungenau, da sie das in Abbildung 3.6 dargestellte Beispiel als Unfall klassifizieren würden, obwohl Auto $k$ nicht auf $k+1$ auffährt. In Jjiang et al., 2003 wurden die Regeln daher, wie in Gleichung 3.10 gezeigt, modifiziert. 


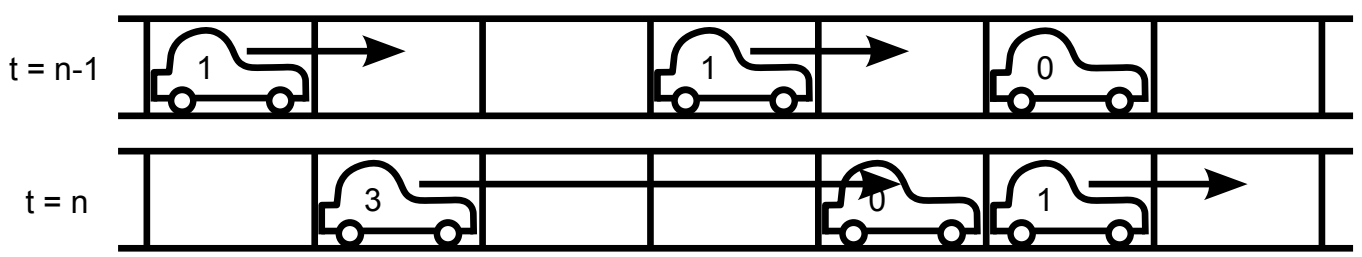

Abbildung 3.5: NSM: Unfälle nach Boccara et al. Die Autos seien von links nach rechts: $k, k+1, k+2$. In Zeitschritt $t=n-1$ beträgt $d(k, n-1)=3=v_{\max }$. Auto $k$ ist in Zeitschritt $t$ unaufmerksam und beschleunigt zweifach. Auto $k+1$ hingegen wird durch Auto $k+2$ behindert und kommt zum Stillstand. Ein Unfall entsteht.

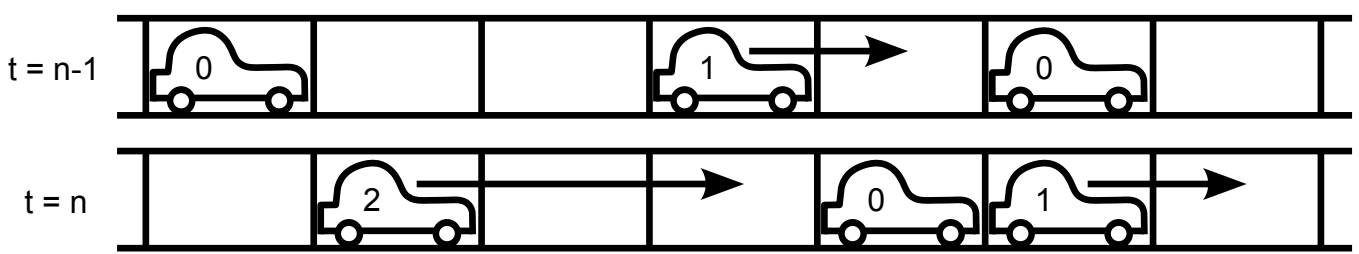

Abbildung 3.6: NSM: Unfälle nach Boccara et al.: Falscher Alarm.

$$
v(k, t-1) \geq d(k, t-1)-1 \quad v(k+1, t-1)>0 \quad v(k+1, t)=0
$$

Unfälle bei geringen Verkehrsdichten und hohen Geschwindigkeitsdifferenzen, ohne dass $v(k+1, t)=0$, werden durch das Modell nicht erzeugt. Die Beschleunigung im Unaufmerksamkeitsfall kann maximal $2 \cdot 7,5 \mathrm{~m} \cdot \mathrm{s}^{-2}$ betragen. Dieser Wert ist unrealistisch hoch. In Moussa, 2003 wird der zweite Beschleunigungsschritt daher entfernt. Ein Unfall entsteht in diesem Modell, falls $k$ unaufmerksam ist und die Bedingungen in Gleichung 3.11 erfüllt sind. Auto $k$ fährt nach dieser Regel zum Zeitpunkt $t$ auf ein stehendes Fahrzeug auf, das zum Zeitpunkt $t-1$ noch fuhr.

$$
\tau \cdot v(k, t-1)>d(k, t-1) \quad v(k+1, t-1)>0 \quad v(k+1, t)=0
$$

Der Faktor $\tau$ bezeichnet die Reaktionszeit, die im Falle einer Unaufmerksamkeit $\tau>1$ wird. Zusätzlich entsteht ein Unfall, wenn die Gleichungen 3.12 und 3.13 erfüllt sind und Auto $k$ unaufmerksam ist.

$$
\tau \cdot v(k, t-1)>d(k, t-1)+v(k+1, t)
$$




$$
v(k+1, t-1)-v(k+1, t) \geq v_{d}
$$

Der Unfall entsteht in diesem Fall durch starkes Abbremsen von Auto $k+1$ über das Bremsmaximum $v_{d}$ hinweg, bis zu dem keine Unfallgefahr bestand.

Einen anderen Weg, die Kollisionsfreiheit des NSM aufzuweichen, geht Lee et al., 2004. Die Bremsleistung der Autos wird hierbei begrenzt, sodass das Verhalten der Fahrzeuge grundlegend überarbeitet werden muss. Jedes Fahrzeug geht stets davon aus, dass sein Vordermann unvermittelt bremsen könnte und hält die Geschwindigkeit in Abhängigkeit zu einer komplexen Ungleichung so, dass sicheres Fahren garantiert ist. In Abhängigkeit zu den Verkehrsbedingungen neigen simulierte Autofahrer dazu, sich zu verschätzen, woraus Unfälle resultieren. Eine vereinfachte Version dieses Vorgehens wird in Li et al., 2009 vorgestellt. Wenn Auto $k$ schneller als Auto $k+1$ fährt, bremst es. Das Modell verwendet eine in Yang und Ma, 2002 vorgestellte Erweiterung zu den Regeln aus Gleichung 3.9. um Unfälle zu simulieren. Unfälle, die entstehen, wenn Auto $k+1$ zum Zeitpunkt $t+1$ nicht steht, können durch dieses Modell nicht abgedeckt werden.

Für innerstädtischen Verkehr wurde das NSM von verschiedenen Arbeitsgruppen angepasst und erweitert. Das Abbiegeverhalten von Autofahrern wurde für Kreisverkehre Wang und Liu, 2005, mehrspurige Kreisverkehre Wang und Ruskin, 2006 und Kreuzungen ohne Ampeln Liu et al., 2005 untersucht. Kreisverkehre werden explizit in Zellen unterteilt. Es müssen jeweils Sicherheitsabstände zu anderen Verkehrsteilnehmern gewahrt werden, deren Größen in den vorgeschlagenen Modellen vom internen Zustand der simulierten Autofahrer abhängen. Normalverteilt um einen Standardwert tolerieren die Autofahrer hierbei größere und kleinere Sicherheitsabstände, die sie anschließend einhalten. Dieses Verhalten lässt sich als Risikobereitschaft beschreiben.

Das NSM wurde in verschiedenen Arbeiten zur Optimierung von Ampelschaltungen verwendet Chowdhury und Schadschneider, 1999. Brockfeld et al., 2001, Fouladvand und Radja, 2001, Fouladvand et al., 2005, Neumann und Wagner, 2008, Bazzan et al., 2008, Bazzan, 2009. Einen Abriss über verschiedene Varianten des NSM gibt Knospe et al., 2004. 


\subsubsection{Diskussion}

In diesem Abschnitt wurden zwei wichtige Modellkategorien aus dem Bereich der mikroskopischen Verkehrssimulationsmodelle vorgestellt. Es existiert zusätzlich die Gruppe der Warteschlangenmodelle. Ein prominenter Vertreter ist das in Gawron, 1998 vorgestellte Modell. Warteschlangenmodelle zeichnen sich durch einen sehr geringen Berechnungsaufwand aus. Heterogene Autos und multimodaler Verkehr werden jedoch nicht modelliert. Weitere abstrakte Ansätze sind nach Schnieder und Becker, 2007 beispielsweise stochastische Petrinetze, sowie Telekommunikations- und Rechnernetze, die jedoch nicht das individuelle Verhalten von Verkehrsteilnehmern abbilden. Sie eignen sich daher nicht für den Rahmen dieser Arbeit. Einen Überblick über weitere Modelle geben Brackstone und McDonald, 1999, Chowdhury et al., 2000.

Fahrzeugfolgemodelle können realistisches Fahrverhalten detailliert abbilden, haben jedoch eine hohe Berechnungskomplexität. ZA-Modelle bilden den Gegenpol: Der Berechnungsaufwand ist sehr gering, das Fahrverhalten ist hingegen einfacher gehalten und viele Details können nicht modelliert werden. Es ist beispielsweise nur bei infinitesimal kleinen Zellen möglich, kontinuierliche Geschwindigkeiten zu ermöglichen. Dies stellt die Nutzung von ZA in Frage, da die Berechnungseffizienz stark darunter leidet.

Im Rahmen dieser Arbeit soll ein raumkontinuierliches Verkehrsmodell auf Basis des NSM entwickelt werden, welches sowohl zur Simulation von Autobahnverkehr, als auch innerstädtischem Verkehr nutzbar ist. Das NSM bietet sich hierfür als Vorlage an, da es sehr gut untersucht wurde und in der Forschung häufig eingesetzt wird. Hierfür werden die grundlegenden Prinzipien aus Abschnitt 3.1.2 erweitert. Von Zellen soll abstrahiert werden, um kontinuierliche Geschwindigkeiten modellieren zu können und somit unterschiedliche Arten von Verkehrsteilnehmern auf den selben Straßen simulieren zu können. High-Fidelity-Modelle, wie das Wiedemann-Modell, aber auch einfachere Fahrzeugfolgemodelle, wie das IDM, sind in der Berechnungseffizienz unterlegen. Dieser Aspekt zeigt sich ebenfalls in den im Kapitel 4 besprochenen Verkehrssimulationssystemen. Je detaillierter das verwendete Simulationsmodell ist, desto weniger Verkehrsteilnehmer können simuliert werden. 


\subsection{Fahrradmodelle}

Fahrräder sind eine Verkehrsteilnehmergruppe, die in bestehenden Simulationssystemen überwiegend vernachlässigt wird. Um ein realistisches Fahrradverkehrsmodell zu erstellen, muss analysiert werden, wie Fahrradfahrer sich im Straßenverkehr verhalten.

In Plate et al., 2001 wird untersucht, wie Anreize für eine weitergehende Nutzung von Fahrrädern geschaffen werden können. Dies könnte zu einer energieeffizienteren Fortbewegung führen. Wichtig hierfür ist es, die Wechselwirkungen der verschiedenen Verkehrsteilnehmertypen zu betrachten, um Risiken im Straßenverkehr abzumildern. Die Wahrscheinlichkeit, bei einem Fahrradunfall zu sterben, ist am höchsten, wenn der Unfall mit einem Auto stattfindet Boström und Nilsson, 2001. Das Zusammenspiel zwischen dem Verhalten von Fahrradfahrern und der Einstellung der anderen Verkehrsteilnehmer zu der Gruppe der Fahrradfahrer sind wichtige Faktoren für die Entstehung von Unfällen mit Fahrradfahren Basford et al., 2002.

Wann immer Fahrräder eine gesonderte Spur in Form eines Fahrradweges haben, ist das Unfallrisiko gering. Im Straßenverkehr gemeinsam mit Autos kommen Wechselwirkungen zum Tragen. In der Literatur findet sich eine Gruppe von Veröffentlichungen, die das Verhalten von Fahrradfahreren an Ampeln untersuchen. Johnsen et al. haben 5.420 Fahrradfahrer an Ampeln videoüberwacht. Insgesamt $11 \%$ der Fahrradfahrer haben rote Ampeln missachtet. Diese Gruppe wird in drei Untergruppenruppen unterteilt [Johnson et al., 2008:

Rennfahrer (25 \%): Fahrradfahrer, die an einer gelben Ampel beschleunigen und anschließend bei rot die Ampel überfahren.

Ungeduldig (33 \%): Fahrradfahrer, die an einer roten Ampel zunächst halten und warten. Anschließend werden sie ungeduldig und suchen nach einer Lücke im Verkehr, um dennoch die Ampel zu passieren. Die Wartezeiten liegen zwischen 2 und $60 \mathrm{~s}(\mu=25 \mathrm{~s}, \sigma=16 \mathrm{~s})$. In $23 \%$ der dokumentierten Ungeduldigkeitsfälle war kein kreuzender Verkehr vorhanden.

Roller (42 \%): Fahrradfahrer, die bei einer roten Ampel sofort nach einer Lücke im Verkehr suchen und diese auch nutzen. Überquerungsdauer zwischen 7 und $24 \mathrm{~s}(\mu=14,6 \mathrm{~s}, \sigma=3,9 \mathrm{~s})$.

In einer späteren Studie haben $7 \%$ der Fahrradfahrer rote Ampeln missachtet Johnson et al., 2011. In Johnson et al., 2009 wird ein umfassender Überblick 


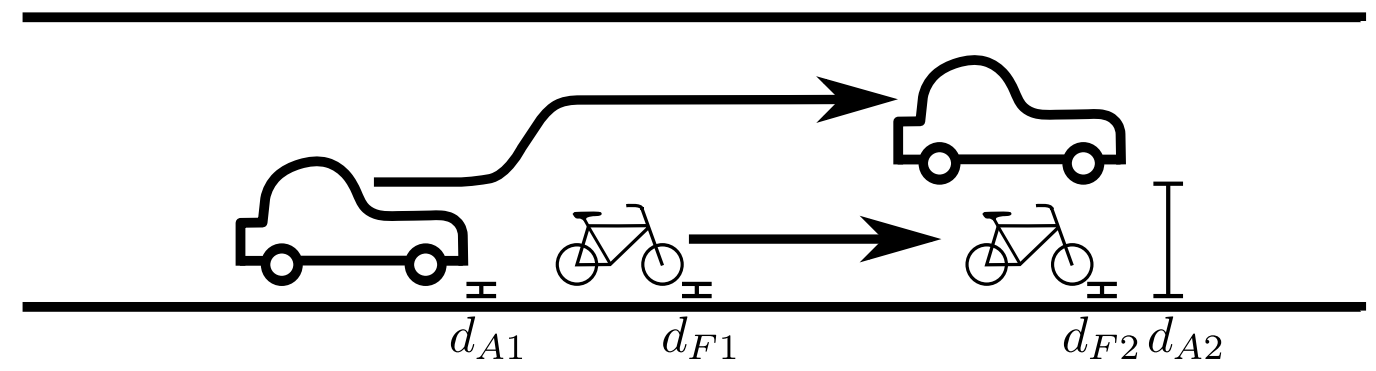

Abbildung 3.7: Überholmanöver: Das überholende Auto hat zuerst einen Abstand $d_{A 1}$ zur Bordsteinkante und schert von seiner Spur aus, um das langsamere Fahrrad zu überholen. Dies führt zu einem Abstand von $d_{A 2}$. Das Fahrrad behält seinen Abstand in etwa bei $d_{F 1} \approx d_{F 2}$.

über das Verhalten von Fahrradpulks gegeben. Dies sind Gruppen von Fahrrädern, die in zwei Reihen fahren. Im Rahmen dieser Arbeit kann dieser Aspekt vernachlässigt werden, da er zu selten in der Realität auftritt.

Oftmals stehen Radfahrern gesonderte Radwege zur Verfügung. Diese können durch eine aufgemalte Spur auf der regulären Straße gekennzeichnet sein oder als separater Pfad zur Verfügung gestellt werden. Die Nutzung von Radwegen hat den Vorteil einer erhöhten Fahrsicherheit. Der Nachteil kann eine längere Reisedauer oder größere Anstrengungen sein. In Larsen und El-Geneidy, 2011 werden Radfahrer nach ihrer Fahrhäufigkeit unterteilt. Freizeitfahrer, die selten Fahrrad fahren, nutzen demnach meist Radwege. Alle anderen Gruppen, vom Freizeitfahrer, der regelmäßig fährt bis zum Berufsfahrer (z.B. Fahrradcourier), nutzen Fahrradwege entweder gar nicht oder nur, wenn sie dadurch keine Umwege in Kauf nehmen müssen.

Das Wechselspiel zwischen Fahrrädern und Autos kommt auch immer dann zum Tragen, wenn Fahrräder langsamer als Autos fahren und Autos Fahrräder überholen möchten. In [Walker, 2007] wird untersucht, welchen Einfluss verschiedene Faktoren auf den seitlichen Abstand haben, den Autos zu Fahrrädern während Überholmanövern halten. Abbildung 3.7 zeigt exemplarisch ein Überholmanöver.

Wenn Fahrradfahrer ohne Helm am Straßenrand fahren, halten Autos durchschnittlich eine Distanz von ca. 1,45 m zum Fahrrad. Diese sinkt mit steigendem $d_{F}$, was sich auf approximativ konstante Überholabstände $d_{A 2}$ zurückführen lässt. Die Abstände von Autos zu Fahrradfahrern sinken, wenn diese einen Helm tragen. Der Abstand zu Frauen ist hingegen größer, als zu Männern Walker, 2007.

Der Bereich der Fahrradsimulationsmodelle findet in der Wissenschaft geringere Beachtung, als der Bereich der Automodelle. In Gould und Karner, 2009 wird 
ein ZA-Modell vorgeschlagen, welches identisch zum NSM ist. Ein Weg wird als mehrspurige Folge von Zellen modelliert. Das Modell wurde im Hinblick auf das Fundamentaldiagramm kalibriert und mit Felddaten verglichen. Interaktion mit Fußgängern und Pkw wurden nicht berücksichtigt. Ein ähnliches Modell wird in Jia et al., 2007 vorgeschlagen.

In Vasić und Ruskin, 2011a, Vasić und Ruskin, 2011b wird ein zeitlich und räumlich diskretes Fahrradmodell auf ZA-Basis vorgeschlagen, das ebenfalls auf dem NSM basiert. Es werden hierbei jedoch grundlegende Verhaltensweisen für urbanen Verkehr und Wechselwirkungen mit Autos berücksichtigt. Die Zellengrößen für Autos sind 7,5 m; für Fahrräder 3,75 m Vasić und Ruskin, 2012. Die Autoren haben ihr Modell ausführlich evaluiert. Es wurde deutlich, dass der Modellierungsaufwand für Straßennetzwerke im beschriebenen System berücksichtigt werden muss. Es wurden keine Szenarien mit einem Straßennetzwerk der realen Welt simuliert.

In Faghri und Egyháziová, 1999 wird ein Fahrzeugfolgemodell verwendet, welches auf die unterschiedlichen Bedingungen für Pkw, Lkw, Busse und Fahrräder angepasst wird. Der Vorteil dieses Ansatzes gegenüber den ZA-Modellen ist, dass kontinuierliche Geschwindigkeiten und somit individuelle Fahreigenschaften simuliert werden könnten, auch wenn dies im vorgeschlagenen Modell nicht geschieht, da alle Fahrräder eine maximale Beschleunigung von $a=1,52 \mathrm{~m} / \mathrm{s}^{2}$ erhalten.

Ein ähnlicher Ansatz wird in Guo et al., 2011 beschrieben. Das verwendete Modell wird in Guo et al., 2012 genutzt, um den Einfluss von Fahrradfahrern auf den Straßenverkehr zu ermitteln. Fahrräder erhielten in einem synthetischen Szenario eine eigene Spur. Mit unterschiedlichen Wahrscheinlichkeiten verlassen Fahrräder diese Spur und wechseln auf die Spur für Autos. Dieses Verhalten soll Hindernisse auf dem Weg modellieren. Mit steigender Wahrscheinlichkeit nimmt der Einfluss der Fahrräder auf die Geschwindigkeiten der Autos zu.

\subsection{Fußgängermodelle}

Die Modellierung von Fußgängern zur Simulation von urbanen Szenarien wurde in der Vergangenheit vernachlässigt Ishaque und Noland, 2008. Dies resultiert einerseits daraus, dass die Verkehrssimulationen für Autobahnszenarien entwickelt wurden und sich dieser Simulationszweig von dort aus weiter entwickelt hat. Andererseits erreichten Verkehrssimulationen früher nicht die feine Granularität, die benötigt wird, um Fußgänger zu modellieren Batty, 2001. Durch gestiegene Rechnerkapazitäten können heute auch Fußgänger in Verkehrssimulationen integriert 


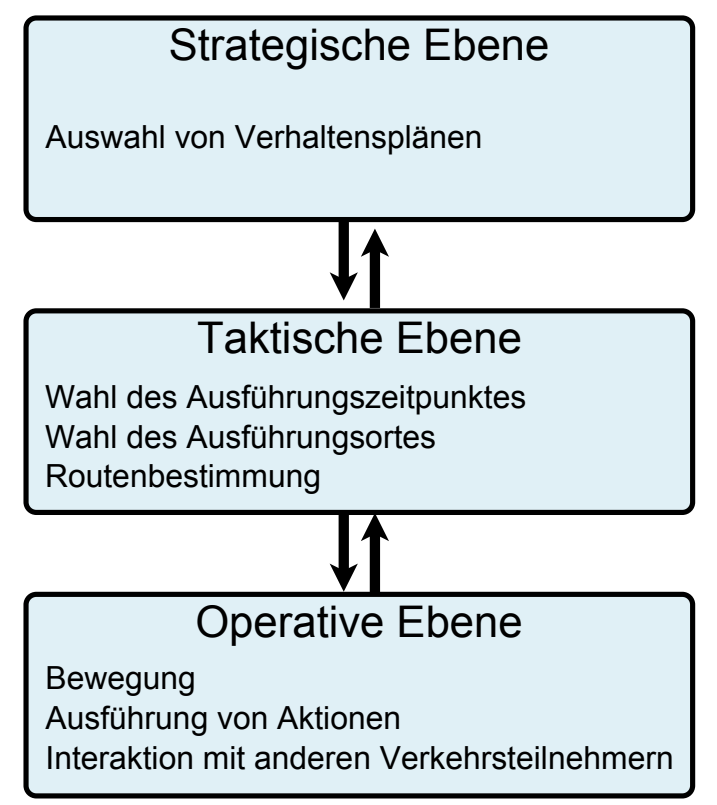

Abbildung 3.8: Verhaltensmodellierungsebenen für Fußgänger, adaptiert nach Daamen, 2004.

werden. Hierfür werden geeignete Modelle benötigt.

Fußgängermodelle können auf unterschiedlichen Ebenen definiert werden. Abbildung 3.8 zeigt eine adaptierte Version der in Hoogendoorn et al., 2002, Daamen, 2004 vorgestellten Einteilung. Fußgänger werden hierbei als Agenten modelliert.

Auf der strategischen Ebene trifft ein Agent grundlegende Entscheidungen, z.B. ob er von seiner aktuellen Position aus zu einer anderen Position laufen soll oder besser mit dem Auto fährt. Wenn er sich für den Fußweg entscheidet, muss er anschließend einen taktischen Plan ausarbeiten. Er muss entscheiden, wann er los läuft und welchen Weg er verwendet. Die operative Ebene betrifft die Durchführung des Planes. Der Agent muss sich im Straßenverkehr bewegen und versuchen, sein Ziel zu erreichen. Die Modellierung des Fußgängerverhaltens kann auf unterschiedlichen Abstraktionsebenen geschehen.

Im Rahmen dieser Arbeit liegt der Fokus auf den Wechselwirkungen der verschiedenen Verkehrsteilnehmertypen untereinander. Im Bereich der Fußgängersimulation sind somit die gelaufenen Geschwindigkeiten (Abschnitt 3.3.1), das Verhalten bei der Überquerung von Straßen (Abschnitt 3.3.2 und Regelverstöße (Abschnitt 3.3.3 wichtige Faktoren (taktische und operative Ebene von Abbildung 3.8). Anschließend beschreibt Abschnitt 3.3 .4 bestehende Fußgängermodelle. 


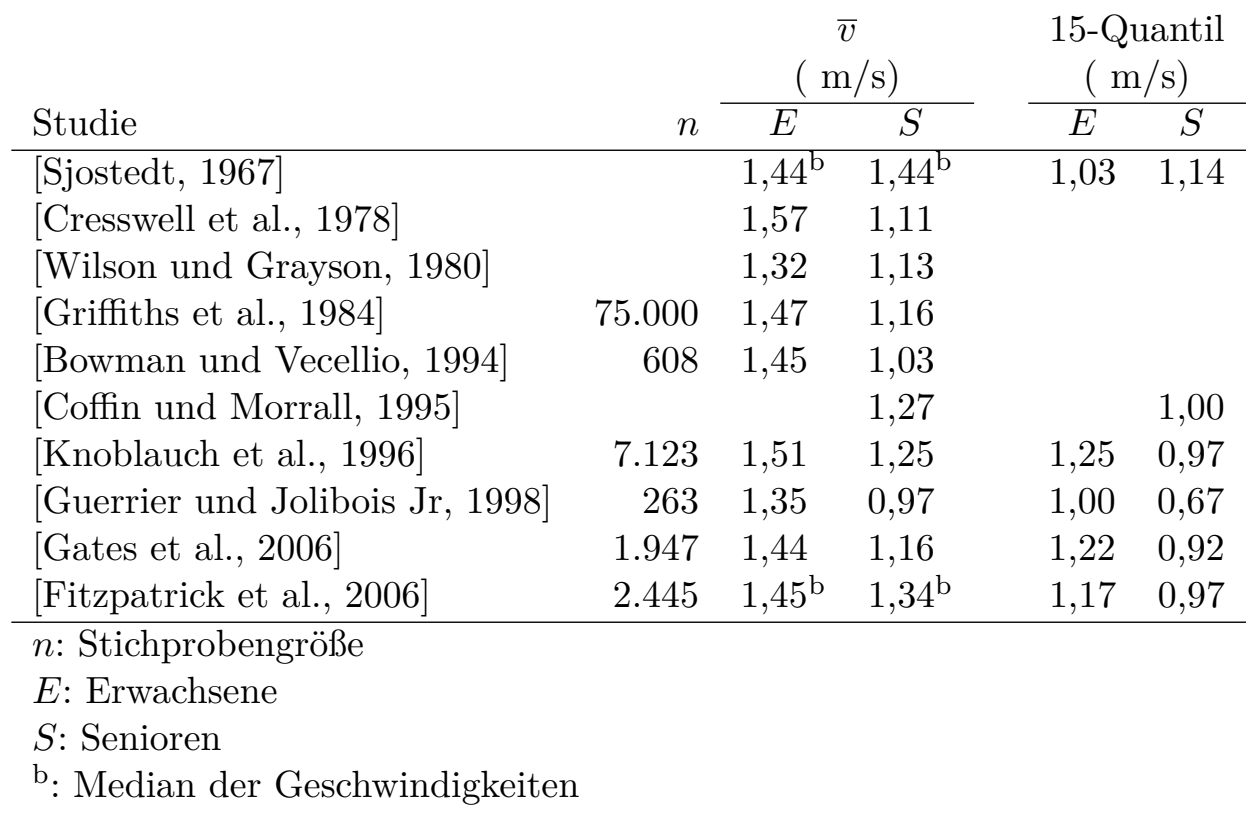

Tabelle 3.5: Fußgängergeschwindigkeiten an Kreuzungen: Erwachsene und Senioren. Nach [shaque und Noland, 2008.

\subsubsection{Laufgeschwindigkeiten}

Es wurden in der Vergangenheit verschiedene Studien zur Bestimmung von Fußgängergeschwindigkeiten durchgeführt. In der Literatur werden Fußgänger zwischen Erwachsenen und Senioren getrennt. Die Ergebnisse einer Studienauswahl über Fußgängergeschwindigkeiten an Straßenkreuzungen werden in Tabelle 3.5 gezeigt.

Die Studien beziehen sich auf die gemessenen Geschwindigkeiten von Fußgängern bei der Überquerung von Straßen und wurden in verschiedenen Ländern durchgeführt. Als Kenngrößen werden die mittleren Geschwindigkeiten der beiden Gruppen, sowie das 15-Quanti $\left.\right|^{3}$ hierzu verwendet. Es ist ersichtlich, dass ältere Menschen langsamer laufen, als jüngere. Die Studien geben unterschiedliche Grenzen zwischen erwachsen und senior.

Die Messungen fanden mit unterschiedlichen Fußgängerdichten statt. Es ist anzunehmen, dass die Fußgängergeschwindigkeiten sich auch je nach Ort unterscheiden. Tabelle 3.5 gibt dennoch einen Überblick über die Spannweite der Fußgängergeschwindigkeiten an Kreuzungen. In den Studien wurden teils weitere Unterschei-

${ }^{3}$ Das 15-Quantil ist der Wert $w$ einer Stichprobe, bei dem $15 \%$ der Einträge der Stichprobe kleiner oder gleich $w$ sind. Siehe Steland, 2010, S. 36ff]. 


\begin{tabular}{|c|c|c|}
\hline Studie & Ort & $\bar{v}[\mathrm{~m} / \mathrm{s}]$ \\
\hline Older, 1968 & London, UK & 1,3 \\
\hline $\mid \overline{\text { Hoel, } 1968}$ & Pittsburg, USA & $1,5^{\mathrm{m}}, 1,41^{\mathrm{w}}$ \\
\hline O'Flaherty und Parkinson, 1972 & Leeds, UK & $1,5^{\mathrm{m}}, 1,36^{\mathrm{w}}$ \\
\hline Tanaboriboon et al., 1986 & Singapur & $\begin{array}{l}1,32^{\mathrm{m}}, 1,15^{\mathrm{w}} \\
1,27^{\mathrm{s}}, 1,23^{\mathrm{e}}, 0,9^{\mathrm{a}}\end{array}$ \\
\hline Tanaboriboon und Guyano, 1991 & Bangkok, Tailand & $\begin{array}{l}1,17^{\mathrm{w}}, 1,27^{\mathrm{mk}} \\
0,85^{\mathrm{ma}}, 0,8^{\mathrm{wa}}\end{array}$ \\
\hline Willis et al., 2004 & York, Edinburgh, UK & $\begin{array}{l}1,52^{\mathrm{m}}, 1,42^{\mathrm{w}}, \\
1,16^{\mathrm{a}}, 1,55^{\mathrm{e}}, 1,53^{\mathrm{k}}\end{array}$ \\
\hline
\end{tabular}

${ }_{\mathrm{m}}$ : männlich
w: weiblich
s: Student
e: erwachsen
k: Kind
a: alt

Tabelle 3.6: Fußgängergeschwindigkeiten auf Gehwegen. Auszug aus Ishaque und Noland, 2008.

dungen nach Geschlecht oder ergänzende Altersstufen eingeführt. Ein Überblick über Fußgängergeschwindigkeiten auf Gehwegen wird in Tabelle 3.6 gegeben.

Teilweise wurden die Geschwindigkeiten zusätzlich nach Uhrzeiten aufgeteilt. Es kam hierbei zu leichten Schwankungen, die jedoch nicht erheblich waren. Weitere Studien haben gezeigt, dass Fußgängergeschwindigkeiten differenzierter zu betrachten sind und der Fokus auf das Verhalten bei der Überquerung von Straßen $\mathrm{zu}$ legen ist. Ishaque beschreibt in Ishaque, 2006 einen Zusammenhang zwischen der Wartedauer von Fußgängern bis zur Straßenüberquerung und der Geschwindigkeit, mit der Fußgänger anschließend die Straße überqueren. Die Geschwindigkeit der Fußgänger legt mit steigender Wartezeit zut Es besteht nach Ishaque, 2006 ein Zusammenhang zwischen der Art des Überwegs und der entstehenden Wartezeit. Fußgänger können Straßen an Zebrastreifen am schnellsten überqueren (Verzögerung durch Straßenüberquerung ca. $4,9 \mathrm{~s}$ ), laufen an diesen allerdings am langsamsten. Ampelgesteuerte Überwege verursachen die längsten durchschnittlichen Überquerungsverzögerungen (ca. 19,9 s) und veranlassen die untersuchten Fußgänger mit hohen Geschwindigkeiten zu laufen. Die maximale Überquerungsgeschwindigkeit tritt bei Straßenüberquerungen ohne Vorfahrt für Fußgänger auf $(\bar{v} \approx 1,8 \mathrm{~m} / \mathrm{s})$. Dies ist auf die Gefahr des Überfahrenwerdens zurückzuführen.

\footnotetext{
${ }^{4}$ Minimale Geschwindigkeit bei Wartezeit $0 \mathrm{~s}: \bar{v} \approx 1,7 \mathrm{~m} / \mathrm{s}$; maximale Geschwindigkeit bei Wartezeit $15 \mathrm{~s}-20 \mathrm{~s}: \bar{v} \approx 2,2 \mathrm{~m} / \mathrm{s}$
} 


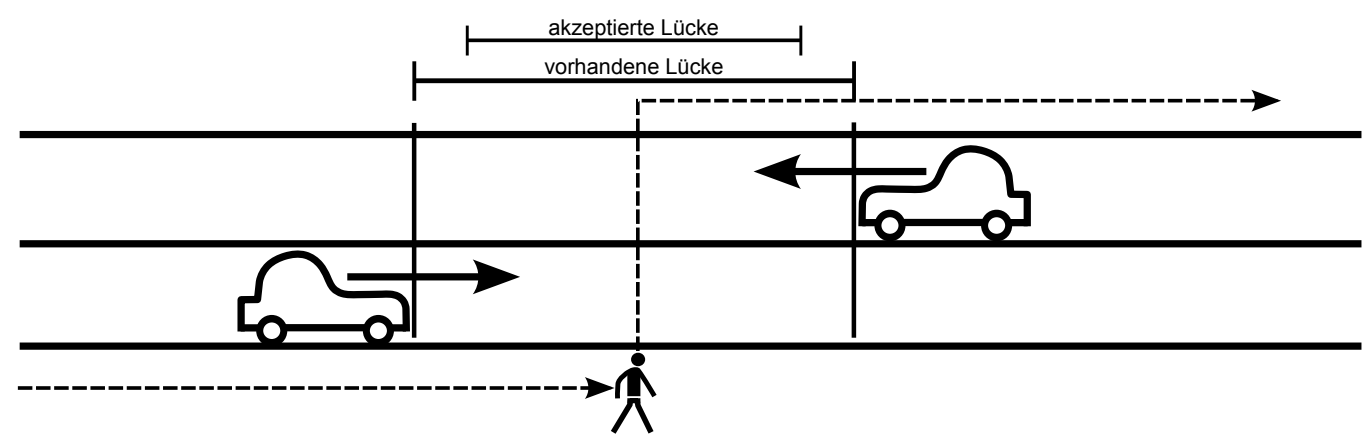

Abbildung 3.9: Straßenüberquerung bei einer Lücke im Verkehr: Fußgänger akzeptieren unterschiedliche Lückengrößen und gehen bei der Straßenüberquerung inkongruente Risiken ein.

\subsubsection{Mut zur Lücke}

Fußgänger überqueren Straßen, wenn sie eine Lücke im Verkehr vorfinden, die sie für ausreichend halten Hamed, 2001 (siehe Abbildung 3.9.

Untersuchungen zum Straßenüberquerungsverhalten im Hinblick auf Alter und Geschlecht werden im medizinischen und psychologischen Bereich durchgeführt (vgl. Holland et al., 2009). Im Rahmen dieser Arbeit genügt die Tatsache, dass unterschiedliches Verhalten vorhanden ist und welche Wahrscheinlichkeiten, in Abhängigkeit zur Lückengröße die Straße zu überqueren, die Realität widerspiegeln.

Himanen und Kulmala haben untersucht, mit welchen Wahrscheinlichkeiten Fußgänger an einem Fußgängerüberweg ohne explizite Vorfahrt für Fußgänger 5 warten oder weiter laufen Himanen und Kulmala, 1988. Sie konnten Unterschiede zwischen verschiedenen Orten feststellen und beeinflussende Faktoren extrahieren (z.B. die Verkehrsdichte auf der Straße). Verallgemeinerbare Wahrscheinlichkeiten konnten nicht ermittelt werden.

In Ottomanelli et al., 2009 wird die Entscheidung, ob ein Fußgänger läuft oder nicht, durch Gleichung 3.14 bestimmt.

$$
G D=\frac{B}{V}+A F
$$

\footnotetext{
${ }^{5}$ Fußgängerüberwege in Form von Zebrastreifen haben nicht in jedem Land die hiesige Bedeutung. In Finnland markieren sie lediglich Positionen, an denen Fußgänger die Straße überqueren dürfen, regeln aber nicht die Vorfahrt. In Spanien und Italien finden sich ähnliche Regelungen.
} 


$$
\text { mit } \begin{aligned}
G D & =\text { Geschätzte Dauer zur Überquerung } \\
B & =\text { Breite der Straße } \\
V & =\text { Fußgängergeschwindigkeit } \\
A F & =\text { Aggresivitätsfaktor }
\end{aligned}
$$

Ein Fußgänger überquert die Straße, wenn $G D$ geringer als die zeitliche Lücke auf der Straße ist. In Brewer et al., 2006 wurde untersucht, welche zeitlichen Lücken Fußgänger akzeptieren, um eine Straße zu überqueren. Bei einer $B=9,1 \mathrm{~m}$ und einer $V=1,2 \mathrm{~m} / \mathrm{s}$ beträgt $G D \approx 7 \mathrm{~s}$. Hieraus ergibt sich ein durchschnittlicher $A F \approx-0,58 \mathrm{~s}$. Somit würden Fußgänger eine Straße überqueren, wenn die Lücke geringer als die benötigte Überquerungsdauer ist. In Das et al., 2005 wird GD durch $G D_{\min }=2 \mathrm{~s}$ und $G D_{\max }=11 \mathrm{~s}$ begrenzt. Als durchschnittlicher Wert kann $G D \approx 6$ s genutzt werden. Die durchschnittliche Laufgeschwindigkeit wird auf $V=1,5 \mathrm{~m} / \mathrm{s}$ geschätzt.

Zusammenfassend sollte die durchschnittlich benötigte zeitliche Lücke zur Überquerung einer Straße zwischen sechs und sieben Sekunden betragen. Verschiedene Fußgänger laufen dabei auch bei geringeren Lücken und können bei der Überquerung den Autoverkehr behindern.

\subsubsection{Regelverstöße}

Fußgänger neigen häufig dazu, Straßen in Abhängigkeit zur Lücke im Verkehr zu überqueren, auch wenn sie damit gegen die Straßenverkehrsordnung verstoßen. Dies ist beispielsweise an roten Ampeln der Fall. Fußgänger können dabei ca. $22 \%$ der Überquerungsdauer einsparen Virkler, 1998. Diese Ersparnis basiert teils auf höheren Laufgeschwindigkeiten und teils auf einer Verringerung der Wartezeiten. Fußgäner überqueren Straßen bei roter Ampel ca. $0,55 \mathrm{~km} / \mathrm{h}$ bis $0,66 \mathrm{~km} / \mathrm{h}$ schneller, als bei einer grünen Ampel Gates et al., 2006.

Ob ein Fußgänger die Straße an einer dafür vorgesehenen Stelle überquert oder auf eine Lücke im Verkehr wartet, hängt unter anderem von der Umweglänge, die zur korrekten Straßenüberquerung in Kauf genommen werden müsste und der persönlichen Einstellung ab. Jährlich finden tausende Unfälle mit Fußgängern auf Straßen statt (vgl. [Jason und Liotta, 1982, King et al., 2008). Ein Fußgängermodell sollte folglich nicht regelkonformes Verhalten berücksichtigen. 


\subsubsection{Simulationsmodelle}

Simulationsmodelle für Fußgänger werden meist zur Simulation bestimmter Szenarien entwickelt. In Ottomanelli et al., 2008 wird beispielsweise ein Modell zur Simulation von Fußgängerüberwegen vorgestellt. Überwege werden in Kanäle zerlegt, die eine Breite von $60 \mathrm{~cm}$ haben (typische Fußgängerbreite inklusive eines Sicherheitsabstandes). Kanäle werden zusätzlich in Zellen mit einer Zelllänge von 2,75 m unterteilt. Fußgänger werden mit $A F=2,5 \mathrm{~s}$ und $V=1,4 \mathrm{~m} / \mathrm{s}$ modelliert. Simulierte Fußgänger wählen vor der Straßenüberquerung einen Kanal und können diesen nicht mehr wechseln. Überholmanöver sind somit nicht möglich. Durch die konstante Modellierung der Geschwindigkeit wären sie ohnehin nicht durchführbar. Eine weiterführende Variante dieses Modells wurde in Blue und Adler, 2001 beschrieben. Fußgänger bewegen sich hierbei ZA-basiert und können Geschwindigkeiten $v \in\{0,1,2,3,4\}\left[\frac{\text { Zelle }}{\text { Zeiteinheit }}\right]$ wählen. Diese Werte lassen sich leicht auf realistische Geschwindigkeiten (siehe Abschnitt 3.3.1) skalieren. Die Vorgehensweise und der dahinterstehende Algorithmus werden in Anhang C] gezeigt.

In Kitazawa und Batty, 2004 und Hoogendoorn und Bovy, 2005 werden komplexe Modelle vorgestellt, in denen Fußgänger ihre Tagesaktivitäten planen und versuchen, ihre Kosten zu minimieren 6 Die Simulationsmodelle sind zur Simulation von Fußgängern in Einkaufszentren (Kitazawa und Batty), bzw. auf öffentlichen Plätzen oder Universitätscampi (Hoogendoorn und Bovy) ausgelegt und stehen dem ZA-Ansatz von Blue und Adler in der Berechnungskomplexität nach.

Detaillierte Modelle beschreiben Bewegungen in zwei Dimensionen. Eine einfache Methode hierfür ist eine Modellierung mittels ZA Weifeng et al., 2003. Eine komplexere Methode ist eine raumkontinuierliche Modellierung. Der bekannteste Vertreter dieser Gattung ist das Social force Modell Helbing und Molnár, 1995, in dem Agentenbewegungen von der gewünschten Geschwindigkeit und Einflüssen (z.B. die Bewegungen anderer Agenten) abhängen. Es konnte gezeigt werden, dass das Modell charakteristische Situationen in Fußgängergruppen abbilden kann. Erweiterungen des Social force Modells werden zur Simulation von Evakuationsszenarien verwendet Helbing und Johansson, 2009. In Johansson et al., 2007 wurde das Modell mit Hilfe von Evolutionären Algorithmen auf die Daten eines Fußgängertrackingsystems kalibriert und zur Simulation von 30.000 Fußgängern verwendet. Das Simulationssystem VISSIM (vgl. Abschnitt 4.5) verwendet eine Variante des Modells zur Fußgängersimulation.

${ }^{6}$ Dies ist ein grundlegend ähnlicher Ansatz zu der Tagesplangenerierung in Transims. Der Fokus liegt hierbei jedoch auf Fußgängern und nicht auf Autos. Vgl. Abschnitt 4.3 
Fluiddynamische Ansätze werden eingesetzt, wenn makroskopische Kenngrößen wie die Verkehrsdichte und die mittlere Geschwindigkeit der Fußgänger bestimmt werden sollen Helbing, 1998. In Anlehnung an Ishaque und Noland, 2008 ist [Henderson, 1974, Helbing, 1992, Hughes, 2003 eine Auswahl bekannter Modelle.

Abseits der Verkehrssimulation mit Fokus auf den Straßenverkehr werden Fußgängermodelle zur Optimierung von großen Fußgängerbereichen, wie z.B. Flughäfen, Bahnhöfen oder Fußgängerzonen genutzt. Sie helfen hierbei den Architekten solcher Gelände, ein besseres Verständnis über Fußgängerströme zu erlangen Usher et al., 2010 und sicherheitsrelevante Schwachstellen im Design frühzeitig zu erkennen Stanton und Wanless, 1995. Narasimhan verwendet eine Fußgängersimulationskomponente, um innerhalb eines Bürogebäudes Agentenarbeitspläne zu optimieren und dabei Staus auf Fußwegen zu vermeiden Narasimhan, 2007.

Für Fußgängerströme lässt sich ähnlich zum Automobilverkehr ein Fundamentaldiagramm (vgl. Abschnitt 2.2 auf Seite 15) erstellen, welches den Verkehrsfluss in Relation zur Verkehrsdichte setzt. Das Diagramm variiert für unterschiedliche Standorte, wie z.B. Treppen, Bürgersteige, Plätze und Engstellen Seyfried et al., 2005.

\subsection{Detailmodellierung}

In diesem Abschnitt wird ein kurzer Überblick über Arbeiten gegeben, die Aspekte des Straßenverkehrs modellieren, die als Details bezeichnet werden können. Diese Aspekte können in Simulationssysteme integriert werden, müssen aber nicht zwangsweise vorhanden sein, um ein valides Modell zu ergeben. In Unterabschnitt 3.4.1 werden die akzeptierten Lückengrößen für Abbiegevorgänge diskutiert. Unterabschnitt 3.4 .2 behandelt Aggressionen im Straßenverkehr. Zuletzt werden in Unterabschnitt 3.4 .3 sonstige Details beschrieben.

\subsubsection{Lückenakzeptanz bei Abbiegevorgängen}

Daganzo untersuchte den Abbiegeprozess von Autofahrern an Kreuzungen Daganzo, 1981. Ein Abbiegeprozess beginnt demnach mit einer mittleren Wartezeit $t_{w}=7,3 \mathrm{~s}$, da Fahrer Zeit benötigen, um die aktuelle Verkehrssituation aufzunehmen. Um abzubiegen, muss durchschnittlich eine zeitliche Lücke der Größe $t_{l}=4,5 \mathrm{~s}$ vorhanden sein. Es sollten weitere Unterscheidungen nach $t_{l}^{l}, t_{l}^{r}$ und $t_{l}^{g}$ getroffen werden (vgl. Abbildung 3.10). 


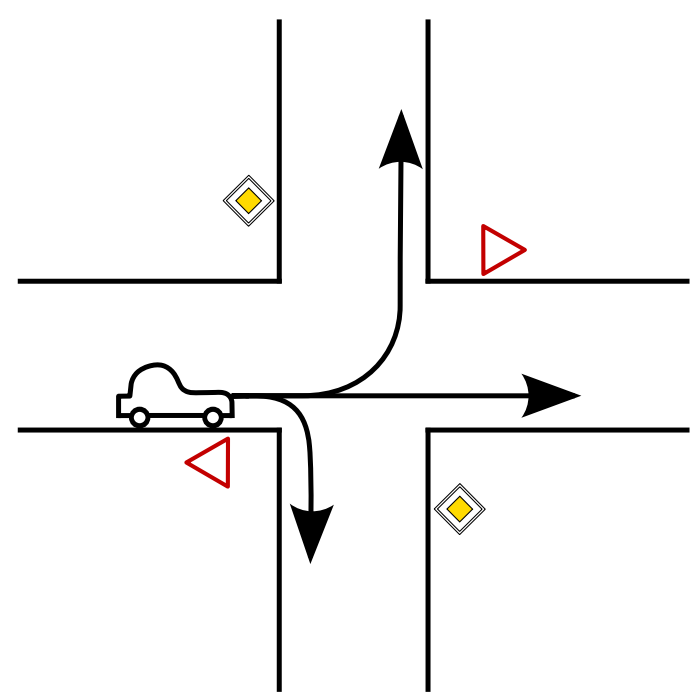

Abbildung 3.10: Abbiegevorgang: Das gezeigte Auto hat Vorfahrt zu achten. Wenn das Auto nach links abbiegt, wartet es auf eine Lücke von $t_{l}^{l}$; nach rechts auf $t_{l}^{r}$ und geradeaus auf $t_{l}^{g}$.

Beim Abbiegevorgang nach rechts ist die zurückzulegende Strecke minimal. Wenn das Auto geradeaus fährt, hat es die vorfahrtberechtigte Straße komplett zu überqueren. Der Linksabbiegevorgang benötigt die größte Lücke, da er eine Kombination der beiden anderen Vorgänge darstellt. Es muss somit Gleichung 3.15 gelten.

$$
t_{l}^{r}<t_{l}^{g}<t_{l}^{l}
$$

Die Bestimmung dieser Kenngrößen ist nicht trivial. In der Literatur werden Diskussionen geführt, nach welchen Versuchsaufbauten und welchen statistischen Methoden die Werte im realen Verkehr ermittelt werden können Brilon et al., 1999. Eine Vereinheitlichung hat bisher nicht stattgefunden, sodass die folgenden Informationen als grobe Richtwerte gesehen werden müssen.

In Ragland et al., 2006 wird der Verlauf des Wertes Accept507 untersucht. An unterschiedlichen Kreuzungen wurden Werte zwischen 5,6 s und 7,6 s ermittelt. Diese Werte beziehen sich jedoch ausschließlich auf $t_{l}^{l}$. In Abdul Kareem, 2002 wurden verschiedene Kreuzungen in Ilorin, Nigeria beobachtet. Mittlere Lückengrößen von 3,8 s wochentags und 4,1 s am Wochenende wurden ermittelt - leider wurde nicht nach Abbiegerichtungen unterschieden. Eine Studie aus Neuseeland vergleicht die verschiedenen Abbiegerichtungen Ashton, 1971. Tabelle 3.7 zeigt

${ }^{7}$ Die Lückengröße, die $50 \%$ der beobachteten Autofahrer akzeptierten. 


\begin{tabular}{lccc}
\hline Verkehrsdichte & Abbiegerichtung & $\mu$ & $\sigma$ \\
\hline Normal & $l_{l}^{l}$ & 3,03 & 0,33 \\
& $l_{l}^{g}$ & 4,29 & 0,18 \\
& $l_{l}^{r}$ & 5,34 & 0,79 \\
\hline Berufsverkehr & $l_{l}^{l}$ & 4,50 & 0,28 \\
& $l_{l}^{g}$ & 5,11 & 0,18 \\
& $l_{l}^{r}$ & 5,48 & 0,40 \\
\hline
\end{tabular}

Tabelle 3.7: Abbiegevorgang: Lückengrößen - Vergleich zwischen Berufsverkehr und normalem Verkehr, sowie zwischen den verschiedenen Abbiegerichtungen. Adaptiert nach Ashton, 1971]. Die Abbiegerichtungen sind vertauscht, da Neuseeland Linksverkehr hat. In der Originalarbeit wird der Begriff "Median gap" verwendet. Dieser wird im Zusammenhang mit Standardabweichung $\sigma$ als Mittelwert $\mu$ interpretiert.

die wichtigsten Ergebnisse.

Ferner beeinflussen Ablenkungen des Fahrers die Bestimmung der Abbiegelücken. Cooper und Zheng ermittelten, dass das Unfallrisiko durch fehlerhafte Lückenwahl bei abgelenkten Fahreren auf das doppelte im Vergleich zu nicht abgelenkten Fahrern steigt Cooper und Zheng, 2002. Nach [Tupper et al., 2010 hängen die Lückengrößen von individuellem Fahrerverhalten, Geschlecht und Alter der Fahrers und der Länge der Warteschlange hinter dem Fahrer ab. Die Modellierung von steigender Aggressivität bei nicht Vorhandensein einer geeigneten Lücke, die zur Akzeptanz geringerer Lückengrößen führt, bietet sich an. Individuelles Fahrverhalten, Geschlecht und Alter des Fahrers lassen sich direkt in die grundlegenden Lückengrößen für eine Auto-Fahrer-Einheit integrieren.

\subsubsection{Aggressionen im Straßenverkehr}

Aggressionen im Straßenverkehr beeinflussen direkt das Fahrverhalten der Autos. Lagland gibt einen Überblick über vorhandene Literatur und beschreibt Aggressivität als:

Ein Fahrverhalten ist aggressiv, wenn es vorsätzlich die Unfallwahrscheinlichkeit steigert und durch Ungeduld, Ärger, Feindseligkeit und / oder den Versuch, Zeit zu sparen, motiviert ist. (Übersetzung nach Laagland, 2005)

Aggressive Handlungen können kommunikativer Natur sein (z.B. schreien, gestikulieren, hupen, lichthupen) oder sich direkt auf das Fahrverhalten auswirken (z.B. dichtes Auffahren, Vorfahrtnahme, schneiden eines anderen Verkehrsteilnehmers 
während eines Überholvorgangs, überhöhte Geschwindigkeiten). Die Ursachen für Aggressionen sind nach Sharkin, 2004 in die folgenden Kategorien zu unterscheiden:

- Situations- und Umgebungsabhängige Aggressionen

- Charakter des Fahrers

- Demographische Eigenschaften

Berufsverkehr ist ein situationsbedingter Stressor. Nach [Tasca, 2000 führt Berufsverkehr nicht immer zu Aggressionen. Ein unerwarteter Stau kann hingegen dazu führen. Unterschiedliche Fahrer neigen auf Grund ihrer Charaktere zu unterschiedlichem Aggressionspotential im Straßenverkehr. Als demographische Unterschiede sind verschiedene Kulturkreise und das Alter des Fahrers entscheidend. Aggressivität im Straßenverkehr ist geschlechtsunabhängig Hennessy und Wiesenthal, 2001; jüngere (18 bis 23 Jahre) Verkehrsteilnehmer neigen zu mehr Aggressivität als ältere (24 bis 66 Jahre) Wiesenthal et al., 2000.

Im Rahmen dieser Arbeit ist zu untersuchen, wie Aggressivität in ein Mikromodell zur Fahrzeugsimulation integriert werden kann. Die Simulationssoftware Paramics 8 verwendet in einem Fahrzeugfolgemodell auf Basis von Fritsche, 1994 Aggressivität als einen Parameter zur Beeinflussung der Fahrereigenschaften. Sie wird jedoch als zeitinvarianter Faktor für einen Fahrer ausschließlich zur Verkürzung des Sicherheitsabstandes genutzt Speirs und Braidwood, 2004.

Laagland schlägt vor, die Aggressivität eines Fahrers in Relation zur Verkehrsdichte und zur Verzögerung, die er erfährt, zu setzen Laagland, 2005. Die Nutzung der Verkehrsdichte scheint nach [Tasca, 2000 nicht uneingeschränkt geeignet zu sein, da demnach lediglich unerwartet hohe Verkehrsdichten zu Aggressionen führen. Eine Verzögerung kann durch die Abweichung der Geschwindigkeit eines Autos von seiner Wunschgeschwindigkeit und durch die Wartedauer bei fehlender Vorfahrt bestimmt werden.

Kommunikative aggressive Handlungen ergeben nur Sinn, wenn Agenten über ein detailliertes Stimmungsmodell verfügen. Dies ist auf Grund der Berechnungskomplexität jedoch nicht zum Einsatz in Simulationsstudien mit 100.000 Verkehrsteilnehmern und mehr geeignet. Eine Verringerung des Sicherheitsabstandes, eine Senkung der im vorherigen Abschnitt 3.4.1 beschriebenen Werte für $l_{l}^{l}, l_{l}^{r}$ und $l_{l}^{g}$ und eine Missachtung von Geschwindigkeitsvorschriften lassen sich im Falle

$\sqrt[8]{\text { http://www.paramics-online.com/ }}$, abgerufen am 15.10.2012 
steigender Aggressionen eines simulierten Verkehrsteilnehmers hingegen mit den meisten mikroskopischen Verkehrsmodellen abbilden.

\subsubsection{Sonstiges}

Unfälle entstehen auf Autobahnen nach Treiber et al., 2005 in Abhängigkeit zur TTC (Time to Collision). Die TTC ist die Zeit, bis - bei sich nicht ändernden Geschwindigkeiten - ein Zusammenstoß mit dem Vordermann auftritt. Werte von weniger als $4 \mathrm{~s}$ gelten als kritisch - die Wahrscheinlichkeit für einen Unfall steigt „rapide“ Hirst und Graham, 1997. Durch Homogenisierung des Verkehrs kann die TTC erhöht werden, z.B. durch Geschwindigkeitsbegrenzungen: Alle Autos fahren langsamer, die Geschwindigkeitsdifferenzen sind geringer, die TTC erhöht sich, es entstehen weniger Unfälle. Insgesamt kann eine Homogenisierung des Verkehrs zu einer Erhöhung der durchschnittlich gefahrenen Geschwindigkeit führen. Das Maß TTC wurde in Minderhoud und Bovy, 2001 auf Basis von Fahrzeugbewegungsprofilen erweitert, um ein besseres Unfallverständnis zu erlangen.

In Tavares et al., 2009b werden Unfälle in urbanen Szenarien in ein ZA-Modell einbezogen. Die Unfallentstehung wird jedoch nicht simuliert. Unfälle geschehen im Modell ereignisorientiert. Das Simulationsmodell wird in Tavares et al., 2009a verwendet, um aus Verkehrsflussinformationen mit annotierten Unfällen einen Klassifikator maschinell zu lernen, der später anhand von Verkehrsflussdaten bestimmen kann, ob ein Unfall vorgelegen hat.

In Dell'Orco et al., 2003 wird die Unsicherheit von Autofahrern bei der Wahl eines geeigneten - legalen, aber auch illegalen - Parkplatzes mittels Fuzzylogik9 modelliert.

Meignan et al. haben ein Simulationsmodell für Busse entwickelt, bei dem Busse mikroskopisch und der restliche Verkehr makroskopisch modelliert werden. Busse interagieren somit nicht mit dem Verkehr und passen ihre Geschwindigkeit an makroskopische Durchflussgeschwindigkeiten an. Fußgänger können an Bushaltestellen zu- und aussteigen Meignan et al., 2006, Meignan et al., 2007. Die hierfür benötigten Zeiten hängen hauptsächlich von der Anzahl an zu- und aussteigenden Fahrgästen ab Rajbhandari et al., 2003, Dueker et al., 2004.

\footnotetext{
${ }^{9}$ Fuzzy knowledge ist ungenaues Wissen. Wenn ein begrenzendes Intervall für einen Messwert bekannt ist, wird der Messwert als unscharf bezeichnet. Fuzzylogik ist eine Erweiterung der booleschen Logik um Unschärfe. Goos und Zimmermann, 2006
} 


\subsection{Diskussion}

Dieses Kapitel hat Modelle zur Simulation von Verkehrsteilnehmern mit Fokus auf Autos, Fahrräder und Fußgänger diskutiert. Diese Auswahl bildet die wichtigsten Verkehrsteilnehmertypen für urbane Szenarien ab. Die Literaturbetrachtung wurde auf Feldstudien zur Beobachtung von Verkehrsteilnehmern ausgedehnt, um einen Überblick über zu modellierende Verhaltensweisen und zur Parametrisierung zu erhalten.

Im Rahmen dieser Arbeit sollen Anleihen beim NSM vorgenommen werden, da dieses für große Szenarien geeignet ist. Die Schwäche des Modells, seine räumliche Diskretisierung, soll behoben werden, um ohne weitere Anpassungen verschiedene Verkehrsteilnehmertypen auf einer Straße simulieren zu können.

Ein Fahrradmodell sollte sich nicht grundlegend vom Automodell unterscheiden, um gemeinsame Funktionen direkt verwenden zu können und lediglich bestimmte Parameter anpassen zu müssen. Verhaltensweisen können je nach Verkehrsteilnehmertyp ergänzt oder entfernt werden.

Im Rahmen dieser Arbeit liegt der Fokus auf den Wechselwirkungen zwischen den verschiedenen Verkehrsteilnehmertypen. Zusätzlich müssen grundlegende makroskopische Zusammenhänge abgebildet werden können. Dieser Anspruch zwingt zur Modellierung des Straßenüberquerungsverhaltens von Fußgängern, da diese Aktionen die einzigen sind, die Wechselwirkungen mit dem Straßenverkehr hervorrufen. Die betrachtete Literatur gibt zudem eine gute Grundlage für die Wahl geeigneter Laufgeschwindigkeiten und Lückenakzeptanzparameter.

In Städten wird ein Teil des Verkehrs durch den ÖPNV (Öffentlicher Personennahverkehr) verursacht. Busse fahren auf vordefinierten Routen zu definierten Zeiten und halten an Bushaltestellen. Dies ließe sich mit einem beliebigen mikroskopischen Verkehrsmodell abbilden. Straßen- und U-Bahnen fahren auf Schienen. Dieser Verkehr soll vorerst außen vor gelassen werden, da der Fokus dieser Arbeit auf dem Straßenverkehr liegt.

In diesem Kapitel wurden bewusst ausschließlich Simulationsmodelle betrachtet. Eine Trennung zwischen Simulationsmodellen und Simulationssystemen ist notwendig, da ein Simulationssystem zwar Simulationsmodelle verwendet, diese jedoch austauschbar sein können. Das nächste Kapitel diskutiert eine Auswahl bekannter Verkehrssimulationssysteme. 


\section{Verkehrssimulationssysteme}

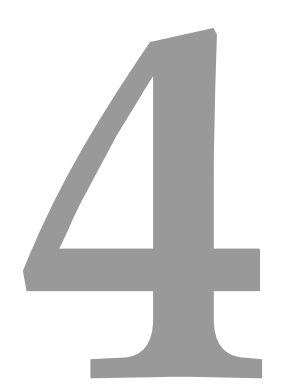

Dieses Kapitel beschreibt bestehende Verkehrssimulationssysteme. Abschnitt 4.1 ordnet die betrachteten Systeme in den wissenschaftlichen Kontext ein. In den Abschnitten 4.2 bis 4.8 werden etablierte Simulationssysteme besprochen. Die dargestellten Systeme stellen eine Auswahl der relevanten Arbeiten dar. Sie modellieren den Verkehrsfluss in unterschiedlicher Detailausprägung und beantworten divergente Fragestellungen. Weniger etablierte Simulationssysteme, die dennoch wichtige Aspekte behandeln, werden in Abschnitt 4.9 kurz eingeführt. Abschließend wird in Abschnitt 4.10 ein Vergleich der Systeme gezogen und besprochen, welche Eignung die genutzten Modelle für das in Abschnitt 1.1 beschriebene motivierende Beispiel haben und inwieweit sie die gestellten Anforderungen (vgl. Abschnitt 1.4) erfüllen.

\subsection{Einordnung in den wissenschaftlichen Kontext}

Es werden in diesem Kapitel ausschließlich Verkehrssimulationssysteme betrachtet, die dynamisch sind (vgl. Kapitel 2.3), da im Bereich der Verkehrssimulation zeitliche Verläufe der Verkehrslage entscheidend sind. Es wird unterschieden, ob die Systeme diskret oder kontinuierlich in Bezug auf Simulationszeit und -raum sind. Es werden ausschließlich Simulationssysteme betrachtet, die über mikroskopische oder mesoskopische Verkehrsmodelle verfügen, da makroskopische Modelle 


\begin{tabular}{c|c|c|c|c} 
System & $\begin{array}{c}\text { Steuerung der } \\
\text { Verkehrsnachfrage }\end{array}$ & Zeit & Raum & Modell \\
\hline \hline Corsim & datengesteuert & ereignisorient. & kontin. & mikroskopisch \\
Transims & agentenbasiert & diskret & diskret & mikroskopisch \\
Matsim & agentenbasiert & ereignisorient. & diskret & mesoskopisch \\
Vissim & datengesteuert & diskret & kontin. & mikroskopisch \\
Integration & datengesteuert & diskret & kontin. & mikroskopisch \\
Hutsim & datengesteuert & diskret & kontin. & mikroskopisch \\
Sumo & agentenbasiert & diskret & kontin. & mikroskopisch \\
\hline
\end{tabular}

Tabelle 4.1: Kontextuelle Einordnung betrachteter Verkehrssimulationssysteme

zeitliche Veränderungen und individuelle Wechselwirkungen zwischen Verkehrsteilnehmern nicht abbilden.

Alle betrachteten Simulationssysteme verfügen über stochastische Komponenten. Es ist offensichtlich, dass stochastische Modelle wiederholte Simulationsläufe benötigen, um sich durch Mittelung der Ergebnisse nach dem Gesetz der großen Zahlen dem Erwartungswert der betrachteten Kenngröße anzunähern. Ein deterministisches Vorgehen kann mit weniger Berechnungsaufwand zu statistisch signifikanten Ergebnissen führen. In Medina et al., 2005 wird ein deterministisches Verkehrsmodell entwickelt, das auf dem NSM basiert und den stochastischen Trödelschritt nicht durchführt. Wenn $\perp=0$ ist, können jedoch keine Staus ohne externe Einflüsse entstehen, obwohl sie ein Charakteristikum des Straßenverkehrs sind.

In Ni, 2006 wird ein historischer Abriss über Verkehrssimulationsarten gegeben. Es wird hierbei zusätzlich zwischen agenten- und objektbasierten Ansätzen unterschieden. Agenten sind als Spezialfälle von Objekten anzusehen. Im Rahmen dieser Arbeit werden diese auf Grund ihrer Ähnlichkeit zu Agenten zusammengefasst. Nach Ni, 2006 kann ein Simulationssystem datengesteuert sein. Ein Beispiel wäre die Nutzung von Induktionsschleifendaten oder Quelle-Ziel-Daten, um simulierte Autos zu erstellen.

Tabelle 4.1 ordnet die in den folgenden Abschnitten 4.2 bis 4.8 diskutierten Verkehrssimulationssysteme in den wissenschaftlichen Kontext ein und stellt eine Extraktion der gezeigten Informationen aus den folgenden Abschnitten dar. Die Verkehrsnachfrage wird bei allen betrachteten Systemen entweder über Eingabedateien oder über agentenbasierte Modelle zur Nachfragegenerierung hervorgerufen. Es werden teils zeitkontinuierliche Simulationsmodelle verwendet - die Simulationssteuerungen arbeiten jedoch mit fixen Zeitschritten. In der räumlichen Detailgenauigkeit unterscheiden sich die betrachteten Systeme eklatant zwischen der Nutzung eines Warteschlangenmodells, eines zellulären Ansatzes, bis hin zu konti- 
nuierlichen Größen. Die verwendeten Fahrzeugmodelle divergieren analog zwischen Reisedauerschätzungen und high-fidelity Ansätzen.

In den folgenden Abschnitten werden die genannten Verkehrssimulationssysteme ausführlicher betrachtet. Es wird hierbei der Fokus auf verwendete Verfahren, Technologien und Modelle gelegt und betrachtet, welche Stärken und Schwächen die jeweiligen Systeme und deren dahinter stehenden Konzepte für die Ziele dieser Arbeit aufweisen.

\subsection{Corsim}

Das Verkehrssimulationssystem Corsim (CORridor SIMulation) wird seit den frühen 1970er Jahren entwickelt. Es besteht aus den Systemen Netsim (NETwork SIMulation) ${ }^{1}$ und FRESIM (FREeway SIMulation).

Netsim ist ein raumkontinuierliches Fahrzeugfolgemodell für urbane Szenarien. Es können verschiedene Verkehrsteilnehmertypen simuliert werden, z.B. Auto, Bus, Lastwagen und Fußgänger. Die simulierten Verhaltensweisen reichen von passiv bis aggressiv. Stehende Autos können zur Spurblockierung genutzt werden. Das Routing von Verkehrsteilnehmern geschieht mittels Abbiegewahrscheinlichkeiten im Straßengraph und QZM ${ }^{2}$ Netsim simuliert Busrouten und die entstehenden Verkehrsbehinderungen durch Busse, die an Bushaltestellen halten. Parkplätze und Straßenrandbereiche, an denen parken erlaubt ist, werden modelliert Paksarsawan et al., 1992.

Außerstädtischer Verkehr wird in Corsim durch das Modul FRESIM modelliert. Zwischen Netsim und FRESIM müssen Übergangskanten im Straßengraph geschaltet werden Minnesota Department of Transportation, 2008. Corsim kann komplizierte Straßenverläufe mit Zu- und Abflüssen modellieren. Eine Nutzung zur Vorhersage von Stauentwicklungen ist möglich. Verschiedene Ampelschaltungen sind implementiert. Zeit-variate Verkehrsaufkommensschwankungen können über einzulesende Stimuli simuliert werden. Der Benutzer muss in Corsim seine Straßenkarte in einem Grapheditor generieren. Er kann dabei auf eine Überlagerung mit einer einzulesenden Bitmap-Datei zurückgreifen, um Straßenzüge nachzuzeichnen Owen et al., 2000.

\footnotetext{
${ }^{1}$ Netsim wurde anfangs unter dem Namen UTCS (Urban Traffic Control System) entwickelt.

${ }^{2}$ Quelle-Ziel-Matrix: Quadratische Matrix $M$, bei der ein Eintrag $M_{i j}$ die Wahrscheinlichkeit angibt, im Straßengraph vom Startknoten $i$ zum Zielknoten $j$ zu fahren.
} 
Corsim wurde in verschiedenen Arbeiten zur Ampelschaltungsoptimierung in einer HiL ${ }^{3}$ Umgebung genutzt Bullock und Catarella, 1998, Stallard und Owen, 1998. Es kann einzelne Autos verfolgen, Wartezeiten aufzeichnen und standardisierte Messgrößen (z.B. Verkehrsdichte und -fluss) protokollieren.

Wu hat verschiedene Verfahren zur Berechnung von QZM verglichen und teilweise mit Hilfe von Corsim bestimmen lassen Wu, 1997. In Chien et al., 2001 werden verschiedene Fahrerpopulationen erstellt und die Auswirkungen der Fahrereigenschaften auf den Verkehrsfluss bestimmt. Es konnte gezeigt werden, dass Corsim bei steigender Geschwindigkeitsvarianz innerhalb der simulierten Fahrzeuge einen sinkenden Gesamtfluss simuliert.

Corsim ist ein Simulationswerkzeug, das hauptsächlich für Ampelschaltungsoptimierungsprobleme genutzt wird (vgl. Bullock und Catarella, 1998, Sacks et al., 2000, Rouphail et al., 2000]). In Wilbur, 2004 wird Corsim um ein Wettermodell ergänzt, um die Auswirkungen verschiedener Witterungsbedingungen auf den Automobilverkehr zu untersuchen. Fußgänger und Autos interagieren, indem Fußgänger Straßen überqueren und Autos zum Halten zwingen Holm et al., 2007.

Corsim wird ausschließlich für Windows angeboten. Lizenzen kosten in verschiedenen Versionen zwischen 500 \$ und 5.000 \$. Die Quelltexte sind nicht zur direkten Verarbeitung freigegeben. Über die Skalierbarkeit des Systems sind keine Informationen vorhanden.

\subsection{Transims}

Transims steht für TRansportation ANalysis SIMulation System. Es handelt sich um ein kostenloses, quelloffenes Simulationswerkzeug, bei dem Agenten komplette Tagesabläufe simulieren. Jeder Agent hat Erwartungen über die aktuelle Verkehrslage (Auslastung der Straßen) und plant auf deren Basis seinen Weg zum Arbeitsplatz und den abendlichen Rückweg. Ein Simulationsszenario wird 50 mal simuliert. Nach jedem Durchlauf passen $10 \%$ der Agenten ihre Erwartungen den „Erlebnissen“ der vorherigen Simulationsläufe an. Das Ziel dieses Verfahrens ist es, dass sich der Verkehr im simulierten Bereich so verteilt, dass ein Nash-

\footnotetext{
${ }^{3}$ Hardware in the Loop: Verfahren, bei dem reale Hardware durch eine Simulationsumgebung ersetzt wird. In diesem Fall wird die Ansteuerung von realen Ampeln simuliert (vgl. Bertsche et al., 2009).
} 
Gleichgewicht ${ }^{4}$ entsteht Nagel et al., 1999. Es lassen sich demnach Auswirkungen von Änderungen im Straßennetz auf den Verkehr simulieren. Zur Simulation von Autos wird eine modifizierte Variante des Nagel-Schreckenberg-Modells verwendet Barrett et al., 1998.

Das Simulationssystem modelliert zusätzlich zu Autos und Bussen auch Fußgänger. Ein Agent, der seinen Weg durch den Simulationsgraphen berechnet, bezieht Buslinien und die Fußwege zu den Bushaltestellen in seine Berechnungen ein. Bei einem gegebenen Busfahrplan kann der Weg von einer Bushaltestelle zur nächsten als Kante mit einem Gewicht entsprechend der angegebenen Dauer des Weges interpretiert werden. Eine modifizierte Variante des Dijkstra-Algorithmus wird verwendet, um Wege zu berechnen. Alle Agentenpläne, Wege durch den Graphen eingeschlossen, werden vorberechnet Nagel, 2001 und stehen während der Simulation zur Verfügung.

Nicht implementierte Mikrosimulationsmodule werden vereinfacht simuliert. Wenn beispielsweise ein Fußweg von einer Position $a$ zu einer Position $b$ gelaufen werden soll, jedoch keine Fußgängersimulationskomponente vorhanden ist, wird die Länge des Weges von $a$ nach $b$ mit einer Geschwindigkeit $v$ skaliert, um die Zeit $t$ abzuschätzen, die der Weg in Anspruch nehmen würde. Der Agent wechselt nach $t$ Zeiteinheiten seine Position von $a$ nach $b$. Es finden keine Wechselwirkungen zwischen Fußgängern und Autos statt. Fahrräder werden ebenfalls nicht beachtet.

Zur Simulation des gesamten Verkehrs in der Schweiz wurde das Modell auf eine reine Auto-Simulation vereinfacht Voellmy et al., 2001. Die Wege, die die Agenten fahren, werden durch Quelle-Ziel-Matrizen bestimmt. Pro Stunde existiert eine Quelle-Ziel-Matrix, sodass die täglichen Dynamiken simuliert werden können. Mit Hilfe einer Variante des Dijkstra-Algorithmus werden die Routen berechnet. Der Algorithmus ist zeitabhängig [Jacob et al., 1999. Die Kantengewichte werden in Abhängigkeit zu den Zeiten gesetzt, die simulierte Autos im Zeitraum der letzten 15 Minuten Simulationszeit durchschnittlich benötigt haben, um die Kanten abzufahren.

Dieses Verfahren wird in Raney und Nagel, 2002 erweitert, sodass während eines ersten Simulationslaufs für jede Kante im Straßengraphen 96 Zeitabschnitte à 15 Minuten definiert werden, über die die Durchfahrtzeiten der simulierten Verkehrsteilnehmer gemittelt werden. Im folgenden Simulationslauf wird für das Routing darauf zurückgegriffen. Die Kantengwichte für den Routingalgorithmus variieren während der für die Fahrt benötigten Zeit. Wenn ein Auto zu einer Uhrzeit von

${ }^{4}$ Kein Agent kann seine eigene Fitness (in diesem Fall Reisezeit) verbessern, ohne dass mindestens ein anderer Agent seine Strategie ändert Russell und Norvig, 2004. 
einer Straße losfährt, kann die geschätzte Dauer für den Weg auf dieser Straße durch eine Mittelung über die angrenzenden Zeitabschnitte aus dem letzten Simulationsdurchlauf abgeschätzt werden. Hieraus kann approximiert werden, wann das Auto die nächste Straße durchfahren wird. Der Algorithmus schätzt auf diese Weise den Verlauf des Verkehrsaufkommens während der Zurücklegung des Weges ab.

Die Schwäche in diesem System ist, dass Fehler sich aufsummieren. Das Auto kann früher oder später an einer Straße ankommen, als geplant. Dies führt zu Schätzfehlern ab dieser Stelle. Die Fehler konnten verringert werden, indem nicht die durchschnittliche Durchfahrtdauer, sondern die maximale Durchfahrtdauer ermittelt wird und bei Routenberechnungen ein zeitliches Offset von 15 Minuten angenommen wird: Wenn eine Fahrt zum Zeitpunkt $t$ beginnt, wird mit Verkehrsinformationen für den Zeitpunkt $t+15$ aus dem letzten Simulationslauf gerechnet. Dieses Verfahren bleibt fehleranfällig, da während der Fahrt keine Routenkorrekturen möglich sind und Deadlocks nach dem erweiterten Verfahren unwahrscheinlicher, aber nicht unmöglich werden. In der Simulation wird angenommen, dass jeder Verkehrsteilnehmer über die Verkehrsinformationen des Vortages verfügt, was als unrealistisch angesehen werden muss. Agenten, die den berechneten Weg nicht fahren können 5 , werden aus der Simulation entfernt, ohne die Möglichkeit zu haben, ihren Plan zu aktualisieren.

Das Simulationssystem wird auf einem Beowulf-Cluster6 ausgeführt. Jeder PC erhält einen Kartenausschnitt und kann über ein MPI-System (Message Passing Interface) Informationen und Fahrzeuge mit PCs, die benachbarte Regionen simulieren, über ein Ethernet interface austauschen. Der Flaschenhals ist an dieser Stelle der Nachrichtenaustausch, der $30 \%$ bis $60 \%$ der Simulationsdauer benötigt Raney et al., 2002b.

Die Simulationsgeschwindigkeit ist ein Augenmerk des Transims Projektes. In Bachem et al., 1994 wurde ein vereinfachtes Verkehrsmodell mit 660.000 Fahrzeugen auf 64 Prozessoren mit einer Geschwindigkeit von zehnfacher Echtzeit simuliert. Der Straßengraph wird in Transims in eine Anzahl an Partitionen zerlegt, die identisch zur Anzahl an verwendeten Prozessoren ist. Jeder Partition wird ein Prozessor zugewiesen, der den Verkehr innerhalb der Partition simuliert.

\footnotetext{
${ }^{5}$ Dies kann beispielsweise auftreten, wenn es aufgrund des Verkehrsaufkommens nicht gelingt, auf eine Abbiegerspur zu wechseln.

${ }^{6}$ Cluster-Computer sind Parallelrechner mit verteiltem Speicher, die über ein Netzwerk kommunizieren und ihre Aufgaben verteilen. Es werden hierfür normale PCs mit freier Software verwendet. Der erste Cluster, der diese Anforderungen erfüllte, war ein von NASA-Wissenschaftlern entwickelter Cluster namens Beowulf Bauke und Mertens, 2005. Sterling et al., 1995.
} 
Kanten, die eine Partitionengrenze überschreiten, werden in der Mitte getrennt, sodass die dazugehörigen Knoten nicht an den Grenzen der Partitionen liegen Nagel und Rickert, 2001. Die Kanten an den Partionengrenzen verfügen über Interaktionsbereich $£^{7}$ von $35 \mathrm{~m}$, was der maximalen Autogeschwindigkeit pro Iteration in $\mathrm{m} / \mathrm{s}$ im Simulationsmodell entspricht Nagel et al., 2001. Die Partitionen werden anfangs mit ca. gleicher Fläche gewählt. Nach jedem Durchlauf wird in Abhängigkeit zur Berechnungsdauer der einzelnen Prozessoren eine erneute Partitionierung des Graphen durchgeführt.

Das Transims Simulationsmodell wurde verwendet, um Abgasemmissionsberechnungen durchzuführen. Da das Mikrosimulationsmodell ausschließlich Schrittweiten von Vielfachen von 7,5 m zulässt, wurde in Williams et al., 1998 ein Ansatz entwickelt, um aus den entstehenden Daten adäquate Mittelungen bezüglich Beschleunigung und Geschwindigkeit zu bestimmen. Das entwickelte Modell ist für Autobahnverkehr ausgelegt und lässt sich auf Grund der starken Vereinfachungen nicht auf die Simulation von Stadtverkehr übertragen.

Zur Simulation des gesamten Straßenverkehrs in der Schweiz wurde Kartenmaterial des Bundesamtes für Raumentwicklung der Schweiz verwendet, das nicht über Informationen bezüglich der Anzahl an Spuren auf Straßen und Lichtsignalanlagen an Kreuzungen verfügt. Um den Verkehr über 24 Stunden Realzeit mit 7,5 Millionen Verkehrsteilnehmern simulieren zu können, wird ein vereinfachtes Warteschlangenmodell nach Gawron, 1998 verwendet, da das komplexere Mikromodell von Transims die fehlenden Informationen benötigt und außerdem einen höheren Berechnungsaufwand bewirkt. Während der Simulation traten in Nebenstraßen Staus auf, während Hauptverkehrsstraßen einen freien Verkehrsfluss hatten. Dies ist darauf zurückzuführen, dass bereits kleine Störungen zu Deadlocks führen können, wenn die Verkehrsteilnehmer nicht umplanen, um die Störung aufzuheben Raney und Nagel, 2002.

Transims ist kostenlos und quelloffen. Die Implementierung geschieht in $\mathrm{C}++$.

${ }^{7}$ Interaktionsbereiche sind die Bereiche, die für mehr als eine Partition relevant sind. Zur Simulation des Verkehrs dieser Bereiche müssen die betreffenden Prozessoren interagieren. 


\subsection{Matsim}

Ein Teil der Entwicklungsgruppe von Transims spaltete sich zu Beginn des neuen Jahrtausends ab und begann, Matsim (Multi-Agent Transport Simulation) zu entwickeln. In Matsim wird nicht mehr das NSM zu Modellierung des Autoverkehrs genutzt, sondern ein vereinfachtes Warteschlangenmodell Cetin et al., 2002. Matsim ist auf large-scale Simulationen ausgerichtet und nimmt dafür in Kauf, keinen multimodalen Verkehr abbilden zu können und in komplexen Szenarien gegenüber anderen Systemen (z.B. Transims oder VISSIM) unterlegen zu sein.

Matsim besteht grundlegend aus vier Modulen. Ein Aktivitätsgenerator steuert die Aktualisierung von Tagesplänen der simulierten Agenten. Ein Routingmodul berechnet die zeitlich (erwarteten) kürzesten Wege auf Basis des Dijkstra Algorithmus und Agentenwissen über Auslastungen von Straßen in vorherigen Durchläufen. Die Mobilitätskomponente führt die berechneten Pläne der Agenten mittels eines Warteschlangenmodells aus. Die vierte Komponente ist eine Agentendatenbank, die für jeden Agentenplan die Fitness speichert Balmer et al., 2004. Der Aufbau von Matsim unterscheidet sich gegenüber Transims hauptsächlich durch ein vereinfachtes Verkehrsmodell.

Der Fokus liegt bei Matsim auf Tagesplanbestimmungen für Agenten. Auf Basis von Zensus-Daten muss jeder Agent verschiedene Aktivitäten abarbeiten, die zu bestimmten Zeiten und Orten stattfinden können bzw. müssen und bestimmte Zeiten in Anspruch nehmen. Die Fitness eines Agenten hängt davon ab, wie viele Aktivitäten er unter seinen Rahmenbedingungen korrekt ausführt. Die Verkehrssimulationskomponente berechnet die Wege, die ein Agent zurücklegen muss. Mit Hilfe eines Genetischen Algorithmus konnte in Charypar und Nagel, 2005 die grundlegende Funktionalität des Konzeptes gezeigt werden.

Im Vergleich zu Transims werden die Möglichkeiten der MAS-Technologie im Hinblick auf die Verwendung mehrerer Strategien pro Agent besser ausgenutzt Raney et al., 2002a. Zusätzlich werden in Transims vorkommende Inkonsistenzen von Tagesplänen behoben Balmer, 2007]. Agenten erhalten die Möglichkeit, ihre Pläne während der Durchführung zu ändern Illenberger und Flötteröd, 2007.

In Raney et al., 2003 wurden erste Ergebnisse für die Simulation der gesamten Schweiz gegeben. Mit Hilfe des beschriebenen Tagesplangenerierungsverfahrens wurde versucht, das Verkehrsaufkommen in der Schweiz nachzubilden. Die grundlegende Funktionalität des Verfahrens konnte im Vergleich zu realen Messdaten gezeigt werden Meister et al., 2008. Die Durchführung von 100 simulier- 
ten Tagen mit je 7,1 $10^{6}$ Trips auf einem Graphen mit 24.000 Knoten benötigt auf einem Hochleistungsrechner mit 8 Doppelrechenkernen à 2,2 GHz 3,2 Tage Balmer et al., 2008.

Matsim kann Fahrzeugbewegungen nur sehr stark vereinfacht simulieren. Es können keine Überholvorgänge oder Spurwechsel modelliert werden [Rieser, 2010. Mit dem Fahrrad zu fahren oder zu laufen wird bei der Wahl der Fortbewegungsmittel durch Agenten berücksichtigt, jedoch bei der Ausführung der Pläne nicht simuliert Ciari et al., 2007. Somit finden keine Interaktionen zwischen Verkehrsteilnehmern unterschiedlichen Typs statt.

In Zilske et al., 2011 wird beschrieben, wie Matsim mittels OSM-Kartenmaterial aufgesetzt werden kann. Durch eine Lookup-Table (LUT) werden im Straßengraphen den Kanten Durchflussdauern und Geschwindigkeiten zugewiesen. Dies geschieht auf Basis des Straßentyps, der in OSM verwaltet wird.

Matsim ist kostenlos und quelloffen. Hierdurch lässt sich das System erweitern. Die Plattformunabhängigkeit wird durch die Verwendung von Java garantiert. Matsim eignet sich jedoch nicht zur realitätsnahen Simulation von Verkehrsszenarien, da das System Szenarien zu stark vereinfacht, um eine hohe Berechnungseffizienz erlangen zu können.

\subsection{Vissim}

Vissim (Verkehr In Städten - SImulationsModell) ist ein von der PTV AG8 entwickeltes, kommerzielles Simulationssystem. Mit Hilfe von Vissim lassen sich sehr detailreiche (High-Fidelity-Simulation) Szenarien im Bereich der multimodalen Verkehrssimulationen analysieren. Das System verwendet eine Variante des Wiedemann-Modells zur mikroskopischen Simulation von Autos mit Zeitschritten von 0,1 s Fellendorf, 1994, PTV AG, 2005, vgl. Abschnitt 3.1.1. Im System werden Straßen detailliert modelliert. Es werden beispielsweise genaue Spurverläufe, Abbiegespuren, Straßenverkehrszeichen, Lichtsignalanlagen, Parkplätze und Induktionsschleifen modelliert. Der hohe Detaillierungsgrad bewirkt einen äquivalenten Aufwand zur Modellierung eines Szenarios, da konventionelle Straßenkarten weniger detaillierte Informationen zur Verfügung stellen.

Das mikroskopische Verkehrsmodell innerhalb von Vissim konnte auf die unterschiedlichen Fahrverhaltensweisen in Deutschland und den USA kalibriert werden

${ }^{8}$ Planung Transport Verkehr AG 
Fellendorf und Vortisch, 2001. Die Kalibrierung fand auf makroskopischer und mikroskopischer Ebene statt. Für die mikroskopische Anpassung des Fahrverhaltens innerhalb des Simulationsmodells wurde das Verhalten von mit Sensoren bestückten Fahrzeugen während realen Testfahrten auf deutschen Autobahnen bzw. US Freeways protokolliert. Das Verhalten der simulierten Fahrzeuge wird von zehn Parametern beeinflusst, die teils subtile Modifikationen des Fahrverhaltens zulassen, deren Einfluss nicht trivial zu analysieren ist Lownes und Machemehl, 2006. Vissim bietet für die implementierten Verkehrsmodelle ein Emissionsmodell PTV AG, 2009.

Vissim kann u.a. Autos, Lkw, Busse, Straßenbahnen, Züge, Fahrräder und Fußgänger simulieren Bloomberg und Dale, 2000. Das implementierte Fußgängermodell wurde in verschiedenen Arbeiten mit Ergebnissen aus Experimenten mit Fußgängern verglichen. In Kretz et al., 2008 wurde gezeigt, dass der Fußgängerfluss an einer Engstelle linear mit der Breite der Engstelle skaliert und dies mit der Simulation reproduziert werden kann.

Das Simulationssystem Vissim wird über Eingabe- und Ausgabedateien angesteuert. Ein direkter Zugriff auf interne Komponenten ist nicht möglich. Eine Ampelschaltungsoptimierung in Straßennetzwerken mit bis zu 12 Kreuzungspunkten wurde in Stevanovic et al., 2008 mittels Genetischer Algorithmen realisiert.

Vissim ist ein international genutztes Simulationssystem Prassas, 2003, dessen graphische Benutzungsoberfläche so weit ausgereift ist, dass erste Tests zur virtuellen Stadtbegehung durchgeführt wurden Arias et al., 2009. In Kobbeloer, 2007 wird ein dezentrales Verfahren zur Ampelschaltungsoptimierung vorgestellt, welches mit Hilfe von Vissim evaluiert wurde. In Hughes et al., 2003 wird Vissim genutzt, um die Interaktionen zwischen Autos und Fußgängern an Kreisverkehren unter besonderer Berücksichtigung von blinden Fußgängern zu modellieren. Zur besseren Berücksichtigung von multimodalem Verkehr, wird in Schönauer und Schrom-Feiertag, 2010 vorgeschlagen, das Social force Modell (siehe Abschnitt 3.3.4 auf Seite 48 für Autobewegungen anzupassen. In Bönisch und Kretz, 2009 werden Straßenüberquerungsdauern von Fußgängern den Wartezeiten von Autofahrern in einer Simulationsstudie anhand einer Kreuzung gegenübergestellt.

Vissim wird für Windows angeboten und kann über eine COM-Schnittstelle angesteuert werden. Direkte Erweiterungen und Veränderungen können nicht implementiert werden, da die kommerzielle Software nicht quelloffen ist. 


\subsection{Integration}

Integration ist ein Simulationssystem, das für Autobahn- und Landstraßenverkehr ausgelegt ist. In das System sind sowohl Komponenten zur Modellierung des Verkehrs, als auch zur Erstellung von Routen integriert |Van Aerde et al., 1996.

Integration benötigt zur Erstellung eines Simulationsmodells 5 Dateien. Die erste Datei beschreibt die Knoten des Simulationsgraphen. Analog werden in der zweiten Datei die Kanten des Graphen definiert. Ampeln werden über die in einer dritten Datei gegebenen Steuerungspläne simuliert. Der Verkehr wird über Quelle-ZielPaare definiert, die zeitabhängig gesetzt werden können und ebenfalls aus einer Datei eingelesen werden. Die letzte Eingabedatei enthält blockierte Spuren und sonstige Störfälle. Diese können zeitabhängig geschaltet werden. In einer Ausgabedatei wird eine Zusammenfassung des Simulationslaufs mittels makroskopischer Kenngrößen gegeben Van Aerde und Associates, 2010a. Optional können mittels weiterer Eingabedateien Zusatzinformationen hinzugefügt werden. Dies können Auf- und Abfahrten, Positionen von Bushaltestellen, Detektoren, Geschwindigkeitsbegrenzungen, usw. sein Van Aerde und Associates, 2010b. Das System ist folglich in der Lage, semiautomatisch ein Simulationsmodell zu berechnen, welches erweiterbar ist. Die Erstellung läuft nicht vollautomatisch, da bereits ein Graph mittels Eingabedateien gegeben sein muss und keine standardisierten Straßenkartenmodelle eingelesen werden können.

Es wurden ein Benzinverbrauchs- und ein Emissionsmodell in Integration integriert und der Einfluss von Ampelschaltungen auf den Verbrauch bzw. Ausstoß ermittelt Rakha et al., 2000. Das Emissionsmodell geht über gemittelte Geschwindigkeiten hinaus und bezieht Geschwindigkeiten und Beschleunigungen mit ein Ahn et al., 2002, Rakha und Ahn, 2004. Das System wurde genutzt, um einen $35 \mathrm{~km}$ Ausschnitt des Highway 401 bei Toronto zu simulieren und konnte auf reale Sensormessdaten kalibriert werden. Um die schwankenden Verkehrsdichten zu modellieren, wurde für je einen 15-minütigen Simulationsabschnitt eine QZM bestimmt Hellinga et al., 1993, Hellinga, 1994, Hellinga und Van Aerde, 1998. In Rakha et al., 1998 wird Integration zur Simulation im Gebiet von Salt Lake City genutzt. Zur Modellierung und Kalibrierung benötigten die Autoren vier Mannjahre. Während der Simulation auf einem Pentium 200 erreichte das Simulationssystem je nach simuliertem Verkehrsaufkommen Simulationsgeschwindigkeiten zwischen $\frac{1}{2}$ und $\frac{1}{17}$ fache Echtzeit.

Das mikroskopische Automodell in Integration wird Van Aerde-Modell genannt und ist ein Fahrzeugfolgemodell mit Spurwechsellogik. Es ist raumkon- 
tinuierlich und zeitdiskret mit Zeitschritten von 0,1 s Van Aerde et al., 1996. Die Autoren konnten das Modell gegen Messdaten von realen StraBen und andere Simulationsmodelle validieren, sowie bekannte Diagramme reproduzieren Van Aerde und Rakha, 1995, Rakha und Van Aerde, 1996 Rakha und Crowther, 2002, Rakha und Zhang, 2003. Durch geeignete Parametrisierung ließen sich Lkw modellieren.

Integration wird kommerziell vertrieben und kann je nach Lizenz zwischen 250 und 10.000 Knoten und Kanten im Graph verwalten Van Aerde und Associates, 2010a. Diese Begrenzung spricht gegen eine gute Skalierbarkeit des Simulationssystems.

\subsection{Hutsim}

Hutsim (Helsinki University of Technology Simulator) ist ein Verkehrssimulationssystem, das seit 1989 an der Helsinki University of Technology entwickelt wird. Es wird die Programmiersprache Delphi verwendet. Hutsim modelliert Autos, Fahrräder und Fußgänger. Autos werden durch das zeitkontinuierliche Fahrzeugfolgemodell von GHR (vgl. Gleichung 3.2 auf Seite 27) mit Erweiterungen simuliert Kosonen, 1999. Fußgänger werden durch ein einfacheres Modell beschrieben, das andere Fußgänger ignoriert. Mehrere Fußgänger können somit auf einer identischen Position stehen. Fußgänger erscheinen in unregelmäßigen Abständen an Kreuzungen und möchten diese überqueren. Fahrräder werden als ein Spezialfall der Fußgänger betrachtet, sodass sie sich schneller als Fußgänger bewegen, jedoch keine Straßen verwenden können. Eine Interaktion zwischen Fußgängern bzw. Fahrrädern und Autos findet lediglich statt, wenn ein Fußgänger eine Straße überquert Kosonen, 1999. Lkw werden nicht explizit erwähnt, lassen sich jedoch durch geeignete Parametrisierung des Fahrzeugmodells abbilden.

Ursprünglich wurde Hutsim zur Ampelschaltungsoptimierung im Hinblick auf eine Minimierung der Wartezeiten und eine Maximierung der Sicherheit entwickelt. Eine Modellannahme ist, dass manche Fußgänger auch bei einer roten Ampel die Straße überqueren, wenn sie zu lange warten müssen Niittymaki und Turunen, 1999. In diesen Situationen blockieren sie die Spur, auf der sie sich befinden und zwingen Autos zu abrupten Bremsmanövern, aus denen Unfälle resultieren können.

Hutsim wurde an Scoot $t^{9}$ angekoppelt, um Echtzeitinformationen über die Ver-

\footnotetext{
${ }^{9}$ Scoot ist ein Akronym für Split Cycle Offset Optimisation Technique und bezeichnet ein
} 
kehrslage zu erhalten und auf deren Basis Simulationsläufe durchführen zu können Kosonen und Bargiela, 1999. Die Genauigkeit hängt stark von der Anzahl an verfügbaren Sensoren auf den Straßen ab [Kosonen und Bargiela, 2000. In Niittymäki und Korkeakoulu, 2002 wird eine Methode zur adaptiven Ampelschaltungsoptimierung vorgestellt, die auf Fuzzylogik basiert und mit Hilfe von Hutsim gegen konventionelle Ampelschaltungsverfahren verglichen wird. In Jokinen et al., 2005 wird eine Fallstudie beschrieben, bei der onlineVerkehrsinformationen genutzt werden, um simulierte Fahrzeuge zu erstellen. Dies stellt einen ersten Schritt zur online-Verkehrsprognose dar.

Hutsim hat das Ziel, Ampelschaltungen zu optimieren. In diesem Zuge finden die Interaktionen zwischen verschiedenen Verkehrsteilnehmertypen (Auto, Fahrrad, Fußgänger) nur an ampelgesteuerten Fußgängerüberwegen statt. Der Einfluss von Fahrrädern auf den Verkehrsfluss an Ampeln wird nicht beachtet, da Fahrräder sich wie Fußgänger verhalten.

In Hutsim muss ein Straßennetz zuerst modelliert werden Kosonen, 1999. Es besteht die Möglichkeit, komplexe Straßenverläufe zu modellieren. Eine Methode zur automatischen Graphgenerierung existiert hingegen nicht. Hutsim verwendet mehrere Fahrzeugfolgemodelle für unterschiedliche Verkehrssituationen. Das System verwendet eine zeitdiskrete Simulationssteuerung. Ein Simulationsupdate berechnet einen Zeitschritt von 0,1 s. Während eines Zeitschritts werden die internen Zustände aller Simulationsentitäten aktualisiert Kosonen, 1999. Die Ausrichtung von Hutsim ist daher die Bereitstellung einer high-fidelity Simulation. Dies führt zu einer schlechten Skalierbarkeit. Es sind keine Studien bekannt, in denen der Verkehr ganzer Ortschaften oder Städte simuliert wurde.

Verkehr kann in Hutsim mittels Eingabedateien erstellt werden. Hierfür können Quelle-Ziel-Dateien oder Verkehrsflussinformationen genutzt werden. Zusätzlich kann über die in Gerlough und Huber, 1975, S. 24] vorgeschlagene Wahrscheinlichkeit zum Zeitpunkt $t$ ein Fahrzeug an einer Eintrittsstelle generiert werden:

$$
\begin{aligned}
P(h>t) & =e^{-\frac{t-t_{0}}{\bar{t}-t_{0}}} \\
\text { mit } h & =\text { Folgedauer (headway) }
\end{aligned}
$$

System, das permanent den Verkehrsfluss und die Verkehrsdichte an innerstädtischen Kreuzungspunkten misst und Ampelschaltungen entsprechend der aktuellen Verkehrslage variiert Robertson, 1983 . 


$$
\begin{aligned}
\bar{t} & =\text { Durchschnittliche Folgedauer } \\
t_{0} & =\text { Minimale Folgedauer }
\end{aligned}
$$

Die dafür benötigten Eingabedaten $t$ und $\bar{t}$ müssen dem System gegeben werden. Falls reale Daten genutzt werden, basieren diese ebenfalls auf Verkehrsflussinformationen. Das System ist somit datengesteuert.

In Zhou et al., 2006 wird die Anbindung von GIS-Technologien an Hutsim beschrieben. Es ist daher von Anreicherungsmöglichkeiten durch zusätzliche Datenquellen auszugehen.

\subsection{Sumo}

Sumo (Simulation of Urban MObility) ist ein kostenloses und quelloffenes Simulationssystem, das in $\mathrm{C}++$ entwickelt wird. Das System soll zu einer Vereinheitlichung von Simulationsergebnissen und Modellen im Verkehrssimulationsbereich führen Behrisch und Krajzewicz, 2008.

Autos werden mit dem in Abschnitt 3.1.1 (Gleichung 3.7) vorgestelltem Modell raumkontinuierlich und zeitdiskret (Zeitschritt: 1 s) simuliert Krajzewicz et al., 2002b. Ähnlich zu Transims und Matsim (vgl. Abschnitt 4.3 und 4.4 verwendet Sumo ein Modell zur Abschätzung der Personenverkehrsnachfrage. Das Modell ermittelt aus soziodemographischen statistischen Daten des Simulationsgebietes Tagespläne, die anschließend durch simulierte Agenten ausgeführt werden können und zu Verkehr führen Varschen und Wagner, 2006. Sumo berücksichtigt multimodalen Verkehr jedoch analog zu Transims und Matsim durch Abschätzung der Reisedauer zwischen Quelle und Ziel ohne Simulation des zurückzulegenden Weges, sofern der Verkehrsteilnehmertyp vom Typen Auto abweicht. Interaktionen zwischen verschiedenen Verkehrsteilnehmertypen können somit nicht berücksichtigt werden.

Sumo wird seit dem Jahr 2001 entwickelt und erweitert. Es verfügt über Importfunktionen von Straßengraphen von Visum 10 Vissim und Matsim. Es kann Graphen aus Kartenmaterial im Shapefile- und OSM-Format generieren Behrisch et al., 2011. Eine Gruppe von Analyseschritten wird bei der Bestimmung eines Simulationsgraphen durchgeführt, um einen Graphen mit Vorfahrts- und Abbiegeregeln, sowie realistischen Anzahlen an Spuren und

\footnotetext{
$\overline{{ }^{10} \text { Verkehrsanalysesystem der PTV AG }}$
} 
Geschwindigkeitsbegrenzungen $\mathrm{zu}$ erhalten, sofern diese nicht gegeben sind Krajzewicz et al., 2005b.

Das verwendete Fahrzeugfolgemodell wurde auf Sensormessdaten eines Autobahnabschnitts kalibriert Krajzewicz et al., 2002a. Sensoren geben Informationen über Anzahl und Geschwindigkeit der passierenden Autos in einer Frequenz von $1 \mathrm{~Hz}$. Der erste Sensor der Messanordnung wird genutzt, um simulierte Autos zu generieren und alle weiteren, um die Daten von simulierten Messpunkten mit realen Messdaten zu vergleichen. Ein Optimierungsverfahren wurde genutzt, um das Modell anzupassen. Die Fehlerrate sank von anfangs $40 \%$ auf $15 \%$.

Sumo wurde während des Weltjugendtages 2005 in Köln zur Vorhersage der Verkehrslage der nächsten halben Stunde anhand von ca. 1.100 Verkehrssensoren genutzt Krajzewicz et al., 2006. In Krajzewicz et al., 2005a wird eine agentenbasierte Ampelsteuerung simuliert, die Kreuzungen mit stark schwankendem Verkehrsaufkommen optimieren kann. Im Car-to-X117. Bereich wird Sumo zur Untersuchung neuer Konzepte eingesetzt [Bauza et al., 2008, Wegener et al., 2008]. In Rieck et al., 2010 wird in diesem Zusammenhang eine Kopplung mit VISSIM (vgl. Abschnitt 4.5) durchgeführt, um wichtige Bereiche des Simulationsumfeldes mit VISSIM und die umliegenden Areale mittels Sumo zu simulieren.

\subsection{Weitere Simulationssysteme}

In diesem Abschnitt werden verschiedene Verkehrssimulationssysteme angesprochen, die weitergehende Ideen berücksichtigen, jedoch keine zu den vorangegangenen Systemem vergleichbare Relevanz besitzen.

In Miller und Horowitz, 2007 wird das System FreeSim ${ }^{12}$ beschrieben, welches kostenlos zur Verfügung gestellt wurde. Benutzer können mit einem Webbrowser ein Flash-Skript ablaufen lassen, welches eine Socketverbindung zu einem Simulationsserver hält. Das System kann aktuelle Geschwindigkeiten auf Schnellstraßen und Autobahnen visualisieren und stellt verschiedene Graphalgorithmen zur Verfügung, um zu diesen Daten die besten Wege im Straßennetz zu bestimmen. Eine Simulation im eigentlichen Sinne wird hierbei nicht durchgeführt.

\footnotetext{
${ }^{11}$ Autos werden mit Funkmodulen ausgestattet und können sich gegenseitig über Vorkommnisse und die aktuelle Verkehrssituation informieren, sowie Informationen mit Funkmasten der Infrastruktur austauschen.

12 http://www.freewaysimulator.com, abgerufen am 31.07.2012
} 
Die Simulationssoftware Pelop: ${ }^{13}$ (Programm zur Entwicklung Längsdynamischer, mikroskopischer Verkehrsprozesse in Systemrelevanter Umgebung) verwendet ein sub-mikroskopisches Automodell auf Basis des Wiedemann-Modells. Es werden verschiedene Größen simuliert (z.B. Lenkeinschlagswinkel, Winkel des Gaspedals), die intern im Auto von Belang sind. Das System eignet sich aufgrund seines detaillierten Modells nicht zur Simulation großer Szenarien. Einen Überblick über sub-mikroskopische Modellierung im Fahrzeugbereich gibt [Schramm et al., 2010].

Félez et al. stellen in Félez et al., 2007 ein Simulationssystem vor, das mittels VR ${ }^{14}$ Technologien zum Training von Berufskraftfahrern genutzt wird. Hierbei wird jeweils nur ein sehr kleiner Bereich um den Übenden herum simuliert, dieser jedoch sehr realistisch. Das System verfügt über ein detailliertes Schadensmodell zur Simulation von Unfällen.

VR-Technologien werden in Wagner et al., 2011 genutzt, um beim Zurücksetzen eines Autos mit Anhänger zu assistieren. Auf einem Bildschirm kann der Fahrer Bilder einer Rückfahrkamera mit eingezeichneten Spuren wahrnehmen, die ihm anzeigen, wohin der Anhänger sich bewegen wird. Vor der realen Umsetzung wurde das System in einem Fahrsimulator getestet.

Das Verkehrssimulationssystem Itsumo (Intelligent Transportation System for Urban MObility) ist ein kostenloses, quelloffenes und in $\mathrm{C}++$ entwickeltes System, welches als Verkehrsmodell das NSM verwendet da Silva et al., 2006b. Das System ist nicht mit dem in Abschnitt 4.8 diskutierten System Sumo verwandt. Die Zellengrößen werden auf $5 \mathrm{~m}$ festgelegt. Die Repräsentation von Fahrer-FahrzeugEinheiten und Ampeln ist agentenorientiert. Itsumo kann Straßenkarten von OSM verwenden Bazzan et al., 2010. In [Bazzan et al., 2009 wird eine Kopplung von Matsim (vgl. Abschnitt 4.4 und Itsumo gezeigt, die sowohl die Tagesplanoptimierungsstrategien aus Matsim, als auch die mikroskopische Verkehrssimulaton aus Itsumo ermöglicht. Itsumo bietet die Möglichkeit, Signalpläne von Ampeln graphisch zu editieren. Online Informationen, z.B. aktuelle Verkehrsflussdaten, können in die Simulation einfließen da Silva et al., 2006a.

Itsumo erfüllt die Anfordernungen AutoGraph, Zusatzmodule, Pkw, Routing, kostenlos und quelloffen. Die Skalierbarkeit konnte in der aufgeführten Literatur nicht gezeigt werden. Die Simulation eines größeren Stadtgebietes wird unter Beschränkung auf Hauptverkehrsstraßen durchgeführt Bazzan et al., 2010]. Die Verwendung von Modellen für Fahrräder und Fußgänger ist nicht vorgesehen. Die Nut-

\footnotetext{
${ }^{13}$ http://www.fka.de/pdf/pelops_whitepaper.pdf, abgerufen am 29.06.2012

${ }^{14}$ Virtuelle Realität: Systeme, die in Echtzeit realistische Visualisierungen und Technologien zur Wahrnehmung der physikalischen Gegebenheiten bereitstellen.
} 
zung des NSM ist für urbane Szenarien nachteilig (vgl. Abschnitt 3.1.3). Daher müssten mehrere Komponenten von Itsumo verändert bzw. ergänzt werden, um für die Ziele dieser Arbeit nutzbar zu werden.

\subsection{Bewertung}

Jedes der besprochenen Simulationssysteme ist grundsätzlich geeignet, verschiedene Fragestellungen des Verkehrs zu untersuchen. Jedes System wurde bzw. wird jedoch vordergründig für bestimmte Teilgebiete der Verkehrsforschung entwickelt. Es sind verschiedene vergleichende Arbeiten publiziert worden, die sich mit den Unterschieden und Gemeinsamkeiten von Teilmengen der vorgestellten Simulationssysteme beschäftigen.

In Wang et al., 1997 werden u.a. Integration und Corsim in Szenarien verglichen, für die exakte Verkehrsdichten und mittlere Geschwindigkeiten bekannt sind. Die Systeme konnten realistische und miteinander vergleichbare Kenngrößen ermitteln. Als Schwäche der Systeme wird in der Arbeit angegeben, dass sie zu viele Parameter haben, die in einer aufwendigen Prozedur kalibriert werden mussten. In einer weiteren Studie Prevedouros et al., 1997 wird bemängelt, dass Integration komplexe Ampelschaltungen nicht modellieren kann und dass Spurwechselaktionen unzulänglich abgebildet werden. Es kann vorkommen, dass Autos den Spurwechsel von einem Beschleunigungsstreifen auf eine Autobahn nicht erreichen können und am Ende des Beschleunigungsstreifens stehen bleiben. Die Parameter von Corsim mussten stark abgeändert werden, um Messdaten widerzuspiegeln. Die QZM-Bestimmung von Integration ist nach Prevedouros et al., 1997 „nervtötend". Nachdem die Prozedur jedoch abgeschlossen ist, ist Integration effizienter und realistischer in den ermittelten Kenngrößen im Vergleich zu Corsim. Nach Rakha und Crowther, 2003 kann Integration über vier Parameter des Van Aerde Modells auf verschiedene Situationen - in diesem Falle Autobahn, Zubringerstraße und Tunnel - kalibriert werden. In Bloomberg und Dale, 2000 werden Vissim und Corsim verglichen. Die Hauptaussage der Studie ist, dass die Ergebnisse der Systeme mehr Gemeinsamkeiten als Unterschiede haben (z.B. ermittelte Reisedauern).

In Tabelle 4.2 wird ein Überblick über die grundlegenden Eigenschaften der betrachteten Systeme gegeben. Es werden die Kriterien der Anforderungsanalyse aus Abschnitt 1.4 zur Bewertung verwendet.

Corsim ist ein Simulationssystem, das sich eignet, um die Wechselwirkungen zwi- 


\begin{tabular}{|c|c|c|c|c|c|c|c|c|}
\hline & 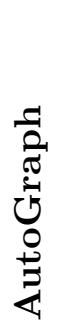 & 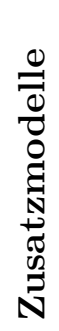 & 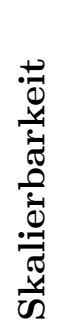 & $\frac{B}{a}$ & $\frac{3}{|c|}$ & 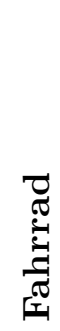 & 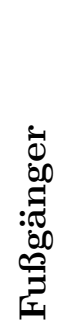 & 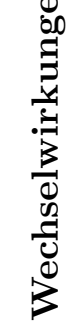 \\
\hline Corsim & $x$ & $\checkmark$ & $?$ & $\checkmark$ & $\checkmark$ & $x$ & $\checkmark$ & $\checkmark$ \\
\hline Transims & $\checkmark$ & $\checkmark$ & $\checkmark$ & $\checkmark$ & $\approx$ & $\approx$ & $\approx$ & $x$ \\
\hline Matsim & $\checkmark$ & $\checkmark$ & $\checkmark$ & $\checkmark$ & $x$ & $x$ & $x$ & $x$ \\
\hline Vissim & $x$ & $x$ & $x$ & $\checkmark$ & $\checkmark$ & $\checkmark$ & $\checkmark$ & $\checkmark$ \\
\hline Integration & $\approx$ & $\checkmark$ & $x$ & $\checkmark$ & $\approx$ & $x$ & $x$ & $x$ \\
\hline Hutsim & $x$ & $\checkmark$ & $x$ & $\checkmark$ & $\approx$ & $\approx$ & $\checkmark$ & $\approx$ \\
\hline Sumo & $\checkmark$ & $\checkmark$ & $\checkmark$ & $\checkmark$ & $\checkmark$ & $x$ & $\approx$ & $x$ \\
\hline
\end{tabular}

Tabelle 4.2: Vergleich der besprochenen Verkehrssimulationssysteme

schen Autos und Fußgängern zu untersuchen. Busrouten können definiert werden. Fahrräder lassen sich nicht abbilden. Die Modellierung ganzer Ortschaften ist aufwendig, da kein Modul zur automatisierten Erstellung des Straßengraphen existiert. Über die grundlegende Eignung zur Simulation großer Szenarien lässt sich keine Aussage treffen, da in der Literatur ausschließlich Szenarien mit wenigen Straßen simuliert wurden und keine Laufzeitanalyse vorhanden ist.

Transims bietet eine innovative Methode zur Tagesablaufsplanung von simulierten Agenten und ist sehr gut skalierbar. Die Skalierbarkeit wird jedoch durch ein Verkehrsmodell erlangt, welches auf die Simulation von Autobahnverkehr optimiert ist und sich schlecht für urbane Szenarien eignet. Fahrräder und Fußgänger werden nicht simuliert, weshalb keine Interaktion der verschiedenen Verkehrsteilnehmertypen stattfindet.

Matsim baut auf Transims auf, legt den Fokus dabei jedoch ausschließlich auf Berechnungseffizienz und Tagesplanoptimierung von Agenten. Zur Bearbeitung von Verkehrsszenarien ist das implementierte Verkehrsmodell auf Basis von Warteschlangen zu einfach gehalten. Die komplexen Wechselwirkungen zwischen verschiedenen Verkehrsteilnehmertypen lassen sich hiermit nicht abbilden.

Vissim modelliert alle geforderten Verkehrsteilnehmertypen in hohem Detaillierungsgrad. Interaktionen lassen sich abbilden. Bei Vissim muss der Benutzer einen Straßengraphen manuell bilden und kann hierfür Satellitenbilder als Grundlage verwenden. Dieser Schritt ermöglicht sehr detaillierte Straßengraphen, führt je- 
doch zu ebenso hohen Modellierungskosten. Das verwendete high-fidelity Modell führt zu langen Berechnungszeiten. Vissim eignet sich aus diesen Gründen nicht zur Simulation ganzer Ortschaften.

Integration stellt interessante Konzepte in Zusammenhang mit der Routenbestimmung für simulierte Autos vor und kann realistische makroskopische Kenngrößen produzieren. Die Generierung von QZM mittels Flussdaten auf Schnellstraßen wurde intensiv untersucht. Es eignet sich hingegen nicht für urbane Szenarien, da der Modellierungsaufwand sehr hoch ist und keine Vorbereitungen für multimodalen Verkehr getroffen wurden.

Hutsim konnte zeigen, dass es zur Optimierung von Ampelschaltungen und Echtzeitsimulation genutzt werden kann. Die Einsatzbereiche sind jedoch stets kleine Ausschnitte von Straßennetzen, die zur Durchführung einer Simulationsstudie per Hand modelliert werden müssen. Die implementierten Verkehrsmodelle sind darauf ausgelegt, detaillierte Informationen über das Straßennetz zur Verfügung zu haben, um sehr genau simulieren zu können. Dies lässt keine automatische Graphgenerierung zu und führt zusätzlich zu hohem Berechnungsaufwand. Simulationsstudien, die eine ganze Stadt umfassen sind daher sowohl vom Modellierungsaufwand, als auch von der Berechnungskomplexität her nicht durchführbar.

Sumo zeichnet sich durch diverse Importfunktionen für Straßennetze, Quelloffenheit und seine Auslegung zur Simulation großer Szenarien aus. Die integrierten Verkehrsmodelle eignen sich nicht für die Ziele dieser Arbeit, wären jedoch austauschbar.

Die Literaturbesprechung hat gezeigt, dass keines der betrachteten Systeme die in Abschnitt 1.4 geforderten Kriterien vollständig erfüllt. Das motivierende Beispiel (vgl. Abschnitt 1.1) kann somit mit keinem der betrachteten Systeme bearbeitet werden. Der folgende Teil III stellt das im Rahmen dieser Arbeit entwickelte Konzept vor. 



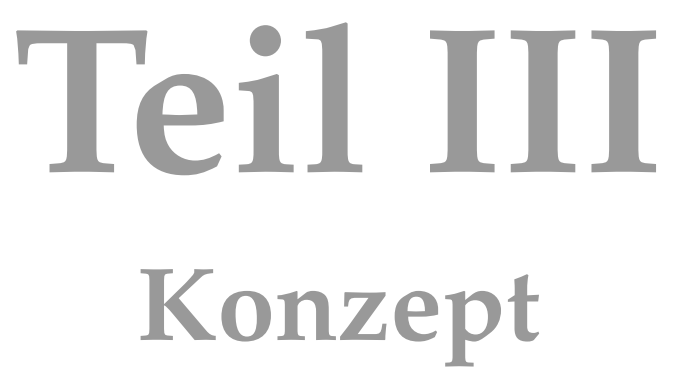





\section{Akteursorientierte multimodale Straßenverkehrssimulation}

Keines der im Stand der Forschung betrachteten Verkehrssimulationssysteme erfüllt die im Abschnitt 1.4 gestellten Anforderungen vollständig. Es muss daher geprüft werden, ob die Erweiterung eines bestehenden Systems zur Erfüllung der Anforderungen oder die Entwicklung eines neuen Konzepts mit vollständiger Implementierung eines Simulationssystems durchgeführt werden muss.

Zur direkten Erweiterung bieten sich vordergründig Transims, Matsim und Sumo an, da diese Systeme quelloffen und skalierbar sind, sowie über eine AutoGraphFunktion verfügen. Keines dieser Systeme ist jedoch dafür vorgesehen, multimodalen Verkehr zu berücksichtigen.

Bestehende mikroskopische Verkehrssimulationsmodelle eignen sich entweder nicht zur Abbildung multimodalen Verkehrs oder sind in ihrer Berechnungskomplexität zu aufwendig, um in Szenarien der geforderten Größenordnung eingesetzt zu werden.

Zur Erfüllung der in Abschnitt 1.3 gestellten Zielsetzung dieser Arbeit wird ein neues Konzept entwickelt. Es werden Methoden generiert, die aus Kartenmaterial einen Simulationsgraphen bestimmen, der die Erfordernisse für urbane multimodale Verkehrssimulationen erfüllt. Es werden mikroskopische Verkehrsmodelle für Autos, Fahrräder und Fußgänger vorgeschlagen, die zur Untersuchung der 
Wechselwirkungen zwischen den Verkehrsteilnehmertypen geeignet sind. Diese Bestandteile werden schließlich in einem Simulationssystem kombiniert, um urbane Verkehrssimulationsstudien durchführen zu können.

Es wird im Rahmen dieser Arbeit ein neues Verkehrssimulationssystem entwickelt, welches die in diesem Teil der Arbeit beschriebenen Konzepte umsetzt. Die Konzepte wurden nicht als Erweiterungen und Modifikationen in ein bestehendes Simulationssystem integriert, da eine Abschätzung des Aufwandes bezüglich Einarbeitung, Restrukturierung und Erweiterung eines bestehenden Simulationssystems höher ausfiel, als die zielgerichtete Entwicklung eines Systems auf die definierten Anforderungen.

Die vorgeschlagene Architektur ergibt ein Verkehrssimulationssystem, das die Verkehrsnachfrage datengesteuert (Stimuli oder QZM) modelliert. Bei diskreter Simulationszeit werden räumlich kontinuierliche mikroskopische Verkehrsmodelle verwendet.

In diesem Kapitel wird der Fokus auf den Simulationsablauf und die Steuerung der Simulation gelegt. Der folgende Abschnitt 5.1 beschreibt die grundlegende Architektur. In Abschnitt 5.2 wird das gewählte Routingkonzept umrissen. Schließlich erläutert Abschnitt 5.3 die wichtigsten Abläufe in den entwickelten Verkehrsmodellen.

\subsection{Architektur}

Dieser Abschnitt beschreibt die in Abbildung 5.1 dargestellte Architektur für das Simulationssystem. Die Kernkomponente zur Durchführung einer Simulationsstudie ist die Simulationssteuerung. Als Eingabe erhält sie den bereits berechneten Straßengraphen und weitere Eingabedaten - z.B. ein DGM oder einen definierten Messbereich für Emissionen. Der Ablauf der Simulation ist iterationsbasiert. In einer Simulationsiteration werden die Zustände aller aktiven Simulationskomponenten aktualisiert.

In jedem Durchlauf generiert die Simulationssteuerung Feedback. Beispiele sind die Anzahl der derzeit aktiven Verkehrsteilnehmer und deren Modal Split, Reisedauer-, Verkehrsdichte- oder Verkehrsflussinformationen für Straßen im Simulationsgebiet. Diese Informationen werden mehreren Komponenten zur Verfügung gestellt. 


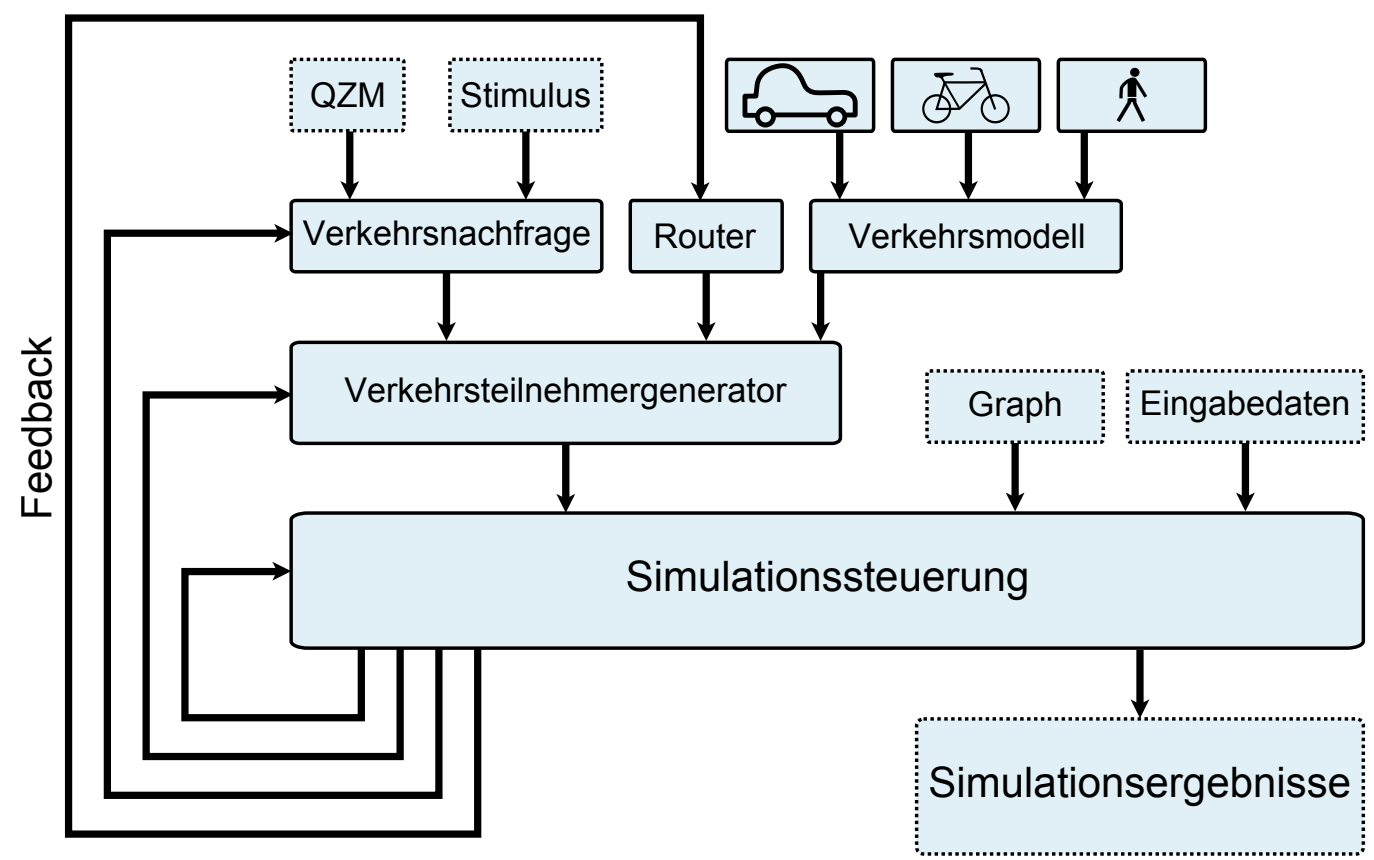

Abbildung 5.1: Architektur

Der Verkehrsteilnehmergenerator erstellt neue Verkehrsteilnehmer. Ob zu einem Zeitpunkt ein Verkehrsteilnehmer erstellt wird, hängt von der Verkehrsnachfrage ab. Diese kann verschiedene Ausprägungen haben. Es können QZM genutzt werden, um realen Verkehr nachzubilden. Alternativ können beliebige Stimuli definiert werden. Ein Beispiel hierfür ist die Vorgabe einer Anzahl an Verkehrsteilnehmern $n$, die über die Simulationsdauer gehalten werden soll. Immer wenn die aktuelle Anzahl der Verkehrsteilnehmer $n^{\prime}$ kleiner als $n$ ist, würden $n-n^{\prime}$ Verkehrsteilnehmer erstellt. $n^{\prime}$ sinkt, wenn Verkehrsteilnehmer ihre Zielposition erreicht haben und aus der Simulation entfernt werden.

Auch das genutzte Verkehrsmodell - Auto, Fahrrad oder Fußgänger, sowie Unteraten (z.B. Lkw) - hängt von der Verkehrsnachfrage ab. Es sind hierfür analog zur Verkehrsnachfrage beliebige Stimuli möglich.

Dem zu erstellenden Verkehrsteilnehmer wird Zugriff auf den Router gegeben, der zur Berechnung von Routen im Straßengraph genutzt wird. Der Router kann aktuelle Verkehrsdaten verarbeiten. Verkehrsteilnehmer können ihre Routen während der Fahrt mittels des Routers umplanen. Je nach Modus kann eine Route einen festen Start- und Zielpunkt haben oder nur einen Startpunkt. Im ersten Fall würde die Route zwischen den Positionen bestimmt. Im letzteren können Abbiegewahrscheinlichkeiten zur Generierung einer Zufallsreise im Straßennetz genutzt werden. 
Je nach Verkehrsmodell müssen bestimmte Straßentypen gemieden werden - z.B. Fußwege für Autos und Autobahnen für Fahrradfahrer. Der Router verwendet dem Verkehrsteilnehmertypen entsprechende Reiseinformationen.

Ein Simulationslauf endet nach einer Abbruchbedingung. Dies könnte eine definierte Anzahl an Simulationsiterationen sein oder das Ende der Fahrt eines ausgewählten Verkehrsteilnehmers. Simulationsergebnisse können von der Simulationssteuerung über Überwachungskomponenten während oder am Ende eines Simulationslaufes exportiert werden. Es sollen zur Visualisierung von Daten GISKomponenten genutzt werden, um beispielsweise Emissionsverteilungen über das Simulationsgebiet darzustellen. Exportformate zur weiteren Verarbeitung in Statistiksoftware ermöglichen wissenschaftliche Auswertungen von Simulationsergebnissen.

\subsection{Router}

In Abbildung 5.2 wird das grundlegende Routingkonzept dargestellt. Als Feedback von der Simulationssteuerung kann der Router aktuelle Reisedaten verwenden. Ein Beispiel hierfür sind gemessene Durchfahrtdauern auf den jeweiligen Straßen getrennt nach Verkehrsteilnehmertypen und Fahrtrichtungen. Die Bewertung potenzieller Straßen kann alternativ über eine Reisedauerschätzung via Nutzung der Kantenlänge und ihrer Geschwindigkeitsbegrenzung geschehen. Für probabilistisches Routingverhalten können Kantennutzungswahrscheinlichkeiten in Abhängigkeit zu verbundenen Knoten genutzt werden. Es wären an dieser Stelle beliebige Bewertungsfunktionen nutzbar (in Abbildung 5.2 durch ??? gekennzeichnet). In der Zukunft könnte beispielsweise die Steigung eines Straßenverlaufs für Fahrräder oder die Enge der Kurven für Lkw ergänzt werden.

Der Router kann einen Pfad zwischen Quelle und Ziel berechnen. Die Positionen können über Knoten und Kanten im Graphen angegeben werden. Falls Kanten verwendet werden, muss der Router geeignete Knoten auswählen und hierbei Einbahnstraßen berücksichtigen. Der berechnete Pfad besteht aus einer Folge von Knoten und Kanten.

Falls der Router von einem Verkehrsteilnehmer während seiner Bewegung im Simulationsgraphen verwendet wird, wird über ein Umplankriterium geprüft, ob durch eine Änderung des Pfades die Güte des Agentenplanes verbessert werden kann. Als Eingabe würden in diesem Fall die aktuelle Position als Quelle und die Zielposition als Ziel verwendet. 


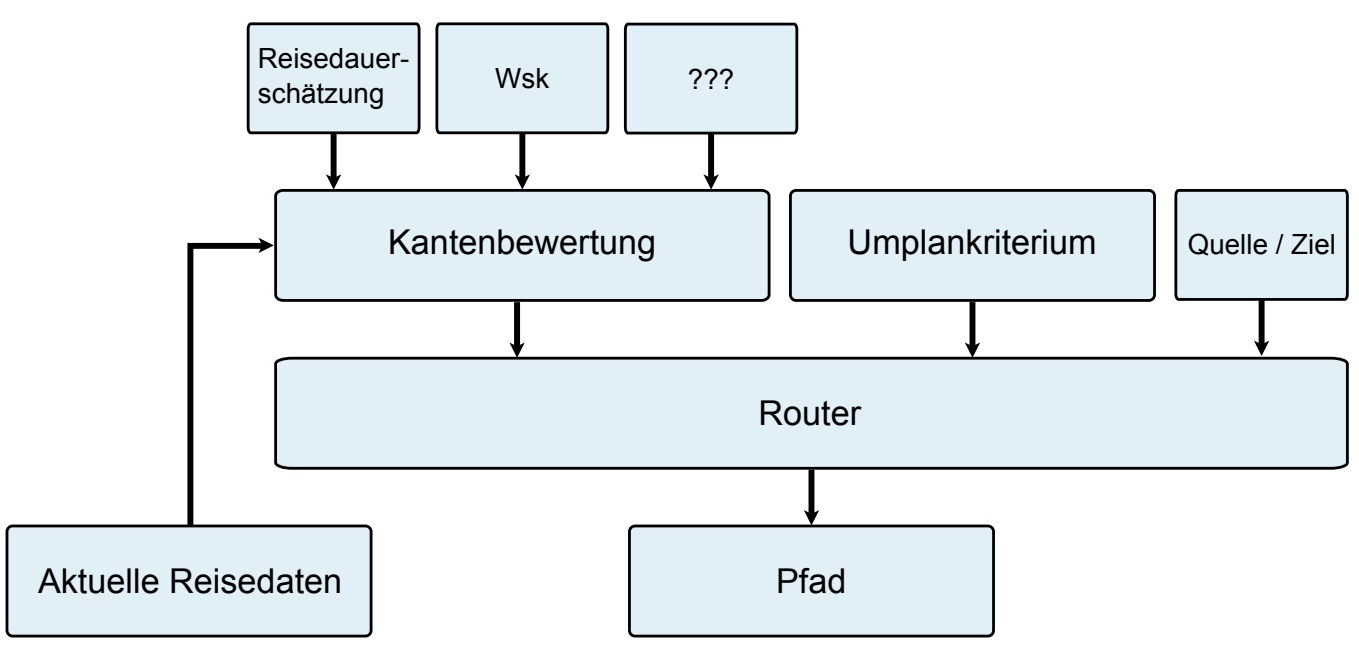

Abbildung 5.2: Architektur: Router

Der Router nimmt eine zentrale Rolle bei der Simulation von Straßenverkehr ein, da er für die Verteilung des Verkehrs zuständig ist. Es genügt nicht, ausschließlich die kürzesten Wege als Route zu verwenden, da bereits bei geringen Verkehrsmengen Staus entstehen würden. Menschen stützen sich bei der Routenplanung auf individuelle Erfahrungen und Vorlieben. Die Wahl einer geeigneten Kantenbewertungsfunktion ist somit essenziell. Es ist möglich, dass Teilgruppen der simulierten Verkehrsteilnehmer unterschiedliche Kantenbewertungsmethoden verwenden.

\subsection{Verkehrsmodell}

Die Verkehrsmodelle für Autos, Fahrräder und Fußgänger unterscheiden sich hinsichtlich ihrer Verhaltensweisen. Zur Simulationssteuerung verfügen sie jedoch über die beiden grundlegenden Schritte update und provide. Abbildung 5.3 skizziert das Vorgehen.

Die Simulationssteuerung ruft in jedem Zeitschritt die update-Methoden aller Verkehrsteilnehmer auf. In diesem Schritt aktualisieren die Verkehrsteilnehmer ihre internen Zustände. Diese bestehen aus ihrer Position, Geschwindigkeit, Spur (falls es sich nicht um Fußgänger handelt) und internen Parametern. Ein interner Parameter kann beispielsweise ein Aggressionspotenzial sein, welches ansteigt, wenn ein Fußgänger eine Straße nicht überqueren kann (vgl. Abschnitt 3.4.1).

In einem zweiten Schritt ruft die Simulationssteuerung provide auf. Die Verkehrsteilnehmer aktualisieren in diesem Schritt ihre öffentlich wahrnehmbaren Zustän- 


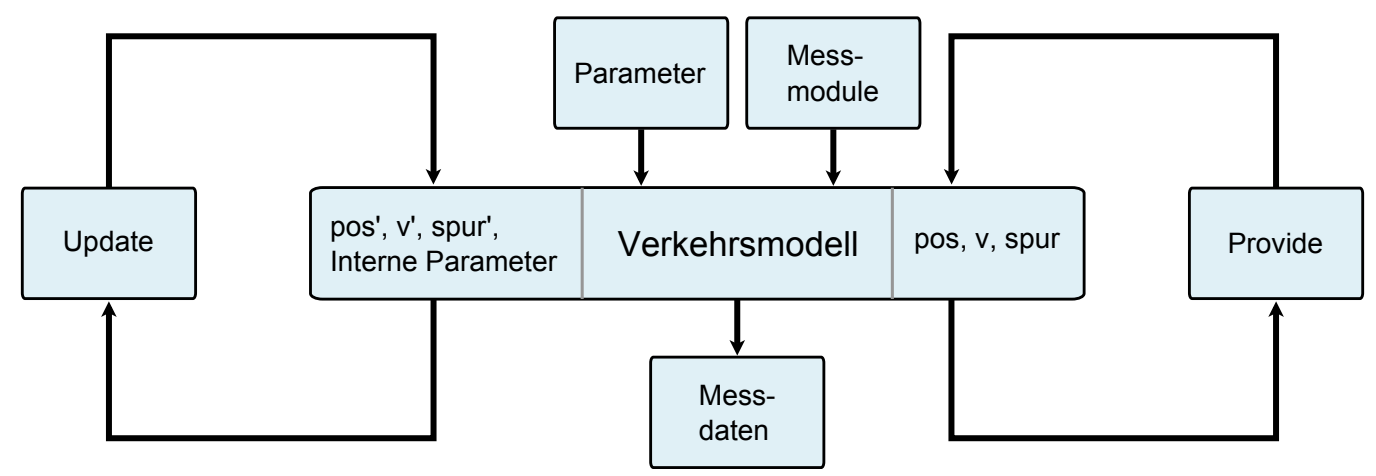

Abbildung 5.3: Architektur: Verkehrsmodell

de. Diese sind beispielsweise ihre Position, Geschwindigkeit, Spur oder auch ein Blinker bzw. Handzeichen, um Abbiegevorhaben anzuzeigen. Während update berechnen alle Verkehrsteilnehmer ihr Folgeverhalten und während provide führen sie ihre Aktionen durch. Im Schema aus Abbildung 5.3 werden im provide-Schritt pos $=p^{\prime} s^{\prime}, v=v^{\prime}$ und spur $=$ spur $^{\prime}$ gesetzt und somit Bewegungen und Geschwindigkeitsänderungen durchgeführt.

Die Trennung zwischen update und provide ist ein Mechanismus, um ein synchrones Update zu garantieren. Es ist auf diese Weise nicht möglich, dass ein Verkehrsteilnehmer mit veralteten Informationen seinen neuen Zustand berechnet. Ansonsten könnten wahrnehmungsbedingte Fahrfehler geschehen, wenn beispielsweise ein Vordermann $n$ seinen Zustand bereits aktualisiert und seine Geschwindigkeit von $v_{n}$ auf $v_{n}^{\prime} \ll v_{n}$ drastisch reduziert hat, der Hintermann $n+1$ jedoch mit der veralteten Geschwindigkeit $v_{n}$ seinen Zustand aktualisiert. Der Synchronisationsschritt ermöglicht in der Folge einfachere Verkehrsmodelle.

Jeder Verkehrsteilnehmer erhält individuelle Parameter. Beispiele hierfür sind seine Maße (Breite und Länge), Maximalgeschwindigkeit und Beschleunigungsvermögen. Über eine geeignete Parametrisierung lassen sich individuelle Fahrweisen und Fahrzeugtypen definieren. Ein Lkw ist beispielsweise länger und breiter als ein Pkw. Er verfügt über ein geringeres Beschleunigungspotenzial und eine niedrigere Maximalgeschwindigkeit. Die grundlegenden Fahrverhaltensweisen sind jedoch mit denen eines Pkw vergleichbar.

Jeder Verkehrsteilnehmer kann durch Messmodule erweitert werden. Es können beispielsweise Reisedauern, Geschwindigkeiten, Beschleunigungs- und Bremswerte extrahiert werden. Mit Hilfe von Messmodulen können Wartezeiten an Ampeln ebenso protokolliert werden, wie interne Parameter - z.B. der zeitliche Verlauf der Aggressivität eines Fußgängers. 


\section{Modellierung von Verkehrswegen}

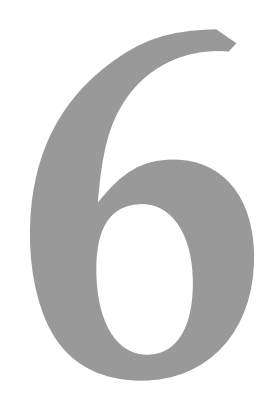

Dieses Kapitel beschreibt den gewählten Ansatz zur Erstellung eines Graphen für die Simulation von Straßenverkehr. Es werden die grundlegenden Vorgehensweisen diskutiert. Weitergehende Informationen werden in Kapitel 8 gegeben. Im Rahmen dieser Arbeit wird das in Abbildung 6.1 gezeigte Verfahren vorgeschlagen.

Eine OpenStreetMap-Datei wird geladen. Sie enthält Geoinformationen in einem XML-Format (a). Die Daten werden unter Nutzung von GeoTools in verschiedene

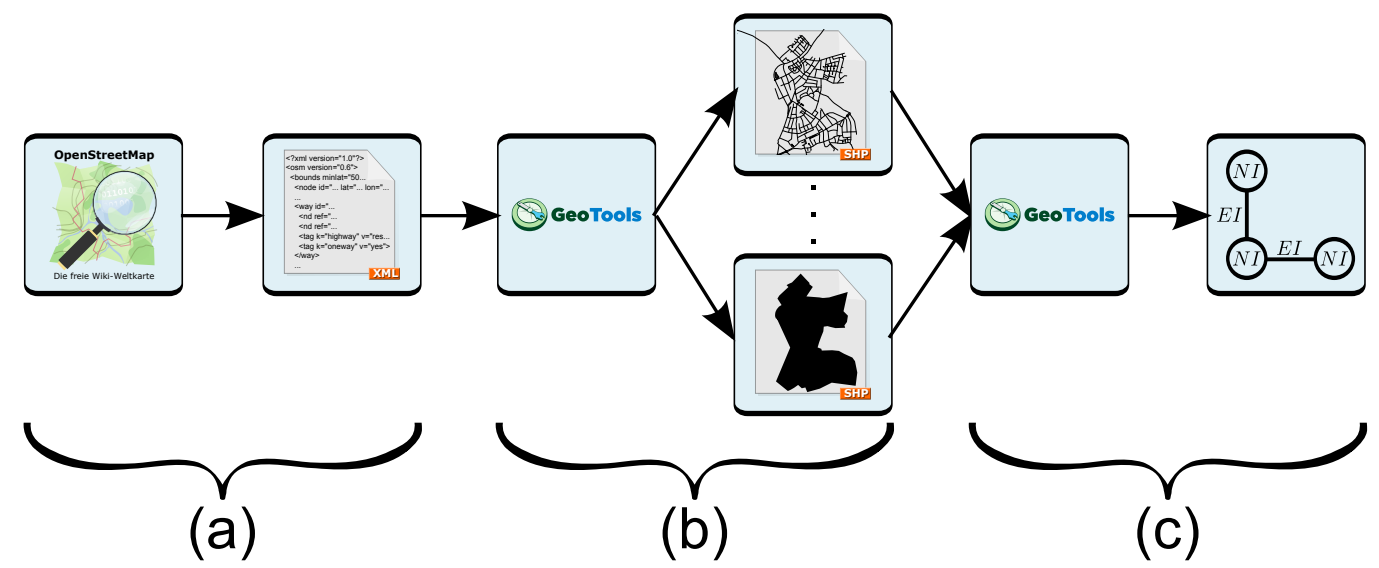

Abbildung 6.1: Vorgehen bei der Generierung eines Graphen aus OSM 
SHP-Layer separiert (b), aus denen anschließend ein Graph generiert werden kann (c). Die einzelnen Schritte und dahinterstehenden Überlegungen werden in den folgenden Abschnitten besprochen.

Abschnitt 6.1 bespricht, wie OSM-Dateien aufgebaut sind. In Abschnitt 6.2 wird gezeigt wie Ausschnitte aus großen Dateien bestimmt werden können. In Abschnitt 6.3 wird beschrieben, wie ein OSM-Ausschnitt weiterverarbeitet werden kann, um ihn anschließend zur Erstellung eines Graphen zu verwenden (Abschnitt 6.4).

\subsection{OpenStreetMap als Datenquelle}

Im Rahmen dieser Arbeit werden Landkarten des OpenStreetMap-Projekt:11 (OSM) genutzt, da dessen Daten offen, kostenlos und dokumentiert sind.

Landkarten werden in OSM von Benutzern erstellt und gewartet. Dieses Vorgehen ist kostengünstig und häufig werden Areale kartographiert, zu denen bisher keine Informationen verfügbar waren Goodchild, 2007. OSM entwickelt sich ähnlich wie die Wikipedia 2 Die Nutzergruppen an verschiedenen Orten kartographieren teils sehr genau und aktuell, teils fehlerhaft. OSM eignet sich immer dann, wenn kein high-fidelity Modell entwickelt werden soll, da Details im Vergleich zu simulationsoptimierten Karten fehlen. Beispielsweise werden keine Beschleunigungsstreifen, Straßenbreiten oder verschiedene Ampeltypen im Kartenmaterial modelliert. Diese Informationen müssen ergänzt werden. Einen Überblick über OSM gibt Bennett, 2010.

Wissenschaftliche Untersuchungen zur Qualität von Kartenmaterial gestalten sich aufwendig. In Neis et al., 2011 wird die Qualität der Geodaten von OSM mit denen des kommerziellen Anbieters TomTom verglichen. Die Menge an verzeichneten Straßen ist demnach bei OSM größer als beim kommerziellen Anbieter. Die Vorteile von TomTom liegen in der genaueren Abbildung der Verbindungen zwischen Straßen und etwaigen kritischen Informationen (z.B. Abbiegeverbote), die zur Navigation benötigt werden.

In Ather, 2009 wurden GPS-Spuren während Autofahrten innerhalb von London protokolliert und die Abweichung der OSM-Daten von den gemessenen Spuren ermittelt. Es wurde eine Genauigkeit von ca. $90 \%$ ermittelt. Es wurden jedoch Schwächen von OSM bei der Vollständigkeit von Straßenattributen ermittelt. Die

1 http://www .openstreetmap.org

2 http://www.wikipedia.de 

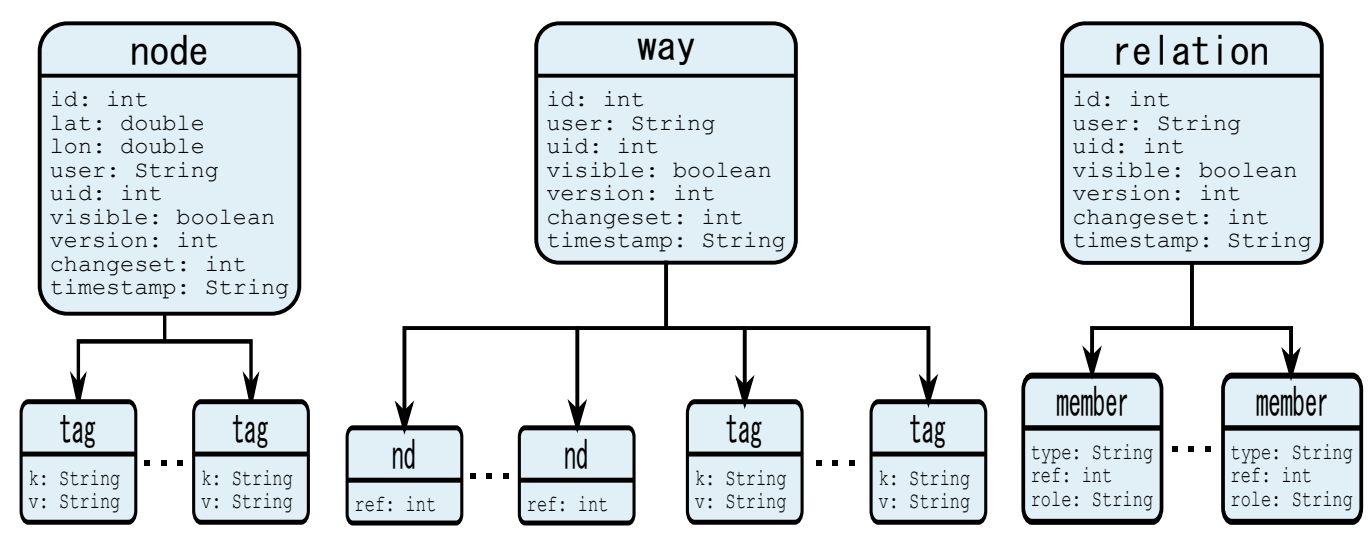

Abbildung 6.2: OSM-Entitäten

Daten von OSM wurden zusätzlich mit Kartenmaterial der National Mapping Agency of Great Britain verglichen und zeigten eine ähnliche Genauigkeit.

Nach Haklay, 2010 waren im Jahr 2008 ca. 29 \% von England in OSM abgebildet. Diese Diskrepanz zu den Daten über London zeigt, dass die Vollständigkeit der Daten stark von den lokalen Kartographen abhängt. Je dichter besiedelt ein Gebiet ist, desto mehr Menschen unterstützen das Projekt in der Regel.

In [Zielstra und Zipf, 2010 werden Karten von OSM mit Karten von TeleAtlas (heute von TomTom übernommen) über einen Zeitraum von knapp einem Jahr verglichen. OSM bietet in absoluten Zahlen gemessen weniger Informationen über deutsche Straßen, verfügt jedoch über eine steile Wachstumskurve. Es muss jedoch beachtet werden, dass auch hier die Vollständigkeit von OSM je nach Kartenausschnitt schwankt, da OSM auf den Einsatz von freiwilligen Helfern angewiesen ist.

In OSM-Dateien wird stets zuerst das umschließende Rechteck angegeben. Der Hauptteil einer OSM-Datei ist in drei Blöcke unterteilt: node-Definitionen, wayDefinitionen und relation-Definitionen. Die Blöcke werden in der Datei in der genannten Reihenfolge strikt voneinander getrennt behandelt. Abbildung 6.2 zeigt den Aufbau der Grundbausteine.

Ein node verfügt über Längen- und Breitengrad, sowie eine id und gegebenenfalls zusätzliche Tags und irrelevante Zusatzinformationen. Ein way führt eine Liste von nd-Elementen, deren ref-Werte den IDs der entsprechenden node-Objekten entsprechen. Ein OSM-way kann zur Definition geometrischer Objekte, die über Punkte hinausgehen und sich aus Punkten zusammensetzen, genutzt werden. relationObjekte dienen vorwiegend zur Beschreibung von Routen (z.B. Radrouten, Wan- 


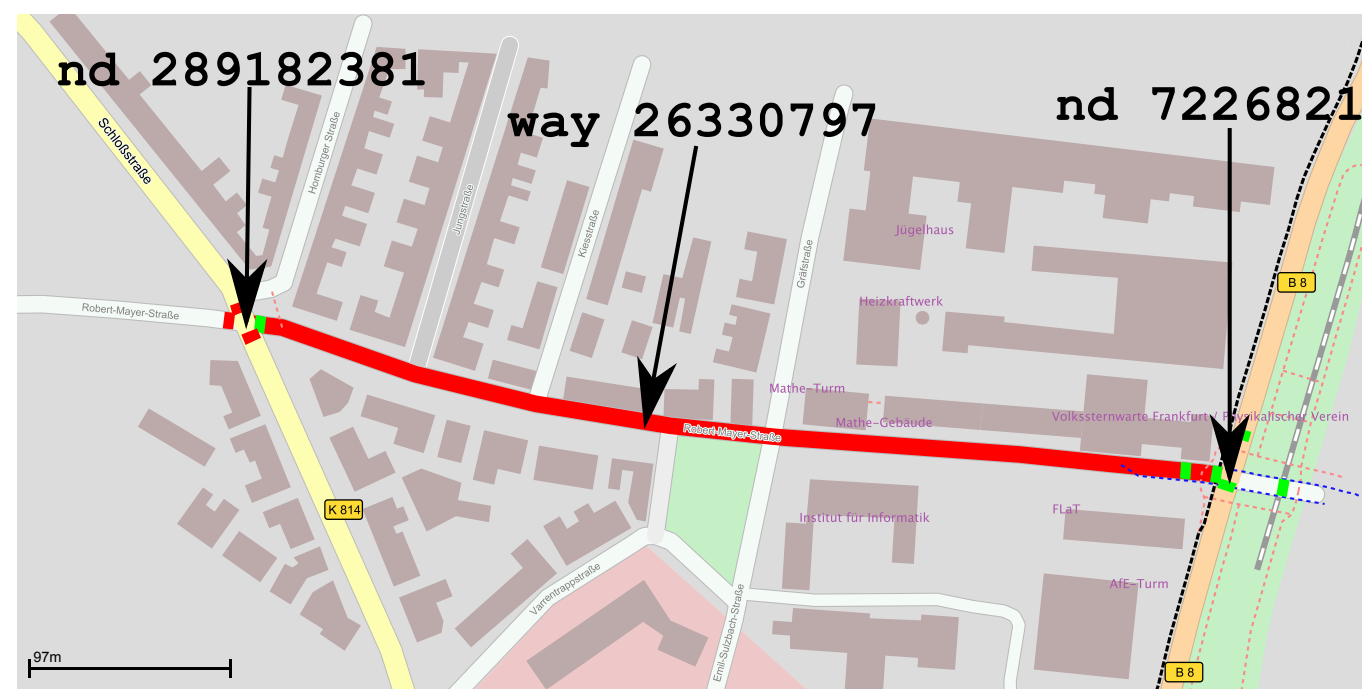

Abbildung 6.3: OSM-Ausschnitt

derwege, Busrouten). Sie können way-Objekte (z.B. Busroute) und node-Objekte (z.B. Bushaltestelle) enthalten. Die folgende Aufstellung gibt einen Überblick über in OSM vorkommende Geometrietypen (in Klammern, welche OSM-Objekte den jeweiligen Typen beschreiben können):

Linienzüge (way, relation) Linienzüge (LineStrings) sind eine Folge von Punkten, die eine Strecke definieren. Sie können u.a. genutzt werden, um Straßen (Wege, Pfade, usw.), Bäche und Bahngleise zu definieren.

Polygone (way) Polygone sind ein Spezialfall eines Linienzuges, wobei der Startund Endpunkt identisch sind. Sie beschreiben Flächen und können u.a. genutzt werden, um Ortsbereiche, Gebäude, Flüsse und landschaftliche Gebiete (z.B. Feld, Wald, Wiese) zu definieren.

Punkte (node) Punkte werden zur Markierung von verschiedenen Dingen auf der Landkarte genutzt (z.B. Ortschaften, Bushaltestellen, Schulen, Museen, usw.).

Über zusätzliche tag-Elemente können beliebige Informationen in nodes, ways und relations gespeichert werden.

Abbildung 6.3 zeigt einen Kartenausschnitt von OSM, der die Robert-MayerStraße in Frankfurt am Main enthält. Die Robert-Mayer-Straße ist rot markiert. Der folgende XML-Code zeigt die OSM-Definitionen des korrespondierenden wayObjekts. 


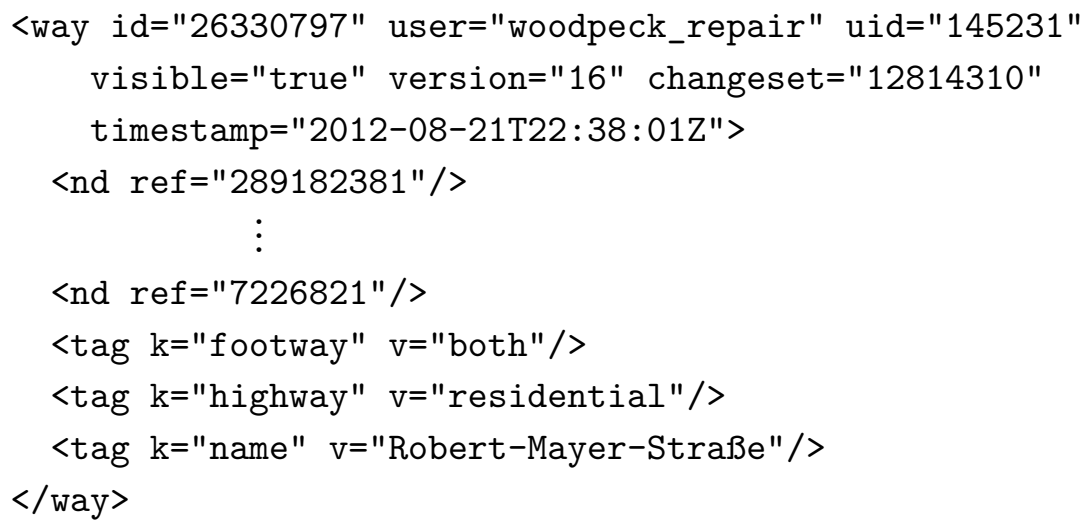

Der Verlauf des way-Objekts wird über die referenzierten nd-Ids definiert. Insgesamt verfügt der way über 14 nd-Objekte, die an Kreuzungen und Positionen von Richtungsänderungen der Straße positioniert sind. Als Zusatzinformation ist angegeben, dass ein beidseitiger Gehweg vorhanden ist, die Straße vom Typ residential (schmalere Straße, häufig in Wohngebieten verwendet) ist und den Namen „Robert-Mayer-Straße“ trägt.

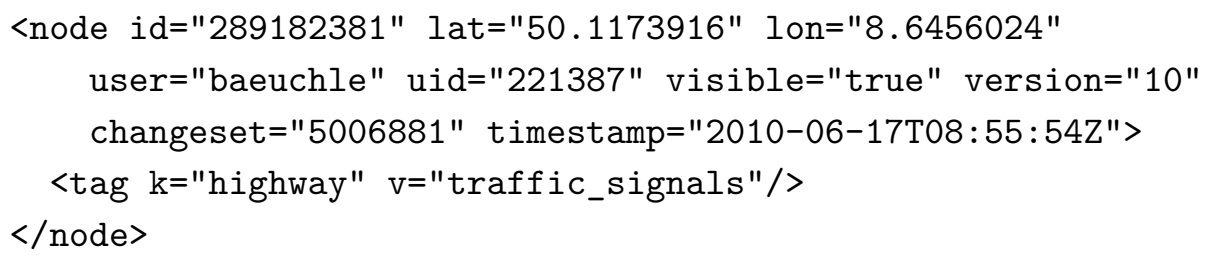

Der erste referenzierte nd mit der Id 289182381 verfügt über lat/lon-Angaben und die Information, dass sich an diesem Knoten eine Ampel befindet (traffic_signals).

OSM-Dateien können einerseits über die Exportfunktion der Webseite bezogen werden und andererseits über regelmäßige Builds ganzer Gebiete (z.B. Hessen). Von der Webseite können nur Gebiete mit maximal 50.000 Knoten heruntergeladen werden. Der OSM-Ausschnitt Hessens hat eine Dateigröße von knapp 2 GB (Stand: 31.07.2012). Dies macht deutlich, dass Ausschnitte aus großen OSM-Dateien bestimmt werden müssen, um anschließend einen Graphen zur Simulation berechnen zu können. 


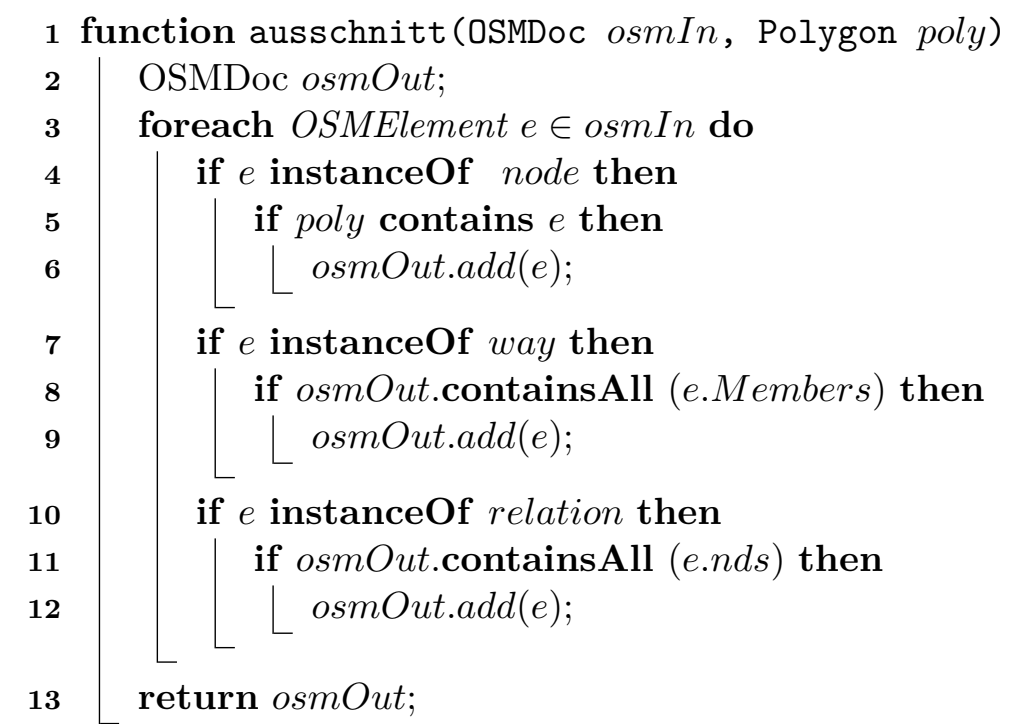

Algorithmus 6.1: Bestimmung eines Kartenausschnitts aus einer OSM-Datei

\begin{tabular}{|c|c|}
\hline$|o s m I n|$ & Anzahl Elemente in OSM-Datei \\
\hline $\mid$ osmIn node $\mid$ & Anzahl Node-Elemente in OSM-Datei \\
\hline$\left|o s m I n_{\text {way }}\right|$ & Anzahl Way-Elemente in OSM-Datei \\
\hline$\left|o s m I n_{\text {rel }}\right|$ & Anzahl Relation-Elemente in OSM-Datei \\
\hline $\mid$ polynode $\mid$ & Anzahl Node-Elemente innerhalb poly \\
\hline
\end{tabular}

Tabelle 6.1: Notation: Laufzeitberechnung für OSM-Ausschnittsbestimmung

\subsection{Ausschnittbestimmung aus OpenStreetMap}

Da bei OSM geometrische Koordinaten ausschließlich in node-Objekten vorkommen und diese den ersten Block des Hauptteils der Datei ausmachen, kann Algorithmus 6.1 einen Ausschnitt bestimmen.

Zur Bestimmung der Laufzeit von Algorithmus 6.1 wird die in Tabelle 6.1 gezeigte Notation verwendet.

Die Laufzeit der containsAll-Operation in den Zeilen 8 und 11 hängt nicht von $\mid$ osmIn $\mid$, sondern von $\mid$ polynode $\mid$ ab. Sie wird in der Implementierung des Algorithmus mit Hilfe einer HashMap durchgeführt, die die id-Werte der node-Elemente verwaltet, die innerhalb poly liegen. Dies reduziert den Auwand einer Einfügeund Lookup-Operation im average case auf $\mathcal{O}(1)$ (vgl. Cormen et al., 2001). Die Gesamtlaufzeit von Algorithmus 6.1 ist 


\begin{tabular}{llr}
\hline $\mid$ osmIn $_{\text {node }} \mid$ & $=$ & 6.220 .319 \\
$\mid$ osmIn & $=$ & 953.806 \\
$\mid$ way & $=$ & 13.893 \\
\hline
\end{tabular}

Tabelle 6.2: OSM: Anzahl Elemente nach Typen

$$
\begin{aligned}
& \mathcal{O}\left(\mid \text { osmIn } \text { node }_{\text {ne }} \mid\right) \cdot \mathcal{O}(k) \\
& +\quad \mathcal{O}\left(\mid \text { poly }_{\text {node }} \mid\right) \cdot \mathcal{O}(1) \\
& +\mathcal{O}\left(\left|o s m I n_{\text {way }}\right|\right) \cdot \mathcal{O}(1) \\
& +\mathcal{O}\left(\left|o s m I n_{\text {rel }}\right|\right) \cdot \mathcal{O}(1) \\
& =\mathcal{O}\left(k \cdot\left|o s m I n_{\text {node }}\right|+\mid \text { poly }_{\text {node }}|+| o s m I n_{\text {way }}|+| o s m I n_{\text {rel }} \mid\right)
\end{aligned}
$$

Gleichung 6.1 behandelt die Zeilen 4 bis 6 des Algorithmus 6.1. Die Prüfung, ob ein Koordinatenpaar innerhalb eines Polygons liegt, geschieht mit einem Punkt-inPolygon-Test (vgl. Bartelme, 2005, S. 103f]), dessen Komplexität $\mathcal{O}(k)$ ist, wobei $k$ die Anzahl an Kanten des Polygons darstellt. Jeder Eintrag aus osmIn node, der innerhalb von poly $y_{\text {node }}$ liegt, wird in $\mathcal{O}(1)$ in die HashMap eingefügt (Gleichung 6.2 . In den Gleichungen 6.3 und 6.4 werden jeweils Lookup-Operationen in der HashMap in $\mathcal{O}(1)$ durchgeführt. Die Gesamtlaufzeit kann auf Gleichung 6.5 zusammengefasst werden. Falls das Polygon ein Rechteck ist, wird für die Prüfung der Inklusion eines Koordinatenpaars konstante Zeit benötigt, sodass

$$
\mathcal{O}(\mid \text { osm In }|+| \text { polynode } \mid)
$$

als Komplexität des Algorithmus angegeben werden kann. Aus der Struktur von OSM-Dateien ergeben sich folgende Aussagen:

$$
\begin{aligned}
& \left|o s m I n_{\text {way }}\right| \ll\left|o s m I n_{\text {node }}\right| \\
& \left|o s m I n_{\text {rel }}\right| \ll\left|o s m I n_{\text {node }}\right|
\end{aligned}
$$

Diese können anhand der Tabelle 6.2 nachvollzogen werden. In der Tabelle werden die Kenngrößen einer OSM-Datei angegeben, die das Bundesland Hessen vollständig abdeckt $t^{3}$

\footnotetext{
${ }^{3}$ Stand: 20.10 .2011
} 


\begin{tabular}{lllc}
\hline Name & Geometrietyp & OSM & Funktion \\
\hline roads & LineString & way & $\checkmark$ \\
routen & LineString & relation & $\times$ \\
buildings & Polygon & way & $\times$ \\
polygone & Polygon & way & $\checkmark$ \\
railways & LineString & way & $\times$ \\
waterwaysPG & Polygon & way & $\times$ \\
waterwaysLS & LineString & way & $\times$ \\
points & Point & node & $\checkmark$ \\
Zebrastreifen & Point & node & $\checkmark$ \\
\hline
\end{tabular}

Tabelle 6.3: Aufteilung der OSM-Daten in Layer. Funktion gibt an, ob der Layer zur Erstellung des Graphen verwendet wird. Falls nicht, wird der Layer ausschließlich zur Visualisierung genutzt.

Im Durchschnitt werden mittels poly Kartenausschnitte gewählt, die deutlich kleiner als die Ursprungskarte sind. In diesem Fall sinkt die Laufzeit von Algorithmus 6.1 approximativ auf $\mathcal{O}(|\operatorname{osm} \operatorname{In}|)$.

Das beschriebene Verfahren integriert nur diejenigen Geometrieobjekte $g$, die vollständig von poly abgedeckt werden. Sollen alle $g$ integriert werden, die zumindest eine Koordinate innerhalb poly haben, so wird ein zweiter Durchlauf der Schleife (Zeile 3 von Algorithmus 6.1] benötigt, was zu einer Verdoppelung der Laufzeit führt. Im ersten Durchlauf werden die IDs der node-Elemente, die durch poly abgedeckt sind, gespeichert. Ein way-Element wird verarbeitet, wenn mindestens eine node-Id des ways gespeichert ist. In diesem Fall werden die restlichen node-Ids ebenfalls gespeichert. Im zweiten Durchlauf werden alle node-Elemente, deren Ids gespeichert sind, in das Ausgabedokument eingefügt. way- und relation-Elemente werden herkömmlich behandelt. Auf diese Weise wird der Kartenausschnitt nicht strikt an den Grenzen von poly beschnitten, sondern aus poly herausragende Geometrien werden vollständig in das Ausgabedokument übernommen.

\subsection{Filterung relevanter Informationen und Aufteilung in Layer}

Das OSM-Format speichert Objekte in einer Struktur, die es nicht erlaubt, Geometrische Operationen auf den Objekten trivial umzusetzen. Es ist von Vorteil, Linienzüge, Polygone usw. in geeigneten Datenstrukturen zu verwalten. Um dies zu erreichen, werden die Daten in verschiedene Layer getrennt, die die unterschiedlichen Geometrietypen aufnehmen. Eine weitere Unterscheidung nach inhaltlichen Kriterien wird durchgeführt. Es werden die in Tabelle 6.3 gezeigten Layer erstellt. 
Es werden Layer im Shapefile-Format erstellt, welches ein Quasi-Standard im Bereich der Geoinformationssysteme ist. Zur Erstellung und Verarbeitung von Shapefiles wird die Java-Klassensammlung GeoTools verwendet, die quelloffen ist. Diese Wahl hat den Vorteil, beliebige Layer in das Programm einbinden zu können. Die verschiedenen OSM-Typen werden nacheinander verarbeitet. Zuerst werden alle Elemente vom Typ way in ihre entsprechenden Layer eingeteilt. Dies geschieht mittels der in den Objekten gespeicherten Tags. Objekte identischen Geometrietyps können in unterschiedlichen Layern verwaltet werden, falls dies die spätere Verarbeitung vereinfacht. Im Layer polygone werden beispielsweise Ortsumrisse, Wälder und Wiesen verwaltet; buildings nimmt ausschließlich Gebäude auf, die beim Rendern über den restlichen Polygonen gezeichnet werden. Es werden zwei Layer zur Darstellung von Gewässern erstellt, um zwischen Linienzügen und Polygonen unterscheiden zu können. Dies geschieht über einen Analyseschritt der Geometrien. Falls Start- und Endpunkt des way-Objektes identisch sind, handelt es sich um ein Polygon, ansonsten um einen Linienzug.

Es folgt die Verarbeitung der relation-Objekte, die z.B. Bus- und Bahnlinien markieren. Zuletzt werden alle node-Objekte im points-Layer gespeichert, die über ausgewählte Attribute verfügen (z.B. traffic_sign für Ampeln). Die Erstellung des Zebrastreifen-Layers wird im folgenden Abschnitt 6.4 beschrieben.

Die Verarbeitung geschieht mit Hilfe eines DOM-Parsers für XML-Daten. Hierfür muss ein DOM-Baum der gesamten OSM-Datei erstellt werden. Dieser Schritt führt zu hohem Speicherbedarf. Er ist jedoch alternativlos, da die node-Objekte, auf die die way- und relation-Objekte verweisen, zur Erstellung der Geometrien benötigt werden und ein sequentielles Parsen der OSM-Datei keinen direkten Zugriff hierauf ermöglicht. Für besonders große Kartenausschnitte, die nicht mehr im Arbeitsspeicher gehalten werden können, wäre die Erstellung einer Datenbank für node-Objekte möglich. Auf diese Weise könnten way- und relation-Objekte erstellt werden, ohne den gesamten DOM-Baum im Speicher zu halten. Die Berechnungsdauer würde sich jedoch erheblich verlängern.

\subsection{Generierung eines ExtendedGraph}

Die GeoTools-Klassensammlung bietet Klassen zum Zugriff auf Shapefiles. Um große Datenbanken verarbeiten zu können, werden nur die Daten im Speicher gehalten, die derzeit verwendet werden. Aus einem LineString-Layer kann unter Nutzung der Klasse FeatureGraphGenerator ein Graph erstellt werden, der edges und nodes enthält. Diese werden anschließend weiter verarbeitet, um zur Nutzung 


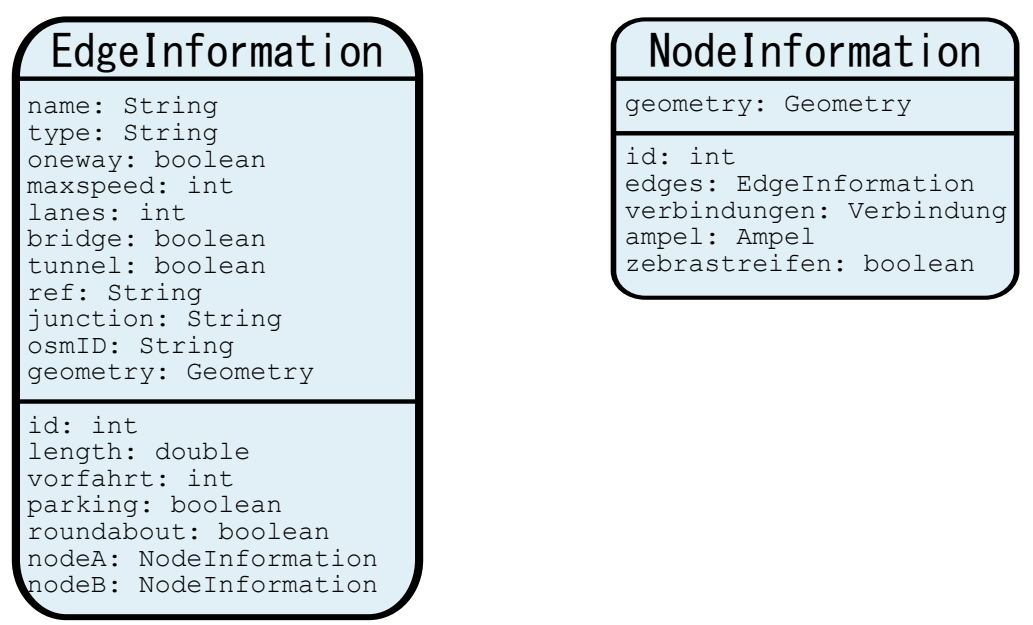

Abbildung 6.4: Attribute von Edgelnformation und Nodelnformation: Unterteilung in zwei Gruppen: Die Attribute der ersten Gruppe können direkt aus dem Kartenmaterial ausgelesen werden, die der zweiten Gruppe entstehen durch Analyseschritte.

in einer Simulation geeignet zu sein. Für jedes Objekt der Klasse edge (node) wird ein Objekt der Klasse EdgeInformation (EI) (NodeInformation (NI)) erstellt, deren grundlegender Aufbau in Abbildung 6.4 gezeigt wird.

$|E I|$ bezeichne die Länge von EI in $[m]$. Sie entspricht der Summe der euklidischen Distanzen zwischen je zwei aufeinanderfolgenden Koordinaten der EI.

Jede Straße in OSM verfügt über die Definition des Straßentyps. Aus dieser lassen sich approximativ fehlende Attribute mittels LUTs ermitteln. Für eine Straße des Typs residential (Wohngebiet), werden per LUT maxspeed $=30$ und lanes $=$ 1 gesetzt, falls die entsprechenden Attribute undefiniert sind. Geschwindigkeiten werden in OSM in $[\mathrm{km} / \mathrm{h}]$ definiert und zur Simulation nach $[\mathrm{m} / \mathrm{s}]$ skaliert.

Die Verbindung zwischen zwei EIs wird über eine NI hergestellt, die eine Menge an EIs verwaltet, die mit NI verbunden sind. Es sei $n=\left|\mathrm{NI}_{\text {edges }}\right|$.

$$
\mathrm{NI}_{\text {edges }}=\left\{\mathrm{EI}_{1} \cdots \mathrm{EI}_{n}\right\}
$$

Es gilt:

$$
\exists \text { Verbindung }\left(\mathrm{EI}_{i}, \mathrm{EI}_{k}\right) \forall i, k \mid(1 \leq i, k \leq n) \wedge(i \neq k)
$$




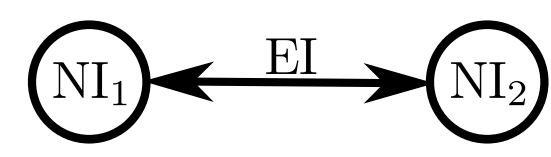

(a) Eine Kante

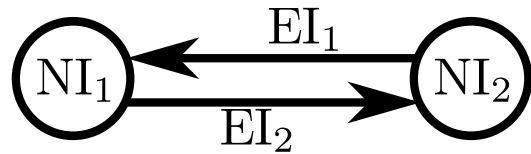

(b) Zwei Kanten

Abbildung 6.5: Modellierung von Straßen mittels Kanten

Ein Verbindungsobjekt verwaltet die Verbindungsrichtung und den Winkel zwischen zwei EIs, sowie ob die Verbindung nutzbar ist. Wenn z.B. $\mathrm{EI}_{k}$ entgegen einer Einbahnstraße befahren würde, ist die korrespondierende Verbindung $\left(\mathrm{EI}_{i}, \mathrm{EI}_{k}\right)$ nicht nutzbar. Die einzelnen Attribute, sowie Erweiterungen der EI - und NI Definitionen werden in Kapitel 8 besprochen.

Eine Straße zwischen zwei Knoten $\mathrm{NI}_{1}$ und $\mathrm{NI}_{2}$ kann auf die in Abbildung 6.5 dargestellten Weisen modelliert werden. Einerseits kann (Teil (a)] eine Kante EI genutzt werden, um die Straße zu modellieren. Andererseits können (Teil (b)] zwei Kanten $\mathrm{EI}_{1}$ und $\mathrm{EI}_{2}$ verwendet werden, um die einzelnen Fahrtrichtungen zu beschreiben. Zwei Kanten zu verwenden hat den Vorteil, dass Einbahnstraßen modelliert werden können, indem eine der Kanten entfernt wird. Wird nur eine Kante verwendet, muss die Richtung einer Einbahnstraße über einen Parameter innerhalb der Kante vermerkt werden. Die Einbahnstraße kann beispielsweise von $\mathrm{NI}_{1}$ ausgehen.

Im Rahmen dieser Arbeit wird nur eine Kante verwendet, um eine Straße zu modellieren (Abbildung 6.5(a)). Dies erleichtert die Modellierung von Interaktionen zwischen verschiedenen Verkehrsteilnehmern auf einer Straße. Ein Fußgänger, der eine Straße EI an Position $p$ überqueren möchte, kann die Verkehrsteilnehmer in seinem Bereich $[p-\Delta \cdots p+\Delta]$ von EI abfragen und muss nicht mehrere EIs berücksichtigen.

Die Fahrtrichtung eines Verkehrsteilnehmers $v$ auf einer EI kann über die NI definiert werden, die $v$ zuletzt passiert hat. Einbahnstraßen erhalten als Attribut oneway = true, um festzulegen, dass sie ausschließlich von nodaA nach nodeB befahren werden können. In OSM werden Straßen stets von ihrem Start- zu ihrem Zielpunkt definiert. Eine Einbahnstraße läuft somit immer von nodaA nach nodeB, was keine weiteren Aktionen notwendig macht.

Ein Straßengraph, der mittels EIs und NIs modelliert ist, wird ExtendedGraph genannt, da er komplexere Informationen speichert, als ein einfacher Graph. Komplexe Straßenkarten können über die beschriebene Graphdatenstruktur definiert 
werden. Mit leichten Modifikationen und Erweiterungen können bekannte Graphalgorithmen (A*, Dijkstra, Floyd, usw.) zur Wegbestimmung im ExtendedGraph verwendet werden.

\subsection{Graphanpassungen für urbane Szenarien}

Um den Verkehr in urbanen Szenarien simulieren zu können, müssen einige Analyse- und Modifikationsschritte am ExtendedGraph vorgenommen werden. Dieser Abschnitt gibt einen kurzen Überblick:

Bushaltestellen sind in OSM als Punkte $\vec{p}$ gespeichert. Sie werden aus dem entsprechenden Layer selektiert. Es wird im Graph nach einer NI gesucht, die die Position $\vec{p}$ hat. Falls notwendig wird eine neue NI angelegt und die geschnittene EI an dieser Stelle aufgeteilt. NI wird als Bushaltestelle markiert.

Ampeln werden identisch zu Bushaltestellen behandelt. In diesem Fall wird jedoch eine Ampel (vgl. Abschnitt 7.5) in die NI integriert. Falls zwei Ampeln über eine EI miteinander verbunden sind und eine Distanz von weniger als $50 \mathrm{~m}$ haben, wird eine der Ampeln entfernt. Es wird davon ausgegangen, dass beide Ampeln in diesem Fall zu einer Kreuzung gehören. Entscheidend ist nicht die Position der Anzeige einer Ampel, sondern welcher Bereich von ihr geregelt werden soll.

Parkplätze speichert OSM als Polygone. Es werden alle innerhalb des Polygons liegenden EIs als Parkplätze deklariert. Wenn keine QZ-Informationen vorhanden sind, werden Autos bevorzugt auf Parkplätzen erstellt.

Kreisverkehre sind im städtischen Straßenverkehr wichtige Vorfahrtsinstrumente. Sie werden in OSM teilweise mittels junction = roundabout markiert. Dies trifft jedoch bei weitem nicht immer zu. Eine Gruppe von EIs wird zusätzlich als Kreisverkehr (höchste Vorfahrtspriorität) markiert, wenn alle enthaltenen EIs Einbahnstraßen sind und identische Kreisverkehreigenschaften haben. Diese sind: Krümmung der Geometrie nach links, Typ, Brücke, Tunnel, Name, Ref, Spuren. Die Verbindungsrichtung von je zwei aufeinanderfolgenden EIs muss links sein.

Endknoten und Endkanten werden bestimmt, um den Verkehr aus dem Simulationsgebiet heraus und in das Gebiet hinein modellieren zu können. Diese Start- und Zielpunkte werden bevorzugt, falls keine QZ-Informationen vor- 
handen sind.

Abzweigungsrichtungen werden benötigt, damit simulierte Verkehrsteilnehmer die Vorfahrtsregeln beachten können. Für jede Verbindung von je zwei EIs über eine NI werden die Richtung und der Winkel in einem Verbindungsobjekt verwaltet.

Vorfahrtsrelevante Verbindungen jede NI weist jeder mit ihr verbundenen EI eine Liste von EIs zu, die Vorfahrt über sie haben. Dies geschieht durch Betrachtung der Vorfahrtswerte der jeweiligen EIs $(0=$ gering (z.B. Feldwege), $1=$ normal, $2=$ Vorfahrtsstraße, $3=$ Kreisverkehr). Die Verbindungsrichtungen finden ebenfalls Beachtung (rechts vor links). Dieser Schritt vereinfacht während der Simulation die Bestimmung von vorfahrtsrelevanten Verkehrsteilnehmern, die von den vorfahrtsrelevanten Straßen abgefragt werden können.

Krümmungen der Geometrien der EIs werden bestimmt, um die auf ihnen fahrbaren Geschwindigkeiten abzuschätzen. Die erlaubte Maximalgeschwindigkeit kann in Folge unvollständiger Angaben in OSM und der daraus resultierenden Abschätzung mittels einer LUT nach dem Straßentypen unrealistisch hoch sein. Die Geometrie einer EI ist eine Folge von Koordinaten $k_{1}, \cdots k_{n}$. Je zwei Koordinaten bilden einen Vektor $\vec{v}=\left(k_{a}, k_{a+1}\right)$. Es werden die Winkel $\alpha_{1}, \cdots \alpha_{n-1}$ der aufeinander folgenden Vektoren bestimmt. Der Krümmungswert $K$ einer EI ist $K=\frac{\sum_{i=1}^{n-1} \alpha_{i}}{(n-1) \cdot \mid \text { EI } \mid}$. Die Krümmungswerte von acht verschiedenen - dem Autor bekannten Straßen - wurden bestimmt und fahrbare Geschwindigkeiten hierfür definiert. Es wurden hierfür Straßen ausgewählt, deren Krümmung von gerade bis sehr enger Kreisel immer weiter anstieg. Die Ergebnisse werden in einer LUT verwaltet. Für jede EI wird $K$ bestimmt und zwischen den nächstliegenden LUT-Einträgen linear die Geschwindigkeit $v_{K}^{\mathrm{EI}}$ interpoliert.

Wartepositionen sind die Abstände, die zu einer NI eingehalten werden müssen, wenn sie nicht überquert werden kann. Für jede mit einer NI verbundenen EI wird das Maximum der Breiten der restlichen mit NI verbundenen EIs als Warteposition genutzt. Als Minimum werden $2 \mathrm{~m}$ verwendet.

Fußgängerüberwege werden an allen NIs für jede verbundene EI gesetzt (vgl. Kapitel 7.3. Zusätzlich werden Fußgängerüberwege für jeden Zebrastreifen platziert.

Zebrastreifen sind eine zentrale Option, um Fußgängern Straßenüberquerungen 
zu ermöglichen. In OSM werden Zebrastreifen als Punkte verwaltet. Die Platzierung eines Zebrastreifens geschieht analog zu Bushaltestellen.

Knoten an Schnittstellen von EIs müssen häufig gesetzt werden, da der Graphgenerator von GeoTools zwar NIs an Endstellen von EIs setzt, jedoch nicht zwei sich überschneidende Straßenzüge auftrennt, um eine verbindende NI zu setzen.

Brücken und Tunnel werden anschließend betrachtet. OSM verfügt über entsprechende Attribute. Der Straßengraph erhält die drei Ebenen Tunnel, normal und Brücke. Alle NIs werden untersucht. Falls eine NI EIs unterschiedlicher Ebenen verbindet, wird eine neue NI auf identischer Position eingefügt und die EIs werden nach Ebenen aufgeteilt. Somit verlaufen Brücken nun über und Tunnel unter normalen Straßen - ohne Verbindung.

Kreisgeometrien sind Geometrien, deren Start- und Endkoordinate identisch ist. Straßen dieses Typs werden in der Mitte aufgeteilt. Es wird eine zusätzliche NI hinzugefügt. Dieser Schritt ist notwendig, da die Fahrtrichtung auf einer EI von der NI abhängt, die zuletzt passiert wurde. Wenn eine EI nur über einen Knoten NI verfügt, lässt sich über diesen Mechanismus keine Fahrtrichtung abbilden.

Knoten mit Grad 2, die nicht in einem der letzten Schritte hinzugefügt wurden, werden entfernt, um Speicherplatz und Rechenzeit zu sparen.

Ortsbereiche verfügen im Normalfall über eine maximale Geschwindigkeit von $50 \mathrm{~km} / \mathrm{h}$. Orte werden in OSM über Polygone umrissen. Die Ränder des Polyongs schneiden EIs. An Schnittstellen werden NIs eingefügt. Alle EIs, die innerhalb eines Ortspolygons liegen, werden auf maximal $50 \mathrm{~km} / \mathrm{h}$ begrenzt, sofern keine anderen Angaben oder darunterliegende Geschwindigkeitsbegrenzungen vorliegen.

Auf- und Abfahrten auf Autobahnen oder autobahnähnlichen Straßen sind in OSM nicht vorhanden. Jeder relevante Straßentyp (z.B. motorway) verfügt jedoch über einen Zubringerstraßentyp (z.B. motorway_link). Alle EIs, deren Typ über das Suffix _link verfügt, werden analysiert. Verbundene EIs des entsprechenden Grundtypen (z.B. motorway) werden um eine Auffahrt erweitert, wenn die Link-EI auf sie führt und um eine Abfahrt, wenn die Link-EI von ihr wegführt. Auf- und Abfahrten werden jeweils gesetzt, indem die entsprechende Straße geteilt wird und ein Abschnitt als Auf- bzw. Abfahrt gekennzeichnet wird. 


\section{Modellierung multimodalen Verkehrs}

Dieses Kapitel beschreibt die entwickelten Simulationsmodelle für Verkehrsteilnehmer. In den folgenden Abschnitten werden die Modelle für Autos (7.1), Fahrräder (7.2) und Fußgänger (7.3) beschrieben. Die Modelle befinden sich auf der operativen Ebene, des in Abbildung 3.8 auf Seite 43 und beschreiben die Bewegungen und Wechselwirkungen zwischen Verkehrsteilnehmern. Abschnitt 7.4 beschreibt, welche Verkehrsregeln in die Modelle integriert wurden. Verschiedene Ampelschaltungen werden in Abschnitt 7.5 diskutiert. Abschließend werden in Abschnitt 7.6 verschiedene Methoden zur Routenbestimmung für Verkehrsteilnehmer vorgestellt, die im Simulationssystem integriert sind (taktische Ebene).

\subsection{Raumkontinuierliche Modellierung des Automobilverkehrs}

Erste Schritte auf dem Weg zur Modellierung des Verhaltens von Autos wurden vor mehr als 35 Jahren gemacht. Eine herausragende Arbeit auf diesem Sektor ist der technische Report Treiterer, 1975. Hierbei wurde zwischen 1966 und 1975 ein Forschungsprojekt durchgeführt, bei dem durch die Analyse von Luftaufnahmen charakteristische Situationen im Verkehrsfluss ermittelt wurden. Ergebnisse von Messreihen wurden als Raum-Zeit-Diagramme aufgetragen und ermöglichten eine einfache Interpretation der Daten. 


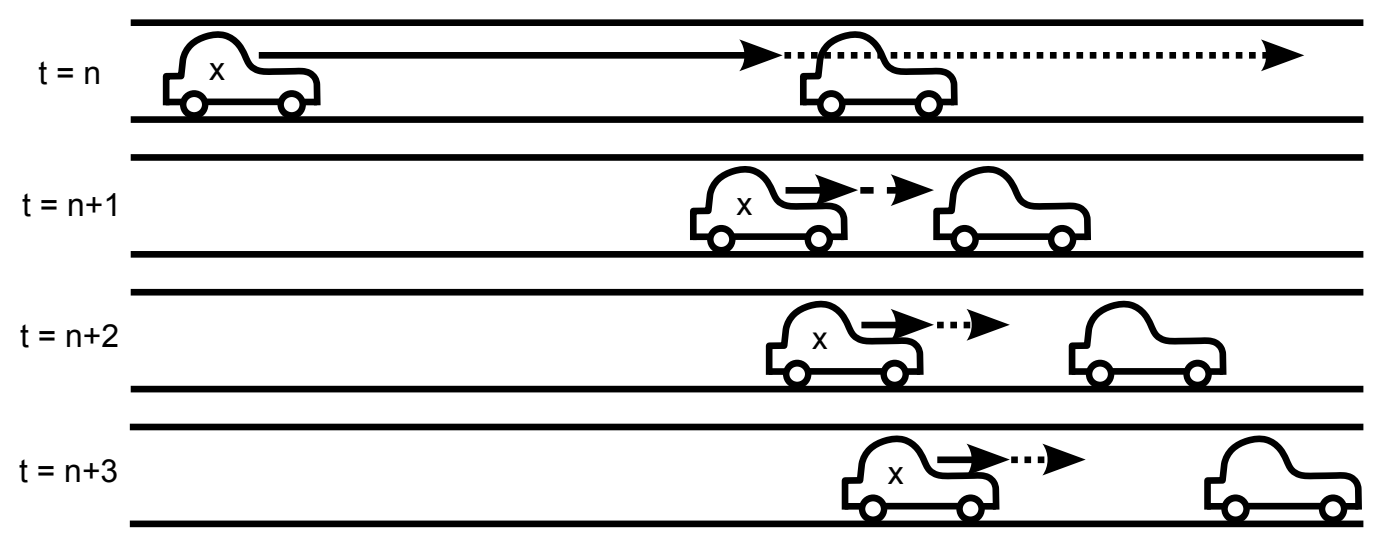

Abbildung 7.1: Das mit einem $\times$ markierte Auto beachtet zum Zeitpunkt $t=n$ seinen Sicherheitsabstand (gepunkteter Vektor) zum Vordermann nicht und fährt mit hoher Geschwindigkeit (durchgezogener Vektor) weiter. Bei $t=n+1$ ist der Abstand zum Vordermann sehr gering und Auto $x$ muss abbremsen, um nicht aufzufahren. In den verbleibenden Zeitschritten fahren beide Autos mit gleichbleibenden Geschwindigkeiten und es zeigt sich, dass Auto $x$ langsamer als sein Vordermann fährt.

Das Phänomen des Staus aus dem Nichts (Phantomstau) wurde identifiziert. Eine Möglichkeit für die Entstehung eines Staus aus dem Nichts ist eine Stauquelle, die zeitlich bereits weit zurückliegt, deren Auswirkungen aber noch zu spüren sind. Ein solcher Stau ist also nur in der subjektiven Wahrnehmung der Autofahrer aus dem Nichts entstanden [Treiber und Kesting, 2010, S. 263f]. Ein alternativer Entstehungsgrund für einen Phantomstau ist eine große Menge an Schätzungsfehlern, die Autofahrer begehen. Wenn ein Autofahrer den Abstand zu seinem Vordermann zu gering schätzt, wird er seine Geschwindigkeit niedriger halten, als dies notwendig ist. Zusätzlich verstärkt wird dieses Phänomen durch einfaches Trödeln Bungartz et al., 2009, S. 189].

Durch Messungen konnte gezeigt werden, dass Autofahrer dazu tendieren, nicht mit konstanter Geschwindigkeit zu fahren, sondern gelegentlich ihre Geschwindigkeit zu variieren. Eine kurze Phase des Ausrollens genügt, um eine kleine Stauwelle loszutreten, die sich durch die angesprochenen Fehleinschätzungen schnell verstärken und im Extremfall zum kompletten Stillstand führen kann.

Zusätzlich führt zu spätes Bremsen beim Auffahren auf einen Vordermann dazu, dass ein Auto weiter abbremsen muss, als notwendig gewesen wäre, wie Abbildung 7.1 verdeutlicht. Hieraus können ebenfalls Stauwellen entstehen. Gründe für zu spätes Bremsen können Unaufmerksamkeit und Schätzfehler bei der Bestimmung der Geschwindigkeitsdifferenz und des Abstands zum Vordermann sein. 
Diese Phänome können in makroskopischen Verkehrssimulationsmodellen nicht berücksichtigt werden und nur durch zeitliche und räumliche Mittelung können die entstehenden Verkehrsstörungen simuliert werden. Mikroskopische Simulationsmodelle können beliebige Effekte berücksichtigen und eignen sich daher besonders für die Simulation von kleinen Ausschnitten des gesamten Straßennetzes. Ein erstes Modell, dass sich zur Berücksichtigung des Stau aus dem Nichts eignete, ist das Nagel-Schreckenberg-Modell (NSM), welches in Abschnitt 3.1.2 behandelt wurde.

Die Schwäche eines zellenbasierten Ansatzes ist, dass die Zellengröße sehr gering gewählt werden muss, um die niedrigen Geschwindigkeiten, die innerorts gefahren werden, modellieren zu können. Ein verkehrsberuhigter Bereich, ugs. Spielstraße, ist auf Schrittgeschwindigkeit (3,6 bis $10 \mathrm{~km} / \mathrm{h})$ begrenzt, sodass hierfür eine Zellengröße von maximal $1 \mathrm{~m}$ benötigt würde. Die Anzahl der Zellen erhöht sich somit drastisch.

Der folgende Abschnitt 7.1.1 stellt ein neues, erweitertes mikroskopisches Modell zur Simulation von Fahrzeugbewegungen vor. Anschließend wird das dazugehörige Spurwechselmodell in Abschnitt 7.1.2 erläutert. Die Modellierung von Überholvorgängen wird in Abschnitt 7.1.3 beschrieben. Abschließend werden Modellierungsdetails in Abschnitt 7.1 .4 aufgelistet.

\subsubsection{Mikroskopisches Automodell}

Im Rahmen dieser Arbeit wurde ein neues mikroskopisches Verkehrsmodell entwickelt, welches auf dem NSM basiert. Die Stärke des zellularen Ansatzes ist seine Berechnungseffizienz. Geschwindigkeiten müssen zur Simulation in urbanen Szenarien feingranular abgebildet werden können, um Verkehrsteilnehmer unterschiedlicher Geschwindigkeit - z.B. Fahrräder und Autos - in einem System modellieren zu können. Hierfür muss die Zellengröße reduziert werden. Dies führt zu einer Erhöhung des Berechnungsaufwandes. Das entwickelte Modell abstrahiert daher von Zellen und führt Bewegungen direkt auf Kanten im Graphen durch. Durch diesen Verallgemeinerungsschritt lassen sich verschiedene Verhaltensweisen einfach modellieren. Unterschiedliche Beschleunigungsfähigkeiten bei verschiedenen Fahrzeugklassen können exakt abgebildet werden und sind nicht auf ZellengröBen limitiert. Dieser Abschnitt beschreibt die grundlegende Funktionsweise des entwickelten Fahrzeugmodells.

Dieser Abschnitt präzisiert die in Abschnitt 5.1 definierte Architektur. Das Simulationssystem wird von einer Steuerungskomponente gelenkt. Eine Iteration in 


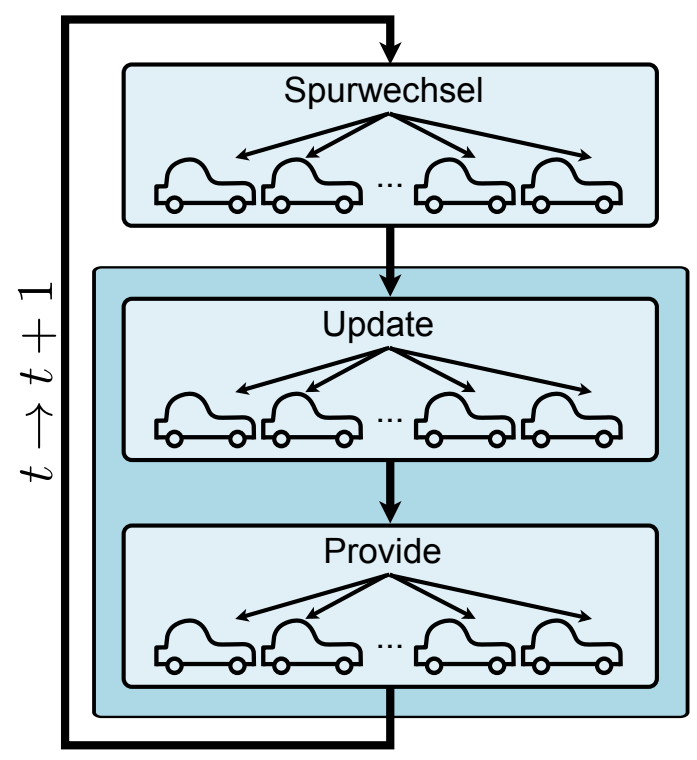

Abbildung 7.2: Updatezyklus der Simulationssteuerung

der Simulation simuliert eine Sekunde Realzeit. Die Simulationssteuerung führt in jeder Iteration die in Abbildung 7.2 gezeigten Schritte aus.

$\mathrm{Zu}$ erst wird die Spurwechselfunktion für jedes Auto parallel aufgerufen. Durchgeführte Spurwechsel werden umgehend wirksam. Anschließend aktualisiert jedes Auto seinen internen Zustand gleichzeitig durch Aufruf der update-Funktion. Die Geschwindigkeitsänderungen und Bewegungen der Autos werden erst öffentlich sichtbar, wenn die Simulationssteuerung die provide-Funktion aufruft. Die Teilung der Zustandsaktualisierung in diese Schritte stellt eine Synchronisierung dar und berücksichtigt Interdependenzen der Zustände der simulierten Autos.

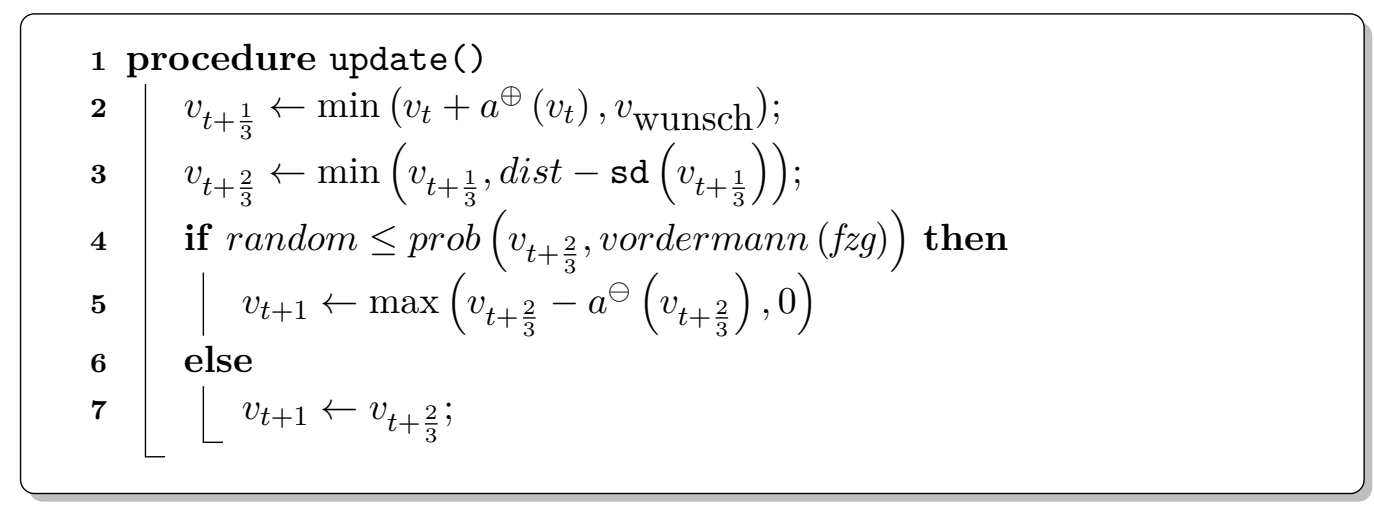

Algorithmus 7.1: Raumkontinuierlicher Ansatz 
Das grundlegende Verhalten der simulierten Autos wird durch die UpdateProzedur beschrieben. Das Update besteht aus den in Algorithmus 7.1 gezeigten Teilschritten. Die Zustandsaktualisierung lässt sich analaog zum NSM in die Schritte „Beschleunigen“, „Sicherheitsabstand wahren“ und „trödeln“ zerlegen. Die folgenden Unterabschnitte vertiefen diese Schritte.

\section{Beschleunigung}

Die Geschwindigkeit wird in Zeile 2 von Algorithmus 7.1 durch die Beschleunigungsfähigkeit $a^{\oplus}(v)$ des Fahrzeugs ausgehend von der aktuellen Geschwindigkeit1], sowie durch die Wunschgeschwindigkeit des Autos begrenzt. Die Maximalgeschwindigkeiten des betreffenden Fahrzeugs $v_{\max }$ und der derzeit genutzten Straße $v$ max müssen berücksichtigt werden. Zusätzlich wird die geschätzt fahrbare Geschwindigkeit $v_{K}^{\mathrm{EI}}$ einbezogen. Es gilt:

$$
v_{\text {wunsch }}=\min \left(v_{\max }, v_{\max }^{\mathrm{EI}}, \psi \cdot v_{K}^{\mathrm{EI}}\right)
$$

Der Verhaltensparameter $\psi$ kann individuell für jedes Fahrzeug bestimmt werden und skaliert den Einfluss von $v_{K}^{\mathrm{EI}}$ auf die Wunschgeschwindigkeit. Falls $\psi>1$ gewählt wird, fährt das Fahrzeug sportlich; $\psi<1$ führt zu defensiver Wahl der Wunschgeschwindigkeit. Die Untersuchung des Einflusses unterschiedlichen Fahrverhaltens mittels Variation von $\psi$ wird somit möglich, steht jedoch nicht im Fokus dieser Arbeit. Als Standardwert wird $\psi=1$ verwendet. Keines der in Teil II diskutierten Modelle oder Systeme berücksichtigt die Krümmung der Straße. Dies ist dennoch notwendig, um bei unvollständigen Informationen über Straßen eine Abschätzung über realistische Geschwindigkeiten zu erlangen.

Dies stellt eine Erweiterung zum NSM dar, da dort konstante Maximalgeschwindigkeiten und Beschleunigungsfähigkeiten für alle Autos festgelegt wurden. Es lassen sich nun unterschiedlich schnelle Verkehrsteilnehmer und variierende Geschwindigkeitsbegrenzungen berücksichtigen. $v_{\max }$ ist eine gleichverteilte Zufallszahl zwischen $33, \overline{3} \mathrm{~m} / \mathrm{s}$ und $55, \overline{5} \mathrm{~m} / \mathrm{s}(120 \mathrm{~km} / \mathrm{h} \cdots 200 \mathrm{~km} / \mathrm{h})$. Die Bestimmung von $v_{\max }^{\mathrm{EI}}$ und $v_{K}^{\mathrm{EI}}$ wird in Kapitel 6 beschrieben.

Die Beschleunigung eines Verkehrsteilnehmers wird durch $a_{\max }^{\oplus}$ begrenzt:

\footnotetext{
${ }^{1}$ Das Symbol $\oplus$ bei $a^{\oplus}(v)$ zeigt auf, dass eine positive Geschwindigkeitsänderung (Beschleunigung) bestimmt wird. In Zeile 5 von Algorithmus 7.1 wird mit $a^{\ominus}(v)$ hingegen eine negative Geschwindigkeitsänderung (Bremsung) bestimmt.
} 


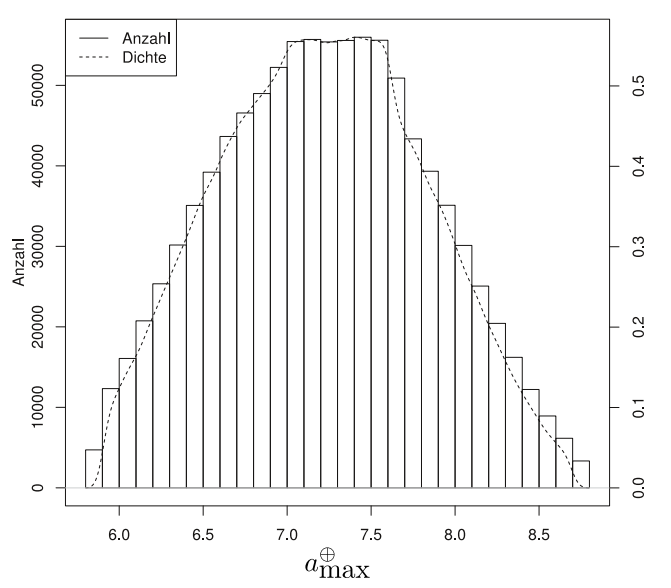

(a)

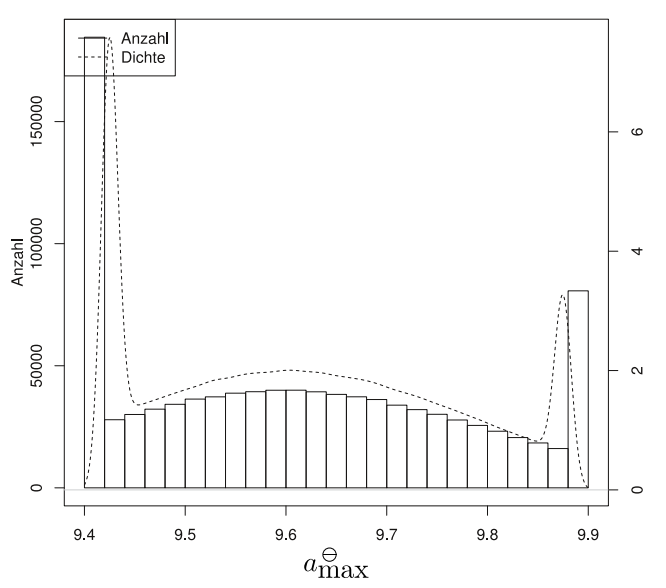

(b)

Abbildung 7.3: Verteilung von $a_{\max }^{\oplus}$ und $a_{\max }^{\ominus}$ für Autos in $\mathrm{m} / \mathrm{s}$.

$$
a_{\max }^{\oplus}=\xi^{\oplus} \cdot \frac{v_{\max }}{55, \overline{\mathrm{m} / \mathrm{s}}}+1-\perp
$$

Der Wert von $a_{\max }^{\oplus}$ hängt linear von $v_{\max }$ (Geschwindigkeit des Autos) und $1-\perp$ (Beschleunigungsvermögen des Fahrers) ab und wird mittels $\xi^{\oplus}$ skaliert. Der Beschleunigungsfaktor $\xi^{\oplus}$ nimmt für Autos $\xi^{\oplus}=4,5$ und für Lkw $\xi^{\oplus}=1,36$ an. Abbildung 7.3(a) zeigt die Werteverteilung von $a_{\max }^{\oplus}$.

Es wird vereinfachend angenommen, dass bis zu einer Grenzgeschwindigkeit $v_{g}$ mit $a_{\max }^{\oplus}$ beschleunigt wird und die Beschleunigung anschließend zu $v_{\max }$ hin linear abnimmt. Gleichung 7.3 beschreibt die resultierende Beschleunigungsfunktion.

$$
a^{\oplus}(v)= \begin{cases}a_{\max }^{\oplus} & \text { falls } v \leq v_{g} \\ a_{\max }^{\oplus} \cdot\left(\frac{v \max -v}{v_{\max }-v_{g}}\right) & \text { sonst }\end{cases}
$$

Die Grenzgeschwindigkeit $v_{g}$ wird auf $v_{g}=0,7 \cdot v_{\max }$ festgelegt. Dies führt $\mathrm{zu}$ gleichverteilten Werten im Bereich $v_{g}=[23, \overline{3} \mathrm{~m} / \mathrm{s} \cdots 38, \overline{8} \mathrm{~m} / \mathrm{s}]$ $(84 \mathrm{~km} / \mathrm{h} \cdots 140 \mathrm{~km} / \mathrm{h})$.

Wenn eine EI als innerstädtisch gekennzeichnet ist, wird die in Gleichung 7.3 bestimmte Beschleunigung um den Faktor $1-\perp$ skaliert. Dies führt zu einer Senkung des Beschleunigungspotentials. Der Parameter $\perp$ ist von zentraler Bedeutung und wird unter dem Punkt „Trödeln“ erläutert. Mit der beschriebenen 


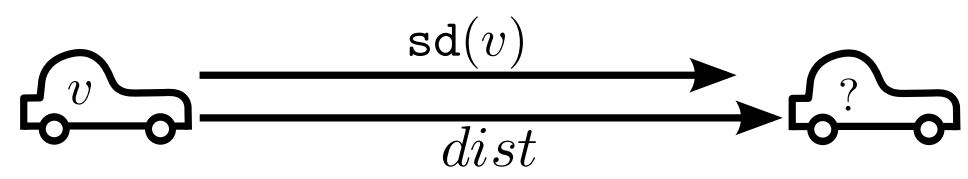

Abbildung 7.4: Beispiel für die Beachtung des Sicherheitsabstands für $v \geq 14$. Das mit $v$ hinterherfahrende Auto muss nicht abbremsen, da $v+\operatorname{sd}(v)<d i s t$.

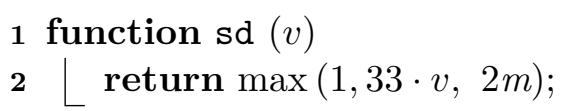

Algorithmus 7.2: Berechnung des Sicherheitsabstands

Beschleunigungsfunktion können heterogene Fahrzeug-/Fahrerkombinationen modelliert werden.

\section{Sicherheitsabstand wahren}

Der Sicherheitsabstand nach vorne wird in $§ 4$ Abs. 1 StVO wie folgt beschrieben:

Der Abstand von einem vorausfahrenden Fahrzeug muß in der Regel so groß sein, daß auch dann hinter ihm gehalten werden kann, wenn es plötzlich gebremst wird. Der Vorausfahrende darf nicht ohne zwingenden Grund stark bremsen.

Dieser Absatz wird meist als „halber Tachoabstand“ ausgelegt und entspricht in etwa der in zwei Sekunden gefahrenen Strecke (siehe Abbildung 7.4). Innerorts wird in der Regel ein geringerer Sicherheitsabstand von ca. einer Sekunde verwendet.

Es hat sich herausgestellt, dass die Einhaltung der Daumenregel „halber Tachoabstand" in Simulationen zu unrealistischen makroskopischen Kenngrößen führt. In der Literatur beschriebene Modelle verwenden geringere Folgezeiten. NSM: $1 \mathrm{~s}$, IDM: $1,5 \mathrm{~s}$ (vgl. Abschnitt 3.1). Der einzuhaltende Sicherheitsabstand $\operatorname{sd}(v)$ in Abhängigkeit zur Geschwindigkeit für das im Rahmen dieser Arbeit entwickelte Modell wird in Algorithmus 7.2 gezeigt. 


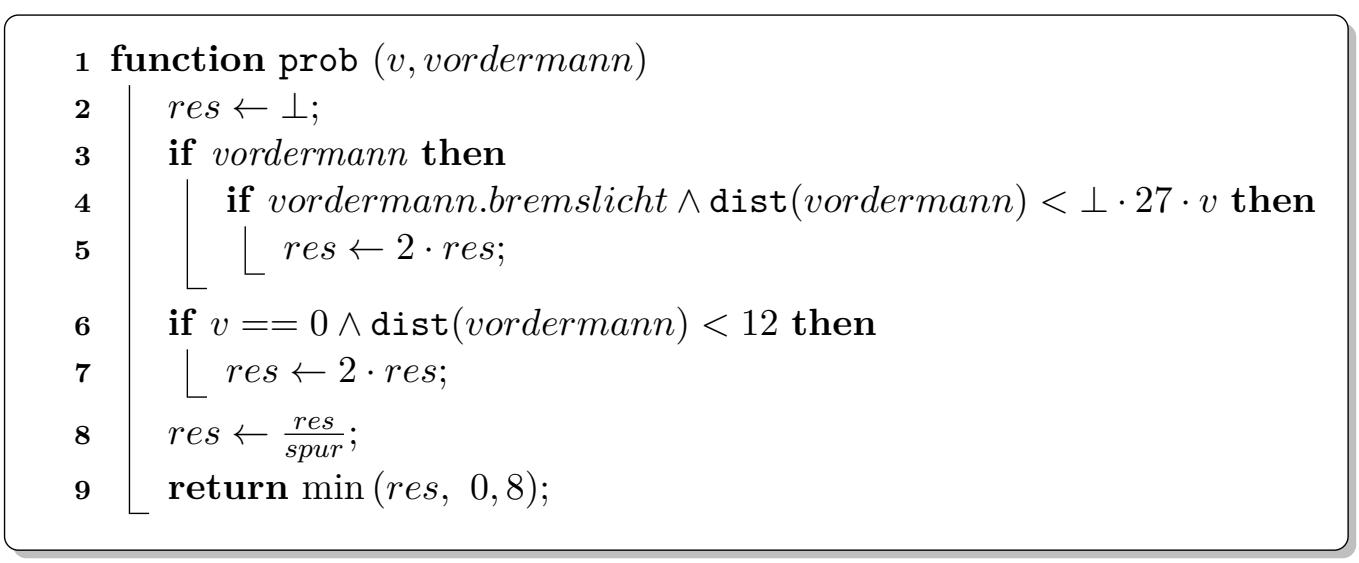

Algorithmus 7.3: Trödelwahrscheinlichkeit prob(v, vordermann)

\section{Trödeln}

Das NSM wurde nicht zuletzt wegen seines Trödelfaktors bekannt. Dieser Faktor ermöglicht die Simulation unvorhersehbarer Geschwindigkeitsschwankungen. Die Wahrscheinlichkeit zu trödeln, wird nach Algorithmus 7.3 bestimmt. Es sei $\mathcal{N}_{a}^{b}(\mu, \sigma)$ eine um $\mu$ mit Standardabweichung $\sigma$ auf das Intervall $[a \cdots b]$ begrenzte, normalverteilte Zufallszahl. Jeder Verkehrsteilnehmer verfügt über einen normalverteilten Trödelfaktor $\perp=\mathcal{N}_{0,05}^{0,3}(0,2,0,1)$. Dies stellt eine Erweiterung zu Algorithmus 3.1 dar, denn dort ist die Trödelwahrscheinlichkeit für jeden Verkehrsteilnehmer identisch. In den Zeilen 3 bis 7 von Algorithmus 7.3 werden die auf Seite 35 besprochenen Erweiterungen um die Beachtung von Bremslichtern und das verspätete Losfahren integriert.

Jeder simulierte Verkehrsteilnehmer aktiviert sein Bremslicht zum Zeitpunkt $t$ im provide-Schritt, falls $v_{t+1}<v_{t}$. Die Trödelwahrscheinlichkeit verdoppelt sich, wenn die Entfernung zum Vordermann gering ist und derzeit sein Bremslicht aufleuchtet2 Wenn der simulierte Verkehrsteilnehmer derzeit steht und die Distanz zum Vordermann gering ist, verdoppelt sich die Wahrscheinlichkeit, nicht loszufahren. Die Grenzdistanz wird in Maerivoet und Moor, 2005 auf zwei Zellen gesetzt. Dies entspricht in etwa $12 \mathrm{~m}$.

Zeile 8 stellt eine Neuerung dar. Die Wahrscheinlichkeit, zu trödeln, wird mit dem Kehrwert der Spur, auf dem der betreffende Verkehrsteilnehmer fährt, skaliert. Dies bewirkt, dass Autos auf einer linken Spur ( spur > 1) weniger trödeln, als auf

${ }^{2}$ Auch an dieser Stelle wird $\perp$ verwendet, um die unterschiedliche Sensibilität verschiedener Fahrertypen auf Bremslichter zu beachten. 
der rechten $($ spur $=1)$. Die Rückgabe des Algorithmus ist die auf 0,8 begrenzte Wahrscheinlichkeit.

Falls ein Verkehrsteilnehmer derzeit nicht überholt, trödelt er mit Wahrscheinlichkeit $p=\operatorname{prob}()$ und verringert seine Geschwindigkeit um $a_{\max }^{\ominus} \cdot \max (p, 0,1)$. Die maximale Bremsintensität wird mit $a_{\max }^{\ominus}=\xi^{\ominus} \cdot(5-\perp)$ beschrieben. Analog zu Beschleunigungen nimmt $\xi^{\ominus}=2$ für Autos und $\xi^{\ominus}=0,8$ für Lkw an. Es entsteht die in Abbildung 7.3(b) gezeigte Verteilung von $a_{\max }^{\ominus}$ für Autos.

\subsubsection{Spurwechselmodell}

Es wird wie bei den im Stand der Forschung diskutierten Modellen (vgl. Abschnitt 3.1.2 zwischen zwei Entscheidungsphasen unterschieden. Zuerst muss geprüft werden, ob ein Auto einen Spurwechsel durchführen möchte. Anschließend wird bestimmt, ob dies unter dem gegebenem Verkehrszustand möglich ist.

Ein Auto möchte seine Spur nach links wechseln, wenn

- außerorts eine Geschwindigkeit gefahren wird, die mehr als $5 \mathrm{~m} / \mathrm{s}$ unter der Wunschgeschwindigkeit liegt und entweder kein Vordermann auf der linken Spur vorhanden ist oder dieser schneller fährt, als der Vordermann auf der rechten Spur

- die linke Spur näher an der bevorzugten Spur liegt, als die aktuelle Spur

- der Vordermann auf der aktuellen Spur einen Unfall hat

- der Vordermann auf der linken Spur langsam fährt und überholt werden soll

Spurbevorzugungen werden eingesetzt, wenn

- von einer Auffahrt auf eine Straße gewechselt wird (Spur 1 wählen)

- von einer Straße auf eine Abfahrt gewechselt wird (Spur 0 wählen)

- die aktuelle Spur auf der Folge-EI nicht mehr existiert

- die Straße sich teilt und das Auto sich einordnen muss:

- Weiterfahrt geradeaus: mittlere Spur bestimmen

- Weiterfahrt rechts: rechte Spur wählen

- Weiterfahrt links: linke Spur wählen

Ein Auto möchte nach rechts wechseln, wenn 


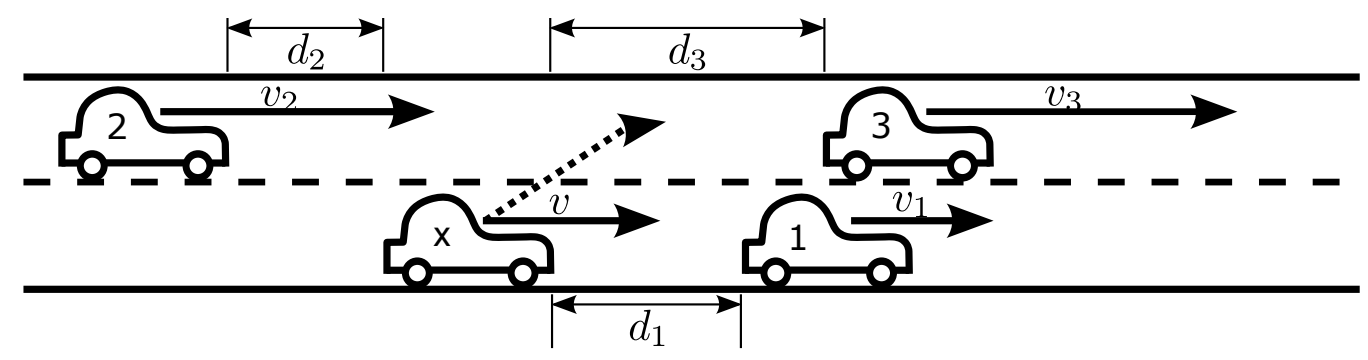

Abbildung 7.5: Spurwechsel

- kein Vordermann auf der aktuellen und der rechten Spur vorhanden ist

- die rechte Spur näher an der bevorzugten Spur liegt, als die aktuelle Spur

- die Vordermänner auf der aktuellen und der rechten Spur mehr als 5 s entfernt sind und zudem schneller fahren

- der Vordermann auf der aktuellen Spur einen Unfall hat

- innerorts auf der rechten Spur eine größere Distanz zum Vordermann ist

Falls ein Auto einen Spurwechsel durchführen möchte, muss geprüft werden, ob dies möglich ist. In Abbildung 7.5 wird ein Beispiel gegeben. Das Auto x möchte nach links wechseln und muss die anderen Autos 1 bis 3 berücksichtigen. Ein Wechsel nach links ist möglich, falls $d_{2}>\operatorname{sd}\left(v_{2}\right)$ und somit Auto 2 nicht behindert würde. Zusätzlich muss eine ausreichende Lücke nach vorne $d_{3}>\mathbf{s d}(v)$ vorhanden sein.

Bei einem Spurwechsel nach rechts muss die Entfernung zum Hintermann auf der rechten Spur mehr als $3 \mathrm{~s}$ betragen, damit dieser nicht behindert wird. Der Vordermann auf der rechten Spur muss weiter als $3 \mathrm{~s}$ entfernt und schneller sein. Zusätzlich ist der Wechsel möglich, wenn der Vordermann mehr als $6 \mathrm{~s}$ entfernt ist.

Um zu vermeiden, dass auf einer Straße mit drei Spuren ein Auto von der rechten Spur und ein Auto von der linken Spur beide auf die mittlere Spur wechseln, werden bei geraden Iterationsnummern nur Spurwechsel nach links und bei ungeraden Iterationsnummern nur Spurwechsel nach rechts zugelassen. Dieser Synchronisierungsschritt vereinfacht die weitere Modellierung von Spurwechseln und stellt keine bedeutende Einschränkung des Modells dar, da innerhalb von $2 \mathrm{~s}$ Realzeit ein Spurwechsel in beide Richtungen möglich ist.

Durch das beschriebene Modell lässt sich Autobahnverkehr unter Berücksichtung von Auf- und Abfahrten ebenso modellieren, wie urbaner Verkehr mit mehrspurigen Straßen. Es stellt im Hinblick auf die im Kapitel 3 diskutierten Modelle die Erweiterung dar, dass eine Spurbevorzugung genutzt werden kann, damit sich 


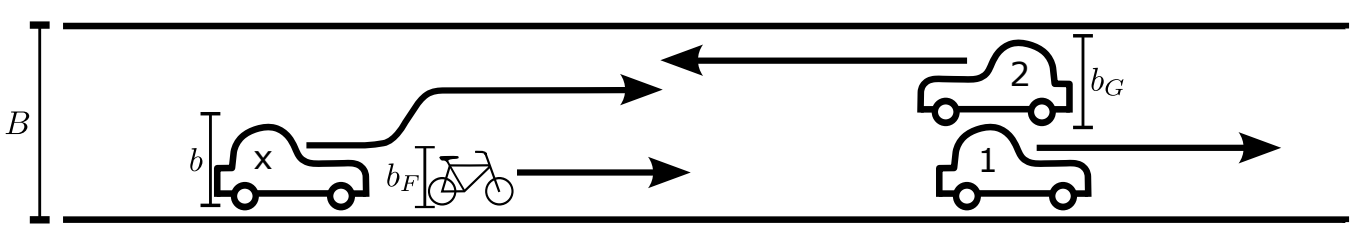

Abbildung 7.6: Überholvorgang

Autos einsortieren. Über diesen Mechanismus können Erweiterungen - z.B. ein Linksfahrgebot - umgesetzt werden. Simulierte Autos fragen bei der Spurwechselplanung zuerst ab, ob ein generelles Überholverbot herrscht. Zusätzlich können Lkw-Überholverbote geschaltet werden.

\subsection{3 Überholvorgänge}

Bei der Literaturbetrachtung konnten keine Studien zum Überholverhalten von Autos an Fahrrädern aufgespürt werden, die über die Betrachtung des seitlichen Abstands hinausgehen. Dennoch muss dieses Verhalten modelliert werden. Würden Autos nicht überholen können, würde sich hinter jedem Fahrrad in einer simulierten Stadt eine Schlange von Autos bilden. Die im Folgenden gewählten Werte für Geschwindigkeiten und Geschwindigkeitsdifferenzen können mangels Feldstudien nicht verifiziert werden. Sie zeigen jedoch eine Möglichkeit zur Modellierung auf, die durch Anpassung dieser Parameter auf die Nachbildung realen Verhaltens kalibriert werden kann.

Wenn ein Auto auf ein Fahrrad aufläuft, möchte es mit Wahrscheinlichkeit prob() überholen, sofern das Fahrrad mindestens 9,9 km/h langsamer als die erlaubte Höchstgeschwindigkeit fährt. Abbildung 7.6 skizziert das Szenario.

Auto x möchte überholen und prüft zuerst, ob das zu überholende Fahrrad derzeit selbst überholt. Falls dies nicht der Fall ist, schätzt x ausgehend von seiner aktuellen Geschwindigkeit, sowie der Geschwindigkeit des Fahrrads ab, wie lange der Überholvorgang dauern würde. Es wird dabei für jede Iteration im Planungszeitraum von einer Beschleunigung von $\mathrm{x}$ und gleich bleibender Geschwindigkeit des Fahrrads ausgegangen. Der Abstand zu Auto 1 ist von Bedeutung, da abschließend wieder eingeschert werden muss. Es wird zum Einscheren ein Abstand von $3 \mathrm{~m}$ zum Fahrrad eingeplant. Die Distanz zwischen Fahrrad und Auto 1 muss die Einhaltung der erforderlichen Sicherheitsabstände ermöglichen.

Zur Bestimmung des Gegenverkehrs werden in diesem Fall ausschließlich Verkehrsteilnehmer des Typs Auto berücksichtigt. Falls Gegenverkehr vorhanden ist, muss 
geprüft werden, ob der Überholvorgang dennoch möglich ist. Jeder Verkehrsteilnehmer hat eine Breite $b$. Die Straße hat eine Gesamtbreite $B$. Falls $B>b+b_{F}+b_{G}$, können alle drei Verkehrsteilnehmer aneinander vorbeifahren, ohne sich zu berühren. Die Breiten der Verkehrsteilnehmer inkludieren hierbei einen seitlichen Sicherheitsabstand (vgl. Abschnitt 3.2). Die Breite eines Autos wird mit $\mathcal{N}_{2,25}^{2,75}(2,5,0,1)$ $\mathrm{m}$ bestimmt und beinhaltet bereits einen seitlichen Sicherheitsabstand. Analog wird die Breite von Fahrrädern mit 1,5 m festgelegt. Insgesamt entstehen somit ca. die in Abschnitt 3.2 ermittelten Abstände.

Ist die Straße nicht breit genug, schätzt Auto x die Bewegungen aller relevanten Verkehrsteilnehmer ab und prüft, ob das Überholmanöver abgeschlossen werden kann, bevor eine Kollision mit Auto 2 auftritt. Es wird ein zeitlicher Sicherheitsabstand von $2 \mathrm{~s}$ hinzugefügt. Der zeitliche Planungshorizont beträgt $5 \mathrm{~s}$. Wenn der Überholvorgang nicht innerhalb dieser Zeit abgeschlossen sein würde, wird er nicht gestartet.

\subsubsection{Detailmodellierungen}

Dieser Abschnitt beschreibt kompakt einige weitere Verhaltensweisen, die in den simulierten Autos von MAINSIM modelliert wurden.

Abbremsen vor Abzweigung Die simulierten Autos können abfragen, ob vorfahrtsrelevanter Verkehr aus einer angrenzenden Straße kommen wird. Reale Autofahrer müssen hingegen häufig abbremsen, um feststellen zu können, ob sie eine Kreuzung passieren dürfen. Wenn die Distanz eines Autos zu einer Kreuzung geringer als $10 \cdot \operatorname{prob}() \cdot v$ ist $(\approx 2 \cdots 6 \mathrm{~s})$ und seine Geschwindigkeit $v>18 \mathrm{~km} / \mathrm{h}$ ist, wird der Trödelmechanismus zusätzlich aufgerufen. Dies geschieht nur bei Straßen mit zulässiger Geschwindigkeit $<70 \mathrm{~km} / \mathrm{h}$, da bei höheren Geschwindigkeiten von einer guten Sicht auszugehen ist.

Einparken Wenn ein Auto das Ziel seiner Fahrt erreicht, wird geprüft, ob dieses am Kartenrand liegt. In diesem Fall fährt das Auto ungebremst weiter und verlässt den Kartenausschnitt. Falls das Ziel jedoch nicht am Rand liegt und die Distanz zum Ziel geringer als $5 \mathrm{~s}$ ist, wird getrödelt, sofern die aktuelle Geschwindigkeit mindestens $1,5 \cdot a^{\oplus}\left(v_{t}\right) \cdot(1-\perp)$ ist. Dies führt zu einem simulierten Halten am Straßenrand.

Ausparken Autos fahren entweder vom Kartenrand in die Simulationsumgebung hinein und haben in diesem Fall bereits eine Geschwindigkeit $>0 \mathrm{~km} / \mathrm{h}$ oder sie starten innerhalb des Simulationsgebietes und müssen zuerst losfahren. In 
diesem Fall warten sie auf eine geeignete Lücke im Verkehr. Der Hintermann muss mindestens $4 \mathrm{~s}$ entfernt sein, damit dieser nicht behindert wird.

Kreisel Kreisverkehre werden in Städten häufig zur Vorfahrtsregelung eingesetzt. Innerhalb von Kreisverkehren können Autos keine Fahrräder überholen. Ein Kreisverkehr kann nur dann betreten werden, wenn seine Verkehrsdichte geringer als $33 \%$ ist. Ohne diese Regel können Staus innerhalb von Kreisverkehren entstehen, die sich nicht mehr aufösen.

Lückenakzeptanz Wenn ein Auto an einer Abzweigung die Vorfahrt eines anderen Verkehrsteilnehmers zu achten hat, bewertet es die Lücken je nach Abbiegerichtung. Die Grundwerte für die Lückenakzeptanz sind an Tabelle 3.7 auf Seite 51 unter normalen Bedingungen angelehnt und werden in Abhängigkeit $\mathrm{zu} \perp$ variiert.

Rechts überholen Autos dürfen außerorts nicht rechts überholen. Sollte ein Auto mit Geschwindigkeit $v_{1}$ ein Auto mit Geschwindigkeit $v_{2}$ von rechts überholen, korrigiert es seine Geschwindigkeit auf $v_{1}=v_{2}$ und trödelt zusätzlich. Falls jedoch $v_{1} \leq 6 \mathrm{~m} / \mathrm{s}$, wird von einer Stop'n'Go-Situation ausgegangen. In diesem Fall darf rechts überholt werden.

Unaufmerksamkeit Autos können nach einer Wahrscheinlichkeitsverteilung unaufmerksam sein. In diesem Fall wird der Sicherheitsabstand nicht gehalten und Überholvorgänge von rechts werden nicht unterdrückt. Es wird in jeder Iteration geprüft, ob der Vordermann unter Unaufmerksamkeit überholt wurde. In diesem Fall ist das Auto durch den Vordermann hindurchgefahren und es wird ein Unfall veranlasst. Beide Autos bleiben für eine definierte Dauer (Standard: 20 min) auf ihrer Position stehen. Die Wahrscheinlichkeit, unaufmerksam zu sein, wird im Normalfall auf $0 \%$ gesetzt.

Verzicht auf Vorfahrt Wenn ein Auto eine Kreuzung nicht passieren kann, verzichtet es mit einer Wahrscheinlichkeit $p$ auf seine eigene Vorfahrt. $p$ steigt innerhalb von $100 \mathrm{~s}$ von $0 \%$ auf sein Maximum $50 \%$. Andere wartende Verkehrsteilnehmer ignorieren das verzichtende Auto in diesem Fall und ein Deadlock an einer Kreuzung kann sich auflösen.

Umplanen Wenn die Wartezeit eines Autos an einer Kreuzung einen linear von $\perp$ abhängigigen Betrag übersteigt, kann die Route zur Zielposition umgeplant werden. Dies tritt in Kraft, wenn die zu überquerende Kreuzung keine Ampel hat, da in diesem Fall eine lange Ampelphase der Wartegrund sein kann. Das Auto muss das erste Auto in der Warteschlange sein. 
Durch geeignete Parametrisierung und Erweiterung können mit dem beschriebenen Modell ebenfalls Lkw, Busse und Lieferwagen simuliert werden. Die Fahrzeuglängen, Beschleunigungsvermögen, Maximalgeschwindigkeiten und Trödelwahrscheinlichkeiten (Lkw verwenden häufig Tempomaten) können angepasst werden, ohne die grundlegenden Abläufe zu beeinflussen.

Die simulierten Autos befinden sich in einer Umgebung, agieren autonom, sind reaktiv, proaktiv und sozial. Ihre Flexibilität und Robustheit entsteht durch die Möglichkeit, umzuplanen. Dies geht Hand in Hand mit der sozialen Fähigkeit, auf die Vorfahrt zu verzichten. In Anlehnung an Definition 2.3.3 auf Seite 18 sind die simulierten Autos somit als intelligente Agenten anzusehen.

\subsection{Modellierung des Fahrradverkehrs}

Aus der Auflistung der Verkehrsmittelwahl verschiedener deutscher Städte (vgl. Tabelle 3.1 auf Seite 24 geht hervor, dass ein signifikanter Anteil an Wegen in Städten mit dem Fahrrad zurückgelegt werden. In MAINSIM wird daher ein Fahrradmodell integriert. Analog zu den in Abschnitt 3.2 besprochenen Modellen, ist auch das im Rahmen dieser Arbeit vorgeschlagene Modell eine Adaption eines mikroskopischen Automodells. Das im vorherigen Abschnitt 7.1 besprochene Modell wird verwendet, verschiedene Kenngrößen auf Fahrräder „herunterskaliert“ und um Verhaltensweisen ergänzt, die typisch für Fahrräder sind.

Fahrräder haben eine Breite von 1,5 m und eine Länge von $2 \mathrm{~m}$. Dies modelliert die Maße eines Fahrrads mit reserviertem Raum für Schwankungen während der Fahrt. Alle weiteren gewählten Belegungen für Parameter wurden mangels gesicherter Daten geschätzt.

Die Maximalgeschwindigkeit $v \max$ wird zufällig gleichverteilt zwischen 12 und $33 \mathrm{~km} / \mathrm{h}$ bestimmt. Es wird ein Beschleunigungsfaktor $a_{f}=\frac{v \max }{33 \mathrm{~km} / \mathrm{h}}$ bestimmt, der die prozentuale Ausnutzung der maximalen Geschwindigkeit für Fahrräder bestimmt. Für Fahrräder werden zwei Beschleunigungsgrenzen $a_{\min }=0,5 \mathrm{~m} / \mathrm{s}^{2}$ und $a_{\max }=\left(a_{\min }+k\right) \cdot a_{f}$ bestimmt. $k$ sei eine gleichverteilte Zufallszahl im Intervall $[0,5 \cdots 1,0]$.

Es sei $r$ eine gleichverteilte Zufallszahl im Intervall $[0 \cdots 1]$. Die Funktion

$$
a^{\oplus}(v)=r \cdot a_{\min }+(1-r) \cdot a_{\max }
$$


bestimmt die Intensität der Beschleunigung eines Fahrrads. Die Trödelwahrscheinlichkeit wird in Relation zu $a_{f}$ als

$$
\perp=\frac{\min \left(\max \left(1-a_{f}, 0\right), 0,7\right)}{3}
$$

gesetzt $(0 \cdots 0,2 \overline{3})$. Die Distanz zu einer Abzweigung, ab der ein Fahrrad seine Geschwindigkeit verringert, wird im Vergleich zum Automodell geringer gewählt. Sie beträgt $\perp \cdot 50(6 \cdots 42 \mathrm{~m})$. Die Wahrscheinlichkeit zu trödeln bleibt stets $\perp$, unabhängig von der Verkehrssituation. Falls ein Fahrrad zur Einhaltung des Sicherheitsabstands bremsen muss, verschätzt es sich mit Wahrscheinlichkeit $3 \cdot \perp$ und trödelt in diesem Fall zusätzlich.

Falls der Vordermann ein Auto ist, welches derzeit überholt, so überholt es mit hoher Wahrscheinlichkeit das entsprechende Fahrrad. In diesem Fall wird der Sicherheitsabstand nicht zum Vordermann, sondern zum Vordermann des Vordermanns gewahrt. Ansonsten würde ein überholtes Fahrrad während des Überholvorgangs eine Vollbremsung durchführen. Fahrräder überholen andere Fahrräder. Es wird in diesem Fall vereinfachend davon ausgegangen, dass dies stets möglich sei und ein Vordermann, der ein Fahrrad ist, nicht beachtet werden muss.

\subsection{Bimodulares Fußgängermodell}

Im Rahmen dieser Arbeit soll der Einfluss von Fußgängern auf den Straßenverkehr berücksichtigt werden. Es erscheint daher sinnvoll, nur diejenigen Schritte mikroskopisch zu modellieren, die einen direkten Einfluss auf den Verkehr haben und alles andere zu vereinfachen. Das entwickelte Fußgängermodell teilt sich in zwei Module, zur Simulation der Bewegungen auf Wegen (Unterabschnitt 7.3.1) und auf Überwegen (Unterabschnitt 7.3.2), z.B. Zebrastreifen. Abschließend werden in Unterabschnitt 7.3.3 Straßenüberquerungskriterien diskutiert.

\subsubsection{Fußgängerbewegung auf Fußwegen}

Die Grundidee des entwickelten Fußgängermodells ist, dass Fußgänger auf Gehwegen relativ ungehindert mit einer mittleren Geschwindigkeit laufen können. Wenn die Route eines Fußgängers dies erfordert, wechselt er die Straßenseite. Dies kann er entweder in deren Verlauf oder an Kreuzungspunkten und Fußgängerüberwegen durchführen. Algorithmus 7.4 beschreibt den grundlegenden Ablauf. 
In Zeile 2 wird die Wunschgeschwindigkeit $v$ des Fußgängers bestimmt. Sie hängt davon ab, ob derzeit eine Straßenüberquerung stattfindet (Zeile 21) oder auf einem Bürgersteig gelaufen wird (Zeile 27). Es wird ferner unterschieden, ob der Fußgänger bei seiner Überquerung Vorfahrt hat oder die Überquerung im Verkehr durchführt (Zeilen 22 bis 27). Die Grundgeschwindigkeiten vNormal, vVorfahrt und vKeineVorfahrt werden für jeden Fußgänger nach den Gleichungen 7.6 bis 7.8 gewählt und basieren auf den Daten aus Abschnitt 3.3 .

$$
\begin{aligned}
\text { vNormal } & =\mathcal{N}_{0,5}^{1,75}(1,33,0,25) \\
\text { vVorfahrt } & =\mathcal{N}_{\text {vNormal }}^{1,5 \cdot v \text { Normal }}(1,5,0,5) \\
\text { vKeineVorfahrt } & =\mathcal{N}_{\text {vNormal }}^{2 \cdot v \text { Normal }}(1,8,0,5)
\end{aligned}
$$

Die bestimmte Grundgeschwindigkeit wird mit dem Faktor $f$ skaliert, der linear zwischen 1,0 und 1,3 verläuft und sein Maximum ab einer standdauer von $15 s$ erhält. Diese Steigerung der Geschwindigkeit um bis zu $30 \%$ lässt sich aus Ishaque, 2006 ableiten (vgl. Abschnitt 3.3.1 auf Seite 45). Die standdauer wird hochgezählt, wenn nach dem update() die Geschwindigkeit des Fußgängers $v=0$ beträgt und zurückgesetzt, falls $v \neq 0$. Auf diese Weise wird Ungeduld modelliert.

Wenn die Bewegung eines Fußgängers auf einem Bürgersteig verläuft, kann er ohne Interferenzen mit anderen Fußgängern seine Bewegung mit $v$ durchführen (Zeile 18), da diese sich über die gelaufene Strecke hinweg mitteln. Überquert er gerade eine Straße, so gilt ueberquerungEI und die Bewegung findet senkrecht zum Straßenverlauf mit $v$ statt (Zeilen 4 bis 10 . Die Variable weite gibt an, wie weit die Überquerung bereits abgeschlossen ist. Übersteigt die weite die breite der Straße, so wird $v$ auf den Teil von $v$ reduziert, der über die Überquerung hinausgeht und die Bewegung in Zeile 18 weitergeführt. Die Variable pos ist hierbei die Position im Straßenverlauf. Die Straßenseite des Fußgängers wird durch seite beschrieben und wechselt, wenn eine Überquerung durchgeführt wurde. Eine Straßenüberquerung kann auch auf einer NI geschehen. Hierfür wird ein detaillierteres Bewegungsmodell verwendet.

\subsubsection{Bewegungsmodell für Nodelnformations}

Hierbei handelt es sich um Stellen, an denen mehrere Fußgänger die Straße gleichzeitig überqueren und sich gegenseitig behindern können. NIs verwalten wayObjekte, die Überwege über adjazente EIs modellieren. Jeder in Abbildung 7.7 gezeigte Überweg wird durch ein way-Objekt modelliert, dessen update()-Methode in Zeile 14 von Algorithmus 7.4 aufgerufen wird. 


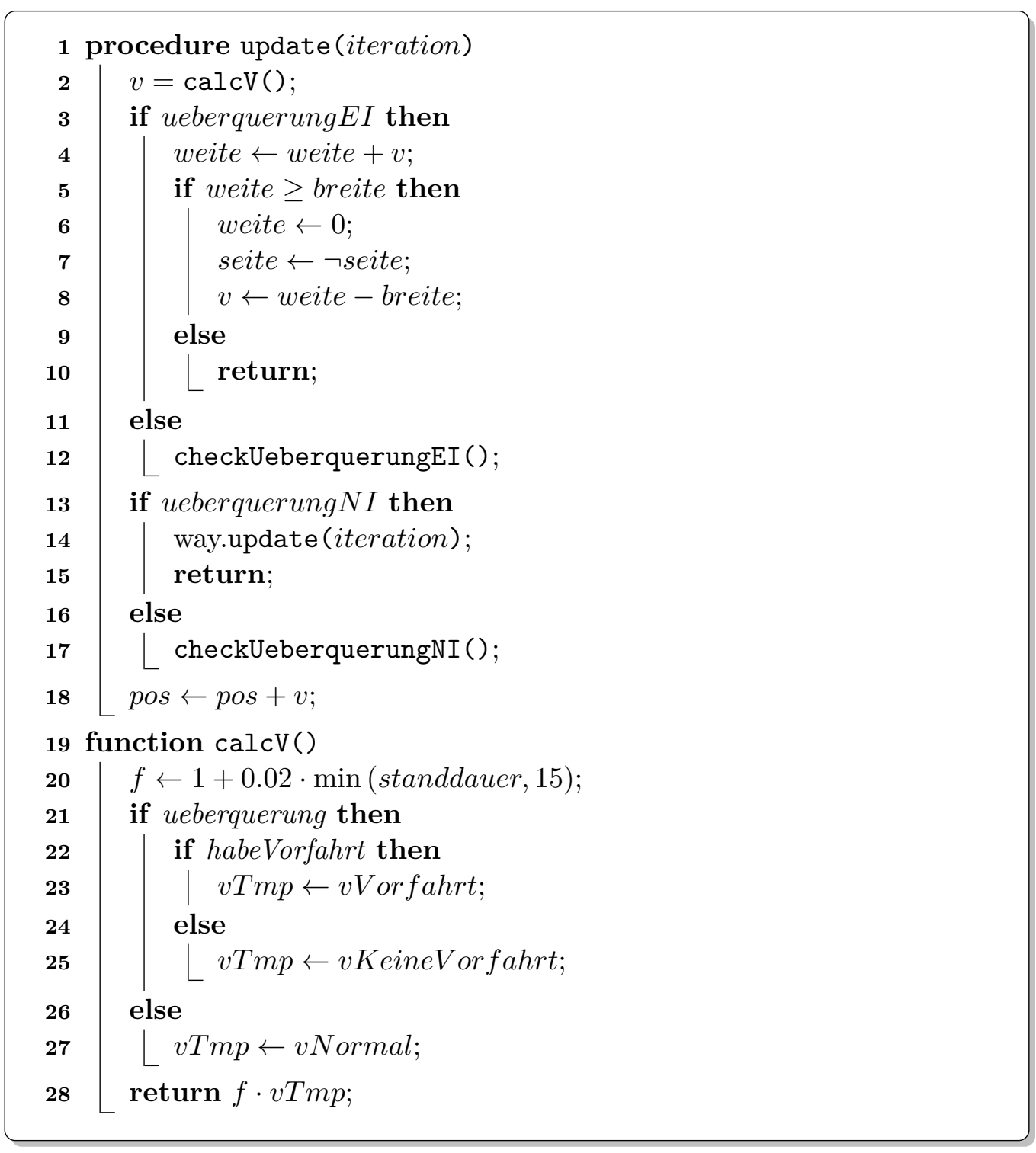

Algorithmus 7.4: Fußgängerbewegung auf EI

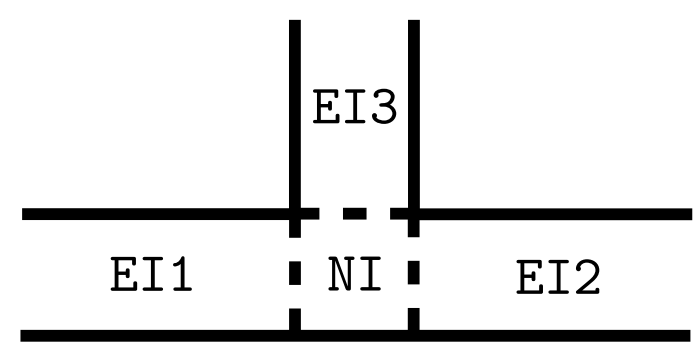

Abbildung 7.7: Positionierung von Fußgängerüberwegen: Die Verläufe der Überwege sind gestrichelt dargestellt. 


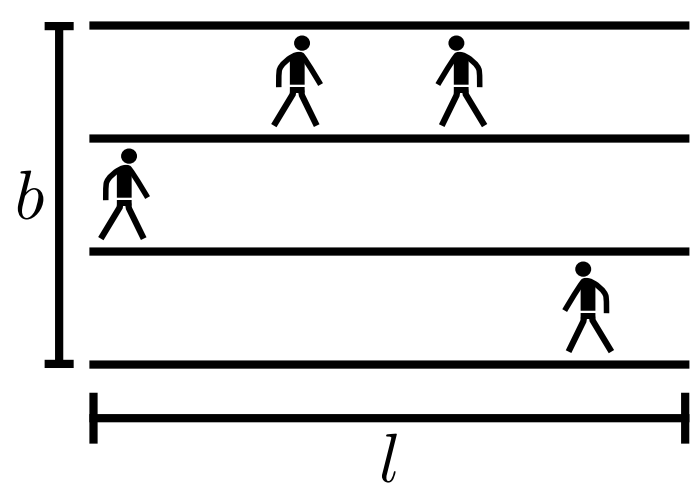

Abbildung 7.8: Fußängerüberweg mit einer Breite $b=3$

In den way-Objekten wird eine raumkontinuierliche Variante des in Blue und Adler, 2001 vorgestellten Fußgängermodells verwendet (vgl. Abschnitt 3.3.4 auf Seite 48, sowie Anhang C). Dies ermöglicht die Verwendung individueller Geschwindigkeiten. Die Überwege werden in Spuren eingeteilt, die von den Fußgängern genutzt werden können. Abbildung 7.8 zeigt einen Fußgängerüberweg mit $b=3$ Spuren. Diese Vereinfachung ermöglicht eine Diskretisierung in der Breite, die zu einer Vereinfachung des Bewegungsmodells führt. Die Länge des Weges ist $l=b r e i t e(\mathrm{EI})$.

Die Update-Methode der way-Objekte wird in Algorithmus 7.5 gezeigt. Da jedes Fußgängerobjekt die update-Methode seines way-Objektes aufruft, wenn es eine Straße überquert, muss Sorge dafür getragen werden, dass ein way nicht mehrfach geupdatet wird. Jeder way speichert daher die iteration seines letzten Updates und verhindert Mehrfachupdates in den Zeilen 2 und 3 . Das beschriebene Verfahren führt zu geringen Aufwendungen für die Verwaltung von way-Objekten, auf denen sich derzeit Fußgänger befinden und die somit aktualisiert werden müssen.

way-Objekte haben eine begrenzte Anzahl an Spuren. Jeder Fußgänger wählt zu Beginn eine Spur mit freier Startposition, sofern vorhanden. Ansonsten wird zufällig eine Spur gewählt. Daher kann es bei starkem Verkehrsaufkommen dazu kommen, dass ein Fußgänger nicht den way betreten kann. In diesem Fall wird er in eine Warteschlange eingefügt, behält jedoch seine anfänglich bestimmte Spur. Bei jedem update() wird in Zeile 4 geprüft, ob ein Fußgänger aus der Warteschlange nun den Weg betreten kann, da seine Spur frei geworden ist.

Für jeden Fußgänger $f$ wird in Zeile 6 nach Gleichung 7.9 ermittelt, welche Spuren in einer Nachbarschaft der aktuellen Spur liegen. Der Parameter $b$ ist die Breite des way-Objektes. 


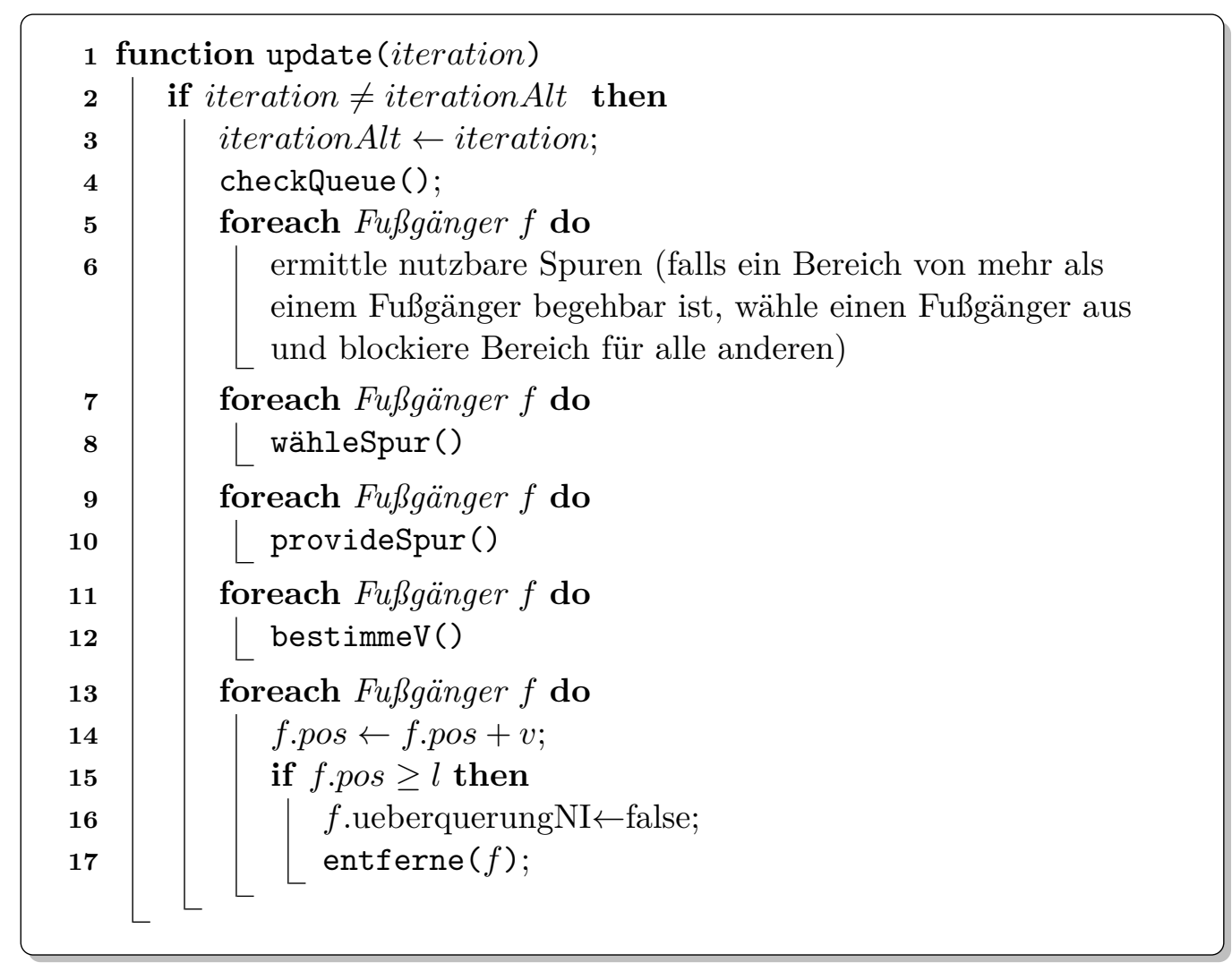

Algorithmus 7.5: update()-Methode der way-Objekte

$$
N_{\text {spur }}=\{f . \text { spur }-1, f . \text { spur, } f . \text { spur }+1\} \cap\{1, \cdots, b\}
$$

Jeder Fußgänger belegt ein Intervall auf seiner Spur. Es können Konflikte entstehen, wenn mehrere Fußgänger bei ähnlicher pos die selben Spuren in ihrer Nachbarschaft haben (vgl. Abbildung 7.9). Konfliktpositionen werden zufällig einem der betroffenen Fußgänger zugewiesen und aus der Nachbarschaft der anderen Fußgänger entfernt.

Die Spurwahl der Fußgänger ist zwecks Vermeidung von Asynchronitäten in die Phasen Spurbestimmung und Spurbekanntgebung getrennt. Zuerst führt in den Zeilen 7 und 8 jeder Fußgänger eine Bewertung der Spuren seiner Nachbarschaft aus. Es werden die Lücken $l(s)$ zum nächsten Fußgänger in Laufrichtung auf der jeweiligen Spur $s \in N_{\text {Spur }}$ bestimmt. Anschließend werden die Lücken bewertet. Die Bewertung einer Spur $\vartheta(s)$ ist identisch zur Lücke und wird halbiert, sofern ein Fußgänger auf dieser Spur in entgegengesetzter Richtung läuft (siehe Abbildung 


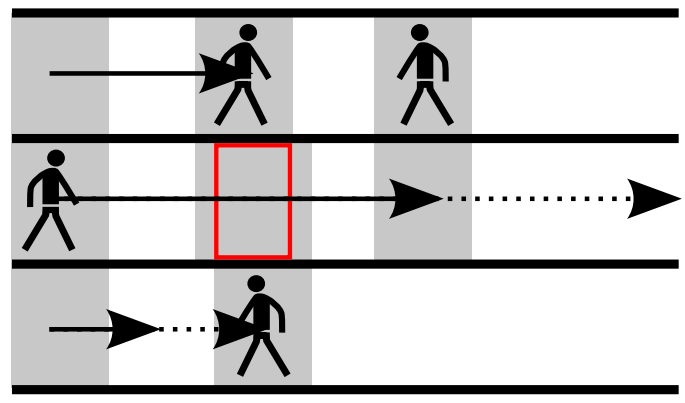

Abbildung 7.9: Nachbarschaft auf Fußgängerüberwegen: Die Nachbarschaften der Fußgänger sind grau markiert. Rot umrandet wurde eine Konfliktstelle, an der zufällig einer der beiden Fußgänger den Vorzug für die mittlere Spur erhält. Der Fußgänger $f$ auf der mittleren Spur bestimmt und bewertet die Lücken in seiner Nachbarschaft. Die gestrichelten Vektoren zeigen die Distanzen bis zum nächsten Fußgänger auf der jeweiligen Spur. Die durchgezogenen Vektoren symbolisieren die Lückenbewertungen von $f$. Auf der mittleren Spur wird die Lücke auf die Sichtweite von $f$ begrenzt. Auf der oberen Spur ist die Lücke identisch zu der Bewertung von $f$. Auf der unteren Spur bewertet $f$ die Lücke halb so groß, wie sie ist, da der Fußgänger auf dieser Spur $f$ entgegenkommt.

7.9). Alle Bewertungen werden auf eine maximale Sichtweite von $10 \mathrm{~m}$ begrenzt. Die Bewertungen werden anschließend auf Wahrscheinlichkeiten zur Nutzung der Spuren abgebildet. Wenn die aktuelle Spur von $f$ eine maximale Bewertung hat, wird sie mit $95 \%$-Wahrscheinlichkeit gewählt und die restlichen $5 \%$ auf die anderen Spuren in $N_{\text {spur verteilt. Ansonsten wird die Wahrscheinlichkeitsverteilung }}$ nach Gleichung 7.10 gebildet.

$$
p_{s}=\vartheta(s)\left(\sum_{s \in N_{\mathrm{Spur}}} \vartheta(s)\right)^{-1} \forall s \in N_{\mathrm{Spur}}
$$

Fußgänger wählen somit bevorzugt die Spur, die die größte Lücke bzw. das schnellste Vorankommen verspricht und bevorzugen die aktuelle Spur, wenn dadurch kein Nachteil entsteht. In den Zeilen 9 und 10 von Algorithmus 7.5 wird der Spurwechsel ausgeführt.

Die Bestimmung der Laufgeschwindigkeiten in den Zeilen 11 und 12 wird nach den Spurwechseln durchgeführt. Die Lückenbewertung $\vartheta(s)$ muss jeder Fußgänger für seine neue Spur durchführen. Die Laufgeschwindigkeiten werden nach Gleichung 7.11 als Minimum der Maximalgeschwindigkeit $v_{\max }=\operatorname{calcV}()$ und $\vartheta(s)$ bestimmt. 


$$
v=\min \left(\vartheta(s), v_{\max }\right)
$$

Wenn durch Gegenverkehr keine Bewegung möglich ist, kann ein Fußgänger mit Wahrscheinlichkeit $p_{\text {tausch }}=0,15$ dennoch am Gegenverkehr vorbeilaufen und erhält als Geschwindigkeit $v=v_{\text {tausch }}=0,25 \cdot v_{\max }$.

In den Zeilen 13 bis 17 wird die Bewegung mit $v$ für jeden Fußgänger $f$ durchgeführt. Wenn $f$ dabei das Ende des Weges erreicht, ist seine Überquerung abgeschlossen und der Überquerungsindikator ueberquerungNI aus Algorithmus 7.4 wird false gesetzt. Das Objekt $f$ wird aus dem way-Objekt entfernt.

Da Fußgänger an Überwegen direkt den Straßenverkehr beeinflussen, muss an diesen Stellen ein Modell verwendet werden, dass auch deren Wechselwirkungen untereinander berücksichtigt, um die Stärke der Verkehrsbeeinflussung realistisch skalieren zu können. Das vorgestellte Modell führt zu geringen Fußgängergeschwindigkeiten an stark genutzen Überwegen und ermöglicht die Nutzung von individuellen Geschwindigkeiten für jeden Fußgänger. Dies führt zu einer Steigerung des Detaillierungsgrades.

Nachdem eine Straßenüberquerung an einer NI abgeschlossen ist, kann sich die Straßenseite des Fußgängers geändert haben. Anhand Abbildung 7.7 werden die unterschiedlichen Fälle in Laufrichtung $\mathrm{EI}_{1} \rightarrow \mathrm{EI}_{2}$ verdeutlicht. Die Straßenseite ändert sich, wenn EI1 oder EI2 überquert wurden, nicht aber wenn EI3 überquert wurde, da hier lediglich dem Verlauf des Bürgersteiges gefolgt wurde und hierfür eine kreuzende Straße überquert werden musste.

Straßenüberquerungen können nun durchgeführt werden. Der folgende Unterabschnitt beschreibt, welche Kriterien erfüllt sein müssen, damit ein Fußgänger eine Überquerung beginnt.

\subsubsection{Straßenüberquerungskriterien}

Wann ein Fußgänger $f$ eine Straße überqueren möchte, legt er in der Taktischen Ebene seines Verhaltensmodells fest. Nachdem $f$ eine Route in dem Straßengraph bestimmt hat, folgt ein Analyseschritt zur Bestimmung der Straßenüberquerungen. Es wird versucht, vor jeder Abzweigung des Weges auf der Straßenseite zu sein, die in Abzweigungsrichtung liegt und Zebrastreifen und Ampeln für die Straßenüberquerungen zu berücksichtigen. Jeder EI auf der Route wird zugewiesen, ob eine Straßenüberquerung beabsichtigt ist. 


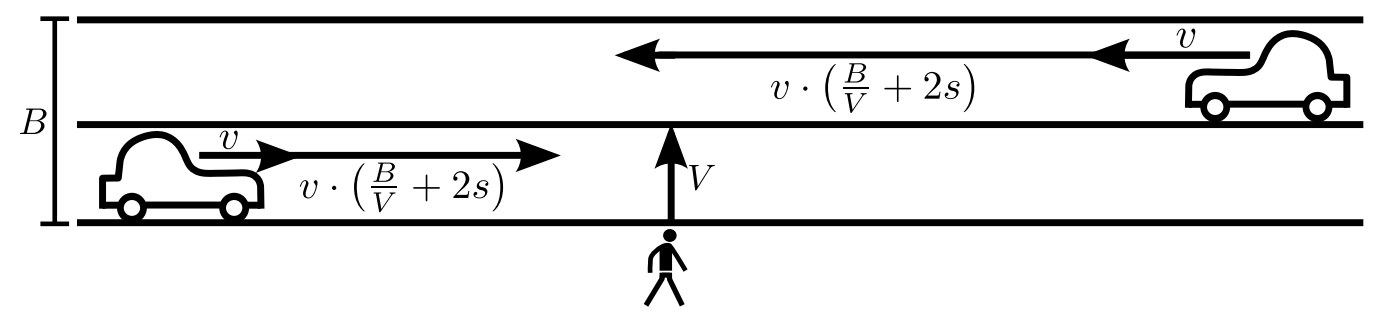

Abbildung 7.10: Straßenüberquerung bei Lücke im Verkehr: Der Fußgänger benötigt $2 s$ Sicherheitsabstand bei der Überquerung der Straße. Ausgehend von den Autos wird der $G D$-Term mit $A F=0$ gezeigt. Er modelliert die geschätzte Position der Autos am Ende der Straßenüberquerung durch $f$. Bei einem negativen $A F$ kann die Lücke noch akzeptiert werden und die Autos treffen gegebenenfalls auf den Fußgänger und müssen bremsen. Bei einem $A F \approx 0$ und größer, wird der Fußgänger auf Grund des Autos von rechts die Straßenseite nicht wechseln.

Auf der operativen Ebene des Fußgängerverhaltensmodells muss während der Durchführung des Plans mittels der Zeilen 12 und 17 aus Algorithmus 7.4 bestimmt werden, wann eine Straßenüberquerung unter Berücksichtigung des Verkehrs durchgeführt werden kann. Die Methode checkUeberquerungEI () prüft, ob eine Überquerung für die aktuelle EI von $f$ vorgesehen ist. Die Straßenüberquerung wird in diesem Fall gestartet, wenn $f$ die Straße überqueren möchte und kann. Ein Fußgänger möchte die Straße mit einer Wahrscheinlichkeit von $p=\frac{f . p o s}{\text { länge(EI) }}$ wechseln. Die Wahrscheinlichkeit steigt folglich, je weiter $f$ auf EI läuft. Wenn $f$ eine EI überqueren möchte, bestimmt $f$ die geschätzte Dauer der Überquerung nach Gleichung 7.12 .

$$
G D=\frac{\text { breite }(\mathrm{EI})}{v}+A F+2 s
$$

$G D$ hängt von der Breite der Straße, der Geschwindigkeit von $f$ bei der Straßenüberquerung, einem Aggressivitätsfaktor $A F=\mathcal{N}_{-3}^{+3}(-0,5,1)$ und einem Sicherheitsabstand von $2 s$ ab. Diese Vorgehensweise basiert auf den in Abschnitt 3.3.2 vorgestellten Überlegungen nach Ottomanelli et al., 2009, Brewer et al., 2006. Zusätzlich steigt $A F$ nach einer standdauer $>5 s$ um $0,1 \cdot($ standdauer $-5 s)$ an, um eine steigende Aggressivität in Relation zur standdauer zu modellieren. Der Fußgänger $f$ prüft für alle Autos, die seine Position auf EI erreichen können, ob die Zeit, die sie dafür voraussichtlich benötigen, größer als $G D$ ist. Falls ja, wird die Überquerung der Straße gestartet. Abbildung 7.10 verdeutlicht die Vorgehensweise exemplarisch.

Die Vorgehensweise für die Überquerung einer NI ist ähnlich. Die Frage, ob $f$ die 
Überquerung durchführen möchte stellt sich in diesem Fall nicht. Eine NI wird entweder überquert, da sie in der Routenbestimmungsphase wegen besonderer Eignung zum Wechsel der Straßenseite ausgewählt wurde (Zebrastreifen, Ampel) oder weil der Wechsel auf der aktuellen EI vorgesehen, aber auf Grund des Verkehrs nicht möglich war und nun am Ende von EI der Wechsel erzwungen ist. Ein weiterer Grund sind kreuzende Straßen. Ob eine NI mittels way-Objekt überquert werden kann, wird analog zur Überquerung einer EI bestimmt. Es werden mittels Gleichung 7.12 alle Straßenverkehrsteilnehmer auf Konflikte bei der Überquerung geprüft, die den Überweg way überfahren werden. Hierbei werden Blinker bzw. Handzeichen von abbiegenden Verkehrsteilnehmern berücksichtigt. Hierbei ist zu beachten, dass nach der StVO § 9(3) ein abbiegender Verkehrsteilnehmer Fußgängern Vorfahrt gewähren muss, denn ,[...] auf Fußgänger muß er besondere Rücksicht nehmen; wenn nötig muß er warten“.

\subsection{Vorfahrtsregeln}

Die simulierten Verkehrsteilnehmer von MAINSIM halten sich an die grundlegenden Verkehrsregeln. Die Verkehrsregeln für Fußgänger wurden direkt in das Fußgängermodell integriert (vgl. Abschnitt 7.3). Dieser Abschnitt beschreibt die Vorfahrtsregeln, die von simulierten Autos und Fahrrädern verwendet werden.

Vorfahrtsregeln müssen immer dann beachtet werden, wenn ein simulierter Verkehrsteilnehmer $T$ von einer $\mathrm{EI}_{1}$ über eine $\mathrm{NI}$ auf eine $\mathrm{EI}_{2}$ wechselt. Er setzt zu diesem Zeitpunkt einen Blinker in die Richtung, in die er fahren möchte. Blinker können links, geradeaus oder rechts geschaltet werden. Fahrräder setzen ebenfalls Blinker - symbolisch für Handzeichen.

Falls NI über eine Ampel verfügt, kann NI überquert werden, wenn diese grün zeigt. Enthält NI keine Ampel, muss geprüft werden, ob die Vorfahrt einem anderen Verkehrsteilnehmer gewährt werden muss. Es werden der Reihe nach alle mit NI verbundenen EIs untersucht und geprüft, ob aus der jeweiligen EI ein Verkehrsteilnehmer in Richtung NI fährt, der nah genug am Ende der EI ist, sodass er NI erreichen wird. In diesem Fall werden die in $\S 8$ StVO beschriebenen Vorfahrtsregeln abgebildet. Hierbei werden Abbiegewünsche (Blinker) anderer Verkehrsteilnehmer und daraus resultierende Möglichkeiten zum Überqueren der NI berücksichtigt.

Vorfahrtsstraßen und Kreisverkehre erhalten entsprechend höhere Vorfahrtsprioritäten. Es werden ausschließlich Verkehrsteilnehmer berücksichtigt, deren EI eine 
identische oder höhere Vorfahrtspriorität zu der EI von $T$ haben.

Falls Verkehrsteilnehmer auf NI zufahren, die Vorfahrt über $T$ haben, bewertet $T$, ob er deswegen warten muss. Dies geschieht unter Berücksichtigung der in Tabelle 3.7 auf Seite 51 dargestellten Lückenakzeptanzwerte. Schwankungen werden über die individuellen Werte von $\perp$ modelliert.

Autos und Fahrräder überqueren keine NI, auf der sich Fußgänger befinden. Es ist unerheblich, ob ein Fußgänger Vorfahrt hat oder nicht - er darf nicht überfahren werden.

Falls $T$ warten muss, so fährt er bis zu einer definierten Warteposition (vgl. Abschnitt 6.5. Diese Regel verhindert ein Warten auf der Mitte der Kreuzung.

\subsection{Ampelschaltungen}

OSM definiert Positionen, an denen sich Ampeln befinden. Die gesetzten Ampeln können in MAINSIM grundlegend drei Arten haben: eine Festzeitsteuerung, eine Fußgängerampel oder eine dynamische Schaltung. Jede der Ampeln kann von Fußgängern gedrückt werden.

Die grundlegende Funktionsweise ist, dass eine Ampel in einer NI eine aktive EI auswählt. Jede nicht aktive EI hat automatisch rot. Die aktive EI durchläuft die grundlegenden Phasen rot, gelb-rot, grün und gelb. Verkehrsteilnehmer werten gelb-rot und gelb jeweils als rot.

Die einfachste Ampel ist die Fußgängerampel. Sie kann ausschließlich an einer NI platziert werden, die nur zwei verbundene EIs hat. Sie ist im Normalfall deaktiviert und somit für Autos und Fahrräder unsichtbar. Wenn ein Fußgänger sie drückt, wird sie aktiviert und schaltet den Straßenverkehr auf rot. Nach einer fixen Dauer wird die Ampel wieder deaktiviert.

Die Festzeitsteuerung verwendet vordefinierte Phasenlängen für jede Phase. Die Phasenlängen bleiben konstant über die Simulationsdauer. Nach einem Phasendurchlauf wird die nächste EI von NI aktiv geschaltet und anschließend identisch verfahren. Keine Seite kann „Verhungern“, da der Reihe nach jede EI von NI aktiviert wird. Diese Schaltung ist nicht effizient, da sie nicht auf die aktuelle Verkehrslage eingeht.

Eine erste dynamische Schaltung ist die lastbasierte Schaltung. Sie schaltet die 
Straße grün, die am meisten wartende Verkehrsteilnehmer hat. Dieses Verfahren ist einfach und führt zu einer Bevorzugung der Hauptverkehrsrichtung. Falls jedoch stets sehr viele Verkehrsteilnehmer aus einer Richtung kommen, kann eine weniger frequentierte Straße „verhungern“, sie erhält niemals grün.

Als standard-Ampel wird bei MAINSIM eine Prioritätsampel verwendet. Die Priorität aller EIs der geschalteten NI ist anfangs 0. Es wird initial eine EI randomisiert ausgewählt, die zuerst grün erhält. In jeder Iteration werden die Prioritäten der EIs, die nicht aktiv sind, um die Anzahl an wartenden Verkehrsteilnehmern inkrementiert. Nach Ablauf einer Phase wird die nächste EI aktiviert. Es wird hierfür die EI mit der höchsten Priorität ausgewählt. Ein „verhungern“ wird ausgeschlossen, da auch eine EI mit wenig Verkehr im Laufe der Zeit Priorität anhäuft. Wenn die Ampel umschaltet und eine andere EI aktiv wird, wird deren Priorität auf 0 gesetzt. Der Mechanismus ist bisher identisch zu der in Fouladvand et al., 2005 beschriebenen Ampelschaltung.

Bei einem Ungleichgewicht an Verkehrsdichte auf den zu schaltenden EIs kann eine dynamische Phasenlänge zu Verbesserungen der Schaltungseffizienz führen. Es sei $p_{g}$ die Regelphasenlänge der grünen Phase. Es sei prio(EI) die Priorität einer EI und EI $a_{a}$ die derzeit aktive EI. Der Mittelwert der Prioritäten aller mit NI verbundenen EIs sei $p_{m}$. Die Phasenlänge $p$ wird durch die folgende Gleichung

$$
p=\min \left(\max \left(\frac{\operatorname{prio}\left(\mathrm{EI}_{a}\right)}{p_{m}}, 0,75\right), 3,0\right) \cdot p_{g}
$$

je nach Prioritätsverteilung variiert. Eine EI mit hoher Priorität erhält länger grün, als eine EI mit geringer Priorität. Die Auflösung eines Staus an einer Ampel wird somit begünstigt.

\subsection{Routing}

Wenn ein Verkehrsteilnehmer von einer Start- zu einer Zielposition möchte, benötigt er eine entsprechende Route. Eine Route besteht aus einer Folge von NIs und EIs, die den Weg beschreiben. In MAINSIM können verschiedene Routingverfahren verwendet werden. Die folgenden Unterabschnitte 7.6.1 bis 7.6.3 geben einen Überblick. 

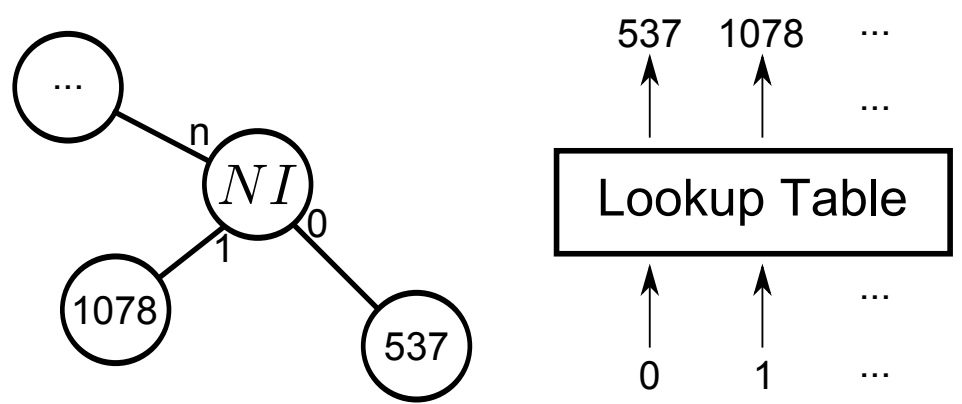

Abbildung 7.11: Lookup table für NI-IDs

\subsubsection{Vorberechnete Routen}

Eine statische Methode ist die Vorberechnung aller möglichen Routen im Graphen. Hierfür wird von jeder NI der Algorithmus von Dijkstra Dijkstra, 1959 berechnet, der das Single-source shortest path (SSSP) - Problem löst. In einem Graphen mit 10.000 NIs existieren $10.000 \cdot 9.999 \approx 10^{8}$ Routen. Hieraus resultieren zwei Probleme: Die Dauer der Berechnung aller Routen steigt schnell an, da Dijkstra eine Laufzeit von $\mathcal{O}(|\mathrm{NIs}| \cdot \lg (|\mathrm{NIs}|)+\mid$ EIs $\mid)$ hat Cormen et al., 2001. Dijkstra muss für jede NI durchgeführt werden, da der ExtendedGraph ein gerichteter Graph ist. Insgesamt ergibt sich eine Laufzeitkomplexität von $\mathcal{O}\left(|\mathrm{NIs}|^{2} \cdot \lg (|\mathrm{NI} \mathbf{s}|)+|\mathrm{NIs}| \cdot|\mathrm{EIs}|\right)$. Im Übrigen müssen alle Routen gespeichert werden.

Vorberechnete Routen werden nach Berechnung des ExtendedGraph berechnet. Anschließend wird der gesamte Graph in einer Datei gespeichert. Auf diese Weise besteht die Laufzeitproblematik nicht mehr. Die Speicherung geschieht wie folgt:

Jede NI verwaltet eine Liste mit Ids. Der Eintrag $i$ der Liste ist die Id der nächsten NI auf dem Pfad zur NI mit der Id $i$. Nach diesem Verfahren müsste jede NI im Beispiel mit 10.000 NIs 9.999 Integer-Zahlen verwalten. Integer benötigt in Java 32 Bit. Es ergibt sich ein Speicherbedarf von ca. 381 MB pro Verkehrsteilnehmertypen. Zur Komprimierung werden nicht die Ids selbst, sondern Ersetzungen davon gespeichert (vgl. Abbildung 7.11).

Dies ist möglich, da jede NI nur sehr wenige Nachbarknoten hat, mit denen sie direkt verbunden ist. Der Datentyp Byte (8 Bits) genügt hierfür. Jede NI erstellt eine LUT, die den IDs der nachbar-NIs eine Byte-Zahl zuweist. Der Speicherbedarf kann somit auf ca. 95MB reduziert werden. Dies geschieht ohne signifikante Einbußen der Berechnungseffizienz. Um den Weg von einer Start-NI zu einer Ziel-NI zu bestimmen, muss jede NI auf dem Weg einen Lookup durchführen. Die zeitliche 
Komplexität ist hierbei insgesamt $\Theta(n)$, wobei $n$ die Anzahl der NIs auf der Route ist.

Die beschriebene Methode führt während der Verkehrssimulation zu Staus, da alle Verkehrsteilnehmer die Routen wählen, die optimal wären, wenn kein Verkehr vorhanden wäre. Ein einfaches Verfahren, um diesem Problem zu begegnen, ist das im folgenden Abschnitt vorgestellte probabilistische Routingverfahren.

\subsubsection{Probabilistisches Routing}

In diesem Ansatz werden Routen zufällig bestimmt. Verkehrsteilnehmer, die mittels dieser Methode ihre Routen bestimmen, haben einen definierten Startpunkt, aber keinen vorgegebenen Zielpunkt. Verkehr dieser Art kann als Hintergrundverkehr genutzt werden. Dies ist Verkehr, der andere Verkehrsteilnehmer beeinflusst, aber nicht im Fokus der Simulation steht.

Es sei $\Omega_{\mathrm{NI}}$ die Menge an EIs, die mit NI verbunden sind. Jede NI des ExtendedGraph speichert eine Abbiegewahrscheinlichkeit $p_{t}\left(E I_{\text {curr }}, E I_{\text {next }}\right)$, für einen Verkehrsteilnehmer des Typs

$$
t \in\{\text { Auto, Fahrrad, Fußgänger }\}
$$

von $\mathrm{EI}_{\text {curr }}$ kommend. $p_{t}$ gibt die Wahrscheinlichkeit, $\mathrm{EI}_{\text {next }}$ als nächste EI zu wählen. Die Funktion $p_{t}$ hält die Gleichungen 7.15 und 7.16 ein.

$$
\begin{aligned}
p_{t}\left(\mathrm{EI}_{\text {curr }}, \mathrm{EI}_{\text {curr }}\right) & =0 \\
\sum_{\mathrm{EI} \in \Omega_{N I}} p_{t}\left(\mathrm{EI}_{\text {curr }}, \mathrm{EI}\right) & =1 \forall t, \text { EIcurr }
\end{aligned}
$$

Mit einer gegebenen $\mathrm{EI}_{\text {curr }}$ kann eine Route durch wiederholte zufällige Wahl der

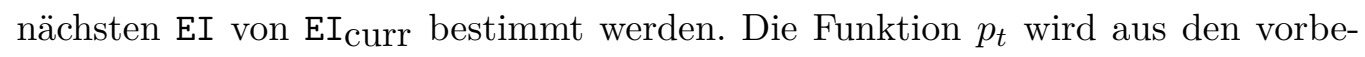
rechneten Routen des vorherigen Abschnitts extrahiert.

Jede NI verwaltet Zähler (initial 0) für jeden Typ $t$ und jede Kombination $E I_{\text {curr }}, E I_{\text {next }} \in \Omega_{N I}$. Die Routen $\zeta\left(N I_{\text {start }}, N I_{\text {dest }}\right)$ zwischen allen nicht identischen Paaren von NIs $N I_{\text {start }}$ zu $N I_{\text {dest }}$ werden als Listen von NIs und EIs bestimmt. Jede Route $\zeta$ wird analysiert und an jeder $N I \in \zeta$ wird der dazugehö- 
rige Zähler für die Verbindung zwischen der aktuellen und der nächsten EI inkrementiert. Dieser Analyseschritt wird für jeden Typen $t$ durchgeführt. Anschließend werden alle Zähler normalisiert, sodass die Wahrscheinlichkeitsverteilungen $p_{t}$ entstehen, die die Bedingungen der Gleichungen 7.15 und 7.16 einhalten.

Die Bestimmung von $p_{t}$ hat eine zeitliche Komplexität von $\mathcal{O}\left(|N I C|^{3}\right)$, da $\mathcal{O}\left(|N I C|^{2}\right)$ Routen mit einer maximalen Pfadlänge von $\mathcal{O}(|N I C|)$ NIs analysiert werden müssen. Der Lookup einer Route mit $n$ NIs geschieht in $\Theta(n)$.

Beide beschriebenen Methoden sind nicht in der Lage, dynamische Veränderungen der Straßenkonditionen zu berücksichtigen. Daher wird im nächsten Abschnitt der bekannte $\mathrm{A}^{*}$-Algorithmus beschrieben.

\subsubsection{Live berechnete Routen}

Innerhalb von MAINSIM wird ein bidirektionaler $\mathrm{A}^{*}$-Algorithmus verwendet. Die Grundlage hierfür ist der A*-Algorithmus Russell und Norvig, 2004 zur Lösung des Single-pair shortest path-Problems zwischen zwei NIs. Der bidirektionale Ansatz führt eine vorwärts gerichtete Suche vom Startknoten zum Zielknoten und gleichzeitig eine rückwärts gerichtete Suche vom Zielknoten zum Startknoten durch. Wenn beide Suchen einen Schnittpunkt ergeben, ist die Suche beendet. Das Vorgehen führt zu einer geringeren Exploration des Suchraums und folglich zu einer Beschleunigung der Suche.

Das Routingproblem wird entscheidend durch die Wahl der Kantenbewertungsfunktion $d(\mathrm{EI})$ beeinflusst. Der einfache Ansatz, die Distanz als $d(\mathrm{EI})=v_{\max }^{\mathrm{EI}}$. $|\mathrm{EI}|^{-1} \mathrm{zu}$ bestimmen, führt zu identischem Routingverhalten wie die in Abschnitt 7.6.1 beschriebene Verwendung vorberechneter Routen. Eine Verteilung des Verkehrs im Simulationsbereich wird durch Einbeziehung von aktuellen Reiseinformationen erzielt.

Ein Verkehrsteilnehmer kann eine eigene Kantenbewertungsfunktion übermitteln und Erfahrungen aus früheren Simulationsläufen aufgreifen. Eine andere Methode ist die Protokollierung von Reisedauern auf EIs. Die Reisedauern der Verkehrsteilnehmer der letzten 15 simulierten Minuten werden pro EI separiert nach Fahrtrichtung und Verkehrsteilnehmertypen protokolliert. Der Mittelwert dieser Protokollreisedauern kann als $d(\mathrm{EI})$ verwendet werden. Wenn auf Grund einer Überlastung einer Straße die Reisedauern der korrespondierenden EI ansteigen, werden simulierte Verkehrsteilnehmer dies einbeziehen und EI tendenziell meiden. 
Als Heuristik $h\left(\mathrm{NI}_{1}, \mathrm{NI}_{2}\right)$ wird die Euklidische Distanz zwischen den Koordinaten der Punkte von $\mathrm{NI}_{1}$ und $\mathrm{NI}_{2}$ verwendet. $h($ ) kann mit einem Überschätzungsfaktor skaliert werden [Jacob et al., 1999]. Dies führt zu nicht optimalen Lösungen bei gleichzeitiger Reduktion der Berechnungsdauer Fu et al., 2006. Als Standard wird keine Skalierung durchgeführt.

Eine in der Literatur diskutierte Methode zur Beschleunigung der Routenfindung ist eine Berechnung von mehreren Hierarchieebenen Sanders und Schultes, 2006. Auf der untersten Ebene sind alle Straßen vorhanden. Bei steigender Ebenennummer werden immer weniger Straßentypen berücksichtigt, bis auf der höchsten Ebene nur noch Autobahnen übrig bleiben. Um eine Route über große Distanzen zu berechnen, müssen auf jeder Ebene die Eintrittspunkte zur nächst höheren Ebene gefunden werden. Von dort kann die Suche mit sehr wenigen Knoten schnell duchgeführt werden. Später muss der Weg auf der obersten Ebene durch die Teilwege der darunterliegenden Ebenen ersetzt werden. Insgesamt führt das Verfahren zu einer Beschleunigung der Routenfindung Sanders und Schultes, 2005.

Verfahren dieser Art können zur Routenfindung in statischen Szenarien ohne starke Schwankungen der Reisedauern auf den jeweiligen Straßen verwendet werden. Im Rahmen eines Verkehrssimulationssystems würde die Verteilung des Verkehrs nicht entstehen, da schwankende Werte von $d()$ zu einer Aktualisierung der Hierarchieebenen führen müssten. Die Neuberechnung der Ebenen wäre zu aufwendig.

Nach Westerdijk, 1990 spielen bei der Routenwahl für Fußgänger und Fahrradfahrer mehrere Faktoren eine Rolle. Eine Auswahl sind Distanz, Anzahl an Kreuzungen, Schönheit des Weges, Attraktionen, Qualität des Straßenbzw. Bürgersteigbelags, Steigung und die Verkehrssicherheit. Die wichtigsten Faktoren für Fahrradfahrer sind jedoch die Distanz und der Straßenverkehr Dill und Gliebe, 2008. Vereinfachend wird in MAINSIM für Fahrräder und Fußgänger als Kantenbewertung die Länge der EI verwendet. Die fahrbare Geschwindigkeit ist für Fußgänger irrelevant. Fahrräder fahren im Normalfall ebenfalls langsamer, als die Straße es zulassen würde. Die Gewichtung der Schönheit einer Straße wird über einen Skalierungsfaktor in Abhängigkeit zum Typ der korrespondierenden EI berücksichtigt. Fahrradwege bzw. Fußwege werden bevorzugt, Wohngebiete erhalten ebenfalls geringere Skalierungsfaktoren als Hauptverkehrsstraßen. Dies führt zu einer Bevorzugung von Straßen mit wenig Automobilverkehr. 



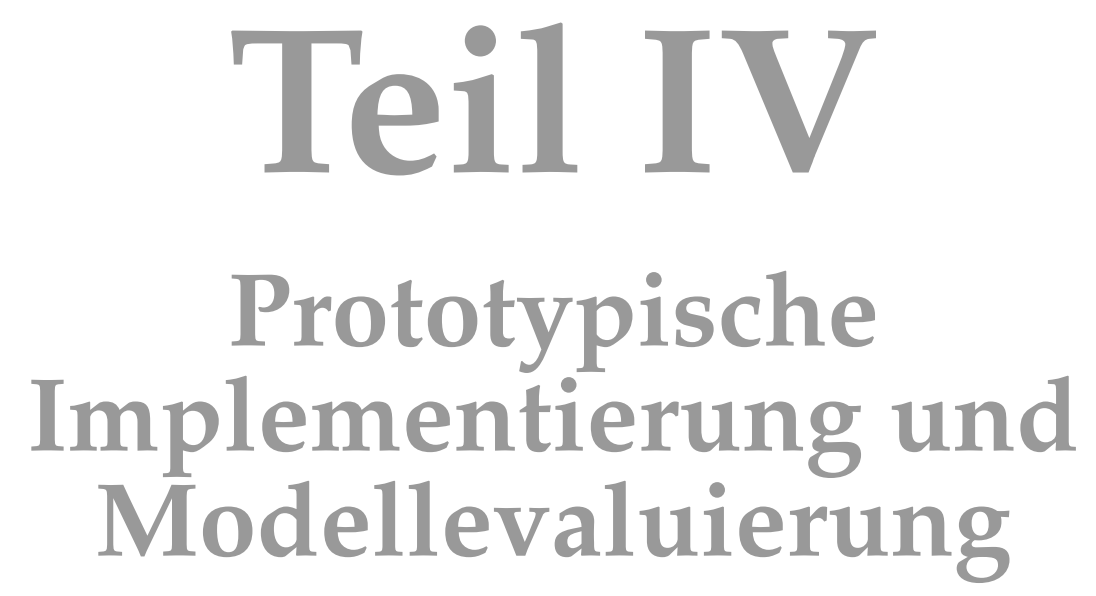





\section{Prototypische Implementierung}

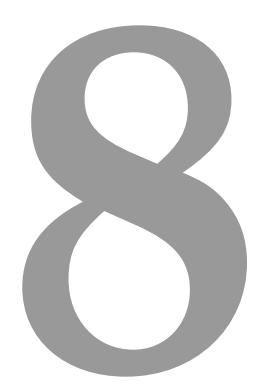

Dieses Kapitel beschreibt grundlegende Implementierungsdetails. Der folgende Abschnitt 8.1 diskutiert fundamentale Designentscheidungen und die wichtigsten Teile der Programmarchitektur. Abschnitt 8.2 zeigt die Vorgehensweise zur Durchführung einer Simulation. Abschnitt 8.3 diskutiert die Zeit- und Speicherkomplexität verschiedener Operationen von MAINSIM. Abschließend wird in Abschnitt 8.4 die Skalierbarkeit des Sytems untersucht.

\subsection{Designentscheidungen und Architektur}

Zuerst musste die Frage nach einer geeigneten Datenquelle für Straßenkarten beantwortet werden. Im Bereich der Geoinformationssysteme (GIS) hat sich das Shapefile-Format als Quasi-Standard durchgesetzt. Ein Aufbau der Simulation auf einem GIS bietet sich daher an. In Kapitel 6 wurde bereits ausgeführt, dass die Java-Klassensammlung GeoTools zur Verarbeitung von Shapefiles genutzt werden kann. Um Funktionalitäten von GeoTools direkt in MAINSIM einbinden zu können, wird die Programmiersprache Java verwendet. Grundlegende Hilfsklassen z.B. zur Verarbeitung von XML-Dateien - sind in Java bereits vorhanden.

Abbildung 8.1 stellt eine Erweiterung von Abbildung 6.1 auf Seite 83 dar. Die 


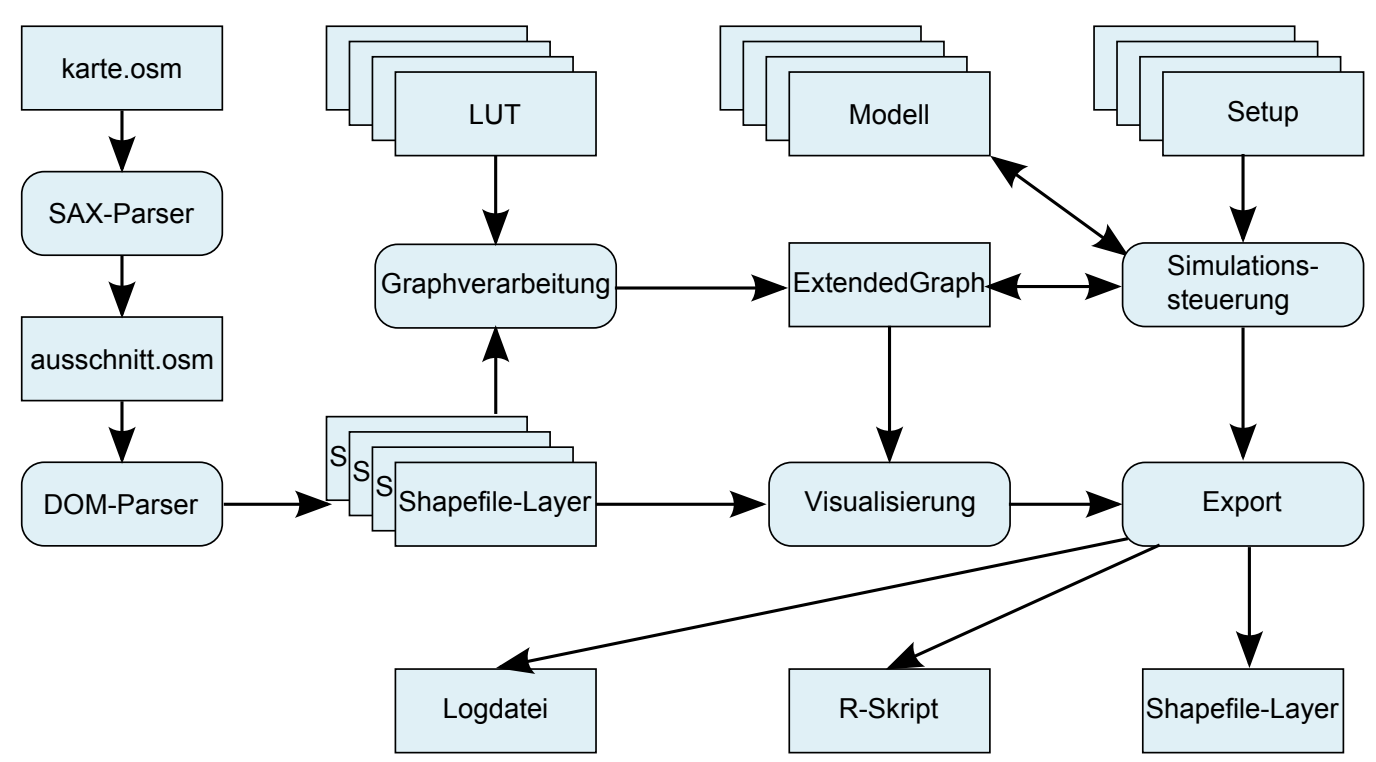

Abbildung 8.1: Architektur von MAINSIM

Datei „karte.osm“ wird mittels eines SAX-XML-Parsers auf einen Ausschnitt beschnitten, der in der Datei „ausschnitt.osm“ gespeichert wird. Die Nutzung eines SAX-Parsers ermöglicht die Verarbeitung beliebig großer XML-Dateien. Ein DOM-Parser wird benötigt, um die einzelnen Elemente der ,ausschnitt.osm“ zu verarbeiten und die enthaltenen Geodaten in Ebenen zu trennen (vgl. Abschnitt 6.1). Die berechneten Ebenen werden im Shapefile-Format gespeichert.

Die Trennung zwischen Ausschnittsbestimmung und der weiteren Verarbeitung der OSM-Daten ist notwendig, da im Format von OSM Geometrien aus NodeElementen zusammengesetzt werden, die verschiedenen Objekten zugehören können. Hieraus folgt, dass ein DOM-Tree benötigt wird. Hierfür muss das gesamte XML-Dokument im Speicher gehalten werden. Dies ist lediglich bei Ausschnitten von OSM-Dateien möglich, da z.B. die Landkarte des Bundeslandes Hessen knapp 2GB (Stand: 31.07.2012) umfasst. Der generierte DOM-Tree benötigt nochmals weit mehr Kapazitäten im Arbeitsspeicher.

Die Shapefile-Layer werden anschließend geladen, um daraus den ExtendedGraph zu berechnen. Es könnten somit auch Geodaten anderer Anbieter als OSM genutzt werden. Die Komponente „Graphverarbeitung“ wird mittels LUTs mit zusätzlichen Informationen angereichert, um lückenhafte Geodaten zu ergänzen. Der ExtendedGraph wird in der Simulationssteuerung genutzt. Angereichert mit Modellen für Verkehrsteilnehmer und Ampeln, sowie Einstellungen zur Durchführung der Simulation, steuert die Simulationssteuerung den Ablauf der Simulation. 
Der aktuelle Zustand (z.B. die Positionen der Verkehrsteilnehmer) kann über eine Visualisierungskomponente dargestellt werden. Die Visualisierung verwendet die berechneten Shapefile-Layer und nutzt eine Renderingkomponente von GeoTools zur Darstellung der Landkarte. Verkehrsteilnehmer und Ampeln werden auf die gerenderte Karte mit einer in MAINSIM implementierten Software-Sprite Technologie gezeichnet (vgl. Watt, 2000).

Eine aktuelle Momentaufnahme und Simulationsergebnisse können exportiert werden. Es stehen eine automatisch geschriebene Logdatei und Exportfunktionen zur Generierung von Shapefiles, Bilddateiformaten und R-Skripten zur Verfügung.

Die grundlegende in Abbildung 8.1 gezeigte Architektur ist stark vereinfacht. MAINSIM verfügt über 32 Pakete mit insgesamt 203 Klassen. Das System besteht aus 35.552 Zeilen Java-Quelltext (Stand: 02.10.2012).

\subsection{Ablauf einer Simulationsstudie}

Nachdem der ExtendedGraph eines gewählten Kartenausschnitts berechnet wurde, kann eine Simulation durchgeführt werden. Die Simulationssteuerung führt in einer Schleife den in Abbildung 7.2 auf Seite 100 gezeigten Updatezyklus durch. Zusätzlich zu den drei Schritten Spurwechsel, Update und Provide werden in jeder Iteration die Zustände der Ampeln aktualisiert.

Eine Methode zum Hinzufügen von neuen Verkehrsteilnehmern wird ausgeführt. Diese kann je nach Simulationsexperiment unterschiedlich agieren. Es kann beispielsweise eine bestimmte Anzahl an Verkehrsteilnehmern nach einer definierten statistischen Verteilung aufrecht erhalten oder pro Iteration eine bestimmte Anzahl an Autos erstellt werden. In einem konkreten Experiment muss diese Methode daher maßgeschneidert werden.

Ein weiterer Schritt, der in jeder Iteration durchgeführt wird, ist die Sortierung der EIs. Die Verkehrsteilnehmer auf jeder EI werden nach aufsteigender Position sortiert, um eine effiziente Bestimmung des Vorder- und Hintermanns zu ermöglichen. Da pro Iteration nur wenige Überholvorgänge auftreten, wird das Sortierverfahren Bubblesort (vgl. Cormen et al., 2001) verwendet. Bubblesort hat im Worstcase eine Laufzeit von $\mathcal{O}\left(n^{2}\right)$ bei $n$ zu sortierenden Elementen. Um den Sortierschritt zu umgehen, könnten alternativ bei den Bewegungen der Verkehrsteilnehmer auch Positionsänderungen detektiert werden. Dies würde jedoch eine komplexere Logik erfordern. Tests haben gezeigt, dass während der Simulation selten mehr als ein 
oder zwei Sortierdurchgänge notwendig sind und der Berechnungsaufwand daher als gering einzustufen ist.

In Abbildung 8.2 wird der vollständige Updatezyklus der Simulationssteuerung gezeigt. Zwischen je zwei Schritten beim Ablauf des Updatezyklus können weitere Methoden integriert werden. Zusätzlich kann in einer Simulationssteuerung für ein Experiment jede der Methoden erweitert werden. Dies ermöglicht eine einfache Umsetzung von Messungen, wie z.B. den Reisedauern(-geschwindigkeiten, -kraftstoffverbrauchswerten, ...) einer bestimmten Verkehrsteilnehmergruppe in einem definierten Messgebiet zu einer bestimmten Messzeit.

Die Simulationssteuerungskomponente wird häufig von einer Experimentensteuerungskomponente aufgerufen. Die Experimentsteuerung führt eine bestimmte Anzahl von Simulationsläufen durch und extrahiert Ergebnisinformationen.

\subsection{Komplexität}

Während der Berechnung des ExtendedGraph wird zuerst ein Ausschnitt aus der „karte.osm“ berechnet, da die Berechnung eines DOM-Trees Zeit und Speicherkapazitäten kostet. MAINSIM arbeitet mit Java 64, da mit 32 Bit nicht genügend Speicher zur Berechnung des DOM-Trees des in Kapitel 15 genutzten Kartenausschnitts zur Verfügung stand.

Während der Berechnung des ExtendedGraph müssen mehrere Arbeitsschritte mit Geodaten durchgeführt werden. Um beispielsweise eine NI an der Schnittstelle einer $\mathrm{EI}_{1}$ mit einer schneidenden $\mathrm{EI}_{2}$ zu setzen, wird über einen Räumlichen Index nach räumlich nahen EIs zu EI 1 gesucht. Der durch GeoTools gegebene Räumliche Index gibt jedoch auch EIs zurück, die weit von $\mathrm{EI}_{1}$ entfernt liegen.

Die Anzahl an zurückgegebenen EIs bei einer Anfrage an den Räumlichen Index kann maximal $|E I C|$ sein. Somit ist die Gesamtlaufzeit der Operation maximal $\mathcal{O}\left(|E I C|^{2}\right)$. Von einer Reimplementierung des Räumlichen Index wird abgesehen, da der ExtendedGraph nur einmalig berechnet werden muss. Er wird in einer Datei per Serialisierung gespeichert und kann zur erneuten Verwendung wieder geladen werden.

Die Simulationsgeschwindigkeit nimmt bei steigender Größe des ExtendedGraph ab. Dies resultiert aus dem steigenden Aufwand zur Berechnung von Pfaden (vgl. Abschnitt 7.6). Die Anzahl der simulierten Verkehrsteilnehmer beeinflusst die 


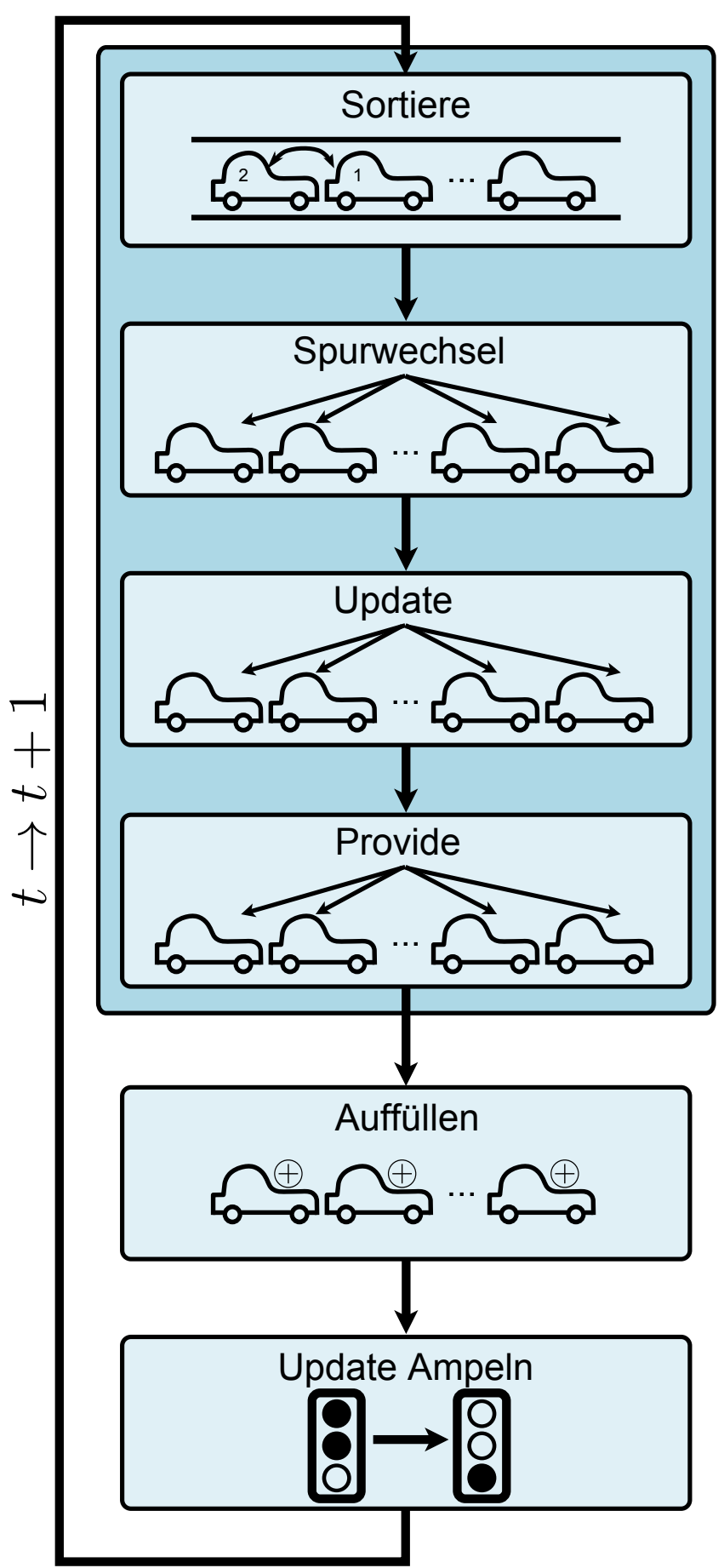

Abbildung 8.2: Vollständiger Updatezyklus der Simulationssteuerung. Der separat gruppierte Block Sortiere, Spurwechsel, Update und Provide muss in der gezeigten Reihenfolge berechnet werden. Weitere Funktionseinheiten können an beliebiger Position innerhalb des Schemas eingefügt werden. 
Simulationsgeschwindigkeit linear. Bei hohen Verkehrsdichten und auftretenden Staus können zusätzliche Kapazitäten für die Berechnung von neuen Routen umplanender Verkehrsteilnehmer benötigt werden.

Bei der Durchführung eines Experiments werden meist wiederholt Simulationsdurchläufe mit identischen Einstellungen durchgeführt, da Simulationsergebnisse auf Grund probabilistischer Einflüsse in den mikroskopischen Verkehrsmodellen streuen. Messgrößen werden daher gemittelt. Um mehrere Simulationsläufe parallel durchführen zu können, verfügt MAINSIM über ein einfaches Client-ServerSystem. Der Server steuert die Simulationsläufe von über TCP/IP verbundenen Clients. Ein Client erhält die Aufforderung, einen Simulationslauf mit einem vom Server gegebenen Seedwert für den Zufallszahlengenerator und Parametern für das Experiment durchzuführen. Der Server verwaltet in Listenstrukturen die von den Clients gesammelten Ergebnisse. Die Auslastung des Servers ist hierbei minimal. Die Clients lasten pro Instanz einen Rechenkern aus. Ein Client-PC kann somit mehrere Client-Instanzen berechnen. Bei Tests wurde mit ca. 20 Rechenkernen parallel simuliert.

\subsection{Skalierbarkeit}

Dieser Abschnitt untersucht den Einfluss der Anzahl an simulierten Verkehrsteilnehmern und der Kartenausschnittsgröße auf die Simulationsgeschwindigkeit. Zur Analyse wird ein Desktop PC mit Intel E6750 (2,66 Ghz) Prozessor genutzt. In den folgenden Experimenten wird stets eine Verkehrszusammensetzung von $50 \%$ Autos, 7,5 \% Fahrrädern und 42,5 \% Fußgängern verwendet.

Die in Abschnitt 7.6 beschriebenen Routingverfahren führen zu divergierenden Simulationsgeschwindigkeiten. Eine Verteilung des Verkehrs im Straßennetz bei gleichzeitiger Berücksichtigung individueller Start- und Zielpunkte wird nur dann erreicht, wenn während eines Simulationslaufes Routen unter Nutzung von aktuellen Reiseinformationen berechnet werden. Es wird daher in den folgenden Experimenten die auf Seite 124 besprochene Protokollierung von Reisedauern genutzt.

Abbildung 8.3 zeigt den Verlauf der Simulationsgeschwindigkeit in Relation zur Anzahl an simulierten Verkehrsteilnehmern in den Städten Erlensee (vgl. Abbildung 13.1 auf Seite 202 und Hanau (vgl. Abbildung 9.9 auf Seite 149. Es wurden hierfür initial $a$ Verkehrsteilnehmer erstellt und nach einer Einschwingphase von 1.000 Iterationen für 4.000 Iterationen die Simulationsgeschwindigkeit gemessen. Anschließend wurde die Anzahl an Verkehrsteilnehmern je in Schritten von $a$ er- 

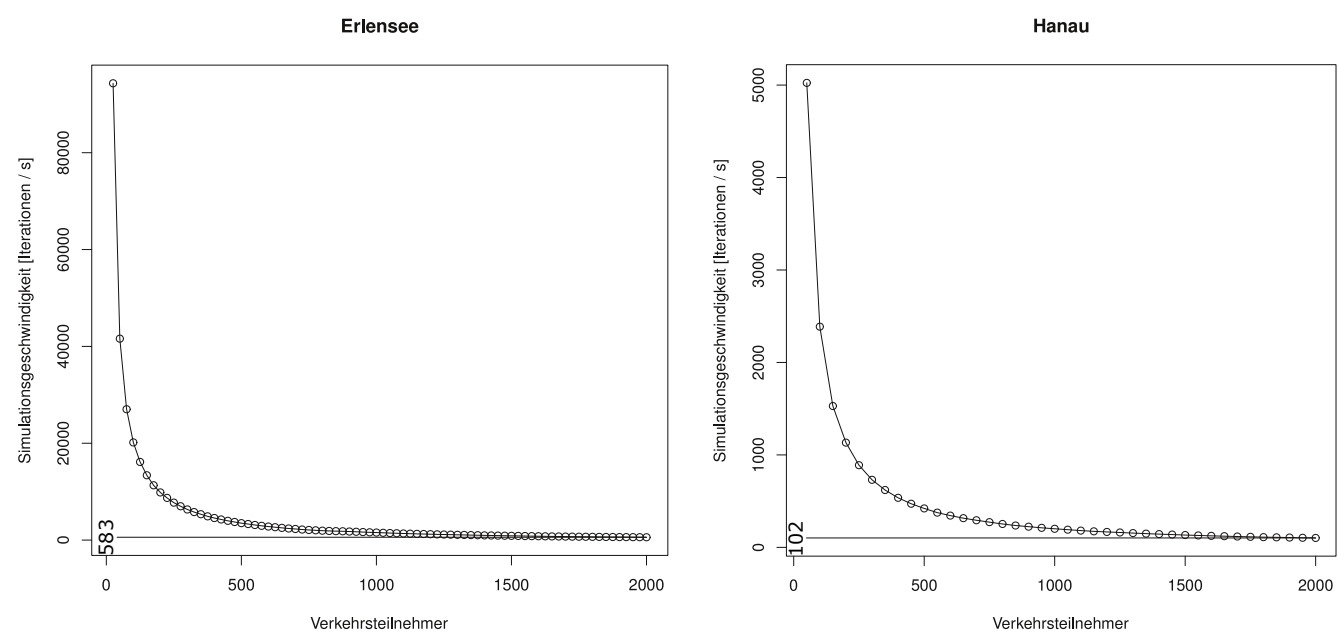

Abbildung 8.3: Simulationsgeschwindigkeitsskalierung nach Verkehrsmengen.

höht und dieses Vorgehen wiederholt, bis eine Anzahl von 2.000 Verkehrsteilnehmern erreicht wurde. Das Verfahren wurde 100 mal wiederholt, um die Ergebnisse mitteln zu können. Für Erlensee wurde $a=25$, für Hanau $a=50$ verwendet.

Bei steigender Anzahl an Verkehrsteilnehmern führen die simulierten Agenten vermehrt Berechnungen durch, die durch das Vorhandensein anderer Agenten hervorgerufen werden (z.B. Überholmanöver). Bei hohen Verkehrsdichten entstehen Staus, die Umplanaktionen hervorrufen. Die Verkehrsdichte im Graphen hängt von der Anzahl an Verkehrsteilnehmern und der Gesamtlänge des Straßennetzes ab. Daher ist in der kleineren Stadt Erlensee ein steilerer Abfall der Simulationsgeschwindigkeit zu beobachten, als in der Stadt Hanau.

Zusätzlich werden die Interaktionen untersucht, die simulierte Autos im Experiment in der Stadt Erlensee mit anderen Verkehrsteilnehmern haben. Exemplarisch wurden folgende Ereignisse protokolliert:

Sicherheitsabstand gewahrt Wenn ein Auto einen Vordermann hat und auf diesen reagieren muss.

Prüfe Fahrradüberholung Wenn ein Auto ein Fahrrad überholen möchte und prüft, ob dies möglich ist.

Überhole Fahrrad Wenn ein Auto ein Fahrrad überholt.

Kann Kreuzung nicht Überqueren Wenn ein Auto eine Kreuzung nicht überqueren konnte. Dies tritt ein, wenn ein vorfahrtsberechtigter anderer Verkehrs- 


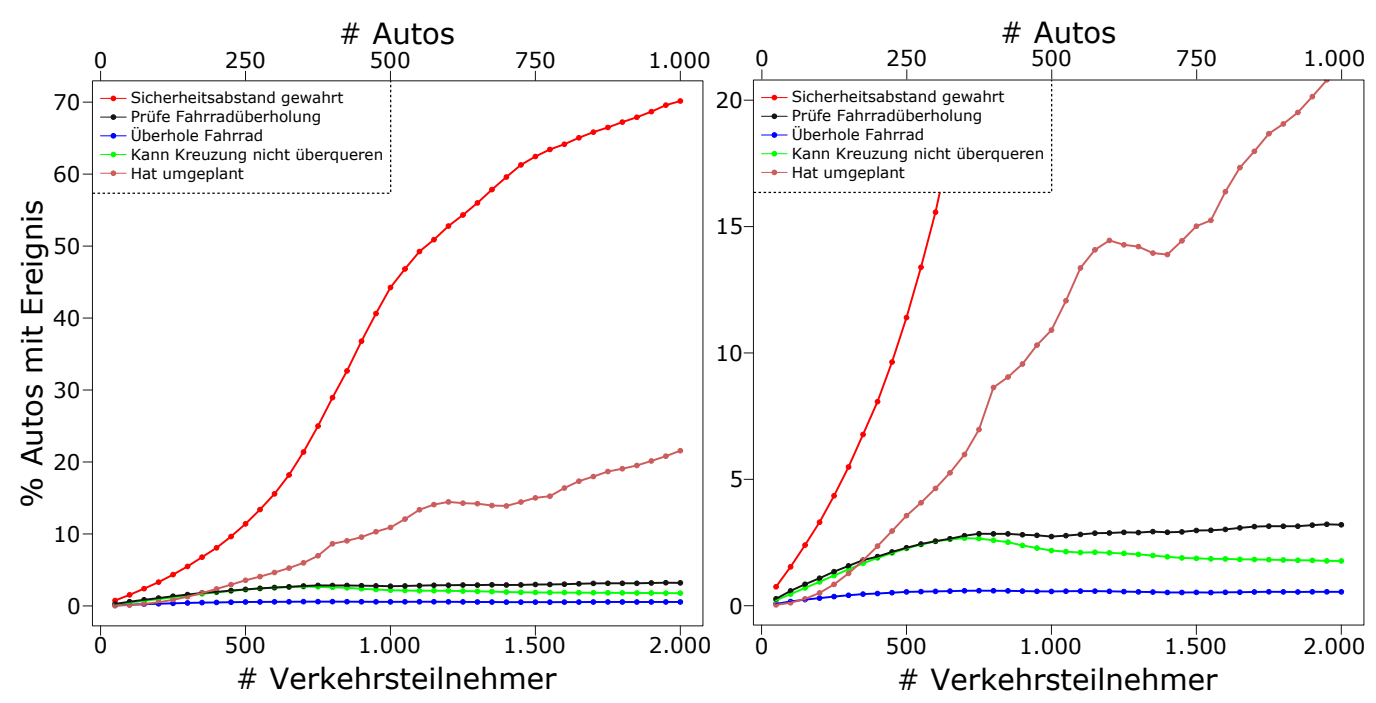

Abbildung 8.4: Menge an Interaktionen von Autos mit anderen Verkehrsteilnehmern.

teilnehmer, eine rote Ampel oder ein Fußgänger auf einem Überweg die Überquerung einer Kreuzung verhinderte.

Hat umgeplant Wenn ein Auto seine Route verändert hat, um Behinderungen zu umgehen. Dies können geänderte Ankunftszeitschätzungen auf der aktuellen Route oder ein Stau an einer Kreuzung sein, der die Wahl einer anderen Abbiegerichtung hervorrief.

Der Simulationsablauf ist identisch zum vorherigen Experiment. Die Verläufe in Abbildung 8.4 sind prozentual nach dem Anteil der Autos skaliert, die bei der jeweiligen Verkehrsmenge die aufgelisteten Ereignisse durchschnittlich pro Iteration hervorriefen. Jeder Messwert ist ein Mittelwert aus 100 Simulationsläufen. Im rechten Teil der Abbildung wird ein Ausschnitt des linken Graphen gezeigt.

Es ist zu erkennen, dass bei 2.000 Verkehrsteilnehmern ca. $70 \%$ der Autos einen Vordermann auf ihrer Straße haben, zu dem sie einen Sicherheitsabstand wahren müssen. Die Anzahl an Umplanmanöver, bei denen Autos neue Routen zu ihren Zielpositionen berechnen, nimmt ebenfalls stark zu. Die Verläufe der weiteren drei protokollierten Ereignisse lassen sich im vergrößerten Ausschnitt der Ergebnisse analysieren.

Die Anteile an Autos, die Überholmanöver an Fahrrädern überprüfen stagniert bei ca. 3,15\%. Tatsächlich überholen durchschnittlich jedoch nur ca. 0,55 \% der Autos pro Iteration. Diese starke Diskrepanz deutet darauf hin, dass Überholmanöver aufgrund geringer Straßenbreiten und vorhandenem Gegenverkehr oftmals nicht 


\begin{tabular}{|l|ccc|}
\hline Stadt & \# NIs & \# EIs & Gesamtlänge [km] \\
\hline Erlensee & 747 & 970 & 142 \\
Hanau & 4.300 & 5.844 & 548 \\
Koblenz & 8.098 & 10.547 & 717 \\
Frankfurt und Umgebung & 52.852 & 66.205 & 6.316 \\
\hline
\end{tabular}

Tabelle 8.1: Kenngrößen der Simulationsgraphen für Skalierungstests. Variierende Mengen an NIs und EIs im Vergleich zu anderen Angaben in dieser Arbeit resultieren aus dem Stand des Kartenmaterials und des Verfahrens der Grapherstellung.

möglich sind. Die Menge an Kreuzungsüberquerungen, die nicht möglich waren, steigt zunächst durch Zunahme an Verkehr. Bei hohen Verkehrsdichten geht diese Kenngröße jedoch zurück, was auf Staus hindeutet.

Abbildung 8.4 zeigt exemplarisch mit fixer prozentualer Verkehrsmittelwahl anhand von Simulationsergebnissen in einem Graphen der Stadt Erlensee, dass eine hohe Verkehrsdichte zu vermehrten Wechselwirkungen zwischen simulierten Verkehrsteilnehmern führt. Hieraus resultieren zusätzliche Berechnungen. Das aufwendigste betrachtete Verfahren ist hierbei die Möglichkeit des Umplanens. Die gezeigten Ergebnisse decken sich mit den Beobachtungen aus Abbildung 8.3.

Der grundlegende Verlauf beider Graphen in Abbildung 8.3 ist vergleichbar. Der größere Simulationsgraph der Stadt Hanau führt jedoch zu geringeren Simulationsgeschwindigkeiten. In einem weiteren Experiment wird daher der Einfluss der Kartenausschnittsgröße anhand der soeben verwendeten Graphen der Städte Erlensee, Hanau, sowie Koblenz ${ }^{1}$ und der Umgebung Frankfurt am Mains (vgl. Abbildung 15.1 auf Seite 224 untersucht. Tabelle 8.1 listet Kenngrößen der Simulationsgraphen auf.

Gegenläufige Kenngrößen sind hierbei die Anzahl an NIs und die Gesamtstraßenlänge. Je mehr NIs im Graphen enthalten sind, desto aufwendiger wird eine Routenbestimmung. Je mehr Gesamtkilometer das modellierte Straßennetz umfasst, desto weniger Wechselwirkungen zwischen den simulierten Verkehrsteilnehmern treten auf. Im Experiment wird die Anzahl an Verkehrsteilnehmern konstant auf 1.000 gehalten. Sobald ein Verkehrsteilnehmer sein Ziel erreicht hat, wird ein neuer erstellt. Zur Mittelung der Ergebnisse wurden ebenfalls 100 Simulationsläufe durchgeführt. Nach einer Einschwingphase von 5.000 Iterationen beginnt eine Messphase mit einer Länge von 20.000 Iterationen. Abbildung 8.5 zeigt den Vergleich der gemessenen Simulationsgeschwindigkeiten.

Es ist zu erkennen, dass die Simulationsgeschwindigkeit bei konstanter Anzahl

\footnotetext{
${ }^{1}$ Grenzen in WGS84: [6.49: 8.44, 49.24 : 50.78]
} 


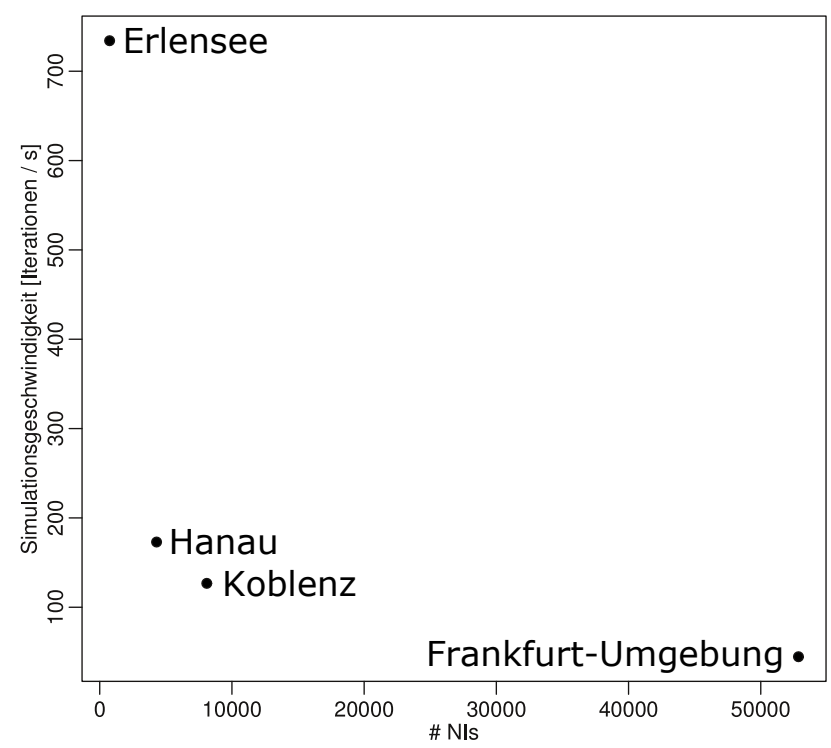

Abbildung 8.5: Simulationsgeschwindigkeitsskalierung bei variierenden Graphgrößen.

an Verkehrsteilnehmern durch Vergrößerung des Simulationsgraphen sinkt. Dies war zu erwarten und ist vorwiegend auf den vermehrten Aufwand bei der Routenbestimmung zurückzuführen. Bei Simulationen mit realistischen Quelle-ZielInformationen ist zu erwarten, dass dieser Effekt geringer ausfällt, da nahe beieinanderliegende Areale größere Verkehrsnachfragen erzeugen.

In Simulationsstudien beeinflussen weitere Faktoren - z.B. Messmodule oder die Anzahl an Ampeln - zusätzlich die Simulationsgeschwindigkeit. 


\section{Evaluierung der Verkehrsmodelle}

Dieser Abschnitt evaluiert die in Kapitel 7 entwickelten Modelle zur Simulation multimodalen Verkehrs. In Abschnitt 9.1 wird eine kurze Einführung in die Modellkalibrierung gegeben. Abschnitt 9.2 zeigt die Plausibilität des Automodells. In Abschnitt 9.3 wird das Fahrradmodell diskutiert. Abschnitt 9.4 evaluiert das Fußgängermodell. Abschließend gibt Abschnitt 9.5 eine kurze Zusammenfassung.

\subsection{Modellkalibrierung}

Um ein Simulationsmodell als Vorhersagewerkzeug nutzen zu können, muss es auf die Gegebenheiten der zu simulierenden Umgebung und Zeit (Tageszeit, Wochentag) kalibriert werden. Die Modellkalibrierung geschieht auf unterschiedlichen Ebenen (Tabelle 9.1).

Es können verschiedene Messwerte der Simulation zur Kalibrierung genutzt werden. Die folgende Auflistung gibt einen groben Überblick. In einer Studie kann jeder einzelne Punkt verfeinert und weiter differenziert werden. Die Varianzen aller Durchschnittswerte können ebenfalls von Bedeutung sein.

- Durchschnittliche Geschwindigkeit auf Straßenabschnitt 


\begin{tabular}{|c|c|c|c|}
\hline Ebene & Parameter & Datenquelle & Anpassungen \\
\hline \multirow[t]{5}{*}{ Straßengraph } & Straßengeometrie & OSM & - \\
\hline & Straßentypen & OSM & - \\
\hline & $v_{\max }$ & OSM & $\begin{array}{l}\text { Ergänzungen } \\
\text { Korrekturen }\end{array}$ \\
\hline & Spuren & OSM & $\begin{array}{l}\text { Ergänzungen } \\
\text { Korrekturen }\end{array}$ \\
\hline & Ampeln & OSM & Signalpläne \\
\hline $\begin{array}{l}\text { Simulations- } \\
\text { modell }\end{array}$ & Fluss-Dichte-Relation & Verkehrszählung & Kalibrierung \\
\hline \multirow[t]{3}{*}{ Routing } & $\begin{array}{l}\text { Abbiegewahrschein- } \\
\text { lichkeiten }\end{array}$ & Verkehrszählung & - \\
\hline & Flussdaten & Verkehrszählung & Kalibrierung \\
\hline & $\begin{array}{l}\text { Quelle-Ziel- } \\
\text { Informationen }\end{array}$ & Verkehrszählung & - \\
\hline
\end{tabular}

Tabelle 9.1: Modellkalibrierungsebenen

- Durchschnittliche Reisezeit zwischen zwei Punkten

- Verkehrsfluss an Messpunkt

- Verkehrsdichte auf Streckenabschnitt

- Durchschnittlicher Benzinverbrauch und Schadstoffausstoß

- Unfallanzahl

Bei Kalibrierungsprozessen im Verkehrssimulationsbereich tritt das Problem auf, dass wenige Messdaten vorhanden sind, die zur Kalibrierung genutzt werden können. Expertenwissen wird benötigt, um zu entscheiden, welche Abweichung zwischen Messdaten und Simulationsergebnissen als kalibriert akzeptiert werden Hellinga, 1998. Die Kalibrierung des Modells geschieht Ebene für Ebene. Nach jedem Kalibrierungsschritt werden die Simulationsergebnisse mit Messdaten verglichen und entschieden, ob weitere Anpassungen am Modell vorgenommen werden müssen. Im Folgenden werden die verschiedenen Kalibrierungsebenen betrachtet.

Die Ebene des Straßengraphen wird im vorgestellten Konzept nahezu vollautomatisch kalibriert. Es werden Straßenkarten von OSM genutzt und analysiert. Ergänzungen und Korrekturen sind nur dann notwendig, wenn die Daten von OSM unvollständig oder fehlerhaft sind. Oftmals fehlen beispielsweise die Positionen von Ampeln und Zebrastreifen und Geschwindigkeitsangaben sind nicht vorhanden.

Die Kalibrierung des Simulationsmodells muss auf das Fahrverhalten der Verkehrsteilnehmer im Bereich des Straßengraphen geschehen. 
Eine wesentliche Ebene der Kalibrierung ist die Routenbestimmung. Eine einfache Methode ist, morgens Wohngebiete als Start- und Industriegebiete als Zielareale zu deklarieren. Es würde ein einfacher Berufsverkehr entstehen. Diese Methode ist jedoch zu einfach, da nicht verzeichnet wird, auf welche Industriegebiete sich die simulierten Verkehrsteilnehmer eines Wohngebietes verteilen. Es helfen QuelleZiel-Matrizen $\left(Q Z M_{i j}\right)$, die jedem Quellareal $i$ für jedes Zielarreal $j$ die Anzahl der Verkehrsteilnehmer zuweisen, die sich von $i$ nach $j$ bewegen. Die Bestimmung der $Q Z M$ kann durch Bevölkerungsbefragungen, Straßenverkehrszählungen, Mikrozensusdaten oder Aggregierung aus Detektordaten geschehen. Tagesabläufe können über $Q Z M_{i j}^{t}$ simuliert werden, die die Anzahl der Verkehrsteilnehmer verzeichnen, die sich zum Zeitpunkt $t$ von $i$ nach $j$ bewegen. Hierbei werden aggregierte Daten über Zeitabschnitte verwendet. Teilweise wird als weiterer Parameter der Verkehrsteilnehmertyp hinzugezogen. Einen Überblick über die Aggregierung von $Q Z M$ geben Hellinga, 1994 und Steierwald et al., 2005.

Hierbei ist zu beachten, dass die Kalibrierung mittels QZM für ein konkretes Szenario notwendig ist, um realistische Verkehrsaufkommen zu erhalten. Um das Verkehrssimulationssystem MAINSIM an sich zu kalibrieren, müssen die entwickelten mikroskopischen Verkehrsmodelle untersucht werden. Das Automodell muss grundlegende Diagramme der Verkehrsforschung reproduzieren können. Das Fahrradmodell muss aus Mangel an Vergleichsdaten auf Plausibilität geprüft werden. Ein Vergleich von Messdaten des Fußgängermodells mit der betrachteten Literatur muss zeigen, ob das Modell die gewünschten Eigenschaften reproduzieren kann.

Nachdem das Simulationssystem kalibriert wurde, kann es genutzt werden, um die Wirkung von beeinflussenden Aktionen vorherzusagen oder Kenngrößen zu extrahieren, die nicht unmittelbar durch den Kalibrierungsprozess bestimmt werden. Es könnte beispielsweise versucht werden, den durchschnittlichen Benzinverbrauch zu senken und gleichzeitig den Verkehrsfluss zu erhöhen.

\subsection{Automodell}

Die Evaluierung des entwickelten mikroskopischen Automodells wird in die Abschnitte „Autobahn- und Landstraßenverkehr“ (Abschnitt 9.2.1), sowie „Innerstädtischer Verkehr" (Abschnitt 9.2.2 unterteilt. In den jeweiligen Abschnitten bestehen unterschiedliche Charakteristika, die einzeln untersucht werden müssen. In Abschnitt 9.2.3 wird eine kurze Vergleichsstudie zu verschiedenen Routingverfahren durchgeführt. 


\subsubsection{Autobahn- und Landstraßenverkehr}

Verkehrsmodelle für Autobahn- und Landstraßenszenarien werden überwiegend an ihren makroskopischen Kenngrößen gemessen. Dies resultiert aus der Tatsache, dass makroskopische Vergleichswerte von realen Messungen verfügbar sind und die meisten mikroskopischen Verkehrsmodelle nicht das Ziel haben, durchgehend realistisches Verhalten zu beschreiben, sondern in der Summe ein stimmiges Bild zu erzeugen.

Zur Evaluierung des im Rahmen dieser Arbeit entwickelten Automodells wird ein Graph verwendet, der aus zwei EIs mit Länge $25.000 \mathrm{~m}$ besteht, die mit zwei NIs verbunden sind. Der Graph modelliert einen Kreisverkehr mit $50 \mathrm{~km}$ Länge. Es entstehen somit periodische Randbedingungen. Dies entspricht dem Standardvorgehen zur Ermittlung anerkannter Kenngrößen. Eine $\mathrm{NI}_{f} \in$ NIs wird zur Ermittlung des Verkehrsflusses verwendet. Immer wenn ein Auto NI $f$ passiert, wird ein Zähler inkrementiert. Es werden unabhängige Simulationsläufe mit steigender Anzahl an Autos durchgeführt. Zu Beginn eines Simulationslaufes werden die zu simulierenden Autos auf zufällige Positionen des Simulationsgraphen platziert. Nach einer Einschwingphase von 1.000 Iterationen beginnen die Messungen der zur Auswertung benötigten Kenngrößen. Nach weiteren 10.000 Iterationen werden gemittelte Ergebnisse für diesen Simulationslauf abgespeichert und der nächste Simulationslauf wird gestartet.

Das identische Vorgehen wird zuerst für eine einspurige Straße und anschließend für eine zweispurige Straße durchgeführt.

\section{Einspuriger Verkehr}

In der Vergangenheit hat sich das Fundamentaldiagramm des Verkehrsflusses als wichtige Kenngröße für die Plausibilität eines Verkehrsmodells etabliert. Bereits in Greenberg, 1959 wird das Diagramm grundlegend beschrieben und einige Jahre später als Fundamentaldiagramm bezeichnet Haight, 1963, Pipes, 1967. Auf einer leeren einspurigen Straße wird die Verkehrsdichte immer weiter erhöht, bis die Straße vollkommen mit Autos belegt ist. Für jede Verkehrsdichte wird der Verkehrsfluss ermittelt. Abbildung 9.1 zeigt das Fundamentaldiagramm des im Rahmen dieser Arbeit entwickelten Modells.

Das gezeigte Diagramm deckt sich mit den in Feldstudien ermittelten Fluss-Dichte- 


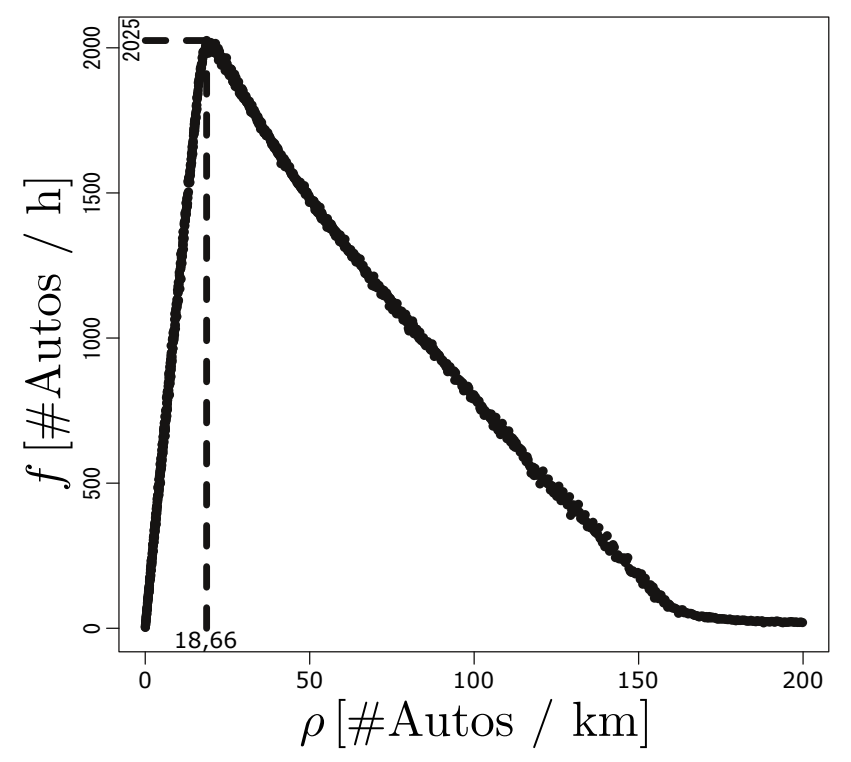

Abbildung 9.1: Fundamentaldiagramm für das entwickelte Simulationsmodell auf einer einspurigen Straße ohne Geschwindigkeitsbegrenzung. Zusammenhang zwischen Verkehrsdichte $\rho$ und Verkehrsfluss $f$.

Diagrammen 1 , Der Verkehrsfluss $f$ auf der Straße steigt bis zu einer Verkehrsdichte von $\rho \approx 18,66$ \#Autos / $\mathrm{km}$ an. Das Maximum von $f$ beschreibt die Verkehrsdichte, ab der die Autos auf der Straße beginnen, sich so stark gegenseitig zu behindern, dass $f$ zu sinken beginnt. Dieses Phänomen resultiert aus den einzuhaltenden Sicherheitsabständen und trödelnden Autos. Der Fluss $f$ sinkt bei steigender Dichte $\rho$ bis zum Nullpunkt, da sich bei vollständiger Auslastung einer Straße kein Auto mehr bewegen kann.

Abbildung 9.2 zeigt den Zusammenhang zwischen $\rho$ und der mittleren Geschwindigkeit $\bar{v}$, die die simulierten Autos fahren. Es ist wie in Abbildung 9.1 die Stelle markiert, an der $\rho \approx 18,66$ ist. Es zeigt sich, dass bei dieser Verkehrsdichte die Werte von $\bar{v}$ bereits geringer als das Maximum sind. Auf einer einspurigen Straße wird $\bar{v}$ durch die Geschwindigkeit des langsamsten Autos dominiert, da keine Überholmöglichkeiten bestehen. Die Werte von $\bar{v}$ sinken daher bereits bei geringen Verkehrsdichten auf Werte von $\bar{v} \approx 120 \mathrm{~km} / \mathrm{h}$.

In Abbildung 9.3 wird der Zusammenhang zwischen $f$ und $\bar{v}$ dargestellt. Die Verkehrsdichte $\rho$ wird nicht explizit aufgetragen, führt jedoch zu den Funktionswerten im Diagramm. Bei sehr niedrigem Verkehrsfluss können die wenigen Autos, die den Messpunkt für $f$ passieren mit großen $\bar{v}$ fahren. Bei steigendem $\rho$ sinkt $\bar{v}$ auf

${ }^{1}$ vgl. Emmerich und Rank, 1997, Nagel et al., 1998, Mahnke et al., 2008 uvm.; siehe auch Abbildung 2.1 auf Seite 15. 


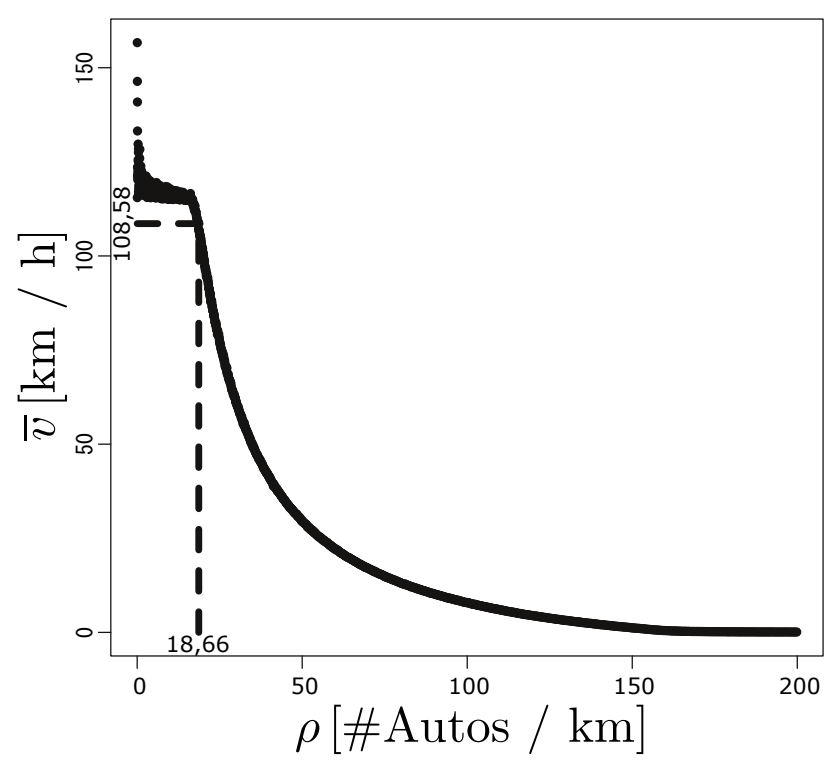

Abbildung 9.2: Zusammenhang zwischen Verkehrsdichte $\rho$ und mittlerer Geschwindigkeit $\bar{v}$ auf einer einspurigen Straße ohne Geschwindigkeitsbegrenzungen.

$\bar{v} \approx 120 \mathrm{~km} / \mathrm{h}$ und bricht beim maximalen $f$ ein. Der Zusammenhang zwischen sinkendem $\bar{v}$ und daraus resultierendem sinkendem $f$ ist ersichtlich.

Die gezeigten Abbildungen 9.1 bis 9.3 verdeutlichen, dass das entwickelte Automodell auf einer einspurigen Straße ohne Geschwindigkeitsbegrenzungen makroskopische Messgrößen korrekt abbilden kann. Ein mikroskopisches Verkehrsmodell muss zusätzlich einen Stau aus dem Nichts entstehen lassen können. Es handelt sich hierbei um ein gut untersuchtes Verkehrsphänomen Sugiyama et al., 2008. Wenn innerhalb einer Kolonne eine situationsabhängige Verkehrsdichte überschritten ist, kann eine kleine Störung genügen, um eine deutlich messbare Störung entstehen zu lassen. Es genügt beispielsweise eine Engstelle oder ein trödelndes Fahrzeug. Durch Schätzfehler der hinterherfahrenden Fahrzeuge, in diesem Fall übermäßiges Bremsen, schaukelt sich die Störung immer weiter hoch. Dies bedeutet auch, dass der Stau nicht für das Fahrzeug spürbar ist, das den Stau ausgelöst hat. Der Stau aus dem Nichts konnte erstmals durch das in Abschnitt 3.1 .2 beschriebene NSM reproduziert werden und entsteht auch in dem in dieser Arbeit entwickelten Modell.

Zur Veranschaulichung dient das in Abbildung 9.4 gezeigte Raum-Zeit-Diagramm. Jeder Punkt im Diagramm zeigt zu einem Zeitpunkt die Position eines Fahrzeuges auf der Straße. Von oben nach unten gelesen, fahren die Fahrzeuge von links nach rechts. Es sind deutlich Stauwellen erkennbar. Auf der Straße existierten keine 


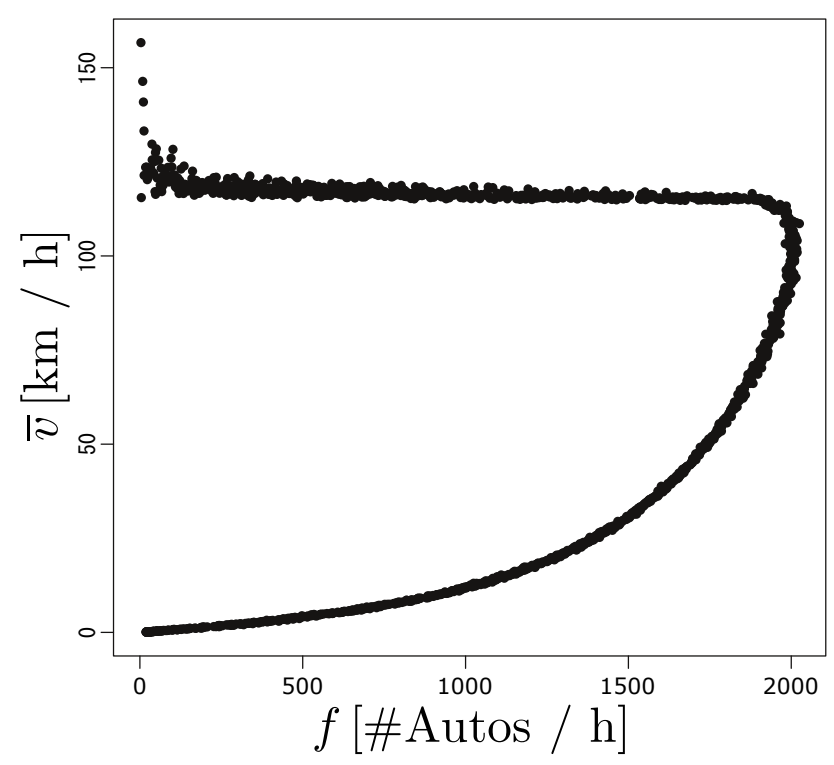

Abbildung 9.3: Zusammenhang zwischen Verkehrsfluss $f$ und mittlerer Geschwindigkeit $\bar{v}$ auf einer einspurigen Straße ohne Geschwindigkeitsbegrenzungen.

Hindernisse. Die Störungen entstanden durch Trödeln. Stauwellen breiten sich entgegen der Fahrtrichtung aus.

\section{Mehrspuriger Verkehr}

Ohne Beschränkung der Allgemeinheit wird mehrpsuriger Autobahnverkehr exemplarisch an zweispurigem Autobahnverkehr evaluiert.

Abbildung 9.5 zeigt das Fundamentaldiagramm für zweispurigen Verkehr. Der maximale Verkehrsfluss hat sich gegenüber Abbildung 9.1 ca. verdoppelt. Der grundlegende Verlauf der Funktion ist identisch zur einspurigen Variante. Dies deckt sich mit in der Literatur gezeigten Diagrammen (vgl. [Nagel et al., 1998]).

Abbildung 9.6 visualisiert die Zusammenhänge zwischen $\rho$ und $\bar{v}$, sowie $f$ und $\bar{v}$. Es zeigt sich, dass gegenüber den Abbildungen 9.2 und 9.3 in Bereichen niedriger $\rho$ höhere $\bar{v}$ erreicht werden, da langsame Autos nun überholt werden können. Dieser Trend setzt sich über den gesamten Dichteverlauf fort.

Abbildung 9.7 zeigt einen Ausschnitt mittlerer Verkehrsdichte, bei der die rechte Spur ein höheres Verkehrsaufkommen und stärkere Störungen in Form von Stauwellen verzeichnet. 


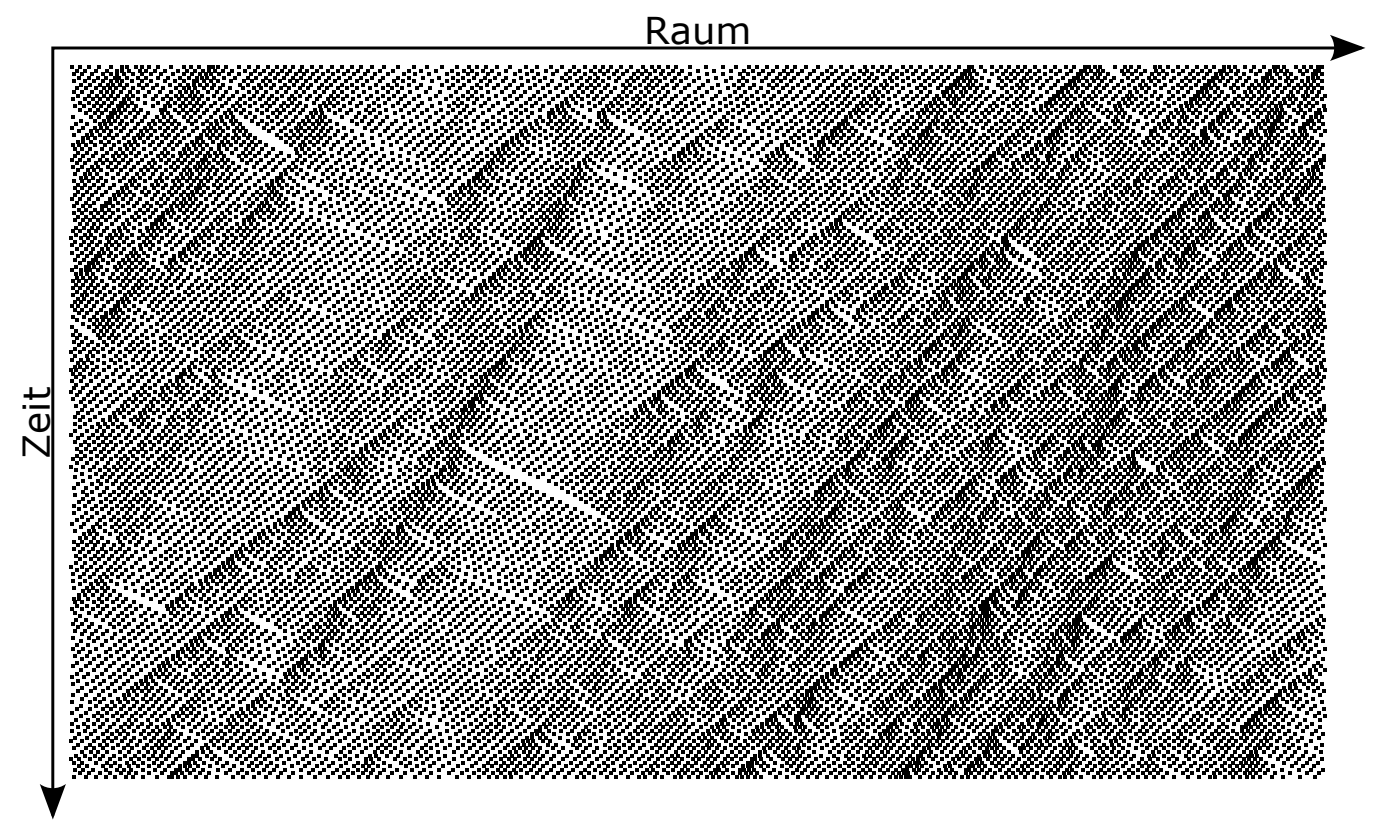

Abbildung 9.4: Raum-Zeit-Diagramm einspurig

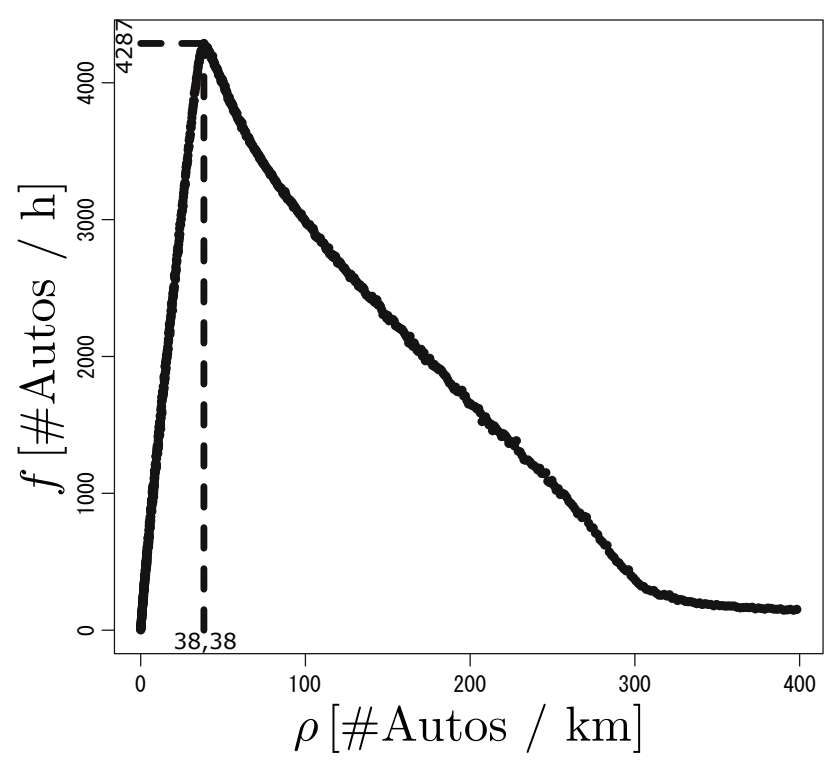

Abbildung 9.5: Fundamentaldiagramm für das entwickelte Simulationsmodell auf einer zweispurigen Straße ohne Geschwindigkeitsbegrenzung. Zusammenhang zwischen Verkehrsdichte $\rho$ und Verkehrsfluss $f$. 

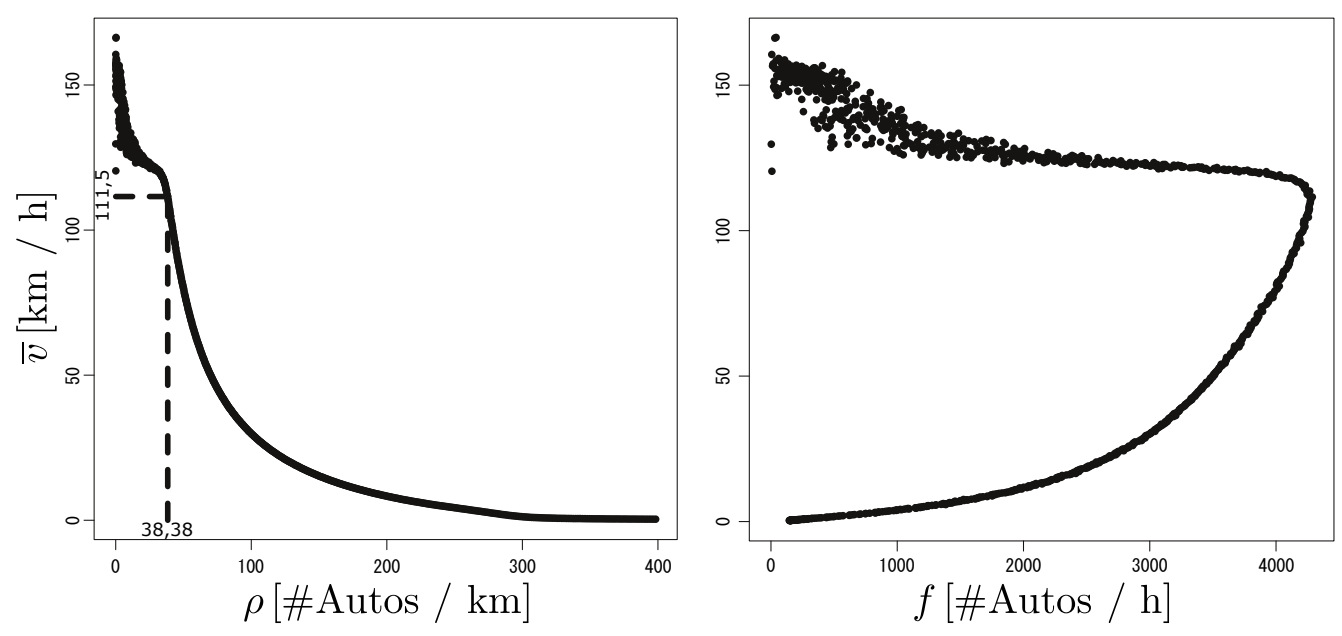

Abbildung 9.6: Zusammenhang zwischen Dichte $\rho$ und mittlerer Geschwindigkeit $\bar{v}$, sowie Fluss $f$ und $\bar{v}$ auf einer zweispurigen Straße.

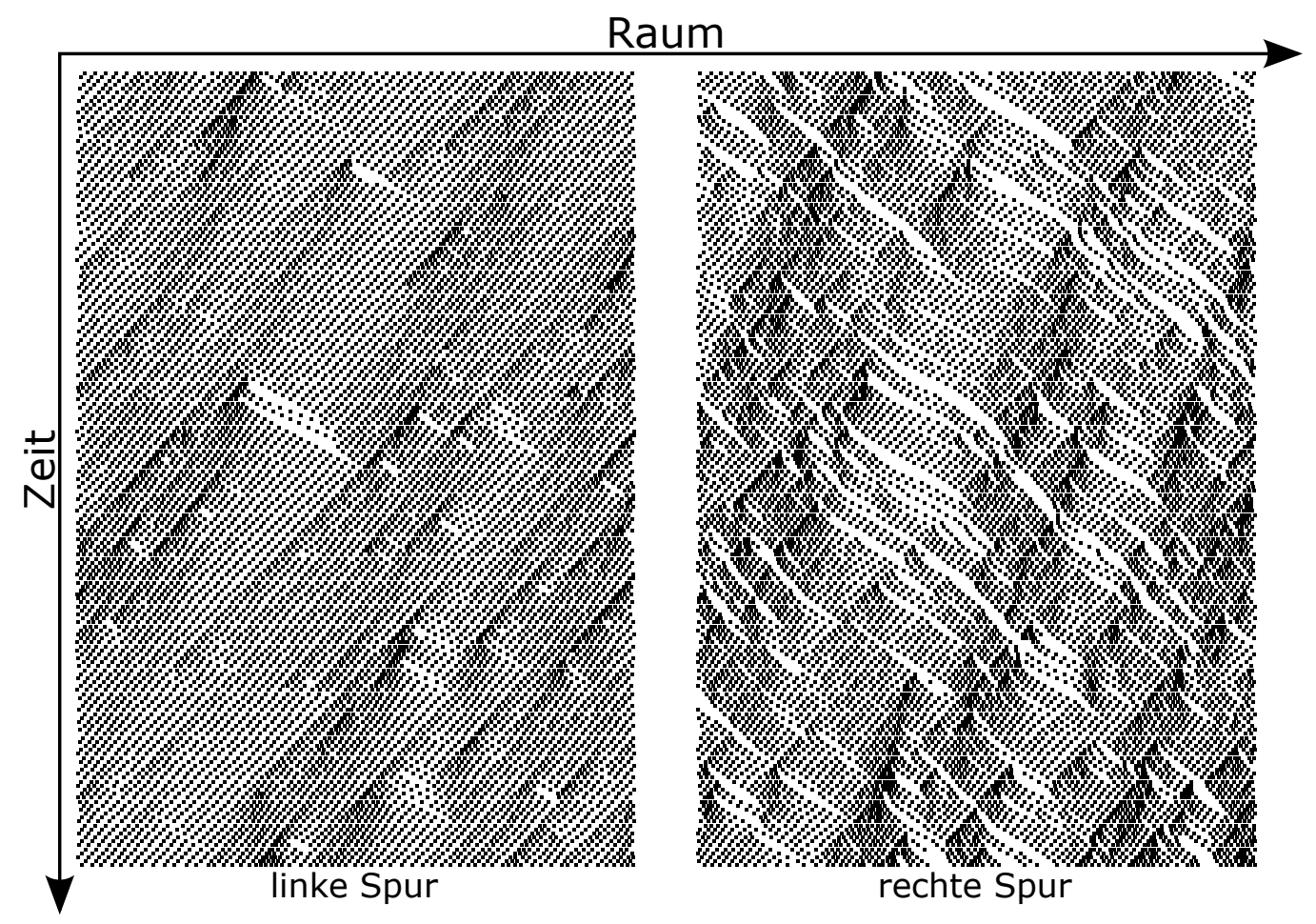

Abbildung 9.7: Raum-Zeit-Diagramm zweispurig 

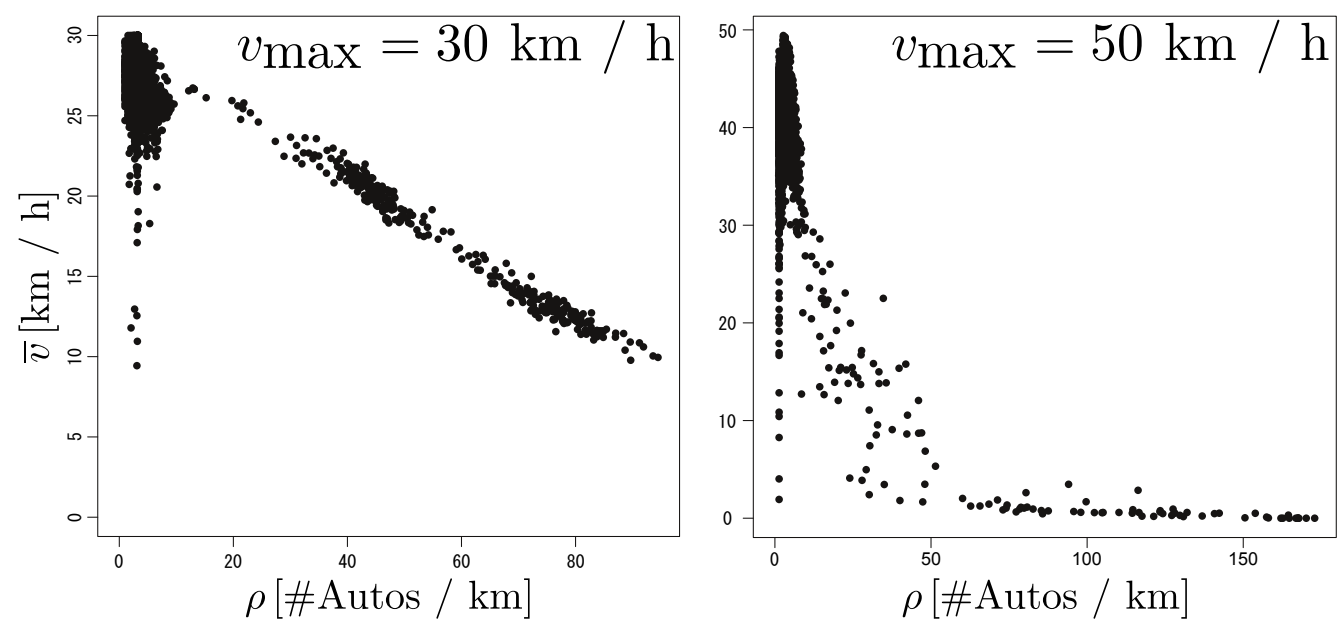

Abbildung 9.8: Zusammenhang zwischen Dichte $\rho$ und mittlerer Geschwindigkeit $\bar{v}$ für urbane Szenarien unter Betrachtung von EIs mit typischen Werten für $v$ max.

\subsubsection{Innerstädtischer Verkehr}

Die fundamentalen Zusammenhänge zwischen $\rho$ und $f$ bzw. $\bar{v}$ gelten auch im urbanen Bereich. Sie sind jedoch weniger gut untersucht, da Messungen in diesem Zusammenhang komplexer ausfallen, als auf Autobahnen und Landstraßen. In jüngerer Zeit wurden Untersuchungen in San Francisco und Yokohama durchgeführt, aus deren Ergebnissen Diagramme analog zu den im vorherigen Abschnitt vorgestellten Diagrammen für $\rho$ und $\bar{v}$ ermittelt werden konnten Daganzo und Geroliminis, 2008.

Abbildung 9.8 zeigt $\rho$ - $\bar{v}$-Diagramme für Straßen mit Maximalgeschwindigkeit $v_{\max }=30 \mathrm{~km} / \mathrm{h}$, bzw. $v_{\max }=50 \mathrm{~km} / \mathrm{h}$. Hierfür wurde der in Abbildung 9.9 gezeigte OSM-Ausschnitt der Stadt Hanau ${ }^{2}$ verwendet.

Die gezeigten Ergebnisse wurden über jeweils 100 Simulationsläufe mit jeweils 5 Messintervallen à 2.000 Iterationen für je 15 betrachtete Straßen ermittelt. Die durchschnittlichen Geschwindigkeiten der Autos auf den jeweiligen Straßen und die dazugehörigen Verkehrsdichten wurden über die Messintervalle gemittelt.

Um repräsentative Ergebnisse erzielen zu können, wurden zur Messung Straßen verwendet, die die für das jeweilige Diagramm vorgesehene Maximalgeschwindigkeit vorgeben. Es wurden ausschließlich Straßen mit einer Länge über $300 \mathrm{~m}$ betrachtet, die für Autos freigegeben sind. Die Auswahl von 15 Straßen aus der

\footnotetext{
${ }^{2}$ Grenzen in WGS84: [8.71: 9.15, 49.97 : 50.19]
} 


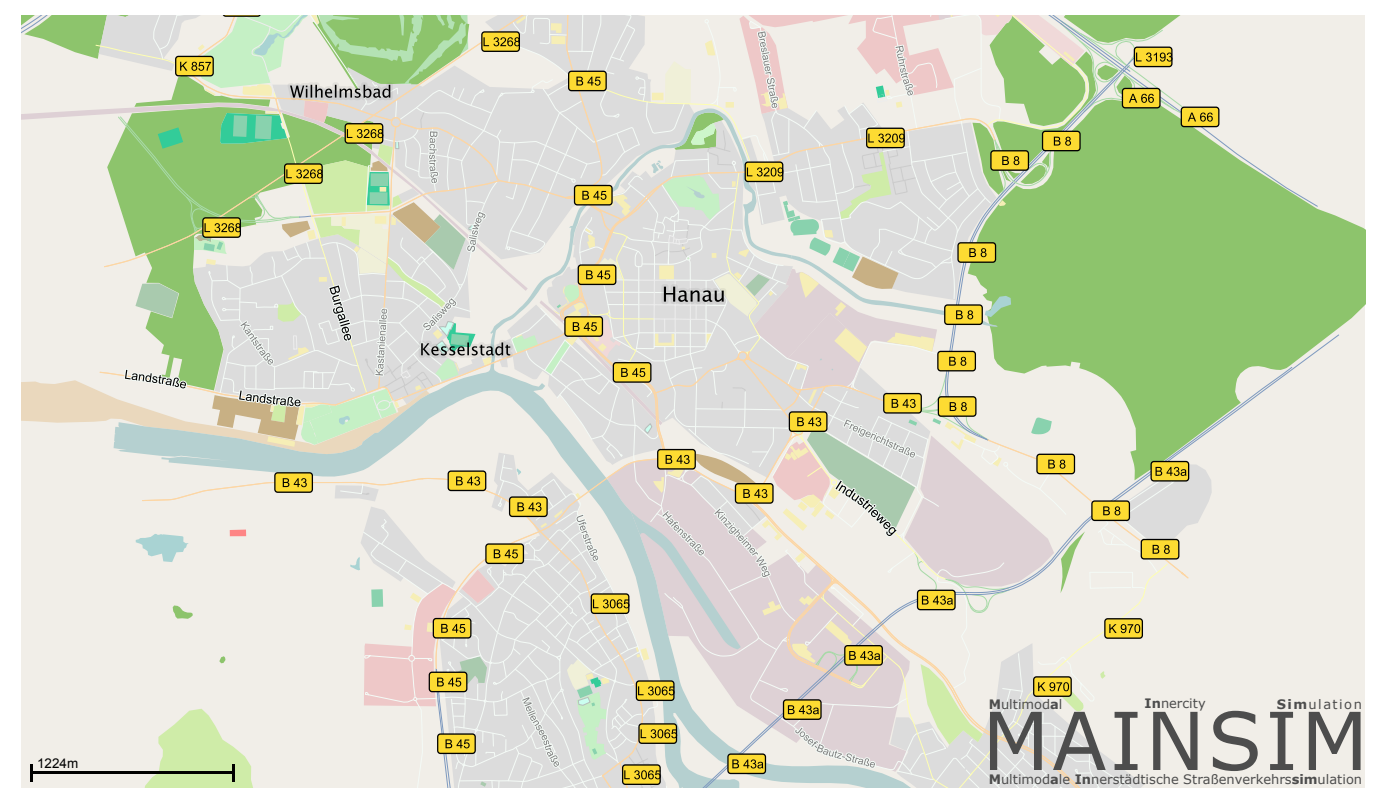

Abbildung 9.9: Visualisierung des Kartenausschnitts der Stadt Hanau. Gesamtlänge der Straßen: 548 km, Anzahl EIs: 5400, Anzahl NIs: 3878

Gruppe mit den beschriebenen Eigenschaften geschah für jeden Simulationslauf zufällig. Somit wird ein Querschnitt über die in der Stadt vorhanden Straßen betrachtet, da die Werte der makroskopischen Kenngrößen für unterschiedliche Straßen in urbanen Szenarien variieren Geroliminis und Sun, 2011. Dies resultiert aus der weitaus größeren Menge an Einflussfaktoren auf urbanen Verkehr im Vergleich zu außerstädtischem Verkehr.

Die ermittelten Diagramme decken sich in ihrem Verlauf mit den in der Literatur gezeigten Geroliminis, 2008, Helbing, 2009. Dieses Ergebnis zeigt, dass das entwickelte Modell grundlegend funktioniert. Das Fundamentaldiagramm für urbanen Verkehr wird in der Realität durch parkende Autos, Fahrräder, Fußgänger, Straßenbahnen und andere Faktoren beeinflusst. Es ist anzunehmen, dass es daher von Ort zu Ort variiert und lediglich als grobe Richtlinie bei der Evaluierung eines Verkehrsmodells zu sehen ist.

In einem weiteren Simulationslauf mit 1095 Verkehrsteilnehmern (50 \% Autos, $25 \%$ Fahrräder, 25 \% Fußgänger) wurde im selben Kartenausschnitt nach einer Einschwingphase von 10.000 Iterationen protokolliert, welche Beschleunigungen die simulierten $\mathrm{Pkw}$ erbrachten. Es wurden $10^{6}$ Beschleunigungen protokolliert. Abbildung 9.10 zeigt das Ergebnis.

Es ist $\mathrm{zu}$ sehen, dass starke Beschleunigungen die Ausnahme sind. In 


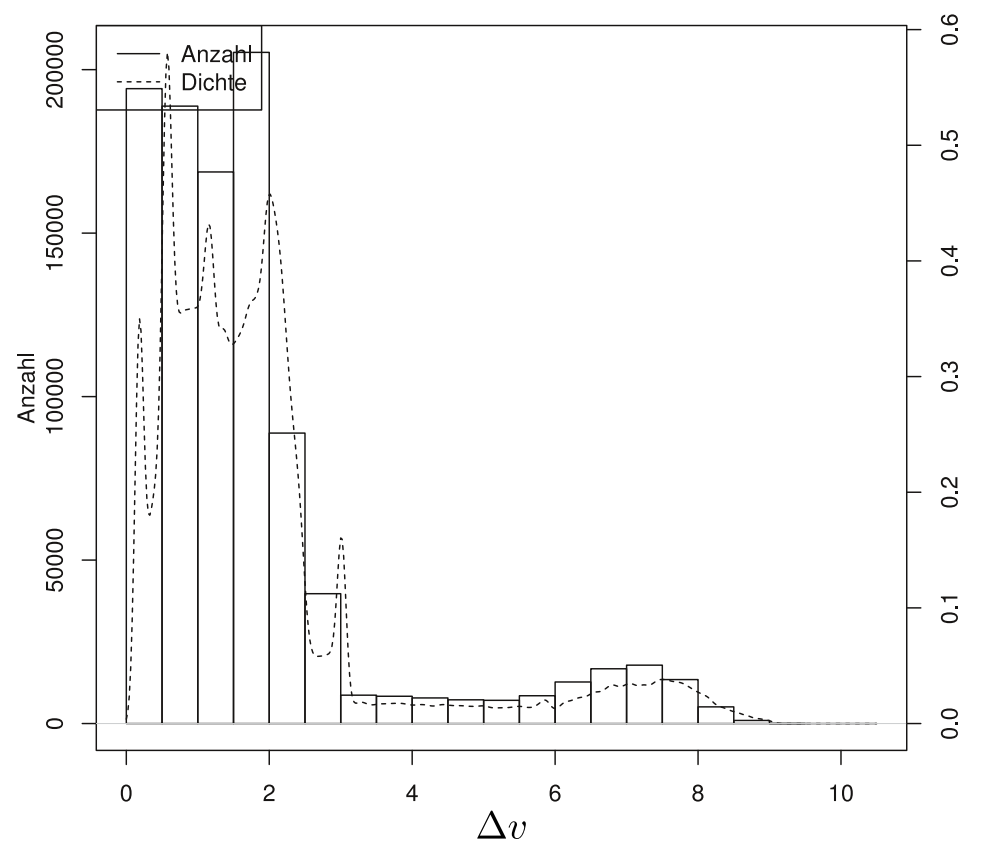

Abbildung 9.10: Beschleunigungsverhalten von Pkw. Angaben in $\mathrm{m} / \mathrm{s}^{2}$.

der Literatur werden verschiedene Beschleunigungsgrößen diskutiert. In Kesting und Treiber, 2008 wird das IDM auf reale Messdaten kalibriert. In mehreren Evaluationsläufen werden Beschleunigungswerte zwischen $a=1,00 \mathrm{~m} / \mathrm{s}^{2}$ und 1,52 m/s $\mathrm{s}^{2}$ ermittelt. Für urbane Szenarien wird in Wang und Liu, 2005 ein ZA-Modell mit konstant $a=1,00 \mathrm{~m} / \mathrm{s}^{2}$ betrieben. Das ursprüngliche NSM für $\mathrm{Au}-$ tobahnverkehr lässt Autos mit $a=7,50 \mathrm{~m} / \mathrm{s}^{2}$ beschleunigen Wahle et al., 2002 . Insgesamt erscheinen die Beschleunigungswerte aus Abbildung 9.10 plausibel.

\subsubsection{Vergleich von Routingverfahren}

Dieser Abschnitt untersucht in einem einfachen Experiment, wie sich der Verkehr im Straßengraphen verteilt, wenn unterschiedliche Routingverfahren eingesetzt werden. Es werden konstant 400 Autos simuliert. Start- und Zielpositionen werden zufällig bestimmt. Nach einer Einschwingphase von 10 Stunden folgt eine Messphase von 8 Stunden Simulationszeit. Jede EI des ExtendedGraph erhält einen Zähler, der mit 0 initialisiert wird. In der Messphase wird nach jeder Iteration für jedes simulierte Auto der Zähler der EI, auf der sich das Auto derzeit befindet, inkrementiert. Abschließend werden die Zählerstände in einem Kartenlayer visualisiert. 


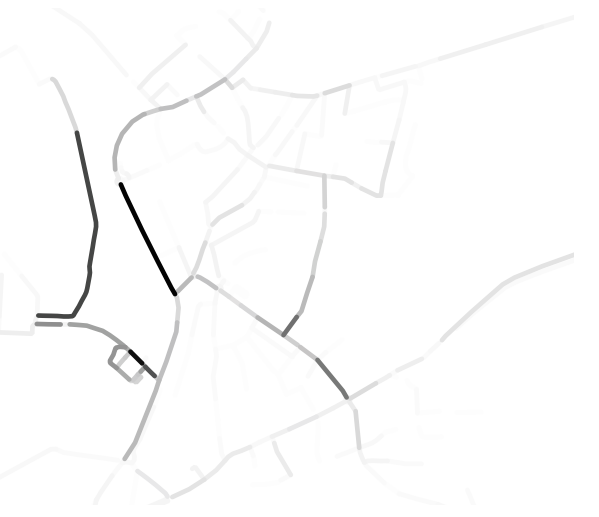

(a) Vorberechnete Routen

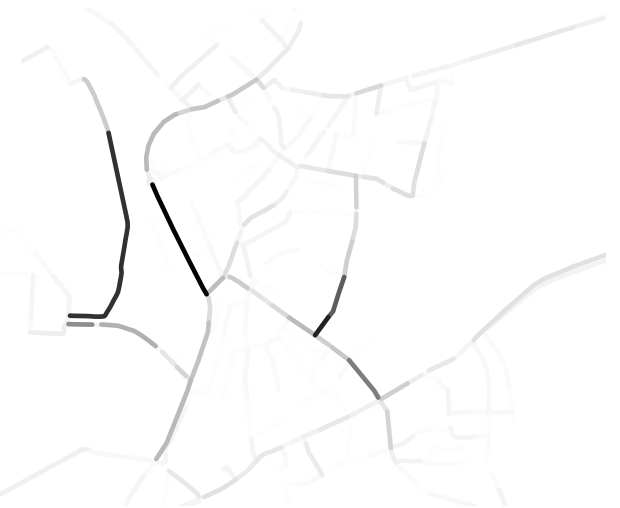

(c) $50 / 50$

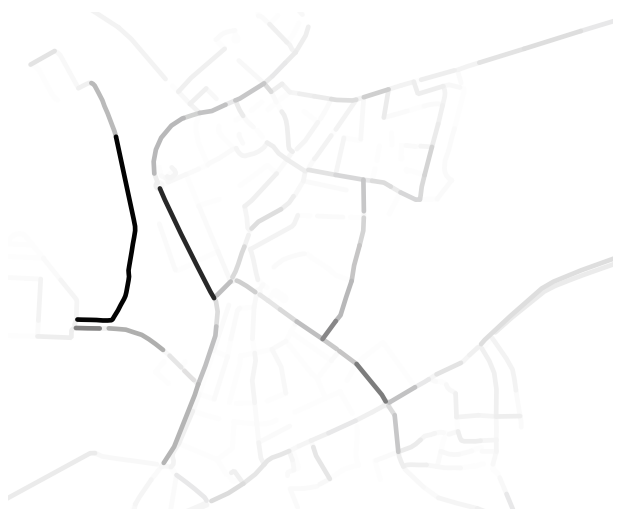

(b) Probabilistisch

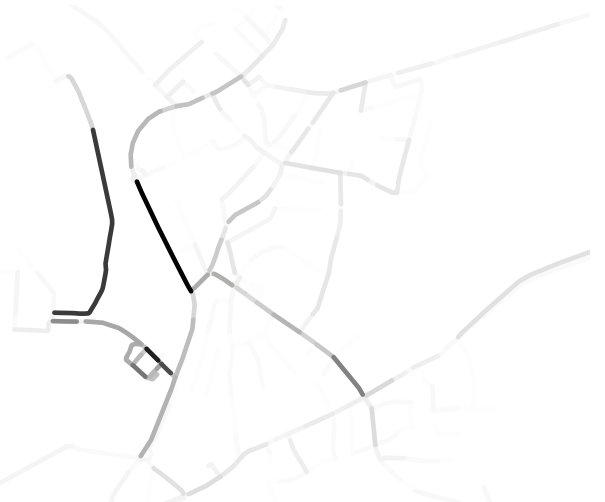

(d) Live

Abbildung 9.11: Vergleich der Straßennutzungshäufigkeiten bei unterschiedlichen Routingverfahren im Straßengraph der Stadt Erlensee. Verlauf der Kantennutzungshäufigkeit von sehr selten (weiß) bis sehr häufig (schwarz).

Abbildung 9.11 zeigt die Ergebnisse. Es werden die in Abschnitt 7.6 beschriebenen Routingverfahren der vorberechneten Routen (Teil (a)p, des probabilistischem Routings (Teil (b)], sowie einer Verteilung von $50 \%$ vorberechneter und $50 \%$ probabilistischer Routen (Teil (c) verglichen. Zusätzlich wird in Teil (d) das Routingverfahren mittels $\mathrm{A}^{*}$-Algorithmus unter Verwendung von Reisedauerinformationen der jeweiligen EIs dargestellt.

Aus Abbildung 9.11 kann nicht gelesen werden, welches der Verfahren am realistischsten ist. Ziel der Abbildung ist aufzuzeigen, wie sich der Verkehr verteilt. Zur Abbildung realen Verkehrs müssen live berechnete Routen verwendet werden, damit Änderungen in der Verkehrslage berücksichtigt werden können. Zur Untersuchung von Fragestellungen wie z.B. der Bestimmung des Einflusses von Fußgängern auf den Straßenverkehr, bieten sich jedoch auch die vorberechneten Routingverfahren an. 


\subsubsection{Zusammenfassung}

Es konnte gezeigt werden, dass das entwickelte Verkehrsmodell für Autoverkehr makroskopische Kenngrößen adäquat wiedergibt und einen Stau aus dem Nichts entstehen lassen kann. Auch in urbanen Szenarien konnten makroskopische Kenngrößen im Vergleich zu realen Messdaten realistische Werte reproduzieren.

Der Sicherheitsabstand wird in Algorithmus 7.2 auf 1,33 s gesetzt und entspricht somit nicht dem „halben Tachoabstand“. In Anhang B wird untersucht, wie sich ein größerer Sicherheitsabstand auf die gemessenen makroskopischen Kenngrößen auswirken würde.

Für unterschiedliche Straßenbeschaffenheiten, Witterungsbedingungen oder lokale Auto- bzw. Fahrereigenschaften sind Schwankungen im Verlauf der gezeigten Diagramme zu erwarten. Abbildung 9.1 gibt nahezu exakt die Messdaten von Hall et al., 1986 wieder (vgl. Abbildung 2.1 auf Seite 15. Entscheidend ist jedoch, dass der Verlauf der Funktion mit Referenzdiagrammen übereinstimmt. Dies gilt für alle in diesem Abschnitt diskutierten Diagramme.

Hieraus wird gefolgert, dass die grundlegenden Wirkungszusammenhänge zwischen den simulierten Fahrzeugen abgebildet werden können und somit das entwickelte Modell plausibel ist. Zur Simulation eines konkreten Szenarios müssen verschiedene Parameter - z.B. Sicherheitsabstand, Beschleunigung, $\perp$ und Lückenakzeptanz an Messdaten angepasst werden.

In weiteren Untersuchungen muss der Einfluss anderer Verkehrsteilnehmertypen auf den urbanen Verkehr untersucht werden. Der nächste Abschnitt 9.3 untersucht daher das im Rahmen dieser Arbeit entwickelte Fahrradmodell.

\subsection{Fahrrad}

Das Fahrradmodell wird in Unterabschnitt 9.3.1 auf die Fahrradgeschwindigkeiten untersucht. Unterabschnitt 9.3.2 analysiert den Einfluss des Fahrradverkehrs auf den Autoverkehr einer Stadt. 


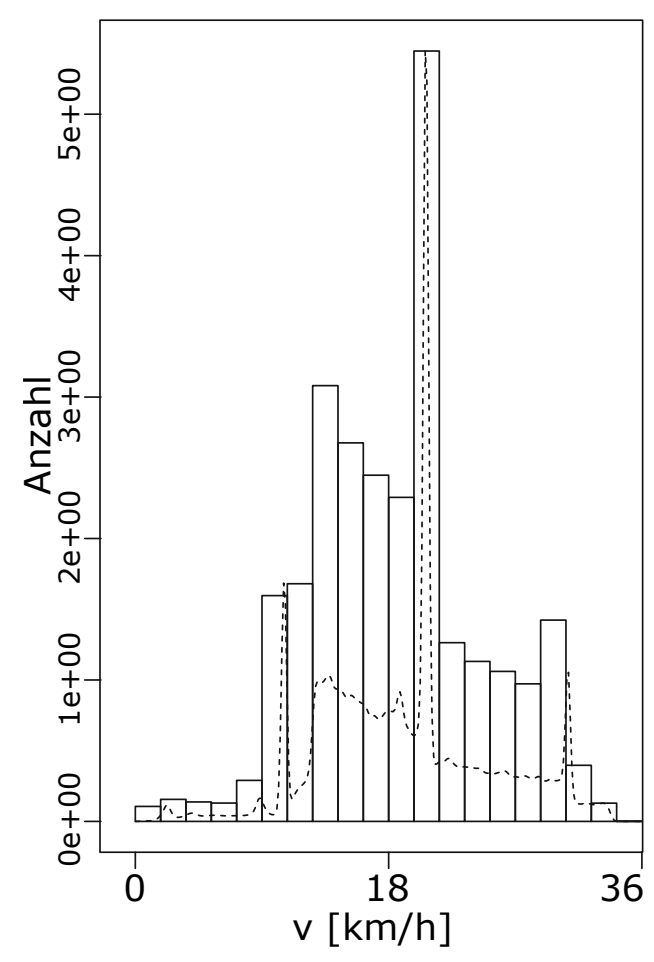

Abbildung 9.12: Fahrradgeschwindigkeiten

\subsubsection{Fahrradgeschwindigkeiten}

Zur Simulation wurde analog zur Analyse innerstädtischen Autoverkehrs der in Abbildung 9.9 auf Seite 149 gezeigte Kartenausschnitt der Stadt Hanau verwendet. Nach einer Einschwingdauer von 2.500 Iterationen begann eine Messdauer von 100.000 Iterationen unter Simulation von 1.500 Verkehrsteilnehmern mit der Zusammensetzung 50 \% Autos, $15 \%$ Fahrräder, $35 \%$ Fußgänger.

Während der Messphase wird in jedem Simulationsschritt von jedem fahrenden $(v>0 \mathrm{~km} / \mathrm{h})$ Fahrrad die Geschwindigkeit protokolliert. Die Verteilung der Geschwindigkeiten gibt Aufschluss über die Plausibilität des Fahrradverhaltens. Abbildung 9.12 zeigt die Ergebnisse.

Der Mittelwert der Geschwindigkeiten liegt bei $\approx 18,46 \mathrm{~km} / \mathrm{h}$, der Median bei $\approx 18,60 \mathrm{~km} / \mathrm{h}$. Es ist zu berücksichtigen, dass auch die geringen Geschwindigkeiten beim Anfahren oder Anhalten protokolliert wurden. Die maximalen Geschwindigkeiten liegen bei ca. $33 \mathrm{~km} / \mathrm{h}$. Diese Werte erscheinen realistisch und decken sich mit dem in Abschnitt 7.2 definierten Bereich. Die gefahrenen Geschwindigkeiten verteilen sich hauptsächlich um ca. $20 \mathrm{~km} / \mathrm{h}$ herum. 


\begin{tabular}{|l|ccccc|}
\hline \#Fahrräder & 0 & 1.000 & 2.000 & 3.000 & 4.000 \\
\hline mittlere Reisedauer [s] & 870,14 & 938,71 & 978,06 & $1.019,96$ & $1.048,37$ \\
\hline
\end{tabular}

Tabelle 9.2: Vergleich der mittleren Reisedauer von Autos in Relation zur Anzahl an Fahrrädern in der Simulation.

\subsubsection{Einfluss auf den Autoverkehr}

Dieser Unterabschnitt untersucht die These, dass Interaktionen mit Fahrädern zu längeren Reisedauern für Autos führen. Hierfür werden 2.000 Verkehrsteilnehmer (95\% Autos und $5 \%$ Fußgänger) auf dem in Abbildung 9.9 gezeigten Kartenausschnitt initialisiert. Nach einer Einschwingdauer von 1.000 Iterationen wird über 10.000 Iterationen die Reisedauer der Autos gemessen, die ihre Fahrt beendet haben. Während der Simulation werden von allen initialisierten Verkehrsteilnehmern Klone in eine Liste eingefügt, die jeder Iteration eine Menge an zu initialisierenden Autos und Fußgängern zuweist.

Anschließend wird die Simulation zurückgesetzt und erneut gestartet. In jeder Iteration werden die Klone der Verkehrsteilnehmer des vorherigen Simulationslaufs initialisiert. Zusätzlich werden Fahrräder initialisiert. Die Messung findet analog statt und berücksichtigt ausschließlich Autos, um eine Aussage über Veränderungen der Reisedauer der Autos treffen zu können. Die Anzahl der Fahrräder wird bei identischem Experimentaufbau zwischen 1.000, 2.000, 3.000 und 4.000 variiert. Durch den Aufbau des Experiments können die Resultate verschiedener Fahrräderdichten paarweise verglichen werden, da die Verteilung der Autos und Fußgänger identisch bleibt.

Das Vorgehen wird in 1.000 Durchläufen wiederholt. Das Resultat eines jeden Laufs ist die mittlere Reisedauer der Autos in der Simulation. Abbildung 9.13 zeigt die Verteilung der Ergebnisse.

Es ist ein klarer Trend zu steigenden Reisedauern von Autos bei Erhöhung der Anzahl an Fahrradfahrern im diskutierten Szenario erkennbar. Die Streuung der Ergebnisse nimmt hierbei zu. Tabelle 9.2 vergleicht die mittleren Reisedauern der Autos. Fahrräder haben somit in MAINSIM einen Einfluss auf den Straßenverkehr. 


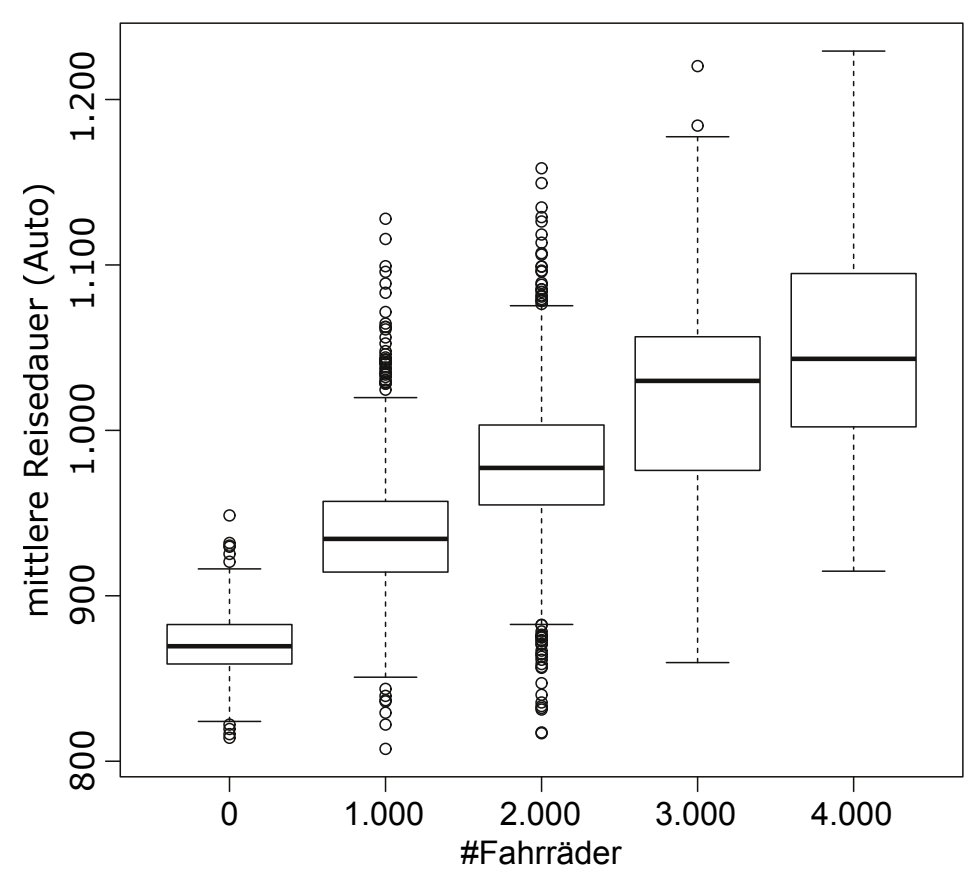

Abbildung 9.13: Fahrradeinfluss auf den Straßenverkehr

\subsection{Fußgänger}

Die Analyse des in Abschnitt 7.3 beschriebenen Modells zur Simulation von Fußgängerbewegungen wird dreigeteilt durchgeführt. Unterabschnitt 9.4 .1 bespricht in der Simulation auftretende Fußgängergeschwindigkeiten und Aggressivitätsfaktoren. Das Modell für Fußgängerbewegungen auf NIs wird in Unterabschnitt 9.4.2 behandelt. Die zwischen Fußgängern und Autos auftretenden Wechselwirkungen werden in Unterabschnitt 9.4 .3 analysiert.

\subsubsection{Laufgeschwindigkeiten und Aggresivität}

In diesem Unterabschnitt werden Fußgängerbewegungen im Hinblick auf Laufgeschwindigkeiten und $A F$ untersucht. Jeder Fußgänger erhält ein Analysemodul, welches gelaufene Geschwindigkeiten und weitere Kennzahlen protokolliert. Es muss geprüft werden, ob die simulierten Fußgänger in Relation zu ihrer Situation ähnliche Geschwindigkeiten, wie in Abschnitt 3.3 ermittelt, laufen.

Es wird zwischen den Geschwindigkeiten auf Bürgersteigen (EIs) und bei Straßenüberquerungen mit Vorfahrt (NIs) und ohne Vorfahrt (EIs und NIs) unter- 


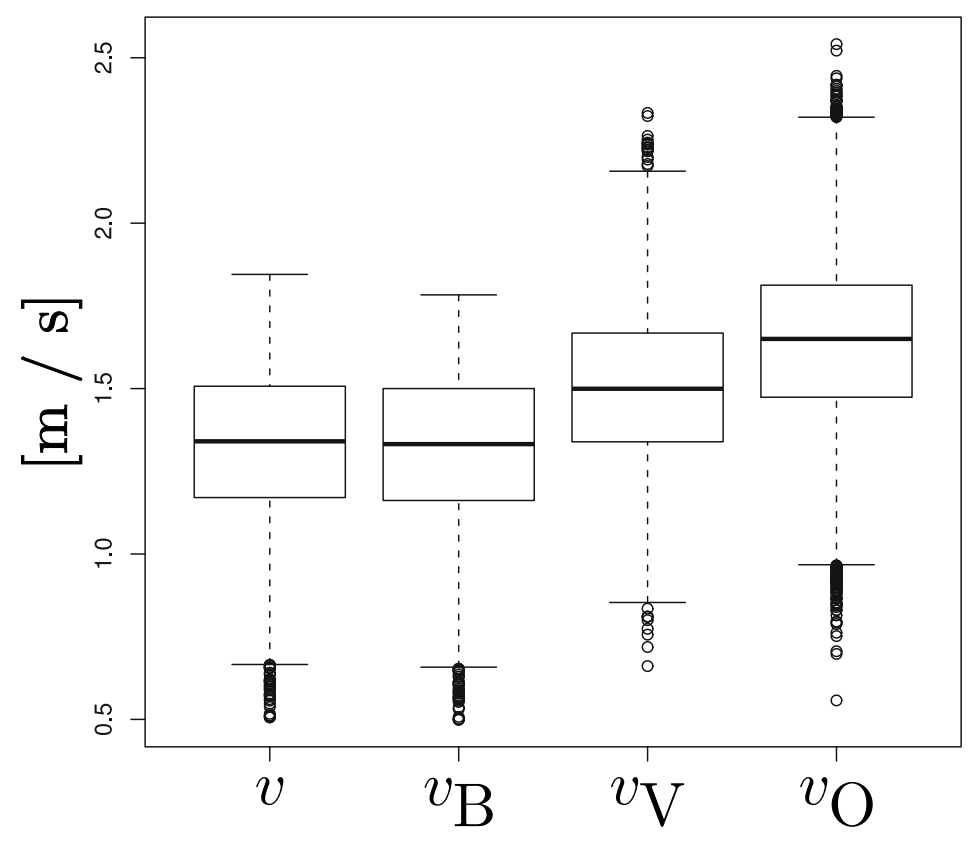

Abbildung 9.14: Verteilung der Fußgängergeschwindigkeiten in der Simulation

schieden. Die Aggressivitätsfaktoren $A F$ (vgl. Gleichung 7.12 auf Seite 118) sind essenziell, da sie belegen, inwiefern simulierte Fußgänger sich bei der Bestimmung der benötigten Lücke für eine Straßenüberquerung verschätzen.

Das Simulationsszenario ist identisch zur Bestimmung der Fahrradgeschwindigkeiten in Unterabschnitt 9.3.1. Die Messung weicht jedoch ab. Von jedem Fußgänger in der Simulation wurden die mittleren Geschwindigkeiten $v$ (insgesamt), $v_{\mathrm{B}}$ (Bürgersteig), $v_{\mathrm{V}}$ (Überquerung mit Vorfahrt) und $v_{\mathrm{O}}$ (Überquerung ohne Vorfahrt) protokolliert. Es entstanden dabei die in Abbildung 9.14 gezeigten Verteilungen.

Es ist ersichtlich, dass die Geschwindigkeiten sich mit dem Stand der Forschung decken (vgl. Abschnitt 3.3.1). Es ist hierbei zu beachten, dass die in der Literatur vorgeschlagenen Geschwindigkeitsverteilungen je nach Untersuchungsgebiet schwanken und die in Abbildung 9.14 gezeigten Verteilungen vorwiegend hinsichtlich der Unterschiede bezüglich $v, v_{\mathrm{B}}, v_{\mathrm{V}}$ und $v_{\mathrm{O}} \mathrm{zu}$ interpretieren sind und plausible Geschwindigkeiten darstellen. Die mittlere Geschwindigkeit $v$ liegt minimal oberhalb der Geschwindigkeit auf Bürgersteigen $v_{\mathrm{B}}$, da Fußgänger die meiste Zeit auf Bürgersteigen verbringen. Sie laufen schneller, wenn sie Straßen überqueren, insbesondere, wenn sie eine Straße ohne Vorfahrt überqueren $\left(v_{\mathrm{V}}\right.$ und $\left.v_{\mathrm{O}}\right)$.

Immer, wenn ein Fußgänger prüft, ob er eine Straße überqueren kann und hierfür seine geschätzte Überquerungsdauer $G D$ bestimmt, wird seine Aggressivität $A F$ 

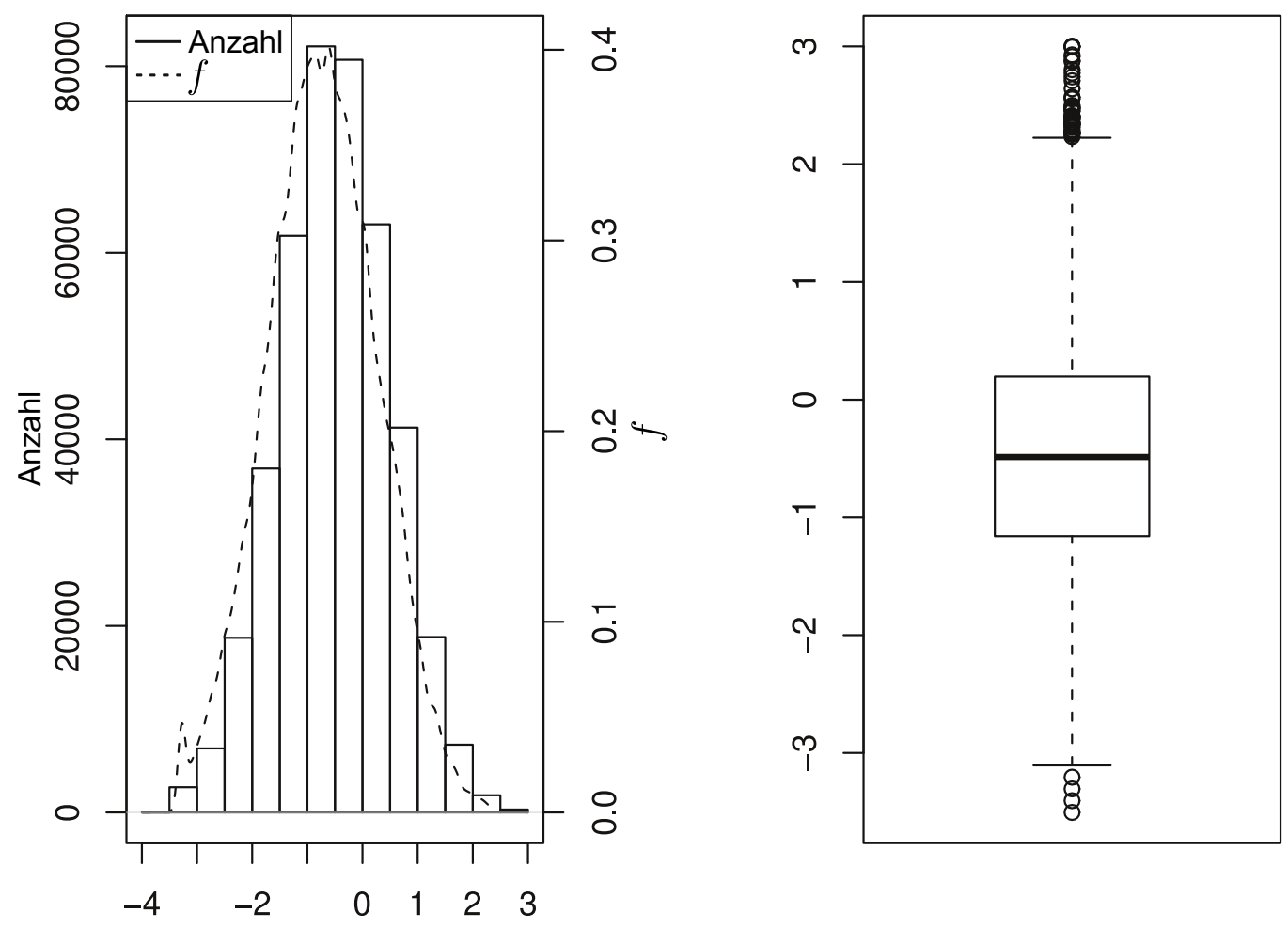

Abbildung 9.15: Verteilung der Aggressivität der Fußgänger

protokolliert. Abbildung 9.15 zeigt die Verteilung aller ermittelten Werte für $A F$.

Es ist ersichtlich, dass $A F$ sich im definierten Bereich (vgl. Abschnite 3.3.2 und 7.3.3 befindet. Ein Fußgänger mit $A F>0$ überschätzt seine Überquerungsdauer und ist somit passiv. Ein $A F<0$ bedeutet hingegen, dass ein Fußgänger die Straße auch bei ungenügend großer Lücke überquert. Der Wert von $A F$ sinkt mit der Wartedauer zur Überquerung einer Straße. Hierdurch können die Ausreißer im negativen Wertebereich von $A F$ erklärt werden. Der Mittelwert von $A F$ liegt bei $\approx-0,48$ und spiegelt somit den in Abschnitt 7.3 .3 auf Seite 118 definierten Bereich wider. Die Differenz von $\approx-0,02$ geht ebenfalls auf Ausreißer im negativen Bereich von $A F$ zurück.

Abbildung 9.16 zeigt den Verlauf des $A F$ exemplarisch anhand eines simulierten Fußgängers. Der Graph zeigt einen passiven Fußgänger, der bis zum Zeitpunkt a nicht auf Straßenüberquerungsmöglichkeiten warten musste. Bei b wurde $1 \mathrm{~s}$ gewartet und $A F$ nimmt mit einer Schrittweite von $0,1 \cdot \mathrm{s}^{-1}$ ab, bis zum Zeitpunkt $\mathrm{d}$ eine ausreichende Lücke vorhanden ist und der Fußgänger die Straße überquert. Sein $A F$ gleicht sich wieder seinem Standardwert an. 


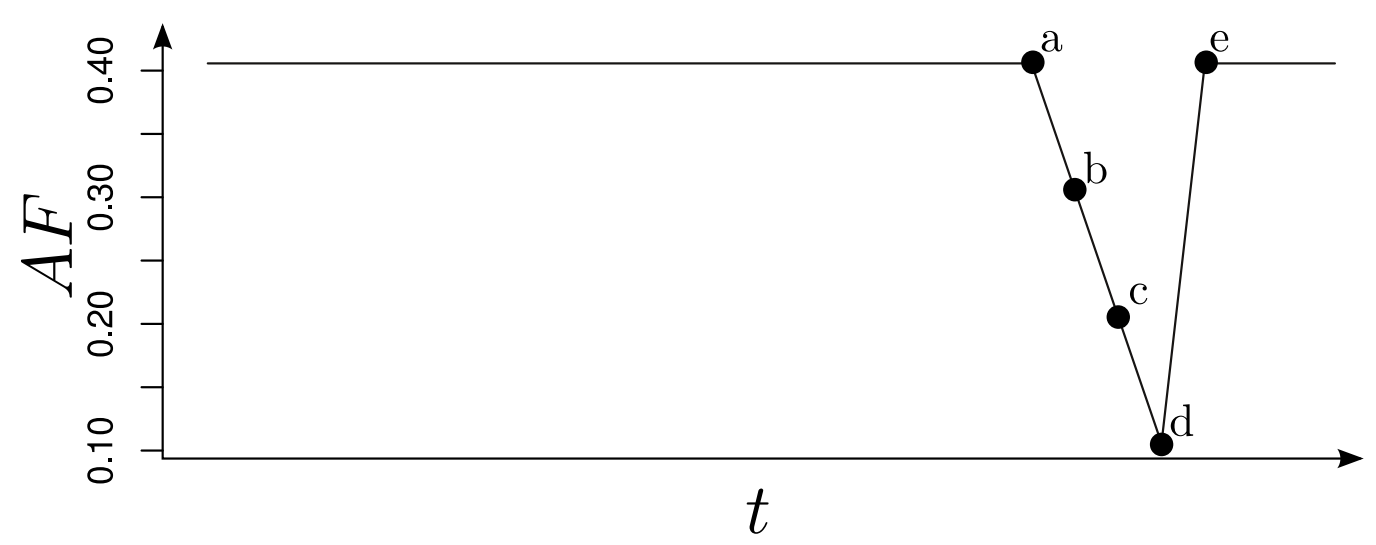

Abbildung 9.16: Exemplarischer Verlauf des $A F$

Die wichtigsten Kenngrößen zur Validierung des Bewegungsmodells für EIs wurden ermittelt und diskutiert. Der folgende Unterabschnitt behandelt das Bewegungsmodell zur Überquerung von NIs.

\subsubsection{Straßenüberquerungen auf Überwegen}

Anhand von Geschwindigkeit-Dichte-Diagrammen kann grundlegend gezeigt werden, wie sich variierende Verkehrsdichten auf Fußgängergeschwindigkeiten auswirken. Das durchgeführte Experiment betrachtet ein way-Objekt (vgl. Abschnitt 7.3.2 mit 5 Spuren und einer Länge von 4,57 m. Dies entspricht in etwa einem Zebrastreifen über eine Straße in einem Wohngebiet. Die Anzahl an Fußgängern wird initial auf 1 gesetzt und pro Lauf um 1 inkrementiert. Pro Anzahl an Fußgängern werden 10 Replikationen durchgeführt und gemittelt. Protokolliert werden die Verkehrsdichte $\rho$ und die mittleren Geschwindigkeiten der Fußgänger $\bar{v}$. Es gilt

$$
\rho=\frac{\# \text { Fußgänger }}{C}
$$

Die Kapazität $C$ eines way-Objektes ist:

$$
\begin{aligned}
C & =\frac{b \cdot l}{0,457} \\
\text { mit } \quad b & =\text { Breite des way-Objektes } \\
l & =\text { Länge des way-Objektes }
\end{aligned}
$$




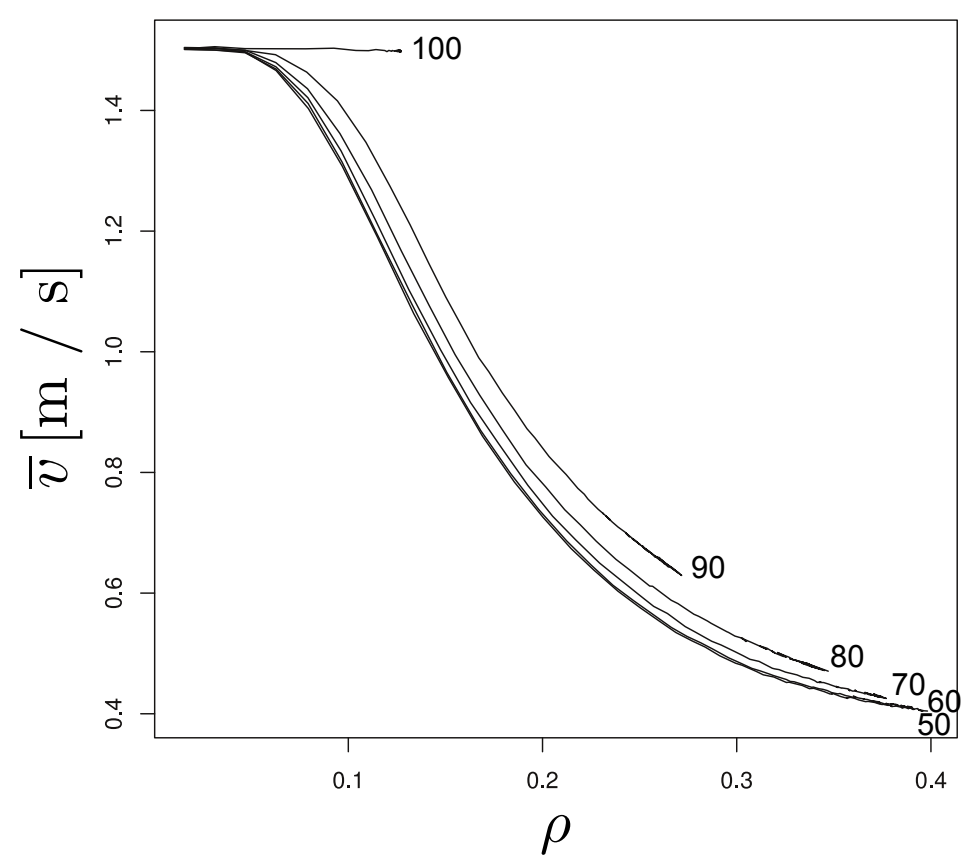

Abbildung 9.17: Geschwindigkeit-Dichte-Diagramm für Fußgängerüberwege. Unterschiedliche Kurven für variierende Verteilungen der Laufrichtung zwischen 100 (alle Fußgänger in selbe Richtung) bis 50 (gleiche Anzahl in beide Richtungen).

Die Breite und Länge eines Weges werden in $[m]$ angegen und sind wie in Abbildung 7.8 auf Seite 114 definiert. Der Skalierungsfaktor 0, 457 resultiert zu Vergleichszwecken daraus, dass in Blue und Adler, 2001] mit Zellen dieser Seitenlänge gearbeitet wird. Ein Fußgänger nimmt folglich eine Fläche von $0,457^{2} \mathrm{~m}^{2}$ ein. Die Anzahl der Fußgänger, die dem Weg folgen und die Anzahl der Fußgänger, die in die Gegenrichtung laufen, werden mit einer Schrittweite von $10 \%$ variiert. Die simulierten Fußgänger wurden mit Geschwindigkeiten nach den Gleichungen 7.6 bis 7.8 in Abschnitt 7.3.1 initialisiert. Es ergibt sich das in Abbildung 9.17 gezeigte Geschwindigkeit-Dichte-Diagramm.

In Blue und Adler, 2001 wurde das vorgestellte Modell systematisch mit Verkehrsdichten zwischen $0 \%$ und $100 \%$ belegte Zellen auf einem Weg mit 10 Spuren und einer Länge von $457 \mathrm{~m}$ mit periodischen Randbedingungen simuliert. Dieses Vorgehen ist für das in dieser Arbeit vorgestellte Modell nicht plausibel. Es wird stattdessen eine Anzahl an Fußgängern erstellt, die den Überweg nutzen möchte. Fußgänger betreten den Weg erst, wenn eine ausreichende Lücke vorhanden ist. Dichten von $100 \%$ entstehen bei der geringen Weglänge nicht. Das simulierte way-Objekt hatte 5 Spuren und eine Länge von 10·0,457 $\mathrm{m}$. Dies sind realistische Werte für einen Überweg. Ein Fußgänger, der das Ende des Weges erreicht hat, 
verlässt die Simulation.

Die maximal erreichbare Dichte nimmt den geringsten Wert an, wenn alle Fußgänger in die selbe Richtung laufen und steigt bei zunehmender Anpassung in Richtung gleicher Anzahl in beide Laufrichtungen. Gleichzeitig nehmen die gelaufenen Geschwindigkeiten immer weiter ab, je heterogener die Menge an Fußgängern ist. Es ist ferner zu erkennen, dass die Werte von $\bar{v}$ mit steigendem $\rho$ sinken. Die Verläufe decken sich grundlegend mit den Ergebnisse aus Blue und Adler, 2001. Die absoluten Geschwindigkeits-Dichte-Verhältnisse weichen jedoch ab, da das Modell im Rahmen dieser Arbeit nicht zur Simulation von Fußgängerzonen und ähnlichen Bereichen genutzt wird, sondern ausschließlich Fußgängerüberwege modelliert.

Abschließend wird aus den Ergebnissen des Experiments des vorherigen Unterabschnitts 9.4.1 ein Geschwindigkeit-Dichte-Diagramm aus allen während der Simulation auftretenden Datenpaaren auf way-Objekten der NIs erstellt. Auf diese Weise kann analysiert werden, welche Bereiche des Diagramms aus Abbildung 9.17 relevant für die Simulation urbaner Szenarien sind. Hierfür wurden die Messwerte in Verkehrsdichteintervalle von jeweils 0,005 Breite gruppiert, um die jeweiligen Maxima und Mittelwerte der gelaufenen Geschwindigkeiten bestimmen zu können. Diese Gruppierung war notwendig, da die Anzahl an Messdaten kein aussagekräftiges Diagramm ermöglicht hätte.

In Abbildung 9.18 werden die berechneten Datenpaare durch Punkte dargestellt. Die Kurven $\bar{v}$ und $v$ max wurden durch eine Regressionsanalyse bestimmt. Die Abbildung zeigt, dass sich $\bar{v}$ und $v_{\max }$ bei steigender Verkehrsdichte $\rho$ annähern. Die schnelleren Fußgänger werden durch die langsameren ausgebremst. Auch insgesamt sinkt die mittlere Geschwindigkeit $\bar{v}$ auf way-Objekten mit steigendem $\rho$. Der Verlauf von $\bar{v}$ ist eine Mischung der in Abbildung 9.17 dargestellten Kurven. Die relative Häufigkeit der auftretenden Verkehrsdichten $h_{n}(\rho)$ belegt, dass die meisten Straßenüberquerungen innerhalb der Simulation bei geringen Verkehrsdichten mit einem oder zwei Fußgängern auf dem way-Objekt stattfinden. Dennoch wird eine maximale Dichte von $\rho_{\max }=0.16$ erreicht. 


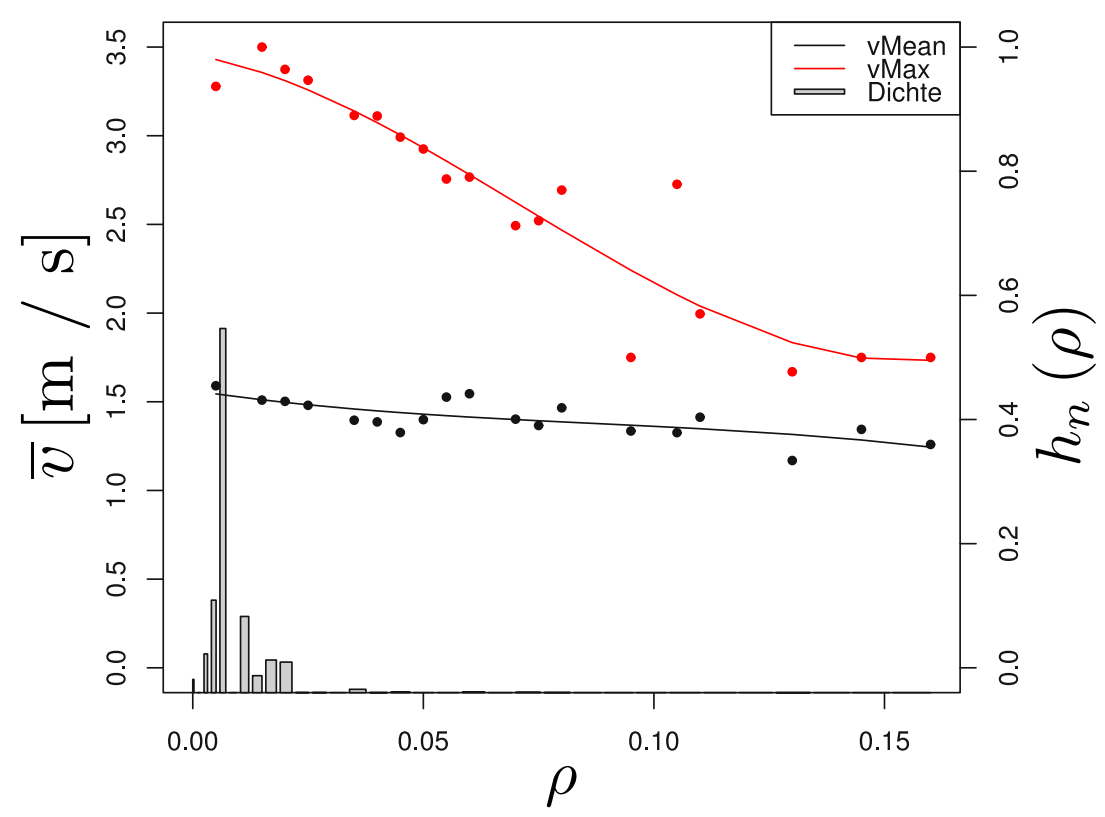

Abbildung 9.18: Geschwindigkeit-Dichte-Diagramm für Fußgängerüberwege innerhalb urbaner Simulation. Gegenüberstellung von $\bar{v}$ (mittlere Geschwindigkeit aller Fußgänger auf way-Objekt) und $v_{\max }$ (maximale gemessene Geschwindigkeit). Die Dichte $h_{n}(\rho)$ zeigt die Verteilung der auftretenden Verkehrsdichten $\rho$ auf den Überwegen.

\begin{tabular}{|l|ccccc|}
\hline \#Fußgänger & 0 & 1.000 & 2.000 & 3.000 & 4.000 \\
\hline mittlere Reisedauer [s] & 410,9319 & 416,3292 & 419,8662 & 423,2393 & 426,7230 \\
\hline
\end{tabular}

Tabelle 9.3: Vergleich der mittleren Reisedauer von Autos in Relation zur Anzahl an Fußgängern in der Simulation.

\subsubsection{Wechselwirkungen mit Autos}

Dieser Unterabschnitt untersucht die These, dass Interaktionen mit Fußgängern zu längeren Reisedauern für Autos führen. Der Aufbau des Experiments ist identisch zur Untersuchung des Fahrradeinflusses in Unterabschnitt 9.3.2. Die AnfangsVerkehrszusammensetzung ist diesmal $95 \%$ Autos und $5 \%$ Fahrräder. Die Anzahl der Fußgänger wird zwischen 0 und 4.000 mit einer Schrittweite von 1.000 erhöht. Das Vorgehen wird in 1.000 Durchläufen wiederholt. Das Resultat eines jeden Laufs ist die mittlere Reisedauer der Autos in der Simulation. Abbildung 9.19 zeigt die Verteilung der Ergebnisse.

Es ist eine Tendenz zur Erhöhung der Reisedauer bei steigender Anzahl an Fußgängern in der Simulation ersichtlich. Die Schwankungen der Resultate durch probabilistische Einflüsse steigen ebenfalls. Tabelle 9.3 vergleicht die mittleren Reisedauern. 


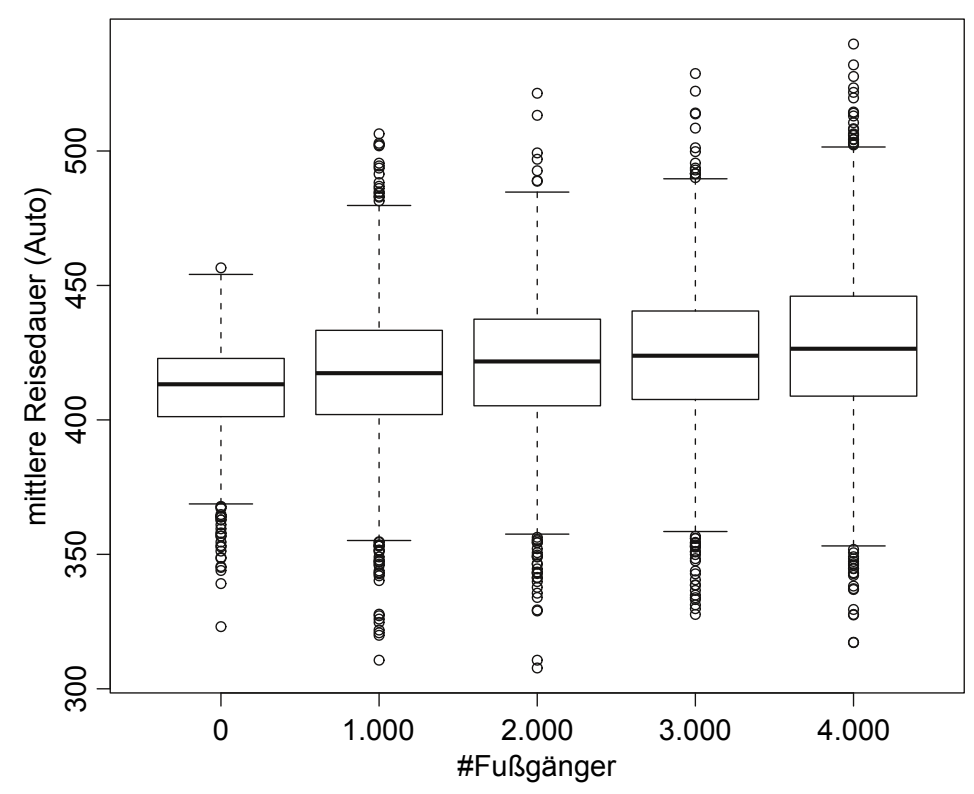

Abbildung 9.19: Fußgängereinfluss auf Straßenverkehr: Vergleich der Reisedauern von Autos bei verschiedenen Fußgängeranzahlen.

Die mittleren Reisedauern sind streng monoton steigend in Relation zur Anzahl an Fußgängern innerhalb der Simulation. Die Steigung ist jedoch nicht vergleichbar mit dem Einfluss von Fahrrädern (vgl. Unterabschnitt 9.3.2). Um die Signifikanz dieser Aussagen nachzuweisen, werden die in Gleichung 9.3 bis 9.6 gezeigten Nullhypothesen aufgestellt.

$$
\begin{array}{ll}
H_{0}^{a} \quad: \quad r d(0) \geq r d(1.000) \\
H_{0}^{b} \quad: \quad r d(1.000) \geq r d(2.000) \\
H_{0}^{c} \quad: \quad r d(2.000) \geq r d(3.000) \\
H_{0}^{d} \quad: \quad r d(3.000) \geq r d(4.000)
\end{array}
$$

Hierbei bezeichnet $r d(x)$ die mittlere Reisedauer bei $x$ Fußgängern. Um ein Signifikanzniveau von $\alpha=0.05$ zu erlangen, muss

$$
\alpha^{*}=\frac{\alpha}{2}=0.025
$$

als Signifikanzniveau verwendet werden, da die Stichproben jeweils maximal zweimal verwendet werden. Tabelle 9.4 zeigt die Resultate der t-Tests. 


\begin{tabular}{clc}
\hline Hypothese & \multicolumn{1}{c}{$p$} & $p<\alpha^{*}$ \\
\hline$H_{0}^{a}$ & $3,157 \cdot 10^{-8}$ & $\checkmark$ \\
$H_{0}^{b}$ & $1,478 \cdot 10^{-4}$ & $\checkmark$ \\
$H_{0}^{c}$ & $5,37 \cdot 10^{-4}$ & $\checkmark$ \\
$H_{0}^{d}$ & $2,803 \cdot 10^{-4}$ & $\checkmark$ \\
\hline
\end{tabular}

Tabelle 9.4: Aufstellung der Irrtumswahrscheinlichkeiten $p$ der t-Tests zur Signifikanzbestimmung der Hypothesen $H_{0}^{a}$ bis $H_{0}^{d}$.

Alle Hypothesen $H_{0}^{a}$ bis $H_{0}^{d}$ können folglich verworfen werden. Hieraus kann Gleichung 9.8 gefolgert werden.

$$
r d(0)<r d(1.000)<r d(2.000)<r d(3.000)<r d(4.000)
$$

Fußgänger haben somit im verwendeten Szenario einen Einfluss auf die Reisedauern von Autos. Dieses Resultat legt einen grundlegenden Nutzen der Berücksichtigung von Fußgängern in urbanen Verkehrssimulationen nahe.

Es konnte in diesem Abschnitt gezeigt werden, dass das in Abschnitt 7.3 entwickelte, bimodulare Fußgängermodell den Stand der Forschung bezüglich Fußgängergeschwindigkeit und -straßenüberquerungsverhalten adäquat abbildet. Die Interaktionen von Fußgängern untereinander während der Überquerung von Straßen zeigen die beobachteten Effekte in Hinblick auf die gelaufenen Geschwindigkeiten in Relation zur Verkehrsdichte auf dem Überweg.

Anhand eines Fallbeispiels konnte gezeigt werden, dass Fußgänger einen signifikanten Einfluss auf den innerstädtischen Straßenverkehr haben. Dies ist darauf zurückzuführen, dass sie bei Straßenüberquerungen Autos behindern.

\subsection{Zusammenfassung}

In diesem Kapitel wurden die verschiedenen Ebenen der Modellkalibrierung besprochen. Die mikroskopischen Verkehrsmodelle wurden untersucht und es konnte gezeigt werden, dass mit Hilfe des Automodells realistische makroskopische Kenngrößen ermittelt werden können. Dies gilt sowohl für den Autobahn-, als auch für den urbanen Verkehr. Eine Plausibilitätsuntersuchung des Fahrradmodells konnte zeigen, dass Fahräder grundlegend realistische Geschwindigkeiten fahren. Das Fußgängermodell ergibt realistische Fußgängergeschwindigkeiten und bildet Straßenüberquerungen adäquat im Vergleich zu den im Stand der Forschung diskutier- 
ten Studien ab. Ein Einfluss von Fahrrädern und Fußgängern auf den Autoverkehr in MAINSIM konnte nachgewiesen werden.

Zur Nutzung von MAINSIM als Werkzeug zur Nachbildung von real gemessenen Verkehrskenngrößen in einem konkreten Szenario müssen die beschriebenen Parameter der mikroskopischen Verkehrsmodelle auf die jeweiligen Untersuchungsraumgegebenheiten kalibriert werden. Hierfür sollten in der Zukunft Kalibrierungsmechanismen implementiert werden. Dies könnte anhand von Flussinformationen geschehen (vgl. z.B. Rakha et al., 1998, Kesting und Treiber, 2008, Duong et al., 2010).

Etwaige schwerwiegende Fehler im Kartenmaterial müssen ebenfalls angepasst werden. Dies könnten beispielsweise fehlerhafte Geschwindigkeitsbegrenzungen oder fehlende Ampeln sein. Diese Modellierungsschritte sind jedoch im Vergleich zur manuellen Grapherstellung mit geringem Zeitaufwand möglich.

Nachdem die mikroskopischen Verkehrsmodelle untersucht wurden, kann MAINSIM nun genutzt werden, um im folgenden Teil $\mathrm{V}$ dieser Arbeit Fallstudien durchzuführen. Die Fallstudien sind an den in Abschnitt 1.3 definierten Zielen dieser Arbeit orientiert und untersuchen die auf Seite 6 aufgelisteten Fragestellungen. Das entwickelte Konzept wird je Studie nach Bedarf erweitert, um resultierende Problemstellungen zu lösen. 

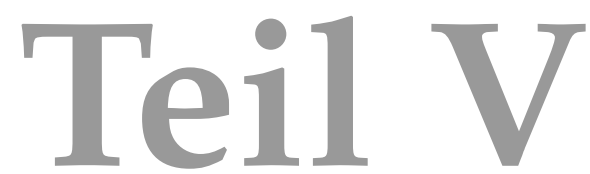

Fallstudien 



\section{Lernen in Verkehrsszenarien}

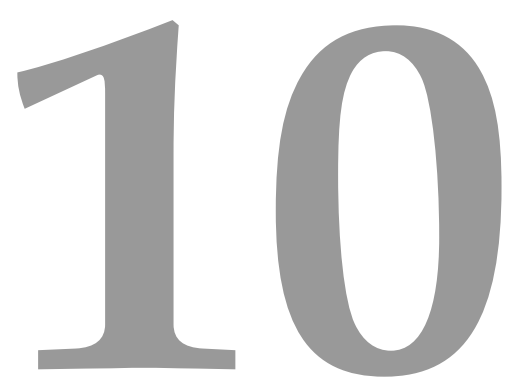

In diesem Kapitel werden Fallstudien zur Anwendung Maschineller Lernverfahren (ML) mit MAINSIM durchgeführt. Dieses Kapitel und die darin gezeigten Abbildungen basieren auf den Arbeiten Lattner et al., 2011, Dallmeyer et al., 2012a, die in Kooperation mit Andreas Lattner und Ingo Timm entstanden sind. Im folgenden Abschnitt 10.1 werden verwandte Arbeiten diskutiert. Abschnitt 10.2 diskutiert die Untersuchung zweier Szenarien für Autobahnverkehr. Eine Methode zur Stauvorhersage in urbanen Bereichen wird in Abschnitt 10.3 beschrieben. Dieses Kapitel schließt in Abschnitt 10.4 mit einer Zusammenfassung und einem Ausblick.

\subsection{Verwandte Arbeiten}

Die Kopplung von ML und Agenten-Simulation wird in der Literatur diskutiert. In Baqueiro et al., 2009 werden zwei Ansätze diskutiert: Einerseits können Lerntechniken in eine Agenten-Simulation integriert werden und andererseits können Simulationsergebnisse verwendet werden, um daraus Regeln zu lernen. Im Bereich der Adaptive Traffic Control Systems (ATCS) werden ML verwendet, um auf Repräsentationen von Verkehrssituationen - z.B. Verkehrsdichte in bestimmten Bereichen einer Autobahn - geeignet zu reagieren. In Bull et al., 2004 werden 
ML genutzt, um adaptive Ampelsteuerungen zu optimieren. Einen Überblick zu ATCS liefert Stevanovic, 2010.

In Gehrke und Wojtusiak, 2008 wird ein Ansatz präsentiert, um online auf Einflüsse durch die Umgebung (z.B. die aktuelle Wetterlage) zu reagieren und diese Informationen zur Lkw-Routenplanung zu nutzen. Der Ansatz sucht die besten Routen unter Berücksichtigung der Straßennässe und der Geschwindigkeitsbegrenzungen. Hierfür wird jeder Lkw durch einen Agenten modelliert, der dynamisch auf neue Ereignisse reagieren kann. Es wird ein Lernverfahren angewendet, um aus den Attributen Tag, Uhrzeit und Wetter eine fahrbare Geschwindigkeit auf einer korrespondierenden Straße zu lernen. Die gelernten Regeln können anschließend genutzt werden, um Routen zu optimieren.

In Bazzan et al., 2008 wird eine Methode zur dezentralen Ampelsteuerung unter Berücksichtigung des Routenplanungsverhaltens simulierter Autos untersucht. Autos und Ampeln werden als Agenten modelliert, die jeweils versuchen, ihre Pläne zu optimieren. Ampeln lernen, wann welche Phasenlängen zu setzen sind und Autos optimieren bei wiederholten Simulationsläufen ihre Routen von ihrer individuellen Start- zur Zielposition. In Bazzan, 2009 wird ein Überblick über Reinforcement learning Methoden im Kontext der Verkehrssteuerung gegeben.

Die Bestimmung einer schnellen Route von einer Start- zu einer Zielposition hängt von den Reisedauern auf den jeweiligen Straßen ab. Diese wiederum sind stark vom aktuellen und zukünftigen Verkehr auf ihnen abhängig. In Fiosins et al., 2011 wird innerhalb einer agentenbasierten Verkehrssimulation versucht, change points zu lernen, also Punkte, an denen der Betrag der Veränderung ein Level überschreitet und damit relevant für die Planung eines Agenten wird. Agenten können in diesem Fall ihre Routen verändern und insgesamt ihre Reisedauer reduzieren.

\subsection{Verkehrsbeeinflussung in Autobahnszenarien}

Dieser Abschnitt beschreibt einen Ansatz, um dynamische Anpassungsstrategien in Abhängigkeit zur Verkehrssituation anzuwenden. Das grundlegende Ziel ist, Muster in Ergebnissen der Verkehrssimulation zu entdecken und diese zu nutzen, um während eines Simulationslaufes Aktionen zur Verkehrsbeeinflussung vorzunehmen. Der folgende Unterabschnitt 10.2.1 beschreibt, wie Muster definiert sind. Abschnitt 10.2.2 beschreibt die Durchführung des Lernvorgangs, dessen Resultat in Abschnitt 10.2.3 angewendet wird. Die Unterabschnitte 10.2.4 und 10.2.5 diskutieren die Anwendung des Konzepts für zwei Simulationsszenarien. 


\subsubsection{Repräsentation}

Der gewählte Ansatz ist eine Anwendung von überwachten Lernverfahren anhand der Beispiele Entscheidungsbaumlernen und Entscheidungsregellernen. Situationen werden durch eine Menge von Attributen definiert, deren Werte für eine konkrete Situation aus dem Simulationssystem extrahiert werden. Attribute können entweder symbolisch oder nummerisch sein. Die Domänen - oder Wertebereiche

- für symbolische Attribute werden durch eine Menge von symbolischen Werten definiert. Die Domäne eines symbolischen Attributes $F_{i, s y m b}$ wird definiert als:

$$
\operatorname{dom}\left(F_{i, s y m b}\right)=\left\{v_{i, 1}, \ldots, v_{i, n}\right\}
$$

Domänen von nummerischen Attributen $F_{i, n u m}$ sind definiert als:

$$
\operatorname{dom}\left(F_{i, \text { num }}\right)=\left[v_{\min , i}, v_{\max , i}\right] \operatorname{mit} v_{\min , i}, v_{\max , i} \in \mathbb{R}
$$

Eine Liste von Attributen $\left(F_{1}, \ldots, F_{n}\right)$ wird zur Repräsentation einer Situation verwendet. Eine konkrete Situation wird nach Gleichung 10.3 definiert.

$$
\left(f_{1}, \ldots, f_{n}\right) \text { mit } f_{i} \in \operatorname{dom}\left(F_{i}\right) \text { für } 1 \leq i \leq n
$$

Aktionen, die in spezifischen Situationen ausgeführt werden können, werden durch eine Menge von Bezeichnern beschrieben:

$$
A=\left\{a_{1}, \ldots, a_{m}\right\}
$$

Mögliche Aktionen sind die Anpassung der Geschwindigkeitsbegrenzungen ausgewählter Straßenabschnitte oder die Aktivierung einer Anzeigetafel.

\subsubsection{Lernen von Strategien}

Abbildung 10.1 skizziert den grundlegenden Lernprozess. In Abhängigkeit zum Seedwert des Zufallsmoduls innerhalb des Simulationssystems entstehen bei identischer Konfiguration der Simulation unterschiedliche Ergebnisse als Resultat probabilistischer Einflüsse innerhalb der Simulationsmodelle (vgl. Kapitel7). Nach einer Simulationsphase, in der mehrere Simulationsläufe durchgeführt werden, folgt eine Lernphase, die eine Menge von Mustern aus den Simulationsergebnissen extrahiert. Ein gelernter Klassifikator kann anschließend genutzt werden, um adaptiv zu Situationen Aktionen durchzuführen oder um zu entscheiden, welche Parameter in zukünftigen Simulationsläufen verstärkt betrachtet werden sollten. 

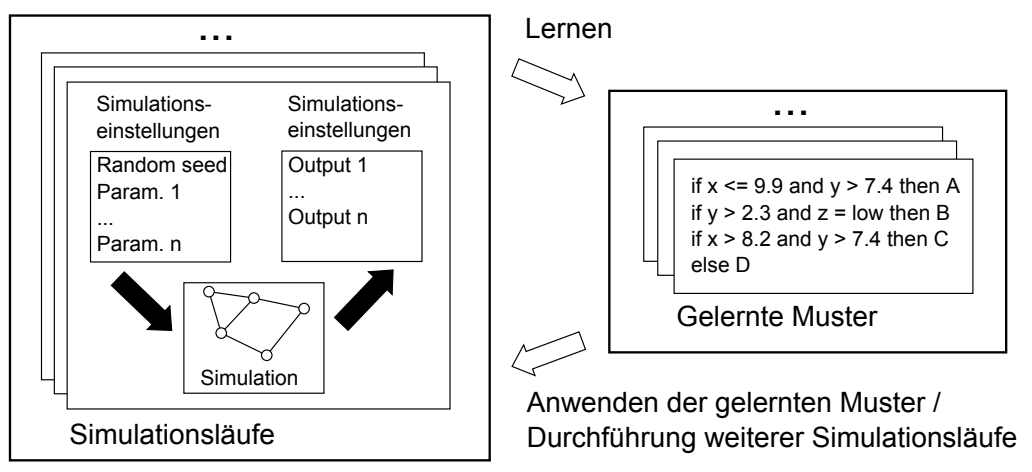

Abbildung 10.1: Ablauf des Lernvorgangs nach [Lattner et al., 2011

Das Verfahren ist nicht auf Klassifizierungsaufgaben der gegenwärtigen oder vergangenen Situation beschränkt - es könnte auch zur Vorhersage wahrscheinlicher, zukünftiger Situationen genutzt werden. In diesem Abschnitt wird versucht, eine Verhaltensstrategie in Abhängigkeit zur gegenwärtigen Situation zu bestimmen. Eine Strategie wird nach Gleichung 10.5 definiert.

$$
\text { Strategie }: \operatorname{dom}\left(F_{1}\right) \times \ldots \times \operatorname{dom}\left(F_{n}\right) \longrightarrow A
$$

Um herauszufinden, welche Aktion bei einer gegebenen Situation am besten abschneidet, wird der aktuelle Simulationszustand gespeichert. Von dort ausgehend werden nacheinander alle möglichen Aktionen auf Funktionalität geprüft. Abbildung 10.2 illustriert das Vorgehen.

Zum Zeitpunkt $t_{n}$ wird eine Beschreibung der Simulationssituation mit $j$ Attributen berechnet $\left(f_{1}, \ldots, f_{j}\right)$. Die verschiedenen Aktionen $a_{i} \in A$ mit $1 \leq i \leq m$ werden ausgeführt und die Simulation wird fortgeführt. Alle Läufe starten von der identischen Startsituation $\left(f_{1}, \ldots, f_{j}\right)$ zum Zeitpunkt $t_{n}$. Zum Zeitpunkt $t_{n+k}$ wird die Aktion $a_{b e s t}$, die zur besten Situation führte, bestimmt und das Tupel $\left(f_{1}, \ldots, f_{j}, a_{\text {best }}\right)$ wird gespeichert. Eine Menge solcher Tupel werden als Trainingsdaten für den Lernvorgang eines Klassifikators genutzt.

\subsubsection{Anwendung der gelernten Strategien}

Die Anwendung einer gelernten Strategie geschieht analog zu Abbildung 10.2, Die Simulation wird bis zu einem Zeitpunkt $t_{n}$ durchgeführt. Die Situationsbeschreibung $\left(f_{1}, \ldots, f_{j}\right)$ wird generiert und genutzt, um die Aktion $a \in A$ zu bestimmen, die durch die trainierte Strategie strategie vorgeschlagen wird. 


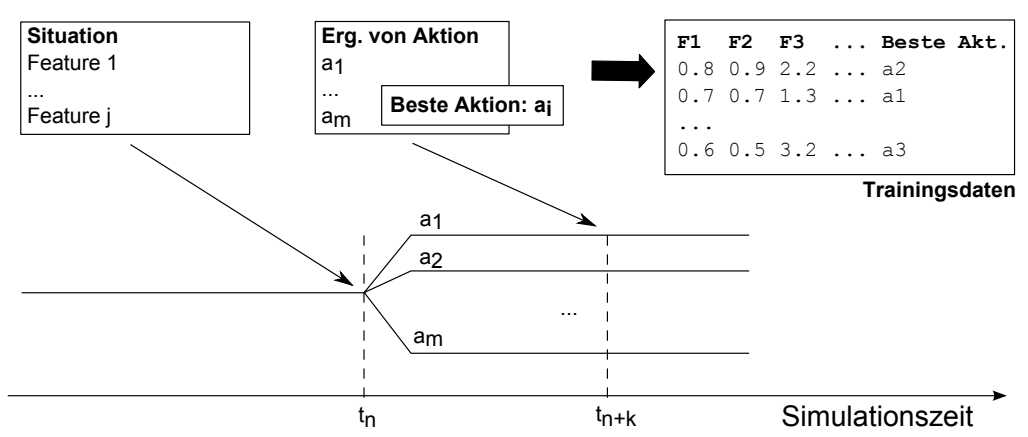

Abbildung 10.2: Generierung von Trainingsdaten nach Lattner et al., 2011

Es ist zu beachten, dass eine wiederholte Anwendung dieser Strategie zu negativen Resultaten führen kann, da eine Einschwingdauer auf die neue Aktion benötigt werden kann. Dies hängt jedoch vom Experimentaufbau ab. Ein zu häufiges Umplanen einer bestimmten Route von einer Startposition zu einer Zielposition innerhalb eines Straßengraphen kann dazu führen, dass sich die Reisedauer erhöht, auch wenn jede einzelne Umplanaktion vorteilhaft war. Oszillierendes Verhalten soll daher vermieden werden. Für ein erstes Experiment wird die zu wählende Aktion alle $60 \mathrm{~s}$ Simulationszeit erneut bestimmt und angewendet, falls eine mehrfache Anwendung vorgesehen ist.

Die Evaluierung des Konzepts wird auf synthetischen Autobahnszenarien durchgeführt. Ein Simulationsserver bestimmt die zu simulierenden Parameter und sendet sie an Simulationsclients, die per TCP/IP verbunden sind. Die Clients führen Simulationsläufe durch und geben Resultate an den Server zurück (vgl. Kapitel 8.3).

\subsubsection{Statisches Szenario: Einführung einer Geschwindigkeitsbegrenzung}

Der Straßengraph ist eine Kreisautobahn mit $24 \mathrm{~km}$ Länge und zwei Spuren. Eine gängige Lehrmeinung der Verkehrsforschung schlägt Geschwindigkeitsbegrenzungen vor, um den Verkehr zu homogenisieren und ihn somit insgesamt schneller fließen zu lassen (vgl. Treiber und Kesting, 2010, Kap. 20]), da Störungen unterdrückt werden, die zu Staus führen können. Bei geringen Verkehrsdichten sind Geschwindigkeitsbegrenzungen nachteilig. Daher soll ein erstes Szenario die Verkehrsdichte bestimmen, ab der sich eine Geschwindigkeitsbegrenzung auf der simulierten Autobahn auf $130 \mathrm{~km} / \mathrm{h}$ lohnt und die mittlere Geschwindigkeit aller simulierten Autos durch die Begrenzung steigt. In diesem Szenario erhalten simulierte Autos die Möglichkeit, unaufmerksam zu sein (vgl. Abschnitt 7.1.4). Die Wahrscheinlichkeit für Unfälle sinkt mit zunehmender Homogenisierung des Ver- 
kehrs. Wenn zwei Autos in einen Unfall verwickelt sind, verweilen sie stehend für 600 Iterationen auf der rechten Spur und fungieren somit als Hindernisse.

Der Simulationsserver lässt seine Clients Experimente mit 4, 6, .., 998, $1000 \mathrm{Au}-$ tos durchführen. Jedes Experiment wird fünfmal durchgeführt, um die Ergebnisse mitteln zu können. Bei der Erstellung eines Verkehrsteilnehmers wird sein Typ mit einer Wahrscheinlichkeit von $p_{\mathrm{Lkw}} \% \mathrm{Lkw}$, ansonsten Pkw. Der Wert von $p_{\mathrm{Lkw}} \%$ wird für jedes Experiment vom Server zwischen 0 und 10 bestimmt. Clients erstellen die gegebene Anzahl an Autos auf zufälligen Positionen auf der simulierten Autobahn und führen anschließend die Simulation nach Abbildung 10.2 durch.

Nach einer Einschwingdauer von 1.000 Iterationen werden für 1.000 Iterationen der Verkehrsfluss und die durschnittliche Geschwindigkeit aller simulierten Fahrzeuge gemittelt. Diese Daten werden zur Situationsbeschreibung des aktuellen Simulationszustandes genutzt. Die folgenden Attribute werden verwendet: Verkehrsfluss, Verkehrsdichte, mittlere Geschwindigkeit $\bar{v}$ aller Fahrzeuge, Standardabweichung von $\bar{v}$ und der Prozentsatz an Lkw. Die Verkehrsdichte und der Prozentsatz an Lkw bleiben während eines Simulationslaufes konstant und werden daher einmalig ermittelt. Der Simulationszustand wird gespeichert.

Die erste Aktion, in diesem Fall $a_{0}=$ „Keine Geschwindigkeitsbegrenzung“ wird durchgeführt. Nach einer weiteren Einschwingphase von 1.000 Iterationen wird die mittlere Geschwindigkeit der simulierten Fahrzeuge für weitere 1.000 Iterationen bestimmt und als Bewertung für die Aktion verwendet. Der gespeicherte Simulationszustand wird wiederhergestellt und die zweite Aktion $a_{1}=$ "Geschwindigkeitsbegrenzung: $130 \mathrm{~km} / \mathrm{h}$ " für die gesamte Autobahn wird durchgeführt. Die Bewertung wird analog durchgeführt. Der Client sendet die Zustandsbeschreibung und die Aktion mit der höchsten mittleren Geschwindigkeit an den Server. Der Server protokolliert alle erhaltenen Ergebnisse und lässt die Clients simulieren, bis alle Experimente durchgeführt wurden.

Der Server nutzt ein Interface zu WEKA $\rrbracket^{1}$ um die Strategie zu erlernen, wann welche Aktion anzuwenden ist. Als Lernverfahren wird von WEKA die J4.8 Implementierung des C4.5 Algorithmus Quinlan, 1993 verwendet. Das Resultat ist:

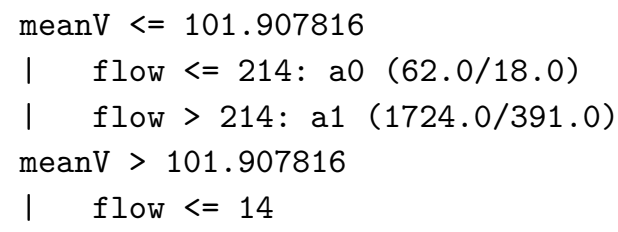

${ }_{1}^{1}$ http://www.cs.waikato.ac.nz/ml/weka/, Hall et al., 2009, Bouckaert et al., 2010. 


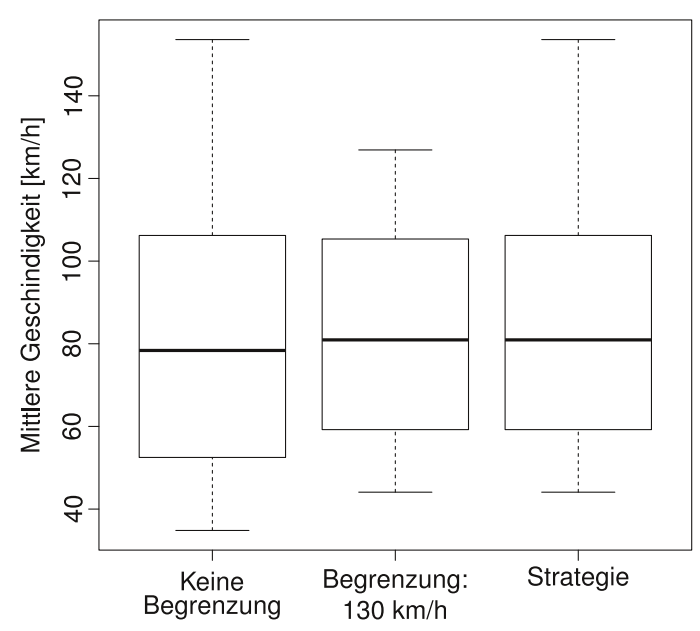

Abbildung 10.3: Ergebnisse: Statisches Szenario

\begin{tabular}{|c|c|c|c|}
\cline { 2 - 4 } $\begin{array}{c}\text { vorgeschlagene Aktion } \\
\text { beste Aktion } \downarrow\end{array}$ & $a_{0}$ & $a_{1}$ & $\sum$ \\
\hline$a_{0}$ & 705 & 352 & $1057(42,60 \%)$ \\
\hline$a_{1}$ & 158 & 1266 & $1424(57,40 \%)$ \\
\hline
\end{tabular}

Korrekt klassifiziert: 1971/2481 (79,44\%)

Tabelle 10.1: Wahrheitsmatrix der gerlernten Strategie im statischen Szenario

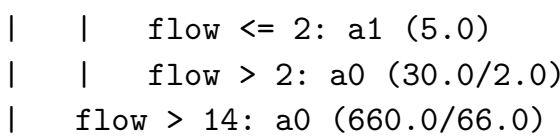

Die verfügbare Verkehrsdichte wurde von $C 4.5$ nicht genutzt. Die gelernte Strategie wird in 497 weiteren Simulationsläufen (mit 4,6, ., 1000 Fahrzeugen) im selben Szenario gegen die konstante Nutzung von $a_{0}$ bzw. $a_{1}$ verglichen. Abbildung 10.3 zeigt den Vergleich der mittleren Geschwindigkeiten. Die Mittelwerte dieser Ergebnisse sind bei „Konstant $a_{0}$ “: $82,71 \mathrm{~km} / \mathrm{h}$, , „Konstant $a_{1} ": 82,83 \mathrm{~km} / \mathrm{h}$, strategie: $85,85 \mathrm{~km} / \mathrm{h}$. Ein einseitiger t-Test mit Signifikanzniveau $\alpha=0.05$ bezeugt höhere mittlere Geschwindigkeiten bei Anwendung der gelernten strategie im Vergleich sowohl zur konstanten Nutzung von $a_{0}$, als auch $a_{1}(p<2.2 e-16$ in beiden Fällen). Hierbei wurden identische Stichproben mehrfach verwendet, weshalb die Methode von Bonferroni verwendet werden muss Abdi, 2007. Die Resultate bleiben jedoch signifikant.

Tabelle 10.1 zeigt die Wahrheitsmatrix, die mit der gelernten Strategie mittels Kreuzvalidierung auf den Trainingsdaten ermittelt wurde. Die gelernte Strategie liefert mehr korrekt klassifizierte Aktionen, als die konstante Anwendung einer Methode oder ein einfaches Münzwerfen der zu wählenden Aktion. 


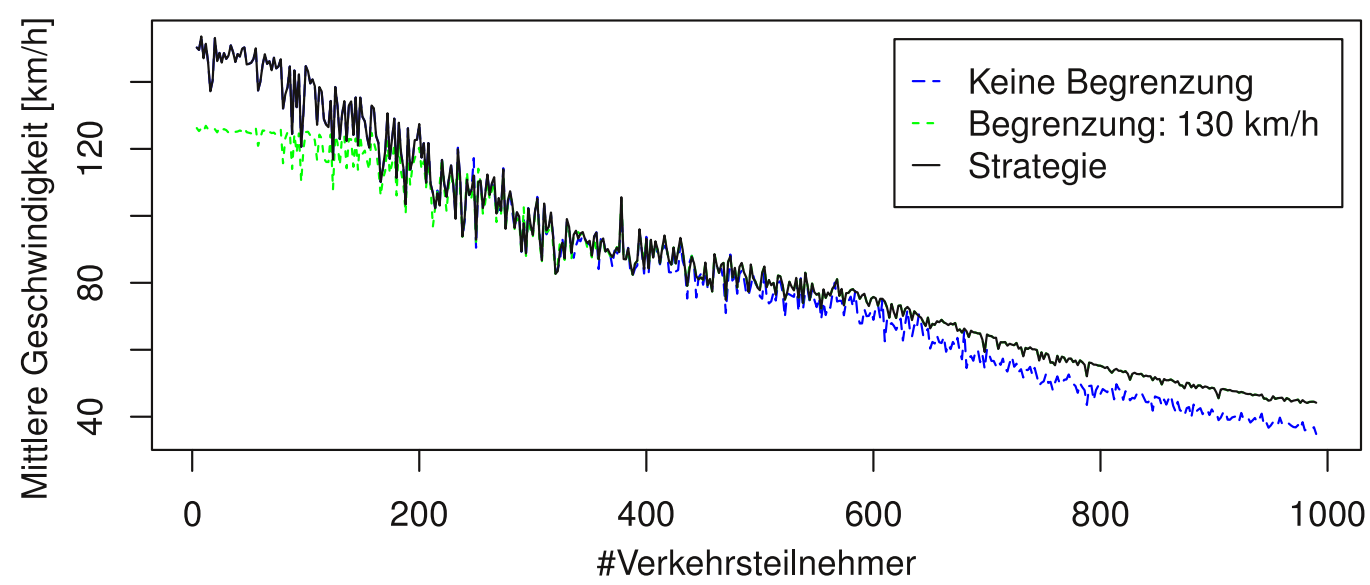

Abbildung 10.4: Vergleich: Gelernte Strategie und konstante Anwendung einer Aktion

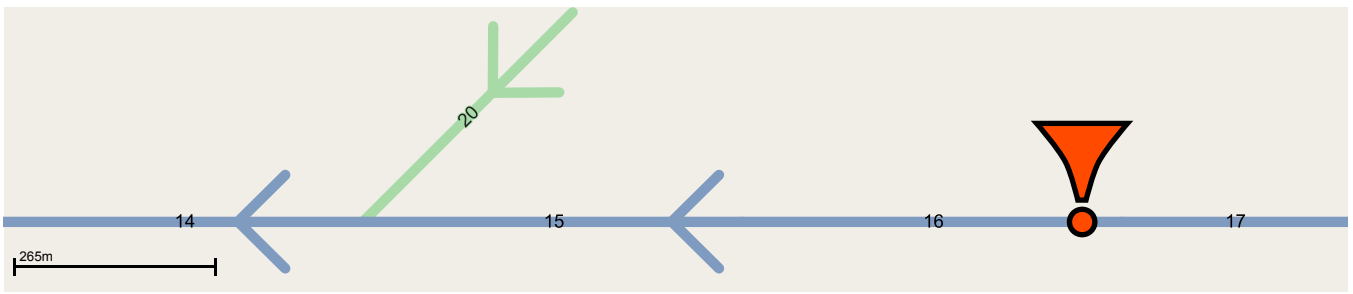

Abbildung 10.5: Kartenausschnitt im Bereich der Einmündung der Zubringerstraße (grün) auf die Autobahn (blau). Die markierte Stelle enthält die Anzeigetafel.

Die bisherigen Ergebnisse wurden stark zusammengefasst. Abbildung 10.4 zeigt die mittleren Geschwindigkeiten bei steigender Verkehrsdichte. Die gelernte Strategie verwendet die Aktion $a_{0}=$ „Keine Geschwindigkeitsbegrenzung“ bei geringen Verkehrsdichten und schaltet bei steigender Verkehrsdichte auf $a_{1}=$ „Geschwindigkeitsbegrenzung: $130 \mathrm{~km} / \mathrm{h}$ “ um. Die gelernte strategie wendet in Abhängigkeit zur Verkehrsdichte die jeweils günstigere Aktion an, obwohl die Verkehrsdichte in den gelernten Regeln nur implizit über die mittlere Geschwindigkeit und den Verkehrsfluss modelliert wurde (vgl. Gleichung 2.1 auf Seite 16).

\subsubsection{Dynamisches Szenario: Einführung eines Linksfahrgebots}

Das zweite Szenario simuliert einen zweispurigen Autobahnausschnitt mit einer Auffahrt. Die Autobahn besteht aus 20 Segmenten $\mathrm{EI}_{0} \cdots \mathrm{EI}_{19}$ mit jeweils 500 m Länge. Der Verbindungsknoten zwischen $\mathrm{EI}_{14}$ und $\mathrm{EI}_{15}$ ist zusätzlich zu der Zuflussstraße $\mathrm{EI}_{20}$ verbunden. Abbildung 10.5 zeigt diesen Ausschnitt.

Einen Kilometer vor der Auffahrt befindet sich eine Anzeigetafel, die in der Ab- 
bildung mit einem Ausrufezeichen markiert ist. Die Anzeigetafel kann genutzt werden, um Fahrzeugen die Anweisung zu erteilen, sich links einzuordnen, um dem zufließenden Verkehr den Wechsel auf die Autobahn zu erleichtern. Wenn die Anzeigetafel aktiviert ist, versuchen alle Fahrzeuge auf den Straßen $\mathrm{EI}_{15}$ und $\mathrm{EI}_{16}$ auf die linke Spur zu wechseln, sofern die notwendigen Abstände dies zulassen. Das Linksfahrgebot gilt bis zur Einmündungsstelle.

Eine Strategie wird gelernt. Die verwendeten Attribute sind die Verkehrsdichten $\rho\left(\mathrm{EI}_{15,16}\right), \rho\left(\mathrm{EI}_{17,18}\right)$ und $\rho\left(\mathrm{EI}_{20}\right)$. Die möglichen Aktionen $a_{0}=$ „Linksfahrgebot" und $a_{1}=$,kein Linksfahrgebot" werden verglichen. Während der Simulation werden die Mengen an Fahrzeugen, die auf den zwei Zuflussstraßen (Beginn der Autobahn und der Zubringerstraße) erscheinen, variiert. Die Simulationsdauer wird in $60 \mathrm{~s}-$ Abschnitte unterteilt. Am Beginn jedes Abschnitts wird analog zu Abbildung 10.2 der aktuelle Simulationszustand gespeichert und beide Aktionen werden separat getestet. Als Bewertung gilt die mittlere Geschwindigkeit aller simulierten Fahrzeuge nach Ablauf der 60 s. Die Clients simulieren, bis der Server 10.000 Trainingsdaten erhalten hat. Ein Auszug aus der gelernten Strategie ist:

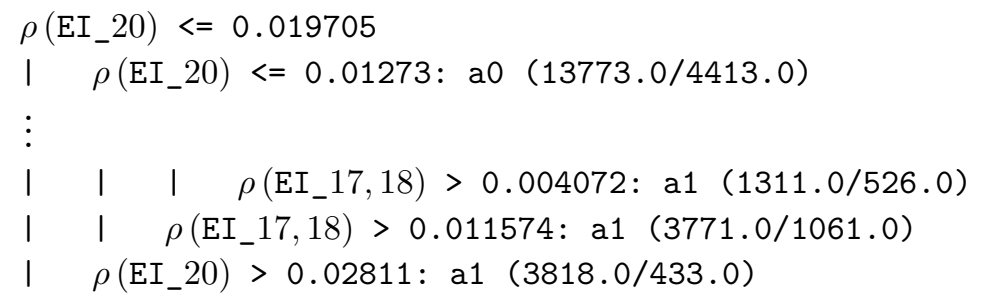

Auf der obersten Entscheidungsebene wird die Verkehrsdichte auf der Zuflussstraße $\mathrm{EI}_{20}$ geprüft. Wenn sie einen Grenzwert übersteigt, ist ein Linksfahrgebot günstig.

Die gelernte Strategie wird im identischen Szenario für 172.800 Iterationen (48 Stunden Realzeit) getestet. Die Verkehrsmengen auf den Zuflussstraßen werden während der Simulationsdauer variiert. Nach jeweils 60 Iterationen wird die aktuelle Situationsbeschreibung von der gelernten Strategie bewertet und die vorgeschlagene Aktion ausgeführt. Als Ergebnis werden die mittleren Geschwindigkeiten und Reisedauern aller Verkehrsteilnehmer über die gesamte Simulationsdauer erhoben. Identische Simulationsläufe werden unter konstanter Nutzung von $a_{0}$, bwz. $a_{1}$ durchgeführt. Abbildung 10.6 vergleicht die genannten Methoden.

Es ist ersichtlich, dass die Anwendung der gelernten Strategie zu einer Erhöhung der mittleren Geschwindigkeiten führt. Die konstante Anwendung von $a_{0}$ führt zu

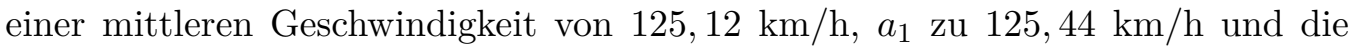
gelernte strategie zu 126, $41 \mathrm{~km} / \mathrm{h}$. Die Unterschiede konnten durch t-Tests mit jeweils $p<2.2 e-16$ bestärkt werden. Die mittleren Reisedauern sind $a_{0}: 289,53 \mathrm{~s}$; 


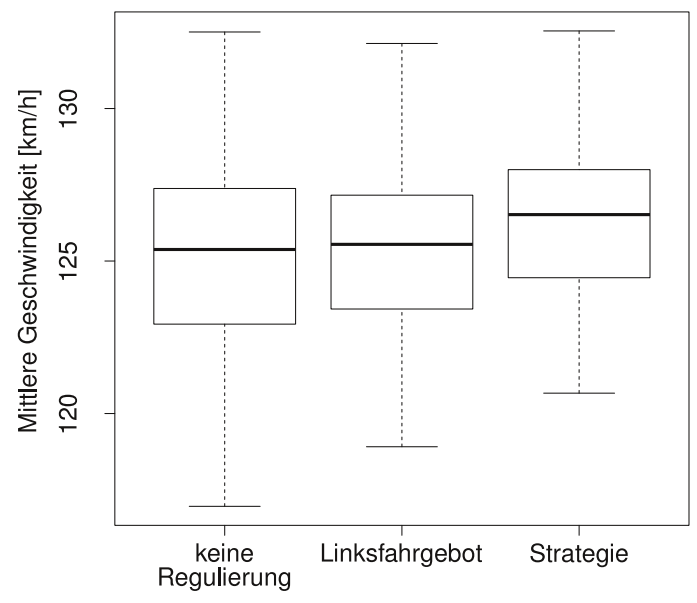

Abbildung 10.6: Ergebnisse: Dynamisches Szenario

\begin{tabular}{|c|c|c|c|}
\cline { 2 - 4 } $\begin{array}{c}\text { vorgeschlagene Aktion } \\
\text { beste Aktion } \downarrow\end{array}$ & $a_{0}$ & $a_{1}$ & $\sum$ \\
\hline$a_{0}$ & 12.185 & 3.049 & $15.234(50,78 \%)$ \\
\hline$a_{1}$ & 6.709 & 8.057 & $14.766(49,22 \%)$ \\
\hline Korrekt klassifiziert: $20242 / 30000(67,47 \%)$ &
\end{tabular}

Tabelle 10.2: Wahrheitsmatrix der gerlernten Strategie im dynamischen Szenario

$a_{1}: 282,15$ s und strategie: $279,89 \mathrm{~s}$. Wenn dieses Resultat auf die Simulationsdauer von 48 Stunden bei durchschnittlich 43148,09 simulierten Fahrzeugen skaliert wird, ergibt sich unter Nutzung der Strategie über 24 Stunden auf dem simulierten Streckenabschnitt eine Gesamtersparnis an Reisedauer von ca. 58 Stunden im Vergleich zur konstanten Nutzung von $a_{0}$ bzw. ca. 14 Stunden zu $a_{1}$.

Tabelle 10.2 zeigt die Wahrheitsmatrix der gelernten Strategie, die per Kreuzvalidierung auf den Trainingsdaten gewonnen wurde. Auch im dynamischen Szenario ist die Wahl des Klassifikators besser als die konstante Nutzung einer Aktion.

\subsection{Stauprognose in urbanen Szenarien}

Im vorangegangenen Abschnitt wurden gelernte Klassifikatoren oder Strategien verwendet, um regelnd in den Verkehrsverlauf einzugreifen. Beide Szenarien spielten auf Autobahnen. MAINSIM eignet sich hingegen ebenso für die Simulation urbaner Szenarien. In diesem Abschnitt wird ein Simulationsszenario mit einem Kartenausschnitt aus dem Osten Frankfurt am Main 2 verwendet. Im Berufsver-

\footnotetext{
${ }^{2}$ Grenzen in WGS84: [8.55: 8.96, 50.08 : 50.21]
} 


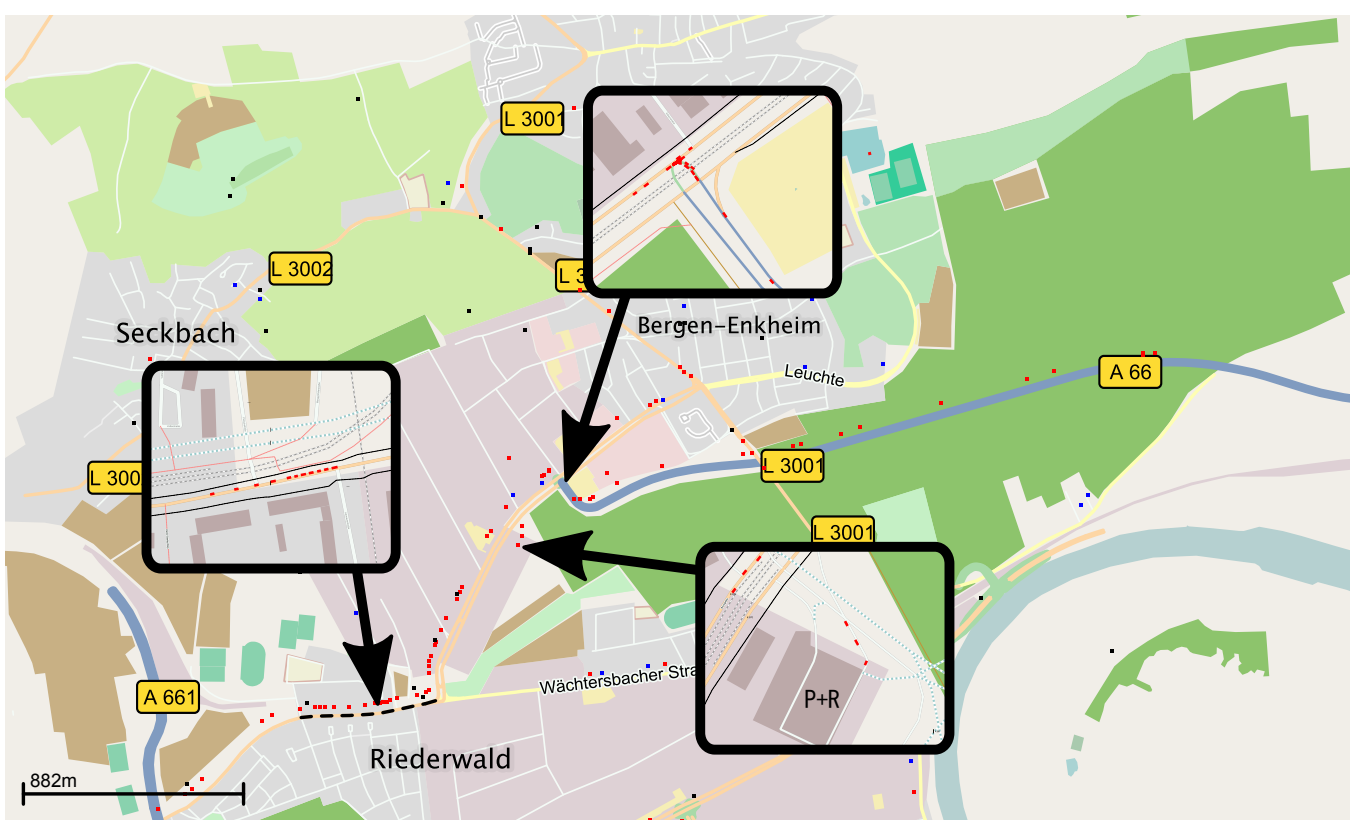

Abbildung 10.7: Kartenausschnitt im Osten Frankfurts

kehr strömen von der A66 große Mengen an Autos in die Stadt. Dies führt - begünstigt durch eine Folge von Ampeln - zu regelmäßigen Staus im Bereich „Borsigallee“ und „Am Erlenbruch“. Abbildung 10.7 zeigt den Kartenausschnitt.

Ein Teil der Autos parkt im P+R Parkhaus an der „Kruppstraße“. Diese Fallstudie untersucht die Möglichkeit, mit einfachen Mitteln abzuschätzen, wann im Messbereich „Am Erlenbruch“ (markiert durch eine gestrichelte Linie) Staus entstehen. Es wird angenommen, dass die Anzahl an Autos pro Stadtgebiet zählbar sei. Dies könnte durch Hochrechnungen von Mobilfunkinformationen oder Kontaktschwelldaten geschehen. Eine Zählung von Fahrrädern und Fußgängern wird nicht vorgenommen, da davon auszugehen ist, dass deren Zählung mit herkömmlichen Mitteln schwieriger zu ermöglichen wäre. Die Stadtgebiete „Bergen-Enkheim“, „Seckbach“ und „Riederwald“ werden unabhängig voneinander betrachtet. Zusätzlich wird die Anzahl an Autos auf der A66 in Richtung Frankfurt gezählt.

Die Attribute zur Bestimmung eines Klassifikators sind (\#Riederwald, \#Bergen - Enkheim, \#A66, \#Seckbach, $\sum$ ), wobei $\sum$ die Summe der Zählungen darstellt und die Gesamtverkehrsmenge angibt. Die gestrichelte Straße aus Abbildung 10.7 ist nicht Teil der zur Attributbestimmung verwendeten Stadtteile. In Verkehrsszenarien ist die Zeit ein wichtiger Faktor. Ein Simulationslauf beginnt mit einer Einschwingphase von 2.000 Iterationen, gefolgt von der Messung der Verkehrsmengen. Wenn in den Messbereichen ein Schnappschuss zum Zeitpunkt $t$ 


\begin{tabular}{|ll|}
\hline$p_{\text {create }}=\mathcal{N}_{0.3}^{0.7}(0.5,0.1)$ & $\begin{array}{l}\text { Wahrscheinlichkeit, einen Verkehrsteilnehmer in } \\
\text { einer Iteration zu erstellen. }\end{array}$ \\
\hline$p_{\text {car }}=\mathcal{N}_{0.5}^{0.8}(0.75,0.1)$ & Wahrscheinlichkeit, dass dieser ein Auto ist. \\
\hline$p_{\text {bicycle }}=\mathcal{N}_{0.5}^{0.9}(0.75,0.1)$ & Wahrscheinlichkeit, dass er ein Fahrrad ist, wenn \\
& er kein Auto ist. Ansonsten wird der Verkehrsteil- \\
& nehmer ein Fußgänger. \\
\hline$p_{\text {A66 }}=\mathcal{N}_{0.67}^{0.9}(0.75,0.1)$ & Wahrscheinlichkeit, für Autos auf der A66 die Si- \\
& mulation zu beginnen. Ansonsten wird eine Zu- \\
& fallsposition bestimmt. \\
\hline$p_{\text {fixDest }}=\mathcal{N}_{0.67}^{0.88}(0.75,0.1)$ & Wahrscheinlichkeit, eine vordefinierte Zielposition \\
& anzufahren. Ansonsten wird die Zielposition zufäl- \\
& lig innerhalb des Stadtgebietes bestimmt. \\
\hline$p_{\text {destInnerCity }}=\mathcal{N}_{0.7}^{0.9}(0.8,0.05)$ & Wenn eine vordefinierte Zielposition verwendet \\
& wird: Wahrscheinlichkeit, in die Innenstadt von \\
Frankfurt am Main zu fahren (führt zur Nutzung \\
der gestrichelten Straße). Ansonsten ist die Ziel- \\
position das P+R Parkhaus. \\
\hline
\end{tabular}

Tabelle 10.3: Verschiedene Wahscheinlichkeitsverteilungen für jeden Simulationslauf führen zu Variationen im Verkehrsaufkommen.

ermittelt wird, wirkt sich diese Situation erst zum Zeitpunkt $t+\Delta$ auf die zu klassifizierende Straße aus. Es wird $\Delta=300 \mathrm{~s}$ als geschätzte durchschnittliche Dauer der Fahrt aus den gewählten Gebieten bis zu „Am Erlenbruch“ gewählt. Dieser Wert ist ein Erfahrungswert und könnte weiter optimiert werden. Nach $\Delta$ wird die mittlere Geschwindigkeit $\bar{v}$ aller Autos innerhalb der gestrichelten Straße nach Gleichung 10.6 ermittelt. Es wird hierbei das zeitliche Mittel über 60 s verwendet.

$$
\text { Klasse }= \begin{cases}\text { Stau } & \text { wenn } \bar{v}<3.6 \mathrm{~m} / \mathrm{s} \\ \text { Frei } & \text { sonst }\end{cases}
$$

Fußgänger und Fahrräder werden durch die Messungen nicht erfasst. Sie beeinflussen dennoch die Simulationsergebnisse und wirken als Unsicherheitsfaktoren. Es werden 20.000 Simulationsläufe durchgeführt. Abbildung 10.8 fasst den grundlegenden Ablauf zusammen.

Jeder Simulationslauf wird mit anderen Wahrscheinlichkeitsverteilungen für die Arten der Verkehrsteilnehmer und deren Quelle-Ziel-Relationen durchgeführt. Tabelle 10.3 fasst die verwendeten Einstellungen zusammen.

Abbildung 10.9 zeigt die Verteilung der Trainingsdaten.

Zum Training des Klassifikators wird analog zu den vorherigen Experimenten $W E$ - 


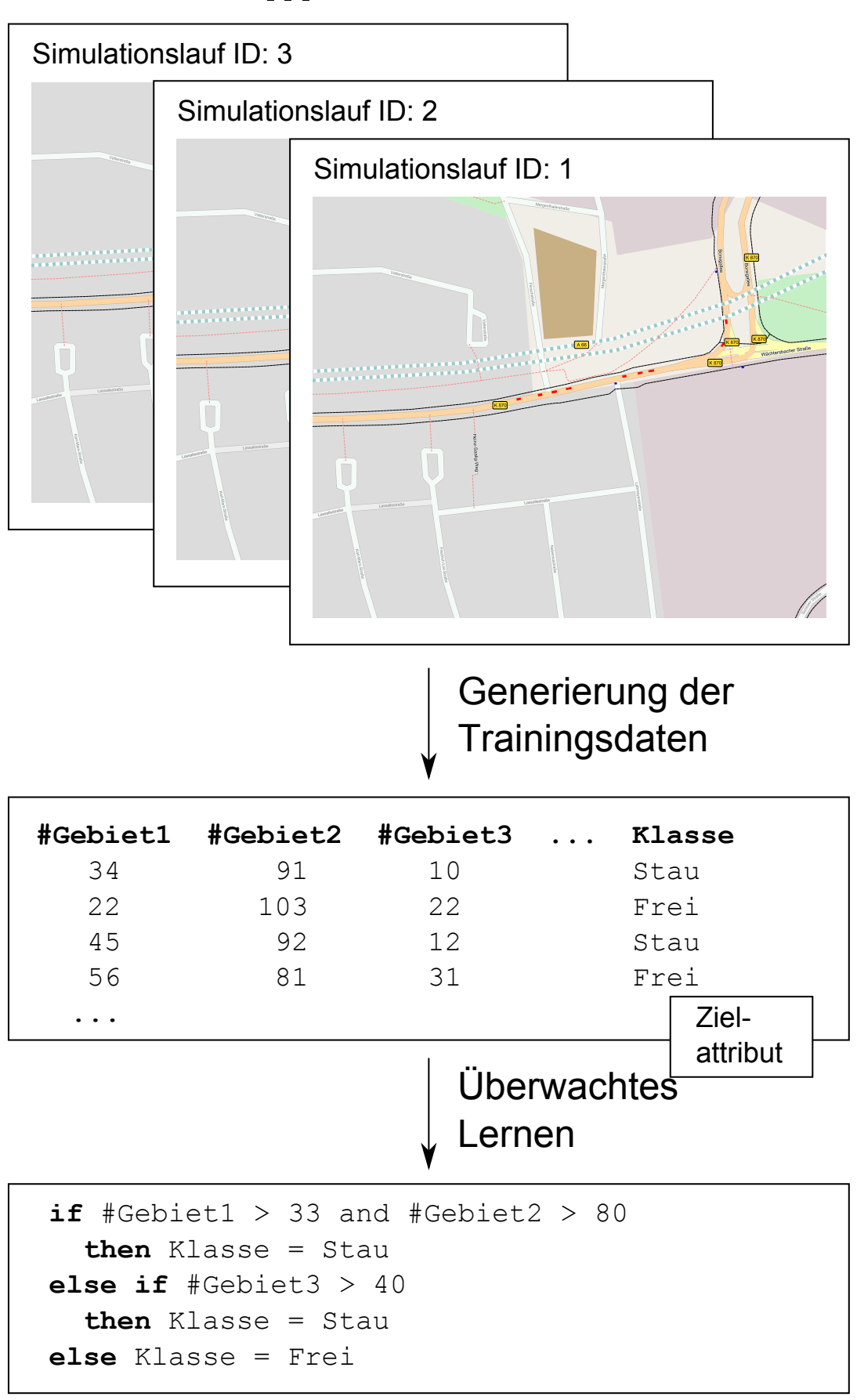

Abbildung 10.8: Lernprozess 


\section{$\times$ Frei \\ $\times$ Stau}
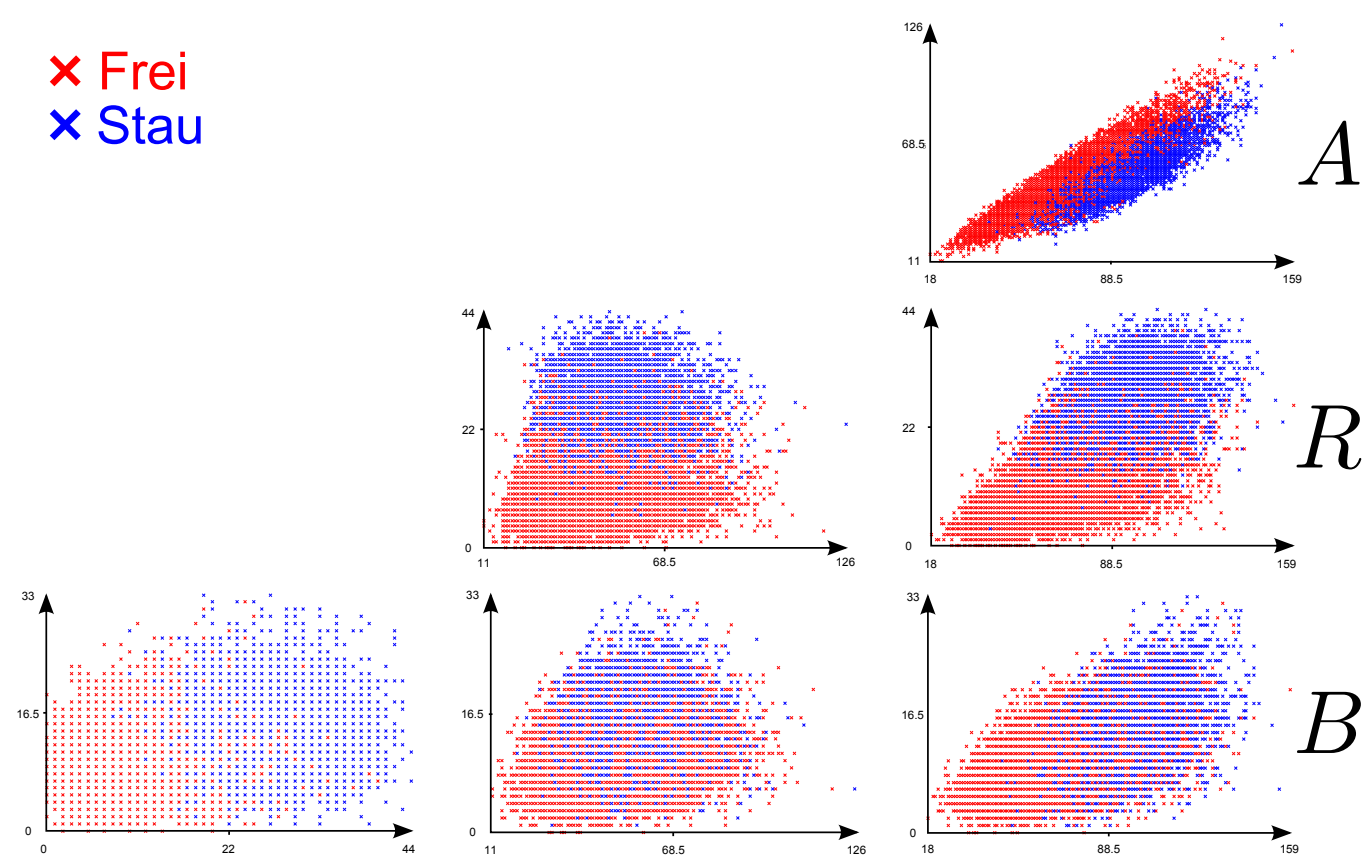

$R$

$A$

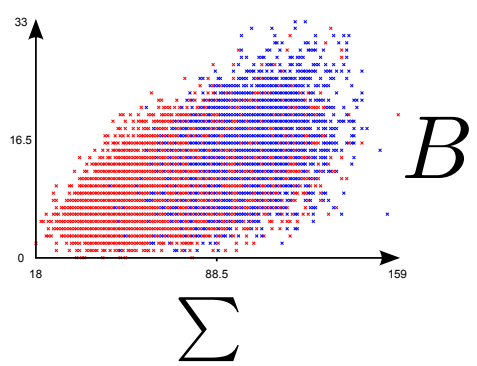

Abbildung 10.9: Verteilung der Trainingsdaten

$K A$ genutzt. Zusätzlich zum Verfahren C4.5 wird die JRIP-Implementierung des Algorithmus RIPPER Cohen, 1995 verwendet. Das Ziel sind Regeln, die einfach verständlich sind. Die Resultate werden von RIPPER und C4.5 unterschiedlich dargestellt. RIPPER gibt eine logische Formel zurück, deren zusammengefasste Form in Gleichung 10.7 dargestellt ist.

$$
\begin{aligned}
& \text { Stau }=(R \geq 22) \\
& \vee \quad(R \geq 19) \wedge(B \geq 10) \\
& \vee \quad(R \geq 17) \wedge(B \geq 15) \\
& \vee \quad(R \geq 21) \wedge(B \geq 6) \wedge(43 \leq A \leq 51) \\
& \vee \quad(R \geq 20) \wedge(B \geq 7) \wedge(58 \leq A \leq 64) \wedge(\Sigma \geq 72) \\
& \vee \quad(R \geq 16) \wedge(B \geq 8) \wedge(42 \leq A \leq 50) \wedge(S \leq 0) \wedge(72 \leq \Sigma \leq 75) \\
& \vee \quad(R \geq 14) \wedge(B \geq 12) \wedge(A \leq 62) \wedge(S \geq 1) \wedge(\Sigma \geq 91) \\
& \vee \quad(14 \leq R \leq 16) \wedge(11 \leq B \leq 17) \wedge(A \leq 50) \wedge(S \leq 1) \wedge(\Sigma \geq 78) \\
& \text { mit } \quad R \quad=\# \text { Riederwald } \\
& B \quad=\# \text { Bergen - Enkheim }
\end{aligned}
$$




\begin{tabular}{|c|c|c|}
\multicolumn{3}{c}{ RIPPER } \\
\cline { 2 - 3 } $\begin{array}{c}\text { klassifiziert } \rightarrow \\
\text { korrekt } \downarrow\end{array}$ & Stau & Frei \\
\hline Stau & 1768 & 313 \\
\hline Frei & 504 & 2415 \\
\hline
\end{tabular}

Korrekt klassifiziert: 4183 (83.66 \%)

\begin{tabular}{|c|c|c|}
\multicolumn{1}{c}{ klassifiziert $\rightarrow$} & \multicolumn{1}{c}{ C4.5 } \\
korrekt $\downarrow$ & Stau & Frei \\
\hline Stau & 1731 & 350 \\
\hline Frei & 476 & 2443 \\
\hline
\end{tabular}

Korrekt klassifiziert: 4174 (83.48 \%)

Tabelle 10.4: Wahrheitsmatrizen berechneter Klassifikatoren auf einem Testset.

$$
\begin{aligned}
A & =\# A 66 \\
S & =\# \text { Seckbach } \\
\Sigma & =B+R+S+A
\end{aligned}
$$

C4.5 berechnet einen Entscheidungsbaum. Im Folgenden wird ein Ausschnit der Rückgabe gezeigt:

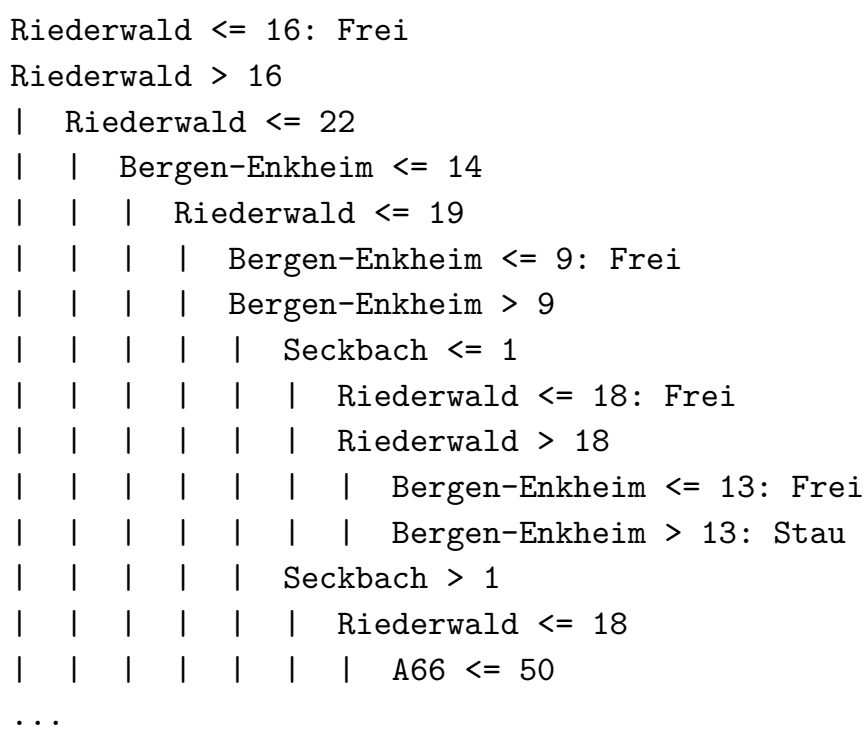

Beide Klassifikatoren funktionieren auf ungesehenen Daten. Tabelle 10.4 zeigt die korrespondierenden Wahrheitstabellen, die auf Testsets mit 5.000 Simulationsläufen generiert wurden. RIPPER erzielt eine Genauigkeit von 83, 66 \%, C4.5 83, 48 \% . Beide Klassifikatoren liefern höhere Trefferquoten, als die konstante Nutzung der wahrscheinlichsten Klasse „Frei“ (58, 38 \%).

Das gezeigte Beispiel beweist, dass mit Hilfe von Data Mining Technologien Vorhersagen über die Stauentwicklung im Messbereich getroffen werden können. Ein nächster Schritt könnte nun sein, den Zufluss auf die gestauten Gebiete frühzeitig zu minimieren. Dies könnte durch verkehrsadaptive Änderungen an den bestehen- 
den Ampelschaltungen geschehen. Ein anderer Ansatz wäre die Abschätzung der Reisedauer durch den gestauten Bereich und der Vorschlag, das $\mathrm{P}+\mathrm{R}$ Parkhaus zu nutzen, wenn diese Dauer einen Grenzwert übersteigen wird.

\subsection{Zusammenfassung und Ausblick}

Dieses Kapitel hat anhand dreier Simulationsstudien die Möglichkeit einer Kopplung eines Verkehrssimulationssystems an Maschinelle Lernverfahren demonstriert und beispielhaft Anwendungsszenarien skizziert. Es konnte gezeigt werden, dass aus einfachen Simulationsdaten Klassifikatoren erstellt werden können, die einerseits verständlich für den Anwender sind und andererseits ausreichende Klassifikationsgüten aufweisen.

Die Lesbarkeit der gelernten Regeln ist wichtig, wenn diese nicht als Blackbox angesehen, sondern praktisch umgesetzt werden können müssen. Außerdem ermöglichen nur lesbare Regeln einen Verständnisgewinn über kausale Zusammenhänge im Straßenverkehr. Aus diesem Grunde wurden Entscheidungsregeln und Entscheidungsbäume gelernt und andere Verfahren außen vorgelassen.

Ein weiteres Anwendungsgebiet maschineller Lernverfahren ist die Erkennung von Sequenzmustern. Es wird hierbei automatisiert versucht, häufig aufeinanderfolgende Situationen zu identifizieren. Ziel des Experimentes ist die Vorhersage von zukünftigen Situationen - z.B. Staus - anhand aktueller Daten. In der Folge könnten gezielte Aktionen zur Verkehrsbeeinflussung durchgeführt werden. Erste Experimente wurden in Zusammenarbeit mit Andreas Lattner durchgeführt und in Lattner, 2012 diskutiert. Im Straßengraph der Stadt Erlensee (vgl. Abbildung 13.1 auf Seite 202 wurden hierbei an verschiedenen Positionen die Verkehrsdichte und der Verkehrsfluss gemessen. Durch wiederholte Messungen konnten zeitliche Verläufe ermittelt werden. Hierfür wurde eine Implementierung $3^{3}$ des PrefixSpanAlgorithmus Pei et al., 2001 verwendet. Als Resultat wurden zahlreiche Muster ermittelt, deren Interpretation derzeit manuelle Bewertungen der Nützlichkeit voraussetzen. In der Zukunft müssen Wege gefunden werden, weniger Muster mit höherer Aussagekraft zu ermitteln. Ein weiteres Problem ist die Komplexität der gelernten Muster. Auch in einfachen Verkehrsszenarien können komplexe Regeln entstehen. Zükunftig sollte nach Möglichkeiten gesucht werden, wenige, einfache Muster zu ermitteln, die bei der Verkehrssteuerung berücksichtigt werden können.

${ }^{3}$ Es wurde $S P M F$ v0.72 von Philippe Fournier-Viger genutzt: http://www philippe-fournierviger.com/spmf/, abgerufen am 07.08.2011. 


\section{Approximation von Fußgängereffekten}

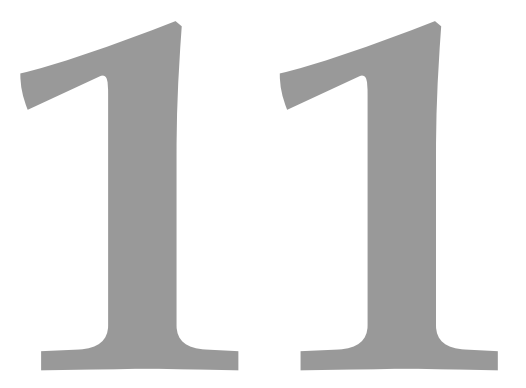

Dieses Kapitel untersucht die Möglichkeit, die Effekte, die Fußgänger auf den Straßenverkehr haben, nachzubilden. Dies hat zwei Ziele: Zum einen kann Simulationszeit eingespart werden, wenn Fußgänger nicht mehr simuliert werden müssen. Zum anderen können Verkehrssimulationsysteme, die nicht über ein Fußgängermodell verfügen, um die Effekte von Fußgängern ergänzt werden. Das Kapitel basiert auf Lattner et al., 2012 in Kooperation mit Andreas Lattner, Dimitrios Paraskevopoulos und Ingo Timm. Es gliedert sich wie folgt: Abschnitt 11.1 beschreibt eine Möglichkeit der Protokollierung von Fußgängereffekten. In Abschnitt 11.2 wird gezeigt, wie hieraus Wahrscheinlichkeitsverteilungen abgeleitet werden können, die in Abschnitt 11.3 angewendet werden. Abschnitt 11.4 fasst die Ergebnisse zusammen und gibt einen Ausblick über zukünftige Untersuchungen.

\subsection{Protokollierung von Fußgängereinflüssen}

Um die Effekte, die Fußgänger auf den Straßenverkehr haben, abzubilden, muss betrachtet werden, wann Interaktionen mit Autos und Fahrrädern auftreten. Dies geschieht ausschließlich, wenn Fußgänger Straßen überqueren. Es existieren hierfür drei Möglichkeiten: auf Zebrastreifen, an Ampeln oder bei Lücken im Verkehr (vgl. Kapitel 7.3.3). 
Straßenüberquerungen geschehen auf EIs oder NIs. Wenn eine Überquerung auf einer EI geschieht, existiert eine korrespondierende Position $p_{\mathrm{EI}}$ auf der Straße. Jede Überquerung hat eine bestimmte Dauer $d$. Zwischen zwei Überquerungen auf einer bestimmten EI oder NI liegt ein zeitliches Intervall $i$.

Wenn simulierte Fußgänger Straßen überqueren, werden Protokolleinträge für $p_{\mathrm{EI}}$, $d$ und $i$ erstellt, die der korrespondierenden EI bzw. NI zugeordnet werden. In einem ersten Experiment werden alle Überquerungen von Fußgängern protokolliert. In einem zweiten Experiment werden nur die Straßenüberquerungen berücksichtigt, bei denen Interaktionen mit Autos stattfanden. Dies geschieht, wenn ein Auto bremsen musste, um einen Unfall mit dem Fußgänger zu vermeiden.

\subsection{Lernen von Wahrscheinlichkeitsverteilungen}

Die protokollierten Zahlen werden anschließend verwendet, um dahinterliegende Wahrscheinlichkeitsverteilungen zu ermitteln. Abschnitt 11.2.1 beschreibt eine Methode, um aus einer Menge von Wahrscheinlichkeitsverteilungen diejenige auszuwählen, die die protokollierten Daten am besten abbildet. Abschnitt 11.2 .2 beschreibt einen histogrammbasierten Ansatz, der beliebige Wahrscheinlichkeitsverteilungen abbilden kann.

\subsubsection{Best-Fit Ansatz}

Der verwendete Ansatz basiert auf der Nutzung der Methode Maximum Likelihood Estimation (MLE) (siehe, z.B. LLongford, 2007). Hierfür wird das MASSPaket der freien Statistiksoftware R Project genutzt Venables und Ripley, 2002, Ricci, 2005, R Development Core Team, 2011. Es wird eine Unterscheidung zwischen Normalverteilung, Exponentialverteilung und Gleichverteilung durchgeführt. Die Approximation der Parameter für eine Gleichverteilung geschieht durch Betrachtung des Minimums und Maximums der Eingabezahlen $x=\left(x_{1}, \cdots x_{l}\right)$. Für die restlichen Wahrscheinlichkeitsverteilungen wird durch R Project eine Optimierung der Verteilungsparameter zur möglichst genauen Nachbildung von $x$ durchgeführt.

Um zu entscheiden, welche Wahrscheinlichkeitsverteilung die Eingabezahlen am besten abbildet, wird ein $\chi^{2}$-Test Steland, 2010 für alle Verteilungen durchgeführt. Die Werte von $x$ werden analog zur Bildung von Histogrammen nach der Formel von Sturges Sturges, 1926 in $n=\left\lceil\log _{2} l+1\right\rceil$ Klassen aufgeteilt. Die- 

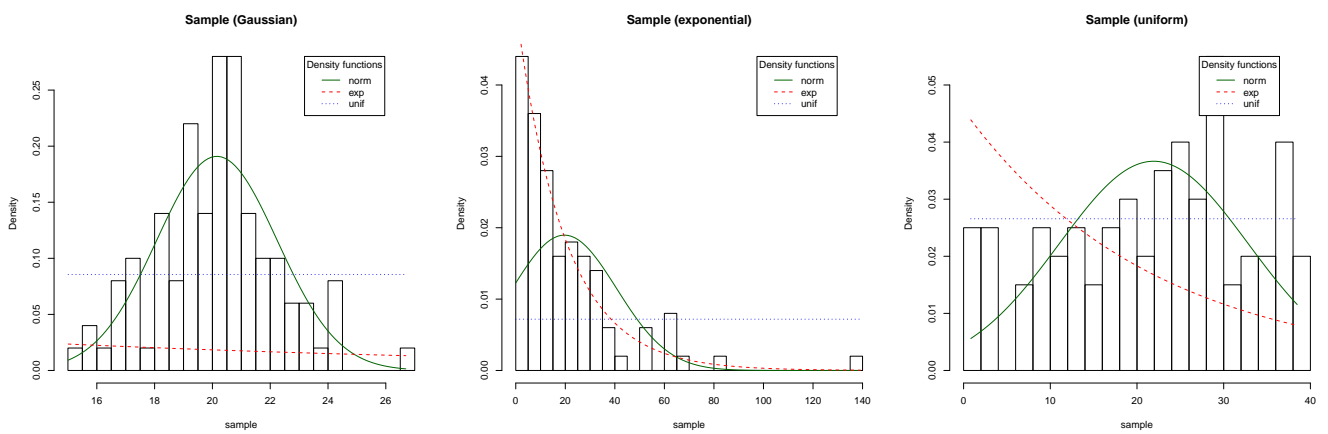

Abbildung 11.1: Wahrscheinlichkeitsverteilungsapproximation [Lattner et al., 2012

se Klassen werden zum Ähnlichkeitsvergleich mit den verwendeten Verteilungen genutzt. Es wird die Nullhypothese

$H_{0}$ : Die Merkmale $x$ und $y$ sind stochastisch unabhängig.

aufgestellt. $y$ ist die verwendete Wahrscheinlichkeitsverteilung, mit der verglichen wird. Das Resultat des $\chi^{2}$-Tests ist die Wahrscheinlichkeit $p$, dass $H_{0}$ verworfen werden kann. Unter den betrachteten Wahrscheinlichkeitsverteilungen wird diejenige verwendet, für die der maximale $p$-Wert ermittelt wurde.

Abbildung 11.1 zeigt die Histogramme verschiedener synthetischer Eingabedaten $x$ und die von R Project optimierten Wahrscheinlichkeitsverteilungen. Es ist ersichtlich, dass die verwendeten Verfahren Wahrscheinlichkeitsverteilungen nachbilden können. Dies funktioniert jedoch nur, wenn die Verteilung, die $x$ zu Grunde liegt, eine der verwendeten Verteilungen ist.

\subsubsection{Histogrammbasierter Ansatz}

Dieser Abschnitt beschreibt eine einfache Methode, beliebige Wahrscheinlichkeitsverteilungen nachzubilden. Die Werte von $x$ werden in $n=$ $\max \left(10\right.$, round $\left.\left(10 \times \log _{10}(l)\right)\right)$ Klassen separiert. Das entstehende Histogramm wird normalisiert. Jede Klasse hat nun eine relative Auftrittshäufigkeit. Hieraus wird ein kumuliertes Histogramm berechnet. Abbildung 11.2 verdeutlicht die Vorgehensweise.

Eine gleichverteilte Zufallszahl $\alpha$ im Intervall $[0,1]$ kann nun genutzt werden, um die Position auf der $y$-Achse zu bestimmen. Der korrespondierende $x$-Wert $f(\alpha)$ ist der Funktionswert der Verteilung. 


\section{Histogram of sample}

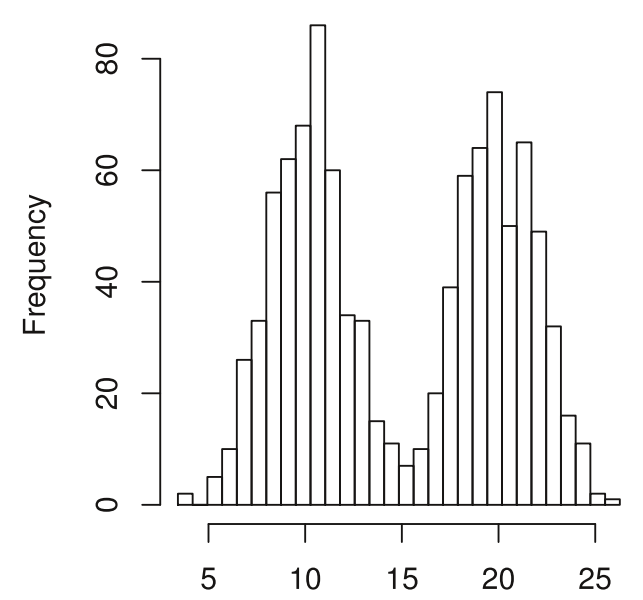

Cumulative distribution function

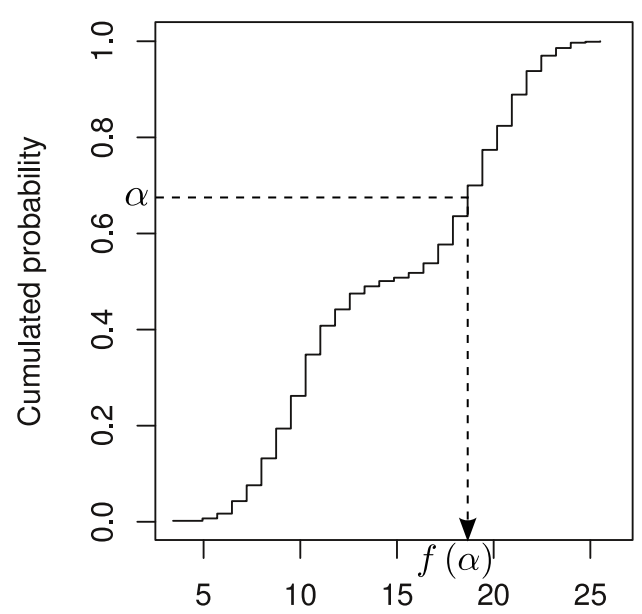

Abbildung 11.2: Histogrammbasierte Wahrscheinlichkeitsverteilungsapproximation, adaptiert nach Lattner et al., 2012. Bildung eines kumulierten Histogramms.

\subsection{Anwendung}

Die beschriebene Methode wird in einer Simulation des in Abbildung 9.9 auf Seite 149 gezeigten Kartenausschnitts der Stadt Hanau angewendet. Nach einer Einschwingphase von 1.000 Iterationen folgt eine Messphase von 50.000 Iterationen. Die Anzahl an Verkehrsteilnehmern wird konstant auf 2.500 gehalten (immer wenn ein Verkehrsteilnehmer seine Zielposition erreicht hat, wird ein neuer Verkehrsteilnehmer erstellt). Die Verteilung der Verkehrsteilnehmertypen ist: 33 \% Autos, 7 \% Fahrräder und $60 \%$ Fußgänger.

Während der Messphase werden die in Abschnitt 11.1 beschriebenen Kenngrößen Position der Überquerung, Dauer der Überquerung und zeitlicher Abstand seit letzter Überquerung für alle Straßenüberquerungsvorgänge von Fußgängern protokolliert. Die dahinterliegenden Wahrscheinlichkeitsverteilungen werden mit beiden in Abschnitt 11.2 beschriebenen Verfahren approximiert.

In einem zweiten Simulationslauf werden identische Kopien der Autos und Fahrräder des Messlaufes verwendet. Anstelle der Fußgänger werden nun Dummies platziert, die auf der Straße nach den gelernten Verteilungen erscheinen und Straßennutzer zum Abbremsen zwingen. Es werden die Reisedauern der simulierten Autos verglichen. Das Verfahren wird 100 mal durchgeführt. Abbildung 11.3 zeigt die Ergebnisse. 


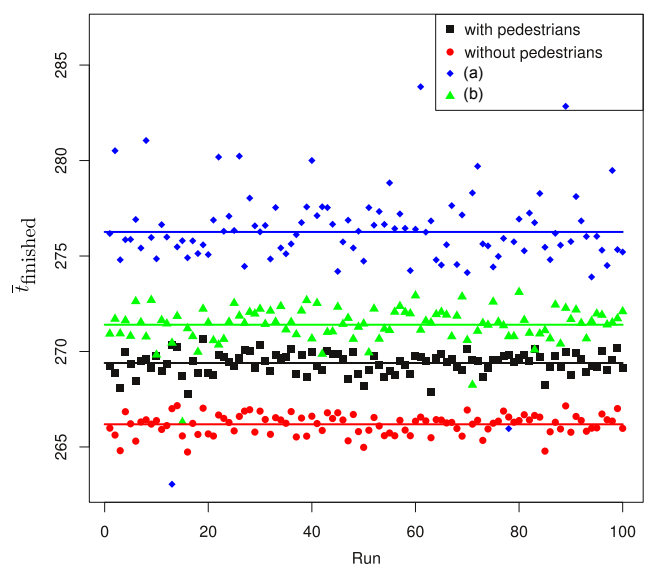

(a) Best-fit

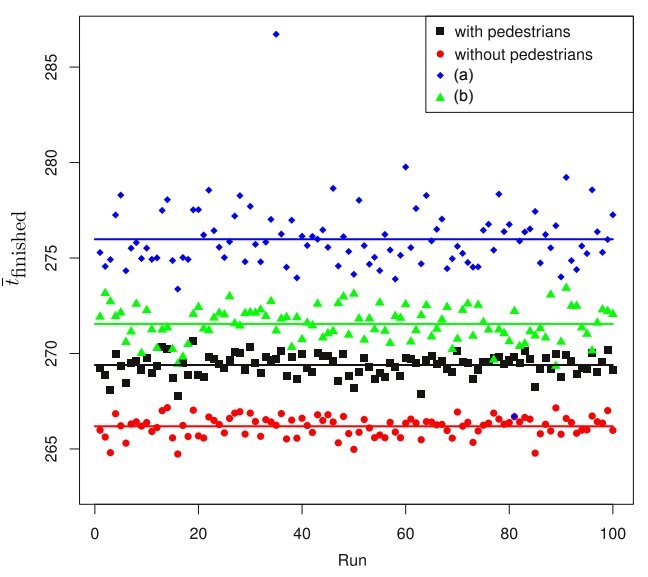

(b) Histogram based

Abbildung 11.3: Vergleich der Reisedauern von Autos $\bar{t}_{\text {finished }}[s]$ bei unterschiedlicher Handhabung von Fußgängern. Linien zeigen Mittelwerte.

\begin{tabular}{|c|c|c|}
\hline & \multicolumn{2}{|c|}{$\bar{t}_{\text {finished }}[s]$} \\
\hline & Best-fit & Histogramm baisert \\
\hline (a) & 276.26 & 275.98 \\
\hline (b) & 271.40 & 271,55 \\
\hline mit Fußgängern & & 269.34 \\
\hline ohne Fußgänger & & 266.19 \\
\hline
\end{tabular}

Tabelle 11.1: Mittlere Reisedauern für Autos und Fahrräder.

Fall (a) zeigt die Ergebnisse, wenn für jede Straßenüberquerung eines simulierten Fußgängers ein Protokolleintrag erstellt wurde. In Fall (b) wurden nur die Überquerungen berücksichtigt, bei denen ein Auto abbremsen musste, um entweder einen Unfall bei zu geringer Lücke im Verkehr zu vermeiden oder die Vorfahrt des Fußgängers zu berücksichtigen. Fall (a) führt zu einer Übersteuerung des Dummyeinflusses, da nicht alle Straßenüberquerungen mit direkten Wechselwirkungen mit dem Straßenverkehr verbunden sind. Zu Vergleichszwecken wurde auch ohne Fußgänger und Dummies simuliert. Die in Abbildung 11.3 gezeigten Reisedauern verdeutlichen, dass die Simulationsergebnisse in Fall (b) näher an den Ergebnissen unter Berücksichtigung des Fußgängermodells liegen, als wenn Fußgänger einfach missachtet werden. Aus der Abbildung ist kein Unterschied zwischen dem Best-fit und dem Histogrammbasierten Ansatz zu erkennen. Tabelle 11.1 vergleicht die mittleren Ergebnisse.

Die mittleren Differenzen von $\bar{t}_{\text {finished }}$ sind für (b) 2,06 s (Best-fit) und 2,21 s (Histogramm basiert). Dieses Ergebnis überrascht, denn die Stärke des Histogramm basierten Ansatzes, beliebige Wahrscheinlichkeitsverteilungen modellieren zu kön- 
nen, kann in diesem Fall nicht ausgespielt werden. Verglichen mit der Abweichung von 3,15 s für die Simulationsläufe ohne Berücksichtigung von Fußgängern, stellen die Simulationsergebnisse mit Dummies eine Verbesserung dar. Die Kernaussage von Abbildung 11.1 ist, dass die Simulationsergebnisse unter Berücksichtigung von Fußgängern zwischen den Ergebnissen ohne Fußgängern und Fall (b) liegen. Somit können diese Ergebnisse zur Eingrenzung genutzt werden.

\subsection{Diskussion und Ausblick}

In diesem Kapitel wurde ein Verfahren diskutiert, um die Einflüsse, die Fußgänger auf den Straßenverkehr haben, in Wahrscheinlichkeitsverteilungen zu überführen und anschließend ohne Nutzung eines Fußgängermodells ein urbanes Verkehrsszenario simulieren zu können. Unter den genannten Gegebenheiten konnte die Simulationsgeschwindigkeit etwa verdoppelt werden. Die Approximationsgüte ist höher, als in den Fällen, in denen Fußgänger schlicht ignoriert werden.

In der Zukunft ist zu prüfen, ob eine Erweiterung der Dummyfunktionen um ein Straßenüberquerungsverhalten weitere Verbesserungen der Approximationsgüte ergeben würden. Dummies sollten nicht plötzlich erscheinen, sondern den $A F$ eines Fußgängers abbilden und sich bei Überquerungen identisch verhalten. Dies würde weiterhin zu einer Erhöhung der Simulationsgeschwindigkeit führen, da keine Routenplanungen für Fußgänger berechnet werden müssten und auch das mikroskopische Fußgängermodell bei Überquerungen an NIs eingespart werden könnte.

Ein grundlegender Nachteil des Verfahrens ist, dass keine dynamischen Änderungen der Verkehrssituation berücksichtigt werden können. Für statische Szenarien könnte das beschriebene Verfahren jedoch genutzt werden, um einerseits bei großen Simulationsszenarien Simulationszeit einzusparen und andererseits Simulationssysteme, die nicht über ein Fußgängermodell verfügen, einfach um die Effekte von Fußgängern zu ergänzen. In diesem Fall würde eine Analyse des Simulationsszenarios mittels MAINSIM genügen, um anschließend die gewonnenen Wahrscheinlichkeitsverteilungen in beliebige Dummystrukturen des Zielsimulationssystems zu überführen. Auf diese Weise könnten die individuellen Stärken anderer Systeme, z.B. ein detaillierteres Automodell, weiterhin genutzt werden und dennoch Fußgänger Berücksichtigung finden. 


\section{Nonkonformismus}

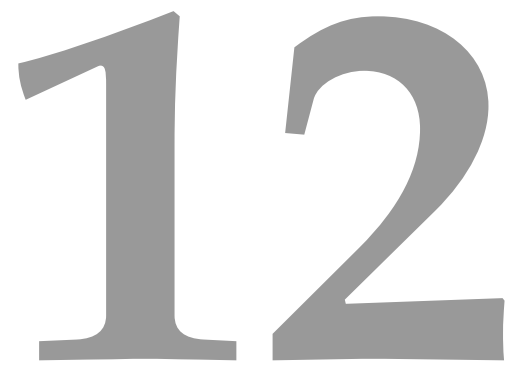

Die Betrachtung relevanter Literatur zum nicht regelkonformen Verhalten von Verkehrsteilnehmern im Straßenverkehr (vgl. Abschnitte 3.2, 3.3.2, 3.3.3 und 3.4.2 hat gezeigt, dass Verkehrsteilnehmer sich aggressiv verhalten können. Häufig ist die Motivation dahinter, ihre eigene Reisedauer zu verkürzen.

In Ehlert und Rothkrantz, 2001 wird ein mikroskopisches Verkehrsmodell für Autos besprochen, welches reaktive Agenten einsetzt. Das Ziel der Arbeit ist die Modellierung menschlichen Fahrverhaltens. Die Agenten können Regeln missachten. Beispiele sind die Ignorierung von Geschwindigkeitsbegrenzungen, Einbahnstraßen und dem Rechtsfahrgebot. Fahrer, die höhere Geschwindigkeiten bevorzugen, werden mit höheren Wahrscheinlichkeiten modelliert, langsamere Autos zu überholen. Das verwendete Verkehrsmodell wurde in der Arbeit jedoch weder beschrieben, noch evaluiert. Dennoch sind die grundlegenden Regelbruchaktionen interessante Studienfelder.

In Yuhara und Tajima, 2006 wird die Notwendigkeit der Nutzung von agententenbasierten Verkehrssimulationssystemen im Hinblick auf Verkehrssicherheit im Feld der Fahrassistenzsysteme diskutiert. Die Entscheidungen der Agenten berücksichtigen hierbei individuelle Einstellungen für die Spurwahl, die Lückenakzeptanz, Sicherheitsabstände und andere Werte. Die Entscheidung bei einer von grün auf gelb umspringenden Ampel zu beschleunigen oder zu bremsen, wird durch Wahrscheinlichkeitsverteilungen modelliert. 
In Baek et al., 2009 wird ein zweidimensionales Zellularautomatenmodell zur Modellierung von Fußgängerbewegungen diskutiert. Wenn zwei Fußgänger aufeinander zugehen, ist die normgerechte Handlung beider Fußgänger, ein Ausweichmanöver einzuleiten. In der Arbeit wird gezeigt, dass unter bestimmten Konstellationen ein maximaler Verkehrsfluss erzielt wird, wenn sich ein Teil der Fußgänger nicht an diese Norm hält.

In diesem Kapitel werden drei Fallstudien zur Thematik Nonkonformismus bezüglich Verkehrsregeln diskutiert. Es wird gezeigt, wie nonkonformes Verhalten mittels MAINSIM modelliert werden kann. Für jeden der drei Verkehrsteilnehmertypen Auto, Fahrrad und Fußgänger wird eine aggressive Verhaltensweise simuliert. Es wird untersucht, welchen Vorteil der aggressive Fahrer erhält und welche Nachteile die restlichen Verkehrsteilnehmer erdulden müssen.

Dieses Kapitel basiert auf der Arbeit Dallmeyer et al., 2012c (in Kooperation mit Andreas Lattner und Ingo Timm). In den Unterabschnitten 12.1 bis 12.4 werden die Fallstudien besprochen und deren Ergebnisse diskutiert. Abschließend wird eine Zusammenfassung und ein Ausblick auf weitere Untersuchungsmöglichkeiten gegeben (Unterabschnitt 12.5).

\section{1 Überholverbot für $L k w$}

Das erste Experiment in diesem Kapitel findet auf einer zweispurigen Autobahn mit $20 \mathrm{~km}$ Länge und einer Geschwindigkeitsbegrenzung von $55 \mathrm{~m} / \mathrm{s}$ statt. Die Autobahn verläuft im Kreis. Es existieren keine Auf- oder Abfahrten. Dies entspricht dem allgemeinem Aufbau zur Untersuchung von Verkehrsmodellen im Autobahnbereich (vgl. Nagel und Schreckenberg, 1992, Emmerich und Rank, 1997, Kerner et al., 2002, Zhu et al., 2009).

Es wird ein Überholverbot für Lkw gesetzt. Lkw dürfen somit nur die rechte Spur nutzen. Es werden 250 Verkehrsteilnehmer (85 \% Pkw, 15 \% Lkw) simuliert. Es werden 100 Gruppen von jeweils 250 individuellen Verkehrsteilnehmern generiert. Jeder Simulationslauf besteht aus einer Einschwingphase von 900 Iterationen und einer Messphase von 18.000 Iterationen (5 Stunden Realzeit). Die durchschnittlichen Geschwindigkeiten der Pkw, der Lkw und beider Gruppen gemeinsam werden erhoben. Als Ergebnis werden die jeweiligen Werte über alle 100 Gruppen gemittelt.

Für jede Gruppe werden 11 Simulationsexperimente durchgeführt. Im ersten Ex- 
Cars

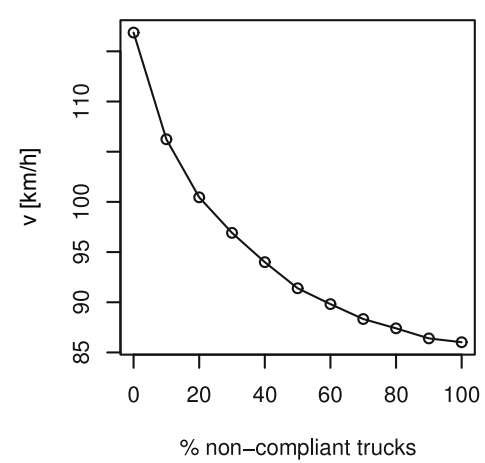

Trucks

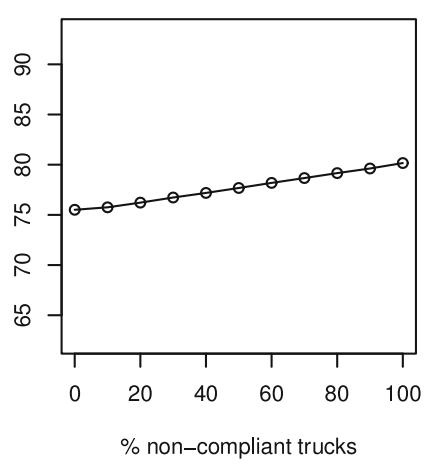

Total

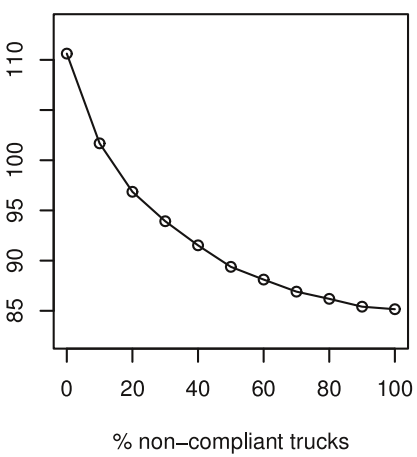

Abbildung 12.1: Simulationsergebnisse: Autobahn

periment ist die Menge der Lkw, die sich nicht an das Überholverbot halten $0 \%$. In Schritten von $10 \%$ wird diese immer weiter erhöht. Pro Experiment werden 5 Replikationen mit identischen Einstellungen zur Mittelung der Ergebnisse durchgeführt. Es werden somit identische Verkehrsteilnehmer in identischen Experimenten simuliert und pro Einstellung ändert sich jeweils nur der Anteil an nicht regelkonform fahrenden Lkw. Auf diese Weise entstehen vergleichbare Ergebnisse. Abbildung 12.1 zeigt den Verlauf der drei Messgrößen bei steigender Anzahl an nonkonformen Lkw.

Die Lkw können zwischen $0 \%$ und 100 \% Nonkonformität ihre Geschwindigkeit um 4,66 km/h erhöhen. Auf der anderen Seite verringert sich die Geschwindigkeit der Pkw in diesem Rahmen um 30,85 km/h. Schon die geringe Menge von $10 \%$ nonkomformen Lkw führt zu einer Verringerung der Pkw-Geschwindigkeit um ca. $10,63 \mathrm{~km} / \mathrm{h}$.

Die Geschwindigkeitsverringerung der Pkw resultiert aus Überholmanövern von Lkw, die im Vergleich zu den Pkw sehr langsam fahren. Die Lkw profitieren, wenn sie sich nicht an das Überholverbot halten, da sie nicht mehr dauerhaft hinter langsameren Lkw bleiben müssen. Die Gesamtdurchschnittsgeschwindigkeit in diesem Beispiel zeigt jedoch deutlich, dass eine Nichteinhaltung des Überholverbots für Lkw insgesamt negative Folgen für den Autobahnverkehr hat. Die Verringerung der Geschwindigkeit ist im Maximum ca. 25, $46 \mathrm{~km} / \mathrm{h}$. 


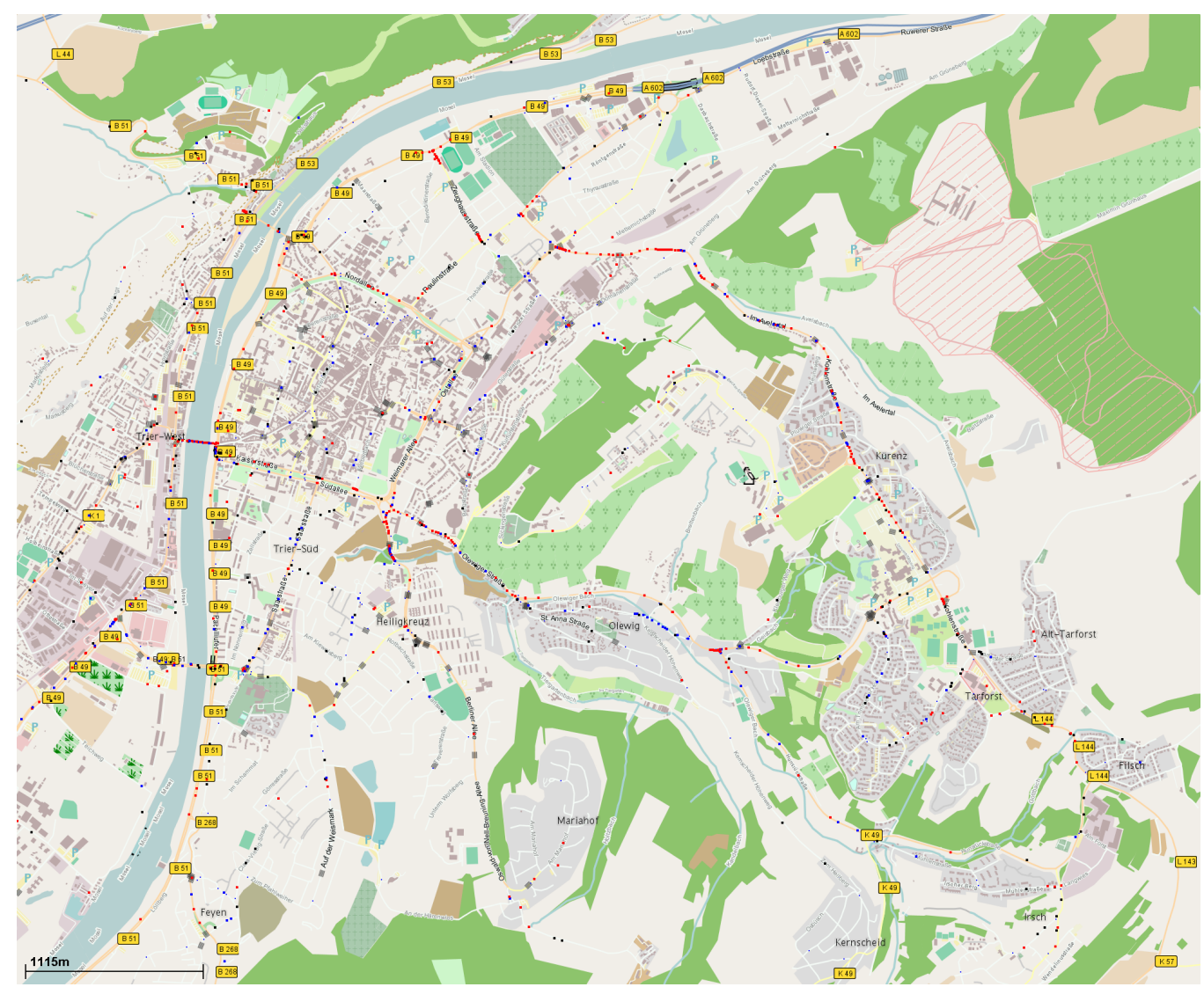

Abbildung 12.2: Kartenausschnitt Trier. Der Graph besteht aus 4.495 NIs und 5.386 EIs und hat eine Gesamtlänge von $485 \mathrm{~km}$.

\subsection{Aufbau der urbanen Studien}

Die Untersuchungen über Nonkonformismus von Fahrrädern und Fußgängern finden in einer urbanen Umgebung statt. Es wird der in Abbildung 12.2 gezeigte Kartenausschnitt der Stadt Trier 1 zur Simulation verwendet.

In den folgenden Unterabschnitten werden jeweils 1.000 Verkehrsteilnehmer (50\% Pkw, $25 \%$ Fahrräder und $25 \%$ Fußgänger) simuliert. Quell- und Zielposition werden zufällig bestimmt. $50 \%$ der Verkehrsteilnehmer nutzen vorberechnete Routen, $50 \%$ die probabilistische Routingmethode. Es entsteht eine Verteilung des Verkehrs in der Stadt mit hohen Verkehrsdichten an zentralen Stellen.

Der grundlegende Simulationsablauf verändert sich gegenüber dem vorangegan-

\footnotetext{
${ }^{1}$ Grenzen in WGS84: [6.32: 8.44, 49.24 : 50.47]
} 
Cars

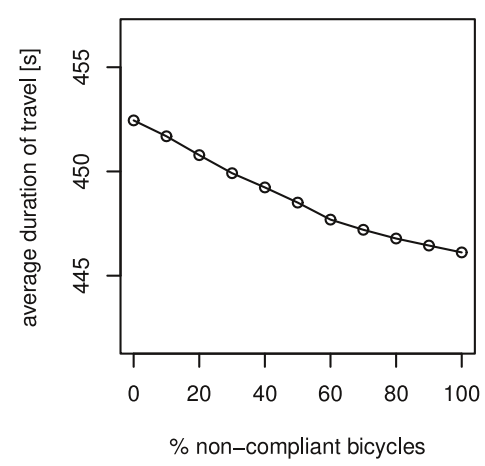

Bicycles

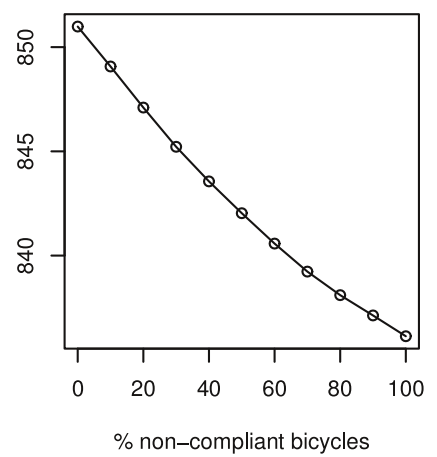

Pedestrians

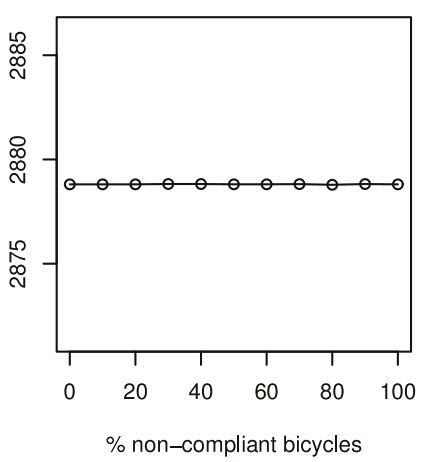

Abbildung 12.3: Simulationsergebnisse: Trier: Fahrrad

genem Experiment nicht. Es werden jeweils identische Verkehrsteilnehmer bei äquidistanter Erhöhung der Nonkonformitätsrate einer Verkehrsteilnehmergruppe simuliert. Als Vergleichsgröße wird die mittlere Reisedauer der verschiedenen Verkehrsteilnehmergruppen betrachtet.

\subsection{Drängelnde Fahrradfahrer}

Im Stadtverkehr ist häufig zu beobachten, dass Fahrradfahrer an roten Ampeln wartende Autos überholen. Dieses Verhalten wird im Rahmen dieser Arbeit als aggressiv eingestuft. Ein nonkonformes Fahrrad drängelt an Ampeln, ein konformes hält seine Position in der Schlange der wartenden Verkehrsteilnehmer. Es ist davon auszugehen, dass drängelnde Fahrradfahrer einen zeitlichen Vorteil erzielen. Für die restlichen Verkehrsteilnehmer könnte das Verhalten jedoch negativ sein, da beispielsweise ein Auto ein Fahrrad mehrmals überholen muss und somit auch mehrfach durch das Fahrrad aufgehalten werden kann. In der Realität tritt dieser Effekt durch ein potentielles Unfallrisiko bei Überholmanövern in den Hintergrund. Abbildung 12.3 zeigt die Simulationsergebnisse.

Fahrräder haben einen Vorteil, wenn sie an Ampeln drängeln. Die Reduzierung ihrer Reisedauer ist 14,85 s, wenn alle Fahrräder nonkonform sind. Insgesamt ist dies nicht viel Ersparnis im Vergleich zur Gesamtfahrtdauer $(\approx 1,75 \%)$, da die meiste Zeit der Fahrt nicht vor Ampeln verbracht wird.

Es ist kein Einfluss auf die Reisedauern von Fußgängern ersichtlich, da der Regelbruch der Fahrräder die möglichen Interaktionen mit Fußgängern nicht beeinflusst. Das überraschende Ergebnis ist, dass Autos in diesem Szenario keine Nachtei- 
le durch drängelnde Fahrräder haben. Autos sparen sogar im Extremfall 6,33 s $(\approx 1,40 \%$ der Reisedauer).

Eine Erklärung für dieses Ergebnis könnte sein, dass Autos und Fahrräder unterschiedliche Routingcharakteristiken haben. Autos bewerten bei einer Kantenbewertung die geschätzte Reisedauer auf der jeweiligen EI und berücksichtigen explizit die erlaubte Höchstgeschwindigkeit. Fahrräder können Geschwindigkeiten über $30 \mathrm{~km} / \mathrm{h}$ selten fahren und bewerten daher die Länge der EI. An einer Ampelkreuzung könnten daher Autos und Fahrräder häufig in unterschiedliche Richtungen abbiegen. Der Effekt, dass ein Auto ein Fahrrad mehrmals überholen muss, bliebe in diesem Fall aus. Zusätzlich verkürzt ein drängelndes Fahrrad die Warteschlange vor der Ampel. Dies kann dazu führen, dass mehr Autos die Ampel in einer Grünphase passieren können und somit insgesamt kürzere Reisedauern erreichen.

Das Gesamtergebnis ist, dass drängelnde Fahrradfahrer ihre eigenen Reisedauern reduzieren können und keine negativen Einflüsse auf andere Verkehrsteilnehmergruppen haben.

\subsection{Aggressive Fußgänger}

Dieses Experiment ist identisch zur vorangegangenen Studie. Anstelle der Fahrräder sind nun Fußgänger aggressiv. Wenn ein Fußgänger $f$ aggressiv ist, wird sein $A F=-3$ s gesetzt (vgl. Abschnitt 7.3.3). Dies führt dazu, dass $f$ eine Straße auch dann überquert, wenn die zeitliche Lücke im Verkehr $3 \mathrm{~s}$ zu klein ist. Straßenverkehrsteilnehmer müssen in diesem Fall bremsen, um eine Kollision zu vermeiden. Zusätzlich ignoriert $f$ rote Ampeln und läuft an Kreuzungen einfach über die Straße. Dieser Effekt ist vergleichbar zur Wahl von $A F$. Die Ignorierung von Ampeln führt dazu, dass $f$ auch keine Ampel drückt. Die Simulationsergebnisse werden in Abbildung 12.4 gezeigt.

Die größte Spannweite zwischen minimaler und maximaler Reisedauer ist 0,31 s bei der Gruppe der Autos. Dies entspricht einer Abweichung von $\approx 0,07 \%$. Das Hauptergebnis dieses Experiments ist daher, dass der Einfluss von aggressiven Fußgängern gering ist. Der Verlauf der Reisedauer der Gruppe der Fußgänger zeigt einen klaren Trend zur Verringerung bei Nonkonformismus. Der Effekt ist jedoch gering, da nur sehr wenig Zeit zur Überquerung von Straßen verwendet und die meiste Zeit auf Gehwegen gelaufen wird. Zusätzlich hat aggressives Verhalten nur dann einen Einfluss, wenn zum entsprechenden Zeitpunkt ein anderer Verkehrsteil- 

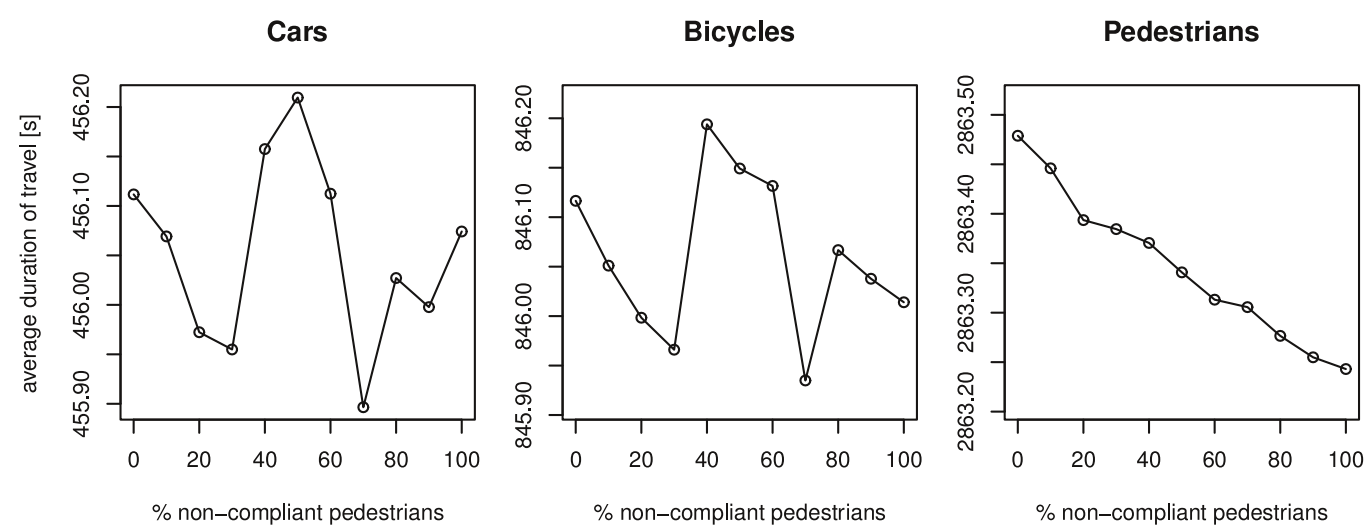

Abbildung 12.4: Simulationsergebnisse: Trier: Fußgänger

nehmer in der Nähe des Fußgängers ist. Ein Teil der aggressiven Überquerungen wäre somit ohne Aggressivität identisch verlaufen. Für die Gruppen der Autos und Fahrräder ist kein klarer Trend erkennbar.

\subsection{Zusammenfassung und Ausblick}

In diesem Kapitel wurden drei Untersuchungen zum Einfluss von RegelNonkonformismus durchgeführt. Es konnte gezeigt werden, dass MAINSIM für derartige Untersuchungen geeignet ist.

Im ersten Experiment wurde untersucht, welchen Einfluss Lkw haben, die sich auf einer Autobahn nicht an ein Lkw-Überholverbot halten. Der Einfluss war stark negativ für die simulierten $\mathrm{Pkw}$, jedoch positiv für die nonkonformen Lkw.

In einem zweiten Experiment wurde ein Straßengraph der Stadt Trier genutzt, um zu untersuchen, welchen Einfluss Fahrräder haben, die sich an roten Ampeln nach vorne drängeln. Entgegen den Erwartungen wurden Pkw, auf ihre gesamte Reisedauer gesehen, dadurch nicht behindert.

Das dritte Szenario untersuchte im selben Straßengraph den Einfluss aggressiver Fußgänger, die ohne Berücksichtigung des Straßenverkehrs Straßen überqueren und somit $\mathrm{Pkw}$ zu starken Bremsungen zwingen, um Unfälle zu vermeiden. Für keinen der betrachteten Verkehrsteilnehmertypen änderten sich die Reisedauern merklich - auch nicht für die Fußgänger, die in der Realität ihr Leben riskiert hätten.

Für die Szenarien innerhalb der Stadt Trier wurde realistisches Kartenmaterial 
genutzt. Um die lokalen Verkehrsströme adäquat abbilden zu können, müssen in der Zukunft QZM für die verschiedenen Verkehrsteilnehmertypen verwendet werden. Die derzeitigen Ergebnisse können daher nicht im Hinblick auf ihre absoluten Werte betrachtet werden.

In der Zukunft sollte genauer untersucht werden, warum sich die Reisezeiten von Autos verringern, wenn Fahrräder drängeln. Es muss geprüft werden, wie häufig Pkw Fahrräder mehrfach überholen müssen, wenn diese an den Ampeln drängeln und welchen zeitlichen Verlust die Pkw dadurch erfahren. Bei der Betrachtung des Stands der Forschung wurde in Abschnitt 3.2 diskutiert, dass Fahrräder teilweise rote Ampeln gänzlich missachten. Diese Form der Aggressivität wurde innerhalb von MAINSIM bisher nicht modelliert. Eine Untersuchung dieses Verhaltens sollte in einer Folgestudie durchgeführt werden.

Das modellierte aggressive Verhalten der Fußgänger soll einen Extremfall aufzeigen. Es erscheint jedoch unrealistisch, dass Fußgänger den Straßenverkehr ignorieren, da die damit verbundenen Gefahren zu groß erscheinen. Zur exakteren Untersuchung würden jedoch weitere Daten von Feldstudien benötigt. Die gewählte Modellierung ermöglichte die Untersuchung, ob tendenziell ein Einfluss dieses Verhaltens auf den Straßenverkehr zu vermuten ist. Nach der durchgeführten Studie ist dies zu verneinen.

Dieses Kapitel schließt mit einer Auflistung weiterer Fragestellungen, die in der Zukunft untersucht werden könnten:

- In Abschnitt 10.2.5 wurde eine Anzeigetafel verwendet, die ein Linksfahrgebot setzte, um Autos von einer Zubringerstraße den Eintritt auf eine Autobahn zu vereinfachen. Wie robust ist dieses System bezüglich Autos, die das Linksfahrgebot ignorieren?

- Geschwindigkeitsbegrenzungen im urbanen Bereich können zu mehr Verkehrssicherheit und einer Verkürzung von Reisedauern führen. Auf Autobahnen wird auf diese Weise der Verkehr homogenisiert. Werden die Vorteile von Geschwindigkeitsbegrenzungen weiterhin erzielt, wenn sich nur noch ein bestimmter Anteil der Verkehrsteilnehmer daran hält?

- Verschiedene Fahrer halten unterschiedliche Sicherheitsabstände. Sehr aggressive Fahrer können Unfälle haben; sehr vorsichtige können Staus hinter sich hervorrufen. Welche Intensität an Aggressivität ist effizient für ein Auto und für den restlichen Verkehr? 
- Welchen Einfluss hat aggressives Verhalten auf Unfälle im Straßenverkehr?

- Wunschgeschwindigkeiten variieren von Fahrer zu Fahrer. Auf einer Autobahn seien drei Spuren vorhanden. Die rechte Spur wird hauptsächlich von Lkw genutzt. Die mittlere Spur wird meist von langsamen Pkw genutzt, die Lkw überholen. Die linke Spur wird von schnellen Pkw genutzt. Je mehr langsame Pkw auf der mittleren Spur fahren, desto mehr Pkw müssen auf die linke Spur ausweichen, um die langsamen Pkw zu überholen. Dies sollte zu einer Verlangsamung des Verkehrs auf der linken Spur führen, da dort nun nicht mehr ausschließlich schnelle Fahrzeuge fahren. Die Menge an Autos auf der linken Spur steigt immer weiter, je langsamer die Geschwindigkeit auf der mittleren Spur wird. Es stellt sich folgende Frage: Wie viele langsame Pkw verträgt der Straßenverkehr? 



\section{Ameisen-inspirierte Routingmethode}

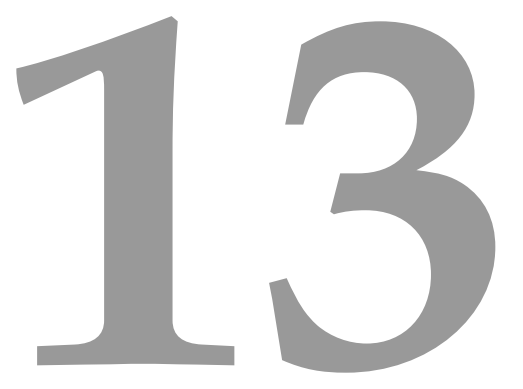

Dieses Kapitel untersucht eine Ameisen-inspirierte Routingmethode, die zu einer effizienteren Nutzung der Verkehrsinfrastruktur beitragen kann. Es basiert auf der Arbeit [Dallmeyer et al., 2012d, die in Kooperation mit René Schumann, Andreas Lattner und Ingo Timm entstanden ist. Die gezeigten Ergebnisse basieren jedoch auf einer neueren Version von MAINSIM, bei der die Analyse des Straßengraphen weiter verfeinert wurde. Das beschriebene Routingverfahren wurde als zusätzliche Erweiterung um die Möglichkeit des Umplanens ergänzt.

Der folgende Abschnitt 13.1 gibt einen Überblick über den relevanten Kontext und die Einordnung dieses Kapitels darin. In Abschnitt 13.2 wird eine Erweiterung einer bestehenden Routingmethode besprochen, um sie durch Ameisen-inspirierte Verhaltensweisen zu ergänzen. Die dabei entstehenden Parameter müssen optimiert werden. Die Vorgehensweise hierfür wird in Abschnitt 13.3 skizziert. Abschnitt 13.4 vergleicht den neuen Routingansatz mit einer Auswahl an bestehenden Verfahren in unterschiedlichen Szenarien. Dieses Kapitel schließt mit einer Diskussion und einem kurzen Ausblick in Abschnitt 13.5 . 


\subsection{Kontextuelle Einordnung}

Ein wichtiger Grundbestandteil bei der Stauentwicklung ist eine Verkehrsdichte, die einen Schwellwert überschreitet, der von Straße zu Straße variieren kann. Einen Überblick über die Stauentstehung geben Kerner und Rehborn, 1997 und Kerner, 2009]. Wenn es möglich wäre, Autos frühzeitig umzuleiten, um den Verkehr effizienter im Straßennetz zu verteilen, könnte die Staugefahr gesenkt werden. Fahrzeug-Fahrzeug-Kommunikation (V2V) und Fahrzeug-InfrastrukturKommunikation (V2I) bilden gemeinsam den Komplex V2X. Die Grundidee ist hierbei, dass Autos miteinander und mit der Infrastruktur mittels Funksignalen kommunizieren können und Informationen über die aktuelle Verkehrslage senden und empfangen können. Unter Annahme dieser Fähigkeiten lassen sich neuartige Routingmethoden entwickeln.

Ameisenalgorithmen wurden entwickelt, um heuristische Lösungen für komplexe Optimierungsprobleme zu ermitteln Dorigo und Blum, 2005. In Alves et al., 2010 wird ein Ant Colony Optimization (ACO) Algorithmus verwendet, um in einer zentralen Verkehrsplanungsstation Reisedauern auf simulierten Straßen zu verarbeiten und Routenvorschläge für simulierte Autos zu bestimmen. Autos werden hierbei zentral gerouted und haben keine individuellen Planungsmöglichkeiten.

In Narzt et al., 2010 wird die Notwendigkeit dezentraler Steuerungsmechanismen thematisiert. Autos werden zu intelligenten Einheiten, die miteinander und mit der Umgebung kommunizieren können. Autos verhalten sich analog zu Ameisen: Sie hinterlassen Pheromonspuren auf ihrem Weg. Die Pheromonintensität wird ähnlich zum Verhalten von Ameisen genutzt, um Routen zu planen. Hohe Pheromonkonzentrationen deuten auf hohe Verkehrsdichten und somit lange Durchfahrtdauern auf der betreffenden Straße hin. Diese Informationen können bei der Routenplanung, z.B. mittels A* berücksichtigt werden. Der beschriebene Ansatz nutzt häufige Umplanungsschritte, damit simulierte Autos sich wechselnden Pheromonkonzentrationen anpassen können. Dieser Schritt ist für große Szenarien mit mehreren Tausend Autos und Graphen mit mehreren Zehntausend Knoten nicht mehr umsetzbar. 


\subsection{Erweiterte Routingmethode}

Um Pheromonkonzentrationen bei der Routenplanung zu berücksichtigen, wird der $\mathrm{A}^{*}$-Algorithmus erweitert. Die Distanzfunktion zwischen zwei Knoten $N I_{a}$ und $N I_{b}$ über die Kante EI wird definiert als:

$$
\begin{aligned}
d\left(N I_{a}, N I_{b}\right) & =\frac{\text { length }(\mathrm{EI})}{v^{*}} \\
v^{*} & =\left(1-\mathrm{EI}_{s i}^{d i r}\right) \cdot \mathrm{EI}_{v M a x}
\end{aligned}
$$

Die Schätzung der fahrbaren Geschwindigkeit auf EI wird durch die erlaubte Geschwindigkeit $\mathrm{EI}_{v M a x}$ und die Pheromonstärke (smell intensity si) $\mathrm{EI}_{s i}^{d i r}$ in Fahrtrichtung dir beeinflusst. Der Wertebereich von $\mathrm{EI}_{s i}$ ist $[0 \cdots 1]$. Der Wertebereich von $v^{*}$ ist somit $\left[0 \cdots \mathrm{EI}_{v M a x}\right]$. Die Pheromonkonzentration wird in jedem Simulationsschritt für jede EI nach Gleichung 13.2 angepasst.

$$
\mathrm{EI}_{s i}^{d i r}=\min \left(\max \left(\mathrm{EI}_{s i}^{d i r}-\kappa+\vartheta \cdot \operatorname{dens}\left(\mathrm{EI}^{d i r}\right), 0\right), 1\right)
$$

Es findet eine Absorption um den Subtrahenden $\kappa$ statt. Die aktuelle Verkehrsdichte $[0 \cdots 1]$ auf der korrespondierenden EI erhöht den Wert von $\mathrm{EI}_{s i}$ und wird dabei durch $\vartheta$ gewichtet. Eine Straße mit hoher Verkehrsdichte wird somit hohe Werte von $\mathrm{EI}_{s i}$ erhalten und umgekehrt. Gleichung 13.1 führt zu einer vermehrten Nutzung von Straßen, die geringe Werte von $\mathrm{EI}_{s i}$ aufweisen. Dieser Effekt sollte eine bessere Ausnutzung des vorhandenen Straßennetzes ermöglichen. Der Zufluss auf einen Stau wird dadurch abgemildert werden. Dies führt zu einer schnelleren Auflösung des Staus (vgl. Lee und Kim, 2011). Das beschriebene Verfahren ist ein Ameisen-inspiriertes Routingverfahren (AIR).

\subsection{Parameterbestimmung}

Die beiden Parameter des Verfahrens, $\kappa$ und $\vartheta$ werden mittels Simulated Annealing (vgl. z.B. Lee und El-Sharkawi, 2005) optimiert. Simulated Annealing wird verwendet, da es sich für komplexe Probleme mit möglichen lokalen Optima eignet. Zur Optimierung werden Simulationsläufe in dem in Abbildung 13.1 dargestellten 


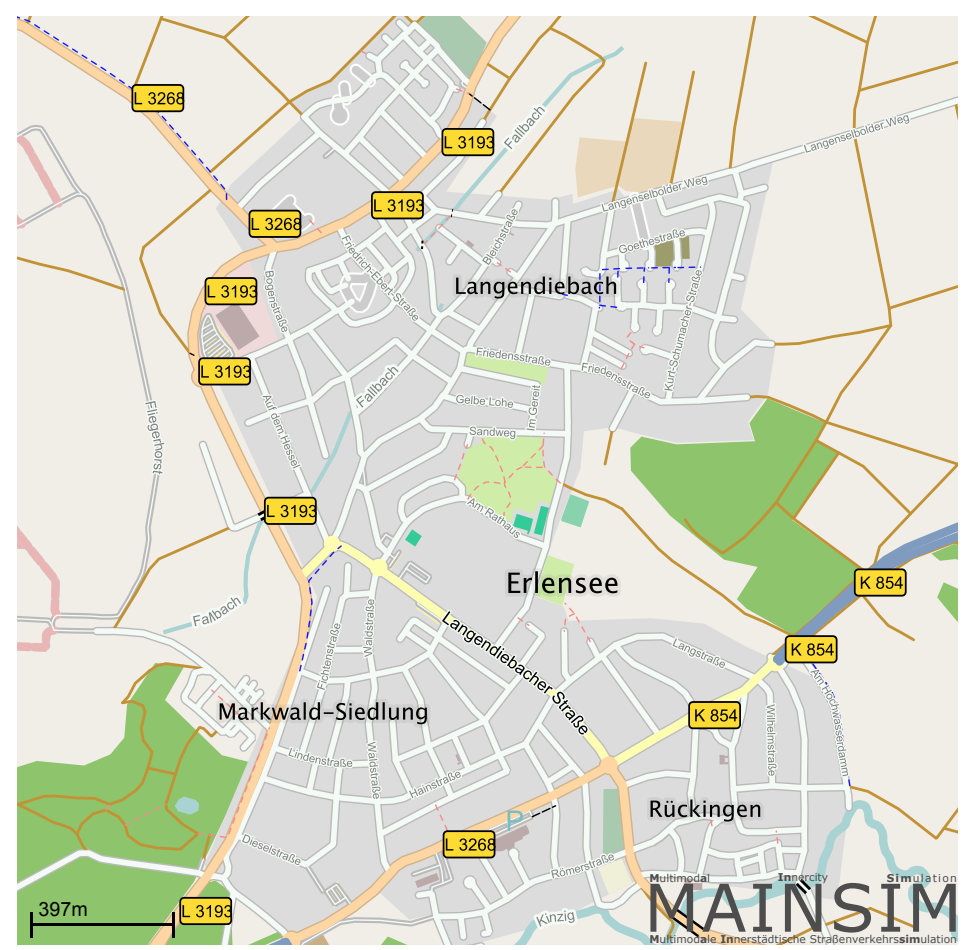

Abbildung 13.1: Stadt Erlensee: Gesamtstraßenlänge 142 km, 937 EIs und 714 NIs.

Kartenausschnitt der Stadt Erlense $\varrho^{1}$ durchgeführt.

Es werden ausschließlich Autos simuliert. Die Anzahl der Autos wird über die Simulationsdauer konstant auf 400 gehalten. Jedes Auto erhält einen zufälligen Start- und Zielpunkt und berechnet die Route zwischen diesen mit Hilfe des erweiterten $A^{*}$-Algorithmus. Nach einer Einschwingphase von 900 Iterationen folgt eine Messphase von 3.600 Iterationen. Mit identischen Autos, aber unterschiedlichen Seedwerten des Zufallsgenerators, werden 5 Replikationen durchgeführt. Die Fitness $f_{i t}$ einer Replikation ist die Anzahl der Autos, die in Replikation $r$ ihre Zielposition erreicht haben. Die Fitness eines Simulationslaufs ist die mittlere negierte Fitness seiner Replikationen $f i t=-\frac{1}{5} \sum_{r=1}^{5} f i t_{r}$. Es entsteht somit ein Minimierungsproblem, welches mittels Simulated Annealing bearbeitet werden kann. Je geringer fit, desto mehr Autos haben ihre Zielposition innerhalb der Messphase erreicht und desto effizienter war somit die Parameterwahl von $\kappa$ und $\vartheta$. Die beste Parameterkonfiguration erzielte $f i t=-4317,6$ mit der in Gleichung 13.3 und 13.4 gezeigten Konfiguration.

\footnotetext{
${ }^{1}$ Grenzen in WGS84: [8.92: 9.08, 50.13 : 50.19]
} 


$$
\begin{aligned}
& \kappa=0,2287046902172737 \\
& \vartheta=0,63524721217982
\end{aligned}
$$

\subsection{Vergleich mit anderen Verfahren}

Das Ameisen-inspirierte Routingverfahren wird mit dem Verfahren der statischen Nutzung (Stat) vorberechneter Routen ohne Anpassung der Kantengewichte und einem iterativen Verfahren verglichen. Das iterative Verfahren berechnet Simulationsläufe mit identischen Einstellungen wiederholt. Nach jedem Lauf werden zufällig $10 \%$ der simulierten Autos ausgewählt und berechnen ihre Route auf Basis der erfahrenen Fahrtdauern auf den EIs des Graphen erneut. Die Methode führt zu einem dynamischen Gleichgewicht Gawron, 1998, Raney und Nagel, 2002. Im Rahmen dieser Arbeit werden 50 Trainingsläufe durchgeführt, um das Verfahren zu kalibrieren. Simulierte Auto-Agenten erhalten im Laufe des Verfahrens Erfahrung über die Verkehrszustände auf den Straßen und optimieren ihre Routen.

Das Routingverfahren AIR wird in $\mathrm{AIR}_{\mathrm{S}}$ und $\mathrm{AIR}_{\mathrm{d}}$ unterschieden. Beim statischen Verfahren AIR $_{S}$ berechnen Autos einmalig eine Route und fahren diese anschließend. Das dynamische Verfahren $\mathrm{AIR}_{\mathrm{S}}$ ermöglicht die Anpassung der Routen. Immer wenn ein simuliertes Auto eine EI verlässt, wird die Reisedauer geschätzt, die unter der aktuellen Kantenbewertungen für die Route des Autos zu erwarten ist. Wenn eine Verdoppelung der Reisedauer im Vergleich zur initial geschätzten Dauer zu erwarten ist, wird eine neue Route geplant. Bei Reisedauern von unter zwei Minuten muss die geschätzte Dauer mindestens fünfmal so lange wie die erste Schätzung sein. Dieses Verfahren soll bei entstehenden Staus ein schnelles Umplanen ermöglichen.

In einem ersten Experiment wird der Straßengraph aus Abbildung 13.1 genutzt, der bereits zur Kalibrierung des Ameisen-inspirierten Verfahrens genutzt wurde. Es werden je 100 Simulationsläufe mit jeweils 10 Replikationen zur Mittelung der Ergebnisse verwendet. Das Experiment wird mit unterschiedlichen Mengen an Autos zwischen 200 und 500 wiederholt. Die Simulationsdauer beträgt 900 (Einschwingphase) plus 3.600 (Messphase) Iterationen und wird verlängert, bis das letzte Auto seine Zielposition erreicht hat. Nach Iteration 4.500 werden keine weiteren Autos mehr hinzugefügt.

Abbildung 13.2 vergleicht die mittleren Reisedauern. In der Abbildung werden 


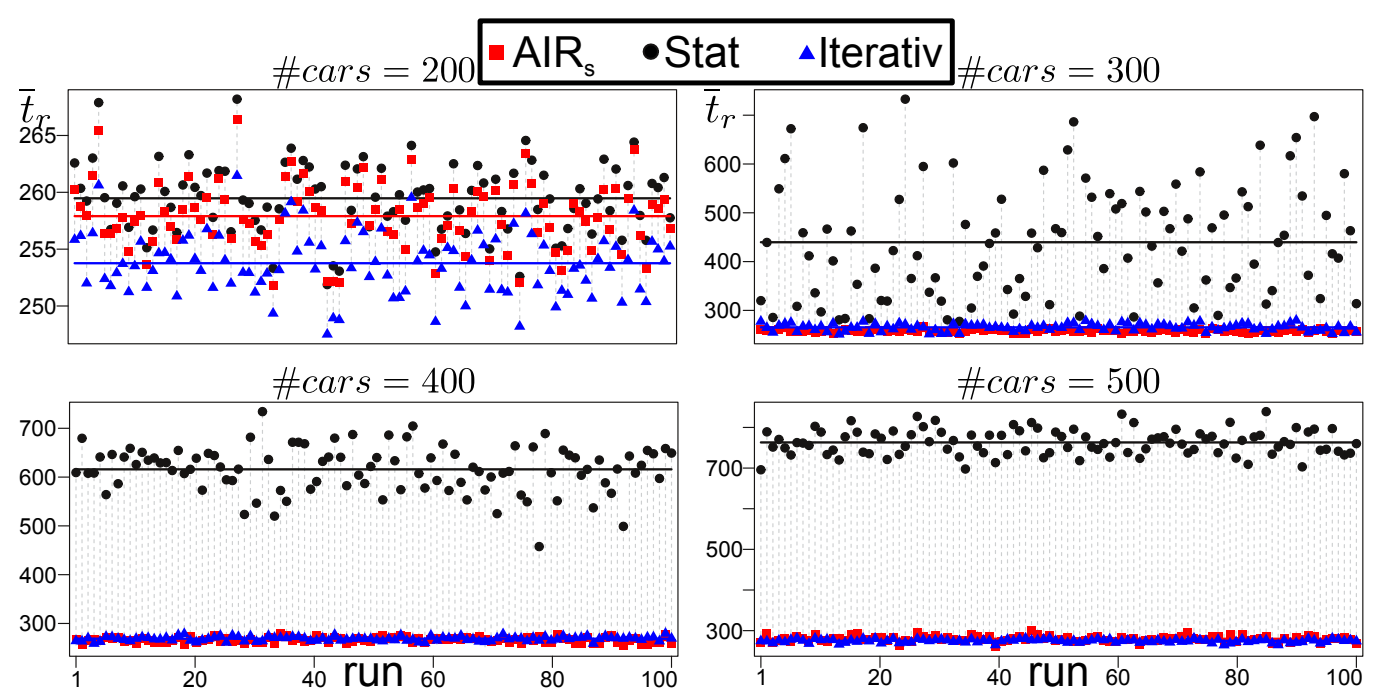

Abbildung 13.2: Vergleich der Routingmethoden: Erlensee

\begin{tabular}{ccllll} 
\#Autos & \#Läufe & \multicolumn{1}{c}{ Stat } & \multicolumn{1}{c}{ AIR $_{\mathrm{S}}$} & \multicolumn{1}{c}{ AIR $_{\mathrm{d}}$} & \multicolumn{1}{c}{ Iterativ } \\
\hline \multirow{2}{*}{200} & \multirow{2}{*}{100} & $\bar{t}=259,47$ & $\bar{t}=257,97$ & $\bar{t}=257,90$ & $\bar{t}=253,76$ \\
& & $\sigma=3,05$ & $\sigma=2,91$ & $\sigma=2,94$ & $\sigma=2,74$ \\
300 & \multirow{2}{*}{100} & $\bar{t}=439,67$ & $\bar{t}=266,32$ & $\bar{t}=266,12$ & $\bar{t}=265,23$ \\
& & $\sigma=115,82$ & $\sigma=5,52$ & $\sigma=5,47$ & $\sigma=7,40$ \\
400 & \multirow{2}{*}{100} & $\bar{t}=615,91$ & $\bar{t}=262,96$ & $\bar{t}=263,03$ & $\bar{t}=263,03$ \\
& & $\sigma=47,05$ & $\sigma=4,54$ & $\sigma=4,89$ & $\sigma=4,55$ \\
500 & \multirow{2}{*}{100} & $\bar{t}=763,14$ & $\bar{t}=279,67$ & $\bar{t}=279,94$ & $\bar{t}=274,85$ \\
& & $\sigma=30,90$ & $\sigma=7,04$ & $\sigma=7,07$ & $\sigma=4,10$
\end{tabular}

Tabelle 13.1: Vergleich: Mittlere Reisedauer $\bar{t}$ und Standardabweichung $\sigma$ im Straßengraphen von Erlensee.

stellvertretend für AIR die Ergebnisse von AIR $_{\mathrm{S}}$ dargestellt. Es ist ersichtlich, dass sowohl das Ameisen-inspirierte, als auch das iterative Routingverfahren zu Verringerungen der Reisedauern im Vergleich zur statischen Methode führen. Beide Verfahren reduzieren die Stauintensitäten bei hohen Verkehrsdichten. Das iterative Verfahren führt - abgesehen von den Simulationsläufen mit 400 Autos - zu den geringsten Reisedauern in diesem Szenario. Tabelle 13.1 fasst die Ergebnisse zusammen. $\mathrm{Zu}$ beachten ist, dass die Streuungen der Reisedauern bei AIR und Iterativ geringer ausfallen.

Die Performanz von $\mathrm{AIR}_{\mathrm{S}}$ ist auf einem Niveau mit $\mathrm{AIR}_{\mathrm{d}}$. Im verwendeten Straßengraphen existieren nur zwei Ampeln, die für unvorhergesehene Verzögerungen sorgen können und die geringe Gesamtlänge der Straßen führt zu geringen Pla- 


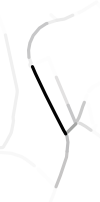

(a) Stat

(b) $\mathrm{AIR}_{\mathrm{s}}$

(c) Iterativ
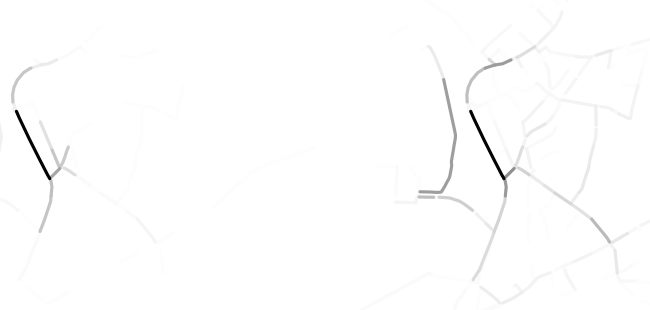

(e) AIR $_{\mathrm{s}}$

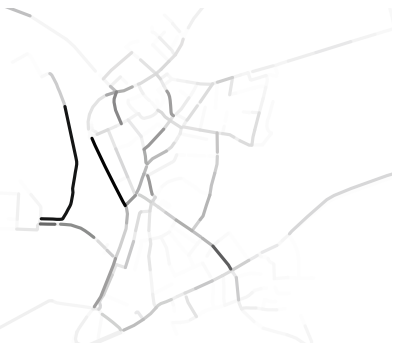

(d) Stat

(f) Iterativ

Abbildung 13.3: Vergleich der Straßennutzungshäufigkeiten bei 400 Autos (weiß: geringe Nutzung; schwarz: häufige Nutzung). Erste Zeile: gleiche Skalierung für alle Methoden; zweite Zeile: individuelle Farbskalierung für jede Methode.

nungshorizonten. Nachdem ein Auto seine Route geplant hat, ändert sich somit im Normalfall die Bewertung der Route während der Fahrt nur geringfügig und ein Umplanen findet selten statt. Beachtlich ist, dass AIR und Iterativ bei 400 simulierten Autos geringere Reisedauern zulassen, als bei 300 Autos. AIR wurde im Gegensatz zu Iterativ bei 400 Autos optimiert. Das iterative Verfahren scheint dennoch bei dieser Verkehrsmenge besonders geringe Fahrtdauern zu ergeben.

Um den Effekt der breiten Nutzung des Straßennetzes zu verdeutlichen, vergleicht Abbildung 13.3 die Staßennutzungsverhältnisse. In der ersten Zeile (Teil (a) bis (c) wird die absolute Spannweite der Kantennutzungswerte unter Betrachtung aller Methoden zur Skalierung der Graustufen genutzt. In der zweiten Zeile (Teil (d) bis (f) wird für jedes Verfahren eine Skalierung nach den Minimum- und Maximumwerten der jeweiligen Verfahren durchgeführt.

Die erste Zeile von Abbildung 13.3 zeigt, dass das Maximum der Kantennutzung des statischen Verfahrens dominiert wird und die verbleibenden Verfahren zu geringeren Verkehrsdichten in diesen Bereichen führen. Die zweite Zeile verdeutlicht, dass die Ameisen-inspirierte und die iterative Routingmethode zu einer deutlicheren Verteilung des Verkehrs führen. Dies bestätigt die Grundannahme, dass das Straßennetz besser genutzt wird. 


\begin{tabular}{ccllll} 
\#Autos & \#Läufe & \multicolumn{1}{c}{ Stat } & \multicolumn{1}{c}{ AIR $_{\mathrm{S}}$} & \multicolumn{1}{c}{ AIR $_{\mathrm{d}}$} & \multicolumn{1}{c}{ Iterativ } \\
\hline \multirow{2}{*}{500} & \multirow{2}{*}{50} & $\bar{t}=491,45$ & $\bar{t}=500,47$ & $\bar{t}=500,78$ & $\bar{t}=516,43$ \\
& & $\sigma=3,83$ & $\sigma=3,90$ & $\sigma=3,89$ & $\sigma=4,06$ \\
750 & \multirow{2}{*}{50} & $\bar{t}=519,08$ & $\bar{t}=524,93$ & $\bar{t}=525,44$ & $\bar{t}=539,82$ \\
& & $\sigma=3,79$ & $\sigma=3,67$ & $\sigma=3,49$ & $\sigma=4,50$ \\
\multirow{2}{*}{1000} & \multirow{2}{*}{50} & $\bar{t}=586,00$ & $\bar{t}=550,86$ & $\bar{t}=552,01$ & $\bar{t}=594,04$ \\
& & $\sigma=9,00$ & $\sigma=3,74$ & $\sigma=3,59$ & $\sigma=10,05$ \\
1500 & \multirow{2}{*}{50} & $\bar{t}=808,09$ & $\bar{t}=635,64$ & $\bar{t}=649,67$ & $\bar{t}=765,43$ \\
& & $\sigma=11,77$ & $\sigma=18,88$ & $\sigma=21,20$ & $\sigma=24,95$
\end{tabular}

Tabelle 13.2: Vergleich: Mittlere Reisedauer $\bar{t}$ und Standardabweichung $\sigma$ im Straßengraphen von Hanau.

In einem weiteren Experiment wird der Straßengraph der Stadt Hanau aus Abbildung 9.9 verwendet auf Seite 149 . Die Ergebnisse werden in Tabelle 13.2 gezeigt. Bei geringen Verkehrsdichten zwischen 500 und 750 Autos sind die mittleren Reisedauern unter Nutzung von $\mathrm{AIR}_{\mathrm{S}}$ länger als unter Nutzung von Stat. Bei steigender Verkehrsdichte ist die Performanz von $\mathrm{AIR}_{\mathrm{S}}$ klar besser als die restlichen zum Vergleich genutzten Verfahren. Die Möglichkeit, umzuplanen, bringt in diesem Szenario keinen Vorteil. Die Resultate von $\operatorname{AIR}_{\mathrm{d}}$ sind über alle betrachteten Verkehrsdichten geringfügig schlechter als AIR $_{S}$. Der Iterative Ansatz kann im Vergleich zu AIR in diesem Szenario nicht überzeugen. Erst bei den Simulationsläufen mit 1500 Autos zahlt sich die Anpassung der Agenten über die 50 Kalibrierungsläufe aus. Unter Nutzung von AIR entstehen jedoch Reisedauern, die über zwei Minuten unter denen des iterativen Ansatzes liegen.

Ein letztes Experiment wird in einem synthetischen Szenario durchgeführt. Abbildung 13.4 zeigt den Straßengraphen. An jeder mit einem Kreis markierten Kreuzung befindet sich eine Ampel. Die maximal erlaubte Geschwindigkeit ist $\mathrm{EI}_{v M a x}=50 \mathrm{~km} / \mathrm{h}$ für alle Kanten im Graphen.

In diesem Szenario liefert AIR über alle Verkehrsdichten die besten Ergebnisse. $\mathrm{AIR}_{\mathrm{S}}$ und $\mathrm{AIR}_{\mathrm{d}}$ sind vergleichbar. Der iterative Ansatz funktioniert in diesem Szenario besser, als in den vorangegangenen. Bei 200 und 300 Autos sind seine Ergebnisse leicht schlechter als Stat. Ab 400 Autos hilft die durch die Agentenerfahrung erzielte Verteilung der Verkehrsteilnehmer im Straßengraphen zur Reduzierung der Reisedauern. AIR kann jedoch mit diesem sehr dynamischen Szenario besser umgehen, da Änderungen der Reisedauern auf den EIs implizit erfasst und sofort verarbeitet werden. 


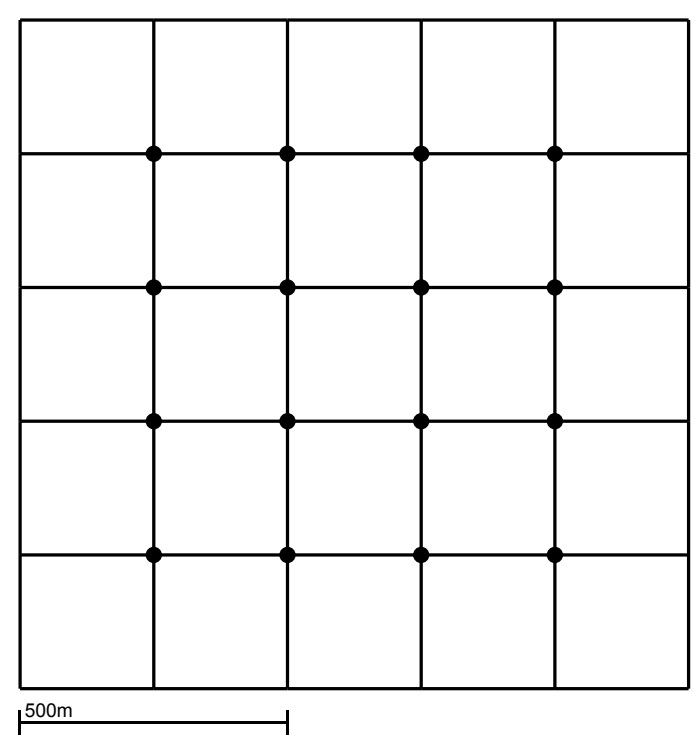

Abbildung 13.4: Synthetischer Straßengraph

\begin{tabular}{ccllll} 
\#Autos & \#Läufe & \multicolumn{1}{c}{ Stat } & \multicolumn{1}{c}{ AIR $_{\mathrm{S}}$} & \multicolumn{1}{c}{ AIR $_{\mathrm{d}}$} & \multicolumn{1}{c}{ Iterativ } \\
\hline \multirow{2}{*}{200} & \multirow{2}{*}{100} & $\bar{t}=255,37$ & $\bar{t}=209,12$ & $\bar{t}=209,11$ & $\bar{t}=258,44$ \\
& & $\sigma=2,95$ & $\sigma=2,39$ & $\sigma=2,43$ & $\sigma=3,30$ \\
300 & \multirow{2}{*}{100} & $\bar{t}=272,91$ & $\bar{t}=215,39$ & $\bar{t}=215,43$ & $\bar{t}=275,18$ \\
& & $\sigma=2,80$ & $\sigma=2,06$ & $\sigma=2,09$ & $\sigma=3,27$ \\
400 & \multirow{2}{*}{100} & $\bar{t}=299,54$ & $\bar{t}=220,49$ & $\bar{t}=220,53$ & $\bar{t}=290,64$ \\
& & $\sigma=3,25$ & $\sigma=1,88$ & $\sigma=1,86$ & $\sigma=2,77$ \\
500 & \multirow{2}{*}{100} & $\bar{t}=354,93$ & $\bar{t}=224,20$ & $\bar{t}=224,24$ & $\bar{t}=301,66$ \\
& & $\sigma=8,63$ & $\sigma=1,83$ & $\sigma=2,35$ & $\sigma=3,42$
\end{tabular}

Tabelle 13.3: Vergleich: Mittlere Reisedauer $\bar{t}$ und Standardabweichung $\sigma$ im synthetischen Graph.

\subsection{Diskussion und Ausblick}

In diesem Kapitel wurde eine Ameisen-inspirierte Routingmethode vorgeschlagen und mit einer Auswahl bestehender Ansätze verglichen. Die Methode konnte sowohl im synthetischen Szenario, als auch auf den Straßengraphen realer Städte Vorteile gegenüber den Vergleichsverfahren aufzeigen. In der Zukunft sollte daher die Robustheit des Verfahrens in weiteren Szenarien überprüft werden. Anschließend könnte die Umsetzung mittels V2X-Technologien angedacht werden.

Zur Verteilung des Verkehrs im Straßengraphen benötigt der iterative Ansatz 50 Kalibrierungsläufe. Die Zahl 50 wurde als Daumenregel aus der Literatur 
entnommen. Es wäre vorteilhaft, die Kalibrierung abzubrechen, wenn sich die Agentenpläne nicht mehr ändern und somit ein Gleichgewicht gefunden wurde Gawron, 1998, Raney und Nagel, 2002.

Bei dynamischen Szenarien mit häufig wechselnden Reisedauern auf den Straßen ist die Nutzung von Real-time Heuristiken (vgl. Kim, 2006) eine viel versprechende Alternative zum vorgestellten Ansatz auf Basis des A*-Algorithmus. Realtime Heuristiken planen nur einen Teil des Weges von Start- zu Zielknoten. Diese Heuristiken führen nicht zu optimalen Routen, benötigen jedoch geringere Rechenkapazitäten und können daher wiederholt angewendet werden. Ein bekannter Vertreter dieser Gattung ist der RTA*-Algorithmus Korf, 1990. Variierende Pheromonkonzentrationen könnten auf diese Weise ebenfalls berücksichtigt werden. Dieser Faktor wird bei größeren Szenarien immer wichtiger, da die Güte einer einmalig geplanten Route sich während der Fahrt ändern kann.

Die dynamische Anpassung der Routen mit dem $\mathrm{AIR}_{\mathrm{d}}$-Verfahren führte in keinem der diskutierten Szenarien zu Verbesserungen gegenüber AIR $_{S}$. Das Kriterium, wann umgeplant werden darf, wurde im Hinblick auf die Berechnungsdauer so gewählt, dass selten umgeplant wird. In einer zukünftigen Studie sollte daher untersucht werden, ob eine Modifikation des Umplanungskriteriums zu Verbesserungen der Reisedauern führt. Ferner muss untersucht werden, ob durch Auswahl einer Teilmenge der Autos zum Umplanen geringere Reisedauern erzielt werden können. 


\section{Benzinverbrauch und Emissionen}

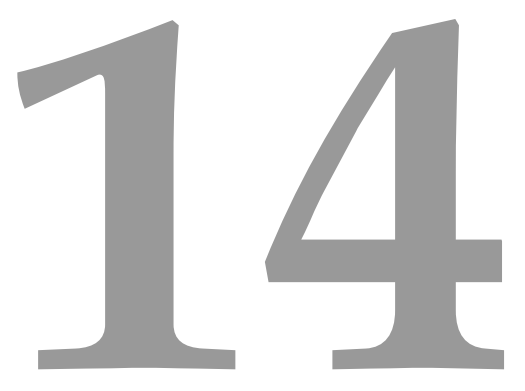

In diesem Kapitel wird eine Ergänzung von MAINSIM um ein Benzinverbrauchsund Emissionsmodell besprochen. Abschnitt 14.1 gibt eine kurze Einleitung in die Thematik. Im folgenden Abschnitt 14.2 wird ein kurzer Überblick über relevante Literatur gegeben. Das gewählte Modell und seine Parametrisierung werden in Abschnitt 14.3 diskutiert. Es folgt ein Vergleich zu Messdaten des NEFZ in Abschnitt 14.4, sowie eine Plausibilitätsprüfung in einem Autobahnszenario (Abschnitt 14.5. In Abschnitt 14.6 wird eine urbane Fallstudie durchgeführt und eine $\mathrm{CO}_{2}$-Landkarte erstellt. Das Kapitel schließt mit einer Zusammenenfassung und einem Ausblick für zukünftige Arbeiten (Abschnitt 14.7).

Die Ergebnisse dieses Kapitels sind größtenteils in Zusammenarbeit mit Carsten Taubert und Andreas Lattner entstanden. Carsten Taubert hat in diesem Kontext seine Masterarbeit Taubert, 2012 verfasst. Teile der Ergebnisse wurden zudem in der Arbeit Dallmeyer et al., 2012e veröffentlicht. 


\subsection{Einleitung}

Verkehrssimulationssysteme können genutzt werden, um Verkehrsphänomene zu verstehen oder den Straßenverkehr in eine bestimmte Richtung zu optimieren. Es liegt nahe, den Benzinverbrauch simulierter Autos zu betrachten, da eine Verringerung des Verbrauchs Kosten einsparen kann. Zusätzlich sollten auch durch den Automobilverkehr verursachte Emissionen Beachtung finden, da diese im Ruf stehen, das Weltklima zu beeinflussen.

Das IPCC1 betrachtet den wissenschaftlichen Stand zu diesem Thema. Es konnte eine globale Erwärmung ermittelt werden, die nicht ausschließlich auf Einflüsse der Sonne zurückzuführen sind. Hieraus wird abgeleitet, dass Treibhausgase einen Einfluss haben WBGU, 2003. Diese Meinung wird von der Mehrheit der Klimaforscher unterstützt Oreskes, 2004. Nach Chapman, 2007 resultieren $26 \%$ der globalen $\mathrm{CO}_{2}$-Emissionen aus Verkehr. In einer Studie einer Forschergruppe der $\mathrm{WHO}^{2}$ wird eine Erhöhung des Lungenkrebsrisikos durch Dieselabgase ermittelt Silverman et al., 2012. Die EU sucht nach Wegen, die $\mathrm{CO}_{2}$-Emissionen pro gefahrenem Kilometer zu reduzieren. Sowohl das Ziel als solches, als auch mögliche Wege dorthin, werden in der Wissenschaft diskutiert (vgl. z.B. [Frondel et al., 2008).

Es kann zusammengefasst werden, dass Benzinverbrauch und Treibhausgasemissionen ein hochbrisantes Thema darstellen, welches im Rahmen dieser Arbeit betrachtet werden soll. Dieses Kapitel ergänzt das in Kapitel 7.1 vorgestellte Fahrzeugmodell durch ein Benzinverbrauchsmodell und ein $\mathrm{CO}_{2}$-Emissionsmodell.

\subsection{Verkehrssimulation und Benzinverbrauch}

Die Einbindung eines Benzinverbrauchsmodells in eine Verkehrssimulation wurde von verschiedenen Autoren vorgeschlagen und umgesetzt (vgl. Ahn, 1998, Pelkmans et al., 2005, Cartenì et al., 2010, Karathodorou et al., 2010]). Nach unserem besten Wissen existieren derzeit keine Studien, die den Einfluss multimodalen Verkehrs auf den Benzinverbrauch simulierter Autos untersuchen. Die in der Literatur diskutierten Verbrauchsmodelle basieren auf den physikalischen Kräften Beschleunigungskraft, Reibungskraft, Hangabtriebskraft und Luftwiderstand. Die physikalischen Zusammenhänge zwischen den zu überwindenden Kräften und dem daraus resultierenden Benzinverbrauch werden detailliert in

\footnotetext{
${ }^{1}$ Intergovernmental Panel on Climate Change

${ }^{2}$ World Health Organization - Weltgesundheitsorganisation
} 
Treiber und Kesting, 2010 und Heißing et al., 2011 besprochen.

In Ross, 1997 werden mehrere simulierte Autos im Hinblick auf ihren Benzinverbrauch untersucht und Strategien zu dessen Reduzierung besprochen. Hierfür werden neue Fahrzeugdesigns vorgeschlagen und die Nutzung hybrider Motoren nahegelegt. In einer weiteren Studie werden die Benzinverbrauchswerte von vier verschiedenen simulierten Fahrzeugen gegenüber Verbrauchswerten aus der Realität verglichen und weichen nur um ca. $1 \%$ ab An et al., 1997. In Cappiello et al., 2002 wird die Funktionsweise des Katalysators nachgebildet und im Hinblick auf toxische Substanzen untersucht, um diese Erkenntnisse in die Entwicklung eines Emissionsmodells einfließen zu lassen.

Die Betrachtung der relevanten Literatur zeigt, dass die Bestimmung von Benzinverbrauchswerten und $\mathrm{CO}_{2}$-Emissionswerten möglich ist. Im Rahmen dieser Arbeit wird ein einfaches Modell verwendet, welches die physikalischen Gegebenheiten abbildet.

\subsection{Verbauchsmodell}

Das Verbrauchs- und Emissionsmodell wird als eigene Komponente entwickelt, die den simulierten Autos bei Bedarf integriert werden kann. Die Komponente erhält vom Auto Informationen über den Typ des Fahrzeugs und seine Länge $l$. Variable Kenngrößen sind ferner die Position auf der Landkarte, die Geschwindigkeit $v$, sowie die Beschleunigung $\dot{v}$ des Autos und der aktuelle Steigungswinkel $\alpha$ der Straße, den das Auto überwinden muss.

Das verwendete Modell wird ausführlich in der Literatur beschrieben Treiber und Kesting, 2010 und basiert auf der physikalischen Energie $P$, die ein Auto pro Zeiteinheit benötigt. Sie hängt von seinem Grundleistungsbedarf $P_{0}$, seiner Momentanleistung $F$, sowie der Geschwindigkeit $v$ ab. $P_{0}$ ist die Energie, die zum Betrieb des Radios, der Klimaanlage, sicherheitsrelevanter Systeme und Steuerelektronik gebraucht wird. Es gilt:

$$
P=P_{0}+\max (F \cdot v, 0)
$$

Ein Auto benötigt also stets mindestens $P_{0}$. $F$ muss begrenzt werden, da bei einer Bremsung oder einer Fahrt bergab negative Werte für $F$ entstehen können. Dies würde einem Energierückgewinnungssystem entsprechen, was nicht als Standard 


\begin{tabular}{|l|}
\hline$\mu_{r}=0,01$ \\
\hline$P_{0}^{\mathrm{Pkw}}=3 \mathrm{~kW}$ \\
$P_{0}^{\mathrm{Lkw}}=6 \mathrm{~kW}$ \\
\hline$c_{w}^{\mathrm{Pkw}}=0,3$ \\
$c_{w}^{\mathrm{Lkw}}=0,8$ \\
\hline$\rho=1,2 \mathrm{~kg} / \mathrm{m}^{2}$ \\
\hline$m^{\mathrm{Pkw}}=1000 \cdot l \cdot \mathcal{N}_{0,26}^{0,49}(0,339 \mathrm{~kg}, 0,34)$ \\
\hline$A^{\mathrm{Pkw}}=\mathcal{N}_{2,3}^{2,81}\left(2,5 \mathrm{~m}^{2}, 0,1\right)$ \\
$A^{\mathrm{Lkw}}=\mathcal{N}_{6,0}^{10,0}\left(8,0 \mathrm{~m}^{2}, 0,25\right)$ \\
\hline
\end{tabular}

Tabelle 14.1: Ermittelte Parameter für das Treibstoffverbrauchsmodell.

angenommen werden kann.

$F$ setzt sich aus der Beschleunigung $m \cdot \dot{v}$, der Rollreibungskraft $m \cdot g \cdot \mu_{r}$, der Hangabtriebskraft $m \cdot g \cdot \sin (\alpha)$ und dem Luftwiderstand $\frac{1}{2} \cdot c_{w} \cdot \rho \cdot A \cdot v^{2}$ zusammen. Dies führt zu:

$$
F=m \cdot \dot{v}+\left[\mu_{r}+\sin (\alpha)\right] \cdot m \cdot g+\frac{1}{2} \cdot c_{w} \cdot \rho \cdot A \cdot v^{2}
$$

Zur Berechnung von $F$ werden die Masse eines Fahrzeugs $m$, der Reibungskoeffizient $\mu_{r}$, der Strömungswiderstandskoeffizient $c_{w}$, die Luftdichte $\rho$ und die Frontfläche des Fahrzeugs $A$ benötigt.

Zur Nachbildung des Individualverkehrs in Deutschland werden Daten des Statistischen Bundesamtes verwendet, die die Verteilung von Fahrzeugen nach Fahrzeugklassen angeben. Um die Masse $m$ eines Fahrzeugs zu bestimmen, werden die Verhältnisse zwischen Länge und Gewicht von 54 typischen Autos untersucht. Die benötigten Daten wurden den Webseiten der jeweiligen Fahrzeughersteller entnommen und sind in Anhang D aufgelistet. Tabelle 14.1 zeigt die resultierenden Parameter.

Die Werte für $\mu_{r}$ und $\rho$ stammen aus Ross, 1997, $P_{0}, c_{w}$ und $A$ wurden aus |Treiber und Kesting, 2010 übernommen. Hierbei werden die Luftdichte $\rho$ und der Reibungskoeffizient $\mu_{r}$ als konstant angenommen. In der Realität variieren sie je nach Höhe über Normalnull und Lufttemperatur, respektive Straßenbelag. Die Varianz von $\rho$ ist jedoch gering. Exakte Werte für $\mu_{r}$ können nicht verwendet werden, da keine Angaben bezüglich des Straßenbelages im OSM-Kartenmaterial enthalten sind. Es wird daher von Teerstraßen ausgegangen, deren $\mu_{r}$ zwischen 0,005 und 0,015 liegt Wallentowitz, 2003. 


\begin{tabular}{|c|c|c|c|}
\hline Kraftstoffart & Heizwert $H$ & Wirkungsgrad $\gamma$ & Emissionen \\
\hline Benzin & $32842,5 \mathrm{kj} / \mathrm{kg}$ & 0.35 & $2,68 \mathrm{~kg} / \mathrm{l}$ \\
Diesel & $35912,5 \mathrm{kj} / \mathrm{kg}$ & 0.42 & $2,32 \mathrm{~kg} / \mathrm{l}$ \\
\hline
\end{tabular}

Tabelle 14.2: Wirkungsgrad und Emissionen nach Kraftstoffart Heißing et al., 2011.

Der Treibstoffverbrauch $C$ kann direkt aus dem Energiebedarf nach Gleichung 14.3 ermittelt werden.

$$
C=\frac{P}{H \cdot \gamma}
$$

Es besteht eine Abhängigkeit zum Heizwert des Kraftstoffs $H$ und zum Wirkungsgrad des Motors $\gamma . H$ gibt die Energiedichte pro Liter Kraftstoff an. $\gamma$ zeigt, welchen Anteil der Motor aus dieser Energie in Kraft umwandeln kann. Es bestehen die in Tabelle 14.2 gezeigten Zusammenhänge. Die Menge an $\mathrm{CO}_{2}$, die ein Fahrzeug emittiert ist direkt proportional abhängig zum Kraftstoffverbrauch. Die Heizwerte entsprechen ca. 9120,4 Wh/l für Benzin und 9971 Wh/l für Diesel.

Das beschriebene Kraftstoffverbrauchs- und Emissionsmodell kann über die von MAINSIM gegebenen Parameter $v, \dot{v}, \alpha$ und $l$ der jeweiligen Fahrzeuge berechnet werden. Die folgenden Abschnitte 14.4 und 14.5 analysieren simulierte Verbrauchswerte.

\subsection{Vergleich zu NEFZ}

Die Europäische Union hat den NEFZ (Neuer Europäischer Fahrzyklus) entwickelt [AblEU, 1970], den jedes neu entwickelte Automodell durchlaufen muss. Auf einem Rollenprüfstand werden nach einem festgelegten Muster Fahrten absolviert und der Benzinverbrauch und die Emissionen des Fahrzeugs ermittelt. Fahrzeughersteller sind gezwungen, die Messdaten zu veröffentlichen. Die Daten bieten sich daher für einen ersten Plausibilitätstest an. Grundlegend können NEFZ-Daten als untere Grenze des Kraftstoffverbrauchs gesehen werden, da die Messdaten nicht auf realen Straßen im Verkehr ermittelt werden und Fahrzeughersteller Optimierungstechniken anwenden, um geringe Werte zu erzielen (z.B. Abschalten aller nicht relevanten Systeme oder Erhöhung des Reifendrucks). Im Amtsblatt der Europäischen Union AblEU, 2008 wird daher vorgeschlagen, den Zyklus in der Zukunft zu überarbeiten. 


\begin{tabular}{|c|c|c|c|c|c|}
\hline Fahrzeug & $m[\mathrm{~kg}]$ & $c_{w}$ & $A\left[\mathrm{~m}^{2}\right]$ & $H[\mathrm{Wh} / \mathrm{l}]$ & $\gamma$ \\
\hline Smart ForTwo & 750 & 0,37 & 1,947 & 9120,4 & 0,35 \\
Audi A4 & 1645 & 0,27 & 2,2 & 9120,4 & 0,35 \\
Porsche 987 & 1405 & 0,29 & 1,98 & 9120,4 & 0,22 \\
Mercedes C180 & 1565 & 0,26 & 2,17 & 9971 & 0,45 \\
\hline
\end{tabular}

Tabelle 14.3: Vergleich mit NEFZ: Verwendete Fahrzeuge. Angegebene Wirkungsgrade sind lediglich Schätzwerte, da diese nicht veröffentlicht werden [Taubert, 2012].

Zum Vergleich zwischen NEFZ und Simulationsergebnissen werden simulierte Fahrten ohne Verkehr in zwei Szenarien durchgeführt. Das erste Szenario ist ein Stadtausschnitt im Bereich der Stadt Rüsselsheim ${ }^{3}$ mit $192 \mathrm{~km}$ Straßenlänge; das zweite Szenario ist ein Mix-Szenario aus Stadt und Landabschnitten im Bereich Rustenfelde $\rfloor^{4}$ mit $65 \mathrm{~km}$ Straßenlänge. Ein einzelnes simuliertes Auto fährt durch den Straßengraphen. Wenn das Auto seine Zielposition erreicht hat, wird ein neues, identisches Auto erstellt. Der Ablauf wird wiederholt, bis die Simulationsdauer von 86.400 Iterationen absolviert wurde. Die Kraftstoffverbrauchswerte werden protokolliert. Das Experiment wird für die in Tabelle 14.3 gezeigten Automodelle wiederholt.

Tabelle 14.4 zeigt den Vergleich der durch die Simulation ermittelten Verbrauchswerte mit den über NEFZ angegebenen Werten. Die Simulationsergebnisse sind jeweils Mittelwerte aus 25 Simulationsläufen. Die Ergebnisse zeigen geringe Abweichungen der Simulationsergebnisse von NEFZ-Werten.

In einem zweiten Test werden nach Tabelle 14.1 parametrisierte Fahrzeuge untersucht. Es werden 500 Autos simuliert. Der mittlere NEFZ-Verbrauch nach Anhang D für urbane Szenarien liegt bei ca. 9 l/100 km. Der durchschnittliche durch Simulation ermittelte Verbrauch ist hingegen ca. 11,2 1/100 km. Im Mix-Straßennetz beträgt der NEFZ-Verbrauch 6,7 l/100 km gegenüber 8,1 l/100 km in der Simulation. Die Resultate erscheinen plausibel, da der NEFZ keine Interaktionen zwischen Verkehrsteilnehmern berücksichtigt.

\footnotetext{
${ }^{3}$ Grenzen in WGS84: [8.29: 8.58, 49.91 : 50.06]. Der Graph besteht aus 1.983 EIs und 1.430 NIs.

${ }^{4}$ Grenzen in WGS84: [9.96: 10.06, $\left.51.38: 51.42\right]$. Der Graph besteht aus 261 EIs und 236 NIs.
} 


\begin{tabular}{|c|c|c|c|c|}
\hline Fahrzeug & $\begin{array}{c}\text { NEFZ } \\
\text { Stadt }\end{array}$ & $\begin{array}{c}\text { SIM } \\
\text { Stadt }\end{array}$ & $\begin{array}{c}\text { NEFZ } \\
\text { Mix }\end{array}$ & $\begin{array}{c}\text { SIM } \\
\text { Mix }\end{array}$ \\
\hline Smart ForTwo & 4,6 & 5,2 & 4,3 & 4,3 \\
\hline Audi A4 & 8,8 & 9,5 & 7,0 & 7,0 \\
\hline Porsche 987 & 13,8 & 13,5 & 9,4 & 9,8 \\
\hline Mercedes C180 & 6,3 & 6,5 & 4,7 & 4,7 \\
\hline
\end{tabular}

Tabelle 14.4: Vergleich mit NEFZ: Simulationsergebnisse Dallmeyer et al., 2012e. Alle Angaben in $1 / 100 \mathrm{~km}$.

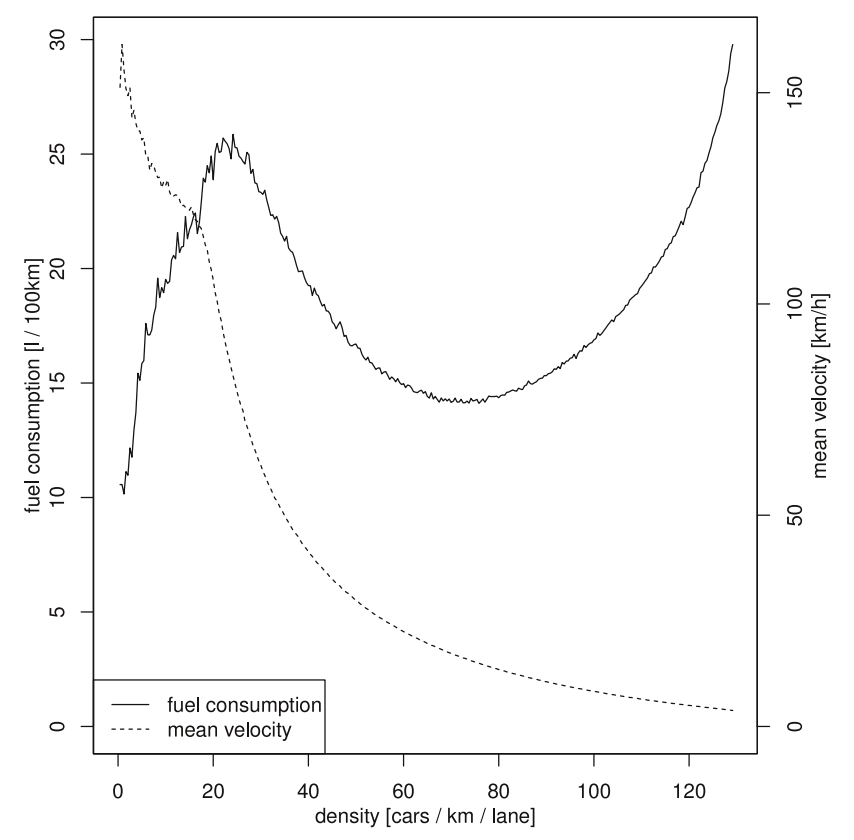

Abbildung 14.1: Kraftstoffverbrauch auf Autobahn bei steigender Verkehrsdichte.

\subsection{Autobahn-Plausibilitätsstudie}

In den Arbeiten Yang et al., 2009 und Madani und Moussa, 2012 wurden Untersuchungen bezüglich Kraftstoffverbrauch mit Hilfe des NSM durchgeführt. Sie bieten sich daher als Vergleichswerte an. Dieser Abschnitt untersucht den Kraftstoffverbrauch auf einer synthetischen zweispurigen Autobahn mit $6 \mathrm{~km}$ Länge und periodischen Grenzbedingungen. Jedes Experiment wird drei mal wiederholt, um Durchschnittswerte ermitteln zu können. Die Anzahl der Autos ist im ersten Experiment 5 und wird pro Experiment um 5 erhöht, bis zu einer Maximalzahl von 1.550. Jedes Experiment hat eine Einschwingphase von 2.000 Iterationen und eine Messphase von 20.000 Iterationen. Als Ergebnis wird der mittlere Kraftstoffverbrauch in $1 / 100 \mathrm{~km}$ angegeben. 
Abbildung 14.1 zeigt die Simulationsergebnisse. Zusätzlich zum Kraftstoffverbrauch wird die mittlere Geschwindigkeit der simulierten Fahrzeuge dargestellt. Bei geringen Verkehrsdichten ist der Kraftstoffverbrauch gering, da wenig Interaktionen zwischen den simulierten Autos stattfinden. Bei steigender Verkehrsdichte steigt auch der Kraftstoffverbrauch, da durch die Natur des NSM und daher auch des in Abschnitt 7.1 beschriebenen mikroskopischen Verkehrsmodells, häufige Geschwindigkeitsänderungen mit abrupten Beschleunigungen zu unrealistisch hohen Verbrauchswerten führen.

Bei weiterer Steigerung der Verkehrsdichte sinkt der Verbrauch nochmals. Dieses Phänomen kann durch die mittleren gefahrenen Geschwindigkeiten erklärt werden: Die simulierten Autos fahren mit effizienten Geschwindigkeiten. Gleichung 14.1 hat durch die Gegenläufigkeit von $P_{0}$ und $F$ ein Minimum. Wenn ein Auto mit $v=0 \mathrm{~km} / \mathrm{h}$ steht, ist sein Verbrauch $\infty \mathrm{l} / 100 \mathrm{~km}$. Bei schneller Fahrt hingegen dominieren die von $v$ abhängigen Parameter aus Gleichung 14.2. Für den in Tabelle 14.3 gezeigten Audi A4 liegt die rechnerisch verbrauchsoptimale Geschwindigkeit bei ca. $52 \mathrm{~km} / \mathrm{h}$. Bei weiterer Steigerung der Verkehrsdichte steigt auch der Kraftstoffverbrauch wieder, da die gefahrenen Geschwindigkeiten sich dem Nullpunkt nähern und Interaktionen zwischen den Autos weiter zunehmen.

Die gezeigten Ergebnisse sind in ihrem Verlauf plausibel. Die absoluten Werte erscheinen hingegen $\mathrm{zu}$ hoch. Im Vergleich $\mathrm{zu}$ den in der Literatur diskutierten Werten zwischen 80 Yang et al., 2009 und 30 l/100 km Madani und Moussa, 2012 sind die ermittelten Verbrauchswerte hingegen gering. In der Studie [Jiang et al., 2007 wird ebenfalls das NSM um ein Verbrauchsmodell erweitert. Die Ergebnisse liegen auf einer Autobahn mit Geschwindigkeitsbegrenzung von $135 \mathrm{~km} / \mathrm{h}$ bei ca. 23, 5 l/100 km, obwohl der Grundleistungsbedarf und der Luftwiderstand im vorgeschlagenen Modell nicht beachtet wurden und jedes Auto nur $1000 \mathrm{~kg}$ wiegt.

Bei identischem Versuchsaufbau werden 200 Fahrzeuge (16, $\overline{6}$ Autos/km/Spur) simuliert und der Anteil an Lkw von $0 \%$ bis $99 \%$ mit einer Schrittweite von $1 \%$ erhöht. Abbildung 14.2 zeigt die ermittelten Verbauchswerte. Der Kraftstoffverbrauch der Gruppe der Pkw erhöht sich nicht bei steigendem Anteil an Lkw, da die Interaktionen zwischen den simulierten Autos bei der angegeben Verkehrsdichte bereits stark ausfallen, wenn noch keine Lkw vorhanden sind. Der Gesamtverbrauch wird bei steigender Anzahl an Lkw durch diese dominiert. Die Verbrauchswerte sind an dieser Stelle zu hoch. Bei Lkw dominiert die Ladung den Verbrauch. Werte von 40 l/100 km können erreicht werden. Die vorhandene Übersteuerung deckt sich mit dem überhöhten Kraftstoffverbrauch der Pkw im Autobahnszenario. 


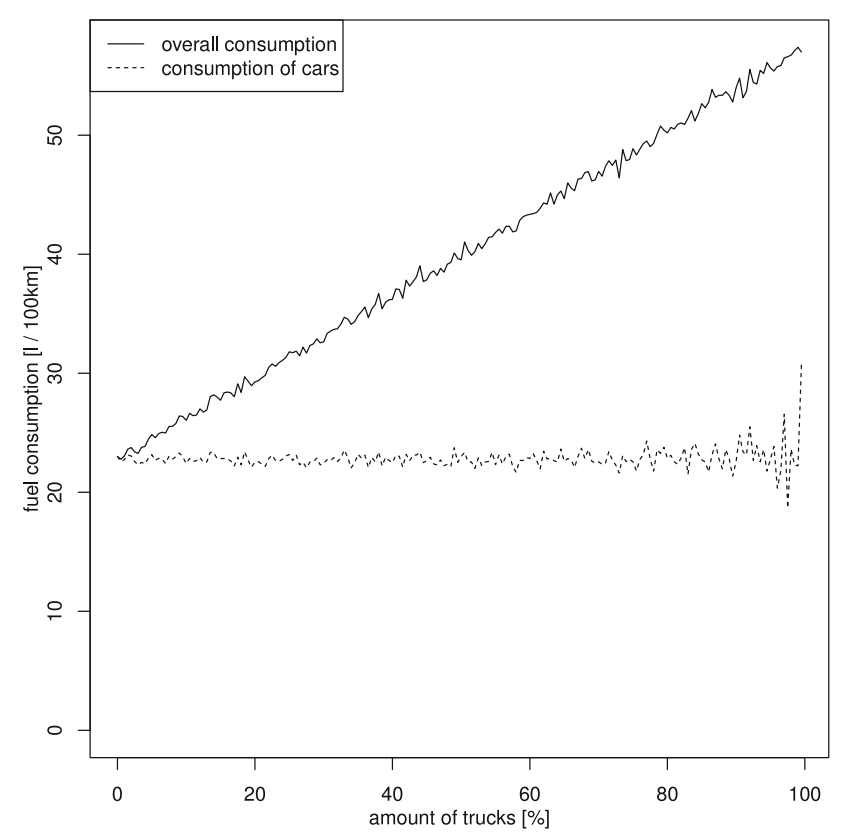

Abbildung 14.2: Kraftstoffverbrauch auf Autobahn: Einfluss von Lkw.

\subsection{Fallstudie im urbanen Verkehr}

Urbane Experimente werden im Kartenausschnitt der Stadt Hanau durchgeführt, der in Abbildung 9.9 dargestellt wurde. Jeder Durchlauf besteht aus drei Experimenten. Im ersten Experiment wird die Anzahl an simulierten Pkw auf 750 festgelegt und über die gesamte Simulationsdauer von 5.000 Iterationen Einschwingphase und 50.000 Iterationen Messphase gehalten. Es werden nur die Verbrauchs- und Emissionswerte in urbanen Bereichen erfasst. Quelle-Ziel-Relationen sind zufällig.

Im zweiten Experiment werden identische Kopien der Pkw verwendet und durch 1.750 Fahrräder und Fußgänger ergänzt (35 \% Fahrräder und 65 \% Fußgänger). Es wird eine Erhöhung des Kraftstoffverbrauchs durch Interaktionen erwartet.

Das dritte Experiment ist identisch zum zweiten Experiment, verwendet jedoch zusätzlich ein digitales Geländemodell, um Höheninformationen und somit $\alpha$ bestimmen zu können. Abbildung 14.3 zeigt einen Ausschnitt des Simulationsbereiches. Es wird abermals eine Erhöhung der Verbrauchswerte erwartet, da bei Bergabfahrten keine Energie zurückgewonnen werden kann und somit Steigungen ab einem bestimmten Winkel einen verbrauchserhöhenden Einfluss haben.

Abbildung 14.4 zeigt die Simulationsergebnisse. Offensichtlich ist der Verbrauch 


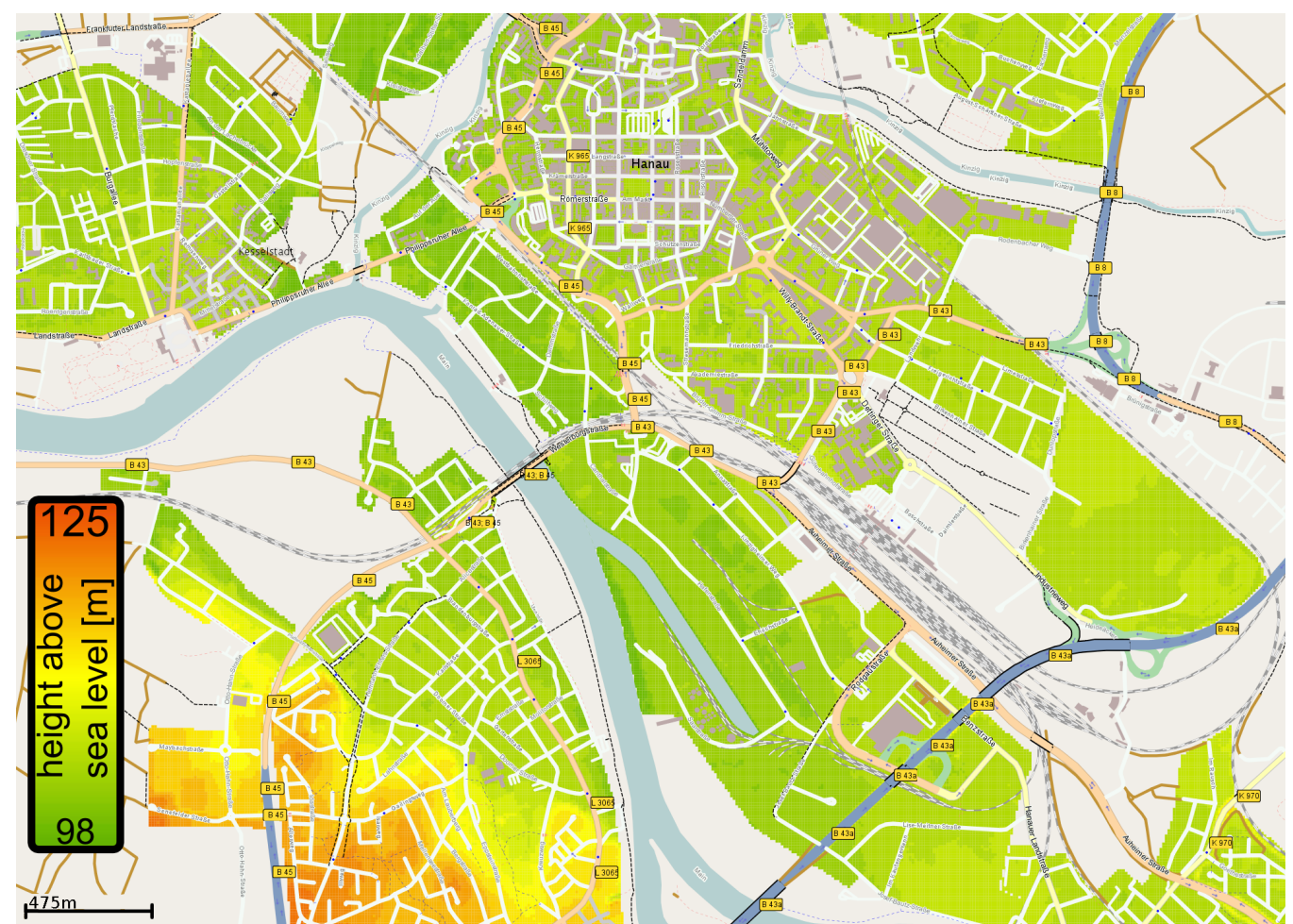

Abbildung 14.3: Kartenausschnitt der Stadt Hanau: Digitales Geländemodell

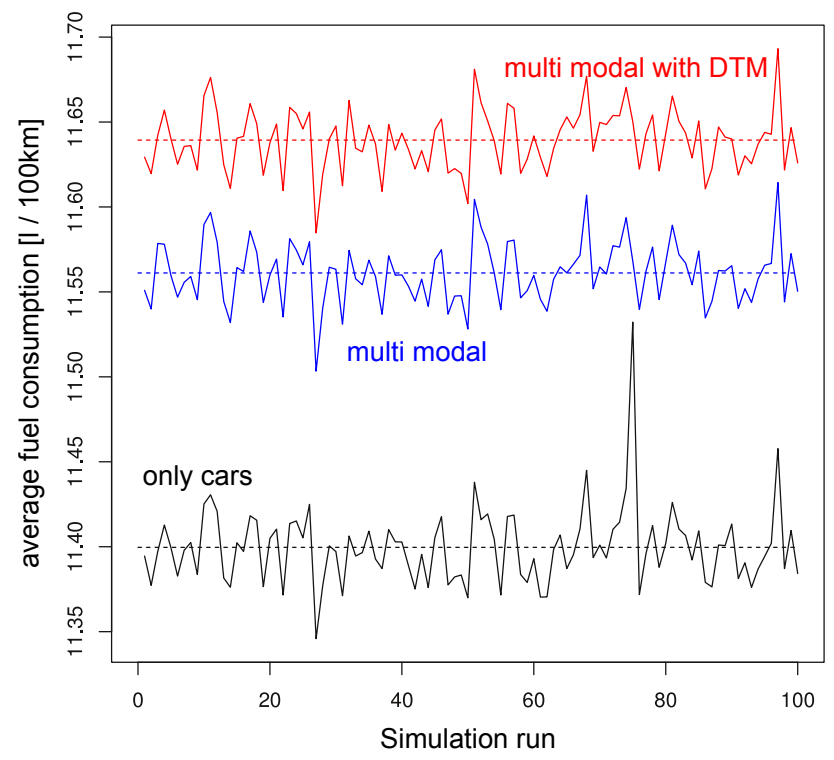

Abbildung 14.4: Vergleich: Kraftstoffverbrauch urban in verschiedenen Experimenten. Gestrichelte Linien zeigen Mittelwerte. 
am geringsten, wenn ausschließlich Autos simuliert werden. Multimodalität erhöht den Verbrauch in diesem Szenario um 0,16 l/100 km. Die Hinzunahme des DGM führt zu einer weiteren Erhöhung des Verbrauchs um 0,08 l/100 km. Wenn Multimodalität und DGM berücksichtigt werden, steigt der Verbrauch insgesamt um 0,24 l/100 km. Statistische Signifikanztests (t-Test mit $\alpha=0,05$ ) bestätigen diese Aussagen mit hoch signifikanten Ergebnissen unter Berücksichtigung von Multiplen Testen.

In Abbildung 14.4 ist im Simulationslauf 75 ein Ausreißer des Kraftstoffverbrauchs im Experiment mit ausschließlich Autos zu erkennen. Die Experimente unter Berücksichtigung multimodalen Verkehrs zeigen diesen Ausreißer nicht. Eine Erklärung hierfür ist ein Stau, der sich bildete und zu hohen Verbrauchswerten führte. Dieser Stau scheint durch Fahrräder und Fußgänger abgeschwächt worden zu sein. Damit ein Stau entsteht, werden im Normalfall je nach Areal im Straßennetz bestimmte Verkehrsdichten benötigt. In diesem Simulationsexperiment scheinen Fahrräder und Fußgänger diejenigen Autos, die auf dem Weg zum Stau waren, behindert zu haben. Ein Stau kann auf diese Weise abgeschwächt oder gar verhindert werden.

Abschließend wird das Simulationsgebiet in 333 Reihen und 333 Spalten geteilt (110.889 Zellen). Jede Zelle protokolliert die $\mathrm{CO}_{2}$-Emissionen, die in ihr stattfinden. Abbildung 14.5 zeigt die mittleren, logarithmierten Emissionen aus allen 100 Simulationsexperimenten, die Multimodalität und das DGM berücksichtigt haben.

Abbildung 14.5 zeigt, dass nur an Straßen Emissionen protokolliert wurden. Häufungen sind an Hauptverkehrsstraßen zu sehen. Es sind zwei Ausschnitte vergrößert dargestellt. Im Norden des Kartenausschnitts ist die Wilhelmbrücke über die Kinzig zu sehen. Beide angrenzenden Kreuzungen haben eine Ampel. Nach Osten fällt das Gelände stark ab. Dadurch, dass Autos, die von Osten kommen, an einer Ampel stehen bleiben müssen und anschließend bergauf beschleunigen müssen, wenn die Ampel grün wird, ist hier ein Hotspot zu sehen. Auf der westlichen Seite der Brücke treffen zwei Straßen mit hohem Verkehrsaufkommen zusammen.

Der andere vergrößerte Ausschnitt zeigt die zweispurige Straße Am Steinheimer Tor. Die Abschnitte mit Hotspots werden jeweils durch Ampelschaltungen gesteuert. Der Bereich zwischen den Kreuzungen hat deutlich geringere Belastungen. Ein Indiz, dass freier Verkehrsfluss einen positiven Effekt auf $\mathrm{CO}_{2}$-Emissionen hat. 


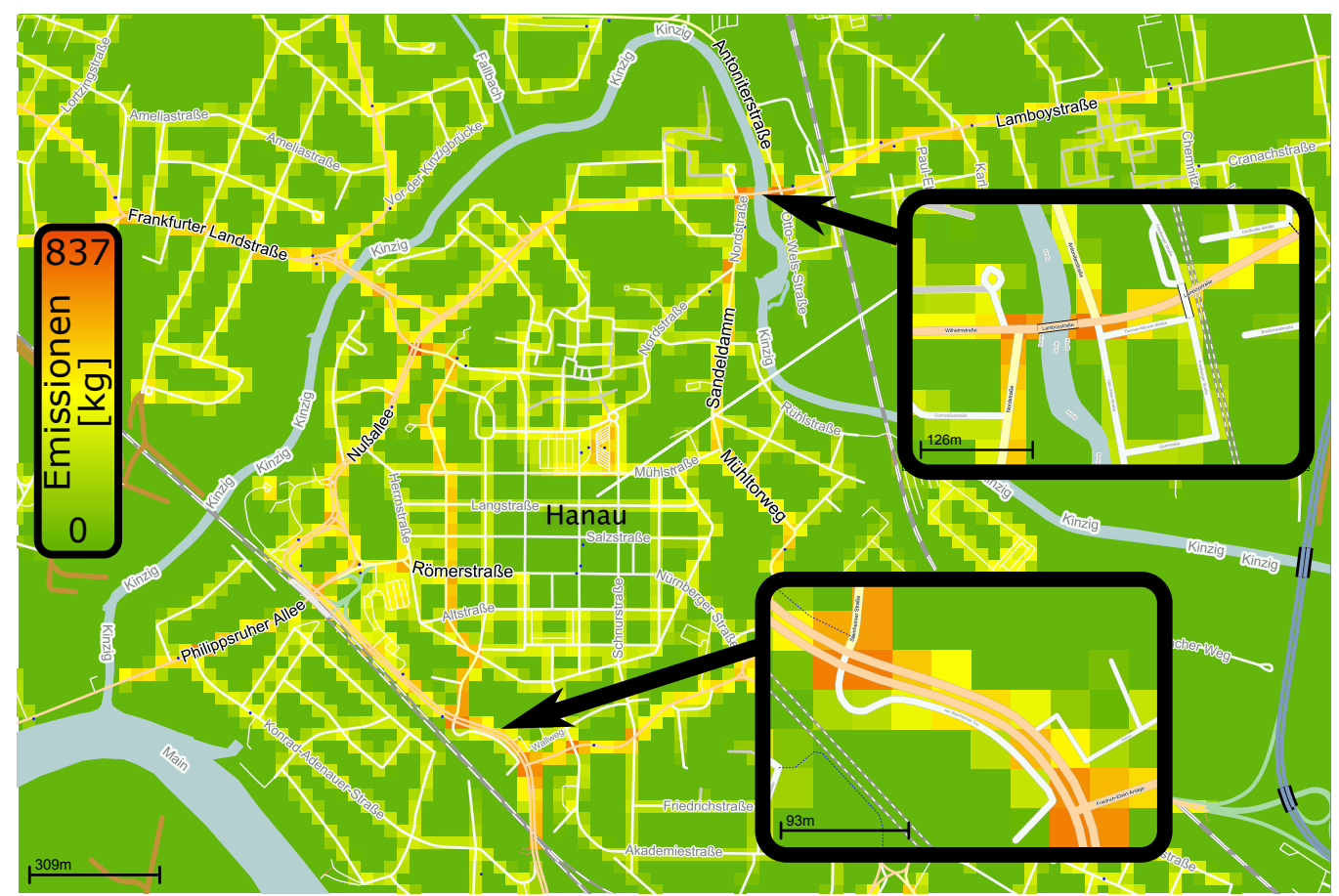

Abbildung 14.5: Kartenausschnitt der Stadt Hanau: Emissionskarte.

\subsection{Zusammenfassung und Ausblick}

In diesem Kapitel wurde gezeigt, wie ein physikalisches Kraftstoffverbauchsmodell in das mikroskopische Autoverkehrsmodell von MAINSIM integriert werden kann. Der Vergleich der ermittelten Verbrauchswerte mit Daten aus NEFZ-Messfahrten zeigte realistische Ergebnisse. Auf Autobahnen werden die grundlegenden Effekte korrekt abgebildet, der Verbrauch wird jedoch zu hoch angegeben. Dies resultiert aus der Art des Verkehrsmodells, welches nicht das Verhalten von Autofahrern realistisch abbildet, sondern makroskopische Kenngrößen korrekt ermittelt. Um bessere Ergebnisse auf Autobahnen zu erzielen, müsste das grundlegende Zusammenspiel zwischen Beschleunigung und Trödeln abgeschwächt werden. In dieser Hinsicht erscheint die Betrachtung von Fahrzeugfolgemodellen (z.B. IDM oder Krauss, vgl. Abschnitt 3.1.1 lohnend, da diese versuchen, abrupte Geschwindigkeitsänderungen zu verhindern. Dieser Vorteil wird jedoch durch einen Verlust an Berechnungseffizienz und Erweiterbarkeit des Modells erkauft.

Im urbanen Bereich wurden realistische Ergebnisse erzielt. Es konnte gezeigt werden, dass Fahrräder und Fußgänger einen Effekt auf den Treibstoffverbrauch der simulierten Fahrzeuge haben und dass eine weitere Steigerung vorliegt, wenn die 
Simulation nicht in der Ebene stattfindet, sondern ein DGM mit Höheninformationen verwendet wird. Dieses Ergebnis zeigt die Stärke eines GIS-basierten Ansatzes: die einfache Erweiterbarkeit des Simulationssystems.

Die Menge der $\mathrm{CO}_{2}$-Emissionen ist proportional zum Treibstoffverbrauch. Eine $\mathrm{CO}_{2}$-Landkarte des Simulationsbereichs wurde erstellt. Die Ergebnisse zeigen klare Hotspots, die durch Betrachtung der Verkehrs- und Höhensituationen der jeweiligen Hotspots erklärt werden können. Die Untersuchung von $\mathrm{CO}_{2}$-Hotspots kann für die Städteplanung interessant sein. Wenn beispielsweise ein neuer Kindergarten gebaut werden soll, wäre eine Position auf der Landkarte mit geringen Emissionswerten zu bevorzugen. Der Einfluss von Eingriffen in den Verkehr kann ebenso mit dieser Technologie untersucht werden. Zukünftige Fragestellungen könnten die folgenden sein:

- Welchen Einfluss auf die $\mathrm{CO}_{2}$-Belastungen haben Umweltzonen, in denen nur noch bestimmte Verkehrsgruppen zugelassen sind? Diese Frage wird kontrovers diskutiert, vgl. Cyrys et al., 2009 und Laberer und Niedermeier, 2009.

- Die Stadt Frankfurt plante zwischen September und Oktober 2012 zwischen 22.00 und 06.00 Uhr in der Nacht auf Hauptverkehrsstraßen Tempo 30 einzuführen.5. Das Vorhaben wurde jedoch kurzfristig verworfen6 Das Ziel war eine Lärmreduzierung für die Anwohner in diesen Gebieten. Es ist jedoch trivial zu zeigen, dass Tempo 30 bei störungsfreier Fahrt nicht Verbrauchsoptimal ist. Welche Auswirkungen hat diese Änderung auf die $\mathrm{CO}_{2}$-Belastungen in diesen Gebieten?

- Deutschland ist das weltweit einzige Land ohne pauschale Tempobegrenzung auf Autobahnen. Ist dies vorteilhaft?

- Angenommen, ein Automobilhersteller entwickelte ein neues Automodell mit Elektroantrieb. Die grundlegenden Zusammenhänge bezüglich Energieverbrauch sind analog zu Verbrennungsmotoren zu bestimmen. Wie viele Kilometer kann ein solches Auto im Stadtverkehr zurücklegen? Genügt die Reichweite, um aus einem Vorort Frankfurts zum Arbeitsplatz innerhalb Frankfurts zu gelangen? An welchen Positionen auf der Landkarte müssten Akkuwechselstationen bzw. -aufladestationen platziert werden? Fragestellungen dieser Art können mit Hilfe von MAINSIM unter Berücksichtigung

$\sqrt[5]{\text { http://heise.de/-1582034, abgerufen am 26.06.2012 }}$

${ }^{6}$ http://www.faz.net/aktuell/rhein-main/frankfurt-landesregierung-stoppt-tempo30-versuch-11871443.html, abgerufen am 18.10.2012 
anderer Verkehrsteilnehmer untersucht werden.

In diesem Kapitel wurden weder Wind noch Wetter (Temperatur, Luftdruck, Regen) berücksichtigt. Emissionen blieben an dem Ort, an dem sie entstanden. Das folgende Kapitel koppelt MAINSIM an ein Simulationsmodell für Gasausbreitungen, um diese wichtigen Einflüsse zu untersuchen. 


\section{Wind und Wetter}

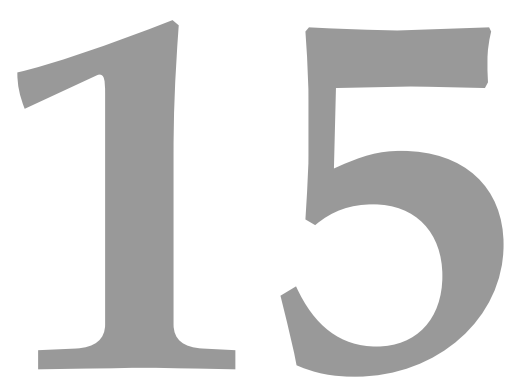

Eine Emissionskarte, wie in Abbildung 14.5 gezeigt, gibt Aufschluss über die Verteilung der Emissionsmengen, die von Autos im Messbereich ausgestoßen wurden. Es ist jedoch anzunehmen, dass das Wetter einen Einfluss auf die Verteilung der Emissionen hat. Aus diesem Grund wird MAINSIM in diesem Kapitel an die atmosphärische Simulation SCIPUFF (Second-order Closure Integrated PUFF Model) Sykes und Gabruk, 1997, Sykes et al., 1984 gekoppelt. Es werden zur Tripgenerierung QZM von Hessen Mobil genutzt. Abbildung 15.1 zeigt den Simulationsbereich 1 . Zur Verdeutlichung der Größe des Szenarios wurden die ungefähren Umrisse der Städte Hanau und Erlensee aufgezeigt, die im Rahmen dieser Arbeit bereits zur Simulation verwendet wurden.

Der Graph besteht aus 60.283 EIs und 46.818 NIs. Die Gesamt-Straßenlänge ist $6.316 \mathrm{~km}$. Der Messbereich ist die Stadt Frankfurt am Main (rot hervorgehoben). Der Kartenausschnitt reicht von Rinderbügen im Norden bis Klein-Ostheim im Süden und von Hofheim im Westen bis Wittgenborn im Osten.

Der folgende Abschnitt 15.1 gibt einen Überblick relevanter Literatur. Abschnitt 15.2 beschreibt die Nutzung von QZM in MAINSIM. Der Versuchsaufbau und die dadurch entstehenden Herausforderungen werden in Abschnitt 15.3 erläutert. Abschnitt 15.4 beschreibt die Ankopplung von MAINSIM an SCIPUFF. Die Verteilung des Windes im Messbereich und die Auswahl bestimmter Tage wird in Ab-

\footnotetext{
${ }^{1}$ Grenzen in WGS84: [8.44: 9.27, 49.98 : 50.34]
} 


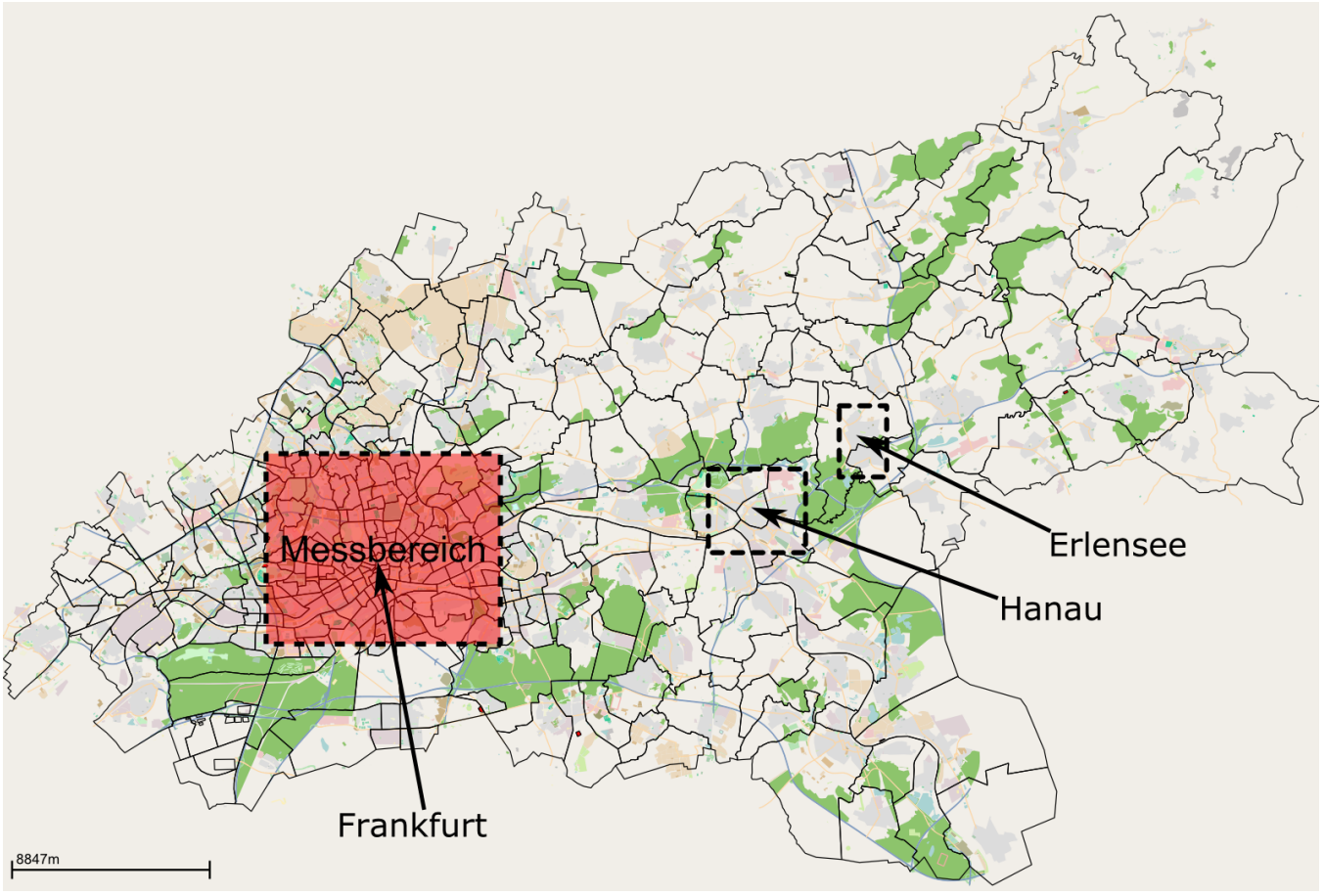

Abbildung 15.1: Simulationsbereich Frankfurt und Umgebung

schnitt 15.5 beschrieben. Die Ergebnisse der atmosphärischen Simulation werden in Abschnitt 15.6 diskutiert. Dieses Kapitel schließt mit einer Zusammenfassung und einem Ausblick in Abschnitt 15.7.

Das Kapitel basiert auf einer Kooperation mit Guido Cervone, Andreas Lattner, Pasquale Franzese und Nigel Waters. Teile der Ergebnisse werden im eingeladenen Buchkapitel Cervone et al., 2013 erscheinen. Die atmosphärischen Simulationen und Auswertungen wurden von Guido Cervone durchgeführt.

\subsection{Relevante Literatur}

Verkehrs- und atmosphärische Ausbreitungsmodelle werden zur Vorhersage der Belastungen für die Luftqualität durch emittierte Schadstoffe genutzt. Es werden hierbei die Verteilung der Emissionskonzentrationen, Spitzenkonzentrationen und Absetzungen berücksichtigt Cervone et al., 2008. Weitere beeinflussende Faktoren sind die Geländetopologie und lokale Mikrometeorologie.

Bei gleichbleibender Verkehrssituation verteilen sich Emissionen in Abhängig- 
keit zum lokalen Wetter unterschiedlich. Wichtige Faktoren sind der durchschnittliche Häuserabstand und die mittlere Gebäudehöhe, sowie Formfaktoren, die den Luftzug beeinflussen. Die durch Fahrzeuge hervorgerufenen Turbulenzen beeinflussen die Emissionsverteilung ebenfalls. Je nach Tageszeit und atmosphärischen Schichtungen kann die Verflüchtigung der Emissionen stark gehemmt werden. Dies führt zu einer signifikanten lokalen Belastung der Luftqualität Franzese und Huq, 2011.

In Di Sabatino et al., 2008 wird eine Studie über die Verteilung von Emissionen in einer Straßenschlucht mit Hilfe des FLUENT22-Modells durchgeführt. Die Ergebnisse werden mit Resultaten des ADMS-Urban Modells Carruthers et al., 1994 verglichen. Die Studie nutzt Abgase einer Abgasquelle und untersucht die Sensitivität der Ergebnisse auf die Windrichtung in Straßenschluchten.

In Kumar et al., 2011 wird ein Überblick über die Simulation der Ausbreitung von Nanopartikeln durch den Straßenverkehr in urbanen Gebieten gegeben. Es werden unterschiedliche Größenordnungen disktutiert: Fahrzeugverwirbelungen, Straßenschluchten, Nachbarschafts-Maßstab, Stadt-Maßstab und Tunnel. Verschiedene aktuelle Ausbreitungsmodelle werden diskutiert und die Relevanz von Transformationsprozessen (Dilution (Verwässerungseffekt), Emission, Nucleation (Zellenbildung), usw.) wird in verschiedenen Skalierungen untersucht.

In Schmidt und Schäfer, 1998] wird ein System zur Analyse von Verkehrsfluss und Luftverschmutzung vorgestellt. Das System nutzt zwei Komponenten: Eine mesoskopische Verkehrssimulation (DYNEMO) und das Imissionsmodell DYMOS. Resultate werden dreidimensional in einem GIS dargestellt. Ein Anwendungsbeispiel in der Nähe von Berlin wird gezeigt. CO-Emissionen von Autos auf verschiedenen Straßen mit unterschiedlichen Ozonkonzentrationen werden berechnet. In einer weiteren Studie wird ein Gebiet um München untersucht [Sydow et al., 1997].

Ein weiteres GIS-Framework wird in Gualtieri und Tartaglia, 1998 vorgeschlagen. Trips werden über QZM generiert. Eine mikroskopische Verkehrssimulation findet nicht statt. Daten eines makroskopischen Modells werden genutzt: Verkehrsfluss $f$, die Anzahl an Fahrzeugen $N$, die durchschnittliche Geschwindigkeit auf der Straße $V_{m}$ und eine Emissionsabschätzungsfunktion $E_{g}$, die nach Fahrzeuggruppen $g$ unterscheidet. $c_{g}$ gibt den prozentualen Anteil der Fahrzeuge der Gruppe $g$ an. Emissionen $Q$ werden nach Gleichung 15.1 abgeschätzt.

${ }^{2}$ http://www .ansys.com/Products/Simulation+Technology/Fluid+Dynamics/ANSYS+Fluent abgerufen am 18.10.2012 


$$
Q=\sum_{g=1}^{N} \frac{c_{g}}{100} \cdot E_{g}\left(V_{m}\right) \cdot f
$$

Das beschriebene System verwendet ein Modell auf Basis der Gaußschen Verteilung von Luftschadstoffen und kann grundlegende Witterungsbedingungen modellieren. Als Simulationsergebnisse können vergleichbare Karten zu Abbildung 14.5 erstellt werden.

Die Arbeit Hatzopoulou et al., 2011 untersucht auf Basis von Abschätzungen der sozioökonomischen Entwicklung der Umgebung von Toronto die Emissionen durch Verkehr im Jahr 2031. Zur Tripgenerierung und Durchführung einer Verkehrssimulation wird Matsim (vgl. Abschnitt 4.4) verwendet. Zur Berechnung der Emissionsverteilung wird ein dreidimensionales Windmodell verwendet.

In Al-Zanaidi et al., 1994 werden CO-Emissionen in Kuwait untersucht. Der Verkehr wird in dieser Studie nicht simuliert, sondern auf Basis von Flussmessungen hochgerechnet. Es werden Emissionsfaktoren pro gefahrenem Kilometer genutzt. Der Wettereinfluss auf die Verbreitung der Emissionen wird anhand gemessener Wetterdaten mit Hilfe des Simulationssystems ITCO berechnet. In Singh et al., 1990 werden Simulationsdaten von ITCO mit Messdaten verglichen. Die vorhergesagten Verläufe der Emissionen über den Tag spiegeln Messdaten wider, weichen jedoch in den Absolutbeträgen ab.

\subsection{Nutzung von Quelle-Ziel-Informationen}

Die in Abbildung 15.1 gezeigten Zellen entsprechen der Einteilung zur Nutzung von Quelle-Ziel-Informationen über den Simulationsbereich. Die QZM wurden von Hessen Mobil zur Verfügung gestellt und sind in Matrizen für Pkw, Lkw und Transporter unterteilt. In diesem Experiment werden daher keine Fahrräder und Fußgänger simuliert.

Der Eintrag $Q Z M_{i j}=n$ gibt an, dass $n$ Verkehrsteilnehmer innerhalb von 24 Stunden von Zelle $i$ nach Zelle $j$ fahren. Es handelt sich somit um statische QZM, die keine tageszeitlichen Schwankungen der Verkehrsmengen modellieren. Die QZM wurden mit Hilfe des EVA-Modells [Schnabel und Lohse, 2011 berechnet. Sie basieren auf den raumstrukturellen Potentialen in den Quell- und Zielbezirken. Die Potentiale basieren auf statistischen Daten über die jeweiligen Zellen - z.B. die Anzahl an Arbeitsplätzen. Jede Zelle $j$ hat auf eine Zelle $i$ eine Anziehungskraft, die 
mit steigender Distanz geringer wird. Dies modelliert den steigenden Aufwand, um von $i$ nach $j$ zu gelangen. Es existieren verschiedene Bewertungsfunktionen, die je nach Verkehrsteilnehmertyp unterschiedliche exponentielle Abklingfunktionen verwenden.

Wenn ein Verkehrsteilnehmer von Zelle $i$ nach Zelle $j$ fahren soll, wird in beiden Zellen eine EI zufällig gewählt, die als Start-, bzw. Zielstraße gilt.

Die Stadt Frankfurt am Main hat morgens eine starke Anziehungskraft auf Berufstätige im Umkreis der Stadt. Die Matrizen werden über ein einfaches Verfahren in dynamische QZM umgewandelt. Es findet eine Aufteilung in Zeitscheiben à eine Stunde statt. Diese Dauer ist willkürlich gesetzt und könnte geändert werden. Sie erscheint fein granular genug, um den Verkehrsverlauf über den Tag nachzubilden. Ohne weitere Skalierung wird die Verkehrsmenge gleichverteilt zu:

$$
Q Z M_{i j}^{t}=\frac{1}{24} \cdot Q Z M_{i j} \forall t \in\{0 \cdots 23\}
$$

Eine nicht gleichmäßige Verteilung bei identischer Verkehrsmenge über 24 Stunden entsteht, wenn mit einem beliebigen 24-elementigen Vektor $\vec{s}$ skaliert wird, für den $\|\vec{s}\|_{1}=1$ und $\vec{s}_{t} \geq 0 \forall t \in\{0 \cdots 23\}$ gilt.

Um Berufsverkehr und sonstigen Verkehr separat betrachten zu können, wird vom Mittelpunkt Frankfurt am Mains mit einem Radius von $5 \mathrm{~km}$ jede in Abbildung 15.1 gezeigte Zelle als Berufsverkehrszone $B Z$ markiert. Tupel $Q Z M_{i j}$ bei denen $j \in B Z$ (Berufsverkehr in Hinrichtung) werden mit $\vec{s}^{\text {th }}$ skaliert, falls $i \in B Z$ (Berufsverkehr in Rückrichtung) wird mit $\vec{s}^{r}$ skaliert. Falls $(i \in B Z) \Leftrightarrow(j \in B Z)$, findet entweder eine Fahrt innerhalb Frankfurts statt oder Frankfurt ist weder Start- noch Zielgebiet. In diesem Fall wird nicht von Frankfurter Berufsverkehr ausgegangen und daher mit $\vec{s}$ skaliert. Abbildung 15.2 zeigt den zeitlichen Verlauf der Skalierung der QZM-Einträge.

Es ist ersichtlich, dass morgens eine starke Verkehrsspitze generiert wird, die anschließend abflaut, bis nachmittags der zweite Berufsverkehr beginnt. Der rückwärtige Berufsverkehr ist stärker gedehnt und dafür weniger intensiv. In den nächtlichen Stunden wird ein Minimum an Verkehr generiert.

Die QZM für Pkw wird wie beschrieben mit $\vec{s}, \vec{s}^{h}$ und $\vec{s}^{r}$ skaliert. Die QZM für Lkw und Transporter werden für alle Tupel $Q Z M_{i j}$ mit $\vec{s}$ skaliert, da diese Fahrzeuggruppen nicht für den klassischen Berufsverkehr genutzt werden. 


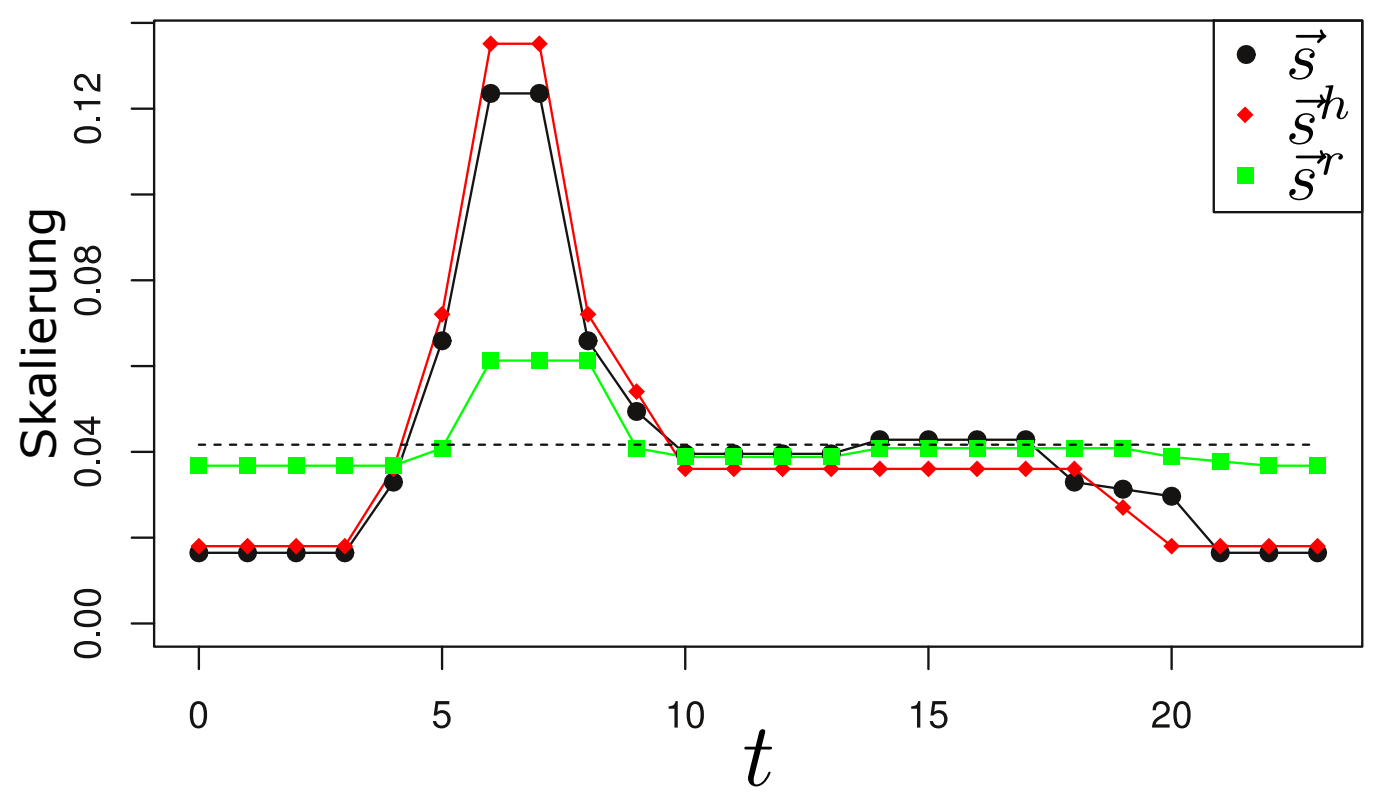

Abbildung 15.2: Skalierungsfaktoren von QZM zur Generierung von dynamischen QZM mit Zeitscheiben.

\subsection{Herausforderungen des Szenarios}

Ein Szenario dieser Größe birgt Herausforderungen. Die Verarbeitung des Kartenausschnitts zur Aufteilung in Layer verwendet knapp 3 GB Arbeitsspeicher. Die Berechnung des Graphen kann über eine Stunde Berechnungsdauer benötigen.

Bei einem Graphen mit knapp 50.000 NIs sind Optimierungen der Algorithmen zur Routenbestimmung notwendig. Ursprünglich wurde in MAINSIM ein gewöhnlicher $\mathrm{A}^{*}$-Algorithmus verwendet. Für dieses Szenario wurde ein bidirektionaler A*-Algorithmus implementiert, mit dem die Simulationsgeschwindigkeit ca. verdoppelt werden konnte.

Die Nutzung statischer QZM führt ähnlich zu dem in Abschnitt 10.3 beschriebenen Szenario zu Staus im Bereich „Am Erlenbruch“ in Frankfurt am Main. Der Verkehr verteilt sich hingegen sehr effizient durch die Nutzung der gleitenden Mittelwerte über Reisedauerinformationen der jeweiligen EIs zur Kantengewichtsbewertung bei der Berechnung von Routen. Die Erweiterung auf dynamische QZM hat sich als schwierig herausgestellt, da die in Abbildung 15.2 gezeigten Skalierungen den Simulationsverlauf empfindlich beeinflussen.

Wenn der morgentliche Berufsverkehr etwas zu stark ausfällt, bilden sich Staus, die sich über Stunden halten können. Wird der abendliche Berufsverkehr zu stark 
gewählt, flaut die Verkehrsmenge in den Abendstunden nicht ab und auch in der Nacht existieren noch Staus. Die gezeigte Skalierung ist das Ergebnis von manueller Anpassung. Ziel der Anpassung war, den erwarteten Verlauf der Verkehrsmengen nachzubilden.

In diesem Szenario werden innerhalb der 24 Stunden Simulationszeit ca. 1,6 Mio. Trips generiert und die dazugehörigen Fahrzeugbewegungen simuliert. Über den Tagesverlauf schwankt die Simulationsgeschwindigkeit. Im Durchschnitt dauert ein Simulationslauf mit 7.200 Iterationen (zwei Stunden - Beginn um 22.00 Uhr Simulationszeit) Einschwingphase und 86.400 Iterationen (24 Stunden - Ende um 24.00 Uhr am simulierten Folgetag) Messphase unter Nutzung eines Intel E6750Prozessors (2,66 Ghz) etwa zwei Tage.

Diese Simulationsdauer bedingt, dass eine Parallelisierung stattfinden muss. In diesem Experiment sind die Ergebnisse die Mengen an $\mathrm{CO}_{2}$-Emissionen im Messbereich, in Zeitscheiben von 15 Minuten unterteilt. Um eine durchschnittliche $\mathrm{CO}_{2}$-Karte des Messbereichs zu erzeugen, werden mehrere Instanzen von MAINSIM gestartet. Der Seed-Wert für den Generator der Zufallszahlen wird in jedem Simulationslauf auf das aktuelle Datum in Millisekunden gesetzt. Die Ergebnisse werden in CSV-Dateien gespeichert. Wenn ein Simulationslauf beendet ist, wird der nächste gestartet. Im Ergebnisordner werden die CSV-Dateien gesammelt.

Zur Auswertung werden die Dateien erneut eingelesen. Es werden eine Datei mit minimalen und eine Datei mit maximalen Emissionen ausgewählt. Die durchschnittlichen Emissionen aller Simulationsläufe werden in einer zusätzlichen Datei gespeichert.

\subsection{Anbindung an atmosphärische Simulation}

Die Emissionen der simulierten Autos werden im Messbereich von Abbildung 15.1 gemessen. Der Messbereich wird analog zum Vorgehen in Kapitel 14 in $100 \times 100$ Zellen zerlegt.

Es besteht keine wechselseitige Beziehung zwischen SCIPUFF und MAINSIM. Es genügt daher, die Ergebnisse der Verkehrssimulation als Eingabedateien für die atmosphärische Simulation zu verwenden. CSV-Ergebnisdateien der Verkehrssimulation werden über einfache Transformationsschritte durch einen Parser so konvertiert, dass SCIPUFF an den entsprechenden Positionen zu den gegebenen Zeitpunkten Gas-Quellen setzt, die mit angegebener Intensität $\mathrm{CO}_{2}$ freigeben. 
SCIPUFF kann sowohl unmittelbare Freisetzungen (z.B. eine Explosion), als auch kontinuierliche Freisetzungen (z.B. Rauch aus einem Kamin) simulieren. In diesem Fall werden kontinuierliche Emissionen angenommen.

Mittels SCIPUFF können anschließend verschiedene Wetterlagen simuliert werden. Die Ausgaben von SCIPUFF bestehen aus zeitabhängigen Konzentrationen. Als Ausgabeformat werden Matrizen verwendet. Mit Hilfe der Statistiksoftware R Project werden hieraus Äquipotentiallinien interpoliert und in einem CSV-Format gespeichert. Diese können anschließend innerhalb von MAINSIM eingelesen und in GIS-Layer exportiert werden. Die Layer können gemeinsam mit den Layern des simulierten Kartenausschnitts gerendert werden, um Erkenntnisse aus den Ergebnissen zu erlangen.

Der folgende Abschnitt beschreibt die Szenarien und deren Ergebnisse.

\subsection{Windverteilung im Messbereich}

Zur atmosphärischen Simulation werden Boden-Messdaten des Flughafens Frankfurt am Main aus dem Jahr 2011 genutzt. Das Jahr 2011 wurde gewählt, da es bei der Durchführung das jüngste vollständige Jahr war. Die Daten wurden heruntergeladen und zu SCIPUFF-Eingabedateien konvertiert. Die verwendeten Wetterdaten beinhalten die Windrichtung und -geschwindigkeit, Temperatur, Luftdruck und Niederschlagsbedingungen (keiner, Regen, Schneeregen, Schnee). Die wichtigsten Einflüsse resultieren aus dem Wind.

Abbildung 15.3 zeigt Messdaten über die Windrichtungen und -stärken im Messbereich als Durchschnitt für das gesamte Jahr 2011 (oben) und für einzelne Tage. Jede Zeile der Abbildung besteht aus zwei Graphen. Der linke Graph zeigt den Verlauf der Windgeschwindigkeit in $\mathrm{m} / \mathrm{s}$ als Funktion über die Zeit. Der rechte Graph visualisiert ein Windrichtungshäufigkeitsdiagramm für den betreffenden Zeitraum.

Die Durchschnittsgraphen für das Jahr 2011 zeigen, dass der Wind im Messbereich hauptsächlich in nordöstlicher und südwestlicher Richtung auftritt. Die Windgeschwindigkeit variiert zwischen Windstille und $14 \mathrm{~m} / \mathrm{s}$ im Maximum. Durchschnittlich wurden Windgeschwindigkeiten zwischen 6 und $8 \mathrm{~m} / \mathrm{s}$ gemessen. Es ist keine klare Trennung der Windgeschwindigkeiten zwischen Tag und Nacht zu erkennen. Die folgenden vier Zeilen der Abbildung zeigen Tage mit unterschiedlichen Windausprägungen. Am 1. März 2011 kam der Wind hauptsächlich aus nordöstlicher 

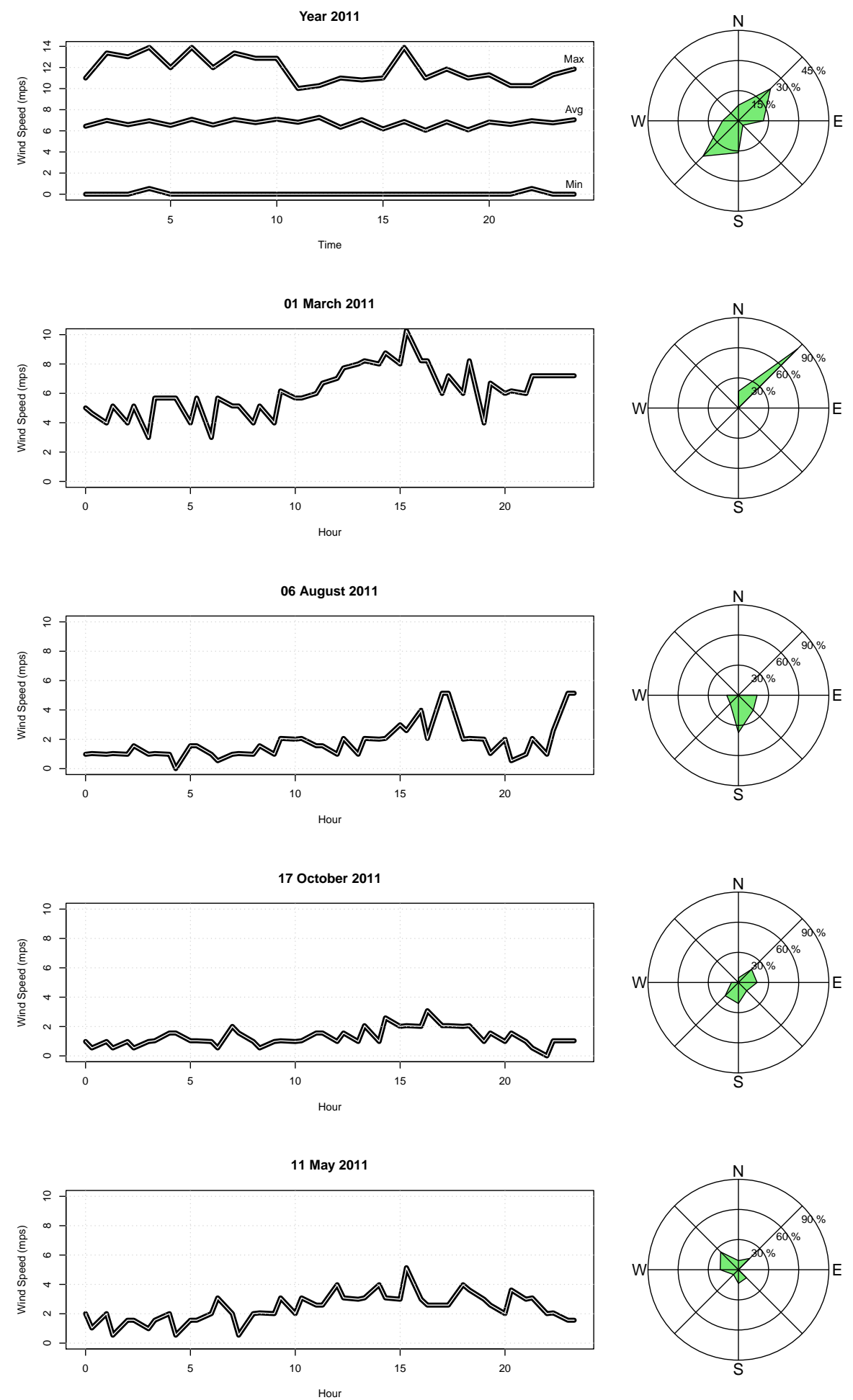

Abbildung 15.3: Windsensordaten Cervone et al., 2013 
Richtung mit einer durchschnittlichen Geschwindigkeit von $6 \mathrm{~m} / \mathrm{s}$ und einer Spitze von $10 \mathrm{~m} / \mathrm{s}$ gegen $16.00 \mathrm{Uhr}$. Am 6. August 2011 dominiert Wind aus dem Süden bei geringer Intensität. Am 17. Oktober 2011 variiert der Wind zwischen nordöstlicher und südwestlicher Richtung bei schwacher Intensität. Am 11. Mai 2011 wurde Wind aus nordwestlicher Richtung bei ebenfalls geringer Intensität gemessen. Dies ist ein für die Messregion ungewöhnlicher Tag.

Abbildung 15.3 verdeutlicht, dass die Wetterbedingungen im Messbereich variieren und somit mehrere Einstellungen mittels SCIPUFF untersucht werden müssen.

\subsection{Ergebnisse}

Es wurden 48 Simulationsläufe von MAINSIM durchgeführt, um den variierenden Ergebnissen durch probabilistische Einflüsse des Verkehrsmodells zu begegnen. Die Simulationsergebnisse wurden anschließend analysiert und minimale, durchschnittliche und maximale Emissionsverläufe über den Messtag extrahiert.

Abbildung 15.4 zeigt die durchschnittlichen kumulierten $\mathrm{CO}_{2}$-Emissionen für die Zellen der simulierten Region am Nachmittag. Es ist zu erkennen, dass Straßen mit hohem Verkehrsaufkommen im Normalfall die höchsten Emissionswerte erzielen. Es wird ein Schnappschuss gezeigt.

Durch Ankopplung an die atmosphärische Simulation entstehen Emissionen über die Zeit. Bei den Abbildungen 15.5 und 15.6 ist zu beachten, dass die Zeiteinheit verschoben ist. Ein Zeitschritt entspricht 30 Minuten. Insgesamt wurden somit nicht 48, sondern 24 Stunden simuliert.

Abbildung 15.5 zeigt die logarithmierten Äquipotentiallinien der durchschnittlichen Emissionen über die Zeit am 1. März 2011. Das Emissionsmaximum wird in dem Bereich gemessen, in dem die stärksten Emissionen während der Verkehrssimulation ermittelt wurden. Mit der Zeit treibt der Wind jedoch die Abgase nach Südwesten. Die nördlichen Areale bleiben unbeeinflusst, da die Windrichtung am 1. März 2011 konstant südwestlich war.

Zum Vergleich zeigt Abbildung 15.6 bei identischer Verkehrssituation die Simulationsergebnisse für den 17. Oktober 2011. Die Windintensität war im Vergleich zum 1. März 2011 geringer, die Windrichtung hingegen variabel. Die Konturlinien zeigen eine im Uhrzeigersinn verlaufende Drehung der Ausbreitungsrichtung der Emissionen. Fast der gesamte Umkreis um das Messgebiet wird in diesem Szenario bis zu einem bestimmten Abstand beeinflusst. 


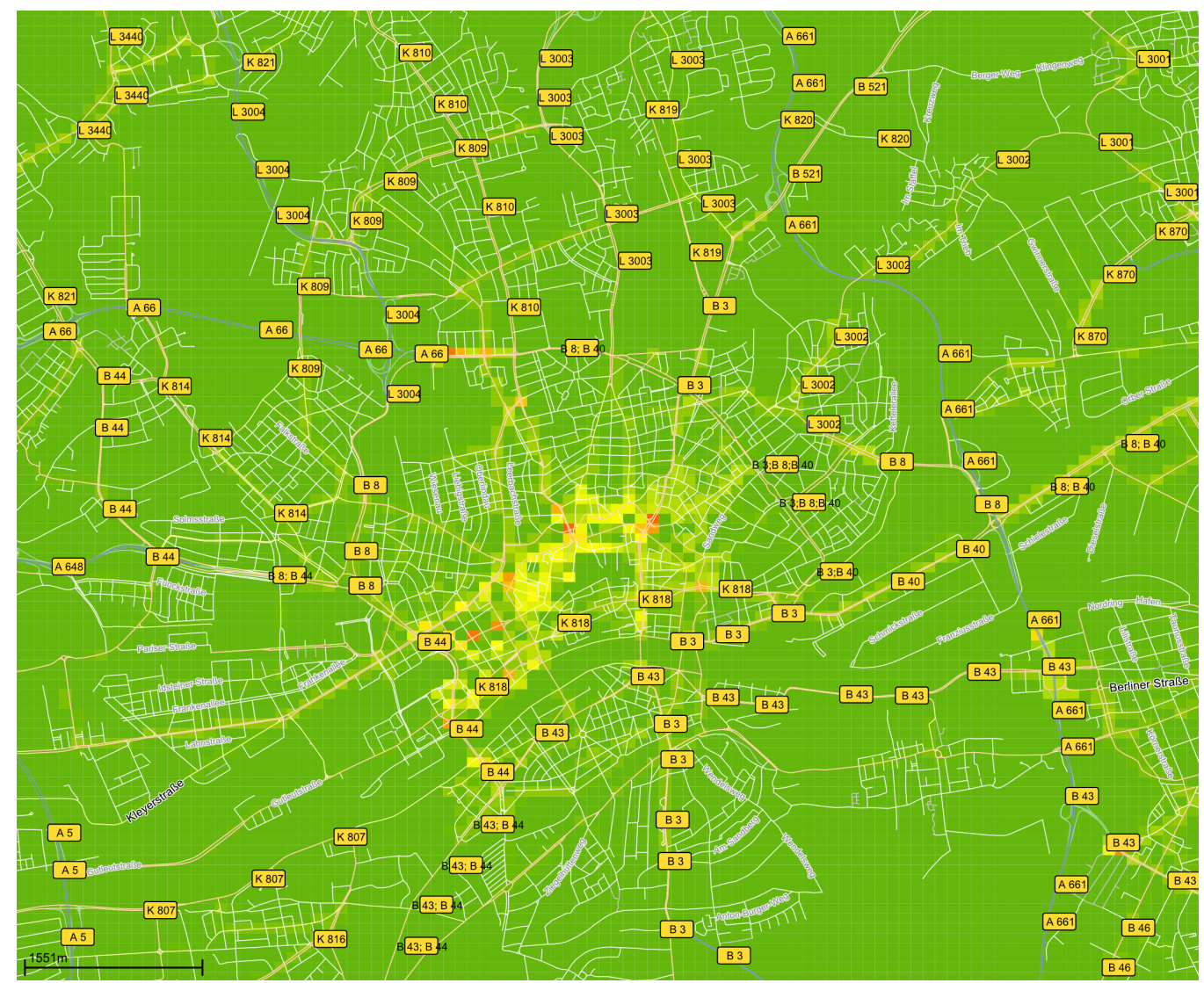

Abbildung 15.4: Emissionen durch Straßenverkehr: Durchschnittliche Emissionen im Zeitabschnitt 17.15 Uhr - 17.30 Uhr. Pro Zelle kumulierte Konzentrationen (grün: Minimum: 0, rot: Maximum: 8,19 kg).

Die interpolierten Äquipotentiallinien werden in den folgenden Abbildungen 15.7 und 15.8 mittels MAINSIM als Layer über den in Abbildung 15.1 gezeigten Kartenausschnitt projiziert. Es wird der Übersicht halber nur die westliche Hälfte des Kartenausschnitts dargestellt und die Darstellung des Bereichs auf die Zellen der QZM reduziert. Die Abbildung ist zeilenweise nach Datum und spaltenweise nach Zeitschritt (20, 30, 40 - entspricht 10, 15, 20 Stunden) unterteilt. Die Abbildung erlaubt die Analyse, welche Gebiete durchschnittlich besonders durch $\mathrm{CO}_{2}$-Emissionen belastet sind und welche Gebiete die höchsten Spitzenwerte erreichen. Diese Informationen könnten später genutzt werden, um die Verkehrsströme hinsichtlich der Zielgrößen „Reisedauer der Verkehrsteilnehmer“ und „Emissionsbelastungen" zu optimieren.

In Abbildung 15.7 werden zur atmosphärischen Simulation die durchschnittlichen Emissionen aus den 48 Simulationsläufen von MAINSIM verwendet. 

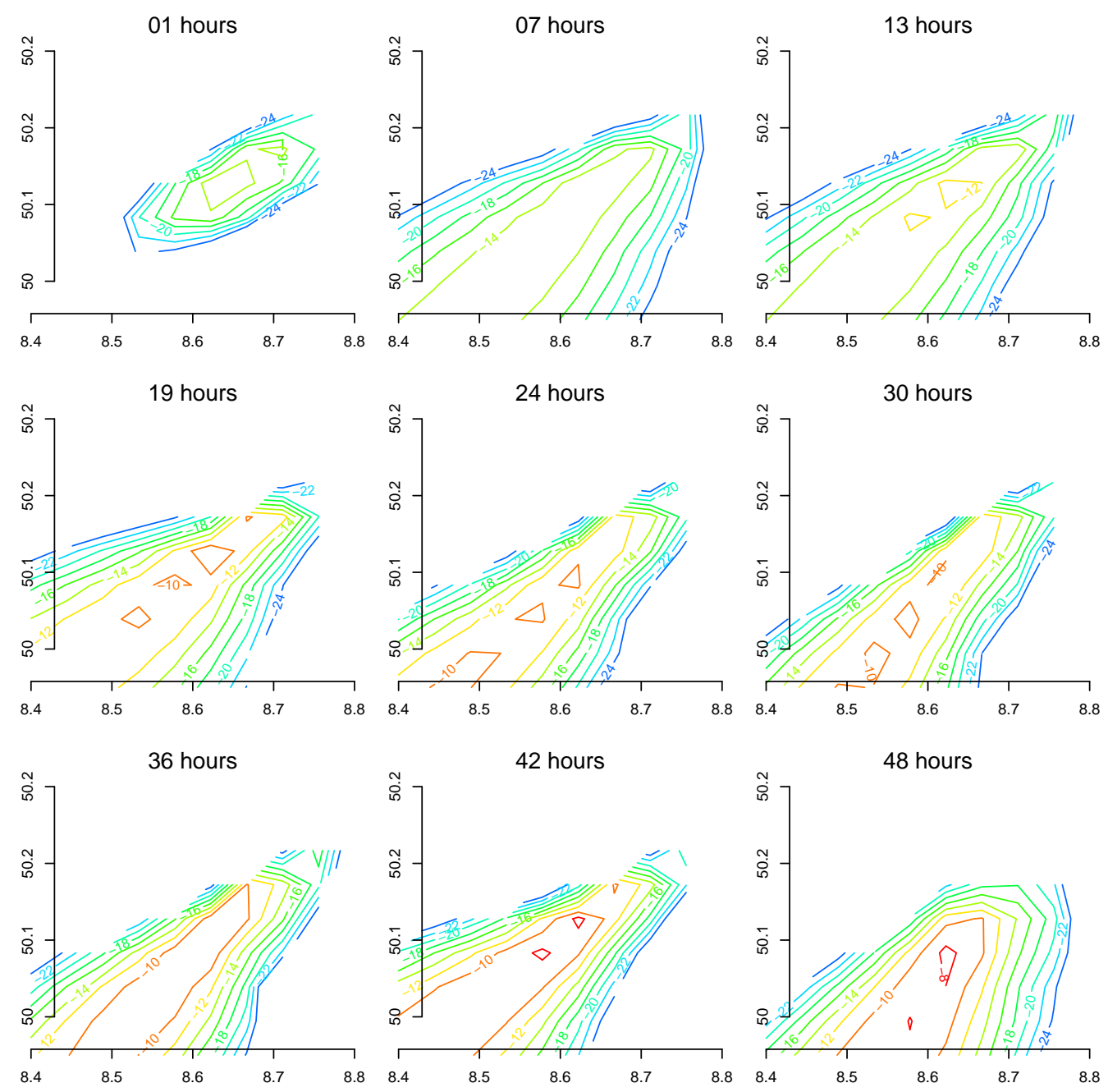

Abbildung 15.5: $\mathrm{CO}_{2}$-Konturen: 1. März $2011(\log (\mathrm{kg}))$ Cervone et al., 2013.

Abbildung 15.8 zeigt den Jahresdurchschnitt des Wetters und die Auswirkungen unterschiedlicher Verkehrsbelastungen anhand der ermittelten minimalen, durchschnittlichen und maximalen Emissionen innerhalb der 48 Verkehrssimulationsläufe. Zur Bestimmung des Jahresdurchschnitts des Wetters wurden die Wettereinflüsse aller Tage des Jahres 2011 mit identischen Emissionswerten simuliert und die resultierenden Ergebnismatrizen gemittelt. Die Ausbreitungsrichtung und -charakteristik der Emissionen ändert sich nicht bei Variation der Emissionsstärke. Das Ausmaß der Konzentrationen ist hingegen proportional zu den ermittelten Emissionen. 

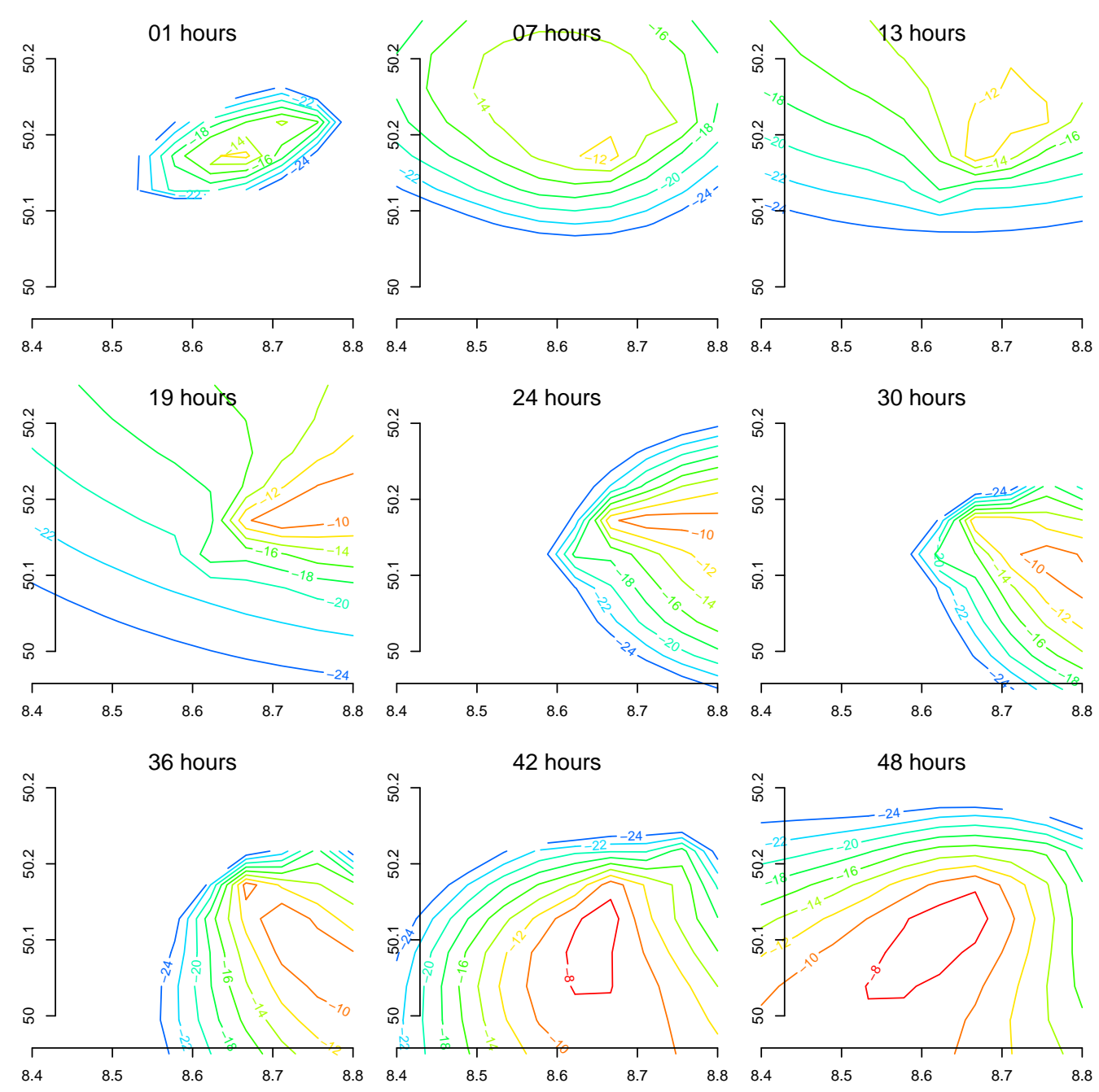

Abbildung 15.6: $\mathrm{CO}_{2}$-Konturen: 17. Oktober $2011(\log (\mathrm{kg}))$ Cervone et al., 2013.

\subsection{Zusammenfassung und Ausblick}

In dieser Fallstudie wurde MAINSIM an die atmosphärische Simulation SCIPUFF angekoppelt, um die atmosphärischen Belastungen unter verschiedenen Wetterbedingungen zu vergleichen. Mit Hilfe der beschriebenen Verfahren können unterschiedliche Verkehrssituationen mit gemessenen oder zu erwartenden Wettercharakteristika kombiniert werden, um Luftverunreinigungen durch den Straßenverkehr vorherzusagen und zu erwartende Überschreitungen von Grenzwerten zu bestimmen.

Der Ansatz wurde im Bereich von Frankfurt am Main getestet. Hierfür wurde 


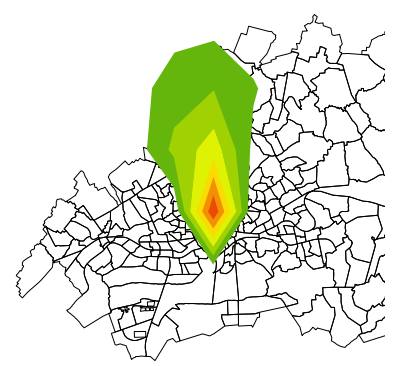

(a) 6. August 2011: 20

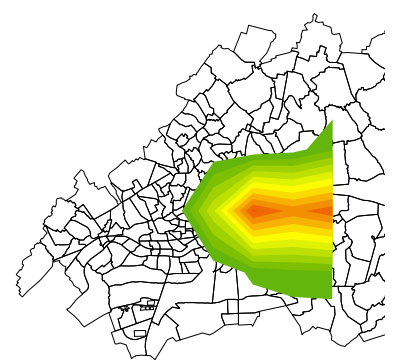

(d) 11. Mai 2011: 20

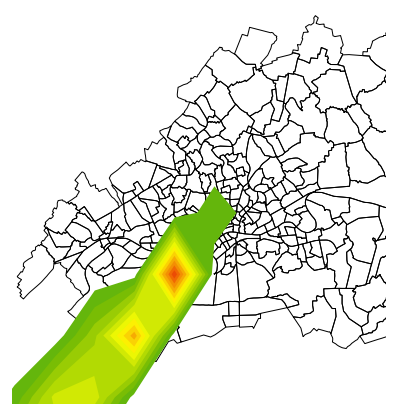

(g) 1. März 2011: 20

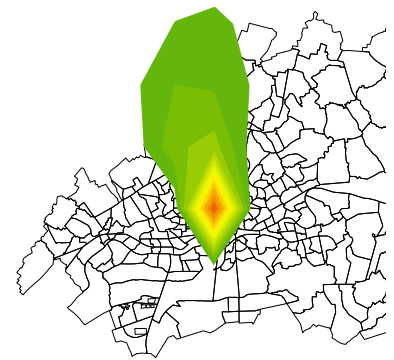

(b) 6. August 2011: 30

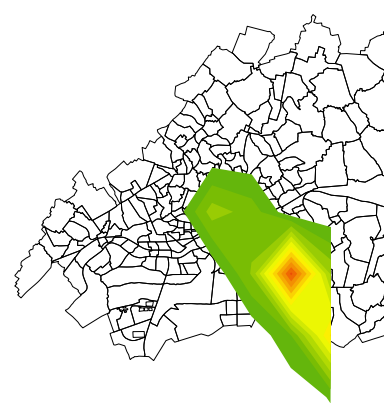

(e) 11. Mai 2011: 30

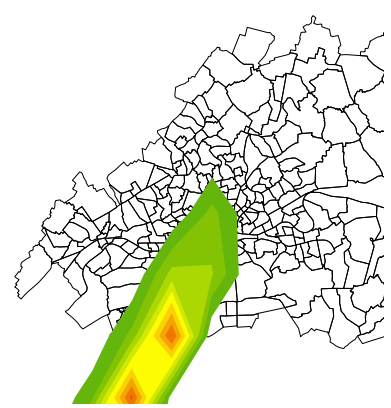

(h) 1. März 2011: 30

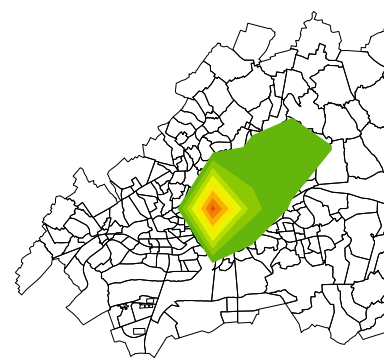

(c) 6. August 2011: 40

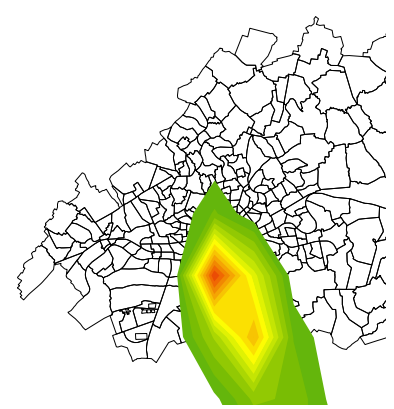

(f) 11. Mai 2011: 40

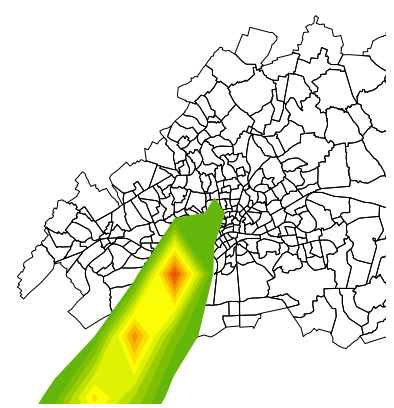

(i) 1. März 2011: 40

Abbildung 15.7: Verteilung der $\mathrm{CO}_{2}$-Emissionen für verschiedene Tage. 


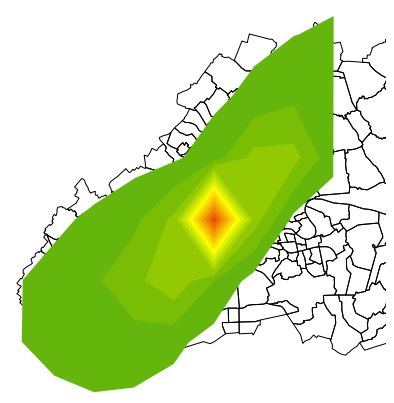

(a) Maximum

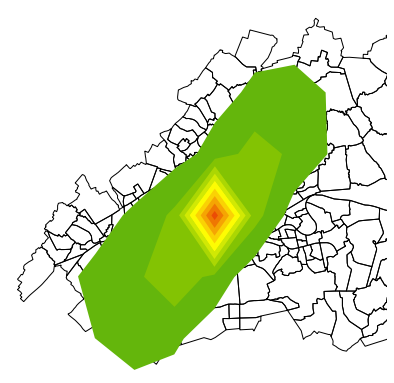

(b) Durchschnitt

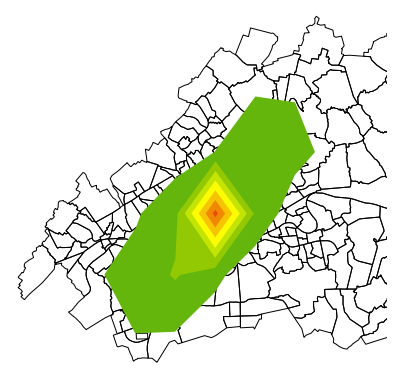

(c) Minimum

Abbildung 15.8: Verteilung der $\mathrm{CO}_{2}$-Emissionen für das Jahr 2011 unter Nutzung maximaler (links), durchschnittlicher (Mitte) und minimaler (rechts) Emissionen.

MAINSIM mit statischen QZM kalibriert, die in dynamische QZM separiert wurden. Das Szenario umfasste einen Straßengraphen mit knapp 50.000 NIs, auf dem innerhalb von 24 Stunden Messphase ca. 1.600.000 Trips von Fahrzeugen simuliert wurden. Die $\mathrm{CO}_{2}$-Emissionen der Fahrzeuge im Messbereich wurden mit Hilfe eines DGM und des im vorherigen Kapitel 14 diskutierten Kraftstoffverbrauchsund Emissionsmodells berechnet.

Zur Analyse des Wettereinflusses wurden reale Messdaten aus dem Jahr 2011 genutzt. Die Ergebnisse zeigen, dass es möglich ist, ein Verkehrssimulationssystem mit einer atmosphärischen Simulation zu koppeln. Verschiedene Verkehrssituationen und variierende atmosphärische Einflüsse führen zu unterschiedlichen Konzentrationen innerhalb und außerhalb des Messbereichs.

Die Ergebnisse zeigen deutlich, dass eine Analyse der $\mathrm{CO}_{2}$-Emissionen innerhalb eines Messbereichs (vgl. Kapitel 14 nur für einen ersten Überblick genügt. Es muss ferner der Verkehr umliegender Bereiche simuliert werden, um die Wechselwirkungen, die durch Wettereinflüsse zwischen den Arealen entstehen, berücksichtigen zu können. Auch Bereiche, in denen kein Straßenverkehr vorhanden ist, können durch dessen Emissionen belastet werden.

Um mit dem beschriebenen Verfahren Vorhersagen treffen zu können, muss in der Zukunft ein Vergleich von ermittelten Konzentrationen mit gemessenen Werten realer Sensoren durchgeführt werden und das System gegebenenfalls kalibriert werden. Die Kalibrierung ist mehrschichtig, da sowohl MAINSIM als auch SCI- 
PUFF an die lokalen Bedingungen angepasst werden müssen. Dies ist aufgrund der Größe des Szenarios eine schwierige Aufgabe. Nachdem dieses Kapitel die Durchführbarkeit dieser Studie in ihrer Größe gezeigt hat, wäre die Nutzung in einem kleineren Szenario der Größenordnung eines Ortes zur Kalibrierung ein nächster Schritt.

In der Zukunft könnte mit dem beschriebenen Ansatz untersucht werden, ob bei unterschiedlichem zu erwartendem Wetter verschiedene Verkehrsleitstrategien notwendig sind, um die Performanz eines städtischen Verkehrssystems zu optimieren. An besonders windstillen Tagen ist zu erwarten, dass die Abgase sich langsam aus der Stadt verflüchtigen und daher die Luftqualität besonders leidet. Es könnten in der Folge bestimmte Areale mit vorgegebenen Grenzwerten belegt werden, die durch die simulierten Emissionen bei Betrachtung des Wetters eines Jahres nur $n$ mal um maximal $p \%$ überschritten werden dürfen. Ein Optimierungsverfahren könnte versuchen, dieses Ziel durch wiederholte Simulationsläufe zu erreichen. 


\section{Teil VI}

\section{Zusammenfassung und Ausblick}





\section{Zusammenfassung und Ausblick}

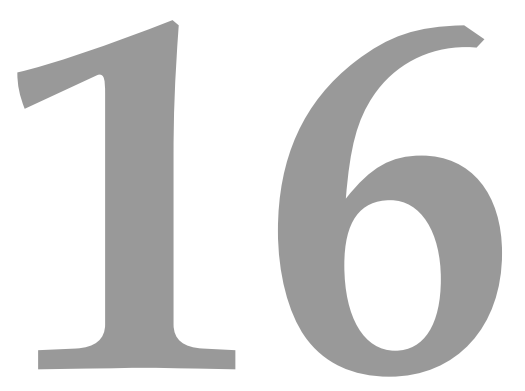

Dieses Kapitel fasst die vorliegende Arbeit zusammen und gibt einen kurzen Ausblick für zukünftige Untersuchungen.

Im Rahmen dieser Arbeit sollte der Verkehr ganzer Städte mit minimalem Modellierungsaufwand untersucht werden können. Das Ziel war die Untersuchung verschiedener Fragestellungen, die erst bei dieser Größenordnung sinnvoll werden. Hierfür wird ein Verkehrssimulationssystem benötigt, das die in Kapitel 11 gestellten Anforderungen erfüllt. Die wichtigsten Eigenschaften sind:

- Eine Methode, um aus Kartenmaterial automatisch einen Simulationsgraphen bestimmen zu können.

- Verkehrsmodelle, die die wichtigsten Verkehrsteilnehmertypen im urbanen Bereich Auto, Fahrrad, Fußgänger und deren Wechselwirkungen abbilden können.

- Das System muss skalierbar sein.

- Es muss über Möglichkeiten der Erweiterbarkeit verfügen.

Die Betrachtung des Stands der Forschung in Teil $\amalg$ hat gezeigt, dass keines der 
bestehenden Verkehrssimulationssysteme große Szenarien unter Berücksichtigung multimodalen Verkehrs unter vertretbarem Aufwand simulieren kann.

Es wurde daher ein Konzept entwickelt, das die durchgängige Modellierung verschiedener Verkehrsteilnehmertypen auf einem Straßengraphen ermöglicht und die obigen Anforderungen erfüllt. Das Konzept wurde im Rahmen einer prototypischen Implementierung namens MAINSIM getestet. Die grundlegende Architektur, wichtige Designentscheidungen und die Funktionsweise der entwickelten mikroskopischen Verkehrsmodelle wurden in Teil III beschrieben.

Nach Evaluierung und Plausibilitätstests der Modelle konnten Fallstudien verschiedener Ausprägungen in Teil V]durchgeführt werden. Sie reichten von der Anwendung von maschinellen Lernverfahren im Bereich der Verkehrssimulation über die Abschätzung der Effekte, die Fußgänger auf den Straßenverkehr haben bis hin zur Berücksichtigung von Verhaltensweisen im Straßenverkehr, die nonkonform bezüglich Normen und Regeln sind. Es wurde ein neuartiges Routingverfahren besprochen. Der Benzinverbrauch der simulierten Autos wurde in Relation zum Einfluss nicht motorisiertem Verkehrs berechnet. Die $\mathrm{CO}_{2}$-Emissionen des Verkehrs konnten genutzt werden, um die Ausbreitung der Gase innerhalb Frankfurt am Mains zu simulieren.

Die Fallstudien zeigten, dass das entwickelte Konzept in die Lage versetzt, große Szenarien zu simulieren und die Wechselwirkungen multimodalen Verkehrs in urbanen Szenarien zu berücksichtigen. Dies stellt eine Erweiterung des Stands der Forschung dar. In den analysierten Szenarien konnte ein Einfluss von Fahrrädern und Fußgängern auf den Automobilverkehr gezeigt werden.

Ein weiteres Ergebnis ist, dass große Szenarien notwendig sind, um bestimmte Fragestellungen untersuchen zu können. Beispiele hierfür sind das in Kapitel 13 entwickelte Routingkonzept zur Optimierung der Verkehrsströme und die Abschätzung der durch den Straßenverkehr verursachten $\mathrm{CO}_{2}$-Verteilung innerhalb einer Stadt (vgl. Kap. 15).

Angereichert mit Quelle-Ziel-Informationen kann MAINSIM genutzt werden, um den Verkehr ganzer Städte zu simulieren und makroskopische Kenngrößen zu ermitteln. Durch die Nutzung mikroskopischer Verkehrsmodelle können zusätzlich individuelle Größen, die z.B. eine bestimmte Verkehrsteilnehmergruppe betreffen, ausgegeben werden.

MAINSIM zeichnet sich dadurch aus, direkt mit Geodaten arbeiten zu können. Dies ermöglicht eine einfache Anreicherung der Simulationsmodelle mit externen 
Informationen.

Die entwickelten Verkehrsmodelle sind nicht darauf ausgelegt, den Verkehr exakt abzubilden, sondern makroskopisch plausible Kenngrößen zu ermitteln. Es wäre z.B. nicht möglich, die optimale Länge einer Auffahrt auf eine Autobahn zu ermitteln. Dies stellt keine Schwäche des Konzepts dar, sondern die Ausrichtung des mikroskopischen Automodells zur Simulation großer Szenarien.

Simulationsergebnisse hängen stets von den Eingabedaten und den verwendeten Simulationsmodellen ab. Einen großen Einfluss hat im Bereich der Verkehrssimulation die Güte des Straßengraphen. Das entwickelte Konzept hat anhand der exemplarischen Entwicklung einer Importfunktion für OSM-Kartenmaterial gezeigt, dass eine automatische Graphgenerierung funktionieren kann. Zur Steigerung der Graphqualität muss in der Zukunft untersucht werden, wie zusätzlich realtätsgetreue Ampelschaltungen auf Basis maschinell lesbarer Schaltpläne in das Konzept integriert werden können.

Der Bereich der Kalibrierung wurde im Rahmen dieser Arbeit diskutiert, um MAINSIM zu evaluieren. In der Literatur werden ausgiebig Verfahren diskutiert, die für verschiedene Bereiche der Kalibrierung genutzt werden können (vgl. Chu et al., 2003, Rakha et al., 1996, Grubb et al., 1999, Duong et al., 2010, Hourdakis et al., 2003, Toledo et al., 2003). Das Grundprinzip ist stets die Minimierung eines Fehlers gegenüber gemessenen Daten (z.B. Verkehrsfluss und Fließgeschwindigkeit). Wenn ein Verkehrsmodell kalibriert ist, können dennoch unrealistische Ergebnisse entstehen, wenn die Tripgenerierung auf Basis von Quelle-ZielInformationen nicht korrekt adjustiert ist. Auch die Kalibrierung bei der Generierung von dynamischen QZM aus statischen QZM könnte anhand von Messdaten automatisiert werden. In der Zukunft muss dieses Thema bearbeitet werden, damit MAINSIM für Planungszwecke in realen Szenarien eingesetzt werden kann.

Die Lösung, nahe beieinander liegende Ampeln auszudünnen, kann nur eine Notlösung sein. Ein weiteres Forschungsthema wäre die Untersuchung, wie die logischen Zusammenhänge an einer Kreuzung anhand der kartographierten Positionen von Ampeln erkannt werden können. Anschließend könnte zumindest für eine einzeln betrachtete Kreuzung eine realistische Ampelschaltung generiert werden, die einen Verbund von Ampel(anzeigen) steuert.

Eine ähnliche Aufgabe ist die Modellierung von U- und Straßenbahnen, Bussen oder gar von Zugverkehr. Die genannten Verkehrsteilnehmertypen können einen Einfluss auf den Straßenverkehr haben, wenn sie beispielsweise an Ampeln bevorzugt werden (Straßenbahn) oder generell grün erhalten (U-Bahn) bzw. eine 
Schranke die Durchfahrt garantiert und gleichzeitig den restlichen Straßen- und Fußverkehr behindert. Fußgänger können in großen Mengen zu- oder aussteigen und lokal Störungen im Straßenverkehr verursachen.

Aggressionen wurden anhand der Fallstudien in Kapitel 12 untersucht. In den zugrundeliegenden mikroskopischen Verkehrsmodellen berücksichtigt jedoch nur das Modell für Fußgängerbewegungen Aggressivität. Die in Abschnitt 3.4 .2 beschriebenen Verhaltensweisen sollten in der Zukunft integriert werden. Kommunikative Aggressionen sind nicht zwangsweise notwendig. Die Variierung der Sicherheitsabstände, Lückenakzeptanzwerte oder gefahrenen Geschwindigkeiten in Relation zur Verkehrssituation könnte hingegen Veränderungen des makroskopischen Verhaltens ergeben.

Die modellierten Verkehrsteilnehmer in MAINSIM halten an Ampeln, wenn diese von grün auf gelb umschalten. Im realen Straßenverkehr tendieren Menschen häufig dazu, zu beschleunigen und nur dann zu halten, wenn ein Passieren der Ampel unrealistisch erscheint. Die Auswirkungen dieses Verhaltens ließen sich über ein komplexeres Aggressionspotential implementieren, welches den Verlauf der Fahrt und die Behinderungen, die ein Verkehrsteilnehmer erfährt, berücksichtigen.

Die Nutzung eines DGM ist nicht immer möglich, da Höheninformationen nicht in OSM verzeichnet werden und somit separat integriert werden müssen. Aus diesem Grund wurde bisher keine Berücksichtigung der Steigungen des Straßenverlaufs für das Beschleunigungs- und Routenplanungsverhalten der Verkehrsteilnehmer berücksichtigt. Fahrräder bevorzugen in der Realität Wege mit wenigen Steigungen. Manche Autos bzw. Lkw können eventuell Steigungen ab einem Grenzwinkel nicht mehr bewältigen. Diese Erweiterungen können über Anpassungen der Kantengewichte bei der Routenplanung, einer Skalierung der maximalen Beschleunigung mit einem vom Steigungswinkel abhängigen Faktor und einer Abfrage der maximalen Steigung für die Routenplanung integriert werden.

Im Patent Aydin, 2004 werden Restzeitampeln beschrieben. Restzeitampeln sind Ampeln, die anzeigen, wie lange sie noch rot sein werden. Für Fußgänger existieren diese z.B. in Hamburg. Es gab zwischen 2006 und 2007 einen Test in Hamburg, auch für Autos diese Zusatzinformation bereitzustellen. Dies sollte den Verkehrsfluss erhöhen, indem beispielsweise der Gang bereits vor Umschalten der Ampeln eingelegt werden kann und ein Bremsmanöver bei rot werdender Ampel rechtzeitig begonnen werden kann. Die Zahl der bei rot überfahrenen Ampeln sank, doch eine spürbare Beschleunigung des Anfahrprozesses bei Grünwerden der Ampeln 
konnte nicht gemessen werden ${ }^{1}$.

Eine Ampel mit Restzeitanzeige könnte zur Reduzierung des Kraftstoffverbrauchs genutzt werden. Autofahrer könnten den Motor abschalten, wenn die Ampel noch genügend lange rot sein wird. Zur Automatisierung könnten Ampeln zusätzlich die Restdauer der aktuellen Phase per Funk an die Fahrzeuge übertragen. Auf diese Weise könnte auch die Emissionsbelastung an Ampelkreuzungen reduziert werden. Den Motor wieder anzuschalten kostet ebenfalls Energie, die durch den Motor erbracht werden muss und somit den Kraftstoffverbrauch erhöht. Die Zeit, ab der sich ein Abschalten des Motors lohnt, müsste somit geklärt werden. Sobald diese Information jedoch vorhanden ist, kann mit Hilfe von MAINSIM untersucht werden, wie viel Ersparnis im Stadtverkehr durch eine Restzeitanzeige erzielt werden könnte.

In Eilts, 2009 wurde untersucht, welche Auswirkungen Straßenverkehrslärm bei Nacht auf die Gesundheit von Kindern hat. Die Ergebnisse der Studie weisen auf eine Gesundheitsschädlichkeit hin. Die Autoren fordern daher Lärmschutzmaßnahmen für Wohngebiete. Die Geräuschemissionen eines Autos hängen bei identischer Straße von seinem Typ, seiner Geschwindigkeit und seiner Beschleunigung ab Coensel und Botteldooren, 2007, Peeters und Blokland, 2007. Ein Korrelationsvergleich zwischen $\mathrm{CO}_{2^{-}}$und Schallemissionen, sowie die Untersuchung der Nutzungsmöglichkeit von Optimierungsverfahren zur Minimierung der Emissionen ist ein wichtiges zukünftiges Forschungsthema.

${ }^{1}$ http://www.abendblatt.de/hamburg/article488326/Behoerde-stoppt-das-Projekt-

Restzeitampel.html abgerufen am 06.08.2012 



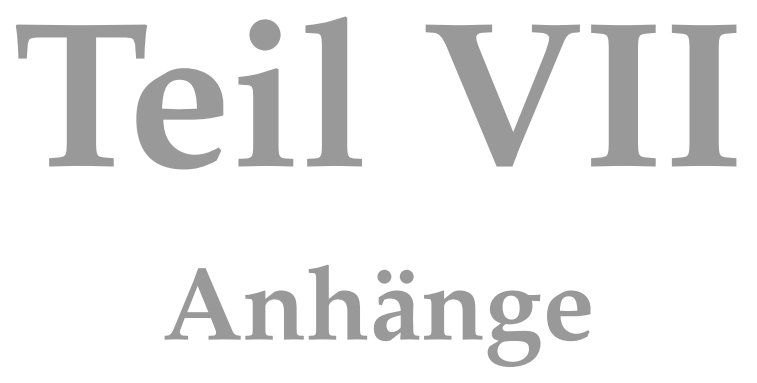





\section{Symbole und Abkürzungen}

\begin{tabular}{|c|c|}
\hline Symbole & \\
\hline$\perp$ & Trödelfaktor \\
\hline$\perp^{\prime}$ & Unachtsamkeitsfaktor \\
\hline$v_{d}$ & Bremsmaximum \\
\hline$v_{K}^{\mathrm{EI}}$ & Maximalgeschwindigkeit von EI durch Krümmung der Straße \\
\hline$\psi$ & Skalierung des Einflusses von $v_{K}^{\mathrm{EI}}$ auf ein Fahrzeug \\
\hline$H_{n}(A)$ & Absolute Häufigkeit von Ereignis $A$ bei Stichprobengröße $n$ \\
\hline$h_{n}(A)$ & Relative Häufigkeit von Ereignis $A$ bei Stichprobengröße $n$ \\
\hline$\xi$ & Beschleunigungsfaktor \\
\hline$\mu$ & Mittelwert \\
\hline$f$ & Wahrscheinlichkeitsdichtefunktion \\
\hline$\sigma$ & Standardabweichung \\
\hline $\mathcal{N}(\mu, \sigma)$ & Normalverteilung um $\mu$ mit $\sigma$ \\
\hline $\mathcal{N}_{a}^{b}(\mu, \sigma)$ & Normalverteilung um $\mu$ mit $\sigma$ begrenzt auf Intervall $[a, b]$ \\
\hline$N$ & Nachbarschaft \\
\hline$\vartheta(s)$ & Bewertung der Spur $s$ \\
\hline$t_{l}^{l}$ & Zeitliche Lücke für Abbiegevorgänge nach links \\
\hline$t_{l}^{r}$ & Zeitliche Lücke für Abbiegevorgänge nach rechts \\
\hline$t_{l}^{g}$ & Zeitliche Lücke für Abbiegevorgänge geradeaus \\
\hline EI & EdgeInformation \\
\hline NI & NodeInformation \\
\hline$|\mathrm{EI}|$ & Länge von EI in $[m]$ \\
\hline
\end{tabular}


Abkürzungen

\begin{tabular}{ll}
\hline GHR & Gazis-Herman-Rothery \\
GIS & Geoinformationssystem \\
IDM & Intelligent Driver Modell \\
LWR & Lighthill-Whitham-Richards \\
LUT & Lookup-Table \\
OSM & OpenStreetMap \\
OVM & Optimal-Velocity Modell \\
MAS & Multi-Agenten-Simulation \\
ML & Maschinelle(s) Lernverfahren \\
NSM & Nagel-Schreckenberg-Modell \\
QZM & Quelle-Ziel-Matrix \\
SHP & Shapefile \\
XML & Extensible Markup Language \\
ZA & Zellulärer Automat
\end{tabular}




\section{Sicherheitsabstand}

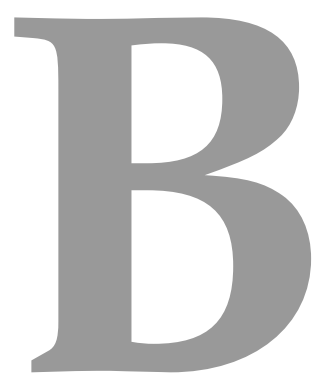

In diesem Anhang wurde das in Abschnitt 9.2.1 durchgeführte Experiment zur Bestimmung grundlegender Diagramme bezüglich Verkehrsdichte, -fluss und -geschwindigkeit auf einer einspurigen Straße ohne Geschwindigkeitsbegrenzung wiederholt. In Abbildung B.1 sind die Diagramme der linken Spalte identisch zu denen in Abschnitt 9.2.1. Zur Bestimmung der rechten Spalte wurde der Sicherheitsabstand auf den „halben Tachoabstand“ erhöht (2 s).

Der Verkehrsfluss bei 2 s Sicherheitsabstand erreicht bei einer Verkehrsdichte von 12,62 Autos / km sein Maximum von 1362,24 Autos / h. Dies stellt eine deutliche Verringerung im Vergleich zu realen Messdaten dar. In der Abbildung wurden zu Vergleichszwecken stets die Funktionswerte bei der Verkehrsdichte mit maximalem Fluss unter Nutzung von 1,33 s Sicherheitsabstand angegeben.

Die weiteren Zeilen von Abbildung B.1 setzen den Trend fort, dass ein größerer Sicherheitsabstand zu höherer Staugefahr führt. Da die Autos bei größerem Sicherheitsabstand mehr Platz benötigen, steigt im übertragenen Sinne die Verkehrsdichte bei gleichbleibender Anzahl an Fahrzeugen, wenn der Sicherheitsabstand erhöht wird.

Der „halbe Tachoabstand“ als Sicherheitsabstand wird in keinem der im Stand der Forschung betrachteten Modelle als Sicherheitsabstand genutzt, da die Ergebnisse der Modelle in diesem Fall unrealistisch werden. 

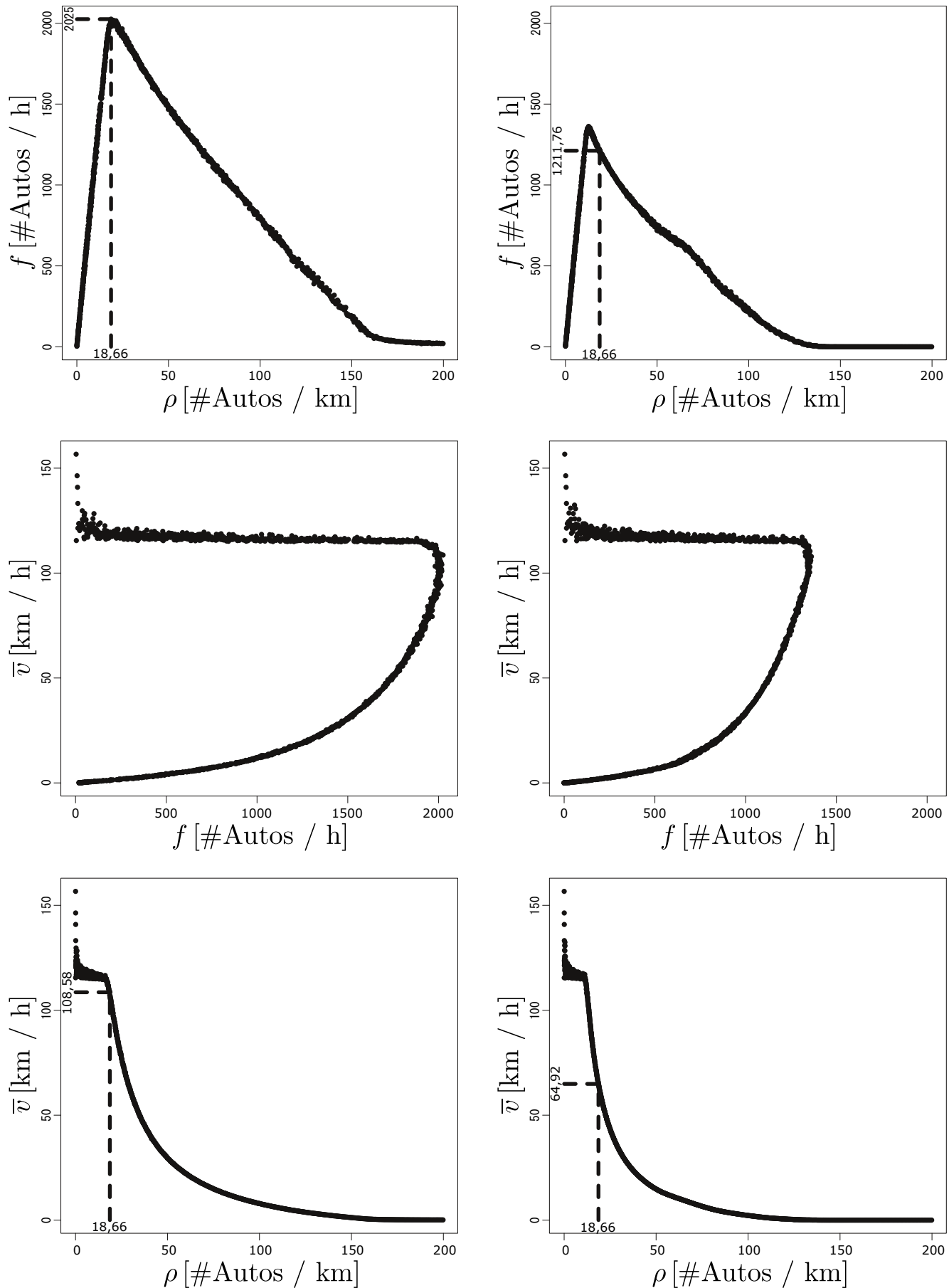

Abbildung B.1: Auswirkung der Wahl des Sicherheitsabstands auf grundlegende makroskopische Kenngrößen. Linke Spalte: 1,33 s. Rechte Spalte: 2 s. 


\section{Fußgängerbewegung: Algorithmus auf ZA-Basis}

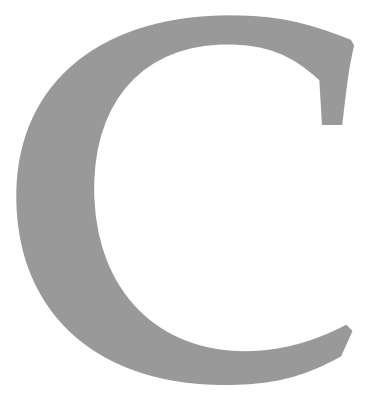

Dieser Anhang beschreibt den in Blue und Adler, 2001 vorgestellten Algorithmus für Fußgängerbewegungen. Die grundlegende Funtkionsweise wird in Abschnitt 3.3.4 auf Seite 48 erläutert. Algorithmus C.1 präzisiert die Funktionsweise in Pseudocode. Die Prozedur update() aktualisiert die Positionen aller simulierten Fußgänger. Im ersten Schritt wird für jeden Fußgänger in Zeile 3 ermittelt, welche Spuren ausgehend von seiner aktuellen Spur derzeit nutzbar sind. Nutzbar sind alle Felder, die nicht belegt und zum aktuellen Feld benachbart sind. Wenn ein Feld von mehr als einem Fußgänger beansprucht wird, wird es zufällig einem von ihnen zugeteilt (siehe Algorithmus C.1).

Die Zeilen 5 und 6 bestimmen, ob ein Spurwechsel durchgeführt wird. Es wird die Spur gewählt, die das beste Vorankommen verspricht. Hierfür wird zuerst mit Hilfe der Prozedur bestimmeLücken () für jeden Fußgänger $f$ geprüft, wie groß die Lücken der benachbarten Spuren zu anderen Fußgängern sind. Bei Fußgängern, die $f$ entgegenkommen, werden Lücken halbiert, um deren Fortbewegung Rechnung zu tragen (Zeile 24).

Es wird in Prozedur wähleSpur() die Spur gewählt, die die größte Lücke enthält. Hierfür wird jeder Spur $s$ eine Nutzungswahrscheinlichkeit $p_{s}$ zugeordnet. Falls auf der aktuellen Spur ein Fußgänger entgegenkommt, wird ein Spurwechsel zu 


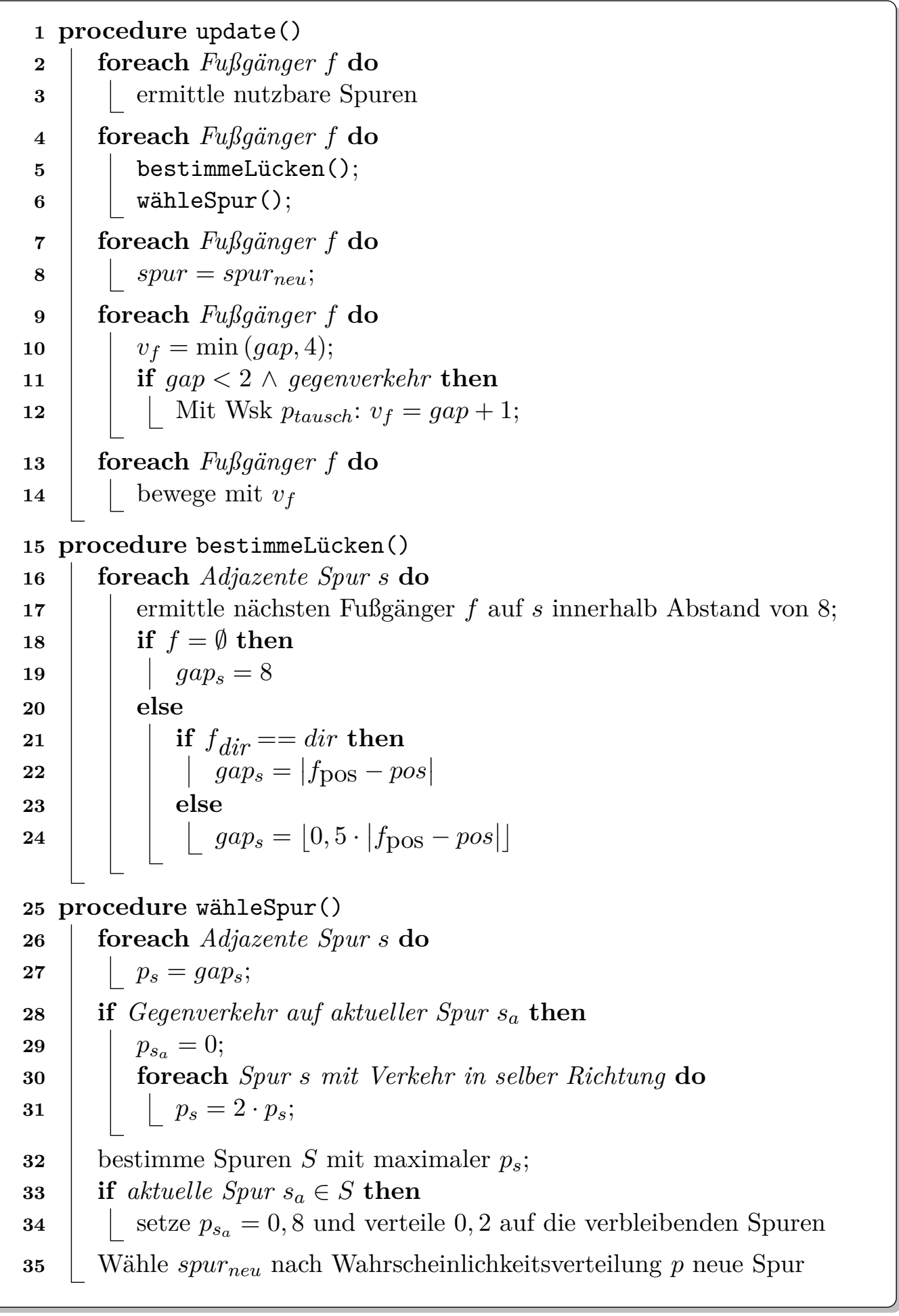

Algorithmus C.1: ZA-Fußgängermodell (adaptiert nach Blue und Adler, 2001). 


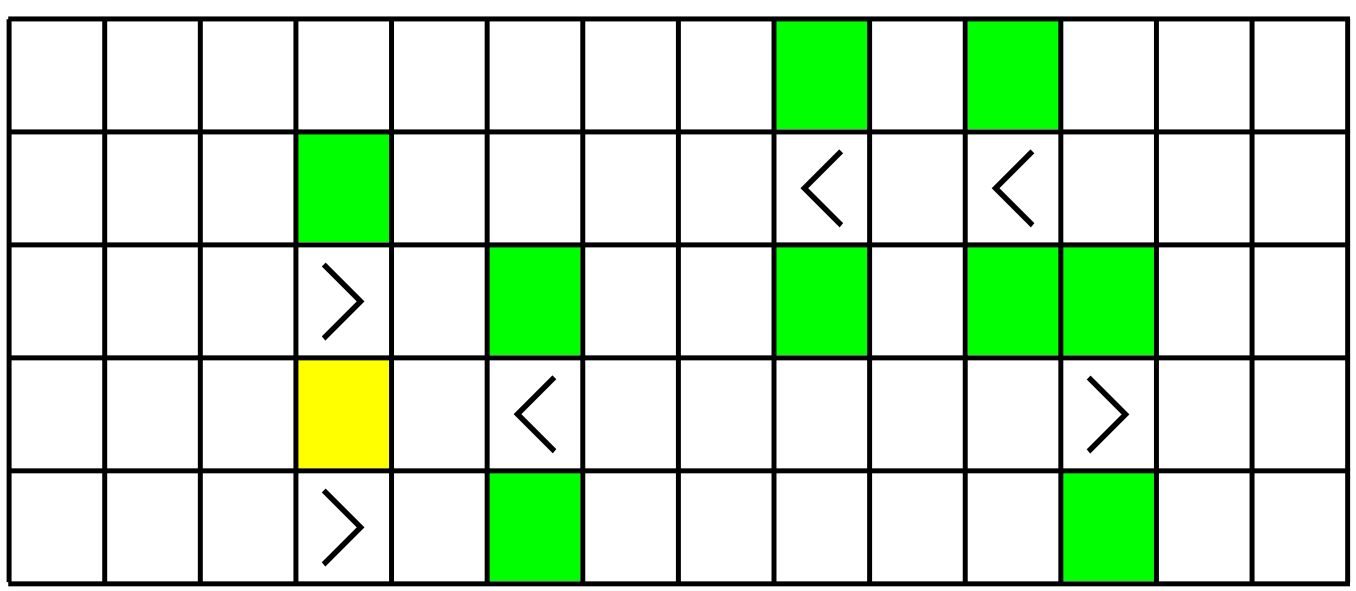

Abbildung C.1: ZA-Fußgängermodell: Spurwechselkonflikte. Fußgänger sind im Hinblick auf ihre Laufrichtung markiert. Felder sind grün markiert, wenn ein Spurwechsel dorthin möglich ist und gelb, falls sie mehrfach beansprucht werden.

einer freien benachbarten Spur erzwungen (Zeile 29). Hierbei werden Spuren, die Verkehr in der selben Richtung wie ein Fußgänger $f$ enthalten, bevorzugt (Zeile 31). Dieser Schritt führt dazu, dass Spuren entstehen, die für jeweils eine Richtung bevorzugt werden. Falls mehrere Spuren die selbe maximale Nutzungswahrscheinlichkeit haben, wird geprüft, ob die aktuelle Spur unter diesen Spuren $S$ enthalten ist und diese bevorzugt (Zeile 34). In Zeile 35 wird spur neu nach der berechneten Wahrscheinlichkeitsverteilung bestimmt.

Nachdem die Schleife über alle Fußgänger (Zeilen 4 bis 6) beendet ist, hat jeder Fußgänger parallel eine Spur bestimmt, die er betreten wird. Dieser Schritt wird nun in den Zeilen 7 und 8 durchgeführt. Die Geschwindigkeiten werden für alle Fußgänger in Abhängigkeit zur vorhandenen Lücke bestimmt (Zeilen 9 bis 12 . Falls die Lücke zwischen zwei Fußgängern weniger als zwei Zellen beträgt, und diese in unterschiedliche Richtungen laufen, können sie mit der Wahrscheinlichkeit $p_{\text {tausch }}$ aneinander vorbeilaufen.

Abschließend bewegen sich alle Fußgänger mit den berechneten Geschwindigkeiten (Zeilen 13 und 14). 



\section{KFZ-NEFZ-Daten}

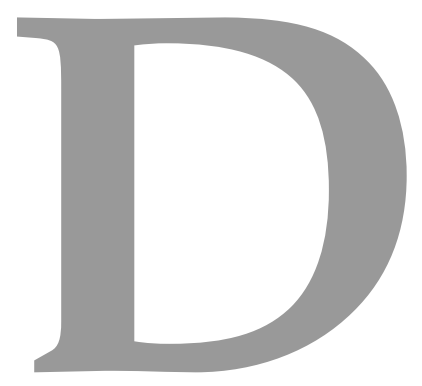

Masse, Länge und NEFZ-Verbauchsdaten in Mix-, Stadt- und Landszenarien von 54 Fahrzeugen (Bj. 2011, 2012), gemäß Herstellerwebseiten nach [Taubert, 2012.

\begin{tabular}{|l|l|l|l|l|l|}
\hline Automodell & $l$ & $m$ & Mix & Stadt & Land \\
\hline BMW 116i & 4239 & 1320 & 6,1 & 7,9 & 5,1 \\
BMW 320i & 4624 & 1505 & 6,4 & 8,3 & 5 \\
BMW 520i & 4899 & 1670 & 7 & 9,1 & 5,7 \\
BMW 740i & 5072 & 1935 & 9,9 & 13,8 & 7,6 \\
BMW Z4 sDrive20i & 4239 & 1470 & 6,8 & 8,9 & 5,6 \\
BMW X3 xDrive20i & 4648 & 1770 & 7,9 & 9,9 & 6,7 \\
BMW X5 xDrive25i & 4857 & 2145 & 10,1 & 13,1 & 8,3 \\
VW take up! & 3540 & 929 & 4,5 & 5,6 & 3,9 \\
VW Polo Trendline & 4064 & 1128 & 5,5 & 7,3 & 4,5 \\
VW Golf TDI Comfortine & 4296 & 1322 & 5,3 & 6,7 & 4,5 \\
VW Passat TSI Comfortline & 4874 & 1626 & 6,9 & 9,6 & 5,3 \\
VW Touareg V8 TDI & 4893 & 2297 & 9,1 & 11,9 & 7,4 \\
Ford Galaxy 1,6-l-EcoBoost & 4819 & 1734 & 7,2 & 9,1 & 6,1 \\
Ford Fiesta 1,25l Duratec & 3950 & 1041 & 5,4 & 7,1 & 4,3 \\
Ford Focus 1,6l Duratec TI-VCT & 4234 & 1307 & 6 & 8,1 & 4,8 \\
Opel Astra 1.6 Turbo & 4419 & 1400 & 6,8 & 8,8 & 5,6 \\
Opel Insignia 1.6 & 4830 & 1503 & 7,4 & 10,3 & 5,7 \\
\hline
\end{tabular}

Fortsetzung auf der nächsten Seite 


\begin{tabular}{|c|c|c|c|c|c|}
\hline Automodell & $l$ & $m$ & Mix & Stadt & Land \\
\hline Opel Antara 2.4 & 4596 & 1800 & 8,8 & 11,7 & 7,1 \\
\hline Opel Meriva 1.4 & 4288 & 1361 & 6,1 & 7,8 & 5,1 \\
\hline Opel Corsa 1.2 ecoFLEX & 3999 & 1163 & 5,5 & 7,2 & 4,5 \\
\hline Mercedes A 160 BlueEFFICENCY & 3883 & 1325 & 6 & 7,5 & 5,1 \\
\hline Mercedes B 180 BlueEFFICENCY & 4359 & 1395 & 5,9 & 8 & 4,7 \\
\hline Mercedes C 180 BlueEFFICENCY & 4591 & 1480 & 6,7 & 9,3 & 5,2 \\
\hline Mercedes ML 350 4MATIC & 4804 & 2130 & 8,5 & 10,9 & 7,1 \\
\hline Mercedes S 350 BlueEFFICENCY & 5096 & 1910 & 7,6 & 10,2 & 6 \\
\hline Mercedes SLK 55 AMG & 4010 & 1610 & 8,4 & 12 & 6,2 \\
\hline Fiat Panda Classic $1.28 \mathrm{~V}$ & 3653 & 1015 & 6 & 7,3 & 5,2 \\
\hline Fiat Punto 1.6 16V & 4065 & 1090 & 5,7 & 7,4 & 4,7 \\
\hline Fiat Bravo 1.4 16V & 4336 & 1350 & 5,7 & 7,3 & 4,6 \\
\hline Fiat Sedici 2.0 MULTIJET & 4115 & 1425 & 5,3 & 6,6 & 4,7 \\
\hline Mitsubishi Colt 1.1 ClearTec Motion & 3880 & 1015 & 4,9 & 6 & 4,3 \\
\hline Mitsubishi Lancer 1.6 ClearTec Inform & 4570 & 1335 & 5,5 & 7 & 4,7 \\
\hline Mitsubishi Pajero 3.2 DI-D Inform & 4385 & 2160 & 7,8 & 9,5 & 6,9 \\
\hline Mitsubishi L200 2.5DI-D Inform & 5040 & 1845 & 7,5 & 8,9 & 6,8 \\
\hline Mazda 2 1.3l MZR & 3920 & 1035 & 5 & 6,2 & 4,3 \\
\hline Mazda 3 2.01 MZR DISI & 4460 & 1258 & 6,7 & 9,1 & 5,3 \\
\hline Mazda $61.8 \mathrm{l} \mathrm{MZR}$ & 4785 & 1425 & 6,6 & 9,3 & 5,0 \\
\hline Mazda CX-7 2.31 MZR DISI & 4700 & 1815 & 10,4 & 14 & 8,4 \\
\hline Renault Twingo 1.2 16V 75 & 3602 & 890 & 5,1 & 7,3 & 4,7 \\
\hline Renault Clio 2.0 16V 200 & 4017 & 1062 & 8,2 & 11,2 & 6,5 \\
\hline Renault Megane dCi 130 & 4295 & 1395 & 5,1 & 6,2 & 4,5 \\
\hline Renault Espace dCi 175 & 4656 & 1807 & 7 & 8,8 & 6,3 \\
\hline Renault Laguna TCe 170 & 4695 & 1377 & 6,5 & 12,5 & 8,7 \\
\hline Citroen C1 1.0 EGS & 3440 & 925 & 5 & 5,4 & 4 \\
\hline Citroen C6 V6 HDi 240 & 4908 & 1995 & 7,3 & 10 & 5,8 \\
\hline Citroen C3 Picasso VTi 120 & 4078 & 1279 & 6,4 & 9 & 4,9 \\
\hline Dacia Sandero 1.6 MPI LPG 85 & 4024 & 1207 & 7,4 & 9,9 & 6,2 \\
\hline Dacia Logan MCV 1.6 MPI 85 & 4450 & 1240 & 7,3 & 9,8 & 6,1 \\
\hline Dacia Duster 1.6 16V 105 & 4315 & 1325 & 8 & 10,4 & 7 \\
\hline Dacia Lodgy 1.6 MPI 85 & 4498 & 1165 & 7,1 & 9,4 & 5,7 \\
\hline Skoda Fabia 1,2 1 51kW & 4111 & 1095 & 5,5 & 7,3 & 4,5 \\
\hline Skoda Yeti 1,2 TSI 77kW & 4360 & 1340 & 6,4 & 7,6 & 5,9 \\
\hline Skoda Octavia 1,8 TSI 118kW & 4666 & 1350 & 6,9 & 9,5 & 5,5 \\
\hline Skoda Superb 2.0 TSI DSG $147 \mathrm{~kW}$ & 4938 & 1555 & 7,9 & 10,6 & 6,3 \\
\hline
\end{tabular}

Tabelle D.1: Länge $[\mathrm{m}]$, Masse $[\mathrm{kg}]$ und NEFZ-Verbrauchsdaten [l] [Taubert, 2012] 


\section{Literaturverzeichnis}

[Abdi, 2007] Abdi, H. (2007). Encyclopedia of Measurement and Statistics, Kapitel Bonferroni and Sidak corrections for multiple comparisons. Salkind, N. J., Herausgeber, Thousand Oaks (CA).

[Abdul Kareem, 2002] Abdul Kareem, Y. (2002). "Comparative Study Of Gap Acceptance At Priority Intersections". Journal Of Science, Engineering And Technology, Enugu, Nigeria, 3(1), S. 1-6.

[AblEU, 1970] AblEU (1970). "Zur Angleichung der Rechtsvorschriften der Mitgliedstaaten über Maßnahmen gegen die Verunreinigung der Luft durch Emissionen von Kraftfahrzeugen". Amtsblatt der Europäischen Union. 70/220/EWG.

[AblEU, 2008] AblEU (2008). "Mitteilung über die Anwendung und die künftige Entwicklung der gemeinschaftlichen Rechtsvorschriften über Emissionen von Fahrzeugen für den Leichtverkehr und über den Zugang zu Reparaturund Wartungsinformationen (Euro 5 und Euro 6)". Amtsblatt der Europäischen Union. 2008/C 182/08, Punkt 10.

[Ahn, 1998] Ahn, K. (1998). Microscopic Fuel Consumption and Emission Modeling. Dissertation, Virginia Polytechnic Institute and State University.

[Ahn et al., 2002] Ahn, K.; Rakha, H.; Trani, A. und Van Aerde, M. (2002). "Estimating Vehicle Fuel Consumption and Emissions based on Instantaneous Speed and Acceleration Levels". Journal of Transportation Engineering, 128(2), S. 182-190.

[Al-Jameel, 2009] Al-Jameel, H. (2009). "Examining and improving the limitations of Gazis-Herman-Rothery car following model". In Salford Postgraduate Annual Research Conference.

[Al-Zanaidi et al., 1994] Al-Zanaidi, M. A.; Singh, M. P. und El-Karim, M. (1994). 
"Traffic CO-Dispersion Pattern in Kuwait". Atmospheric Environment, 25A, 5/6, S. 909-914.

[Alves et al., 2010] Alves, D.; van Ast, J.; Cong, Z.; De Schutter, B. und Babuška, R. (2010). "Ant Colony Optimization for traffic dispersion routing". In Intelligent Transportation Systems (ITSC), 2010 13th International IEEE Conference on, S. $683-688$.

[An et al., 1997] An, F.; Barth, M.; Norbeck, J. und Ross, M. (1997). "Development of Comprehensive Modal Emissions Model Operating Under HotStabilized Conditionss". Transportation Research Record, 1587(1), S. 5262 .

[Arias et al., 2009] Arias, A. P.; Hanebeck, U. D.; Ehrhardt, P.; Hengst, S.; Kretz, T. und Vortisch, P. (2009). "A Framework for Evaluating the VISSIM Traffic Simulation with Extended Range Telepresence". In Proceedings of the 22nd Annual Conference on Computer Animation and Social Agents (CASA 2009), S. 13-16, Amsterdam.

[Ashton, 1971] Ashton, W. D. (1971). "Gap-Acceptance Problems at a Traffic Intersection". Journal of the Royal Statistical Society. Series C (Applied Statistics), 20(2), S. 130-138.

[Ather, 2009] Ather, A. (2009). A Quality Analysis of OpenStreetMap Data. PhD thesis, M. Eng. Dissertation, Department of Civil, Environmental \& Geomatic Engineering, University College London.

[Aydin, 2004] Aydin, M. O. (2004). "Traffic Light Displaying Remaining Time". United States Patent 2004/0189491 A1.

[Bachem et al., 1994] Bachem, A.; Nagel, K. und Rickert, M. (1994). "Ultraschnelle mikroskopische Verkehrssimulationen". Parallele Datenverarbeitung aktuell: TAT 9́4, 173, S. 460-469.

[Baek et al., 2009] Baek, S. K.; Minnhagen, P.; Bernhardsson, S.; Choi, K. und Kim, B. J. (2009). "Flow improvement caused by agents who ignore traffic rules". Physical Review E (Statistical, Nonlinear, and Soft Matter Physics), 80(1).

[Balmer, 2007] Balmer, M. (2007). Travel demand modeling for multi-agent traffic simulations: Algorithms and systems. Dissertation, ETH Zürich, Switzerland. 
[Balmer et al., 2004] Balmer, M.; Raney, B. und Nagel, K. (2004). ”Agent-Based Activities Planning for an Iterative Traffic Simulation of Switzerland Activity Time Allocation". 4th Swiss Transport Research Conference.

[Balmer et al., 2008] Balmer, M.; Rieser, M.; Meister, K.; Charypar, D.; Lefebvre, N.; Nagel, K. und Axhausen, K. (2008). "MATSim-T: Architecture and Simulation Times". In Bazzan, A. L. C. und Klügl, F., Herausgeber, MultiAgent Systems for Traffic and Transportation Engineering.

[Bando et al., 1998] Bando, M.; Hasebe, K.; Nakanishi, K. und Nakayama, A. (1998). "Analysis of optimal velocity model with explicit delay". Physical Review E, 58, S. 5429-5435.

[Bando et al., 1994] Bando, M.; Hasebe, K.; Nakayama, A.; Shibata, A. und Sugiyama, Y. (1994). "Structure stability of congestion in traffic dynamics". Japan Journal of Industrial and Applied Mathematics, 11, S. 203-223. 10.1007/BF03167222.

[Bando et al., 1995] Bando, M.; Hasebe, K.; Nakayama, A.; Shibata, A. und Sugiyama, Y. (1995). "Dynamical model of traffic congestion and numerical simulation". Phys. Rev. E, 51(2), S. 1035-1042.

[Baqueiro et al., 2009] Baqueiro, O.; Wang, Y. J.; McBurney, P. und Coenen, F. (2009). "Integrating Data Mining and Agent Based Modeling and Simulation". In Advances in Data Mining. Applications and Theoretical Aspects, 9th Industrial Conference, ICDM 2009, 5633 Auflage von Lecture Notes in Computer Science, S. 220-231. Springer.

[Barrett et al., 1998] Barrett, C. L.; Eubank, S.; Nagel, K.; Rasmussen, S.; Riordan, J. und Wolinsky, M. (1998). "Issues in the representation of traffic using multi-resolution cellular automata". Technical report, Los Alamos National Laboratory Transims Report Series.

[Bartelme, 2005] Bartelme, N. (2005). Geoinformatik: Modelle, Strukturen, Funktionen. Springer, Berlin, 4. Auflage.

[Basford et al., 2002] Basford, L.; Reid, S.; Lester, T.; Thomson, J. und A., T. (2002). "Drivers' perceptions of cyclists". Technical Report 549, Prepared for Charging and Local Transport Division, Department for Transport. ISSN: 0968-4107.

[Batty, 2001] Batty, M. (2001). "Agent-based pedestrian modelling”. Environment 
and Planning B: Planning and Design, 28, S. 321-326.

[Batty, 2007] Batty, M. (2007). Cities and Complexity: Understanding Cities with Cellular Automata, Agent-Based Models, and Fractals. The MIT Press.

[Bauke und Mertens, 2005] Bauke, H. und Mertens, S. (2005). Cluster Computing : Praktische Einführung in das Hochleistungsrechnen auf Linux-Clustern (X.systems.press). Springer.

[Bauza et al., 2008] Bauza, R.; Gozalvez, J. und Sepulcre, M. (2008). "Operation and Performance of Vehicular Ad-hoc Routing Protocols in Realistic Environments". In Proceedings of the 2nd IEEE International Symposium on Wireless Vehicular Communications (WiVeC).

[Bazzan et al., 2008] Bazzan, A.; de Oliveira, D.; Klügl, F. und Nagel, K. (2008). "To Adapt or Not to Adapt - Consequences of Adapting Driver and Traffic Light Agents". In Tuyls, K.; Nowe, A.; Guessoum, Z. und Kudenko, D., Herausgeber, Adaptive Agents and Multi-Agent Systems III. Adaptation and Multi-Agent Learning, 4865 Auflage von Lecture Notes in Computer Science, S. 1-14. Springer Berlin / Heidelberg.

[Bazzan, 2009] Bazzan, A. L. (2009). "Opportunities for multiagent systems and multiagent reinforcement learning in traffic control". Autonomous Agents and Multi-Agent Systems, 18, S. 342-375.

[Bazzan et al., 2010] Bazzan, A. L. C.; de Brito do Amarante, M.; Sommer, T. und Benavides, A. J. (2010). "ITSUMO: an Agent-Based Simulator for ITS Applications". In Rossetti, R.; Liu, H. und Tang, S., Herausgeber, Proc. of the 4 th Workshop on Artificial Transportation Systems and Simulation. IEEE.

[Bazzan et al., 2009] Bazzan, A. L. C.; Nagel, K. und Klügl, F. (2009). "Integrating MATSim and ITSUMO for Daily Replanning Under Congestion". In Proceedings of the 35th Latin-American Informatics Conference, CLEI, Pelotas, Brazil.

[Behrisch et al., 2011] Behrisch, M.; Bieker, L.; Erdmann, J. und Krajzewicz, D. (2011). "SUMO - Simulation of Urban MObility: An Overview". In SIMUL 2011, The Third International Conference on Advances in System Simulation, S. 63-68, Barcelona, Spain. ISBN 978-1-61208-169-4.

[Behrisch und Krajzewicz, 2008] Behrisch, M. und Krajzewicz, D. (2008). "'Si- 
mulation of Urban MObility“ - SUMO Eine freie Verkehrssimulation". Technical report, Institut für Verkehrssystemtechnik.

[Bennett, 2010] Bennett, J. (2010). OpenStreetMap. Packt Publishing Ltd, Olton Birmingham, GBR. ISBN: 978-1-84719-750-4.

[Bertsche et al., 2009] Bertsche, B.; Göhner, P.; Jensen, U.; Schinköthe, W.; Wunderlich, H.-J.; Göhner, P.; Beck, A.; Kunz, S. und Wedel, M. (2009). "Zuverlässigkeit der Software in mechatronischen Systemen". In Zuverlässigkeit mechatronischer Systeme, VDI-Buch, S. 317-389. Springer Berlin Heidelberg. 10.1007/978-3-540-85091-5_7.

[Bloomberg und Dale, 2000] Bloomberg, L. und Dale, J. (2000). "A Comparison of the VISSIM and CORSIM Traffic Simulation Models". Institute of Transportation Engineers Annual Meeting, 1727, S. 52-60.

[Blue und Adler, 2001] Blue, V. J. und Adler, J. L. (2001). "Cellular automata microsimulation for modeling bi-directional pedestrian walkways". Transportation Research Part B: Methodological, 35(3), S. 293 - 312.

[Boccara et al., 1997] Boccara, N.; Fuks, H. und Zeng, Q. (1997). "Car accidents and number of stopped cars due to road blockage on a one-lane highway". MATH.GEN., 30, S. 3329.

[Bönisch und Kretz, 2009] Bönisch, C. und Kretz, T. (2009). "Simulation of Pedestrians Crossing a Street". Proceedings of the Eighth International Conference on Traffic and Granular Flow, 8.

[Bossel, 2004] Bossel, H. (2004). Systeme Dynamik Simulation: Modellbildung, Analyse und Simulation komplexer Systemen. Books on Demand GmbH, Norderstedt. ISBN: 3-8334-0984-3.

[Boström und Nilsson, 2001] Boström, L. und Nilsson, B. (2001). "A review of serious injuries and deaths from bicycle accidents in Sweden from 1987 to 1994.”. The Journal of Trauma, 50(5), S. 900.

[Bouckaert et al., 2010] Bouckaert, R. R.; Frank, E.; Hall, M. A.; Holmes, G.; Pfahringer, B.; Reutemann, P. und Witten, I. H. (2010). "WEKAExperiences with a Java Open-Source Project". Journal of Machine Learning Research, 11, S. 2533-2541.

[Bowman und Vecellio, 1994] Bowman, B. L. und Vecellio, R. L. (1994). "Pede- 
strian walking speeds and conflicts at urban median locations". Transportation Research Record: Journal of the Transportation Research Board, 1438, S. $67-73$.

[Brackstone und McDonald, 1999] Brackstone, M. und McDonald, M. (1999). "Car-following: a historical review". Transportation Research Part F: Traffic Psychology and Behaviour, 2(4), S. 181 - 196.

[Brewer et al., 2006] Brewer, M. A.; Fitzpatrick, K.; Whitacre, J. A. und Lord, D. (2006). "Exploration of Pedestrian Gap-Acceptance Behavior at Selected Locations". Transportation Research Record: Journal of the Transportation Research Board, 1982, S. 132-140. ISSN: 0361-1981.

[Brilon et al., 1999] Brilon, W.; Koenig, R. und Troutbeck, R. J. (1999). "Useful estimation procedures for critical gaps". Transportation Research Part A: Policy and Practice, 33(3-4), S. 161 - 186.

[Brockfeld et al., 2001] Brockfeld, E.; Barlovic, R.; Schadschneider, A. und Schreckenberg, M. (2001). "Optimizing Traffic Lights in a Cellular Automaton Model for City Traffic". Physical Review E, 64. 056132.

[Bull et al., 2004] Bull, L.; Sha'Aban, J.; Tomlinson, A.; Addison, J. und Heydecker, B. (2004). Towards distributed adaptive control for road traffic junction signals using learning classifier systems, S. 279-299. Springer: New York. ISBN: 3540211098.

[Bullock und Catarella, 1998] Bullock, D. und Catarella, A. (1998). "A Real-Time Simulation Environment for Evaluating Traffic Signal Systems". In Transportation Research Record, TRB, National Research Council, S. 130-135. Nr. 1634.

[Bungartz et al., 2009] Bungartz, H.-J.; Zimmer, S.; Buchholz, M. und Pflüger, D. (2009). Modellbildung und Simulation. eXamen.press. Springer Verlag, 1 Auflage. ISBN 978-3-540798095.

[Cappiello et al., 2002] Cappiello, A.; Chabini, I.; Nam, E. K.; Lue, A. und Abou Zeid, M. (2002). "A statistical model of vehicle emissions and fuel consumption". Proceedings of the IEEE 5th International Conference on Intelligent Transportation Systems, 617, S. 801-809.

[Carruthers et al., 1994] Carruthers, D.; Holroyd, R.; Hunt, J.; Weng, W.; Robins, A.; Apsley, D.; Thompson, D. und Smith, F. (1994). "UK-ADMS: A new 
approach to modelling dispersion in the earth's atmospheric boundary layer". Journal of Wind Engineering and Industrial Aerodynamics, 52(0), S. $139-153$.

[Cartenì et al., 2010] Cartenì, A.; Cantarella, G. E. und Luca, S. D. (2010). "A Methodology for Estimating Traffic Fuel Consumption and Vehicle Emissions for Urban Planning". 12th World Conference for Transportation Research.

[Cervone et al., 2013] Cervone, G.; Dallmeyer, J.; Lattner, A. D.; Franzese, P. und Waters, N. (2013). CyberGIS: Fostering a New Wave of Geospatial Discovery and Innovation, Kapitel Coupling Traffic Simulation and Gas Dispersion Simulation for Atmospheric Pollution Estimation. Wang, Shaowen und Goodchild, Michael F. (Herausgeber) Springer. (eingeladener Buchbeitrag, Veröffentlichung für Anfang 2013 geplant).

[Cervone et al., 2008] Cervone, G.; Franzese, P.; Ezber, Y. und Boybeyi, Z. (2008). "Risk assessment of atmospheric emissions using machine learning". $\mathrm{Na}$ tural Hazards and Earth System Science, 8(5), S. 991-1000.

[Cetin et al., 2002] Cetin, N.; Burri, A. und Nagel, K. (2002). "Parallel Queue Model Approach to Traffic Microsimulations". In In Proceedings of Swiss Transportation Research Conference.

[Chapman, 2007] Chapman, L. (2007). "Transport and Climate Change: A Review". Journal of Transport Geography,, 15(5), S. 354-367.

[Charypar und Nagel, 2005] Charypar, D. und Nagel, K. (2005). "Generating complete all-day activity plans with genetic algorithms". Transportation, 32(4).

[Chen et al., 2005] Chen, D.; Yuan, Y.; Li, B. und Wu, J. (2005). "Validation and Comparison of Microscopic Car-Following Models Using Beijing Traffic Flow Data". In Wang, L. und Jin, Y., Herausgeber, Fuzzy Systems and Knowledge Discovery, 3614 Auflage von Lecture Notes in Computer Science, S. 491-491. Springer Berlin / Heidelberg. 10.1007/11540007_128.

[Chien et al., 2001] Chien, S. I.-J.; Mouskos, K. C. und Chowdhury, S. M. (2001). "Generating Driver Population for the Microscopic Simulation Model (CORSIM)". Simulation, 76(1), S. 40-45.

[Chowdhury et al., 2000] Chowdhury, D.; Santen, L. und Schadschneider, A. 
(2000). "Statistical Physics of Vehicular Traffic and Some Related Systems". Physics Reports, 329.

[Chowdhury und Schadschneider, 1999] Chowdhury, D. und Schadschneider, A. (1999). "Self-organization of traffic jams in cities: Effects of stochastic dynamics and signal periods". Phys. Rev. E, 59(2), S. 1311-1314.

[Chu et al., 2003] Chu, L.; Liu, H. X.; Oh, J.-S. und Recker, W. (2003). "A Calibration Procedure for Microscopic Traffic Simulation". Proceedings of Intelligent Transportation Systems, 2, S. 1574-1579. ISBN: 0-7803-81254 .

[Ciari et al., 2007] Ciari, F.; Balmer, M. und Axhausen, K. (2007). "Mobility Tool Ownership and Mode Choice Decision Processes in MultiAgent Transportation Simulation". Swiss Transport Research Conference, 7.

[Coensel und Botteldooren, 2007] Coensel, B. D. und Botteldooren, D. (2007). "Microsimulation Based Corrections on the Road Traffic Noise Emission Near Intersections". Acta Acustica United With Acustica, 93, S. 241-252.

[Coffin und Morrall, 1995] Coffin, A. und Morrall, J. (1995). "Walking speeds of elderly pedestrians at crosswalks". Transportation Research Record: Journal of the Transportation Research Board, 1487, S. 63-67.

[Cohen, 1995] Cohen, W. W. (1995). "Fast Effective Rule Induction". In Proceedings of the 12th International Conference on Machine Learning, Lake Taho, California.

[Cooper und Zheng, 2002] Cooper, P. J. und Zheng, Y. (2002). "Turning gap acceptance decision-making: the impact of driver distraction". Journal of Safety Research, 33(3), S. $321-335$.

[Cormen et al., 2001] Cormen, T. H.; Stein, C.; Rivest, R. L. und Leiserson, C. E. (2001). Introduction to Algorithms. McGraw-Hill Higher Education, 2nd Auflage. ISBN 0-262-03293-7.

[Cresswell et al., 1978] Cresswell, C.; Griffiths, J. D. und Hunt, J. G. (1978). "Site evaluation of a pelican crossing simulation model". Traffic Engineering and Control, 19, S. 546-549.

[Cyrys et al., 2009] Cyrys, J.; Peters, A. und Wichmann, H.-E. (2009). "Umweltzone München - Eine erste Bilanz". Umweltmedizin in Forschung und 
Praxis, 14 (3), S. 127-132.

[da Silva et al., 2006a] da Silva, B.; Bazzan, A.; Andriotti, G.; Lopes, F. und de Oliveira, D. (2006a). "ITSUMO: An Intelligent Transportation System for Urban Mobility". In Böhme, T.; Larios Rosillo, V.; Unger, H. und Unger, H., Herausgeber, Innovative Internet Community Systems, 3473 Auflage von Lecture Notes in Computer Science, S. 224-235. Springer Berlin / Heidelberg.

[da Silva et al., 2006b] da Silva, B. C.; Junges, R.; de Oliveira, D. und Bazzan, A. L. C. (2006b). "ITSUMO: an Intelligent Transportation System for Urban Mobility". In Proceedings of the fifth international joint conference on Autonomous agents and multiagent systems, AAMAS '06, S. 14711472, New York, NY, USA. ACM.

[Daamen, 2004] Daamen, W. (2004). Modelling passenger flows in public transport facilities. PhD thesis, Delft University. Dissertation, ISBN: 90-407-2521-7.

[Daganzo, 1981] Daganzo, C. F. (1981). "Estimation of gap acceptance parameters within and across the population from direct roadside observation". Transportation Research Part B: Methodological, 15(1), S. 1 - 15.

[Daganzo und Geroliminis, 2008] Daganzo, C. F. und Geroliminis, N. (2008). "An analytical approximation for the macroscopic fundamental diagram of urban traffic". Transportation Research Part B-Methodological, 42(9), S. $771-781$.

[Dallmeyer et al., 2011] Dallmeyer, J.; Lattner, A. D. und Timm, I. J. (2011). "From GIS to Mixed Traffic Simulation in Urban Scenarios". In Liu, J.; Quaglia, F.; Eidenbenz, S. und Gilmore, S., Herausgeber, 4th International ICST Conference on Simulation Tools and Techniques, SIMUTools '11, Barcelona, Spain, March 22 - 24, 2011, S. 134-143. ICST (Institute for Computer Sciences, Social-Informatics and Telecommunications Engineering), Brüssel. ISBN 978-1-936968-00-8.

[Dallmeyer et al., 2012a] Dallmeyer, J.; Lattner, A. D. und Timm, I. J. (2012a). Data Mining for Geoinformatics: Methods and Applications, Kapitel GISbased Traffic Simulation using OSM. Cervone, Guido; Lin, Jessica und Waters, Nigel (Herausgeber) Springer. (akzeptierter Beitrag).

[Dallmeyer et al., 2012b] Dallmeyer, J.; Lattner, A. D. und Timm, I. J. (2012b). "Pedestrian Simulation for Urban Traffic Scenarios". In Bruzzone, A. G., 
editor, Proceedings of the Summer Computer Simulation Conference 2012. 44 rd Summer Simulation Multi-Conference (SummerSim'12), S. 414-421. Curran Associates, Inc.

[Dallmeyer et al., 2012c] Dallmeyer, J.; Lattner, A. D. und Timm, I. J. (2012c). "Selfish Road Users - Case Studies on Rule Breaking Agents for Traffic Simulation". In Timm, I. J. und Guttmann, C., Herausgeber, Lecture Notes in Artificial Intelligence 7598, S. 83 - 95. Springer.

[Dallmeyer et al., 2012d] Dallmeyer, J.; Schumann, R.; Lattner, A. D. und Timm, I. J. (2012d). "Don't Go with the Ant Flow: Ant-inspired Traffic Routing in Urban Environments". Seventh International Workshop on Agents in Traffic and Transportation (ATT 2012), Valencia, Spain.

[Dallmeyer et al., 2012e] Dallmeyer, J.; Taubert, C.; Lattner, A. D. und Timm, I. J. (2012e). "Fuel Consumption and Emission Modeling for Urban Scenarios". In Troitzsch, K. G.; Möhring, M. und Lotzmann, U., Herausgeber, Proceedings of the 26th EUROPEAN Conference on Modelling and Simulation, S. 567-573. ISBN 978-0-9564944-4-3.

[Dallmeyer und Timm, 2012] Dallmeyer, J. und Timm, I. J. (2012). "MAINSIM - MultimodAl INnercity SIMulation". Poster and Demo Track of the 35th German Conference on Artificial Intelligence (KI-2012), S. 125-129.

[Das et al., 2005] Das, S.; Manski, C. F. und Manuszak, M. D. (2005). "Walk or wait? An empirical analysis of street crossing decisions". Journal of Applied Econometrics, 20(4), S. 529-548.

[Dell'Orco et al., 2003] Dell'Orco, M.; Ottomanelli, M. und Sassanelli, D. (2003). "Modelling uncertainty in parking choice behaviour". 82nd Annual Meeting of the Transportation Research Board, 82, S. Paper no. 03-3776.

[Di Sabatino et al., 2008] Di Sabatino, S.; Buccolieri, R.; Pulvirenti, B. und Britter, R. (2008). "Flow and Pollutant Dispersion in Street Canyons using FLUENT and ADMS-Urban". Environmental Modeling and Assessment, 13, S. 369-381. 10.1007/s10666-007-9106-6.

[Dijkstra, 1959] Dijkstra, E. W. (1959). "A note on two problems in connexion with graphs". Numerische Mathematik, 1, S. 269-271. 10.1007/BF01386390.

[Dill und Gliebe, 2008] Dill, J. und Gliebe, J. (2008). "Understanding and measu- 
ring bicycling behavior: A focus on travel time and route choice". Technical report, Oregon Transportation Research and Education Consortium, Portland State University, Portland, OR.

[Dorigo und Blum, 2005] Dorigo, M. und Blum, C. (2005). "Ant colony optimization theory: A survey". Theoretical Computer Science, 344(2-3), S. $243-278$.

[Dueker et al., 2004] Dueker, K. J.; Kimpel, T. J.; Strathman, J. G. und Trimet, S. C. (2004). "Determinants of Bus Dwell Time Determinants of Bus Dwell Time". Journal of Public Transportation, 7(1), S. 21-40.

[Duong et al., 2010] Duong, D. D. Q.; Saccomanno, F. F. und Hellinga, B. R. (2010). "Calibration of microscopic traffic model for simulating safety performance". Compendium of papers of the 89th Annual Transportation Research Board Conference.

[Ehlert und Rothkrantz, 2001] Ehlert, P. A. M. und Rothkrantz, L. J. M. (2001). "Microscopic traffic simulation with reactive driving agents". ITSC 20012001 IEEE Intelligent Transportation Systems Proceedings Cat No01TH8585, 40(5), S. 860-865.

[Eilts, 2009] Eilts, M. (2009). Erhöhung des Risikos für Bronchitis im Kindesalter durch Lärm und Abgase aus dem Straßenverkehr. $\mathrm{PhD}$ thesis, Medizinische Fakultät Charité - Universitätsmedizin Berlin.

[Emmerich und Rank, 1997] Emmerich, H. und Rank, E. (1997). "An improved cellular automaton model for traffic flow simulation". Physica A: Statistical and Theoretical Physics, 234(3-4), S. $676-686$.

[Esser und Schreckenberg, 1997] Esser, J. und Schreckenberg, M. (1997). "Microscopic Simulation of Urban Traffic Based on Cellular Automata". In International Journal of Modern Physics C, 8, 5 Auflage, S. 1025-1036. World Scientific Publishing Company.

[Faghri und Egyháziová, 1999] Faghri, A. und Egyháziová (1999). ”Development of a Computer Simulation Model of Mixed Motor Vehicle and Bicycle Traffic on an Urban Road Network". Transportation Research Record, 1674, S. 86-93.

[Félez et al., 2007] Félez, J.; Maroto, J.; Romero, G. und Cabanellas, J. M. (2007). "A Full Driving Simulator of Urban Traffic including Traffic Accidents". 
Simulation, 83(5), S. 415-431.

[Fellendorf, 1994] Fellendorf, M. (1994). "VISSIM: A microscopic Simulation Tool to Evaluate Actuated Signal Control including Bus Priority". 64th Institute of Transportation Engineers Annual Meeting.

[Fellendorf und Vortisch, 2001] Fellendorf, M. und Vortisch, P. (2001). "Validation of the Microscopic Traffic Flow Model VISSIM in Different Real-World Situations". Annual Meeting, TRB, Washington, D. C.

[Fiosins et al., 2011] Fiosins, M.; Fiosina, J. und Müller, J. P. (2011). "Change Point Analysis for Intelligent Agents in City Traffic". In The Seventh International Workshop on Agents and Data Mining Interaction (ADMI2011) at the 10th International Conference on Autonomous Agents and Multiagent Systems (AAMAS2011). Springer-Verlag.

[Fitzpatrick et al., 2006] Fitzpatrick, K.; Brewer, M. A. und Turner, S. (2006). "Another look at pedestrian walking speed.". In Proceedings of the 85th Annual Meeting of the Transportation Research Board, Washington, DC, USA.

[Fouladvand und Radja, 2001] Fouladvand, E. M. und Radja, N. H. (2001). "Optimised Traffic Flow at a Single Urban Crossroads: Dynamical Symmetry Breaking". Technical Report cond-mat/0108149, Institute for Studies in Theoretical Physics and Mathematics, P.O. Box 19395-5531, Tehran, Iran.

[Fouladvand et al., 2005] Fouladvand, E. M.; Shaebani, R. M. und Sadjadi, Z. (2005). "Intelligent Controlling Simulation of Traffic Flow in a Small City Network". Journal of Physics, Soc. Japan, 73(physics/0511141), S. 32093214 .

[Franzese und Huq, 2011] Franzese, P. und Huq, P. (2011). "Urban Dispersion Modelling and Experiments in the Daytime and Nighttime Atmosphere". Boundary-Layer Meteorology, 139, S. 395-409. 10.1007/s10546-011-95935 .

[Frisch, 2004] Frisch, N. (2004). Verfahren zur Unterstützung der Arbeitsabläufe bei der Crash-Simulation im Fahrzeugbau. PhD thesis, Universität Stuttgart.

[Fritsche, 1994] Fritsche, H.-T. (1994). "A model for traffic simulation". Traffic Engineering, 35, S. 317-321. 
[Frondel et al., 2008] Frondel, M.; Schmidt, C. M. und Vance, C. (2008). "A Regression on Climate Policy - The European Commission's Proposal to Reduce $\mathrm{CO}_{2}$ Emissions from Transport". In Transportation Research Part A: Policy and Practice, 45(10) Auflage, S. 1043-1051. ISBN 978-3-86788045-9.

[Fu et al., 2006] Fu, L.; Sun, D. und Rilett, L. (2006). "Heuristic shortest path algorithms for transportation applications: State of the art". Computers EJ Operations Research, 33(11), S. 3324-3343.

[Fuks und Boccara, 1998] Fuks, H. und Boccara, N. (1998). "Generalized Deterministic Traffic Rules". International Journal of Modern Physics C, 9, S. 1.

[Fukui und Ishibashi, 1996] Fukui, M. und Ishibashi, Y. (1996). "Traffic Flow in 1D Cellular Automaton Model Including Cars Moving with High Speed". Journal of the Physical Society of Japan, 65(6), S. 1868-1870.

[Gates et al., 2006] Gates, T. J.; Noyce, D. A.; Bill, A. R. und Van Ee, N. (2006). "Recommended Walking Speeds for Pedestrian Clearance Timing Based on Pedestrian Characteristics". Transportation Research Record, 6.

[Gawron, 1998] Gawron, C. (1998). "An Iterative Algorithm to Determine the Dynamic User Equilibrium in a Traffic Simulation Model". International Journal of Modern Physics C (IJMPC), 9(3), S. 393-408.

[Gazis et al., 1959] Gazis, D. C.; Herman, R. und Potts, R. B. (1959). "CarFollowing Theory of Steady-State Traffic Flow". Operations Research, 7(4), S. 499-505.

[Gazis et al., 1961] Gazis, D. C.; Herman, R. und Rothery, R. W. (1961). "Nonlinear Follow-The-Leader Models of Traffic Flow". Operations Research, 9(4), S. pp. 545-567.

[Gehrke und Wojtusiak, 2008] Gehrke, J. D. und Wojtusiak, J. (2008). "Traffic Prediction for Agent Route Planning". In Proceedings of the 8th international conference on Computational Science, Part III, ICCS '08, S. 692701, Berlin, Heidelberg. Springer-Verlag.

[Gerlough und Huber, 1975] Gerlough, D. L. und Huber, M. J. (1975). "Traffic flow theory". Transportation Research Board, S. 199-201. 
[Geroliminis, 2008] Geroliminis, N. (2008). "A Macroscopic Fundamental Diagram of Urban Traffic: Recent Findings". In Transportation Research Board: Symposium on the Fundamental Diagram: 75 Years (Greenshields 75 Symposium).

[Geroliminis und Sun, 2011] Geroliminis, N. und Sun, J. (2011). "Properties of a well-defined macroscopic fundamental diagram for urban traffic". Transportation Research Part B: Methodological, 45(3), S. 605-617.

[Gilbert, 2007] Gilbert, N. (2007). Agent-Based Modelling and Simulation, Kapitel 5: Computational Social Science, S. 115-133. The Bardwell Press. Phan, Denis and Amblard, Frédéric (Herausgeber), ISBN 978-1-905622-01-6.

[Goodchild, 2007] Goodchild, M. F. (2007). "Citizens as sensors: the world of volunteered geography". GeoJournal, 69, S. 211-221.

[Goos, 1998] Goos, G. (1998). Vorlesungen über Informatik Band 4. eXamen.press. Springer-Verlag GmbH, Heidelberg, 1 Auflage. ISBN: 3-54060650-5.

[Goos und Zimmermann, 2006] Goos, G. und Zimmermann, W. (2006). Vorlesungen über Informatik Band 1. eXamen.press. Springer-Verlag GmbH, Heidelberg, 4 Auflage. ISBN: 978-3-540-24405-9.

[Gould und Karner, 2009] Gould, G. und Karner, A. (2009). "Modeling Bicycle Facility Operation: a Cellular Automaton Approach". Transportation Research Record: Journal of the Transportation Research Board, 2140, S. 157-164.

[Greenberg, 1959] Greenberg, H. (1959). "An Analysis of Traffic Flow". Operations Research, 7(1), S. 79-85.

[Greenshield, 1935] Greenshield, B. D. (1935). "A Study of Traffic Capacity". Proceedings of the 14th Annual Meeting Highway Research Board 14, 468, S. $448-477$.

[Griffiths et al., 1984] Griffiths, J.; Hunt, J. und Marlow, M. (1984). "Delays at pedestrian crossings: 1 . Site observations and the interpretation of data". Traffic Engineering and Control, 25, S. 355-371.

[Grubb et al., 1999] Grubb, P. E.; Hellinga, B. und Mallett, J. (1999). "Using Micro-Simulation Tools to Improve Transportation Decisions: Case Stu- 
dies". Proceedings of the AQTR-ITE Conference.

[Gualtieri und Tartaglia, 1998] Gualtieri, G. und Tartaglia, M. (1998). "Predicting urban traffic air pollution: A GIS framework". Transportation Research Part D: Transport and Environment, 3(5), S. $329-336$.

[Guerrier und Jolibois Jr, 1998] Guerrier, J. H. und Jolibois Jr, S. C. (1998). "The safety of elderly pedestrians at five urban intersections in Miami". In Proceedings of the Human Factors and Ergonomics Society 42nd Annual Meeting, Chicago, IL, USA.

[Guo et al., 2011] Guo, H.-W.; Gao, Z.-Y.; Zhao, X.-M. und Xie, D.-F. (2011). "Dynamics of Motorized Vehicle Flow under Mixed Traffic Circumstance". Commun. Theor. Phys., 55, S. 719-724.

[Guo et al., 2012] Guo, H.-w.; Wang, W.-h.; Zhao, F.-c. und Guo, W.-w. (2012). "Analyzing influence of bicycles on traffic flow using microscopic simulation approach". Journal of Beijing Institute of Technology, 21(2), S. 210-215.

[Hafstein et al., 2003] Hafstein, S. F.; Pottmeier, A.; Wahle, J. und Schreckenberg, M. (2003). "Cellular Automaton Modeling of the Autobahn Traffic in North Rhine-Westphalia". In I. Troch and F. Breitenecker (Eds.), Proc. of the 4th MATHMOD, S. 1322-1331.

[Haight, 1963] Haight, F. A. (1963). Mathematical Theories of Traffic Flow (Mathematics in Science and Engineering, Vol. 7). Mathematics in Science and Engineering. Academic Press New York/London.

[Haklay, 2010] Haklay, M. (2010). "How good is volunteered geographical information? A comparative study of OpenStreetMap and Ordnance Survey datasets". Environment and Planning B: Planning and Design, 37(4), S. 682-703.

[Hall et al., 1986] Hall, F. L.; Allen, B. L. und Gunter, M. A. (1986). "Empirical analysis of freeway flow-density relationships". Transportation Research Part A: General, 20(3), S. $197-210$.

[Hall et al., 2009] Hall, M.; Frank, E.; Holmes, G.; Pfahringer, B.; Reutemann, P. und Witten, I. H. (2009). "The WEKA Data Mining Software: An Update". SIGKDD Explorations, 11(1), S. 10-18. 
[Hamed, 2001] Hamed, M. M. (2001). "Analysis of pedestrians' behavior at pedestrian crossings". Safety Science, 38(1), S. $63-82$.

[Hatzopoulou et al., 2011] Hatzopoulou, M.; Hao, J. und Miller, E. (2011). "Simulating the impacts of household travel on greenhouse gas emissions, urban air quality, and population exposure". Transportation, 38, S. 871-887.

[Heißing et al., 2011] Heißing, B.; Ersoy, M. und Gies, S. (2011). Fahrwerkhandbuch: Grundlagen, Fahrdynamik, Komponenten, Systeme, Mechatronik, Perspektiven, dritte Auflage. Vieweg+Teubner Verlag.

[Helbing, 1992] Helbing, D. (1992). "A Fluid Dynamic Model for the Movement of Pedestrians". COMPLEX SYSTEMS, 6, S. 391.

[Helbing, 1997a] Helbing, D. (1997a). "Empirical traffic data and their implications for traffic modeling". Phys. Rev. E, 55(1), S. R25-R28.

[Helbing, 1997b] Helbing, D. (1997b). Verkehrsdynamik: Neue physikalische Modellierungskonzepte. Springer Berlin. ISBN 978-3-54061-927-7.

[Helbing, 1998] Helbing, D. (1998). "Models for Pedestrian Behavior". ArXiv Condensed Matter e-prints.

[Helbing, 2001] Helbing, D. (2001). "Traffic and related self-driven many-particle systems". Rev. Mod. Phys., 73(4), S. 1067-1141.

[Helbing, 2009] Helbing, D. (2009). "Derivation of a fundamental diagram for urban traffic flow". The European Physical Journal B - Condensed Matter and Complex Systems, 70, S. 229-241. 10.1140/epjb/e2009-00093-7.

[Helbing und Johansson, 2009] Helbing, D. und Johansson, A. (2009). "Pedestrian, Crowd and Evacuation Dynamics.". In Meyers, R. A., editor, Encyclopedia of Complexity and Systems Science, S. 6476-6495. Springer.

[Helbing und Molnár, 1995] Helbing, D. und Molnár, P. (1995). "Social force model for pedestrian dynamics". Physical Review E, 51(5), S. 4282-4286.

[Helbing und Tilch, 1998] Helbing, D. und Tilch, B. (1998). "Generalized force model of traffic dynamics". Phys. Rev. E, 58(1), S. 133-138.

[Hellinga, 1998] Hellinga, B. (1998). "Requirements for the Validation and Calibration of Traffic Simulation Models". Proceedings of the Canadian Society for Civil Engineering Annual Conference held in Halifax, Nova Scotia, 
IVb, S. 211-222. ISBN 0-921303-86-6.

[Hellinga et al., 1993] Hellinga, B.; Baker, M.; Van Aerde, M.; Aultman-Hall, L. und Masters, P. (1993). "Overview of a Simulation Study of the Highway 401 FTMS". Technical report, Transportation Systems Research Group Queen's University, Kingston, ON K7L 3N6 and Freeway Traffic Management Section Ontario Ministry of Transportation, Downsview, ON.

[Hellinga und Van Aerde, 1998] Hellinga, B. und Van Aerde, M. (1998). "Estimating Dynamic O-D Demands for a Freeway Corridor using Loop Detector Data". Proceedings of the Canadian Society for Civil Engineering 1998 Annual Conference held in Halifax, Nova Scotia, IVb, S. 185-197. ISBN: 0-921303-86-6.

[Hellinga, 1994] Hellinga, B. R. (1994). Estimating Dynamic Origin-Destination Demands from Link and Probe Counts. Dissertation, Queen's University Kingston, Ontario, Canada.

[Henderson, 1974] Henderson, L. F. (1974). "On the fluid mechanics of human crowd motion". Transportation Research, 8(6), S. 509 - 515.

[Hennermann, 2006] Hennermann, K. (2006). Kartographie und GIS. Eine Einführung. Wissenschaftliche Buchgesellschaft. ISBN 978-3-534-19692-0.

[Hennessy und Wiesenthal, 2001] Hennessy, D. A. und Wiesenthal, D. L. (2001). "Gender, Driver Aggression, and Driver Violence: An Applied Evaluation". Sex Roles, 44, S. 661-676. 10.1023/A:1012246213617.

[Himanen und Kulmala, 1988] Himanen, V. und Kulmala, R. (1988). "An application of logit models in analysing the behaviour of pedestrians and car drivers on pedestrian crossings". Accident Analysis \& Prevention, 20(3), S. $187-197$.

[Hirst und Graham, 1997] Hirst, S. und Graham, R. (1997). The format and presentation of collision warnings, Kapitel 11, S. 203-219. L. Erlbaum Associates Inc., Hillsdale, NJ, USA. ISBN: 0-8058-1956-8.

[Hoel, 1968] Hoel, L. A. (1968). "Pedestrian Travel Rates in Central Business Districts". Traffic Engineering, 38, S. 10-13.

[Holland et al., 2009] Holland, C. A.; Hill, R. und Cooke, R. (2009). "Understanding the role of self-identity in habitual risky behaviours: pedestrian 
road-crossing decisions across the lifespan". HEALTH EDUCATION RESEARCH, 24, S. 674-685.

[Holm et al., 2007] Holm, P.; Tomich, D.; Sloboden, J. und Lowrance, C. (2007).

"Traffic Analysis Toolbox Volume IV: Guidelines for Applying CORSIM Microsimulation Modeling Software". Report no. fhwa-hop-07-079, U.S. Department of Transportation.

[Hoogendoorn et al., 2002] Hoogendoorn, S.; Bovy, P. und Daamen, W. (2002). Pedestrian and Evacuation Dynamics, Kapitel Microscopic pedestrian wayfinding and dynamics modelling, S. 124-154. Schreckenberg, M. und Sharma, S. D. (Herausgeber) Springer.

[Hoogendoorn und Bovy, 2005] Hoogendoorn, S. P. und Bovy, P. H. L. (2005). "Pedestrian Travel Behavior Modeling". Networks and Spatial Economics, 5, S. 193-216. 10.1007/s11067-005-2629-y.

[Hourdakis et al., 2003] Hourdakis, J.; Michalopoulos, P. G. und Kottommannil, J. (2003). "A Practical Procedure for Calibrating Microscopic Traffic Simulation Models". Transportation Research Board Annual Meeting.

[Hughes et al., 2003] Hughes, R.; Rouphail, N. und Chae, K. (2003). "Exploratory Simulation of Pedestrian Crossings at Roundabouts". ASCE Journal of Transportation Engineering.

[Hughes, 2003] Hughes, R. L. (2003). "The Flow of Human Crowds". Annual Review of Fluid Mechanics, 35(1), S. 169-182.

[Illenberger und Flötteröd, 2007] Illenberger, J. und Flötteröd, Gunnar Nagel, K. (2007). "Enhancing MATSim with capabilities of within-day re-planning". IEEE Intelligent Transportation Systems Conference.

[Ishaque, 2006] Ishaque, M. M. (2006). Policies for Pedestrian Access: MultiModal Trade-off analysis using Micro-Simulation Techniques. PhD thesis, Centre for Transport Studies Imperial College London.

[Ishaque und Noland, 2008] Ishaque, M. M. und Noland, R. B. (2008). "Behavioural Issues in Pedestrian Speed Choice and Street Crossing Behaviour: A Review". In Transport Reviews: A Transnational Transdisciplinary Journal, 28(1) Auflage, S. 61-85.

[Jacob et al., 1999] Jacob, R.; Marathe, M. und Nagel, K. (1999). "A computa- 
tional study of routing algorithms for realistic transportation networks". J. Exp. Algorithmics, 4.

[Jason und Liotta, 1982] Jason, L. A. und Liotta, R. (1982). "Pedestrian jaywalking under facilitating and nonfacilitating conditions". Journal of Applied Behavior Analysis, 15(15), S. 469-473.

[Jia et al., 2007] Jia, B.; Li, X.-G.; Jiang, R. und Gao, Z.-Y. (2007). "Multi-value cellular automata model for mixed bicycle flow". The European Physical Journal B - Condensed Matter and Complex Systems, 56, S. 247-252.

[Jiang et al., 2007] Jiang, R.; Hu, M.-B. und Wu, Q.-S. (2007). "NagelSchreckenberg traffic flow model based fuel consumption analysis". In 5th Asia Pacific conference on Transportation and Environment (5th APTE 2007), Singapore.

[Jiang et al., 2003] Jiang, R.; Wang, X.-L. und Wu, Q.-S. (2003). "Dangerous situations within the framework of the Nagel-Schreckenberg model". Journal of Physics A: Mathematical and General, 36(17), S. 4763-4769.

[Johansson et al., 2007] Johansson, A.; Helbing, D. und Shukla, P. K. (2007). "Specification Of The Social Force Pedestrian Model By Evolutionary Adjustment To Video Tracking Data”. Advances in Complex Systems (ACS), 10(su), S. 271-288.

[Johnson et al., 2008] Johnson, M.; Charlton, J. und Oxley, J. (2008). "Cyclists and red lights - a study of behaviour of commuter cyclists in Melbourne". In 2008 Australasian Road Safety Research, Policing and Education Conference.

[Johnson et al., 2011] Johnson, M.; Newstead, S.; Charlton, J. und Oxley, J. (2011). "Riding through red lights: The rate, characteristics and risk factors of non-compliant urban commuter cyclists". Accident Analysis $\&$ Prevention, 43(1), S. 323-328.

[Johnson et al., 2009] Johnson, M.; Oxley, J. und Cameron, M. (2009). "Cyclist bunch riding: A review of the literature". Technical report, Monash University Accident Research Centre. ISBN: 0-7326-2355-3.

[Jokinen et al., 2005] Jokinen, R.; Kosonen, I. und Luttinen, T. (2005). "An Integrated On-Line Simulation of Signalized Intersections". Transportation Research Board, 84th Annual Meeting. 
[Karathodorou et al., 2010] Karathodorou, N.; Graham, D. J. und Noland, R. B. (2010). "Estimating the effect of urban density on fuel demand". Energy Economics, 32(1), S. 86-92.

[Kennedy et al., 2001] Kennedy, J.; Kennedy, J.; Eberhart, R. und Shi, Y. (2001). Swarm Intelligence. The Morgan Kaufmann Series in Evolutionary Computation. Morgan Kaufmann Publishers. ISBN 978-1-55860-595-4.

[Kerner, 2009] Kerner, B. S. (2009). Introduction to Modern Traffic Flow Theory and Control: The Long Road to Three-Phase Traffic Theory. Springer. ISBN: 978-3-64202-604-1.

[Kerner et al., 2002] Kerner, B. S.; Klenov, S. L. und Wolf, D. E. (2002). "Cellular automata approach to three-phase traffic theory". Journal of Physics A: Mathematical and General, 35(47), S. 9971.

[Kerner und Konhäuser, 1994] Kerner, B. S. und Konhäuser, P. (1994). "Structure and parameters of clusters in traffic flow". Phys. Rev. E, 50(1), S. 54-83.

[Kerner und Rehborn, 1997] Kerner, B. S. und Rehborn, H. (1997). "Experimental Properties of Phase Transitions in Traffic Flow". Phys. Rev. Lett., 79(20), S. 4030-4033.

[Kesting und Treiber, 2008] Kesting, A. und Treiber, M. (2008). "Calibrating Car-Following Models using Trajectory Data: Methodological Study". Transportation Research Record: Journal of the Transportation Research Board, Volume, 2088, S. 148-156.

[Kesting et al., 2008] Kesting, A.; Treiber, M. und Helbing, D. (2008). "Agents for Traffic Simulation". Arxiv preprint arXiv08050300, physics.so, S. 325-356.

[Kesting et al., 2009] Kesting, A.; Treiber, M. und Helbing, D. (2009). MultiAgent Systems, Kapitel 11: Agents for Traffic Simulation, S. 325-356. Uhrmacher, Adelinde M. und Weyns, Danny (Herausgeber) CRC Press, Taylor and Francis Group. ISBN: 978-1-4200-7023-1.

[Kesting et al., 2010] Kesting, A.; Treiber, M. und Helbing, D. (2010). "Enhanced intelligent driver model to access the impact of driving strategies on traffic capacity." Philosophical Transactions of the Royal Society - Series A: Mathematical, Physical and Engineering Sciences, 368(1928), S. 4585-605.

[Kim, 2006] Kim, I.-C. (2006). "Real-Time Search Algorithms for Exploration 
and Mapping". In Gabrys, B.; Howlett, R. und Jain, L., Herausgeber, Knowledge-Based Intelligent Information and Engineering Systems, 4251 Auflage von Lecture Notes in Computer Science, S. 244-251. Springer Berlin / Heidelberg.

[King et al., 2008] King, M. J.; Soole, D. W. und Ghafourian, A. (2008). "Relative risk of illegal pedestrian behaviours". Australasian Road Safety Research, Policing and Education Conference, S. 768-778.

[Kitazawa und Batty, 2004] Kitazawa, K. und Batty, M. (2004). "Pedestrian Behavior Modelling: An Application to Retail Movements using a Genetic Algorithm". Proceedings of the 7th International Conference on Design and Descision Support Systems in Architecture and Urban Planning.

[Knoblauch et al., 1996] Knoblauch, R. L.; Pietrucha, M. T. und Nitzburg, M. (1996). "Field Studies of Pedestrian Walking Speed and Start-Up Time". Transportation Research Record, 1538, S. 27-38.

[Knospe et al., 2000] Knospe, W.; Santen, L.; Schadschneider, A. und Schreckenberg, M. (2000). "Towards a realistic microscopic description of highway traffic". Journal of Physics A: Mathematical and General, 33(48).

[Knospe et al., 2002] Knospe, W.; Santen, L.; Schadschneider, A. und Schreckenberg, M. (2002). "A realistic two-lane traffic model for highway traffic". Journal of Physics A: Mathematical and General, 35(15), S. 3369-3388.

[Knospe et al., 2004] Knospe, W.; Santen, L.; Schadschneider, A. und Schreckenberg, M. (2004). "Empirical test for cellular automaton models of traffic flow". Phys. Rev. E, 70(016115).

[Kobbeloer, 2007] Kobbeloer, D. (2007). Dezentrale Steuerung von Lichtsignalanlagen in urbanen Verkehrsnetzen, 18 Auflage von Kasseler Dissertation Schriftenreihe Verkehr. Institut für Verkehrswesen Universität Kassel. ISBN 978-3-89958-323-6.

[Koizumi et al., 2010] Koizumi, Y.; Nishida, Y.; Motomura, Y.; Miyazaki, Y. und Mizoguchi, H. (2010). "Presenting Potential Injury Risk by Biomechanical Simulation Based on Bodygraphic Injury Data". SIMUTools'10 3rd International ICST Conference on Simulation Tools and Techniques.

[Kolodziej, 2009] Kolodziej, A. (2009). "Umweltbundesamt: Daten zum Verkehr Ausgabe 2009". http://www. umweltdaten.de/publikationen/fpdf-l/ 
3880.pdf, abgerufen am 31.07.2012.

[Kolodziej et al., 2009] Kolodziej, A.; Richter, N. und Erdmenger, C. (2009). "Daten zum Verkehr". Technical report, Bundesministeriums für Umwelt, Naturschutz und Reaktorsicherheit.

[Korf, 1990] Korf, R. E. (1990). "Real-time heuristic search". Artificial Intelligence, $42(2-3)$, S. 189-211.

[Kosonen, 1999] Kosonen, I. (1999). HUTSIM - Urban Traffic Simulation and Control Model: Principles and Applications. Dissertation, Helsinki University of Technology Transportation Engineering, Teknillinen korkeakoulu Liikennetekniikka Julkaisu 100 Espoo 1999. ISBN 951-22-4778-x.

[Kosonen und Bargiela, 1999] Kosonen, I. und Bargiela, A. (1999). "A Distributed Traffic Monitoring and Information System". In Journal of Geographic Information and Decision Analysis, 3(1) Auflage, S. 30-39.

[Kosonen und Bargiela, 2000] Kosonen, I. und Bargiela, A. (2000). "Simulation Based Traffic Information System". ITS World Congress in Torino, 7.

[Krajzewicz et al., 2006] Krajzewicz, D.; Bonert, M. und Wagner, P. (2006). "The Open Source Traffic Simulation Package SUMO". In RoboCup 2006 Infrastructure Simulation Competition, RoboCup 2006, Germany, Bremen.

[Krajzewicz et al., 2005a] Krajzewicz, D.; Brockfeld, E.; Mikat, J.; Ringel, J.; Rössel, C.; Tuchscheerer, W.; Wagner, P. und Wösler, R. (2005a). "Simulation of modern Traffic Lights Control Systems using the open source Traffic Simulation SUMO". In Proceedings of the 3rd Industrial Simulation Conference 2005; J. Krüger, A. Lisounkin, G. Schreck (Herausgeber); EUROSIS-ETI, S. 299-302. ISBN: 90-77381-18-X.

[Krajzewicz et al., 2005b] Krajzewicz, D.; Hertkorn, G.; Ringel, J. und Wagner, P. (2005b). "Preparation of Digital Maps for Traffic Simulation; Part 1: Approach and Algorithms". In Proceedings of the 3rd Industrial Simulation Conference 2005, EUROSIS-ETI, J. Krüger, A. Lisounkin, G. Schreck (Herausgeber), S. 285-290. ISBN 90-77381-18-X.

[Krajzewicz et al., 2002a] Krajzewicz, D.; Hertkorn, G.; Rössel, C. und Wagner, P. (2002a). "An Example of Microscopic Car Models Validation using the open source Traffic Simulation SUMO". In Proceedings of Simulation in Industry, 14th European Simulation Symposium. 
[Krajzewicz et al., 2002b] Krajzewicz, D.; Hertkorn, G.; Rössel, C. und Wagner, P. (2002b). "SUMO (Simulation of Urban MObility); An open-source traffic simulation". In Al-Akaidi, A. (Herausgeber) Proceedings of the 4th Middle East Symposium on Simulation and Modelling (MESM2002), SCS European Publishing House, S. 183-187.

[Krauß, 1997] Krauß, S. (1997). "Towards a Unified View of Microscopic Traffic Flow Theories". In IFAC Transportation Systems, S. 941-946.

[Krauß, 1998] Krauß, S. (1998). Microscopic Modeling of Traffic Flow: Investigation of Collision Free Vehicle Dynamics. PhD thesis, Deutsches Zentrum fürr Luft- und Raumfahrt Hauptabteilung Mobilität und Systemtechnik, Köln.

[Krauß et al., 1997] Krauß, S.; Wagner, P. und Gawron, C. (1997). "Metastable States in a Microscopic Model of Traffic Flow". Phys. Rev. E, 55(304), S. $55-97$.

[Krauss et al., 1996] Krauss, S.; Wagner, P. und Gawron, C. (1996). "Continuous limit of the Nagel-Schreckenberg model". Phys. Rev. E, 54(4), S. 37073712 .

[Kretz et al., 2008] Kretz, T.; Hengst, S. und Vortisch, P. (2008). "Pedestrian Flow at Bottlenecks - Validation and Calibration of Vissim's Social Force Model of Pedestrian Traffic and its Empirical Foundations". CoRR, abs/0805.1788.

[Kumar et al., 2011] Kumar, P.; Ketzel, M.; Vardoulakis, S.; Pirjola, L. und Britter, R. (2011). "Dynamics and dispersion modelling of nanoparticles from road traffic in the urban atmospheric environmental review". Journal of Aerosol Science, 42(9), S. $580-603$.

[Laagland, 2005] Laagland, J. (2005). "How To Model Aggressive Behavior In Traffic simulation". Twente Student Conference on IT, Enschede, 3.

[Laberer und Niedermeier, 2009] Laberer, C. und Niedermeier, M. (2009). "Wirksamkeit von Umweltzonen". ADAC-Untersuchung.

[Larsen und El-Geneidy, 2011] Larsen, J. und El-Geneidy, A. (2011). "A travel behavior analysis of urban cycling facilities in Montréal, Canada". Transportation Research Part D: Transport and Environment, 16(2), S. 172-177. 
[Lattner, 2012] Lattner, A. D. (2012). "Towards Automation of Simulation Studies - Artificial Intelligence Methodologies for the Control and Analysis of Simulation Experiments". Habilitationsschrift zur Erlangung der Lehrbefähigung für das Fach Informatik.

[Lattner et al., 2012] Lattner, A. D.; Dallmeyer, J.; Paraskevopoulos, D. und Timm, I. J. (2012). "Approximation of Pedestrian Effects in Urban Traffic Simulation by Distribution Fitting". In Troitzsch, K. G.; Möhring, M. und Lotzmann, U., Herausgeber, Proceedings of the 26th EUROPEAN Conference on Modelling and Simulation, S. 574-580. ISBN 978-0-9564944-4-3.

[Lattner et al., 2011] Lattner, A. D.; Dallmeyer, J. und Timm, I. J. (2011). "Learning Dynamic Adaptation Strategies in Agent-based Traffic Simulation Experiments". In Ninth German Conference on Multi-Agent System Technologies (MATES 2011), S. 77-88. Springer: Berlin, LNCS 6973, Klügl, F.; Ossowski, S. ISBN: 978-3-642-24602-9.

[Law, 2007] Law, A. M. (2007). Simulation Modeling And Analysis. McGraw-Hill International Edition. McGraw-Hill. ISBN 978-007-125519-6.

[Lee et al., 2004] Lee, H. K.; Barlovic, R.; Schreckenberg, M. und Kim, D. (2004). "Mechanical Restriction versus Human Overreaction Triggering Congested Traffic States". Phys. Rev. Lett., 92(23), S. 238702.

[Lee und Kim, 2011] Lee, H. K. und Kim, B. J. (2011). "Dissolution of traffic jam via additional local interactions". Physica A: Statistical Mechanics and its Applications, 390(23-24), S. 4555 - 4561.

[Lee und El-Sharkawi, 2005] Lee, K. Y. und El-Sharkawi, M. A., Herausgeber (2005). Modern Heuristic Optimization Techniques With Applications To Power Systems. John Wiley \& Sons. ISBN: 978-0471-45711-4.

[Li et al., 2009] Li, X.-G.; Gao, Z.-Y.; Jia, B. und Jiang, R. (2009). "Deceleration in advance in the Nagel-Schreckenberg traffic flow model". Physica A: Statistical Mechanics and its Applications, 388(10), S. 2051 - 2060.

[Lighthill und Whitham, 1955] Lighthill, M. J. und Whitham, G. B. (1955). "On Kinematic Waves. II. A Theory of Traffic Flow on Long Crowded Roads". Proceedings of the Royal Society of London. Series A, Mathematical and Physical Sciences, 229(1178), S. 317-345.

[Liu et al., 2005] Liu, M.; Wang, R. und Kemp, R. (2005). "Towards a Realistic 
Microscopic Traffic Simulation at an Unsignalised Intersection". In Gervasi, O.; Gavrilova, M.; Kumar, V.; Laganà, A.; Lee, H.; Mun, Y.; Taniar, D. und Tan, C., Herausgeber, Computational Science and Its Applications - ICCSA 2005, 3481 Auflage von Lecture Notes in Computer Science, S. 1187-1196. Springer Berlin / Heidelberg. 10.1007/11424826_126.

[Longford, 2007] Longford, N. T. (2007). Studying Human Populations An Advanced Course in Statistics, Kapitel Maximum Likelihood Estimation, S. 37-66. Springer New York. ISBN 978-0-387-98735-4.

[Lownes und Machemehl, 2006] Lownes, N. E. und Machemehl, R. B. (2006). "VISSIM: a multi-parameter sensitivity analysis". In Proceedings of the 38th conference on Winter simulation, WSC '06, S. 1406-1413. Winter Simulation Conference.

[Madani und Moussa, 2012] Madani, A. und Moussa, N. (2012). "Simulation of Fuel Consumption and Engine Pollutant in Cellular Automaton". In Journal of Theoretical and Applied Information Technology, 35 (2) Auflage. ISSN: 1992-8645.

[Maerivoet und Moor, 2005] Maerivoet, S. und Moor, B. D. (2005). "Cellular automata models of road traffic". Physics Reports, 419(1), S. $1-64$.

[Mahnke et al., 2008] Mahnke, R.; Liebe, C.; Kuhne, R. D. und Wang, H. (2008). "Traffic Flow Prospectives: From Fundamental Diagram to Energy Balance". In Transportation Research Board: Symposium on the Fundamental Diagram: 75 Years (Greenshields 75 Symposium).

[McCartney und Carey, 1999] McCartney, M. und Carey, M. (1999). "Follow that Car! Investigating a Simple Class of Car Following Model". In Teaching Mathematics and its Applications, 19(2) Auflage. Oxford University Press. ISSN 0268-3679, Online ISSN: 1471-6976.

[Medina et al., 2005] Medina, J.; Moreno, M. und Royo, E. (2005). "Stochastic Vs Deterministic Traffic Simulator. Comparative Study for Its Use Within a Traffic Light Cycles Optimization Architecture". In Mira, J. und Álvarez, J., Herausgeber, Artificial Intelligence and Knowledge Engineering Applications: A Bioinspired Approach, 3562 Auflage von Lecture Notes in Computer Science, S. 99-111. Springer Berlin / Heidelberg.

[Meignan et al., 2006] Meignan, D.; Simonin, O. und Koukam, A. (2006). "MultiAgent Approach for Simulation and Evaluation of Urban Bus Networks". 
Agents in Traffic and Transportation (ATT06), S. 50-56.

[Meignan et al., 2007] Meignan, D.; Simonin, O. und Koukam, A. (2007). "Simulation and evaluation of urban bus-networks using a multiagent approach". Simulation Modelling Practice and Theory, 15(6), S. 659-671.

[Meister et al., 2008] Meister, K.; Rieser, M.; Ciari, F.; Horni, A.; Balmer, M. und Axhausen, K. (2008). "Anwendung eines agentenbasierten Modells der Verkehrsnachfrage auf die Schweiz". Heureka '08.

[Miller und Horowitz, 2007] Miller, J. und Horowitz, E. (2007). "Freesim - a free real-time freeway traffic simulator". Intelligent Transportation Systems Conference ITSC 2007 IEEE, S. 18-23.

[Minderhoud und Bovy, 2001] Minderhoud, M. M. und Bovy, P. H. L. (2001). "Extended time-to-collision measures for road traffic safety assessment". Accident Analysis 83 Prevention, 33(1), S. 89 - 97.

[Minnesota Department of Transportation, 2008] Minnesota Department of Transportation (2008). Advanced CORSIM Training Manual. SEH No. A-MNDOT0318.00.

[Moussa, 2003] Moussa, N. (2003). "Car accidents in cellular automata models for one-lane traffic flow". Phys. Rev. E, 68(3), S. 036127.

[Nagatani, 1996] Nagatani, T. (1996). "Effect of car acceleration on traffic flow in 1D stochastic CA model". Physica A: Statistical and Theoretical Physics, 223(1-2), S. $137-148$.

[Nagel, 1996] Nagel, K. (1996). "Particle hopping models and traffic flow theory". Phys. Rev. E, 53(5), S. 4655-4672.

[Nagel, 2001] Nagel, K. (2001). "Multi-modal traffic in TRANSIMS". In in: Schreckenberg, M.; Sharma, S.D. (eds.), Pedestrian and Evacuation Dynamics, S. 161-172. Springer.

[Nagel et al., 1999] Nagel, K.; Beckman, R. J. und Barrett, C. L. (1999). "TRANSIMS for urban planning". Los Alamos Unclassified Report LA-UR 984389 .

[Nagel et al., 2001] Nagel, K.; Frye, R.; Jakob, R.; Rickert, M. und Stretz, P. (2001). "Dynamic traffic assignment on parallel computers". In IN: FUTURE GENERATION COMPUTER SYSTEMS, S. 637-648. 
[Nagel und Rickert, 2001] Nagel, K. und Rickert, M. (2001). "Parallel implementation of the TRANSIMS micro-simulation". CoRR, cs.CE/0105004.

[Nagel und Schreckenberg, 1992] Nagel, K. und Schreckenberg, M. (1992). "A cellular automaton model for freeway traffic". In Journal de Physique I, 2(12) Auflage, S. 2221-2229. ISSN 1155-4304.

[Nagel et al., 1998] Nagel, K.; Wolf, D. E.; Wagner, P. und Simon, P. (1998). "Two-lane traffic rules for cellular automata: A systematic approach". Physical Review E, 58(2), S. 1425-1437.

[Narasimhan, 2007] Narasimhan, S. (2007). Simulation and Optimized Scheduling of Pedestrian Traffic. Dissertation, IPVS, Universität Stuttgart.

[Narzt et al., 2010] Narzt, W.; Wilflingseder, U.; Pomberger, G.; Kolb, D. und Hörtner, H. (2010). "Self-organising congestion evasion strategies using ant-based pheromones". Intelligent Transport Systems, IET, 4(1), S. 93102.

[Neis et al., 2011] Neis, P.; Zielstra, D. und Zipf, A. (2011). "The Street Network Evolution of Crowdsourced Maps: OpenStreetMap in Germany 2007-2011". Future Internet, 4(1), S. 1-21.

[Neis und Zipf, 2008] Neis, P. und Zipf, A. (2008). "Zur Kopplung von OpenSource, OpenLS und OpenStreetMaps in OpenRouteService.org". In Blaschke, T.; Griesebner, G. und Strobl, J., Herausgeber, Angewandte Geoinformatik 2008 Beiträge zum 20. AGIT-Symposium Salzburg, Austria, S. 158-163, Salzburg. Wichmann Verlag, Heidelberg.

[Neumann und Wagner, 2008] Neumann, T. und Wagner, P. (2008). "Delay times in a cellular traffic flow model for road sections with periodic outflow". The European Physical Journal B - Condensed Matter and Complex Systems, 63, S. 255-264. 10.1140/epjb/e2008-00234-6.

[Ni, 2006] Ni, D. (2006). "A framework for new generation transportation simulation". In Proceedings of the 38th conference on Winter simulation, WSC '06, S. 1508-1514. Winter Simulation Conference.

[Niittymaki und Turunen, 1999] Niittymaki, J. und Turunen, E. (1999). "ManyValued Similarity Modelling Traffic Signal Control". In In: Mayor G, Suner J (Eds) Proceedings of the EUSFLAT-ESTYLF Joint Conference. Universitat de les Illes Balears, European Society of Fuzzy Logic and Tech- 
nology, S. 22-25.

[Niittymäki und Korkeakoulu, 2002] Niittymäki, J. und Korkeakoulu, T. (2002). Fuzzy Traffic Signal Control-Principles and Experiences. In: Grahne G. (Ed. PhD thesis, Helsinki University of Technology Transportation Engineering.

[O'Flaherty und Parkinson, 1972] O'Flaherty, C. A. und Parkinson, M. H. (1972). "Movement on a City Centre Footway". Traffic Engineering and Control, 2, S. 434-438.

[Older, 1968] Older, S. J. (1968). "Movement of pedestrians on footways in shopping streets". Traffic Engineering and Control, 10, S. 160-163.

[Oreskes, 2004] Oreskes, N. (2004). "The Scientific Consensus on Climate Change". In Science, 306. Auflage.

[Ottomanelli et al., 2009] Ottomanelli, M.; Caggiani, L.; Giuseppe, I. und Sassanelli, D. (2009). "An Adaptive Neuro-Fuzzy Inference System for simulation of pedestrians behaviour at unsignalized roadway crossings". 14th Online World Conference on Soft Computing in Industrial Application, 14.

[Ottomanelli et al., 2008] Ottomanelli, M.; Iannucci, G. und Sassanelli, D. (2008). Studies in Computational Intelligence, Kapitel 1: A discrete events based system for modelling pedestrian-vehicle interactions at pedestrian crossing. Adamski, Marian Andrzej, Springer. ISBN: 1860-949X.

[Owen et al., 2000] Owen, L. E.; Zhang, Y.; Rao, L. und McHale, G. (2000). "Traffic Flow Simulation Using Corsim". In Joines, J. A.; Barton, R. R.; Kang, K. und Fishwick, P. A., Herausgeber, Proceedings of the 2000 Winter Simulation Conference.

[Paksarsawan et al., 1992] Paksarsawan, S.; Montgomery, F. und Clark, S. (1992). "How TRAF-NETSIM Works". Technical report, Institute of Transport Studies, University of Leeds, Leeds, UK. Working Paper 380, ISSN 01428942.

[Peeters und Blokland, 2007] Peeters, B. und Blokland, G. v. (2007). "The Noise Emission Model For European Road Traffic". Deliverable 11 of the IMAGINE project. 
[Pei et al., 2001] Pei, J.; Han, J.; Mortazavi-Asl, B. und Pinto, H. (2001). "PrefixSpan: Mining Sequential Patterns Efficiently by Prefix-Projected Pattern Growth". In Proceedings of the 17th International Conference on Data Engineering, ICDE '01, S. 215-224, Washington, DC, USA. IEEE Computer Society.

[Pelkmans et al., 2005] Pelkmans, L.; Verhaeven, E.; Spleesters, G.; Kumra, S. und Schaerf, A. (2005). "Simulations of fuel consumption and emissions in typical traffic circumstances". SAE Technical Paper.

[Pipes, 1953] Pipes, L. A. (1953). "An Operational Analysis of Traffic Dynamics". Journal of Applied Physics, 24, S. 274.

[Pipes, 1967] Pipes, L. A. (1967). "Car following models and the fundamental diagram of road traffic". Transportation Research, 1(1), S. $21-29$.

[Plate et al., 2001] Plate, E.; Steinberg, G.; Haase, M. und Brunsing, J. (2001). "Chancen des Rad- und Fußverkehrs als Beitrag zur Umweltentlastung". Ein Leitfaden für die kommunale Praxis in kleinen und mittleren Kommunen 32, Bundesministeriums für Umwelt, Naturschutz und Reaktorsicherheit. Förderkennzeichen: 29896112.

[Prassas, 2003] Prassas, E. S. (2003). Kutz, Myer (Herausgeber) Handbook of Transportation Engineering, Kapitel 4: Software Systems and Simulation for Transportation Applications, S. 93-116. McGraw-Hill Professional Publishing. ISBN 978-0-07150-095-1.

[Prevedouros et al., 1997] Prevedouros, P. D.; D, P. und Wang, Y. (1997). "Simulation of a Large Freeway/Arterial Network with CORSIM, INTEGRATION and WATSim". In Transportation Research Record: Journal of the Transportation Research Board, 1678 / 1999 Auflage, S. 197-202. ISSN 0361-1981.

[PTV AG, 2005] PTV AG (2005). "VISSIM 4.10 User Manual". Technical report, PTV Planung Transport Verkehr AG.

[PTV AG, 2009] PTV AG (2009). "Neue Funktionalität in VISSIM 5.20". Technical report, PTV Planung Transport Verkehr AG.

[Quinlan, 1993] Quinlan, J. R. (1993). C4.5 - Programs for Machine Learning. Morgan Kaufmann Publishers, Inc. 
[R Development Core Team, 2011] R Development Core Team (2011). R: A Language and Environment for Statistical Computing. R Foundation for Statistical Computing, Vienna, Austria. ISBN 3-900051-07-0.

[Ragland et al., 2006] Ragland, D. R.; Arroyo, S.; Shladover, S. E.; Misener, J. A. und Chan, C.-Y. (2006). "Gap acceptance for vehicles turning left across on-coming traffic: Implications for Intersection Decision Support design". Technical report, UC Berkeley: Safe Transportation Research \& Education Center.

[Rajbhandari et al., 2003] Rajbhandari, R.; Chien, S. I. und Daniel, J. R. (2003). "Estimation of Bus Dwell Times with Automatic Passenger Counter Information". Transportation Research Record: Journal of the Transportation Research Board, 1841, S. 120-127. ISSN: 0361-1981.

[Rakha und Ahn, 2004] Rakha, H. und Ahn, K. (2004). "Integration Modeling Framework for Estimating Mobile Source Emissions". Journal of Transportation Engineering, 130(2), S. 183-193.

[Rakha und Crowther, 2002] Rakha, H. und Crowther, B. (2002). "A Comparison of the Greenshields, Pipes, and Van Aerde Car-Following and Traffic Stream Models". Transportation Research Record, Traffic Flow Theory and Highway Capacity, 1802.

[Rakha und Crowther, 2003] Rakha, H. und Crowther, B. (2003). "Comparison and calibration of FRESIM and INTEGRATION steady-state carfollowing behavior". Transportation Research Part A: Policy and Practice, $37(1)$, S. $1-27$.

[Rakha et al., 1996] Rakha, H.; Hellinga, B.; Van Aerde, M. und Perez, W. (1996). "Systematic Verification, Validation and Calibration of Traffic Simulation Models". Transportation Research Board Annual Meeting, 75.

[Rakha et al., 2000] Rakha, H.; Van Aerde, M.; Ahn, K. und Trani, A. A. (2000). "Requirements for Evaluating Traffic Signal Control Impacts on Energy and Emissions based on Instantaneous Speed and Acceleration Measurements". Transportation Research Board 79th Annual Meeting, 79.

[Rakha et al., 1998] Rakha, H.; Van Aerde, M.; Bloomberg, L. und Huang, X. (1998). "Construction and calibration of large scale microsimulation model of the salt lake area". Transportation Research Record, 1644, S. 93-102. 
[Rakha und Zhang, 2003] Rakha, H. und Zhang, Y. (2003). "The INTEGRATION 2.30 Framework for Modeling Lane-Changing Behavior in Weaving Sections". 83rd Transportation Research Board Meeting, 83.

[Rakha und Van Aerde, 1996] Rakha, H. A. und Van Aerde, M. W. (1996). "A Comparison of the Simulation Modules of the TRANSYT and INTEGRATION Models". In Transportation Research Record, 1566 Auflage, S. 1-7, Washington, DC 20001 USA. Transportation Research Board. ISSN: 0361-1981.

[Raney et al., 2002a] Raney, B.; Cetin, N.; Völlmy, A.; Vrtic, M.; Axhausen, K. und Nagel, K. (2002a). "Truly Agent-Based Strategy Selection for Transportation Simulations". Proceedings of the 82th Annual Meeting of the Transportation Research Board, 82.

[Raney et al., 2003] Raney, B.; Cetin, N.; Vollmy, A.; Vrtic, M.; Axhausen, K. und Nagel, K. (2003). "An agent-based microsimulation model of Swiss travel: First results". Networks and Spatial Economics, 3, S. 23-41.

[Raney und Nagel, 2002] Raney, B. und Nagel, K. (2002). "Iterative Route Planning for Modular Transportation Simulation". In in Proceedings of the Swiss Transport Research Conference, Monte Verita.

[Raney et al., 2002b] Raney, B.; Voellmy, A.; Cetin, N.; Vrtic, M. und Nagel, K. (2002b). Towards a Microscopic Traffic Simulation of All of Switzerland, S. 371-380. P.M.A. Sloot et al. (Herausgeber), Computational Science - ICCS 2002 Part I, Lecture Notes in Computer Science 2329, Springer Heidelberg.

[Reuschel, 1950a] Reuschel, A. (1950a). "Fahrzeugbewegungen in der Kolonne". Oesterreich. Ing. Archiv., 4, S. 193.

[Reuschel, 1950b] Reuschel, A. (1950b). "Fahrzeugbewegungen in der Kolonne bei gleichförmig beschleunigtem oder verzögertem Leitfahrzeug". Zeitschrift des Österreichischen Ingenieur- und Architektur Vereines, 95, S. 59-73.

[Ricci, 2005] Ricci, V. (2005). "Fitting Distributions with R". Release 0.4-21, http://cran.r-project.org/doc/contrib/Ricci-distributionsen.pdf, abgerufen am 13.04.2012.

[Richards, 1956] Richards, P. I. (1956). "Shock Waves on the Highway". Operations Research, 4(1), S. 42-51. 
[Rickert und Wagner, 1996] Rickert, M. und Wagner, P. (1996). "Parallel Realtime Implementation of Large-scale, Route-plan-driven Traffic Simulation". International Journal of Modern Physics C, 7(308), S. 133-153.

[Rickert et al., 1996] Rickert, M.; Wagner, P. und Gawron, C. (1996). "Real-Time Traffic Simulation of the German Autobahn Network". In Proceedings by World Scientific Publishing Singapore.

[Rieck et al., 2010] Rieck, D.; Schünemann, B.; Radusch, I. und Meinel, C. (2010). "Efficient Traffic Simulator Coupling in a Distributed V2X Simulation Environment". In 3rd International Conference on Simulation Tools and Techniques, SIMUTools '10, Malaga, Spain - March 16 - 18, 2010. ISBN 78-963-9799-87-5.

[Rieser, 2010] Rieser, M. (2010). Adding Transit to an Agent-Based Transportation Simulation Concepts and Implementation. Dissertation, Technische Universität Berlin.

[Robertson, 1983] Robertson, D. I. (1983). "Esso Energy Award Lecture, 1982: Coordinating Traffic Signals to Reduce Fuel Consumption". Proceedings of the Royal Society of London. Series A, Mathematical and Physical Sciences, 387(1792), S. 1-19.

[Rose, 2003] Rose, M. (2003). Modellbildung und Simulation von Autobahnverkehr. Dissertation, Universität Hannover.

[Ross, 1997] Ross, M. (1997). "Fuel Efficiency and the Physics of Automobiles". Contemporary Physics, 38(6), S. 381-394.

[Ross, 2006] Ross, S. M. (2006). Simulation. Elsevier Academic Press. Singer, Tom (Herausgeber), London, UK, 4 Auflage. ISBN: 978-0-12-598063-0.

[Rouphail et al., 2000] Rouphail, N. M.; Park, B. B. und Sacks, J. (2000). "Direct Signal Timing Optimization: Strategy Development and Results". Technical report, In XI Pan American Conference in Traffic and Transportation Engineering.

[Russell und Norvig, 2004] Russell, S. und Norvig, P. (2004). Künstliche Intelligenz. Ein moderner Ansatz, 2 Auflage. Pearson Studium. ISBN 978-38273-7089-1.

[Sacks et al., 2000] Sacks, J.; Rouphail, N. M.; Park, B. B. und Thakuriah, P. 
(2000). "Statistically-Based Validation of Computer Simulation Models in Traffic Operations and Management". Journal Transportation and Statistics, 5(1).

[Sanders, 2007] Sanders, L. (2007). Agent-based Modelling and Simulation in the Social and Human Sciences, Phan, Denis and Amblard, Frédéric (Herausgeber), Kapitel 7: Agent Models in Urban Geography, S. 147-168. The Bardwell Press. ISBN: 978-1-905622-01-6.

[Sanders und Schultes, 2005] Sanders, P. und Schultes, D. (2005). "Highway Hierarchies Hasten Exact Shortest Path Queries.". In Brodal, G. S. und Leonardi, S., Herausgeber, Algorithms - ESA 2005, 13th Annual European Symposium, Palma de Mallorca, Spain, October 3-6, 2005, Proceedings., 3669 Auflage von Lecture Notes in Computer Science, S. 568-579. Springer. ISBN 3-540-29118-0.

[Sanders und Schultes, 2006] Sanders, P. und Schultes, D. (2006). "Engineering highway hierarchies". In Proceedings of the 14th conference on Annual European Symposium - Volume 14, ESA'06, S. 804-816, London, UK, UK. Springer-Verlag.

[Satava, 2001] Satava, M.D., R. M. (2001). "Surgical Education and Surgical Simulation". World Journal of Surgery, 25, S. 1484-1489. 10.1007/s00268001-0134-0.

[Schmidt und Schäfer, 1998] Schmidt, M. und Schäfer, R.-P. (1998). "An integrated simulation system for traffic induced air pollution". Environmental Modelling \& Software, 13(3-4), S. 295 - 303.

[Schnabel und Lohse, 2011] Schnabel, W. und Lohse, D. (2011). Grundlagen der Straßenverkehrstechnik und der Verkehrsplanung - Band 2 Verkehrsplanung, dritte Auflage. Beutz Verlag Gmbh Berlin Wien Zürich. ISBN 978-3-410-17272-7.

[Schönauer und Schrom-Feiertag, 2010] Schönauer, R. und Schrom-Feiertag, H. (2010). "Mikrosimulation von Mischverkehr - Konzept MiMiSim und Ausblick auf MixME". In SCHRENK, M.; POPOVICH, V. V. und ZEILE, P., Herausgeber, REAL CORP 2010 Proceedings, S. 1157-1161.

[Schnieder und Becker, 2007] Schnieder, E. H. und Becker, U. (2007). Verkehrsleittechnik: Automatisierung des Straßen- und Schienenverkehrs. SpringerVerlag Berlin Heidelberg. ISBN 978-3-540-48541-4. 
[Schramm et al., 2010] Schramm, D.; Hiller, M. und Bardini, R. (2010). Modellbildung und Simulation der Dynamik von Kraftfahrzeugen. Springer-Verlag Berlin Heidelberg. ISBN 978-3-540-89315-8.

[Schüle et al., 2004] Schüle, M.; Herrler, R. und Klügl, F. (2004). "Coupling GIS and Multi-agent Simulation - Towards Infrastructure for Realistic Simulation". In Multiagent System Technologies, Second German Conference, MATES 2004, Erfurt, Germany, September 29-30, 2004, Proceedings, S. $228-242$.

[Seyfried et al., 2005] Seyfried, A.; Steffen, B.; Klingsch, W. und Boltes, M. (2005). "The fundamental diagram of pedestrian movement revisited". Journal of Statistical Mechanics: Theory and Experiment, 10.

[Sharkin, 2004] Sharkin, B. S. (2004). "Road Rage: Risk Factors, Assessment, and Intervention Strategies". Journal of Counseling and Development, 82, S. 191-199.

[Silverman et al., 2012] Silverman, D. T.; Samanic, C. M.; Lubin, J. H.; Blair, A. E.; Stewart, P. A.; Vermeulen, R.; Coble, J. B.; Rothman, N.; Schleiff, P. L.; Travis, W. D.; Ziegler, R. G.; Wacholder, S. und Attfield, M. D. (2012). "The Diesel Exhaust in Miners Study: A Nested Case-Control Study of Lung Cancer and Diesel Exhaust". Journal of the National Cancer Institute.

[Singh et al., 1990] Singh, M. P.; Goyal, P.; Basu, S.; Agarwal, P.; Nigam, S.; Kumari, M. und Panwar, T. S. (1990). "Predicted and Measured Concentrations of Traffic Carbon Monoxide over Delhi". Atmospheric Environment, 24A, 4, S. 801-810.

[Sjostedt, 1967] Sjostedt, L. (1967). "Behaviour of Pedestrians at Pedestrian Crossings". Stockholm: National Swedish Road Research Institute.

[Speirs und Braidwood, 2004] Speirs, E. und Braidwood, R. (2004). "Quadstone Paramics V5.0 Technical Notes". Technical report, Quadstone Paramics Ltd.

[Stallard und Owen, 1998] Stallard, C. und Owen, L. E. (1998). "Evaluating adaptive signal control using CORSIM". In Proceedings of the 30th conference on Winter simulation, WSC '98, S. 1147-1154, Los Alamitos, CA, USA. IEEE Computer Society Press. 
[Stanton und Wanless, 1995] Stanton, R. J. C. und Wanless, G. K. (1995). "Pedestrian movement". Safety Science, 18(4), S. 291 - 300. Engineering for Crowd Safety.

[Steierwald et al., 2005] Steierwald, G.; Kiinne, H. D. und Vogt, W. (2005). Stadtverkehrsplanung. Grundlagen, Methoden, Ziele. Springer-Verlag Berlin Heidelberg, 2., neu bearbeitete und erweiterte auflage Auflage. ISBN: 3-540-40588-7.

[Steland, 2010] Steland, A. (2010). Basiswissen Statistik. Springer-Verlag Berlin Heidelberg. ISBN: 978-3-642-02666-9.

[Sterling et al., 1995] Sterling, T.; Becker, D. J.; Savarese, D.; Dorband, J. E.; Ranawake, U. A. und Packer, C. V. (1995). "Beowulf: A Parallel Workstation For Scientific Computation". In In Proceedings of the 24th International Conference on Parallel Processing, S. 11-14. CRC Press.

[Stevanovic, 2010] Stevanovic, A. (2010). Adaptive Traffic Control Systems: Domestic and Foreign State of Practice (NCHRP Synthesis 403, Transportation Research Board). National Academy of Sciences, Washington D.C.

[Stevanovic et al., 2008] Stevanovic, J.; Stevanovic, A.; Martin, P. T. und Bauer, T. (2008). "Stochastic optimization of traffic control and transit priority settings in VISSIM". Transportation Research Part C: Emerging Technologies, 16(3), S. 332 - 349. Emerging Commercial Technologies.

[Sturges, 1926] Sturges, H. A. (1926). "The Choice of a Class Interval". Journal of the American Statistical Association, 21(153), S. 65-66.

[Sugiyama et al., 2008] Sugiyama, Y.; Fukui, M.; Kikuchi, M.; Hasebe, K.; Nakayama, A.; Nishinari, K.; Tadaki, S. und Yukawa, S. (2008). "Traffic jams without bottlenecks - experimental evidence for the physical mechanism of the formation of a jam". New Journal of Physics, 10(3).

[Suhl und Mellouli, 2007] Suhl, L. und Mellouli, T. (2007). Optimierungssysteme: Modelle, Verfahren, Software, Anwendungen (Springer-Lehrbuch). Springer-Verlag New York, Inc., Secaucus, NJ, USA. ISBN: 978-3-54026119-3.

[Sydow et al., 1997] Sydow, A.; Lux, T.; Mieth, P. und Schäfer, R.-P. (1997). "Simulation of traffic-induced air pollution for mesoscale applications". Math. Comput. Simul., 43(3-6), S. 285-290. 
[Sykes und Gabruk, 1997] Sykes, R. I. und Gabruk, R. S. (1997). "A secondorder closure model for the effect of averaging time on turbulent plume dispersion". Journal of Applied Meteorology, 36, S. 165-184.

[Sykes et al., 1984] Sykes, R. I.; Lewellen, W. S. und Parker, S. F. (1984). "A turbulent transport model for concentration fluctuation and fluxes". $J$. Fluid Mech., 139, S. 193-218.

[Takayasu und Takayasu, 1993] Takayasu, M. und Takayasu, H. (1993). "1/f noise in a traffic model". Fractals, 1(4), S. 860-866.

[Tanaboriboon und Guyano, 1991] Tanaboriboon, Y. und Guyano, J. A. (1991). "Analysis of pedestrian movements in Bangkok". Transportation Research Record: Journal of the Transportation Research Board, 1294, S. 52-56.

[Tanaboriboon et al., 1986] Tanaboriboon, Y.; Hwa, S. S. und Chor, C. H. (1986). "Pedestrian characteristics study in Singapore". Journal of Transportation Engineering, 112, S. 229-235.

[Tasca, 2000] Tasca, L. (2000). "A Review of the Literature on Aggressive Driving Research". Aggressive Driving Issues Conference.

[Taubert, 2012] Taubert, C. (2012). "Entwicklung eines Treibstoffverbrauchsmodells zur mikroskopischen Verkehrssimulation". Masterarbeit, Goethe Universität Frankfurt am Main.

[Tavares et al., 2009a] Tavares, L. D.; Vieira, D. A.; Saldanha, R. R. und Caminhas, W. M. (2009a). "Detecting Car Accidents based on Traffic Flow Measurements using Machine Learning Techniques". In Anais do IX Congresso Brasileiro de Redes Neurais / Inteligencia Computacional (IX CBRN).

[Tavares et al., 2009b] Tavares, L. D.; Vieira, D. A.; Saldanha, R. R. und Caminhas, W. M. (2009b). "Simulating car accidents with Cellular Automata traffic flow model". In de Oliveira, P. P. und Kari, J., Herausgeber, AUTOMATA 2009, 15th International Workshop on Cellular Automata and Discrete Complex Systems, S. 272-281. ISBN: 978-1-905986-21-7.

[Timm, 2004] Timm, I. J. (2004). Dynamisches Konfliktmanagement als Verhaltenssteuerung intelligenter Agenten. $\mathrm{PhD}$ thesis, University of Bremen. ISBN: 3-89838-283-4.

[Toledo et al., 2003] Toledo, T.; Koutsopoulos, H. N.; Davol, A.; Ben-Akiva, 
M. E.; Burghout, W.; Andréasson, I.; Johansson, T. und Lundin, C. (2003). "Calibration and Validation of Microscopic Traffic Simulation Tools: Stockholm Case Study". Journal of the Transportation Research Board, 1831, S. 65-75. ISSN 0361-1981.

[Treiber, 2011] Treiber, M. (2011). "Der simulierte Stau". Spektrum der Wissenschaft, Verlagsgruppe Georg von Holtzbrinck, 9, S. 82 - 85. ISSN 01702971.

[Treiber und Helbing, 1999] Treiber, M. und Helbing, D. (1999). "Explanation of Observed Features of Self-Organization in Traffic Flow". preprint condmat/9901239.

[Treiber und Helbing, 2002] Treiber, M. und Helbing, D. (2002). "Realistische Mikrosimulation von Straßenverkehr mit einem einfachen Modell". 16. Symposium Simulationstechnik (ASIM 2002), Rostock, Germany.

[Treiber et al., 1999] Treiber, M.; Hennecke, A. und Helbing, D. (1999). "Derivation, properties, and simulation of a gas-kinetic-based, nonlocal traffic model". Phys. Rev. E, 59(1), S. 239-253.

[Treiber et al., 2000] Treiber, M.; Hennecke, A. und Helbing, D. (2000). "Congested Traffic States in Empirical Observations and Microscopic Simulations". PHYSICAL REVIEW E, 62, S. 1805.

[Treiber und Kesting, 2010] Treiber, M. und Kesting, A. (2010). Verkehrsdynamik und -simulation. Springer-Verlag Berlin Heidelberg. ISBN 978-3-64205228-6.

[Treiber et al., 2005] Treiber, M.; Kesting, A. und Helbing, D. (2005). Für eine neue deutsche Verkehrspolitik: Mobilität braucht Kommunikation, Stopka, Ulrike and Pällmann, Wilhelm (Herausgeber), Kapitel : Verkehr verstehen und beherrschen. Deutscher Verkehrs-Verlag, Internationales Verkehrswesen Auflage. ISBN 978-3-87154-335-7.

[Treiber et al., 2006] Treiber, M.; Kesting, A. und Helbing, D. (2006). "Delays, inaccuracies and anticipation in microscopic traffic models". Physica A: Statistical Mechanics and its Applications, 360(1), S. $71-88$.

[Treiterer, 1975] Treiterer, J. (1975). "Investigation of Traffic Dynamics by Aerial Photogrammetry Techniques". Technical Report Ohio-Dot-09-75, Ohio State University, Engineering Experiment Station 2070 Neil Avenue Co- 
lumbus, Ohio 43210. Final Report.

[Tupper et al., 2010] Tupper, S.; Knodler, M. A. und Hurwitz, D. S. (2010). "Revisiting Gap Acceptance". ITE 2010 Annual Meeting and Exhibit Presentations.

[Usher et al., 2010] Usher, J. M.; Liu, X. und Kolstad, E. (2010). "Simulation of Pedestrian Behavior in Intermodal Facilities". Proceedings of SpringSim2010, 2, S. 127-134. ISBN: 1-56555-342-X.

[Van Aerde und Associates, 2010a] Van Aerde, M. und Associates (2010a). INTEGRATIONC RELEASE 2.30 FOR WINDOWS: User's Guide - Volume I: Fundamental Model Features. M. Van Aerde \& Assoc., Ltd.

[Van Aerde und Associates, 2010b] Van Aerde, M. und Associates (2010b). INTEGRATIONC RELEASE 2.30 FOR WINDOWS: User's GuideVolume II: Advanced Model Features. M. Van Aerde \& Assoc., Ltd.

[Van Aerde et al., 1996] Van Aerde, M.; Hellinga, B.; Baker, M. und Rakha, H. (1996). "INTEGRATION: An Overview of Simulation Features". Transportation Research Board Annual Meeting.

[Van Aerde und Rakha, 1995] Van Aerde, M. und Rakha, H. (1995). "Multivariate calibration of single regime speed-flow-density relationships". Proceedings of 6th International Vehicle Navigation Information Systems Conference, IEEE, 6 .

[Varschen und Wagner, 2006] Varschen, C. und Wagner, P. (2006). "Mikroskopische Modellierung der Personenverkehrsnachfrage auf Basis von Zeitverwendungstagebüchern". Tagungsband AMUS, 81, S. 63-69.

[Vasić und Ruskin, 2011a] Vasić, J. und Ruskin, H. (2011a). "A Discrete Flow Simulation Model for Urban Road Networks, with Application to Combined Car and Single-File Bicycle Traffic". In Murgante, B.; Gervasi, O.; Iglesias, A.; Taniar, D. und Apduhan, B., Herausgeber, Computational Science and Its Applications - ICCSA 2011, 6782 Auflage von Lecture Notes in Computer Science, S. 602-614. Springer Berlin / Heidelberg. ISBN: 978-3-642-21928-3.

[Vasić und Ruskin, 2011b] Vasić, J. und Ruskin, H. (2011b). "Throughput and Delay in a Discrete Simulation Model for Traffic Including Bicycles on Urban Networks". Proceedings of the ITRN2011. 
[Vasić und Ruskin, 2012] Vasić, J. und Ruskin, H. (2012). Urban Development, Kapitel 5: A CA-Based Model for City Traffic Including Bicycles, S. 7992. Polyzos, Serafeim (Herausgeber) InTech. ISBN: 978-953-51-0442-1.

[Venables und Ripley, 2002] Venables, W. N. und Ripley, B. D. (2002). Modern Applied Statistics with S. Springer, New York, 4. Auflage.

[Virkler, 1998] Virkler, M. R. (1998). "Prediction and Measurement of Travel Time along Pedestrian Routes". Transportation Research Record No. 1636, Bicycle and Pedestrian Research, 1636, S. 37-42. ISSN: 0361-1981.

[Voellmy et al., 2001] Voellmy, A.; Vrtic, M.; Raney, B.; Axhausen, K. und Nagel, K. (2001). "Status of a TRANSIMS implementation for Switzerland".

[Wagner et al., 2011] Wagner, M.; Zöbel, D. und Meroth, A. (2011). "An adaptive Software and Systems Architecture for Driver Assistance Systems based on service orientation". International Journal of Machine Learning and Computing, 1(4), S. 359-366.

[Wagner, 1995] Wagner, P. (1995). "Traffic Simulations Using Cellular Automata: Comparison With Reality". In Traffic and Granular Flow. World Scientific.

[Wagner et al., 1996] Wagner, P.; Nagel, K. und Wolf, D. E. (1996). "Realistic Multi-Lane Traffic Rules for Cellular Automata". PHYSICA A, 234, S. 96-2586.

[Wahle et al., 2002] Wahle, J.; Chrobok, R.; Pottmeier, A. und Schreckenberg, M. (2002). "A Microscopic Simulator for Freeway Traffic". Networks and Spatial Economics, 2, S. 371-386. 10.1023/A:1020899528337.

[Walker, 2007] Walker, I. (2007). "Drivers overtaking bicyclists: Objective data on the effects of riding position, helmet use, vehicle type and apparent gender". Accident Analysis 83 Prevention, 39(2), S. 417 - 425.

[Wallentowitz, 2003] Wallentowitz, H. (2003). Längsdynamik von Kraftfahrzeugen: Verkehrssystem Kraftfahrzeug, Leistungs- und Energiebedarf, Antriebsstrang, Fahrzeugdynamik. Vorlesungsumdruck Fahrzeugtechnik, zweite Auflage von IKA-Schriftenreihe Automobiltechnik. Forschungsgesellschaft Kraftfahrwesen. (Zitiert nach Heißing et al., 2011, S. 44]).

[Wang et al., 1998] Wang, B.-H.; Kwong, Y.-R. und Hui, P.-M. (1998). "Statistical mechanical approach to Fukui-Ishibashi traffic flow models". Phys. Rev. 
E, 57(3), S. 2568-2573.

[Wang und Liu, 2005] Wang, R. und Liu, M. (2005). "A Realistic Cellular Automata Model to Simulate Traffic Flow at Urban Roundabouts". In Sunderam, V. S.; Albada, G. D. v.; Sloot, P. M. A. und Dongarra, J. J., Herausgeber, Computational Science - ICCS 2005, 3515 Auflage von Lecture Notes in Computer Science, S. 420-427. Springer Berlin / Heidelberg. 10.1007/11428848_56.

[Wang und Ruskin, 2006] Wang, R. und Ruskin, H. J. (2006). "Modelling Traffic Flow at Multi-Lane Urban Roundabouts". International Journal of Modern Physics C, 17(5), S. 693-710.

[Wang et al., 1997] Wang, Y.; Prevedouros, P. D. und D, P. (1997). "Comparison of INTEGRATION, TSIS/CORSIM and WATSim in Replicating Volumes and Speeds on Three Small Networks". 1998 Annual Meeting of the Transportation Research Board.

[Wardrop, 1952] Wardrop, J. (1952). "Some theoretical aspects of road traffic research". Proceedings of the Institution of Civil Engineers, Part II, 1(36), S. $325-362$.

[Watt, 2000] Watt, A. (2000). 3D Computer Graphics, 3 Auflage. Addison-Wesley. ISBN 0-201-39855-9.

[WBGU, 2003] WBGU (2003). Welt im Wandel - Energiewende zur Nachhaltigkeit, Hauptgutachten 2003. Springer-Verlag, Berlin-Heidelberg. Wissenschaftlicher Beirat der Bundesregierung Globale Umweltveränderungen, ISBN 3-540-40160-1.

[Wegener et al., 2008] Wegener, A.; Piórkowski, M.; Raya, M.; Hellbrück, H.; Fischer, S. und Hubaux, J.-P. (2008). "TraCI: an interface for coupling road traffic and network simulators". In Proceedings of the 11th communications and networking simulation symposium, CNS '08, S. 155-163, New York, NY, USA. ACM.

[Weifeng et al., 2003] Weifeng, F.; Lizhong, Y. und Weicheng, F. (2003). "Simulation of bi-direction pedestrian movement using a cellular automata model". Physica A: Statistical Mechanics and its Applications, 321(3-4), S. $633-640$.

[Westerdijk, 1990] Westerdijk, P. K. (1990). "Pedestrian and Pedal Cyclist Rou- 
te Choice Criteria". Technical Report Working Paper 302, Institute of Transport Studies, University of Leeds.

[Wiedemann, 1974] Wiedemann, R. (1974). Simulation des Straßenverkehrsflusses. Institut für Verkehrswesen. Schriftenreihe. Heft 8. Institut für Verkehrswesen der Universität Karlsruhe. ISSN 0341-5503.

[Wiesenthal et al., 2000] Wiesenthal, D. L.; Hennessy, D. und Gibson, P. M. (2000). "The Driving Vengeance Questionnaire (DVQ): The Development of a Scale to Measure Deviant Drivers' Attitudes". Violence and Victims, $15(2)$, S. $115-136$.

[Wilbur, 2004] Wilbur, T. (2004). "Identifying and Assessing Key WeatherRelated Parameters and Their Impacts on Traffic Operations Using Simulation". Publication number: Fhwa-hrt-04-131, U.S. Department of Transportation.

[Williams et al., 1998] Williams, M. D.; Williams, M. D.; Thayer, G.; Thayer, G.; Smith, L. und Smith, L. (1998). "A Comparison of Emissions Estimated in the Transims Approach with those Estimated from Continuous Speeds and Accelerations".

[Willis et al., 2004] Willis, A.; Gjersoe, N.; Havard, C.; Kerridge, J. und Kukla, R. (2004). "Human movement behaviour in urban spaces: Implications for the design and modelling of effective pedestrian environments.". Environment and Planning B Planning and Design, 31(6), S. 805-828.

[Wilson und Grayson, 1980] Wilson, D. G. und Grayson, G. B. (1980). "AgeRelated Differences in the Road Crossing Behaviour of Adult Pedestrians". Technical report, Laboratory Report 933, Transport and Road Research Laboratory, Crowthorne.

[Wooldridge und Jennings, 1995] Wooldridge, M. und Jennings, N. R. (1995). "Intelligent Agents: Theory and Practice". Knowledge Engineering Review, 10, S. $115-152$.

[Wooldridge, 2009] Wooldridge, M. J. (2009). An Introduction to MultiAgent Systems (second edition). Wiley \& Sons. ISBN: 978-0-47051-946-2.

[Wu, 1997] Wu, J. (1997). "A real-time origin-destination matrix updating algorithm for on-line applications". Transportation Research Part B: Methodological, 31(5), S. 381-396. 
[Yang et al., 2009] Yang, M.; Liu, Y. und You, Z. (2009). "Investigation of Fuel Consumption and Pollution Emissions in Cellular Automata". In Chinese Journal of Physics, 47 (5) Auflage, S. 589-597.

[Yang und Ma, 2002] Yang, X. und Ma, Y. (2002). "Car accidents determined by stopped cars and traffic flow". Journal of Physics A: Mathematical and General, 35(49), S. 10539-10547.

[Yilmaz und Ören, 2009] Yilmaz, L. und Ören, T. (2009). Agent-Directed Simulation and Systems Engineering. Wiley, Weinheim. ISBN: 978-3-527-407811 .

[Yuhara und Tajima, 2006] Yuhara, N. und Tajima, J. (2006). "Multi-driver agent-based traffic simulation systems for evaluating the effects of advanced driver assistance systems on road traffic accidents". Cognition, Technology \& Work, 8, S. 283-300. 10.1007/s10111-006-0045-9.

[Zhao und Gao, 2005] Zhao, X. und Gao, Z. (2005). "A new car-following model: full velocity and acceleration difference model". Eur. Phys. J. B, 47(1), S. $145-150$.

[Zhou et al., 2006] Zhou, M.; Korhonen, A.; Malmi, L.; Kosonen, I. und Luttinen, T. (2006). "Integration of Geographic Information System for Transportation with Real-Time Traffic Simulation System: Application Framework". In Transportation Research Record: Journal of the Transportation Research Board, 1972 (1) Auflage, S. 78-84.

[Zhu et al., 2009] Zhu, H.; Lei, L. und Dai, S. (2009). "Two-lane traffic simulations with a blockage induced by an accident car". Physica A: Statistical Mechanics and its Applications, 388(14), S. 2903 - 2910.

[Zielstra und Zipf, 2010] Zielstra, D. und Zipf, A. (2010). "A Comparative Study of Proprietary Geodata and Volunteered Geographic Information for Germany". In 13th AGILE International Conference on Geographic Information Science 2010 Guimarães, Portugal.

[Zilske et al., 2011] Zilske, M.; Neumann, A. und Nagel, K. (2011). "OpenStreetMap for Traffic Simulation". State of the map Europe (SOTM-EU). 


\title{
Lebenslauf
}

\author{
Jörg Dallmeyer \\ geboren am 3. Februar 1986 in Hanau \\ 2009 - 2012 Promotionsstudium im Fachgebiet Informatik an der Goethe- \\ Universität Frankfurt am Main. \\ 2008 - 2009 Studium der Informatik mit Anwendungsfach Medizin an der \\ Goethe-Universität Frankfurt am Main. Abschluss Master of \\ Science im November 2009. Masterarbeit an der Professur für \\ Wirtschaftsinformatik und Simulation: „Potentialfeldabhängige \\ Simulation von Menschenwanderungen auf Basis von Partikel \\ Schwarm Optimierung“ (Betreuer: Prof. Dr.-Ing. Ingo J. Timm). \\ 2005 - 2008 Studium der Informatik mit Anwendungsfach Medizin an der \\ Goethe-Universität Frankfurt am Main. Abschluss Bachelor of \\ Science im März 2008. Bachelorarbeit an der Professur für Ent- \\ wurfsmethodik: „Heuristiken für das Optimierungsproblem einer \\ vollständigen graphbasierten Traversierung des Zustandsraums in \\ der formalen Verifikation analoger Schaltungen“ (Betreuer: Prof. \\ Dr.-Ing. Lars Hedrich).
}

1996 - 2005 Besuch der Hohen Landesschule Hanau, Abschluss Abitur (Allgemeine Hochschulreife) im Juni 2005. 